\title{
NIST Technical Note 1863-3
}
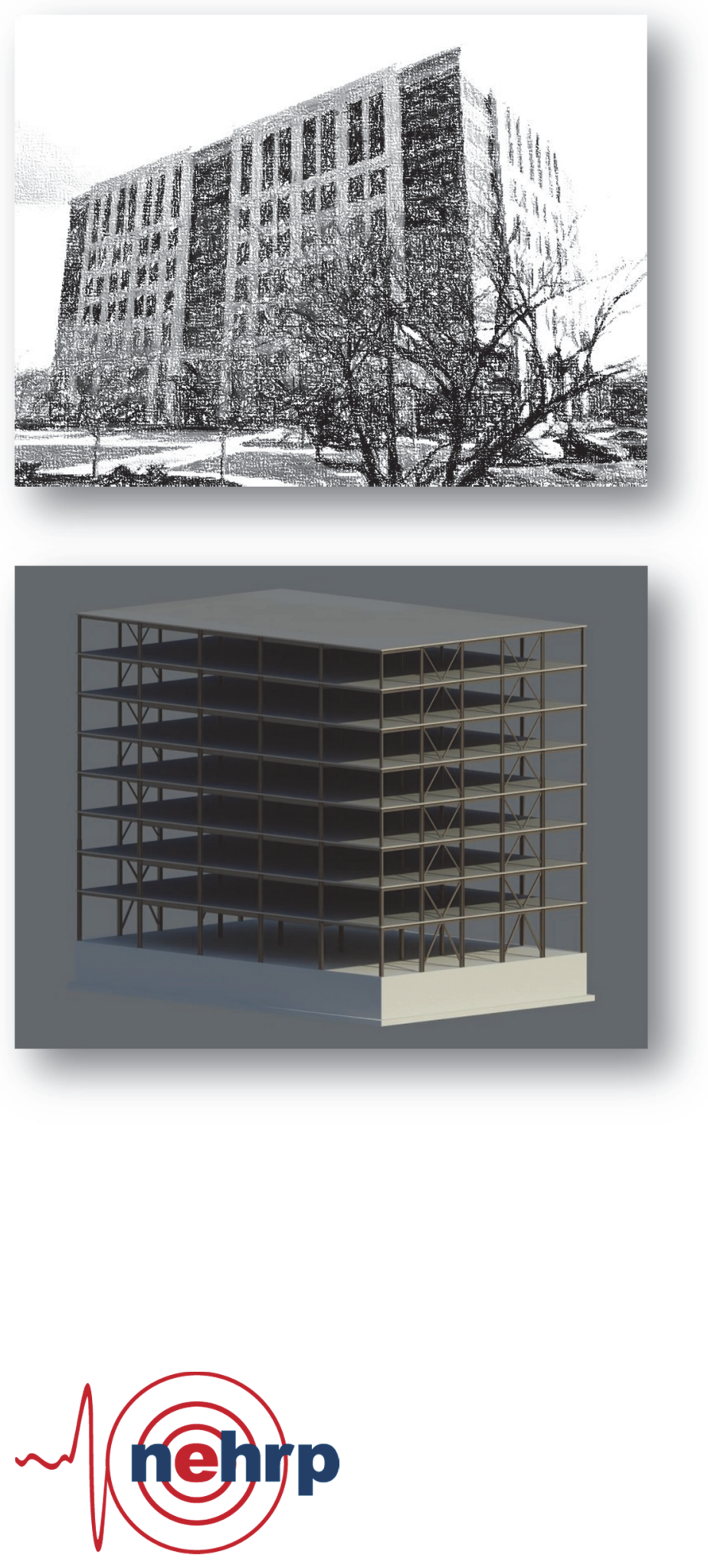

\author{
Assessment of \\ First Generation \\ Performance-Based \\ Seismic Design \\ Methods for \\ New Steel Buildings
}

\section{Volume 3: Eccentrically Braced Frames}

\author{
John L. Harris III \\ Matthew S. Speicher
}

This publication is available free of charge from: http://dx.doi.org/10.6028/NIST.TN.1863-3

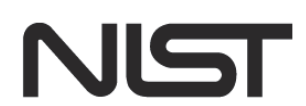

National Institute of Standards and Technology U.S. Department of Commerce 


\title{
NIST Technical Note 1863-3
}

\section{Assessment of First Generation Performance- Based Seismic Design Methods for New Steel Buildings}

\section{Volume 3: Eccentrically Braced Frames}

\author{
John L. Harris III \\ Matthew S. Speicher \\ Materials and Structural Systems Division \\ Engineering Laboratory
}

This publication is available free of charge from: http://dx.doi.org/10.6028/NIST.TN.1863-3

February 2015

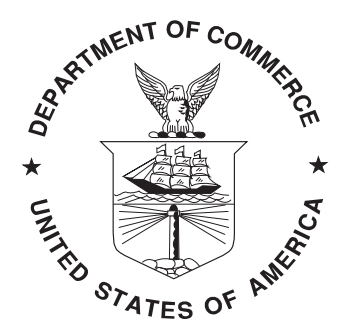

U.S. Department of Commerce Penny Pritzker, Secretary

National Institute of Standards and Technology Willie May, Acting Under Secretary of Commerce for Standards and Technology and Acting Director 


\section{Disclaimers}

Certain commercial software, equipment, instruments, or materials may have been used in the preparation of information contributing to this report. Identification in this report is not intended to imply recommendation or endorsement by NIST, nor is it intended to imply that such software, equipment, instruments, or materials are necessarily the best available for the purpose.

NIST policy is to use the International System of Units (metric units) in all its publications. In this report, however, information is presented in U.S. Customary Units (inch-pound), as this is the preferred system of units in the U.S. earthquake engineering industry.

National Institute of Standards and Technology Technical Note 1863-3 Natl. Inst. Stand. Technol. Tech Note 1863-3, 279 pages (February 2015) CODEN: NTNOEF

This publication is available free for charge from: http://dx.doi.org/10.6028/NIST.TN.1863-3 



\section{Preface}

In June 2008, the National Earthquake Hazards Reduction Program (NEHRP) sponsored a PerformanceBased Seismic Design (PBSD) workshop for leading practitioners and researchers from around the United States to develop a comprehensive list of research needs to foster full development and implementation of PBSD. From this workshop, the Building Seismic Safety Council (BSSC) reported a prioritized list of key PBSD research and implementation needs in NIST GCR 09-917-2: Research Required to Support Full Implementation of Performance-Based Seismic Design (NIST 2009a). The highest priority need identified in this report was to "benchmark" current PBSD methodologies (e.g., ASCE/SEI 41-06: Seismic Rehabilitation of Existing Buildings (ASCE 2006) - hereafter referred to as ASCE 41) with code procedures for design of new buildings. Two observations from the report were that among workshop participants (1) ASCE 41 procedures are perceived to be overly conservative and (2) existing PBSD methods are not accepted by practitioners as providing a uniform level of confidence. A supporting reason for these two observations was that no systematic effort had been undertaken to benchmark structural performance as determined using ASCE 41 procedures, together with widely accepted procedures for designing new buildings using ASCE/SEI 7-10: Minimum Design Loads for Buildings and Other Structures (ASCE 2010)-hereafter referred to as ASCE 7.

Work was initiated at the National Institute of Standards and Technology (NIST) to support this priority study under the Assessment of Design Methods in Existing PBSD Standards Project. This task was conducted by the Earthquake Risk Reduction in Buildings and Infrastructure group, the research division of the NEHRP Office at NIST. This research involves problem-focused studies in PBSD assessing the applicability and accuracy of implementing first-generation PBSD analysis methods now used for evaluating existing buildings in the performance-based design of new buildings. The current study focuses on buildings with lateral systems utilizing structural steel frames. This project evaluates the results of the studies and identifies changes to current model building codes and standards provisions that will encourage more universal use of PBSD. The volume of data required to illustrate the results and conclusions necessitated three separate reports, as follows:

- Volume 1: Special Moment Frames

- Volume 2: Special Concentrically Braced Frames

- Volume 3: Eccentrically Braced Frames (this report)

A fourth report, Volume 4: Buckling Restrained Braced Frames, is under development.

A peer review panel (PRP) was engaged under a contract with the NEHRP Consultants Joint Venture (NCJV) - www.nehrp-consultants.org. The PRP was tasked to complement the NIST project by providing, where needed, individual subject matter expert guidance to improve the technical detail and outcome of the project; provide technical support on specific topics on seismic hazard analysis, ground motion scaling for 
varying return periods, nonlinear dynamic analysis, structural behavior and design of building structures; and provide peer review services. The review panel for this project was structured as follows:

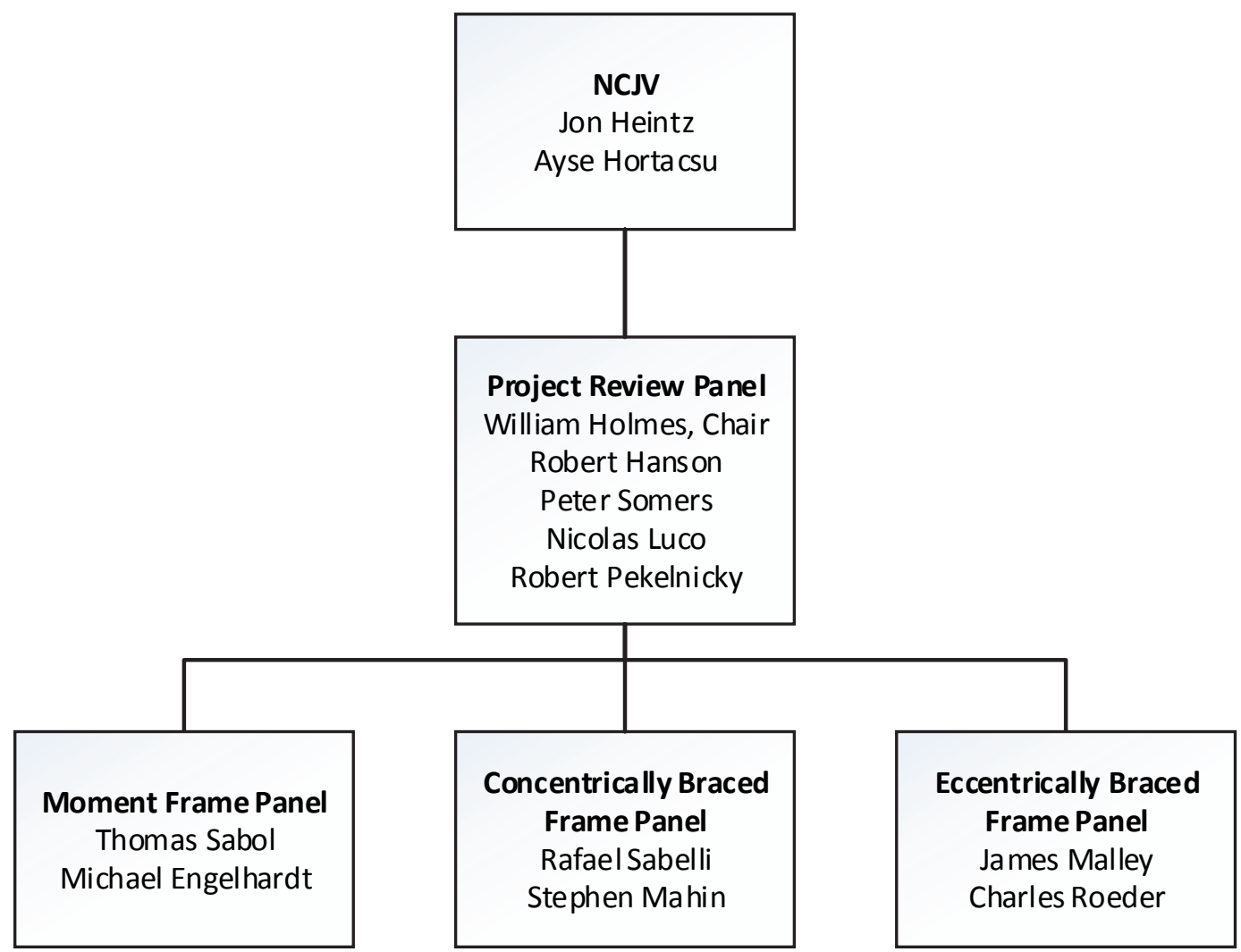

Jon Heintz (Program Manager)

Applied Technology Council

201 Redwood Shores Parkway, Suite 240

Redwood City, California 94065

Michael Engelhardt

The University of Texas at Austin Dept. of Civil, Arch. and Environ. Eng.

301 East Dean Keeton St., Stop C1747

Austin, TX 78712

William Holmes (PRP Chair)

Rutherford + Chekene

55 Second Street, Suite 600

San Francisco, California 94105

Stephen Mahin

University of California, Berkeley

Dept. of Civil and Environ. Eng.

777 Davis Hall

Berkeley, California 94720
Ayse Hortacsu (Project Manager)

Applied Technology Council

201 Redwood Shores Parkway, Suite 240

Redwood City, California 94065

Robert Hanson

University of Michigan, Emeritus

5885 Dunabbey Loop

Dublin, Ohio 43017

Nicolas Luco

U.S. Geological Survey

Box 25046 - DFC - MS 966

Denver, Colorado 80225

James Malley

Degenkolb Engineers

235 Montgomery St., Suite 500

San Francisco, California 94104 
Robert Pekelnicky

Degenkolb Engineers

235 Montgomery St., Suite 500

San Francisco, California 94104

Rafael Sabelli

Walter P. Moore

595 Market St., Suite 2130

San Francisco, California 94105
Charles Roeder

University of Washington

Dept. of Civil and Environ. Eng.

Box 352700

Seattle, WA 98195

Thomas Sabol

Englekirk Institutional

2116 Arlington Ave.

Los Angeles, California 90018

Peter Somers

Magnusson Klemencic Associates

1301 Fifth Ave., Suite 3200

Seattle, WA 98101

The authors gratefully acknowledge the members of the PRP for their input and guidance during this project and in the preparation of report volumes 1-3. Further, the authors wish to thank Jon Heintz and Ayse Hortacsu at Applied Technology Council for management of Task Order 12. Special thanks are also extended to Jack Hayes (NEHRP Director), Steve McCabe (NEHRP Deputy Director), and Bob Pekelnicky for reviewing the final NIST reports.

John L. Harris III

Research Structural Engineer

jay.harris@nist.gov

Matthew S. Speicher

Research Structural Engineer

matthew.speicher@nist.gov

National Institute of Standards and Technology

Engineering Laboratory

National Earthquake Hazards Reduction Program Office

100 Bureau Dr., MS 8604

Gaithersburg, MD 20899 


\section{Table of Contents}

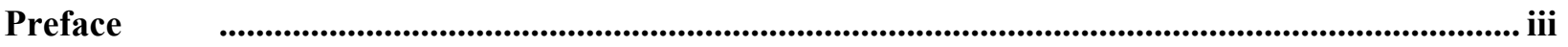

Table of Contents............................................................................................................................................... vii

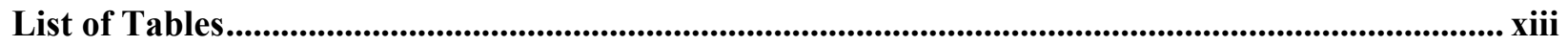

List of Figures ................................................................................................................................................................. xvii

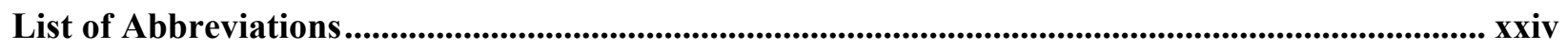

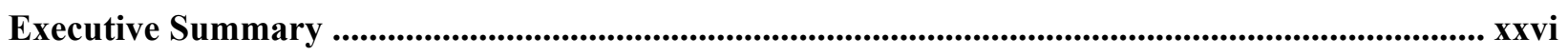

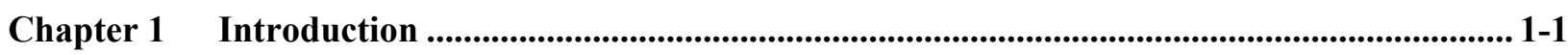

$1.1 \quad$ Project Motivation and Background........................................................................ 1-3

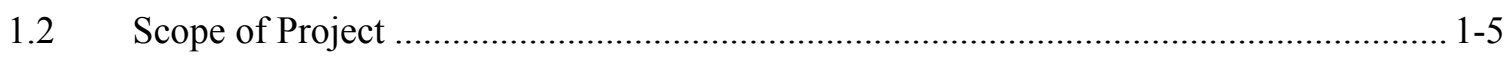

Chapter 2 Overview and Design of Archetype Buildings............................................................. 2-1

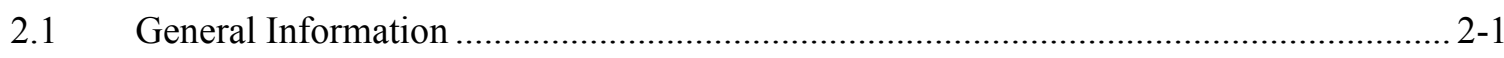

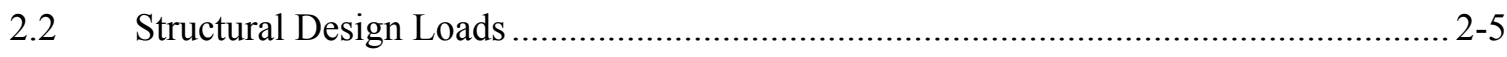

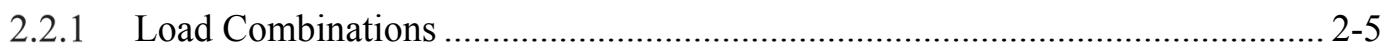

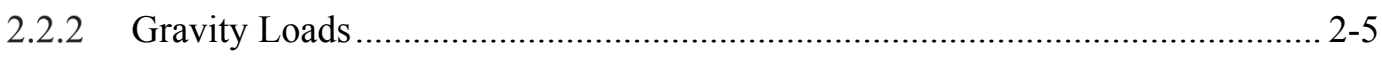

2.2.3 Environmental Loads .......................................................................... 2-6

2.2.3.1 Earthquake Forces ........................................................................... 2-6

2.2.3.2 Wind Forces ............................................................................. 2-8

2.3 Structural Analysis and Mathematical Model .................................................................... 2-9

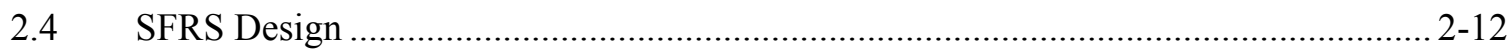

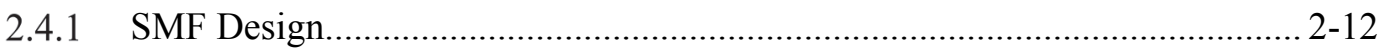

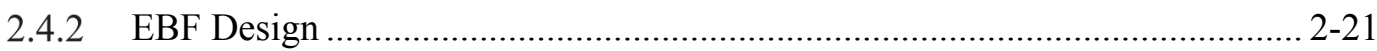

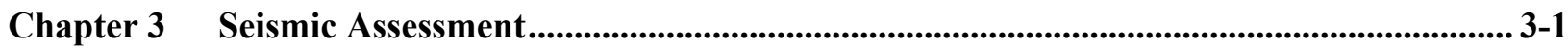

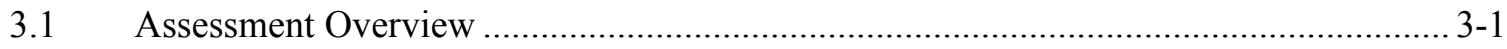

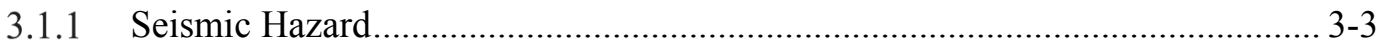

3.1.2 General Analysis Requirements .......................................................... 3-3

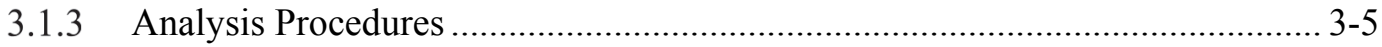

3.1.3.1 Linear Analysis Procedures............................................................. 3-5

3.1.3.1.1 Linear Static Procedure ............................................ 3-7 
3.1.3.1.2 Linear Dynamic Procedure......................................... 3-8

3.1.3.2 Nonlinear Analysis Procedures .......................................................... 3-8

3.1.3.2.1 Nonlinear Static Procedure...................................... 3-10

3.1.3.2.2 Nonlinear Dynamic Procedure ................................. 3-13

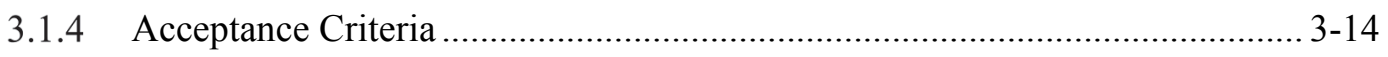

3.1.4.1 Linear Procedures................................................................................ 3-15

3.1.4.1.1 Calculating Component Assessment Results ............ 3-15

3.1.4.2 Nonlinear Procedures ..................................................................... 3-16

3.1.4.2.1 Calculating Component Assessment Results ............ 3-17

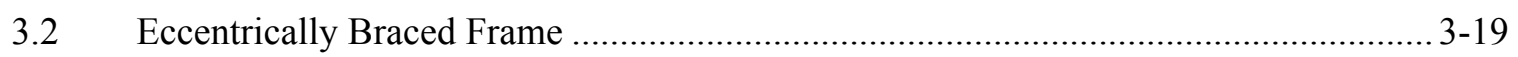

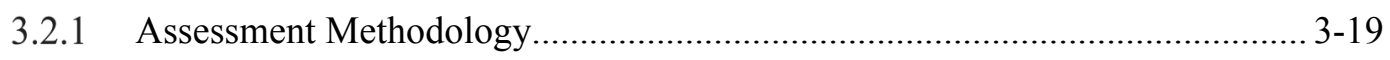

3.2.1.1 Linear Procedures ..................................................................... 3-19

3.2.1.2 Nonlinear Procedures ................................................................ 3-31

3.2.2 Seismic Assessment Results.................................................................... 3-45

3.2.2.1 Four-Story Eccentrically Braced Frame............................................ 3-46

3.2.2.1.1 Linear Static Procedure ............................................ 3-46

3.2.2.1.1.1 ..... BSE-1 Earthquake Hazard Level (LS BPL).............. 3-46

3.2.2.1.1.2 ..... BSE-2 Earthquake Hazard Level (CP BPL).............. 3-47

3.2.2.1.2 Linear Dynamic Procedure........................................ 3-49

3.2.2.1.2.1 ..... BSE-1 Earthquake Hazard Level (LS BPL).............. 3-49

3.2.2.1.2.2 ..... BSE-2 Earthquake Hazard Level (CP BPL)............. 3-50

3.2.2.1.3 Nonlinear Static Procedure...................................... 3-52

3.2.2.1.4 Nonlinear Dynamic Procedure ................................. 3-56

3.2.2.2 Eight-Story Eccentric Braced Frame............................................. 3-62

3.2.2.2.1 Linear Static Procedure ........................................... 3-62

3.2.2.2.1.1 ..... BSE-1 Earthquake Hazard Level (LS BPL)............. 3-62

3.2.2.2.1.2 ..... BSE-2 Earthquake Hazard Level (CP BPL)............. 3-65

3.2.2.2.2 Linear Dynamic Procedure...................................... 3-67

3.2.2.2.2.1 ..... BSE-1 Earthquake Hazard Level (LS BPL)............. 3-67

3.2.2.2.2.2 ..... BSE-2 Earthquake Hazard Level (CP BPL).............. 3-69

3.2.2.2.3 Nonlinear Static Procedure........................................ 3-71

3.2.2.2.4 Nonlinear Dynamic Procedure ................................. 3-76

3.2.2.3 Sixteen-Story Eccentrically Braced Frame ..................................... 3-82

3.2.2.3.1 Linear Static Procedure ........................................... 3-82

3.2.2.3.1.1 ..... BSE-1 Earthquake Hazard Level (LS BPL).............. 3-82 viii 
3.2.2.3.1.2 ..... BSE-2 Earthquake Hazard Level (CP BPL) ............. 3-86

3.2.2.3.2 Linear Dynamic Procedure ...................................... 3-89

3.2.2.3.2.1 ..... BSE-1 Earthquake Hazard Level (LS BPL)............. 3-89

3.2.2.3.2.2 .... BSE-2 Earthquake Hazard Level (CP BPL) ............ 3-92

3.2.2.3.3 Nonlinear Static Procedure ..................................... 3-95

3.2.2.3.4 Nonlinear Dynamic Procedure ................................ 3-101

3.2.3 Seismic Assessment Discussion ........................................................... 3-108

3.2.3.1 Linear Procedures.......................................................................... 3-108

3.2.3.1.1 Link Beams ....................................................... 3-108

3.2.3.1.2 Member Cross-section Strength (flexural hinge) and Global Strength (member stability)........................ 3-110

3.2.3.1.3 Summary ........................................................ 3-113

3.2.3.2 Nonlinear Procedures ....................................................................... 3-115

3.2.3.2.1 Link Beams .......................................................... 3-115

3.2.3.2.2 Member Cross-section Strength (flexural hinge) and Global Strength (member stability)....................... 3-116

3.2.3.2.3 Summary .................................................... 3-118

3.2.3.3 Comparison between Linear and Nonlinear Assessment Results ..... 3-119

Chapter 4 Summary, Conclusions, and Recommendations............................................................... 4-1

4.1 Summary of Project Work ........................................................................................ 4

4.2 Assumptions and Limitations of this Study............................................................. 4-3

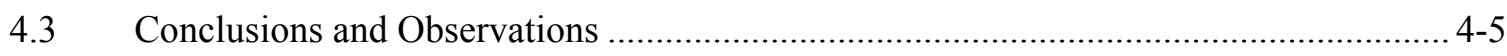

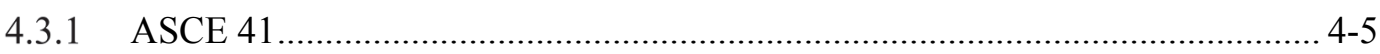

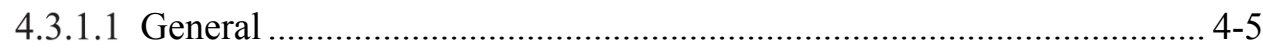

4.3.1.2 Eccentrically Braced Frame ......................................................... 4-6

4.4 Recommendations for Future Research................................................................... 4-7

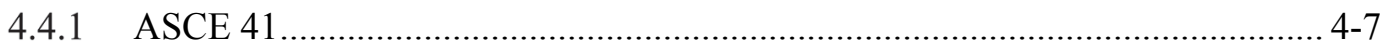

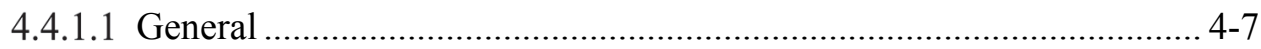

4.4.1.2 Eccentrically Braced Frames........................................................... 4-8

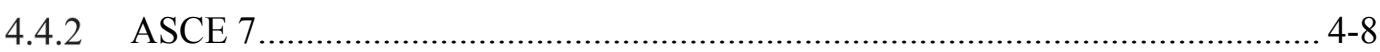

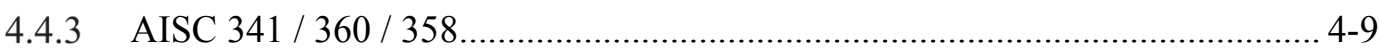

4.4.3.1 Eccentrically Braced Frames............................................................ 4-9

Appendix A Ground Motions for Response History Analysis .................................................... A-1

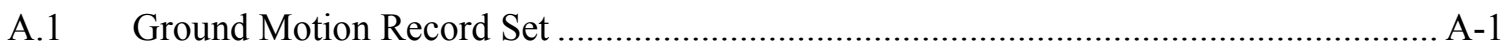

A.2 Ground Motion Selection and Scaling …................................................................ A-2 


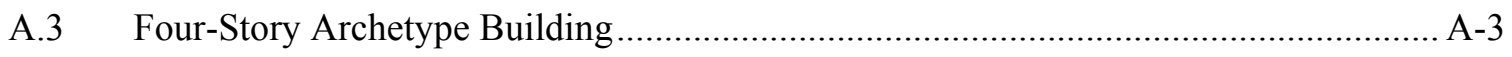

A.3.1 4-Story Eccentrically Braced Frame …........................................................ A-3

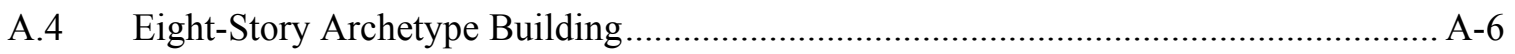

A.4.1 8-Story Eccentrically Braced Frame ……................................................... A-6

A.5 Sixteen-Story Archetype Building ……................................................................ A-9

A.5.1 16-Story Eccentrically Braced Frame ………............................................. A-9

A.6 FEMA P-695 Far-Field Record Set.................................................................. A-12

Appendix B Supplemental Design Information and Design Examples............................................ B-1

B.1 Horizontal Design Forces ................................................................................. B-1

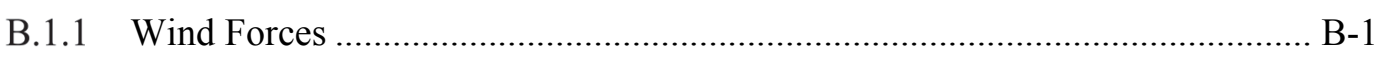

B.1.2 Seismic Forces.............................................................................. B-3

B.1.2.1 Effective Seismic Weights and Story Gravity Forces ........................ B-3

B.1.2.2 Horizontal Seismic Forces, E-W Direction ....................................... B-4

B.1.2.2.1 ME4 (Special Moment Frame)................................. B-4

B.1.2.2.2 ME8 (Special Moment Frame)................................ B-7

B.1.2.2.3 ME16 (Special Moment Frame)............................. B-10

B.1.2.3 Horizontal Seismic Forces, N-S Direction ...................................... B-13

B.1.2.3.1 ME4 (Eccentrically Braced Frame)......................... B-13

B.1.2.3.2 ME8 (Eccentrically Braced Frame)........................ B-15

B.1.2.3.3 ME16 (Eccentrically Braced Frame)....................... B-18

B.2 Horizontal and Vertical Irregularities................................................................ B-21

B.2.1 Special Moment Frame ............................................................................... B-21

B.2.2 Eccentrically Braced Frame …................................................................. B-23

B.3 SMF AISC Frame Stability (Effective Length Method) …....................................... B-25

B.4 Eccentrically Braced Frame Plastic Link Rotation................................................... B-28

B.5 Example Design Calculations ........................................................................... B-33

B.5.1 Eccentrically Braced Frame Example …….............................................. B-33

B.5.1.1 Member Selection ...................................................................... B-33

B.5.1.2 EBF Link Beam..................................................................... B-34

B.5.1.2.1 Shear Demand ........................................................ B-34

B.5.1.2.2 Shear Strength ......................................................... B-34

B.5.1.2.3 Strength Check ......................................................... B-34

B.5.1.2.4 Link Rotation Angle Verification ........................... B-34

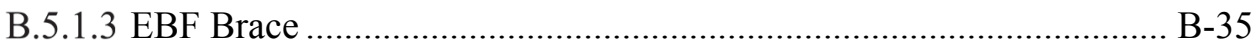




\begin{tabular}{|c|c|}
\hline B.5.1.3.1 & Axial and Flexure Demand .......................................... 35 \\
\hline B.5.1.3.2 & Axial Strength. \\
\hline B.5.1.3.3 & Strength Check. \\
\hline 5.1.4 EBF Colu & 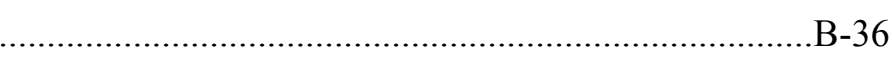 \\
\hline B.5.1.4.1 & Axial and Flexural Demand .......... \\
\hline B.5.1.4.2 & Axial Strength ......... \\
\hline B.5.1.4.3 & Strength Check.. \\
\hline
\end{tabular}

Appendix C Example Assessment Calculations ............................................................................

C.1 Linear Assessment Examples ............................................................................. C-1

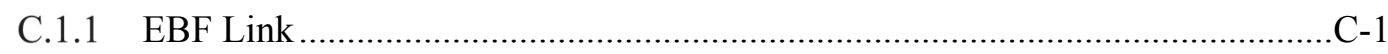

C.1.1.1 Shear Demand ...............................................................................

C. 1.1.2 Shear Strength ............................................................................. -1

C.1.1.3 Acceptance Criteria .....................................................................

C.1.1.4 Acceptance Criteria Check ...............................................................

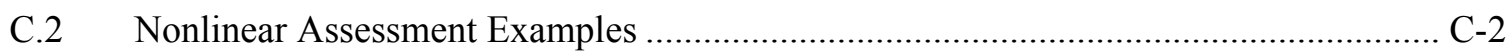

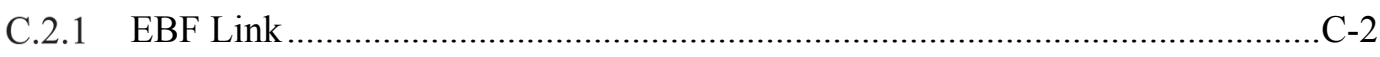

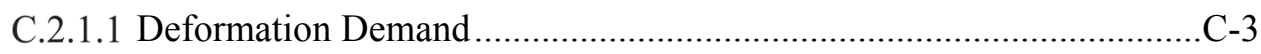

C.2.1.2 Acceptance Criteria .......................................................................

C.2.1.3 Acceptance Criteria Check ............................................................

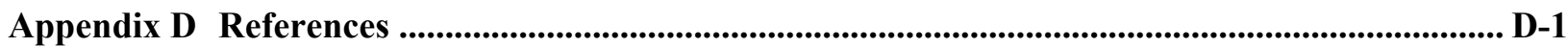




\section{List of Tables}

Table 1-1. Comparison of Seismic Hazard and Associated Performance for ASCE 7 and ASCE 41 ..... 1-4

Table 1-2. Performance Comparison between IBC and ASCE 41 - (From IEBC Table 301.1.4.1) ....... 1-4

Table 2-1. Structural Characteristics of Archetype Buildings............................................................ 2-1

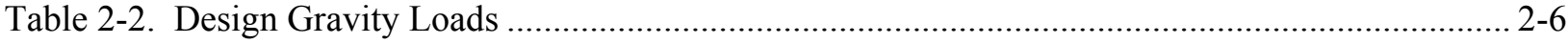

Table 2-3. Spectral Response Acceleration Parameters ................................................................... 2-7

Table 2-4. Seismic Analysis and Design Parameters, E-W ........................................................... 2-8

Table 2-5. Seismic Analysis and Design Parameters, N-S .............................................................. 2-9

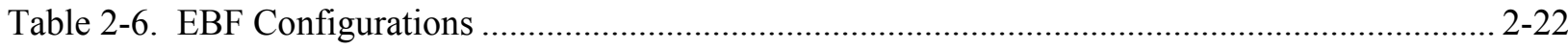

Table 3-1. Seismic Performance Targets (from ASCE 41-06) ............................................................. 3-2

Table 3-2. Seismic Performance Targets (from ASCE 41-13) ............................................................ 3-2

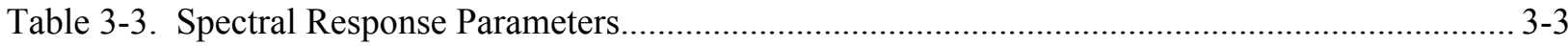

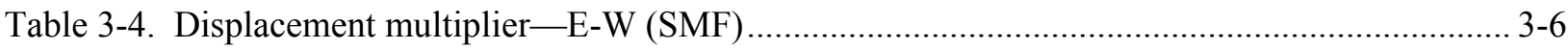

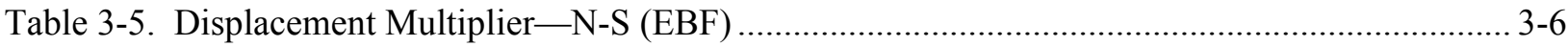

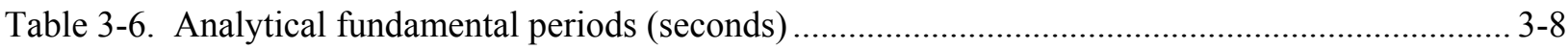

Table 3-7. Empirical fundamental periods (seconds) ........................................................................ 3-8

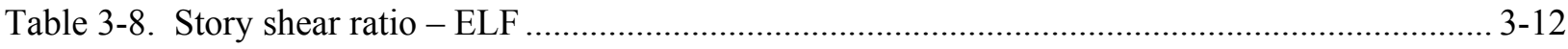

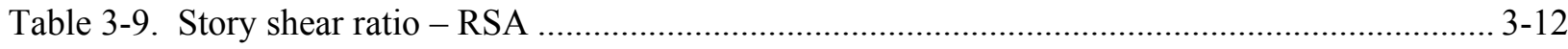

Table 3-10. Basic $m$-factors for Linear Procedures, EBF ................................................................. 3-28

Table 3-11. NSP General Information, 4-Story EBF (kip, inch) .................................................. 3-52

Table 3-12. CP NSP Analysis Parameters, 4-Story EBF BSE-2 CP (kip, inch) ................................. 3-52

Table 3-13. LS NSP Analysis Parameters, 4-Story EBF BSE-1 LS (kip, inch) ................................. 3-52

Table 3-14. NSP General Information, 8-Story EBF (kip, inch) ................................................... 3-71

Table 3-15. CP NSP Analysis Parameters, 8-Story EBF BSE-2 CP (kip, inch) ................................ 3-72

Table 3-16. LS NSP Analysis Parameters, 8-Story EBF BSE-1 LS (kip, inch).................................. 3-72

Table 3-17. NSP General Information, 16-Story EBF (kip, inch) ................................................... 3-96

Table 3-18. CP NSP Analysis Parameters, 16-Story EBF BSE-2 CP (kip, inch) ............................... 3-96

Table 3-19. LS NSP Analysis Parameters, 16-Story EBF BSE-1 LS (kip, inch)................................ 3-96

Table 3-20. Performance Summary of Shear Links (SL) per Frame, Linear Procedures.................... 3-109

Table 3-21. Effect of Design and Assessment Provisions on $D C R_{N}$ for LS at BSE-1 ...................... 3-109

Table 3-22. Performance Summary of Column Members (CM) per Frame, Linear Procedures ......... 3-110

Table 3-23. Performance Summary of Brace Members (BM) per Frame, Linear Procedures............. 3-112 
Table 3-24. BSO Performance Summary of Archetype Buildings, Linear Procedures 3-114

Table 3-25. Summary of Base Shears, Linear Procedures (kips) ...................................................... 3-114

Table 3-26. Performance Summary of Shear Links (SL) per Frame, Nonlinear Procedures ............... 3-115

Table 3-27. Performance Summary of Column Hinges (CH) per Frame, Nonlinear Procedures ........ 3-116

Table 3-28. Performance Summary of Column Members (CM) per Frame, Nonlinear Procedures .... 3-117

Table 3-29. Performance Summary of Brace Hinges (BH) per Frame, Nonlinear Procedures............ 3-117

Table 3-30. Performance Summary of Brace Members (BM) per frame, Nonlinear Procedures ........ 3-118

Table 3-31. BSO Performance Summary of Archetype Buildings, Nonlinear Procedures .................. 3-118

Table 3-32. BSO Performance Summary of Archetype Buildings.................................................... 3-119

Table A-1. Ground Motion Records for N-S Direction of ME4 ....................................................... A-3

Table A-2. Ground Motion Records for N-S Direction of ME8 …..................................................... A-6

Table A-3. Ground Motion Records for N-S Direction of ME16 ....................................................... A-9

Table B-1. Wind Forces on ME4 (kips) …............................................................................... B-2

Table B-2. Wind Forces on ME8 (kips) …............................................................................. B-2

Table B-3. Wind Forces on ME16 (kips) ….............................................................................. B-2

Table B-4. Effect Seismic Weights and Story Gravity Forces, ME4 (kips) ...................................... B-3

Table B-5. Effect Seismic Weights and Story Gravity Forces, ME8 (kips) ....................................... B-3

Table B-6. Effect Seismic Weights and Story Gravity Forces, ME16 (kips) ...................................... B-4

Table B-7. Seismic Strength Design Forces, E-W ME4 .................................................................... B-4

Table B-8. Seismic Drift Forces, E-W ME4 _............................................................................... B-5

Table B-9. ASCE 7 Allowable Drift and Stability Verification, E-W ME4 RSA................................ B-6

Table B-10. ASCE 7 Allowable Drift and Stability Verification, E-W ME4 ELF .............................. B-7

Table B-11. Seismic Strength Design Forces, E-W ME8 …............................................................ B-7

Table B-12. Seismic Drift Forces, E-W ME8 …....................................................................... B-8

Table B-13. ASCE 7 Allowable Drift and Stability Verification, E-W ME8 RSA.............................. B-9

Table B-14. ASCE 7 Allowable Drift and Stability Verification, E-W ME8 ELF .............................. B-9

Table B-15. Summary of Seismic Strength Design Forces, E-W ME16 …….................................... B-10

Table B-16. Summary of Seismic Drift Forces, E-W ME16 …......................................................... B-11

Table B-17. ASCE 7 Allowable Drift and Stability Verification, E-W ME16 RSA........................... B-12

Table B-18. ASCE 7 Allowable Drift and Stability Verification, E-W ME16 RSA........................... B-13

Table B-19. Seismic Strength Design Forces, N-S ME4 ................................................................ B-13

Table B-20. Seismic Drift Forces, N-S ME4 ….......................................................................... B-14

Table B-21. ASCE 7 Allowable Drift and Stability Verification, N-S ME4 RSA .............................. B-15

Table B-22. ASCE 7 Allowable Drift and Stability Verification, N-S ME4 ELF ............................. B-15

Table B-23. Seismic Strength Design Forces, N-S ME8 …............................................................ B-16 
Table B-24. Seismic Drift Forces, N-S ME8

B-16

Table B-25. ASCE 7 Allowable Drift and Stability Verification, N-S ME8 RSA................................B-18

Table B-26. ASCE 7 Allowable Drift and Stability Verification, N-S ME8 ELF ................................B-18

Table B-27. Seismic Strength Design Forces, N-S ME16 …............................................................. 18

Table B-28. Summary of Seismic Drift Forces, N-S ME16 ….............................................................. 19

Table B-29. ASCE 7 Allowable Drift and Stability Verification, N-S ME16 RSA............................. B-21

Table B-30. ASCE 7 Allowable Drift and Stability Verification, N-S ME16 ELF .............................. B-21

Table B-31. Horizontal Irregularity Type 1 (a and b) Verification .....................................................

Table B-32. Vertical Irregularity Type 1 ( $a$ and b) Verification (Exception 1).....................................-22

Table B-33. Vertical Irregularity Type 5 ( $\mathrm{a}$ and b) Verification, ELF (kip, feet)................................. B-23

Table B-34. Vertical Irregularity Type 5 ( $\mathrm{a}$ and b) Verification, RSA................................................

Table B-35. Horizontal Irregularity Type 1 (a and b) Verification .....................................................

Table B-36. Vertical Irregularity Type 1 ( $a$ and b) Verification (Exception 1).....................................24

Table B-37. Vertical Irregularity Type 5 (a and b) Verification, ELF (kip, feet)..................................25

Table B-38. Vertical Irregularity Type 5 ( $a$ and b) Verification, RSA (kip, feet)................................

Table B-39. AISC 360 Frame Stability (Effective Length Method), 4-Story SMF ELF (kip, inch)......B-26

Table B-40. AISC 360 Frame Stability (Effective Length Method), 4-Story SMF RSA (kip, inch) .....B-26

Table B-41. AISC 360 Frame Stability (Effective Length Method), 8-Story SMF ELF (kip, inch)......B-26

Table B-42. AISC 360 Frame Stability (Effective Length Method), 8-Story SMF RSA (kip, inch) .....B-26

Table B-43. AISC 360 Frame Stability (Effective Length Method), 16-Story SMF ELF (kip, inch)....B-27

Table B-44. AISC 360 Frame Stability (Effective Length Method), 16-Story SMF RSA (kip, inch)...B-27

Table B-45. Adjusted Effective Length Factors - 4-Story SMF ........................................................

Table B-46. Adjusted Effective Length Factors - 8-Story SMF ........................................................

Table B-47. Adjusted Effective Length Factors - 16-Story SMF .....................................................

Table B-48. 4-Story EBF ELF, Shear Link Parameters ......................................................................29

Table B-49. 4-Story EBF RSA, Shear Link Parameters.....................................................................

Table B-50. 8-Story EBF ELF, Shear Link Parameters ...................................................................

Table B-51. 8-Story EBF RSA, Shear Link Parameters...........................................................................

Table B-52. 16-Story EBF ELF, Shear Link Parameters ................................................................

Table B-53. 16-Story EBF RSA, Shear Link Parameters.................................................................

Table B-54. Controlling Load Combinations (kips, feet) .................................................................35

Table B-55. Controlling Load Combinations (kips, feet) ..................................................................36 


\section{List of Figures}

Figure 2-1. Isometric View of ME4 Archetype Building ................................................................. 2-2

Figure 2-2. Isometric View of ME8 Archetype Building ................................................................. 2-2

Figure 2-3. Isometric View of ME16 Archetype Building ............................................................ 2-3

Figure 2-4. Typical Floor Framing Plan, ME4 and ME8 ….......................................................... 2-4

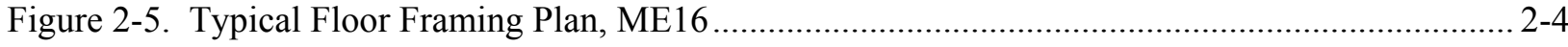

Figure 2-6. Typical Type FR RBS Beam-to-Column Connection Assembly .................................... 2-10

Figure 2-7. Type FR RBS Beam-To-Column Connection Subassembly Model for Linear Analysis.... 2-11

Figure 2-8. Brace-to-Beam Connection Subassembly Model for Linear Analysis .............................. 2-11

Figure 2-9. Flow Chart of SMF Design Process ......................................................................... 2-13

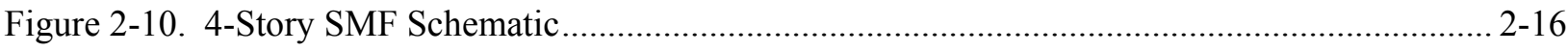

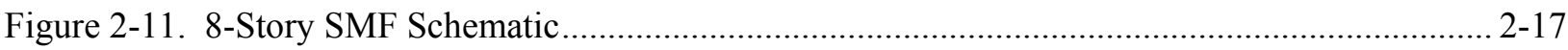

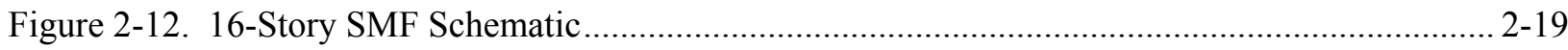

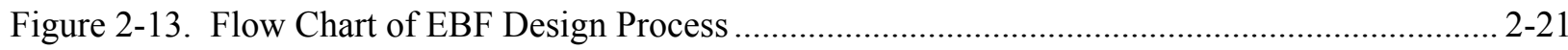

Figure 2-14. Story Shear Demands and Nominal Strengths, 16-story EBF (N-S ME16) ................... 2-23

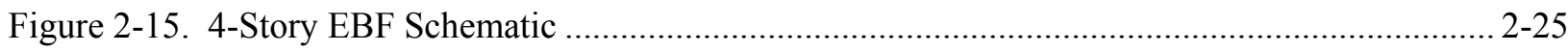

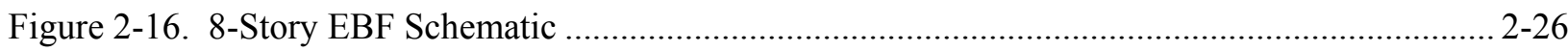

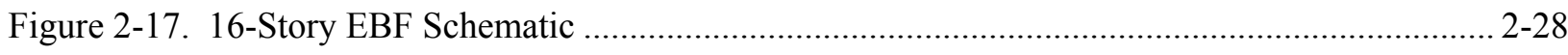

Figure 3-1. Acceleration Response Spectra........................................................................... 3-3

Figure 3-2. Generalized Component Backbone Curve (adopted from ASCE 41 Figure C2-1) .............. 3-9

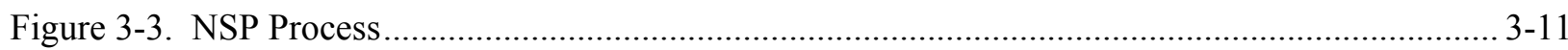

Figure 3-4. $P-M$ Interaction on Section $m$-factor (in-plane) and Member Instability (Primary Component)

Figure 3-5. Compactness Requirements as a Function of Axial Load Ratio, LS Acceptance Criteria .. 3-26

Figure 3-6. Acceptance Criteria as a Function of Axial Load Ratio and Section Compactness, LS

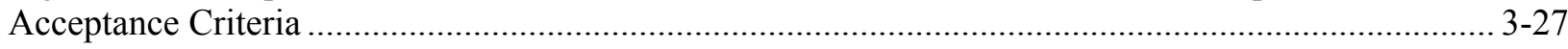

Figure 3-7. Frame Capacity Schematic ( $m$-factor), LS and CP, 4-Story EBF ELF and RSA ............... 3-29

Figure 3-8. Frame Capacity Schematic ( $m$-factor), LS and CP, 8-Story EBF ELF and RSA ............... 3-29

Figure 3-9. Frame Capacity Schematic ( $m$-factor), LS and CP, 16-Story EBF ELF and RSA ............. 3-30

Figure 3-10. EBF Analytical Modeling Schematic .......................................................................... 3-32

Figure 3-11. PERFORM-3D Link Beam Shear Hinge Calibration...................................................... 3-33

Figure 3-12. In-plane Flexural Hinge Yield Surface Model (Including Force-Controlled Response)... 3-36

Figure 3-13. Variation in Acceptance Criteria and Hinge Model for Axial Force................................. 3-37 
Figure 3-14. $P-M$ Interaction on Plastic Rotation, LS Acceptance Criteria (Primary Component) ....... 3-38

Figure 3-15. $P-M$ Interaction Curve (Section Yield Surface)............................................................ 3-39

Figure 3-16. $P$ - $M$ Interaction Curve (Member Instability) .............................................................. 3-39

Figure 3-17. Frame Capacity Schematic (Inelastic), LS and CP, 4-Story EBF - ELF....................... 3-40

Figure 3-18. Frame Capacity Schematic (Inelastic), LS and CP, 4-Story EBF - RSA ...................... 3-40

Figure 3-19. Frame Capacity Schematic (Inelastic), LS and CP, 8-Story EBF - ELF........................ 3-41

Figure 3-20. Frame Capacity Schematic (Inelastic), LS and CP, 8-Story EBF - ELF........................ 3-42

Figure 3-21. Frame Capacity Schematic (Inelastic), LS and CP, 16-Story EBF - ELF...................... 3-43

Figure 3-22. Frame Capacity Schematic (Inelastic), LS and CP, 16-Story EBF - RSA ..................... 3-44

Figure 3-23. LSP Assessment Results, 4-Story EBF ELF, BSE-1 LS ............................................. 3-46

Figure 3-24. LSP Assessment Results, 4-Story EBF RSA, BSE-1 LS............................................. 3-46

Figure 3-25. LSP Assessment Results, Compression in Exterior Columns, 4-Story EBF, BSE-1 ........ 3-47

Figure 3-26. LSP Assessment Results, 4-Story EBF ELF, BSE-2 CP .............................................. 3-48

Figure 3-27. LSP Assessment Results, 4-Story EBF RSA, BSE-2 CP ............................................. 3-48

Figure 3-28. LSP Assessment Results, Compression in Exterior Columns, 4-Story EBF, BSE-2 ........ 3-48

Figure 3-29. LDP Assessment Results, 4-Story EBF ELF, BSE-1 LS................................................. 3-49

Figure 3-30. LDP Assessment Results, 4-Story EBF RSA, BSE-1 LS ................................................ 3-49

Figure 3-31. LDP Assessment Results, Compression in Exterior Columns, 4-Story EBF, BSE-1 ........ 3-50

Figure 3-32. LDP Assessment Results, 4-Story EBF ELF, BSE-2 CP ............................................. 3-51

Figure 3-33. LDP Assessment Results, 4-Story EBF RSA, BSE-2 CP ............................................ 3-51

Figure 3-34. LDP Assessment Results, Compression in Exterior Columns, 4-Story EBF, BSE-2 ........ 3-51

Figure 3-35. 4-Story EBF ELF Pushover, BSE-2 …............................................................ 3-53

Figure 3-36. 4-Story EBF RSA Pushover, BSE-2 ..................................................................... 3-53

Figure 3-37. 4-Story EBF ELF Pushover - Story Drift Ratios - BSE-2 …..................................... 3-54

Figure 3-38. 4-Story EBF RSA Pushover - Story Drift Ratios - BSE-2 ....................................... 3-54

Figure 3-39. Schematic of Flexural Actions in Components, 4-Story EBF (NSP and NDP) ............... 3-55

Figure 3-40. NSP Assessment Results, 4-Story EBF, BSE-1 LS (+push to left) ................................ 3-56

Figure 3-41. NSP Assessment Results, 4-Story EBF, BSE-2 CP (+push to left)................................ 3-56

Figure 3-42. NDP Assessment Results, Shear Hinges, 4-Story EBF BSE-1 LS ................................ 3-57

Figure 3-43. NDP Assessment Results, Shear Hinges, 4-Story EBF BSE-2 CP............................... 3-57

Figure 3-44. NDP Assessment Results, Column Hinges, 4-Story EBF ELF, BSE-2 Yield.................. 3-58

Figure 3-45. NDP Assessment Results, Column Hinges, 4-Story EBF RSA, BSE-2 Yield ................. 3-59

Figure 3-46. NDP Assessment Results, Column Members, 4-Story EBF ELF, BSE-2 ...................... 3-59

Figure 3-47. NDP Assessment Results, Column Members, 4-Story EBF RSA, BSE-2 …................. 3-60

Figure 3-48. NDP Assessment Results, Brace Hinges, 4-Story EBF ELF, BSE-2 Yield .................... 3-60 
Figure 3-49. NDP Assessment Results, Brace Hinges, 4-Story EBF RSA, BSE-2 Yield.................... 3-61

Figure 3-50. NDP Assessment Results, Brace Members, 4-Story EBF ELF, BSE-2 ....................... 3-61

Figure 3-51. NDP Assessment Results, Brace Members, 4-Story EBF RSA, BSE-2 ….................... 3-62

Figure 3-52. LSP Assessment Results, 8-Story EBF ELF, BSE-1 LS ............................................ 3-63

Figure 3-53. LSP Assessment Results, 8-Story EBF RSA, BSE-1 LS............................................ 3-63

Figure 3-54. LSP Assessment Results, Compression in Exterior Columns, 8-Story EBF, BSE-1 ........ 3-64

Figure 3-55. LSP Assessment Results, 8-Story EBF ELF, BSE-2 CP ............................................ 3-66

Figure 3-56. LSP Assessment Results, 8-Story EBF RSA, BSE-2 CP …....................................... 3-66

Figure 3-57. LSP Assessment Results, Compression in Exterior Columns, 8-Story EBF, BSE-2 ........ 3-67

Figure 3-58. LDP Assessment Results, 8-Story EBF ELF, BSE-1 LS............................................ 3-68

Figure 3-59. LDP Assessment Results, 8-Story EBF RSA, BSE-1 LS .......................................... 3-68

Figure 3-60. LDP Assessment Results, Compression in Exterior Columns, 8-Story EBF, BSE-1 ........ 3-69

Figure 3-61. LDP Assessment Results, 8-Story EBF ELF, BSE-2 CP ............................................. 3-70

Figure 3-62. LDP Assessment Results, 8-Story EBF RSA, BSE-2 CP ............................................. 3-70

Figure 3-63. LDP Assessment Results, Compression in Exterior Columns, 8-Story EBF, BSE-2 ........ 3-71

Figure 3-64. 8-Story EBF ELF Pushover, BSE-2 …....................................................................... 3-72

Figure 3-65. 8-Story RSA ELF Pushover, BSE-2 …......................................................................... 3-73

Figure 3-66. 8-Story EBF ELF Pushover - Story Drift Ratios - BSE-2 ............................................. 3-73

Figure 3-67. 8-Story EBF RSA Pushover - Story Drift Ratios - BSE-2 …....................................... 3-74

Figure 3-68. Schematic of Flexural Actions in Components, 8-Story EBF (NSP and NDP) ............... 3-75

Figure 3-69. NSP Assessment Results, 8-Story EBF, BSE-1 LS (+push to left) ................................ 3-75

Figure 3-70. NSP Assessment Results, 8-Story EBF, BSE-2 CP (+push to left)............................... 3-76

Figure 3-71. NDP Assessment Results, Shear Hinges, 8-Story EBF, BSE-1 LS .............................. 3-77

Figure 3-72. NDP Assessment Results, Shear Hinges, 8-Story EBF, BSE-2 CP............................. 3-77

Figure 3-73. NDP Assessment Results, Column Hinges, 8-Story EBF ELF, BSE-2 Yield.................. 3-78

Figure 3-74. NDP Assessment Results, Column Hinges, 8-Story EBF RSA, BSE-2 Yield ................. 3-79

Figure 3-75. NDP Assessment Results, Column Members, 8-Story EBF ELF, BSE-2 ….................. 3-79

Figure 3-76. NDP Assessment Results, Column Members, 8-Story EBF RSA, BSE-2 ..................... 3-80

Figure 3-77. NDP Assessment Results, Brace Hinges, 8-Story EBF ELF, BSE-2 Yield .................... 3-80

Figure 3-78. NDP Assessment Results, Brace Hinges, 8-Story EBF RSA, BSE-2 Yield.................... 3-81

Figure 3-79. NDP Assessment Results, Brace Members, 8-Story EBF ELF, BSE-2 ........................ 3-81

Figure 3-80. NDP Assessment Results, Brace Members, 8-Story EBF RSA, BSE-2 ….................... 3-82

Figure 3-81. LSP Assessment Results, 16-Story EBF ELF, BSE-1 LS ............................................. 3-84

Figure 3-82. LSP Assessment Results, 16-Story EBF RSA, BSE-1 LS.......................................... 3-85

Figure 3-83. LSP Assessment Results, Compression in Exterior Columns, 16-Story EBF, BSE-1 ...... 3-86 
Figure 3-84. LSP Assessment Results, 16-Story EBF ELF, BSE-2 CP ............................................. 3-87

Figure 3-85. LSP Assessment Results, 16-Story EBF RSA, BSE-2 CP …...................................... 3-88

Figure 3-86. LSP Assessment Results, Compression in Exterior Columns, 16-Story EBF, BSE-2 ...... 3-89

Figure 3-87. LDP Assessment Results, 16-Story EBF ELF, BSE-1 LS........................................... 3-90

Figure 3-88. LDP Assessment Results, 16-Story EBF RSA, BSE-1 LS ........................................... 3-91

Figure 3-89. LDP Assessment Results, Compression in Exterior Columns, 16-Story EBF, BSE-1 ...... 3-92

Figure 3-90. LDP Assessment Results, 16-Story EBF ELF, BSE-2 CP ........................................... 3-93

Figure 3-91. LDP Assessment Results, 16-Story EBF RSA, BSE-2 CP .......................................... 3-94

Figure 3-92. LDP Assessment Results, Compression in Exterior Columns, 16-Story EBF, BSE-2 ...... 3-95

Figure 3-93. 16-Story EBF ELF Pushover, BSE-2 ….................................................................. 3-96

Figure 3-94. 16-Story EBF RSA Pushover, BSE-2 ..................................................................... 3-97

Figure 3-95. 16-Story EBF ELF Pushover - Story Drift Ratios - BSE-2 …..................................... 3-98

Figure 3-96. 16-Story EBF RSA Pushover - Story Drift Ratios - BSE-2 ......................................... 3-98

Figure 3-97. Schematic of Flexural Actions in Components, 16-Story EBF (NSP and NDP) ............. 3-99

Figure 3-98. NSP Assessment Results, 16-Story EBF ELF, BSE-1 LS (+push to left) ...................... 3-100

Figure 3-99. NSP Assessment Results, 16-Story EBF RSA, BSE-2 CP (+push to left) ..................... 3-101

Figure 3-100. NDP Assessment Results, Shear Hinges, 16-Story EBF, BSE-1 LS .......................... 3-102

Figure 3-101. NDP Assessment Results, Shear Hinges, 16-Story EBF, BSE-2 CP.......................... 3-103

Figure 3-102. NDP Assessment Results, Column Hinges, 16-Story EBF ELF, BSE-2 Yield............. 3-104

Figure 3-103. NDP Assessment Results, Column Hinges, 16-Story EBF RSA, BSE-2 Yield ............ 3-104

Figure 3-104. NDP Assessment Results, Column Members, 16-Story EBF ELF, BSE-2 2................. 3-105

Figure 3-105. NDP Assessment Results, Column Members, 16-Story EBF RSA, BSE-2 ................. 3-105

Figure 3-106. NDP Assessment Results, Brace Hinges, 16-Story EBF ELF, BSE-2 Yield ............... 3-106

Figure 3-107. NDP Assessment Results, Brace Hinges, 16-Story EBF RSA, BSE-2 Yield................ 3-106

Figure 3-108. NDP Assessment Results, Brace Members, 16-Story EBF ELF, BSE-2 …............... 3-107

Figure 3-109. NDP Assessment Results, Brace Members, 16-Story EBF RSA, BSE-2 ................... 3-107

Figure 3-110. $D C R_{N}$ values for EBF columns and braces using LSP, BSE-1, LS BPL .................... 3-113

Figure A-1. Acceleration Response Spectra: Original, Scaled, and Scaled Average Spectrum for N-S

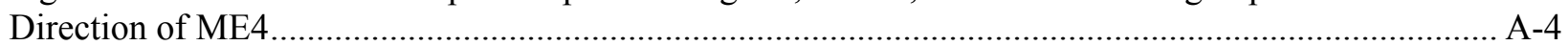

Figure A-2. Acceleration Response Spectra: Original and Scaled for Each Selected Record for N-S

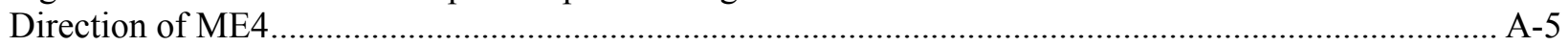

Figure A-3. Acceleration Response Spectra: Original, Scaled, and Scaled Average Spectrum for N-S Direction of ME8.

Figure A-4. Acceleration Response Spectra: Original and Scaled for Each Selected Record for N-S Direction of ME8. 
Figure A-5. Acceleration Response Spectra: Original, Scaled, and Scaled Average Spectrum for N-S Direction of ME16 A-10

Figure A-6. Acceleration Response Spectra: Original and Scaled for Each Selected Record for N-S Direction of ME16. A-11

Figure A-7. 1994 Northridge Earthquake at Beverly Hills, Mulholland Drive Station, Comp. 009.... A-12 Figure A-8. 1994 Northridge Earthquake at Beverly Hills, Mulholland Drive Station, Comp. 279..... A-12 Figure A-9. 1994 Northridge Earthquake at Canyon Country WLC Station, Comp. 000................... A-13 Figure A-10. 1994 Northridge Earthquake at Canyon Country WLC Station, Comp. 270................. A-13

Figure A-11. 1999 Duzce, Turkey Earthquake at Bolu Station, Comp. 000 ...................................... A-14

Figure A-12. 1999 Duzce, Turkey Earthquake at Bolu Station, Comp. 090 ...................................... A-14

Figure A-13. 1999 Hector Mine Earthquake at Hector Station, Comp. 000 ...................................... A-15

Figure A-14. 1999 Hector Mine Earthquake at Hector Station, Comp. 090 ...................................... A-15

Figure A-15. 1979 Imperial Valley Earthquake at Delta Station, Comp. 262 ................................... A-16

Figure A-16. 1979 Imperial Valley Earthquake at Delta Station, Comp. 352 .................................. A-16

Figure A-17. 1979 Imperial Valley Earthquake at El Centro Array Station \#11, Comp. 140.............. A-17

Figure A-18. 1979 Imperial Valley Earthquake at El Centro Array Station \#11, Comp. 230.............. A-17

Figure A-19. 1995 Kobe, Japan Earthquake at Nishi-Akashi Station, Comp. 000 ............................ A-18

Figure A-20. 1995 Kobe, Japan Earthquake at Nishi-Akashi Station, Comp. 090 ............................. A-18

Figure A-21. 1995 Kobe, Japan Earthquake at Shin-Osaka Station, Comp. 000 ................................ A-19

Figure A-22. 1995 Kobe, Japan Earthquake at Shin-Osaka Station, Comp. 090 _............................... A-19

Figure A-23. 1999 Kocaeli, Turkey Earthquake at Duzce Station, Comp. 180 ................................... A-20

Figure A-24. 1999 Kocaeli, Turkey Earthquake at Duzce Station, Comp. 270 ................................. A-20

Figure A-25. 1999 Kocaeli, Turkey Earthquake at Arcelik Station, Comp. 000................................ A-21

Figure A-26. 1999 Kocaeli, Turkey Earthquake at Arcelik Station, Comp. 090................................ A-21

Figure A-27. 1992 Landers Earthquake at Yermo Fire Station, Comp. 270 ...................................... A-22

Figure A-28. 1992 Landers Earthquake at Yermo Fire Station, Comp. 360 ..................................... A-22

Figure A-29. 1992 Landers Earthquake at Coolwater Station, Longitudinal Direction...................... A-23

Figure A-30. 1992 Landers Earthquake at Coolwater Station, Transverse Direction ......................... A-23

Figure A-31. 1989 Loma Prieta Earthquake at Capitola Station, Comp. 000 ................................... A-24

Figure A-32. 1989 Loma Prieta Earthquake at Capitola Station, Comp. 090 .................................... A-24

Figure A-33. 1989 Loma Prieta Earthquake at Gilroy Array Station \#3, Comp. 000 ......................... A-25

Figure A-34. 1989 Loma Prieta Earthquake at Gilroy Array Station \#3, Comp. 090 ......................... A-25

Figure A-35. 1990 Manjil, Iran Earthquake at Abbar Station, Longitudinal Direction ...................... A-26

Figure A-36. 1990 Manjil, Iran Earthquake at Abbar Station, Transverse Direction.......................... A-26

Figure A-37. 1987 Superstition Hills Earthquake at El Centro, Imperial County, Comp. 000 ............ A-27 
Figure A-38. 1987 Superstition Hills Earthquake at El Centro, Imperial County, Comp. 090 .....

Figure A-39. 1987 Superstition Hills Earthquake at Poe Road, Comp. 270 ..................................... A-28

Figure A-40. 1987 Superstition Hills Earthquake at Poe Road, Comp. 360 ...................................... A-28

Figure A-41. 1992 Cape Mendocino Earthquake at Rio Dell Overpass, Comp. 270 ......................... A-29

Figure A-42. 1992 Cape Mendocino Earthquake at Rio Dell Overpass, Comp. 360 ......................... A-29

Figure A-43. 1999 Chi-Chi, Taiwan Earthquake at CHY101 Station, E-W Component..................... A-30

Figure A-44. 1999 Chi-Chi, Taiwan Earthquake at CHY101 Station, N-S Component ...................... A-30

Figure A-45. 1999 Chi-Chi, Taiwan Earthquake at TCU045 Station, E-W Component ..................... A-31

Figure A-46. 1999 Chi-Chi, Taiwan Earthquake at TCU045 Station, N-S Component ..................... A-31

Figure A-47. 1971 San Fernando Earthquake at Los Angeles Hollywood Store Station, Comp. 090 .. A-32

Figure A-48. 1971 San Fernando Earthquake at Los Angeles Hollywood Store Station, Comp. 180 .. A-32

Figure A-49. 1976 Fruili, Italy Earthquake at Tolmezzo Station, Comp. 000 .................................. A-33

Figure A-50. 1976 Fruili, Italy Earthquake at Tolmezzo Station, Comp. 270 ................................... A-33

Figure B-1. Strength Design Lateral Forces and Story Shears ......................................................... B-5

Figure B-2. Drift Design Lateral Forces and Story Shears.......................................................... B-5

Figure B-3. Story Drift Ratios and Deflected Shape ................................................................. B-6

Figure B-4. Strength Design Lateral Forces and Story Shears ......................................................... B-7

Figure B-5. Drift Design Lateral Forces and Story Shears.............................................................. B-8

Figure B-6. Story Drift Ratios and Deflected Shape ......................................................................... B-9

Figure B-7. Strength Design Lateral Forces and Story Shears ...................................................... B-10

Figure B-8. Drift Design Lateral Forces and Story Shears.......................................................... B-11

Figure B-9. Story Drift Ratios and Deflected Shape .................................................................... B-12

Figure B-10. Strength Design Lateral Forces and Story Shears .................................................... B-14

Figure B-11. Drift Design Lateral Forces and Story Shears........................................................ B-14

Figure B-12. Story Drift Ratios and Deflected Shape ................................................................ B-15

Figure B-13. Strength Design Lateral Forces and Story Shears ..................................................... B-16

Figure B-14. Drift Design Lateral Forces and Story Shears.......................................................... B-17

Figure B-15. Story Drift Ratios and Deflected Shape .................................................................... B-17

Figure B-16. Strength Design Lateral Forces and Story Shears ...................................................... B-19

Figure B-17. Drift Design Lateral Forces and Story Shears......................................................... B-20

Figure B-18. Story Drift Ratios and Deflected Shape .................................................................. B-20

Figure B-19. Link Rotation Angle, 4-Story EBF ELF and RSA …................................................ B-29

Figure B-20. Link Rotation Angle, 8-Story EBF ELF and RSA …............................................... B-30

Figure B-21. Link Rotation Angle, 16-Story EBF ELF and RSA ................................................ B-32

Figure B-22. EBF member sizes, 8-story RSA …..................................................................... B-33 
xxiii 


\section{List of Abbreviations}

\begin{tabular}{|c|c|}
\hline AISC & American Institute of Steel Construction \\
\hline ASCE & American Society of Civil Engineers \\
\hline ASTM & American Society for Testing and Materials \\
\hline ATC & Applied Technology Council \\
\hline BPL & Building Performance Level \\
\hline BSE & Basic Safety Earthquake \\
\hline $\mathrm{BSO}$ & Basic Safety Objective \\
\hline BSSC & Building Seismic Safety Council \\
\hline $\mathrm{CBF}$ & Concentrically Braced Frame \\
\hline $\mathrm{CP}$ & Collapse Prevention \\
\hline $\mathrm{DC}$ & Deformation-Controlled \\
\hline DCR & Demand to capacity ratio \\
\hline EBF & Eccentrically Braced Frame \\
\hline EHL & Earthquake Hazard Level \\
\hline ELF & Equivalent Lateral Force \\
\hline $\mathrm{E}-\mathrm{W}$ & East-West \\
\hline $\mathrm{FC}$ & Force-Controlled \\
\hline FEMA & Federal Emergency Management Agency \\
\hline FR & Fully Restrained \\
\hline GSA & General Services Administration \\
\hline GCR & Grant/Contract Report \\
\hline HD & Highly Ductile \\
\hline HSS & Hollow Structural Section \\
\hline IBC & International Building Code \\
\hline $\mathrm{ICC}$ & International Code Council \\
\hline IEBC & International Existing Building Code \\
\hline IO & Immediate Occupancy \\
\hline LC & Load Combination \\
\hline LDP & Linear Dynamic Procedure \\
\hline LFRS & Lateral Force Resisting System \\
\hline LRFD & Load and Resistance Factor Design \\
\hline
\end{tabular}

xxiv 


\begin{tabular}{|c|c|}
\hline LS & Life Safety \\
\hline LSP & Linear Static Procedure \\
\hline LTB & Lateral Torsional Buckling \\
\hline $\mathrm{MC}$ & Moment Curvature \\
\hline $\operatorname{MCE}\left(\mathrm{MCE}_{\mathrm{R}}\right)$ & Maximum Considered Earthquake \\
\hline MD & Moderately Ductile \\
\hline MR & Moment Rotation \\
\hline MRSA & Modal Response Spectrum Analysis \\
\hline MWFRS & Main Wind Force Resisting System \\
\hline NDP & Nonlinear Dynamic Procedure \\
\hline NEHRP & National Earthquake Hazards Reduction Program \\
\hline NIBS & National Institute of Building Sciences \\
\hline NIST & National Institute of Standards and Technology \\
\hline NPL & Nonstructural Performance Level \\
\hline $\mathrm{N}-\mathrm{S}$ & North-South \\
\hline NSP & Nonlinear Static Procedure \\
\hline PBS & Public Buildings Service \\
\hline PBSD & Performance-Based Seismic Design \\
\hline RBS & Reduced Beam Section \\
\hline RHA & Response History Analysis \\
\hline RSA & Response Spectrum Analysis \\
\hline $\mathrm{SCBF}$ & Special Concentrically Braced Frame \\
\hline SCWB & Strong Column Weak Beam \\
\hline SDC & Seismic Design Category \\
\hline SDOF & Single Degree of Freedom \\
\hline SEAONC & Structural Engineers Association of Northern California \\
\hline SEI & Structural Engineering Institute \\
\hline SFRS & Seismic Force Resisting System \\
\hline SMF & Special Moment Frame \\
\hline SPL & Structural Performance Level \\
\hline SRSS & Square Root Sum of the Squares \\
\hline W.P. & Work Point \\
\hline
\end{tabular}




\section{Executive Summary}

This report presents the results of a study investigating the correlation between the seismic performance of an ASCE 7 code-compliant building and its performance as quantified using ASCE 41 analysis procedures and structural performance metrics. This investigation is performed by evaluating a suite of structural steel buildings in a high seismicity region that are designed using ASCE 7 and evaluated using ASCE 41. The basic question is whether the standards for designing new steel buildings and assessing existing steel buildings provide consistent levels of performance. An additional outcome of this research is to advance the state-of-knowledge in PBSD and assessment of buildings using ASCE 41. Further, results provide the technical background for provisions that target equivalent seismic performance between a new building and an existing building that is required to meet the seismic performance objective of a new building.

This report presents the results of a structural seismic performance assessment using ASCE 41 procedures and performance measures of buildings utilizing steel eccentrically braced frames (EBF) as the lateral forceresisting system (LFRS).

A suite of archetype buildings that incorporate EBFs along one principal direction of the buildings is designed in accordance with ASCE 7. The suite consists of 4-, 8-, and 16-story buildings designed using both the Equivalent Lateral Force (ELF) Procedure and Modal Response Spectrum Analysis (RSA). Both analysis procedures are used to provide a generally applicable range of LFRS strength within the selected seismic intensity region. As such, an LFRS may include significant overstrength to resist nonseismic loads or to satisfy other design criteria. A design space is created to investigate the effects of design methodology, building height and other LFRS-specific geometric modifications on seismic performance. In reality, the design space is infinitely large and many design choices made in this study can also have different configurations to evaluate the variation in performance specific to a design choice.

The seismic performance assessment of the building suite is conducted using both linear and nonlinear analysis procedures prescribed in ASCE 41:

- Linear Static Procedure (LSP)

- Linear Dynamic Procedure (Response Spectrum) (LDP)

- Nonlinear Static Procedure (NSP)

- Nonlinear Dynamic Procedure (NDP)

For this study, the performance assessment targets the Basic Safety Objective (BSO) prescribed in ASCE 41. This objective includes the interrelated goals of Life Safety (LS) Building Performance Level (BPL) at the Basic Safety Earthquake-1 (BSE-1) earthquake hazard level (EHL) and Collapse Prevention (CP) BPL at the BSE-2 EHL. This performance objective is chosen to align with the intended structural performance objective of an ordinary building in ASCE 7, which is qualitatively defined here as "life safety" provided by collapse prevention of the building, given a maximum considered earthquake (MCE) event. 
To evaluate seismic assessment criteria, each component of the EBFs is designated as a primary or component in accordance with ASCE 41 §2.2.5 (and ASCE 41 §2.4.4.2). Similarly, quantitative performance measures (i.e., acceptance criteria) for primary components are used for all assessment procedures, although performance measures for secondary components are permitted by ASCE 41 for some primary components. The consistent use of primary acceptance criteria keeps all components and associated assessment results correlated among the assessment procedures for this study.

The goals of this research are as follows:

- Assess new structural steel buildings utilizing EBFs designed per ASCE 7 requirements and, in turn, evaluated using ASCE 41,

- Develop a qualitative link between the performance implied in ASCE 7 in light of the performance identified by ASCE 41 procedures and performance measures,

- Provide guidance or technical support for improved or new provisions in ASCE 41 (and to a lesser extent, ASCE 7),

- Reduce uncertainty in first-generation PBSD procedures for performance-based seismic assessment, and

- Identify any inconsistencies, ambiguities, and confusing provisions in ASCE 41.

The primary conclusions of this research can be divided into two parts: General Observations and Specific Observations about ASCE 41 analytical procedures.

\section{A. General Observations for Eccentrically Braced Frames:}

- The LSP generally results in more conservative normalized demand to capacity ratios, $D C R_{N}$, values than that of the LDP, because of the differences in the distribution of seismic demands and the lack of modal representation other than the fundamental mode in the LSP.

- The NSP generally results in less conservative $D C R_{N}$ values than that of the NDP, contrary to what would be expected with increasing the analytical complexity, because of the differences in the distribution of seismic demands and the lack of modal representation other than the fundamental mode in the NSP.

- The nonlinear procedures provide a more rigorous assessment approach as compared to the linear procedures. The results from the LSP, and to a lesser extent the LDP, indicate more performance failures in force-controlled components than identified using the nonlinear procedures. The results presented emphasize the inherent conservatism in the linear procedures. However, this conservatism is accompanied by a reduction in required analytical resources and proficiency of the analyst.

- The linear procedures can illustrate the trend in demands but may fail to highlight critical performance zones within a given frame.

- No clear consistency exists between linear assessment results and nonlinear assessment results for link beams. Contrary performance results indicate acceptance criteria that need further calibration. 


\section{B. Specific Observations for Eccentrically Braced Frames:}

The following significant observations and conclusions are based on the collective results obtained from the assessment of the EBFs. More details about the specific items are in the relevant sections of the assessment discussion in Chapter 3.

- Analytical results based on component-level performances indicate that new long-period EBFs designed in accordance with ASCE 7, and its referenced standards, have difficulty achieving the ASCE 41 BSO for an existing building intended to be equivalent to a new building. This observation is driven by the performance of the link beams and, to a lesser extent, the columns and braces. Assessment results for the link beams from the nonlinear procedures provide a conclusion opposite to that provided for the linear procedures in that nonlinear assessment reveals higher $D C R_{N}$ values than the linear assessment.

- Assuming the archetype buildings meet the collapse performance objective of ASCE 7, the results of the assessment procedures indicate that ASCE 41 is generally conservative for EBFs. ASCE 41 analysis would require retrofit or replacement of specific components of a code-compliant SFRS to satisfy the CP BPL, given an MCE event. The results highlight that columns (i.e., beam-columns) with high axial and flexural demands and link beams have difficulty in satisfying the performance criteria in ASCE 41. Future research is needed to couple the collapse performance objectives of the two standards, as well as other performance objectives associated with a seismic hazard with a lower return period.

- Analysis results indicate that the linear procedures generally give smaller $D C R_{N}$ values than that obtained from nonlinear procedures, contrary to what would be expected with increasing the analytical complexity. Given a properly designed new link beam, the linear procedures will result in a maximum $D C R_{N}$ value for a primary component of 0.72 for the BSE-1 EHL $(0.76$ for the BSE2 EHL).

- A significant number of link beams did not satisfy the acceptance criteria for the nonlinear procedures. This result was particularly problematic for EBFs as they quickly progressed towards a collapse state after the ultimate link capacity in the second story was reached. Though link beam performance is based on high fidelity analytical modeling parameters, enhanced modeling practices should be investigated. Research should investigate the influence of the loading protocol adopted to establish the deterministic acceptance criteria for short link beams.

- A significant number of columns, primarily at the base of the frame, did not satisfy the ASCE 41 acceptance criteria (force-controlled). The results for columns can be enhanced by more mechanistically consistent assessment provisions and analytical modeling parameters for columns (e.g., column/brace-to-base connection modeling).

- Components of the EBFs that do not satisfy the CP acceptance criteria would need to be strengthened to achieve the performance required by ASCE 41. However, the results from the various assessment procedures were seen to be inconsistent in some cases for a given design routine (i.e., LSP vs. NDP) or the same assessment procedure was inconsistent between design routines (i.e., ELF and RSA). This makes it difficult to definitively suggest that using ASCE 41 to design a new EBF would produce a system capable of achieving the seismic performance objective of ASCE 7. Future research is needed to evaluate the collapse probability of a new system strengthened by ASCE 41 relative to the seismic performance objective of ASCE 7. The same is required for a new 
system that has component strengths reduced from that required by ASCE 7 to meet an ASCE 41 performance objective. Further, the adequacy of the components of the enhanced frame (those required to satisfy ASCE 41) would be dependent upon which analysis procedure is used to iterate between design and assessment, and therefore the fidelity of the analytical model and analysis parameters.

- Results of this study indicate that for ASCE 41 to be used as a seismic design procedure for new steel buildings, as a performance-based alternative to ASCE 7 (see ASCE 7 §1.3.1.3), acceptance criteria for the various analysis methods must be calibrated to each other to consistently result in a uniform collapse risk. Additionally, ASCE 41 would need to reference material-specific design standards (e.g., AISC 341) for their seismic design requirements, as well as consistent requirements for defining acceptance criteria for a component (e.g., plastic rotation). 


\section{Chapter 1 Introduction}

In 1997, the Federal Emergency Management Agency (FEMA) published FEMA 273: NEHRP Guidelines for the Seismic Rehabilitation of Buildings (FEMA 1997) as a first step towards standardizing seismic performance assessment procedures for existing buildings. This effort, produced under the Applied Technology Council's project 33 (ATC-33), was the first significant step in implementing performancebased seismic design (PBSD) into practice. Subsequently in 2000, FEMA and the American Society of Civil Engineers (ASCE) published FEMA 356: Prestandard and Commentary for the Seismic Rehabilitation of Buildings (FEMA 2000e). This publication introduced many changes to FEMA 273 to refine the accuracy and applicability of the provisions. The changes are chronicled in FEMA 357: Global Topics Report on the Prestandard and Commentary for the Seismic Rehabilitation of Buildings (FEMA 2000f). In 2006 ASCE published ASCE/SEI 41-06: Seismic Rehabilitation of Existing Buildings (ASCE 2006) as an ASCE Standard-hereafter referred to as ASCE 41. This document is referenced by the International Existing Building Code (IEBC) published by the International Code Council (ICC) (ICC 2012a).

ASCE 41 represents the current state-of-practice in seismic evaluation and rehabilitation of existing buildings. This standard is referenced by the California Building Standards Code (CBSC 2010), Federal government building standards (e.g., NIST 2011a), and a number of other local jurisdictions. ASCE 41 provides analytical procedures and criteria for evaluating buildings and designing seismic retrofits based on a defined performance goal (i.e., Life Safety and Collapse Prevention). This ability to explicitly define a performance goal and then assess a building design against that goal has led practitioners to adapt ASCE 41 methodology for use in new building design. The performance-based methodologies in ASCE 41 provide an alternative to the traditional prescriptive approaches used in the current standard for new buildings, ASCE/SEI 7-10: Minimum Design Loads for Buildings and Other Structures (ASCE 2010) - hereafter referred to as ASCE 7. Referenced by the International Building Code (IBC) (ICC 2012b), ASCE 7 is widely used throughout the country for seismic design of new buildings. However, with the trend toward performance-based design, the correlation between the performance of a building designed with the prescriptive provisions of ASCE 7 and assessed with the performance-based provisions of ASCE 41 is largely unknown.

The next version of ASCE 41 (ASCE 41-13 ${ }^{1}$ ) will offer a new track for application of the provisions to existing buildings whose performance goal is equivalent to that of a building designed with the new building standard. Consequently, this new track will allow direct seismic performance assessment of new buildings or, alternatively, a substitute seismic design approach via Chapter 1 of ASCE 7. For example, the PBSP100: Facility Standards for the Public Buildings Service (GSA 2012) prescribes that ASCE 41-06 shall

${ }^{1}$ ASCE 41-13: Seismic Evaluation and Retrofit of Existing Buildings (ASCE 2014) was being developed during this project period. As such, new or updated provisions in ASCE 41-13 were not incorporated, except where changes were required to align with the seismic hazard prescribed in ASCE 7-10. 
be used for the seismic design of new GSA facilities ${ }^{2}$ and that the guidelines from ASCE 41 are intended to be applied to new buildings. This document does not permit a building to be designed for seismic performance below the minimum level specified by IBC. The National Institute of Building Sciences (NIBS) is using PBS-P100 as the basis for developing their National Performance Based Design Guide (NIBS 2013). Further, the Provisions Update Committee for the Building Seismic Safety Council is currently deliberating expanded provisions for performing nonlinear response history analysis (Chapter 16 in ASCE 7) for the 2015 edition of the NEHRP Recommend Provisions for Seismic Regulations for New Buildings and Other Structures (FEMA 2015). These expanded provisions reference ASCE 41-13 in the commentary for modeling and acceptance criteria for the design of new buildings.

This report presents the results of a study investigating the correlation between the seismic performance of an ASCE 7 code-compliant building and its performance as quantified using ASCE 41 analysis procedures and structural performance metrics. This investigation is performed by evaluating a suite of structural steel buildings in a high seismicity region that are designed using ASCE 7 and evaluated using ASCE 41. The basic question is whether the standards for designing new steel buildings and assessing existing steel buildings provide consistent levels of performance. The intended outcome of this research is to advance the state-of-knowledge in performance-based seismic design and assessment of buildings using ASCE 41. Further, results provide the technical background for provisions that target equivalent seismic performance between a new building and an existing building that is required to meet the seismic performance objective of a new building.

\section{Applicability of ASCE 41-13 to this Study}

During this project, ASCE/SEI 41-13, Seismic Evaluation and Retrofit of Existing Buildings (ASCE 2014), completed committee balloting and was sent out for public comment as well as for approval for inclusion in the IEBC. Any significant differences between ASCE 41-06 and ASCE 41-13 regarding assessment of steel structural systems will be highlighted in the discussions where applicable - unless otherwise noted, reference to ASCE 41 refers only to ASCE 41-06.

One significant addition to ASCE 41-13 is a process for applying the provisions for the seismic assessment of existing buildings where the intended performance is equivalent to that which is intended for new buildings designed in accordance with ASCE 7, including a correlation matrix between the two standards. While ASCE 41-06 is being used currently in practice to justify seismic performance of new buildings in compliance with ASCE 7 (as well as to identify noncompliance), this addition is the first step in conceptually aligning future editions of ASCE 41 and ASCE 7 so that ASCE 41 can be used for the seismic design of new buildings. Still, there are variations between material-specific provisions in ASCE 41 and provisions in material design standards referenced in ASCE 7 (e.g., AISC 360 and ACI 318) that need to be resolved.

\footnotetext{
${ }^{2}$ The seismic hazard used to characterize the design basis earthquake is that with a $10 \%$ probability of exceedence in 50 years. This differs from that used in ASCE/SEI 7-05 (ASCE 2005) - two-thirds of that with a 2\% probability of exceedence in 50 years-and ASCE 7-10 - two-thirds of that producing a 1\% probability of collapse in 50 years.
} 
In terms of assessment of steel systems, the technical content in ASCE 41-13 did not change in any significant manner that invalidates the results presented in this report. The few changes that would affect the results of individual components are highlighted where applicable. In fact, data from this study instigated some of these changes.

\subsection{Project Motivation and Background}

Traditional prescriptive seismic provisions for new buildings principally concentrate on the Life Safety objective applied to all-encompassing arrangements of similar lateral force-resisting systems. Little consideration is given to either the actual performance of individual buildings or the economic loss and occupancy interruption that may occur after an earthquake. Thus, a need arises for seismic provisions that allow engineers to design buildings and assess them against varying levels of performance associated with varying levels of earthquake hazard. So doing provides a method where desired building damage levels can be coupled to both quantitative and qualitative definitions of performance so that building and operational stakeholders are integrated into a project. Conceptually, PBSD was conceived to satisfy this need. The objective of PBSD is to provide a means of integrating additional performance objectives into the seismic design of new buildings that explicitly measure and account for risk of casualties, occupancy interruption, and economic loss including repair costs.

Prescriptive building code procedures, such as those found in ASCE 7, tend to restrict design innovation and can lead to inefficient structural designs and higher construction costs. In lieu of its prescriptive provisions, ASCE 7 allows alternative "rational" design methods, such as PBSD, to be used in new building design. PBSD affords the designer the freedom to bypass prescriptive building code provisions by demonstrating that a building performs to an explicitly defined performance target that equals or exceeds the life safety objective in prescriptive provisions. The use of such methods must be approved by the local authority having jurisdiction and typically requires rigorous structural analysis coupled with a high level of expertise.

Although ASCE 7 allows PBSD (see ASCE 7 §1.3.1.3) to be used in new building design, it provides no substantial guidance on implementing PBSD for this purpose. Therefore, many practitioners and local authorities have turned to the provisions in ASCE 41 as a way of implementing PBSD into new building design. These provisions, widely considered to be "first generation" PBSD principles, were originally intended to be used in the evaluation of existing buildings by assessing performance compliance with a selected rehabilitation objective. Since ASCE 41 is applicable to existing buildings, it does not provide a direct correlation between the rehabilitation objective and the intended performance of an ASCE 7 codecompliant new building (see Table 1-1). However, the IEBC does provide a correlation between ASCE 41 performance levels and IBC (and thus ASCE 7) Risk Categories, thus providing the link between the prescriptive requirements for new building design and the nonprescriptive requirements of existing building assessment and PBSD. A matrix showing this correlation is shown in Table 1-2. Still, this matrix has not been comprehensively validated nor have the seismic performance expectations for new buildings been quantitatively assessed to standardize acceptable performance within the framework of ASCE 41, or vice versa. ASCE 7 has not expressly adopted Table 1-2 for seismic design. 
Table 1-1. Comparison of Seismic Hazard and Associated Performance for ASCE 7 and ASCE 41

\begin{tabular}{|c|c|c|c|c|c|}
\hline & & \multicolumn{4}{|c|}{ Target Building Performance Level ${ }^{1}$} \\
\hline & & Operational & $\begin{array}{l}\text { Immediate } \\
\text { Occupancy } \\
\text { (IO) }\end{array}$ & $\begin{array}{l}\text { Life Safety } \\
\text { (LS) }\end{array}$ & $\begin{array}{l}\text { Collapse } \\
\text { Prevention } \\
\text { (CP) }\end{array}$ \\
\hline \multirow{7}{*}{ 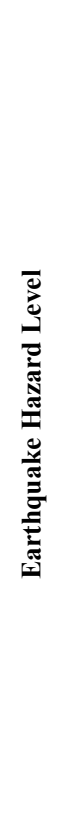 } & $\begin{array}{c}\text { ASCE } 41 \\
50 \% / 50 \text { year }^{2}\end{array}$ & $\begin{array}{c}\text { ASCE } 41 \\
\text { (nonstructural) }\end{array}$ & ASCE 41 Limited & ASCE 41 Limited & ASCE 41 Limited \\
\hline & $\begin{array}{c}\text { ASCE } 41 \\
20 \% / 50 \text { year }^{2}\end{array}$ & $\begin{array}{l}\text { ASCE } 41 \\
\text { Enhanced }\end{array}$ & ASCE 41 & $\begin{array}{l}\text { ASCE } 41 \\
\text { Limited }\end{array}$ & $\begin{array}{c}\text { ASCE } 41 \\
\text { Limited }\end{array}$ \\
\hline & $\begin{array}{l}\text { ASCE } 7 \\
\text { "Frequent" }\end{array}$ & $\begin{array}{c}\text { ASCE } 7 \\
\text { Risk Category III } \\
\text { \& IV }\end{array}$ & $\begin{array}{c}\text { ASCE } 7 \text { Risk } \\
\text { Category I \& II } \\
\text { (anticipated) }^{3}\end{array}$ & N.A. & N.A. \\
\hline & $\begin{array}{c}\text { ASCE } 41 \\
\text { BSE-1 } \\
\sim 10 \% / 50 \text { year }^{2}\end{array}$ & $\begin{array}{l}\text { ASCE } 41 \\
\text { Enhanced }\end{array}$ & $\begin{array}{l}\text { ASCE } 41 \\
\text { Enhanced }\end{array}$ & ASCE 41 BSO & $\begin{array}{l}\text { ASCE } 41 \\
\text { Limited }\end{array}$ \\
\hline & $\begin{array}{c}\text { ASCE } 7 \\
2 / 3 \times \mathrm{MCE}_{\mathrm{R}}^{1}\end{array}$ & N.A. & $\begin{array}{c}\text { ASCE } 7 \\
\text { Risk Category III } \\
\text { \& IV }\end{array}$ & $\begin{array}{l}\text { ASCE } 7 \text { Risk } \\
\text { Category I \& II } \\
\text { (design) }\end{array}$ & N.A. \\
\hline & $\begin{array}{c}\text { ASCE } 41 \\
\text { BSE-2 } \\
\sim 2 \% / 50 \text { year }^{2}\end{array}$ & $\begin{array}{l}\text { ASCE } 41 \\
\text { Enhanced }\end{array}$ & $\begin{array}{l}\text { ASCE } 41 \\
\text { Enhanced }\end{array}$ & $\begin{array}{l}\text { ASCE } 41 \\
\text { Enhanced }\end{array}$ & ASCE 41 BSO \\
\hline & $\begin{array}{l}\text { ASCE } 7 \\
M_{R} 1\end{array}$ & N.A. & N.A. & $\begin{array}{c}\text { ASCE } 7 \\
\text { Risk Category III } \\
\text { \& IV }\end{array}$ & $\begin{array}{c}\text { ASCE } 7 \text { Risk } \\
\text { Category I \& II } \\
\text { (objective) }^{3}\end{array}$ \\
\hline
\end{tabular}

Table 1-2. Performance Comparison between IBC and ASCE 41 - (From IEBC Table 301.1.4.1)

\begin{tabular}{ccc}
\hline $\begin{array}{c}\text { Risk Category } \\
\text { (Based on IBC Table 1604.5) }\end{array}$ & $\begin{array}{c}\text { Performance Level for use with ASCE } \\
\text { 41 BSE-1 Earthquake Hazard Level }\end{array}$ & $\begin{array}{c}\text { Performance Level for use with ASCE } \\
\text { 41 BSE-2 Earthquake Hazard Level }\end{array}$ \\
\hline I & Life Safety (LS) & Collapse Prevention (CP) \\
II & Life Safety (LS) & Collapse Prevention (CP) \\
III & Note a & Note a \\
IV & Immediate Occupancy (IO) & Immediate Occupancy (IO) \\
\hline $\begin{array}{l}\text { a. Acceptance criteria for Risk Category III shall be taken as 80 percent of the acceptance criteria specified for Risk Category II performance levels, but } \\
\text { need not be less than the acceptance criteria specified for Risk Category IV performance levels }\end{array}$
\end{tabular}

In June 2008 the National Earthquake Hazards Reduction Program (NEHRP) sponsored a PBSD workshop for leading practitioners and researchers from around the United States to develop a comprehensive list of research needs to foster full development and implementation of PBSD. From this workshop, the Building Seismic Safety Council (BSSC) reported a prioritized list of key PBSD research and implementation needs in NIST GCR 09-917-2: Research Required to Support Full Implementation of Performance-Based Seismic Design (NIST 2009a). The highest priority need identified in this report was to "benchmark" current PBSD methodologies (e.g., ASCE 41) with code procedures for design of new buildings. Two observations from the report were that among workshop participants (1) ASCE 41 procedures are perceived to be overly 
conservative and (2) existing PBSD methods are not accepted by practitioners as providing a uniform level of confidence. A supporting reason for these two observations was that no systematic effort had been undertaken to benchmark structural performance as determined using ASCE 41 procedures, together with widely accepted procedures for designing new buildings using ASCE 7.

Additionally, needs for the advancement of PBSD have been highlighted by other researchers and practitioners (Toranzo-Dianderas 2009, SEAONC 2010, Paret, Searer, and Freeman 2011, and Pekelnicky and Poland 2012). The needs identified include, but are not limited to the following:

- Calibration / comparison of ASCE 41 to ASCE 7

- Reduced conservatism in linear procedures and acceptance criteria

- Better clarification of provisions and intent

Therefore, the research study presented in this report was undertaken in an effort to address some of these needs.

\subsection{Scope of Project}

This report presents the results of a structural seismic performance assessment using ASCE 41 procedures and performance measures of buildings utilizing steel eccentrically braced frames (EBF) as the lateral forceresisting system (LFRS) ${ }^{3}$.

A suite of archetype buildings that incorporate EBFs along one principal direction of the buildings is designed in accordance with ASCE 7. The suite consists of 4-, 8-, and 16-story buildings designed using both the Equivalent Lateral Force (ELF) Procedure and Modal Response Spectrum Analysis (RSA). Both analysis procedures are used to provide a generally applicable range of LFRS strength within the selected seismic intensity region. As such, components of an LFRS may include significant overstrength ${ }^{4}$ to resist nonseismic loads or to satisfy other design criteria. A design space is created to investigate the effects of design methodology, building height, and other LFRS-specific geometric modifications on seismic performance. In reality, the design space is infinitely large and many design choices made in this study can also have different configurations to evaluate the variation in performance specific to a design choice (e.g., study of a range of doubler plate thicknesses in an SMF and the influence on frame column performance).

The seismic performance assessment of the building suite is conducted using both linear and nonlinear analysis procedures prescribed in ASCE 41:

- $\quad$ Linear Static Procedure (LSP)

\footnotetext{
${ }^{3}$ Although the LFRS is a component of a cohesive three-dimensional building system that includes structural framing intended to primarily resist gravity loads and nonstructural components, only the performance of the LFRS as identified by ASCE 41 procedures and measures is presented. The performance of an LFRS can be influenced by the inclusion of gravity framing in a analysis. Based on the analytical modeling used in this study, this interaction is deemed to be negligible because the LFRS resists nearly all forces and deformations resulting from lateral loads and movement.

${ }^{4}$ Overstrength is defined here as the additional elastic strength in a component that is in excess of the required minimum seismic strength.
} 
- Linear Dynamic Procedure (Response Spectrum) (LDP)

- Nonlinear Static Procedure (NSP)

- Nonlinear Dynamic Procedure (NDP)

For this study, the performance assessment targets the Basic Safety Objective (BSO) prescribed in ASCE 41. This objective includes the interrelated goals of Life Safety (LS) Building Performance Level (BPL) at the Basic Safety Earthquake-1 (BSE-1) earthquake hazard level (EHL) and Collapse Prevention (CP) BPL at the BSE-2 EHL (see Table 1-2 above). This performance objective is chosen to align with the intended structural performance objective of an ordinary building ${ }^{5}$ in ASCE 7, which is qualitatively defined here as "life safety" provided by collapse prevention of the building, given a maximum considered earthquake (MCE) event.

To evaluate seismic assessment criteria, each component of the EBFs is designated as a primary member (or component) in accordance with ASCE 41 §2.2.5 (and ASCE 41 §2.4.4.2). Similarly, quantitative performance measures (i.e., acceptance criteria) for primary components are used for all assessment procedures, although performance measures for secondary components are permitted by ASCE 41 for some primary components. The consistent use of primary acceptance criteria keeps all components and associated assessment results correlated among the assessment procedures for this study.

The goals of this research are as follows:

- Assess new structural steel buildings utilizing EBFs designed per ASCE 7 requirements and, in turn, evaluated using ASCE 41,

- Develop a qualitative link between the performance implied in ASCE 7 in light of the performance identified by ASCE 41 procedures and performance measures,

- Provide guidance or technical support for improved or new provisions in ASCE 41 (and to a lesser extent, ASCE 7),

- Reduce uncertainty in first-generation PBSD procedures for performance-based seismic assessment, and

- Identify any inconsistencies, ambiguities, and confusing provisions in ASCE 41.

This report does not discuss the correlation between deterministic performance metrics for components (e.g., $m$-factor in ASCE 41) and the system (e.g., $R$-factor in ASCE 7) - a topic for future research and one that is required to establish the link between the performance of an ASCE 7 code-compliant design and the associated performance identified by ASCE 41 procedures and performance measures. Here, it is assumed that the acceptance criteria for each BPL in ASCE 41 Chapter 5 are quantitatively rational and accurate interpretations of what deformations / actions are appropriate for the intended structural performance (see ASCE 41 Tables C1-2, C1-3, and C1-4). Clearly, this is a subjective and, at times, controversial topic, as some component actions are physically qualified only by experimental test results (i.e., SAC project, see FEMA 351 (FEMA 2000b)), but not necessarily for all performance levels.

\footnotetext{
${ }^{5}$ Buildings assigned Risk Category I or II.
} 
In this report, the archetype building designs are presented in Chapter 2. Next, the results from the seismic assessment in accordance with ASCE 41 and supplementary discussions are presented in Chapter 3. In the discussion of the assessment results, special focus is given to highlighting any notable differences or similarities between ASCE 41 and ASCE 7 (including the referenced design standards in ASCE 7 for the design of structural steel buildings) and to making suggestions for improvements in ASCE 41. Conclusions are provided in Chapter 4. Selection and scaling of ground motions, including pertinent ground motion data, for the NDP are provided in Appendix A. Additional information applicable to the design of the archetype buildings and detailed design calculations for a few example members and connections are provided in Appendix B. Detailed assessment calculations for a few example members and connections are provided in Appendix C.

The hope is that other follow-on projects will be undertaken by NIST and / or other organizations to provide additional data sets that advance the state-of-practice and state-of-knowledge and facilitate the implementation of performance-based seismic engineering in design of buildings. Further studies of structural steel systems (e.g., buckling-restrained braced frames), as well as studies of reinforced concrete moment frame and shear wall buildings, are planned as next steps at NIST. 


\section{Chapter 2 Overview and Design of Archetype Buildings}

This chapter presents the design of the archetype buildings. General discussions are provided in Section 2.1 on the geometry of the buildings and the design criteria, including sizes of structural members not considered to principally resist lateral loads. Section 2.2 discusses the structural design loads and associated design criteria specific to them. Section 2.3 presents information regarding the structural analysis and mathematical model used in the structural member selection process. Section 2.4 provides the design of the structural systems principally required to resist lateral loads and stabilize the buildings.

\subsection{General Information}

A suite of three steel-framed office buildings is investigated in this study. It is presumed that the archetype buildings will be constructed in a high seismicity area (e.g., somewhere along the west coast of the United States - see Earthquake Forces section below). Building stability and resistance to environmental loads and deformations is provided by special moment frames along the East-West (E-W) direction and eccentrically braced frames along the North-South (N-S) direction. All lateral force-resisting systems (LFRS) are symmetrically located at the perimeter of the building and orthogonal. For purposes of design, the identified LFRS acts as both the seismic force-resisting system (SFRS) and the main wind force-resisting system (MWFRS). For completeness of the full design of the archetype buildings, design of both LFRSs is presented below. However, only the assessment of the braced frames will be presented in this report. Assessment of the moment frames is presented in NIST TN 1863-1: Assessment of First Generation Performance-Based Design Methods for New Steel Buildings, Volume 1: Special Moment Frames (Harris and Speicher 2015a).

Each building is rectangular in plan, with five 30-foot bays in the E-W direction and generally five 20-foot bays in the N-S direction. The plan dimensions for all floors and roofs are 152 feet in the E-W direction and 102 feet in the N-S direction. For all buildings, the height of the first story is 18 feet and the remaining story heights are 14 feet. A summary of the geometric structural characteristics of each building is provided in Table 2-1. Building schematics are shown in Figure 2-1 through Figure 2-3. The typical floor framing plan is shown in Figure 2-4 and Figure 2-5. For brevity, the building schematics do not show symmetrical elevator core or stairwell diaphragm openings.

Table 2-1. Structural Characteristics of Archetype Buildings

\begin{tabular}{|c|c|c|c|c|c|c|}
\hline Bldg. ID & Stories & $\begin{array}{c}\text { E-W } \\
\text { Dimension }\end{array}$ & $\begin{array}{c}\text { N-S } \\
\text { Dimension }\end{array}$ & $\begin{array}{l}\text { E-W } \\
\text { LFRS }\end{array}$ & $\begin{array}{c}\mathrm{N}-\mathrm{S} \\
\text { LFRS }\end{array}$ & Notes \\
\hline ME4 & 4 & $\begin{array}{l}150^{\prime}=5 \text { bays } \\
\text { @ 30’ }\end{array}$ & $\begin{array}{l}100^{\prime}=5 \text { bays } \\
\text { @ 20’ }\end{array}$ & SMF & $\mathrm{EBF}$ & $\begin{array}{l}\text { SMF: } 3-30 \text {-foot bays } \\
\text { EBF: } 1-20 \text {-foot bay short link with HSS } \\
\text { braces }\end{array}$ \\
\hline ME8 & 8 & $\begin{array}{c}150^{\prime}=5 \text { bays } \\
\text { @ } 30^{\prime}\end{array}$ & $\begin{array}{l}100^{\prime}=5 \text { bays } \\
\text { @ } 20^{\prime}\end{array}$ & SMF & $\mathrm{EBF}$ & $\begin{array}{l}\text { SMF: } 3-30 \text {-foot bays } \\
\text { EBF: } 1-20 \text {-foot bay short link with HSS } \\
\text { braces }\end{array}$ \\
\hline ME16 & 16 & $\begin{array}{l}150^{\prime}=5 \text { bays } \\
\text { (a) 30' }\end{array}$ & $\begin{array}{l}100^{\prime}=\text { Varies - } \\
\text { see Figure 2-5 }\end{array}$ & SMF & $\mathrm{EBF}$ & $\begin{array}{l}\text { SMF: } 3-30 \text {-foot bays } \\
\text { EBF: } 1-30 \text {-foot bay short link with W- } \\
\text { Section braces }\end{array}$ \\
\hline
\end{tabular}




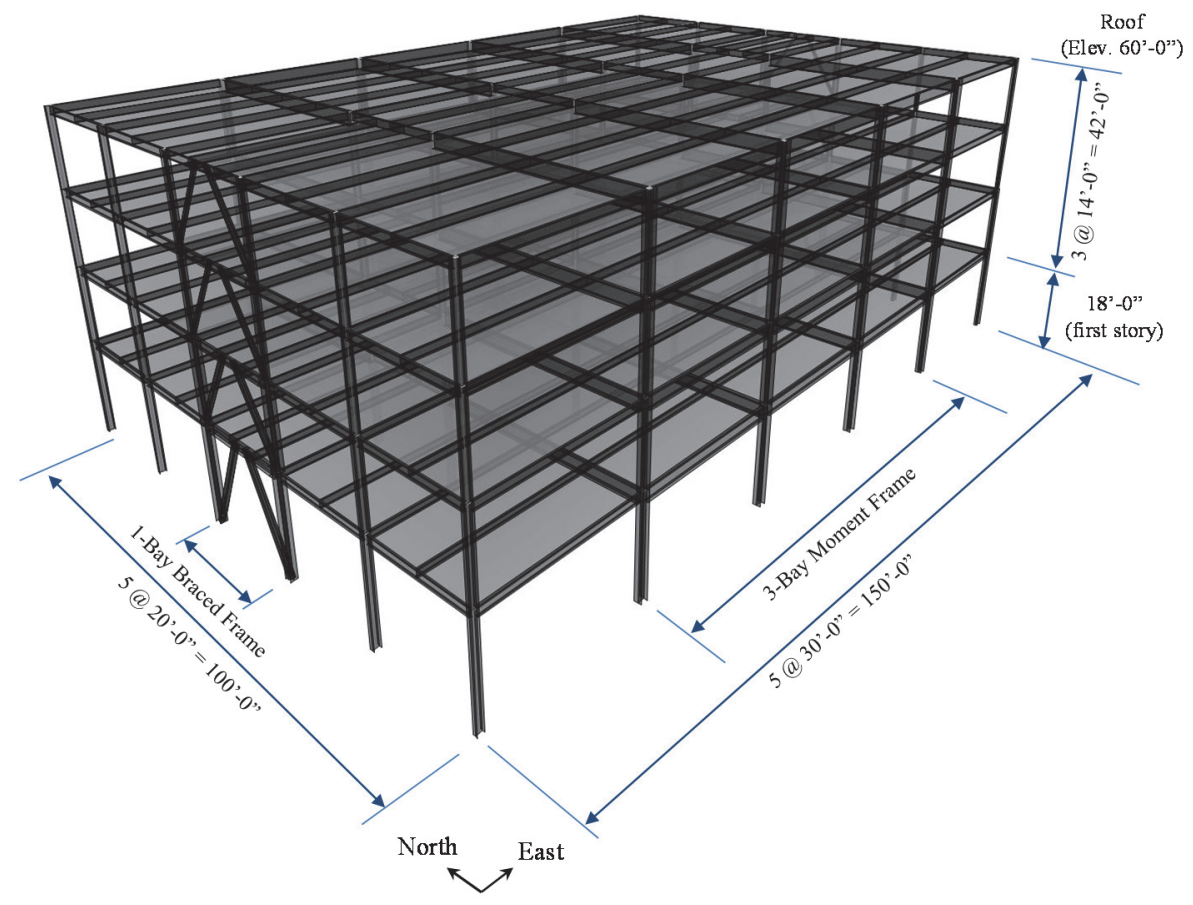

Figure 2-1. Isometric View of ME4 Archetype Building

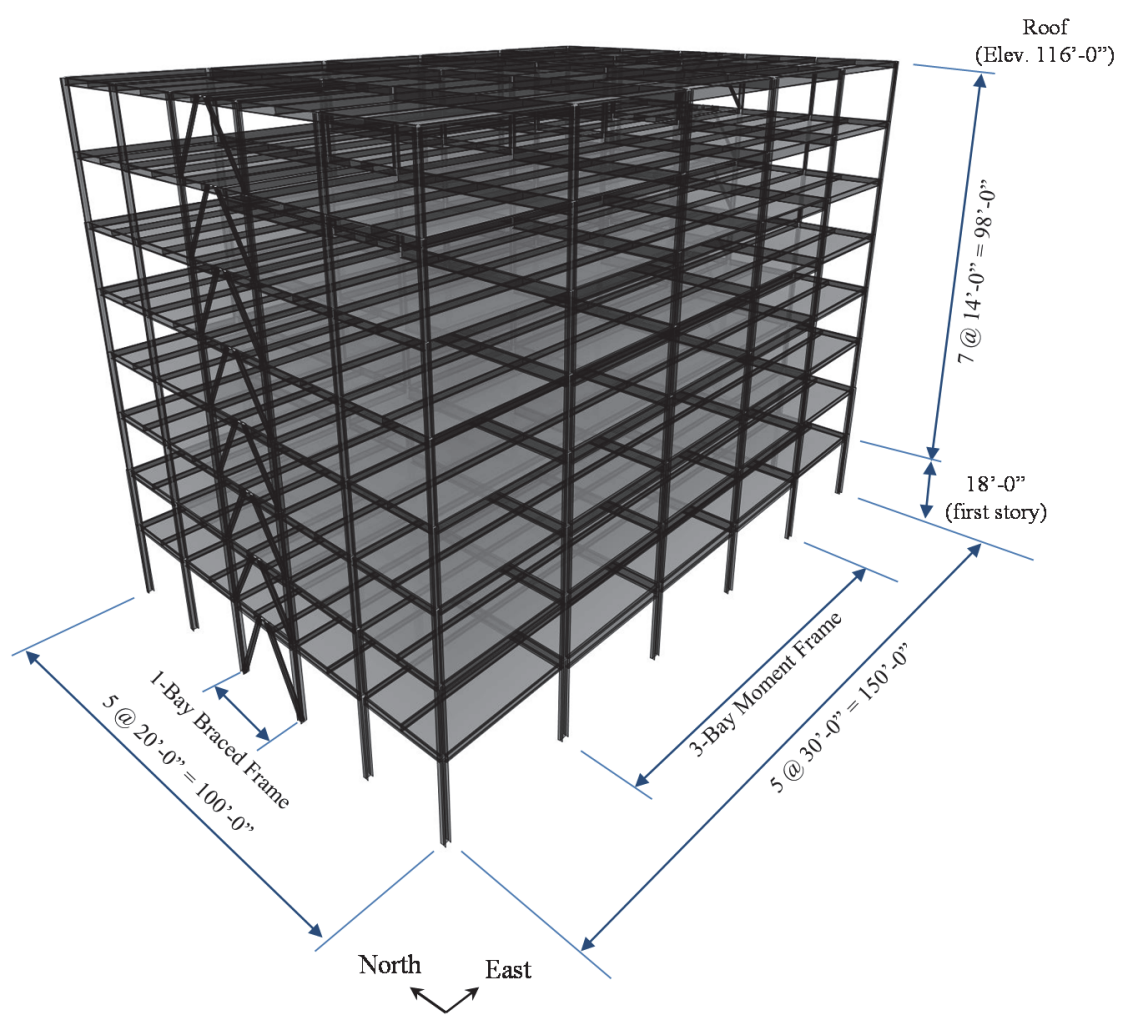

Figure 2-2. Isometric View of ME8 Archetype Building 


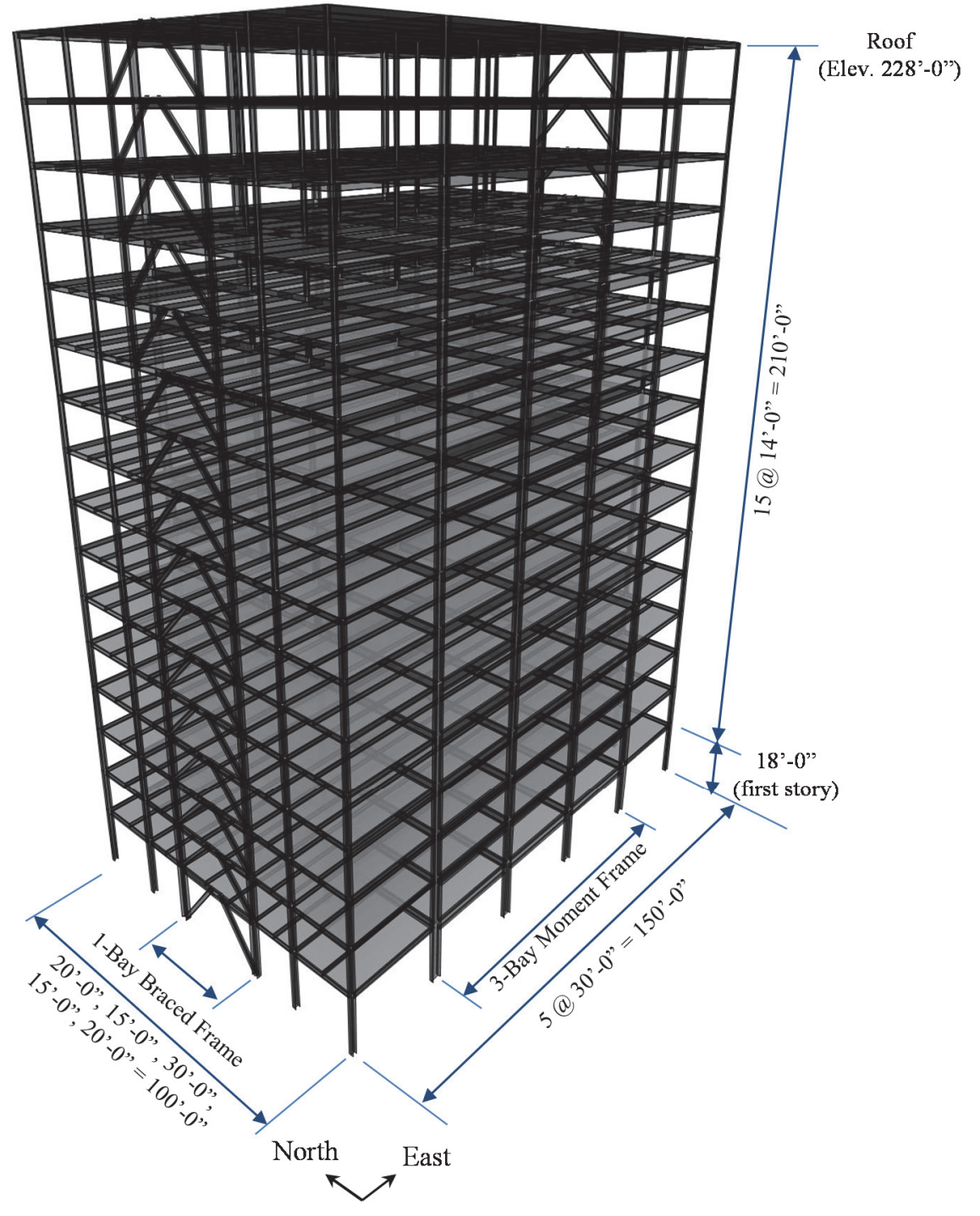

Figure 2-3. Isometric View of ME16 Archetype Building 


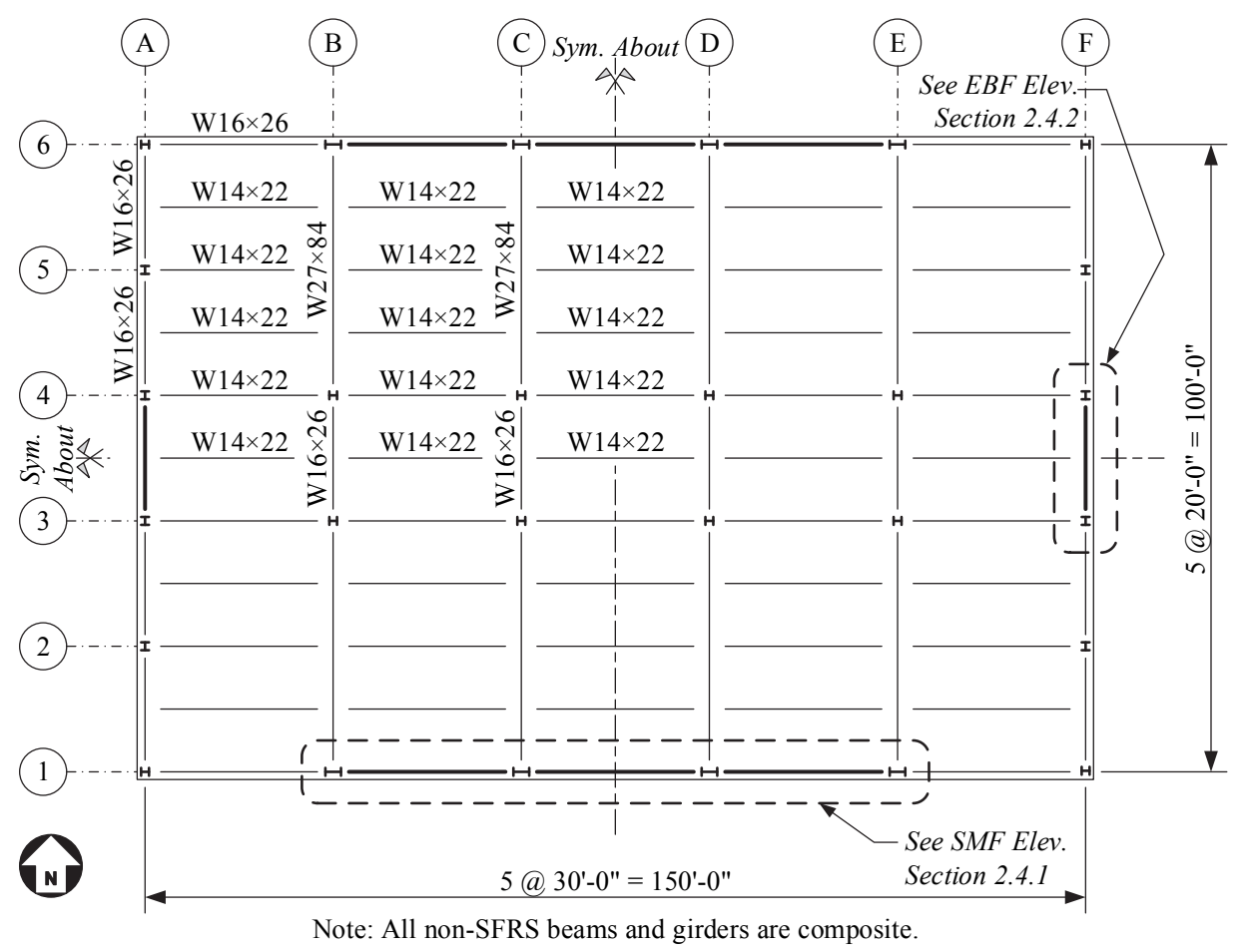

Figure 2-4. Typical Floor Framing Plan, ME4 and ME8

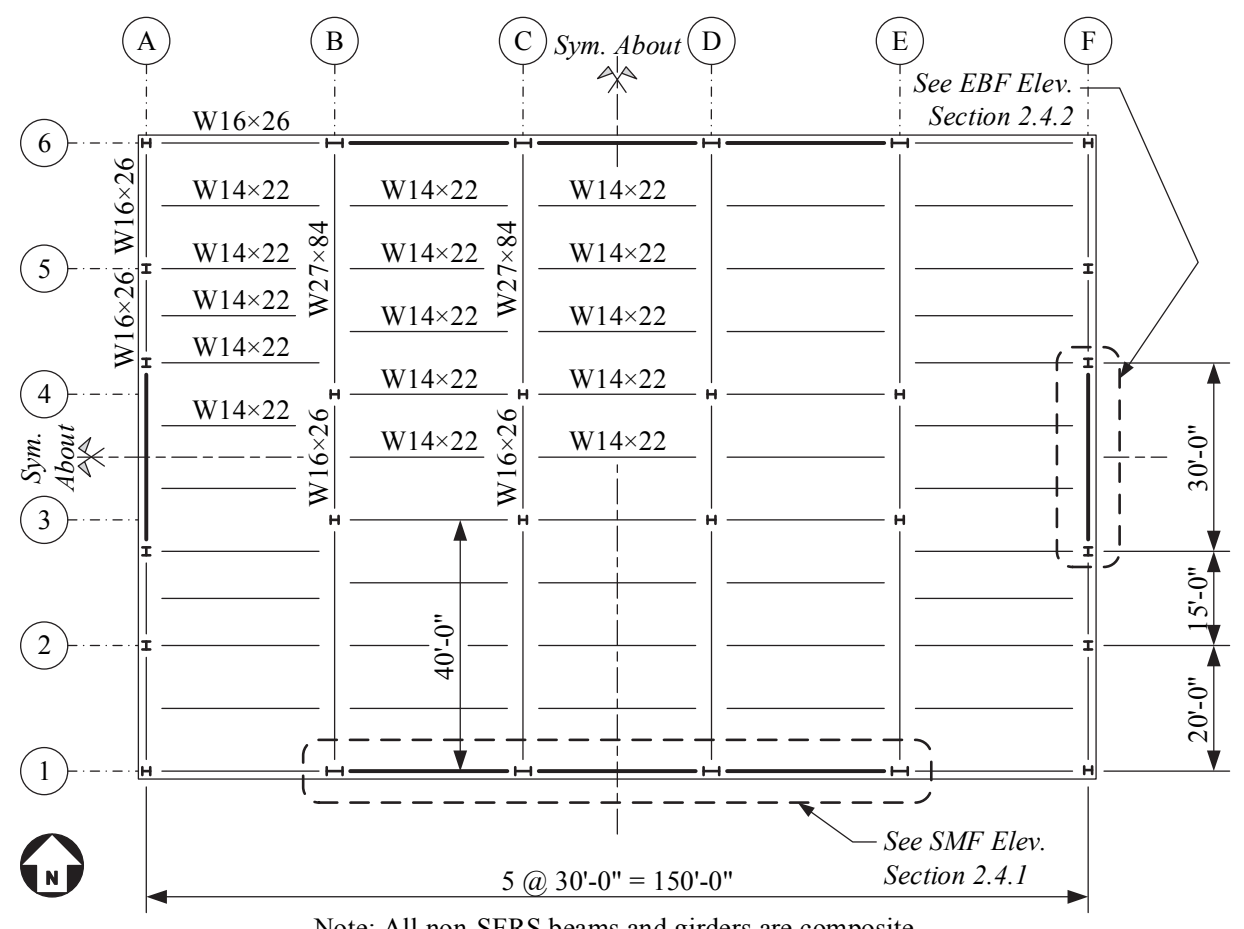

Figure 2-5. Typical Floor Framing Plan, ME16 
The archetype buildings are analyzed and designed for all load effects in accordance with the following:

- IBC 2012: International Building Code (ICC 2012b)

- ASCE 7-10: Minimum Design Loads for Buildings and Other Structures (ASCE 2010)

- AISC 360-10: Specification for Structural Steel Buildings (AISC 2010a)

- AISC 341-10: Seismic Provisions for Structural Steel Building (AISC 2010b)

- AISC 358-10: Prequalified Connections for Special and Intermediate Steel Moment Frames for Seismic Applications (AISC 2010c)

The following material types and corresponding nominal properties were assumed in design:

- Wide-Flange Sections: A992 Grade $50, F_{y}=50 \mathrm{ksi}, R_{y}=1.1$

- HSS Sections: A500 Grade B, $F_{y}=46 \mathrm{ksi}, R_{y}=1.4$

- Connections: A572 Grade 50, $F_{y}=50 \mathrm{ksi}, R_{y}=1.1$

- $E=29000 \mathrm{ksi}, G=11200 \mathrm{ksi}, \nu=0.3$

The archetype buildings do not contain any geometry-based or configuration-based horizontal irregularities, Type 2, 3, 4, or 5 as defined in ASCE 7 Table 12.3-1. Horizontal irregularity Type 1 is dependent on postdesign analysis verification, and is presented in Appendix B. Similarly, the archetype buildings do not contain any geometry-based or configuration-based vertical irregularities, Type 2, 3, or 4 as defined in ASCE 7 Table 12.3-2. Vertical irregularity Types 1 and 5 are dependent on post-design analysis verification and are presented in Appendix B. The buildings are classified as Risk Category II structures in accordance with ASCE $7 \S 1.5$.

\subsection{Structural Design Loads}

\subsubsection{Load Combinations}

Loads and load combinations used for analysis and strength design of members and connections are in accordance with ASCE 7 §2.3, including modifications to these combinations prescribed in ASCE 7 §12.4. This resulted in 189 load combinations for design of each component. Capacity design provisions for each SFRS type prescribed in AISC 341 as well as for beam-to-column connections in an SMF prescribed in AISC 358 provided several design load combinations in addition to those from ASCE 7.

Loads and load combinations for serviceability analysis and verification (e.g., wind drift), and seismic drift analysis and allowable drift compliance verification are discussed subsequently under Environmental Loads, §2.2.3.

\subsubsection{Gravity Loads}

The floor and roof dead load consists of the weight of the steel members, metal deck, and concrete slab weight ( $3 \frac{1 / 4}{4}$ inch lightweight concrete at $110 \mathrm{pcf}$ on 18 -gage, 3 inch metal deck $\left.\approx 46 \mathrm{psf}\right)$. Superimposed dead loads are taken as 15 psf for floors and 10 psf for the roof, representing mechanical, electrical, plumbing, and miscellaneous dead loads. A 250 plf superimposed dead load is also applied to the perimeter 
horizontal framing to account for façade (curtain wall) weight. The edge of the slab is 1 foot from the perimeter framing. The design live load (unreduced) is $50 \mathrm{psf}$ for floors and $30 \mathrm{psf}$ for the roof (increased live load within egress areas is neglected in this study). A summary of the design gravity loads is presented in Table 2-2.

Table 2-2. Design Gravity Loads

\begin{tabular}{ccc}
\hline Load & Load Type & Magnitude \\
\hline Dead, $D$ & Dead & $46 \mathrm{psf}^{1}$ \\
Floor Superimposed Dead, $S D$ & Dead & $15 \mathrm{psf}$ \\
Roof Superimposed Dead, $S D$ & Dead & $10 \mathrm{psf}$ \\
Façade Dead (Curtain Wall), $S D$ & Dead & $250 \mathrm{plf}$ \\
Unreduced Design Floor Live, $L_{o}$ & Floor Live & 50 psf $($ Office $)$ \\
Unreduced Design Roof Live, $L_{o}$ & Roof Live & 30 psf $^{2}$ \\
\hline Theight of slab and metal deck only. Self-weight of steel components are included automatically in the structural analysis. \\
${ }^{2} 10$ psf was added to the roof live load to represent non-inertial service equipment weight.
\end{tabular}

Verification of serviceability criteria under gravity loads is performed per IBC $\S 1604.3$, ASCE $7 \S 1.3 .2$, and AISC 360 Chapter L.

\subsubsection{Environmental Loads}

\subsubsection{Earthquake Forces}

The archetype buildings are located where it is assumed they would be assigned a Seismic Design Category (SDC) at the upper limit of D (i.e., $D_{\max }$ - see footnote 6). Two designs are produced for each archetype building height and frame type as follows:

- One design using the ELF procedure per ASCE $7 \S 12.8$ to determine the equivalent seismic effects.

- One design using the RSA procedure per ASCE 7 \$12.9 to determine the equivalent seismic effects.

Two designs are performed to provide a common range of potential system strengths for seismic assessment using ASCE 41, and to a lesser extent, provide comparison points between the two design methodologies. There are cases when the two designs do not result in different member sizes because of material-specific minimum requirements. Further, wind effects are determined from statically applied design forces and thus the analysis method for wind does not vary between the two seismic analyses.

For the RSA procedure, enough modes are included in each principal direction to exceed 90 percent mass participation in both horizontal orthogonal directions. Masses were not modeled in the analysis to address vertical accelerations. Design forces determined from the RSA are scaled up so that the total modal base shear for design is equal to 85 percent of the corresponding base shear from applying the ELF procedure; story drifts are not scaled for verifying seismic drift compliance. Application of orthogonal seismic forces and accidental eccentricity prescribed in ASCE $7 \S 12.5$ and ASCE $7 \S 12.8 .4$, respectively, are considered in the strength design analysis. The redundancy factor, $\rho$, is taken as 1.0 for each SFRS, and therefore does not affect the allowable seismic drift limits along the E-W direction (moment frames).

\footnotetext{
${ }^{6}$ See FEMA P695: Quantification of Building Seismic Performance Factors (FEMA 2009a) for further information.
} 
Effective seismic weights for computing the horizontal earthquake forces are determined from dead loads plus 20 percent of the unreduced design floor live loads to represent partition weight (i.e., $0.2 \times 50 \mathrm{psf}=10$ psf). The effective seismic weights (lumped at each level) are tabulated in Appendix B. It is assumed in this study that there is no snow load on the building.

The story gravity loads for seismic drift analysis prescribed in ASCE 7 \$12.8.6 (including period calculation) and stability verification prescribed in ASCE $7 \$ 12.8 .7$ are determined from dead loads plus 25 percent of the unreduced floor live loads (i.e., $0.25 \times L_{o} \approx 0.5 L$ where $L$ is the reduced floor live loads). Roof live loads are considered not to be present for seismic drift analysis. The effective lumped gravity load acting on a story is tabulated in Appendix B. Vertical seismic loads are considered for strength design but not for drift or stability compliance. Similarly, application of orthogonal seismic forces and accidental eccentricity are not considered in the drift analysis because story drifts are computed at the center of mass (which aligns with the center of stiffness) of each story because of building symmetry and regularity. The centers of mass for all stories are vertically aligned.

The seismic hazard in ASCE 7 is based on a risk-targeted design philosophy and is defined as ground motions having a one percent probability of causing total or partial structural collapse (i.e., "risk") of an appropriately designed structure in 50 years (except in areas controlled by the deterministic cap ${ }^{7}$ on ground

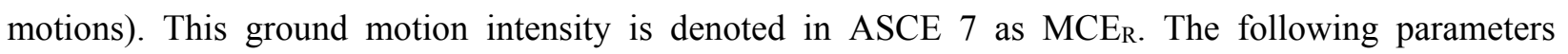
summarize the seismic hazard used for design:

- Building Risk Category: II

- Site Soil Conditions: Site Class D, Stiff Soil - ASCE 7 Table 20.3-1

- Spectral Response Acceleration Parameters: shown in Table 2-3

- $\quad$ SDC: D-taken as $D_{\max }$ as used in FEMA P695

Table 2-3. Spectral Response Acceleration Parameters

\begin{tabular}{cccccccccc}
\hline $\mathrm{SDC}$ & $\begin{array}{c}S_{s} \\
(g)\end{array}$ & $\begin{array}{c}S_{1} \\
(g)\end{array}$ & $F_{a}$ & $F_{v}$ & $\begin{array}{c}S_{M S} \\
=F_{a} S_{s} \\
(g)\end{array}$ & $\begin{array}{c}S_{M 1} \\
=F_{v} S_{l} \\
(g)\end{array}$ & $\begin{array}{c}S_{D S} \\
=2 / 3 S_{M S} \\
(g)\end{array}$ & $\begin{array}{c}S_{D 1} \\
=2 / 3 S_{M 1} \\
(g)\end{array}$ & $\begin{array}{c}3.5 \times T_{S} \\
(\mathrm{sec})\end{array}$ \\
\hline $\mathrm{D}_{\max }$ & 1.50 & $0.60^{1}$ & 1.00 & 1.50 & 1.50 & 0.90 & 1.00 & 0.60 & 2.1 \\
\hline 1. & $S_{1}$ is actually just under $0.60(i . e ., 0.599)$
\end{tabular}

Allowable seismic drift limit is set to $h_{s x} / 50$ (for amplified story drifts, see ASCE 7 §12.12) where $h_{s x}$ is the story height below the level under consideration. Composite action between the beams of the SFRS and the concrete slab is not considered for checking seismic drifts or when computing the fundamental period, $T_{1}$. This action is commonly neglected in seismic analysis and design because research has shown that the slab does not contribute significantly to the strength or stiffness of the assembly at significant inelastic deformations (see FEMA 355D (FEMA 2000c)).

The seismic analysis and design parameters for each archetype building are provided in Table 2-4 for the E-W direction and Table 2-5 for the N-S direction. There are two archetype building systems that are not

\footnotetext{
${ }^{7}$ Regions where probabilistic-based ground motion parameters exceed those resulting from deterministic ground motions based on the characteristic magnitudes of earthquakes from well-defined active fault systems.
} 
permitted to be designed with the ELF procedure because its design period, $C_{u} T_{a}$, is greater than $3.5 \times T_{s}$ (see ASCE 7 §12.6): E-W component (SMF) and N-S component (EBF) of ME16 - these systems are shaded in Table 2-4 and Table 2-5. These frames are included to make a seismic performance comparison. Furthermore, ASCE 7 is vague about which $T$ is referenced in ASCE 7 \$12.6. For example, although the capped fundamental period $\left(T=C_{u} T_{a}\right)$ may satisfy $3.5 \times T_{s}$, the actual fundamental period $\left(T=T_{1}\right)$ may not, indicating that the ELF procedure may be used for strength design but not used for drift verification per ASCE 7 12.8.6.2. Consequently, the same analysis procedure was used for both strength design and computation of the design story drifts in this study.

Table 2-4. Seismic Analysis and Design Parameters, E-W

\begin{tabular}{|c|c|c|c|c|c|c|}
\hline Building & \multicolumn{2}{|c|}{ ME4 } & \multicolumn{2}{|c|}{ ME8 } & \multicolumn{2}{|c|}{ ME16 } \\
\hline SFRS & \multicolumn{2}{|c|}{ SMF } & \multicolumn{2}{|c|}{ SMF } & \multicolumn{2}{|c|}{ SMF } \\
\hline$R, C_{d}, \Omega_{o}$ & \multicolumn{2}{|c|}{$8,5.5,3$} & \multicolumn{2}{|c|}{$8,5.5,3$} & \multicolumn{2}{|c|}{$8,5.5,3$} \\
\hline$C_{u} T_{a}$ (seconds) & \multicolumn{2}{|c|}{1.04} & \multicolumn{2}{|c|}{1.76} & \multicolumn{2}{|c|}{3.02} \\
\hline ELF Permitted? & \multicolumn{2}{|c|}{ Yes } & \multicolumn{2}{|c|}{ Yes } & \multicolumn{2}{|c|}{$\mathrm{No}^{8}$} \\
\hline Height Limit (feet) & \multicolumn{2}{|c|}{ No Limit } & \multicolumn{2}{|c|}{ No Limit } & \multicolumn{2}{|c|}{ No Limit } \\
\hline Analysis Procedure & ELF & RSA & ELF & RSA & ELF & RSA \\
\hline$W^{1}$ (kips) & 5132 & 5103 & 10566 & 10460 & 21900 & 21659 \\
\hline$V_{b}{ }^{1}$ Design (kips) & 374 & 316 & $467^{2}$ & $394^{2}$ & $958^{2}$ & $810^{2}$ \\
\hline$V_{b}{ }^{1}$ Drift (kips) & 213 & 166 & 273 & 192 & 375 & 295 \\
\hline RSA Scaling Factor ${ }^{3}$ & NA & $\begin{array}{c}\text { Design }=93 \\
\text { Drift }=266\end{array}$ & NA & $\begin{array}{c}\text { Design }=101 \\
\text { Drift }=266\end{array}$ & NA & $\begin{array}{c}\text { Design }=134 \\
\text { Drift }=266\end{array}$ \\
\hline$T_{1}^{4}$ (seconds) & 1.82 & 2.22 & 2.91 & 3.81 & 4.36 & 5.01 \\
\hline$T_{2}{ }^{4}$ (seconds) & 0.55 & 0.61 & 1.02 & 1.30 & 1.58 & 1.83 \\
\hline$T_{3}{ }^{4}$ (seconds) & 0.26 & 0.29 & 0.56 & 0.68 & 0.91 & 1.05 \\
\hline$T_{1}^{5}$ (seconds) & 1.83 & 2.24 & 2.94 & 3.86 & 4.40 & 5.07 \\
\hline$T_{1}{ }^{6}$ (seconds) & 1.77 & 2.12 & 2.79 & 3.55 & 4.15 & 4.70 \\
\hline Steel Wgt. ${ }^{7}$ (tons) & 37 & 29 & 74 & 53 & 193 & 163 \\
\hline \\
\hline \multicolumn{7}{|c|}{$\begin{array}{l}\text { Inertial mass computed from Dead + Superimposed Dead }+0.2 \times \text { Floor Live. } W \text { for ELF and RSA differ because of member size differences. } \\
0.044 S_{D S} I_{e} \text { min } \text { controls strength design (not applied for drift). }\end{array}$} \\
\hline \multicolumn{7}{|c|}{$\begin{array}{l}\text { 0.044S } \text { DSS }_{e} \text { min. controls strength design (not applied for drift). } \\
\text { Scaling for design }=g \times I_{e} / R \times\left(0.85 \times V_{b, E L F}\right) / V_{b, R S A} \text {. Scaling for drift }=g \times I_{e} / R \times C_{d} / I_{e} \text {. Scaling assumes the spectrum is defined as a function of } g \text {. }\end{array}$} \\
\hline \multicolumn{7}{|c|}{$\begin{array}{l}\text { Scaling for design }=g \times I_{e} / R \times\left(0.85 \times V_{b, E L F}\right) / V_{b, R S A} \text {. Scaling for drift }=g \times I_{e} / R \times C_{d} / I_{e} \text {. Scaling assumes the spectrum is defined as a function of } g \text {. } \\
\text { Computed from a second-order eigenvalue analysis with Dead }+ \text { Superimposed Dead }+0.25 \times \text { Floor Live gravity load. }\end{array}$} \\
\hline \multicolumn{7}{|c|}{$\begin{array}{l}\text { Computed from a second-order eigenvalue analysis with Dead + Superimposed Dead + } 0.25 \times \text { Floor Live gravity load. } \\
\text { Computed from a second-order eigenvalue analysis with } 1.2 \times \text { Dead }+1.2 \times \text { Superimposed Dead }+0.25 \times \text { Floor Live gravity load. }\end{array}$} \\
\hline \multicolumn{7}{|c|}{ Computed from a first-order eigenvalue analysis. } \\
\hline \multicolumn{7}{|c|}{ Per single SFRS (see Table 1-1). Does not include connection or miscellaneous steel. } \\
\hline \multicolumn{7}{|c|}{$\begin{array}{l}\text { Per single SFRS (see Table 1-1). Does not include connection or miscellaneous steel. } \\
\text { Analysis procedure not permitted per ASCE } 7 \text { §12.6. Shaded Area: design is included for seismic performance comparison purposes. }\end{array}$} \\
\hline
\end{tabular}

The difference in the stiffness and strength of the EBFs provided by the ELF and RSA procedures is negligible when $h_{n}$ is less than say $60 \mathrm{ft}$. This effect is primarily due to design provisions prescribed in AISC 341 (e.g., minimum width-to-thickness ratios). A summary of the equivalent seismic forces for each archetype building is provided in Appendix B.

\subsubsection{Wind Forces}

Basic wind speeds are taken from the ASCE 7 wind maps based on locations along the west coast that would have a high probability of producing structures assigned to SDC D. The basic wind speed is taken to be $110 \mathrm{mph}$ for the 700 -year wind for strength design of components and $72 \mathrm{mph}$ for the 10 -year wind for verifying story drifts (serviceability). Each archetype building is assigned to Exposure B and is not considered rigid, with gust factors, $G_{f}$, for each principal direction computed assuming two percent damping. Torsional wind effects are considered, and the directionality factor, $k_{d}$, is 0.85 . A summary of the wind forces for each archetype building is provided in Appendix B.

Allowable wind drift limit is set to $h_{s x} / 400$ (elastic) for the 10-year wind. Composite action between the beams of the MWFRS and the concrete slab is considered for checking wind drift and when computing the 
fundamental period, $T_{1}$, for wind vibrations using an average $I_{\text {eff }}$ as recommended in AISC 360 commentary for Chapter I. The same gravity load combination used for the seismic drift analysis is used in the wind drift analysis (see previous discussion under Earthquake Forces—§2.2.3.1).

Not all practitioners will use the 10-year wind to verify drift compliance (see ASCE 7 commentary for Appendix C); the 25-year or 50-year may be used, depending on project-specific requirements. The 10-year wind is considered appropriate for these structures, as the façade (curtain wall) is designed to accommodate large in-plane seismic movements, and period control (i.e., acceleration) is typically not a concern for building geometries in the range used for this investigation.

Table 2-5. Seismic Analysis and Design Parameters, N-S

\begin{tabular}{|c|c|c|c|c|c|c|}
\hline Building & \multicolumn{2}{|c|}{ ME4 } & \multicolumn{2}{|c|}{ ME8 } & \multicolumn{2}{|c|}{ ME16 } \\
\hline SFRS & \multicolumn{2}{|c|}{ EBF } & \multicolumn{2}{|c|}{ EBF } & \multicolumn{2}{|c|}{$\mathrm{EBF}$} \\
\hline$R, C_{d}, \Omega_{o}$ & \multicolumn{2}{|c|}{$8,4,2$} & \multicolumn{2}{|c|}{$8,4,2$} & \multicolumn{2}{|c|}{$8,4,2$} \\
\hline$C_{u} T_{a}$ (seconds) & \multicolumn{2}{|c|}{0.90} & \multicolumn{2}{|c|}{1.48} & \multicolumn{2}{|c|}{2.46} \\
\hline ELF Permitted? & \multicolumn{2}{|c|}{ Yes } & \multicolumn{2}{|c|}{ Yes } & \multicolumn{2}{|c|}{$\mathrm{No}^{8}$} \\
\hline Height Limit (feet) & \multicolumn{2}{|c|}{240} & \multicolumn{2}{|c|}{240} & \multicolumn{2}{|c|}{240} \\
\hline Analysis Procedure & ELF & RSA & ELF & RSA & ELF & RSA \\
\hline$W^{1}$ (kips) & 5132 & 5103 & 10566 & 10460 & 21900 & 21659 \\
\hline$V_{b}{ }^{1}$ Design (kips) & 425 & 359 & 534 & 449 & $964^{2}$ & $810^{2}$ \\
\hline$V_{b}{ }^{1}$ Drift (kips) & 370 & 312 & 370 & 315 & 505 & 474 \\
\hline RSA Scaling Factor ${ }^{3}$ & NA & $\begin{array}{c}\text { Design }=56 \\
\text { Drift }=193\end{array}$ & NA & $\begin{array}{l}\text { Design }=69 \\
\text { Drift }=193\end{array}$ & NA & $\begin{array}{c}\text { Design }=83 \\
\text { Drift }=193\end{array}$ \\
\hline$T_{1}^{4}$ (seconds) & 1.04 & 1.08 & 2.14 & 2.46 & 3.19 & 3.83 \\
\hline$T_{2}{ }^{4}$ (seconds) & 0.41 & 0.42 & 0.72 & 0.79 & 0.99 & 1.11 \\
\hline$T_{3}{ }^{4}$ (seconds) & 0.24 & 0.25 & 0.40 & 0.44 & 0.53 & 0.58 \\
\hline$T_{1}^{5}$ (seconds) & 1.04 & 1.09 & 2.15 & 2.47 & 3.21 & 3.85 \\
\hline$T_{1}^{6}$ (seconds) & 1.03 & 1.07 & 2.09 & 2.38 & 3.11 & 3.68 \\
\hline Steel Wgt. ${ }^{7}$ (tons) & 11 & 11 & 28 & 21 & 123 & 88 \\
\hline \\
\hline \multicolumn{7}{|l|}{$\frac{\text { Notes: }}{1 \quad \text { Inerti: }}$} \\
\hline \multicolumn{7}{|c|}{$20.044 S_{D S} I_{e}$ min. controls strength design (not applied for drift). } \\
\hline \multirow{2}{*}{\multicolumn{7}{|c|}{3 Scaling for design $=g \times I_{e} / R \times\left(0.85 \times V_{b, E L F}\right) / V_{b, R S A}$. Scaling for drift $=g \times I_{e} / R \times C_{d} / I_{e}$. Scaling assumes the spectrum is defined as a function of $g$. }} \\
\hline & & & & & & \\
\hline \multicolumn{7}{|c|}{$\begin{array}{l}4 \text { Computed from a second-order eigenvalue analysis with Dead + Superimposed Dead }+0.25 \times \text { Floor Live gravity load. } \\
5 \\
\text { Computed from a second-order eigenvalue analysis with } 1.2 \times \text { Dead }+1.2 \times \text { Superimposed Dead }+0.25 \times \text { Floor Live gravity load. }\end{array}$} \\
\hline \multicolumn{7}{|c|}{$\begin{array}{l}5 \text { Computed from a second-order eigenvalue analysis with } 1.2 \times \text { Dead }+1.2 \times \text { Superimposed Dead }+0.25 \times \text { Floor Live gravity load. } \\
6 \text { Computed from a first-order eigenvalue analysis. }\end{array}$} \\
\hline \multicolumn{7}{|c|}{$\begin{array}{l}\text { Computed from a first-order eigenvalue analysis. } \\
\text { Per single SFRS (see Table 1-1). Does not include connection or miscellaneous steel. }\end{array}$} \\
\hline \multicolumn{7}{|c|}{$\begin{array}{l}7 \text { Per single SFRS (see Table 1-1). Does not include connection or miscellaneous steel. } \\
8 \text { Analysis procedure not permitted per ASCE } 7 \S 12.6 \text {. Shaded Area: design is included for seismic performance comparison purposes. }\end{array}$} \\
\hline
\end{tabular}

\subsection{Structural Analysis and Mathematical Model}

The archetype buildings are analyzed in ETABS, ver. 9.7.4 (CSI 2011a). A conventional second-order elastic analysis is used to determine the required strength of components, and member and story deformation demands (elastic and virtual inelastic). This type of analysis uses a constant reduced stiffness matrix based on an initial gravity (i.e., $P-\Delta$ ) load combination applied in a pre-analysis as follows:

- $\quad P-\Delta$ load combination for strength analysis: $1.2 \times$ Dead $+0.25 \times$ Floor Live

- $P-\Delta$ load combination for drift and stability verification analysis: $1.0 \times$ Dead $+0.25 \times$ Floor Live

The analyses do not account for material nonlinearity or geometric imperfections (except for gravity-only load combinations - see AISC 360 Chapter C). Because the stiffness matrix remains constant for all loads, superposition of individual load effects in load combinations is applicable. 
Each building is considered globally restrained horizontally, vertically, and rotationally at the seismic base (taken at grade level). The base columns of the LFRSs are embedded into the foundation wall. As such, column bases are modeled as rotationally restrained in the plane of the frames and rotationally unrestrained out-of-plane. Non-SFRS gravity column bases are considered rotationally unrestrained in both orthogonal directions.

The mathematical models are based on centerline dimensions with rigid end offsets at the beam-to-column, brace-to-beam / column, and brace-to-beam joints with panel zones explicitly modeled based on column web and doubler plate (if required) geometry. The slabs are modeled as semi-rigid membrane diaphragms (no out-of-plane bending stiffness) with a 0.5 in-plane stiffness modifier to account for cracking at the design loads-see NIST GCR 11-917-10: Seismic Design of Composite Steel Deck and Concrete-filled Diaphragms: A Guide for Practicing Engineers (NIST 2011b).

In the moment frames, reduced beam sections (RBS) are explicitly modeled in all SMF beams by subdividing each member into seven segments; the RBS is modeled as two linearly-tapered sections. The typical fully restrained (FR) beam-to-column connection is illustrated in Figure 2-6, and the equivalent assembly model for linear analysis is shown in Figure 2-7.

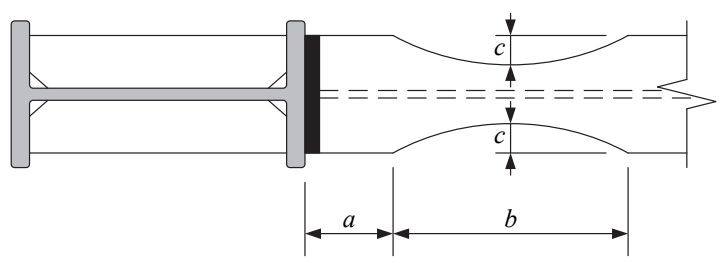

$\underline{\text { Section A-A }}$

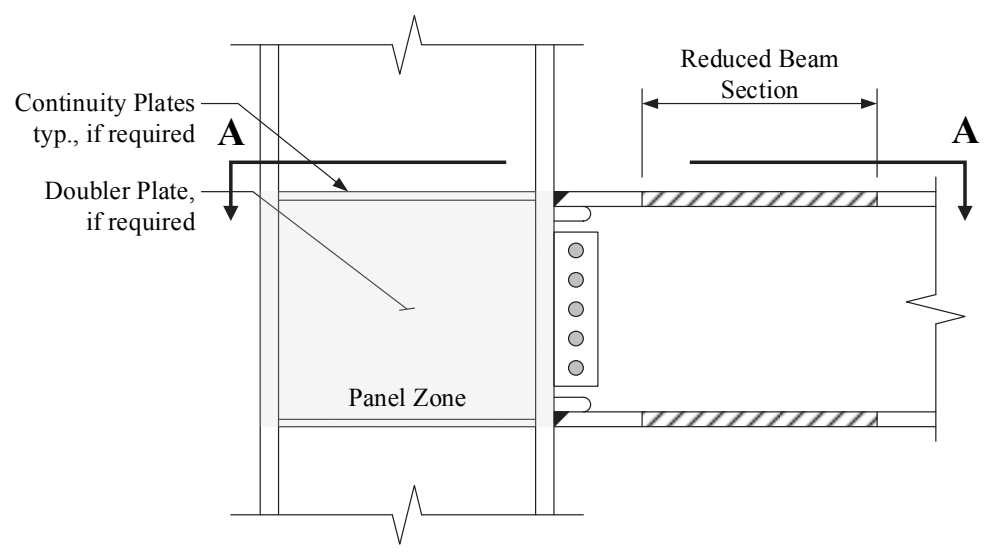

Figure 2-6. Typical Type FR RBS Beam-to-Column Connection Assembly

In the eccentrically braced frame, links beams are modeled with standard beam sections that span between the two work points. Rigid end zones are included to capture gusset plate rigidity outside the link - see Figure 2-8. The member ends of the EBF diagonal braces are rotationally unrestrained out-of-plane and restrained in-plane. Similarly, rigid end zones are included to capture gusset plate rigidity; the tangible length of EBF braces is approximately equal to 90 percent of the distance between work points, $L_{w p}$. Beams 
adjacent to links are fully restrained in the plane of the frame to the adjacent columns. All members were analyzed with gravity loads.

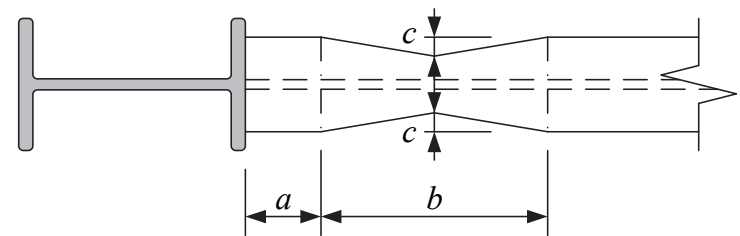

Section A-A

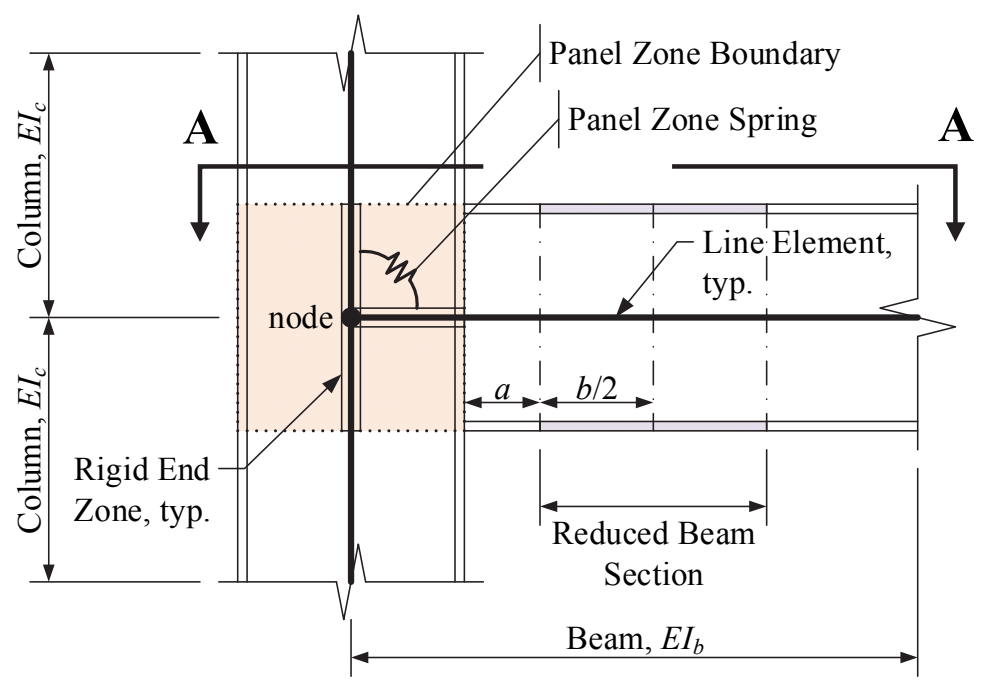

Figure 2-7. Type FR RBS Beam-To-Column Connection Subassembly Model for Linear Analysis

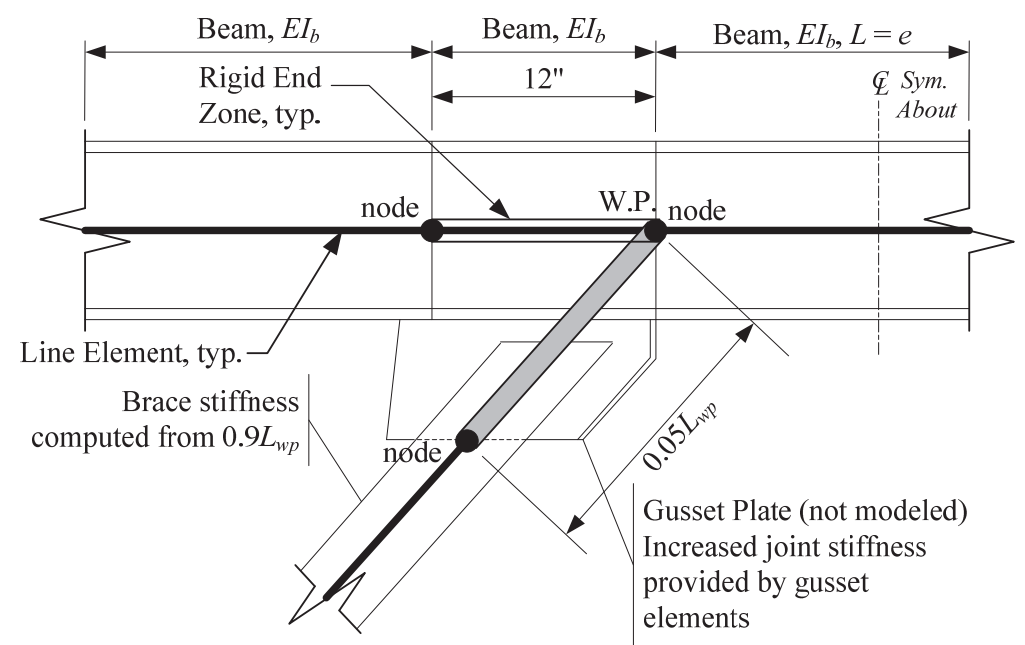

Figure 2-8. Brace-to-Beam Connection Subassembly Model for Linear Analysis

Non-LFRS framing that primarily supports gravity loads is included in the mathematical models to capture the "leaning column" effect. The gravity beams are modeled as composite beams with rotationally 
unrestrained member ends (i.e., shear tab connections). The gravity columns are modeled as continuous along the height of the building and designed for deformation compatibility. The increase in lateral stiffness along the two principal axes due to these columns is negligible. Other than the columns, no other forms of lateral stiffness attributed to non-SFRS framing (e.g., stairs) and nonstructural components are considered in the mathematical model.

\subsection{SFRS Design}

\subsubsection{SMF Design}

SMF designs are included here to provide the required information of the building for EBF designs. For the SMF designs, seismic drift criteria prescribed in ASCE $7 \S 12.12$ tended to control member sizes for both 4-story archetype buildings and the 8-story ELF-designed archetype building. The seismic stability criteria prescribed in ASCE $7 \S 12.8 .7$ tended to control member sizes for the 8-story RSA-designed archetype building and both 16-story archetype buildings (ELF design was more drift controlled than the RSA design). The strong-column / weak-beam (SCWB) criteria using an amplified seismic load (axial) and section compactness requirements provided additional constraint on column sizes where required.

The RBS beam-to-column connections were designed in accordance with AISC 358 with the flange cut out dimensions optimized to produce the lowest probable moment, $M_{p r}$. Because the bay length did not change, the same beam depth was used for a given floor for uniform connection constructability and plastic hinge sequencing optimization (i.e., yield rotation is theoretically equal). The panel zones are designed for demands based on the probable moment of the RBS projected to the column face using nominal material properties. AISC $360 \S \mathrm{J} 10.6$ (b) is applicable for panel zone design because the effect of panel zone deformations on frame stability is explicitly considered in the analysis. The flow chart in Figure 2-9 illustrates the analysis and design process for an SMF. Additional details on design and construction of special moment frames can be found in NIST GCR 09-917-3: Seismic Design of Steel Special Moment Frames: A Guide for Practicing Engineers (NIST 2009b).

To reflect industry practice and reduce fabrication costs, column sizes were increased from that required by analysis rather than adding doubler plates. AISC Design Guide 13 (AISC 1999) and Troup (1999) showed that increasing the column size by up to 100 plf to eliminate both continuity plates and doubler plates was often the more economical alternative. Some seismic specifications (e.g., AISC 341), however, either require or suggest the use of transverse stiffeners in all high seismic applications. For these cases, eliminating the need for doubler plates alone may still prove to be the economical choice (Lee et al. 2002). In this study, this increase typically occurred at interior frame columns and on average was 100 plf for the frame as a whole.

The Effective Length Method (see AISC $360 \S \mathrm{C} 1$ ) is used for design of the SMF columns, with the Story Buckling Method (see commentary for AISC 360 Appendix 7) used to compute the effective length, $K L=$ 
$K_{2} L$ (see footnote 8) for determining the nominal compression strength, $P_{n}$, of the column in the plane of the frame. In all but one SMF, at least one story had $B_{2}$, as defined in AISC 360 Appendix 8, greater than 1.1; all stories were less than 1.5 (see AISC 360 Appendix 7). Tabulated values for $B_{2}$ are provided in Appendix B. $K L=L$ was adopted for determining $P_{n}$ of the column out-of-plane of the frame.

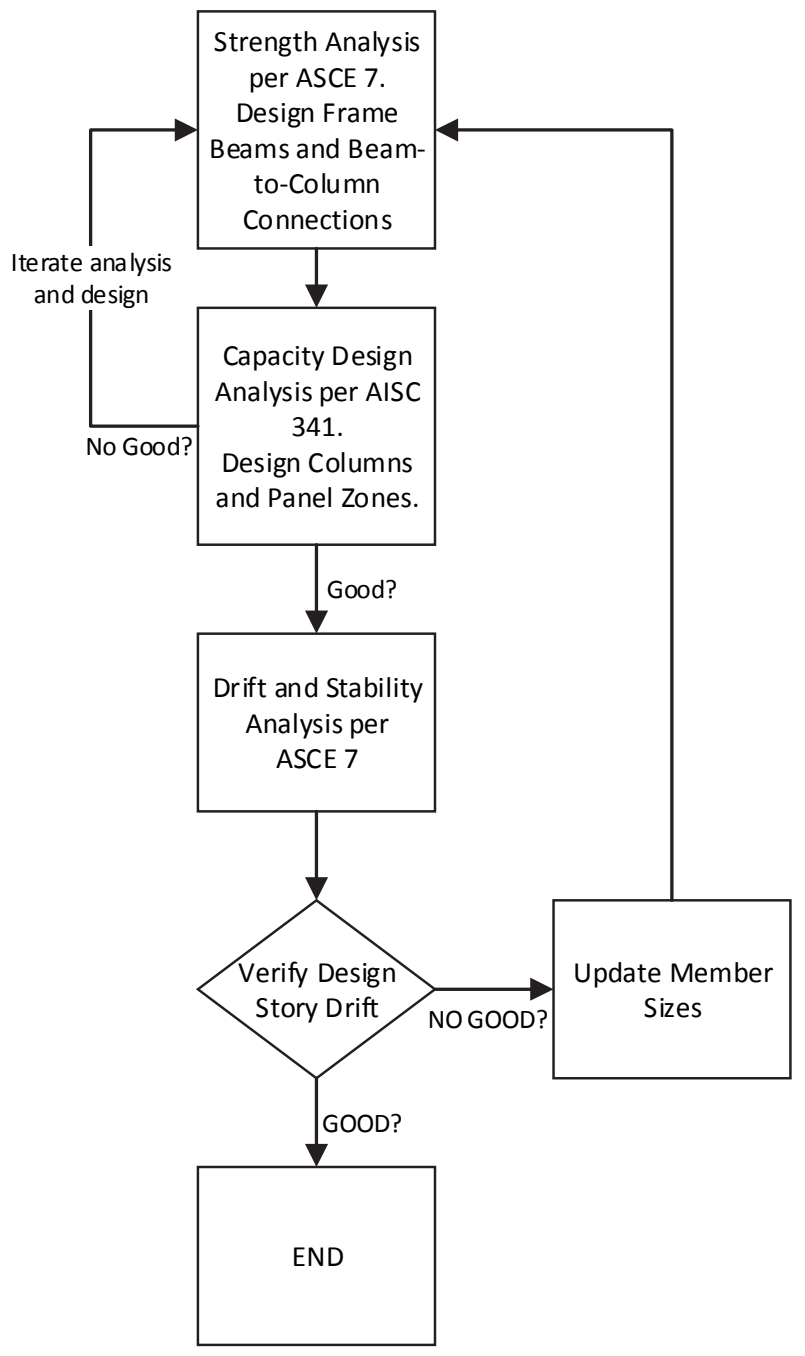

Figure 2-9. Flow Chart of SMF Design Process

Computation of in-plane effective length factors is generally considered to be not required for columns in regular moment frames in high seismicity areas (i.e., adopting $K L=L$ ). Seismic story drift and stability requirements in ASCE 7 typically result in significant in-plane story strength and stiffness above that required by the analysis. However, the concept of story drift control without consideration of its effects on a moment frame is a nontechnical rationale for neglecting the effective length factors for in-plane seismic design of frame columns. Essentially, the effects of drift control reduce the error in calculating the beam-

${ }^{8}$ AISC 360-05 (AISC 2005) used the term $K_{2}$ to define the in-plane effective length factor. AISC 360-10 Appendix 7 uses $K$ in lieu of $K_{2}$. However, $K_{2}$ is still used in the commentary of Appendix 7. Therefore, $K_{2}$ is also used here. Additional information can be found in Steel Design Guide 28: Stability Design of Steel Buildings (AISC 2013). 
column strength for load combinations including lateral forces because of the following (taken from White and Hajjar 1997):

- The in-plane, strong-axis radius of gyration, $r_{x}$, is increased thus lowering $K L / r_{x}$ in the design equation. Column strength varies little with large variations of $K L / r_{x}$.

- The columns are heavily restrained at each end by deep beams, and subjected to nearly doublecurvature bending under sideway of the frame.

- The beam-column interaction check for the lateral force-resisting columns is dominated by the moment term.

Designers must also consider the vertical strength of a story ${ }^{9}$ loaded only with gravity-type loads, as well as the correlation of the actual demand relative to this vertical strength (e.g., $P_{\text {story }} / P_{e, s t o r y}$ as defined in AISC 360 Appendix 8). This story strength would represent the minimum value of $P_{n}$ (in the absence of moment) for construction of the $P-M$ interaction diagram for the in-plane strength of a frame column in the story. Fundamentally, the effective length factor for a column is a function of the frame deformation demands and the loading on the column. Consequently, a $P-M$ interaction diagram would need to be constructed for each category of load combination (e.g., vertical load, vertical plus horizontal loads) considering the influence of the ratio of vertical to horizontal loading demands as well as which loading is being incremented until incipient buckling. Theoretically, $K L=L$ should be adopted when the physical behavior of the column at its ultimate strength aligns with the theoretical definition of the compression strength. Future studies should investigate designing the selected frames using the Direct Analysis Method prescribed in AISC 360 Chapter C.

In proportioning frame members for this study, section depths were selected to maintain a low in-plane relative beam-to-column stiffness ratio, G. AISC 360 commentary for Appendix 7 defines $G$ for bending about the strong-axis as Equation (2-1) - see AISC 360 for definitions of the variables and subscripts.

$$
G=\frac{\sum \tau_{b} \frac{E I_{x, c}}{L_{c}}}{\sum \gamma \frac{E I_{x, b}}{L_{b}}}
$$

Other relationships can be used to show that $G$ can be computed as function of the in-plane flexural section strength, $Z_{x} \times F_{y}$, independent of the in-plane flexural stiffness, $E \times I_{x}$. Equation (2-2) shows that the strongaxis plastic section modulus, $Z_{x}$, is a function of the strong-axis moment of inertia, $I_{x}$, and section depth, $d$. Equation (2-3) rewrites the SCWB equation in AISC 341 (see $\S \mathrm{E} 3.4 \mathrm{a}$ ) for a wide-flange section.

$$
Z_{x}=\frac{2 I_{x}}{d}
$$

\footnotetext{
${ }^{9}$ The vertical story strength, $P_{e, s t o r y}$, is a function of the elastic lateral stiffness of the story.
} 


$$
\begin{gathered}
\frac{M_{p, c}}{M_{p, b}}=\frac{\min \left[1.18\left(1-\frac{P_{u, \Omega}}{P_{y}}\right), 1.0\right] Z_{x, c} F_{y}}{Z_{x, b} F_{y}} \geq 1 \rightarrow \therefore Z_{x, b} \leq \min \left[1.18\left(1-\frac{P_{u, \Omega}}{P_{y}}\right), 1.0\right] Z_{x, c} \\
\therefore G=\frac{\tau_{b}}{\min \left[1.18\left(1-\frac{P_{u, \Omega}}{P_{y}}\right), 1.0\right]} \frac{d_{c}}{d_{b}} \frac{L_{b}}{L_{c}}
\end{gathered}
$$

In the previous equations, $P_{u, \Omega}$ is the axial load demand in the column including the system overstrength factor, $\Omega_{o}$, in ASCE 7 as required by AISC $341 \S E 3.4 \mathrm{a}$. An additional rule of thumb is that $d_{c} \geq d_{b} / 2$ and $d_{c} \leq d_{b}$. For example, an 18-inch deep column and 24-inch deep beam were selected for the SMF in the 8story building. Conservatively assuming a 15 percent reduction in plastic flexural strength of the column leads to $G=1.9$ (see Equation (2-5)). A 27-inch deep column and 36-inch deep beam could also have been selected, but this would have been at the expense of architectural constraints and construction budget.

$$
G=\frac{\tau_{b}}{1.18\left(1-\frac{P_{u, \Omega_{o}}}{P_{y}}\right)} \frac{d_{c}}{d_{b}} \frac{L_{b}}{L_{c}}=\frac{1.0}{0.85} \frac{d_{c}}{d_{b}} \frac{360}{168}=2.52 \frac{18}{24}=1.9
$$

This approach assists in selecting member depths while attempting to minimize relative elastic flexural stiffness variations between the beams and columns within a story, and between adjacent stories. As discussed previously, computing $G$ is not required unless the size of the frame column is governed by strength requirements and one is using the Effective Length Method; tabulated values for $K$ are provided in Appendix B.

The lateral force distributions and story shears for each archetype building are provided in Appendix B. Allowable drift compliance verification is provided in Appendix B. Similarly, verification for drift amplification from global p-delta $(P-\Delta)$ effects and ASCE 7 stability verification are also provided in Appendix B. Design calculations for select members and connections are provided to illustrate the design process in Appendix B.

The 4-, 8-, and 16-story SMF frame designs are shown in Figure 2-10 through Figure 2-12. Each figure shows both the RSA design (a) and the ELF design (b) - underlined member sizes indicate changes from the RSA design. These figures also provide the RBS dimensions and fundamental periods of the archetype building for various gravity loads (e.g., drift and strength design). 


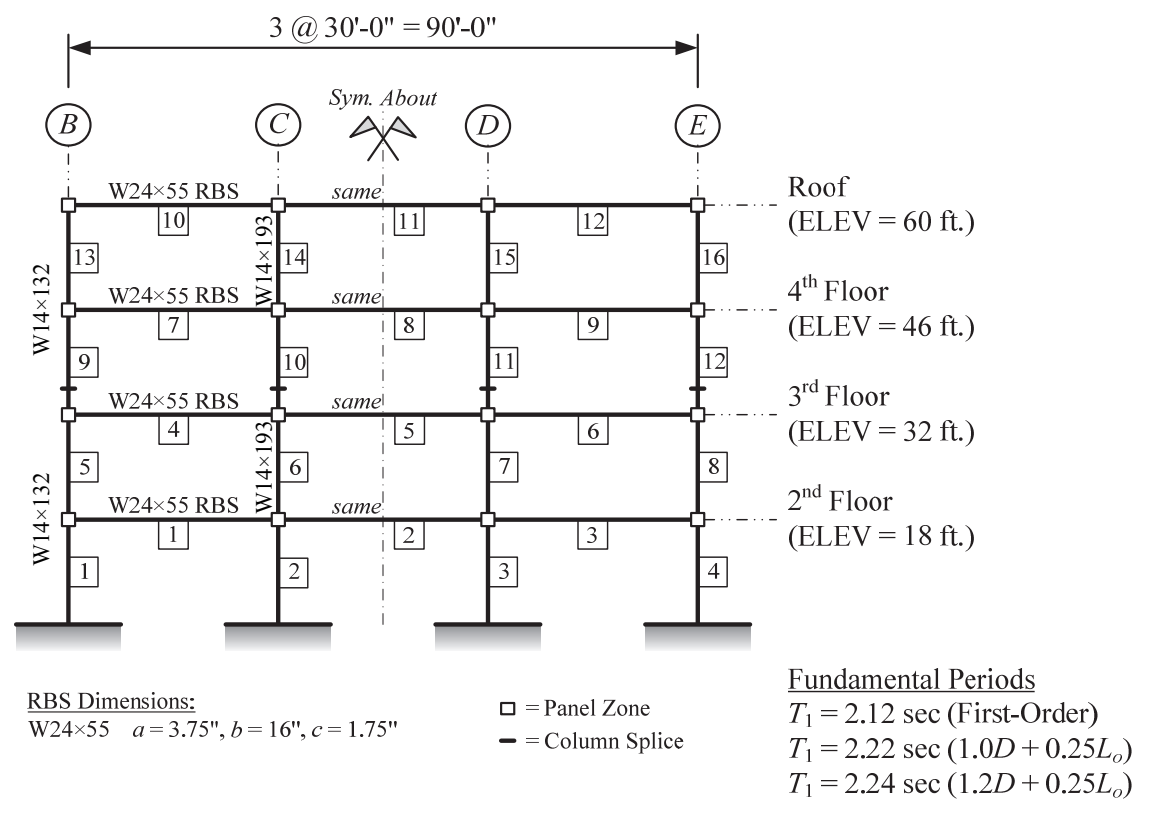

(a) RSA

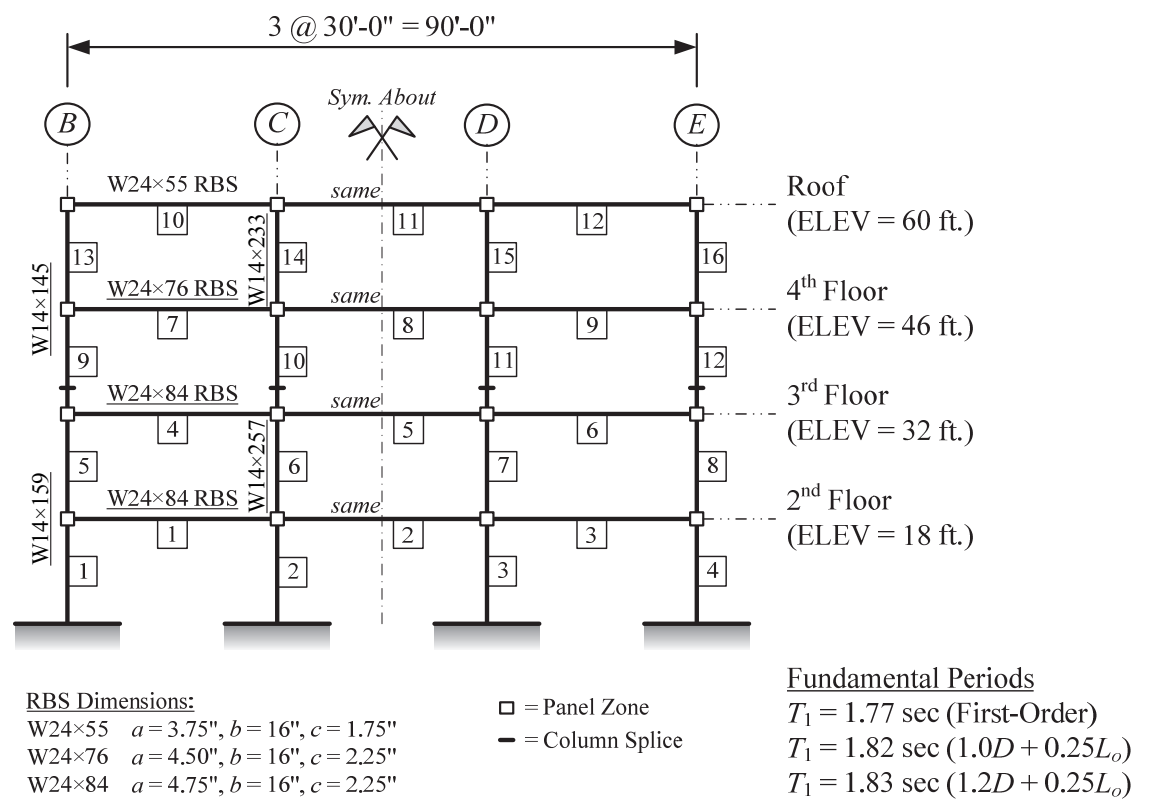

(b) ELF

Figure 2-10. 4-Story SMF Schematic 


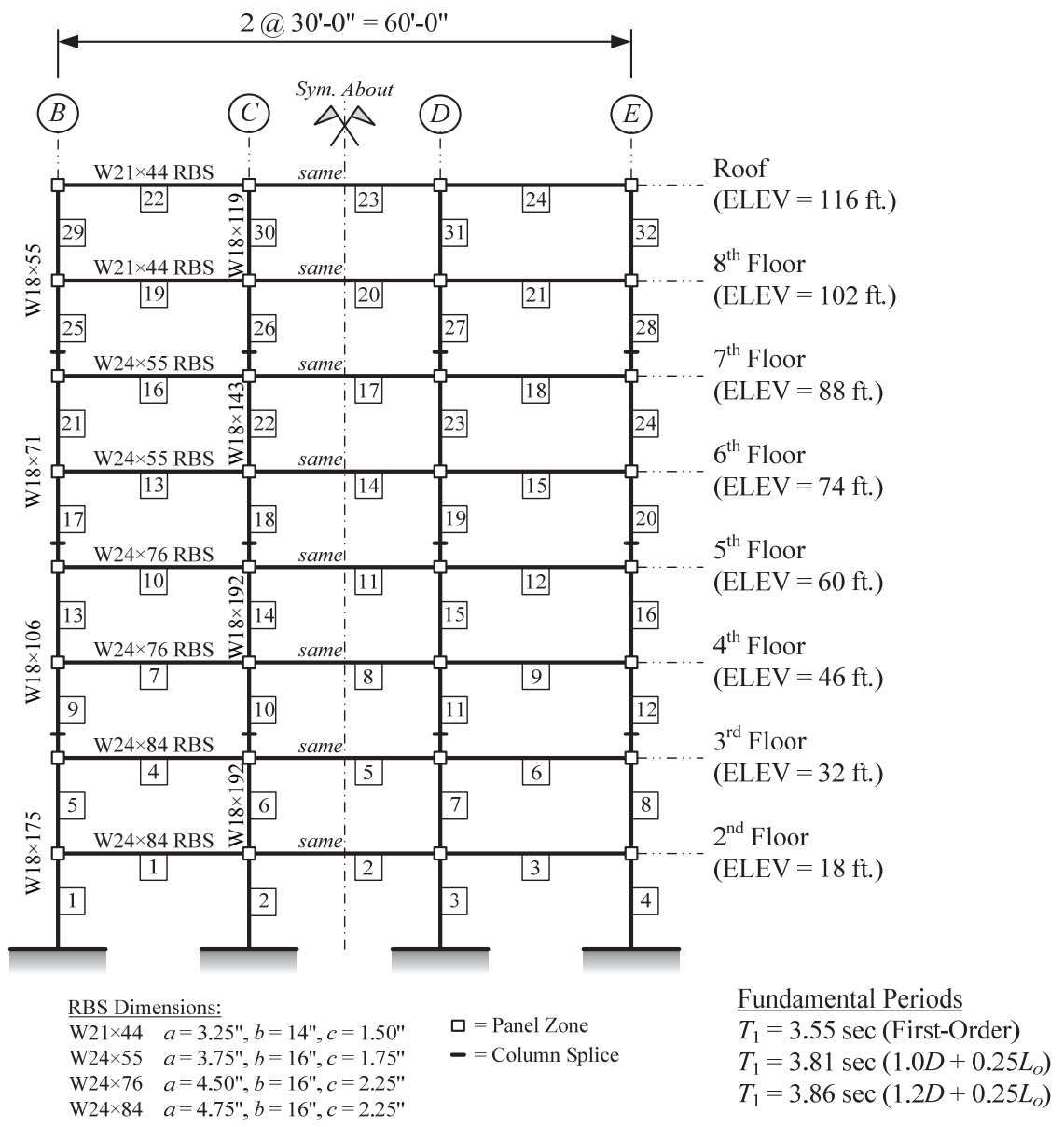

(a) RSA

Figure 2-11. 8-Story SMF Schematic 


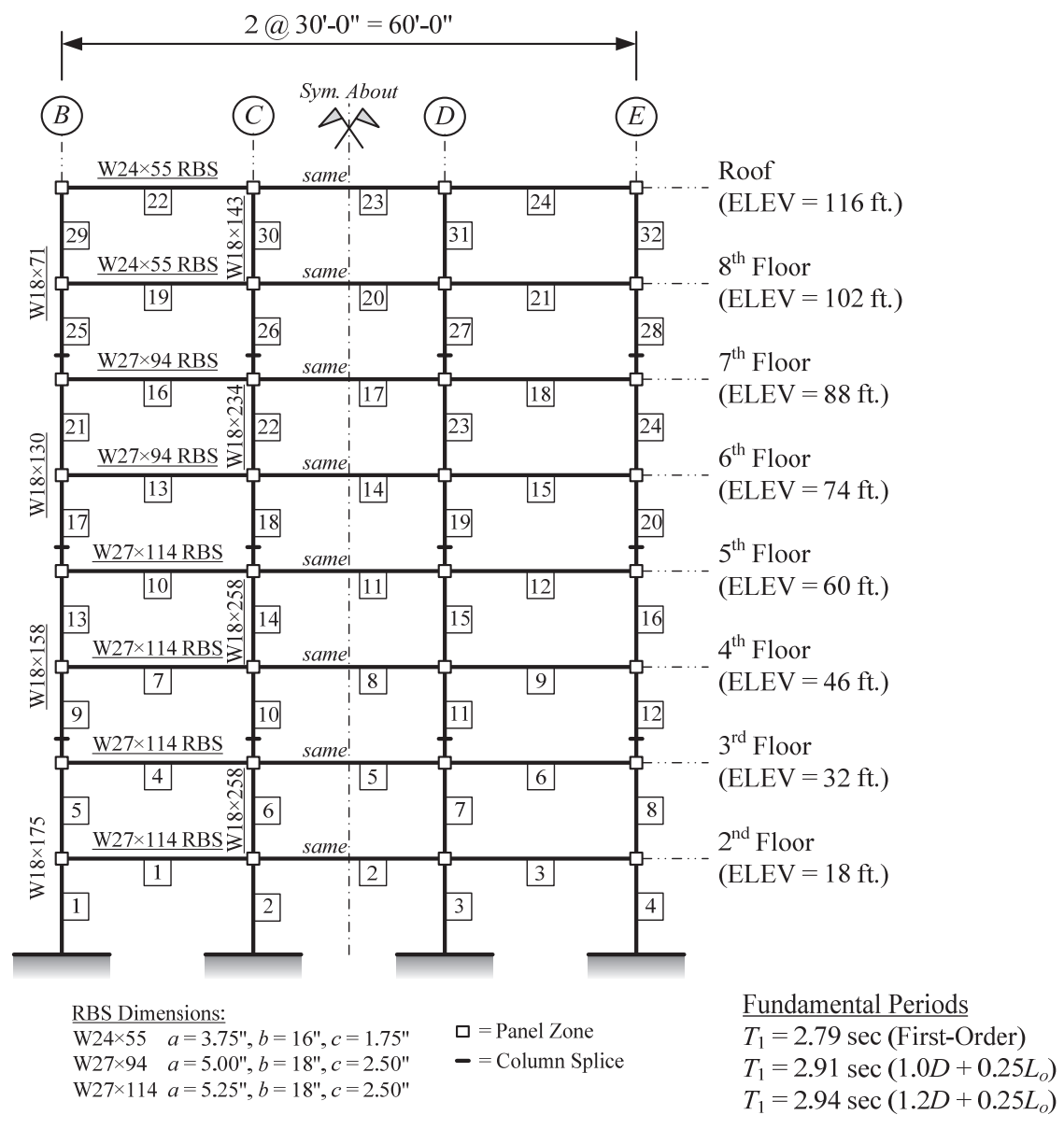

(b) ELF

Figure 2-12. 8-Story SMF Schematic, Cont'd 


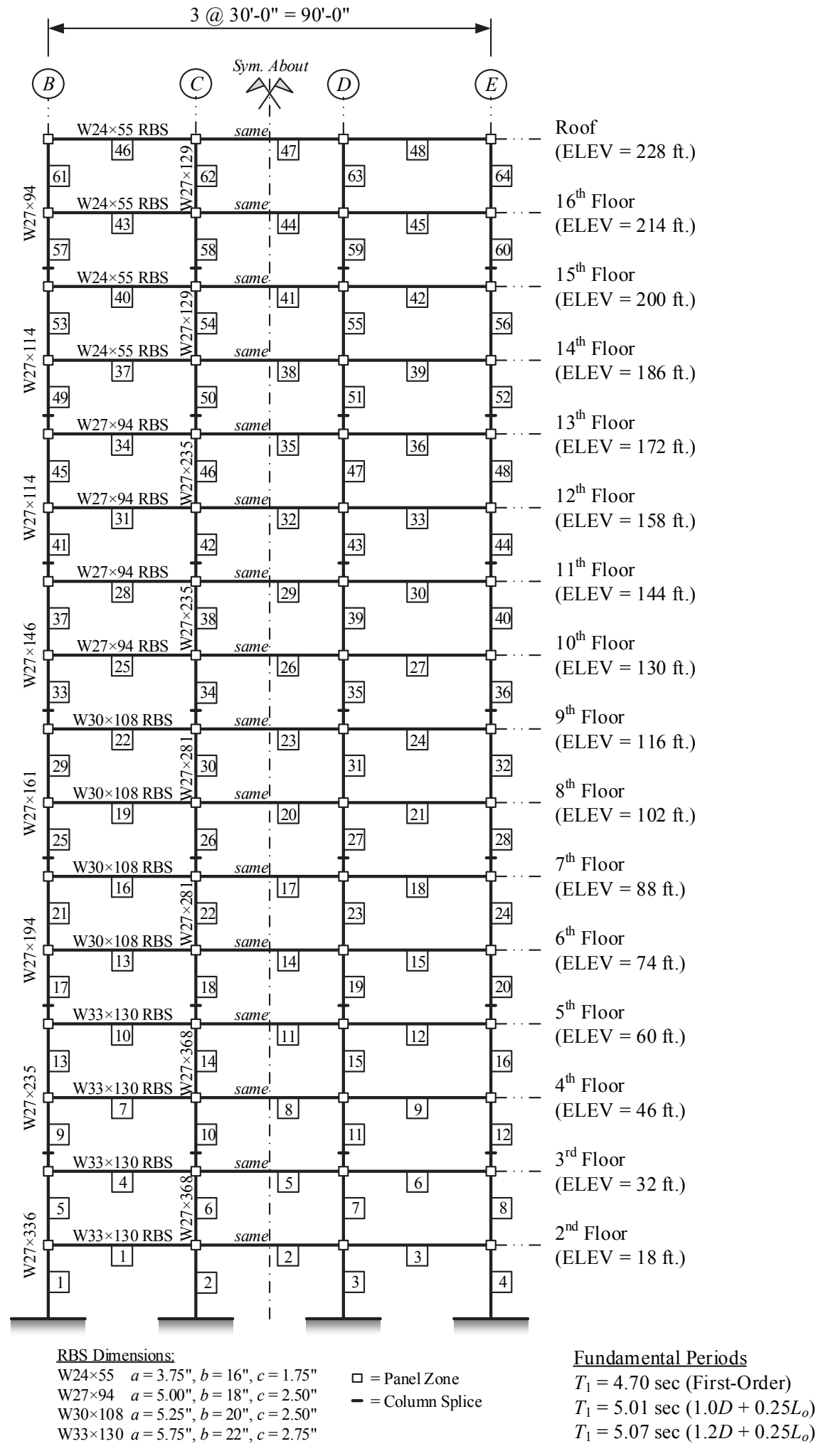

(a) RSA

Figure 2-12. 16-Story SMF Schematic 


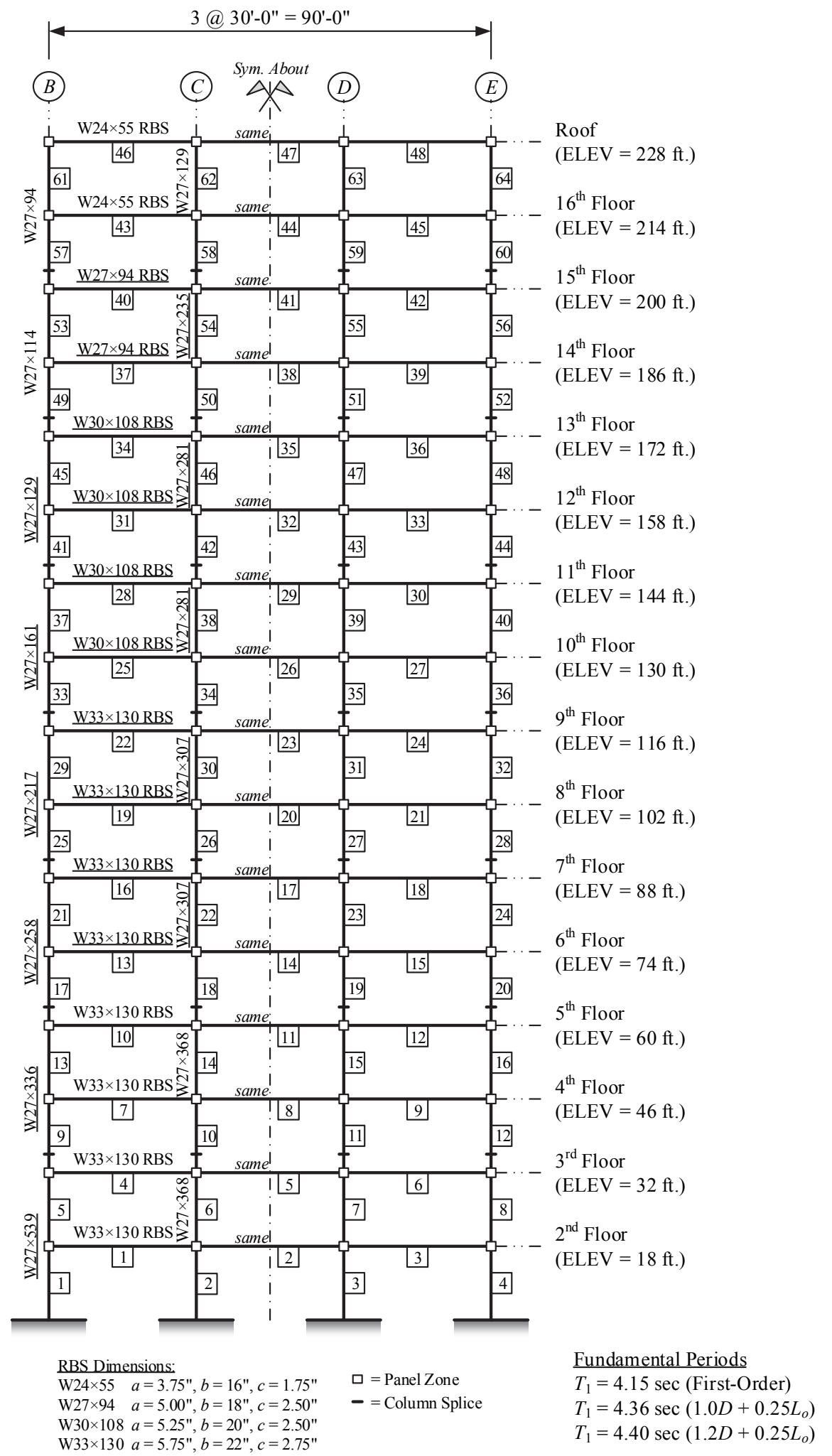

(b) ELF

Figure 2-13. 16-Story SMF Schematic, Cont'd 


\subsubsection{EBF Design}

For the EBF designs, the seismic strength requirements prescribed in ASCE 7 controlled the link beam sizes for all archetype buildings. Section compactness and capacity design requirements prescribed in AISC 341 governed brace sizes and initial column sizes. Limiting the plastic link rotation angle (i.e., story drift) to the allowable permitted by AISC 341 governed the size of columns in the 8- and 16-story ELF-designed frames. These columns are oversized from that required by ASCE 7 analysis to increase the flexural stiffness of these frames. The elastic lateral drift of a slender braced frame can be dominated by the global flexural mode of deformation more than the shear mode because of the prescribed lateral force distribution in ASCE 7. This deformation patter can result in apparent high link rotation demands in the upper stories of tall frames, e.g., 8-stories or higher. Figure 2-13 illustrates the analysis and design process for an EBF.

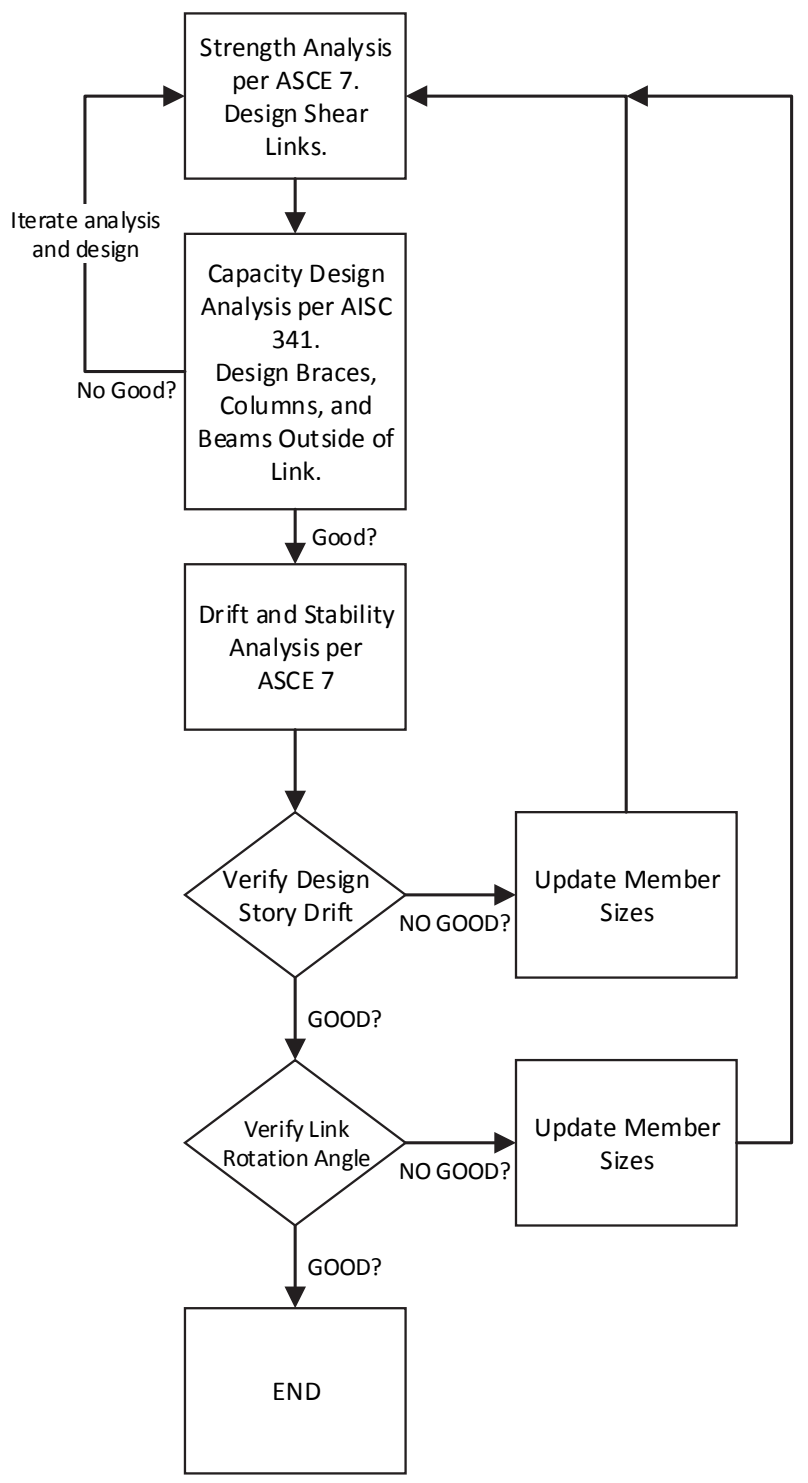

Figure 2-13. Flow Chart of EBF Design Process 
The Effective Length Method (see AISC $360 § C 1$ ) is used for design of the EBF braces, beams (adjacent to shear link beams), and columns. The effective length factor, $K$, was conservatively taken as unity for determining the nominal compression strength, $P_{n}$, of the EBF members, although a lower value could be justified by analysis.

Table 2-6 summarizes the EBF layout for each building. Using a single bay for the SFRS results in $\rho=1.3$ per ASCE 7 \$12.3.4. However, the increase of $\rho$ to 1.3 was neglected in the design to minimize member overstrength contributions in the seismic performance assessment.

Table 2-6. EBF Configurations

\begin{tabular}{cccccc}
\hline Stories & Design & $\begin{array}{c}\text { No. of bays / } \\
\text { frame line }\end{array}$ & $\begin{array}{c}\text { No. of frame } \\
\text { lines }\end{array}$ & $\begin{array}{c}\text { Bay width, } L_{b} \\
\text { (ft.) }\end{array}$ & $\begin{array}{c}\text { Link length, } e \\
\text { (in.) }\end{array}$ \\
\hline \multirow{2}{*}{4} & ELF & 1 & 2 (exterior) & 20 & 30 \\
& RSA & 1 & 2 (exterior) & 20 & 30 \\
8 & ELF & 1 & 2 (exterior) & 20 & 30 \\
& RSA & 1 & 2 (exterior) & 20 & 30 \\
16 & ELF & 1 & 2 (exterior) & 30 & 39 \\
& RSA & 1 & 2 (exterior) & 30 & 39 \\
\hline
\end{tabular}

A 30-foot bay was adopted to minimize nonseismic contributions to member selections. This configuration change is similar to that done for the design of the 16-story SCBF in NIST TN 1863-2: Assessment of First Generation Performance-Based Design Methods for New Steel Buildings, Volume 2: Special Concentrically Braced Frames (Harris and Speicher 2015b). However, wind strength requirements governed link beam design within the lower stories of the 16-story archetype buildings, as shown in Figure 2-14. Required shear strengths of link beams governed by seismic or wind necessitated two different design equations for computing the nominal shear strength, $\phi_{v} V_{n}$, depending on the controlling load. Switching equations also assisted in minimizing nonseismic contributions to link beam design.

$$
\begin{gathered}
\text { Design for Wind (per AISC 360) } \rightarrow \phi_{v} V_{n}=(1.0) 0.6 F_{y} d t_{w} \\
\text { Design for Seismic (per AISC 341) } \rightarrow \phi_{v} V_{n}=(0.9) 0.6 F_{y}\left(d-2 t_{f}\right) t_{w}
\end{gathered}
$$

where $d$ section depth, $t_{f}=$ flange thickness, and $t_{w}=$ web thickness. Using AISC 341 purposefully to design for wind-controlled required strengths would have resulted in approximately a 20 percent increase in member design overstrength of the link as compared to that required for seismic design alone. In Figure 2-14, the nominal story shear strength, $V_{n, \text { story }}$, is computed using Equation (2-8).

$$
V_{n, s t o r y}=\sum \phi_{v} V_{n} \frac{L_{b}}{h_{s x}}
$$

where $L_{b}=$ the bay width and $h_{s x}=$ story height. Short links ( $e \leq 1.6 M_{p} / V_{p}$, see AISC 341 Chapter F3) are selected to achieve maximum shear strain ductility for each design; link beam length is kept constant over the height of the building for constructability and architectural considerations. A minimum link depth of 14 inches (W14) is selected for connection constructability and gravity-load carrying capacity. Consequently, 
this minimum depth contributes to design overstrength of the link beams in the upper stories where story shear demands are relatively low.

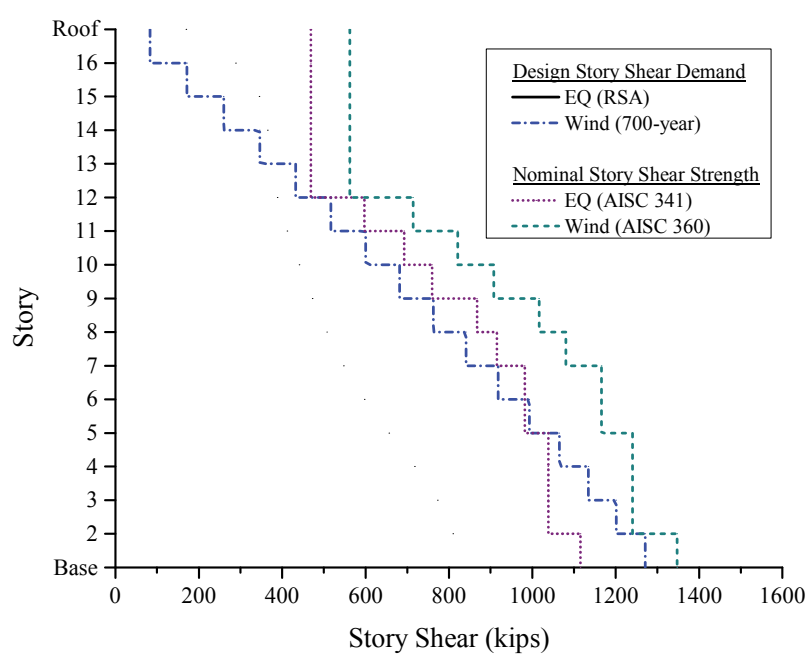

(a) RSA

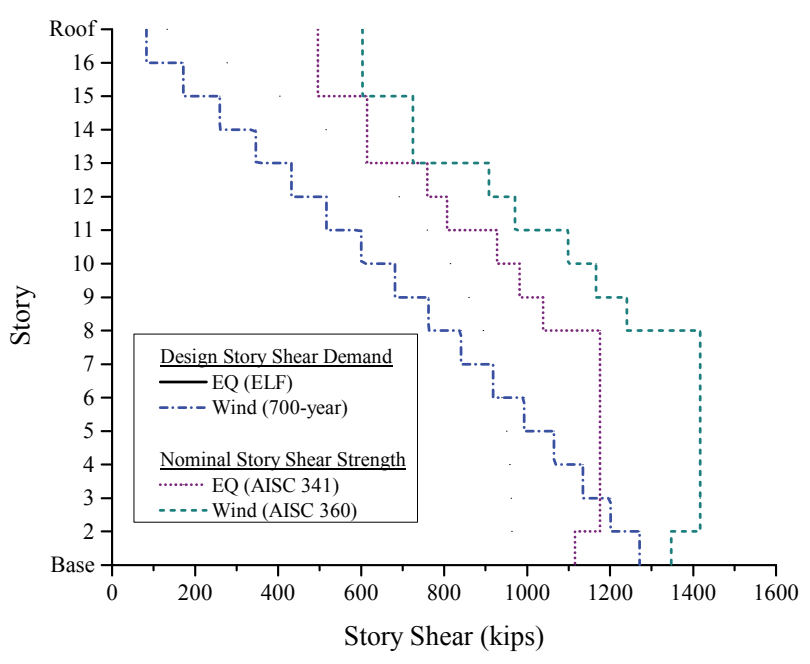

(b) ELF

Figure 2-14. Story Shear Demands and Nominal Strengths, 16-story EBF (N-S ME16)

In reference to the allowable story drift, assuming a rigid-plastic story mechanism, the (plastic) link rotation angle, $\gamma_{p}$, in a given story is

$$
\gamma_{p}=\frac{L_{b}}{e} \frac{\Delta_{x p, s}}{h_{s x}}
$$

where $L_{b}=$ the bay width, $e=$ the link length, $h_{s x}=$ story height, $\Delta_{x p, s}=$ the global plastic story shear distortion contribution of the design story drift.

In reference to ASCE 7, the design story drift of story $x, \Delta_{x}$, can be computed from Equation (2-10), which is the summation of the elastic, $\Delta_{x e}$, and plastic, $\Delta_{x p}$, story drifts. Each story drift component can be divided into a global story shear and flexural deformation components, denoted in Equation (2-10) using the subscript $s$ and $f$, respectively.

$$
\frac{\text { design }, \Delta_{x}}{\frac{C_{d}}{I_{e}}\left(\Delta_{x e, s}+\Delta_{x e, f}\right)}=\frac{\text { elastic }, \Delta_{x e}}{\Delta_{x e, s}+\Delta_{x e, f}}+\frac{\text { plastic }, \Delta_{x p}}{\Delta_{x p, s}+\Delta_{x p, f}}
$$

The plastic story drift component is therefore equal to the difference between the design story drift and the elastic story drift component. 


$$
\Delta_{x p}=\Delta_{x p, s}+\Delta_{x p, f}=\left(\frac{C_{d}}{I_{e}}-1\right)\left(\Delta_{x e, s}+\Delta_{x e, f}\right)
$$

Global flexural deformations do not theoretically contribute to link rotations. Ideally plastic story flexural deformations can be removed to get the story shear deformations.

$$
\Delta_{x p}=\Delta_{x p, s}=\left(\frac{C_{d}}{I_{e}}-1\right)\left(\Delta_{x e, s}+\Delta_{x e, f}\right)-\Delta_{x p, f}
$$

Assuming that elastic displacement is equivalent to yield displacement, it can rationally be assumed that the contribution of the plastic story flexural deformations to plastic story drift is theoretically zero.

$$
\begin{aligned}
& \Delta_{x p}=\Delta_{x p, s}=\left(\frac{C_{d}}{I_{e}}-1\right) \Delta_{x e} \\
& \therefore \Delta_{x p, s}=\Delta_{x}-\frac{\Delta_{x}}{4}=\frac{3}{4} \Delta_{x}
\end{aligned}
$$

Design link rotation angles are provided in Appendix B.

HSS are used as diagonal braces in the 4- and 8-story archetype buildings, while keeping face dimensions within the adjacent column flange widths. Wide-flange sections are used as diagonal braces in the 16-story archetype building. The tangible length of the braces is taken equal to 90 percent of the distance between work points, $L_{w p}$. The effective length factor, $K$, was conservatively taken as unity for all members, although a lower value can be justified for computing the brace design strength for buckling in the plane of the frame. Diagonal braces are designed to carry gravity loads—see $\S 2.3$.

The lateral force distributions and story shears for each archetype building are provided in Appendix B. Allowable drift compliance verification is provided in Appendix B. Similarly, verification for drift amplification from global p-delta $(P-\Delta)$ effects and ASCE 7 stability verification are also provided in Appendix B. Design calculations for select members and connections are provided to illustrate the design process in Appendix B.

The 4-, 8-, and 16-story EBF frame designs are shown in Figure 2-15 through Figure 2-17, respectively. Each figure shows both the RSA design (a) and the ELF design (b) - underlined member sizes indicate changes from the RSA design. These figures also provide the fundamental periods of the archetype building for various gravity loads (e.g., drift and strength design). 


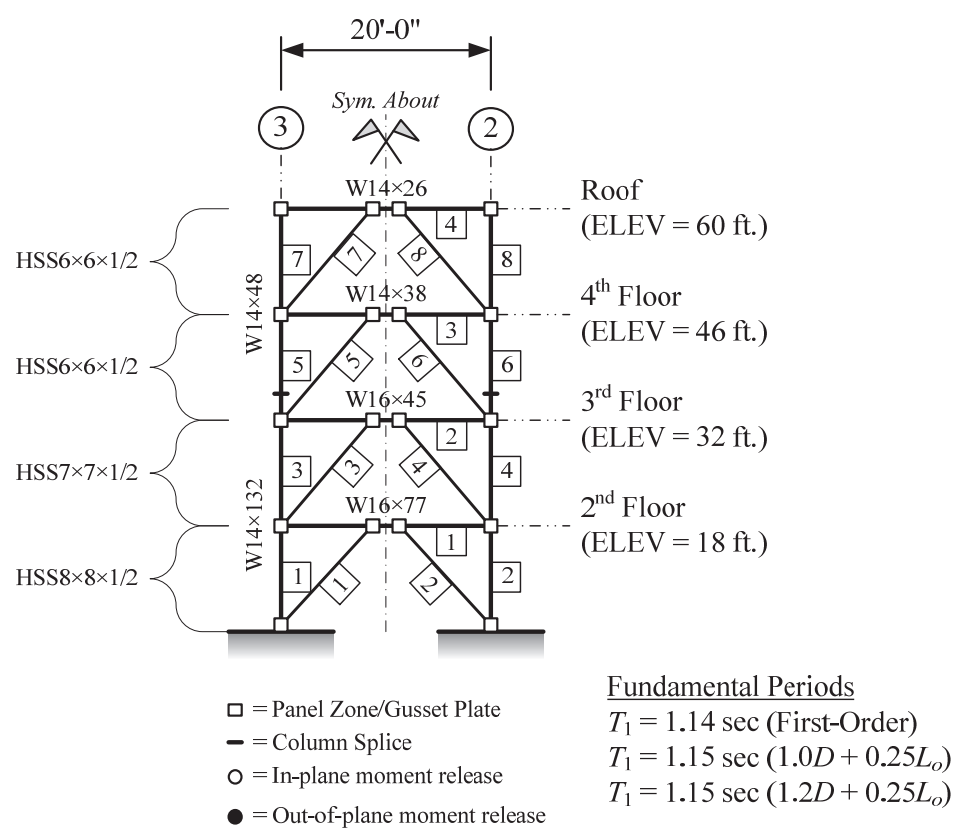

(a) RSA

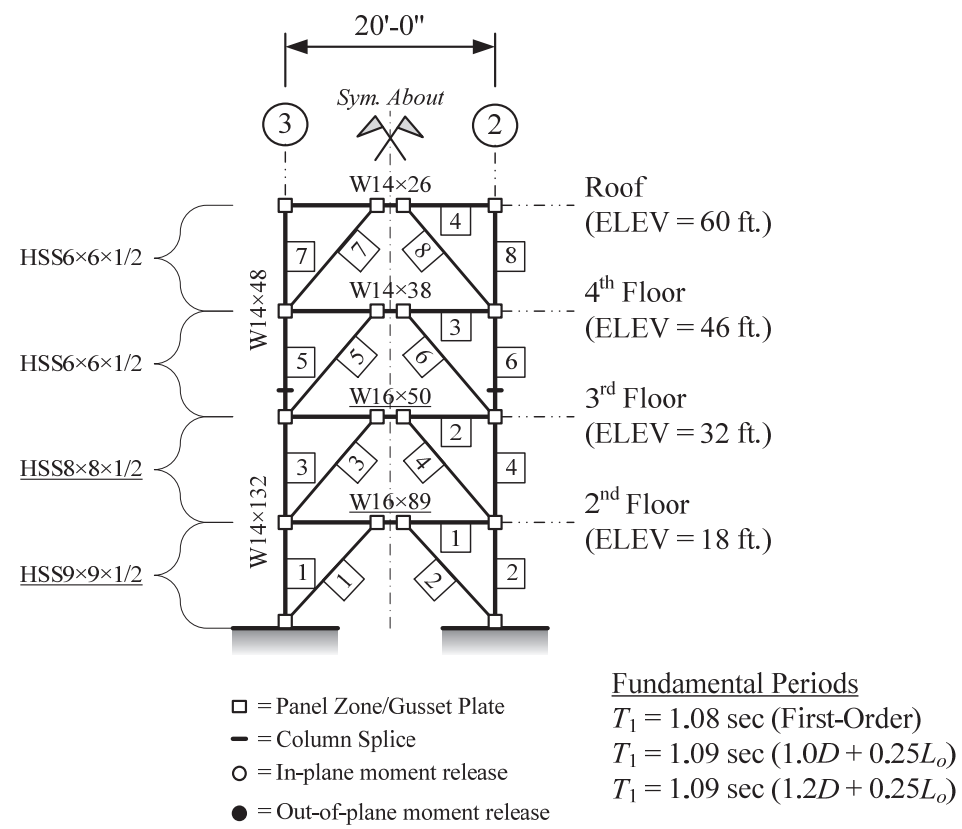

(b) ELF

Figure 2-15. 4-Story EBF Schematic 


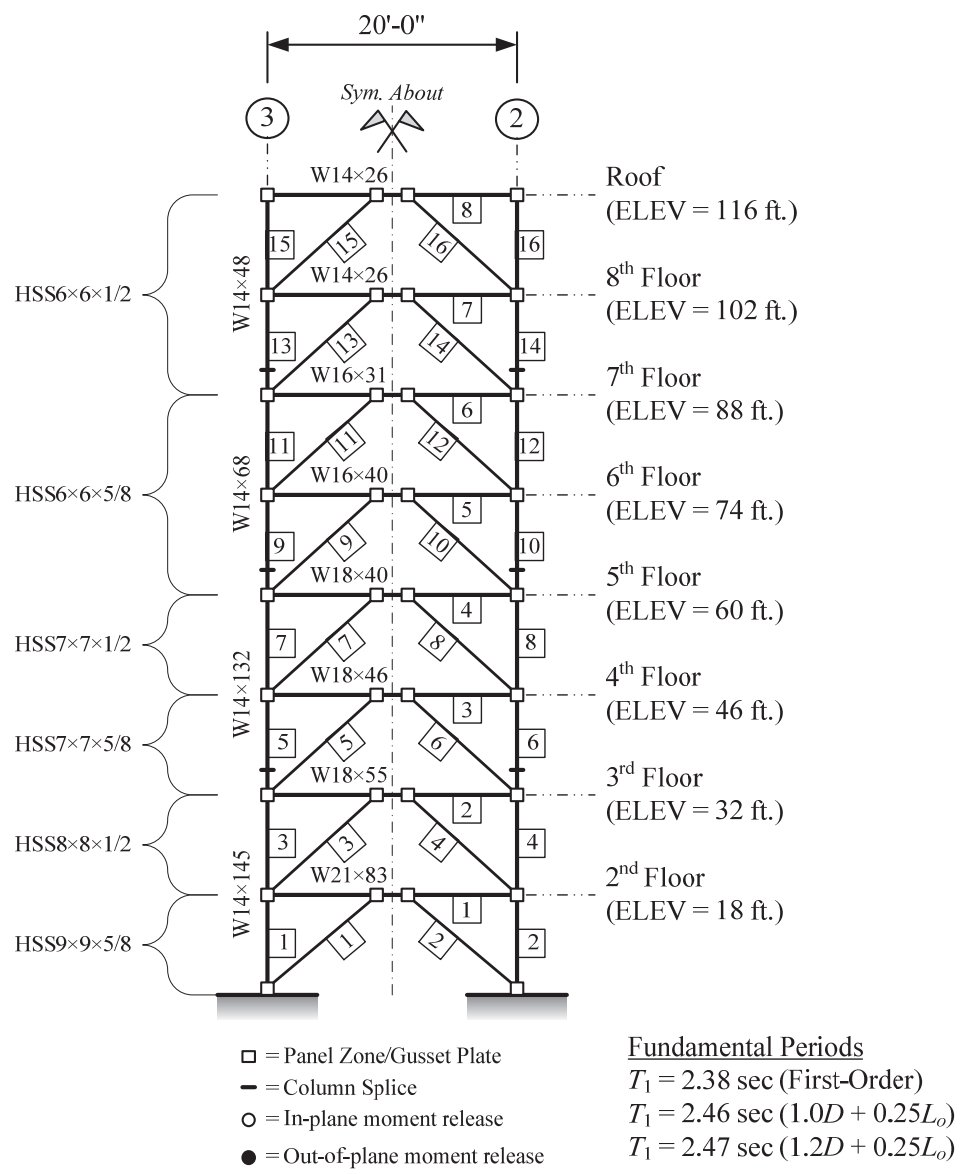

(a) RSA

Figure 2-16. 8-Story EBF Schematic 


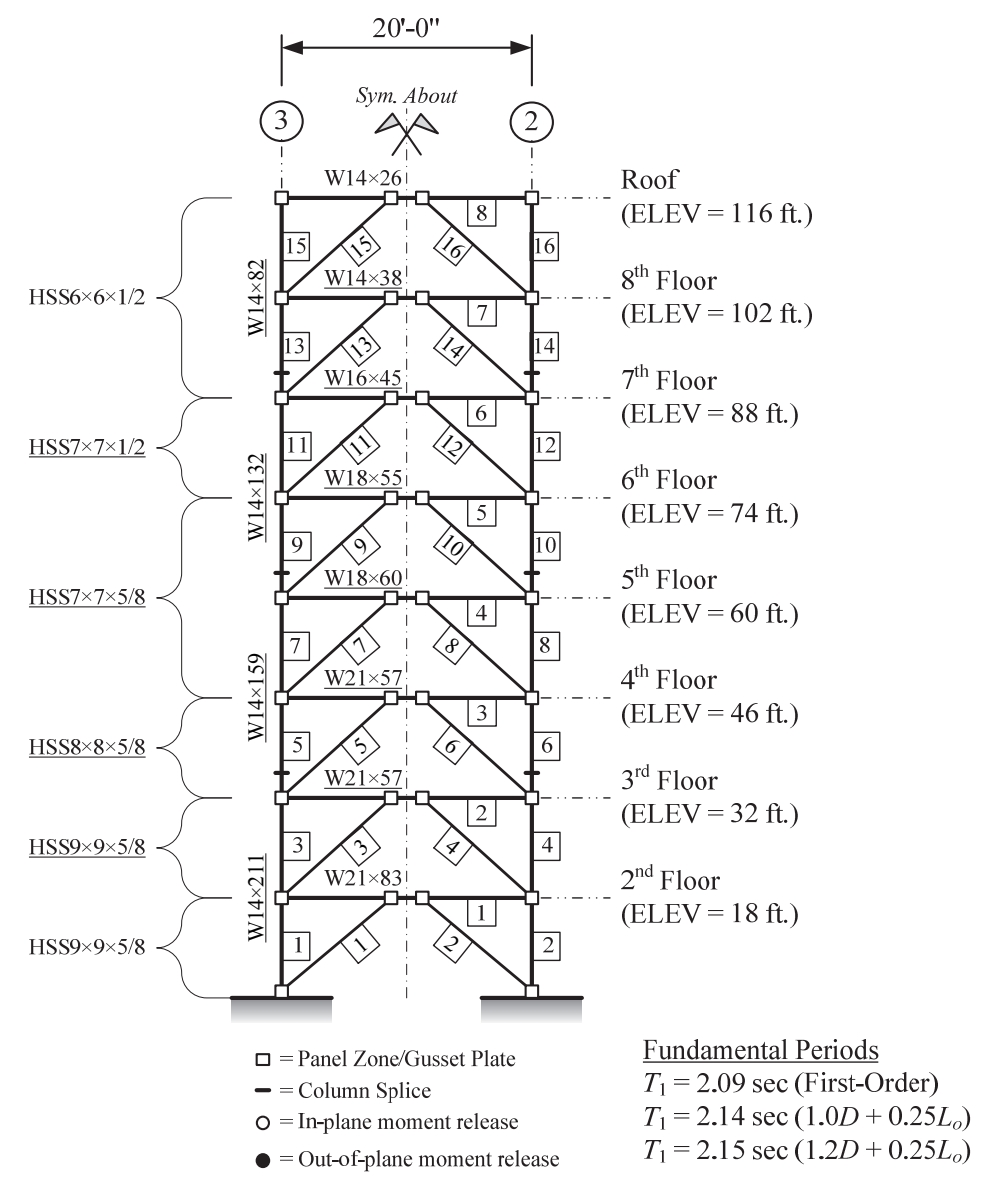

(b) ELF

Figure 2-16. 8-Story EBF Schematic, Cont'd 


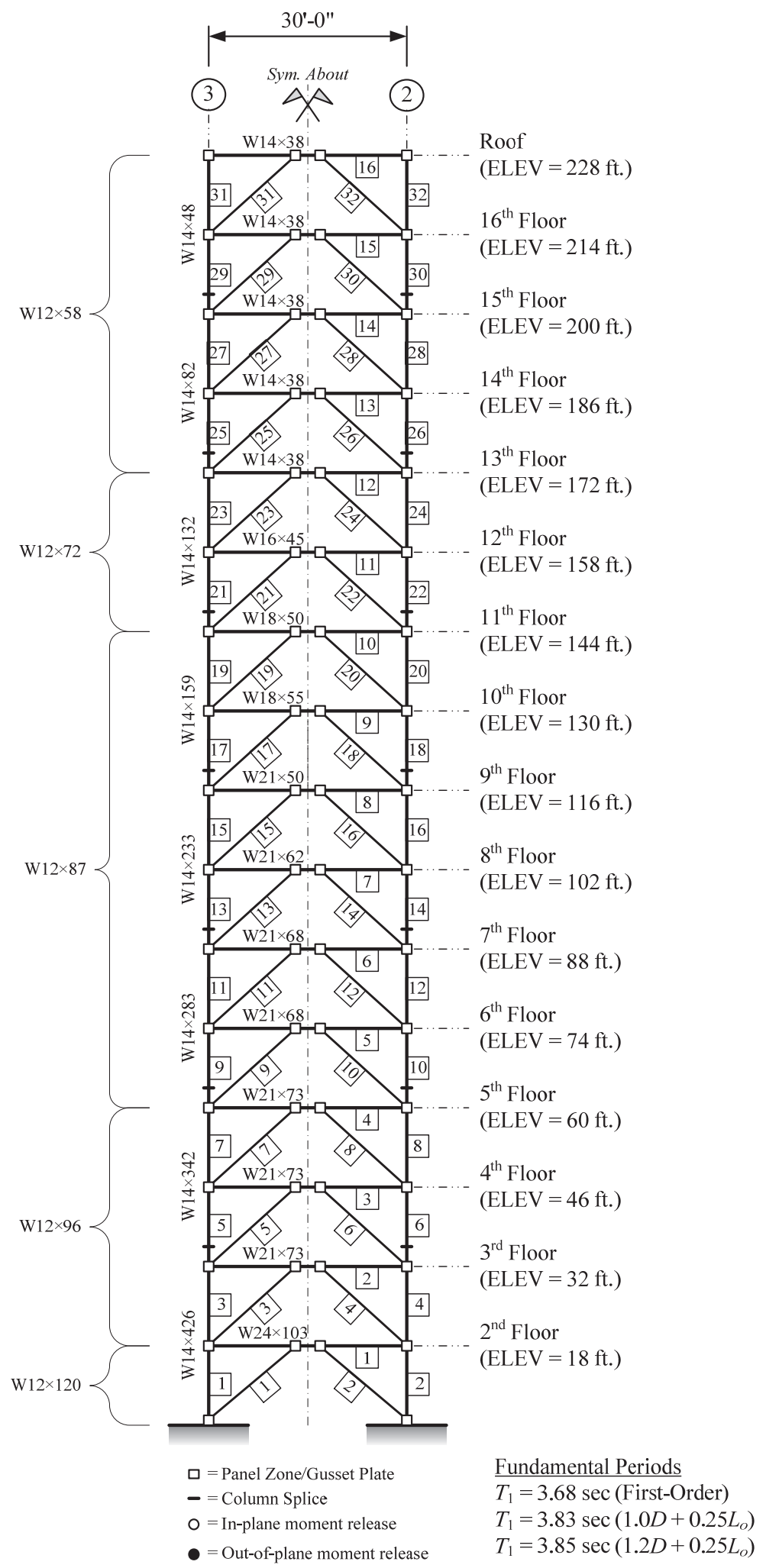

(a) RSA

Figure 2-17. 16-Story EBF Schematic 


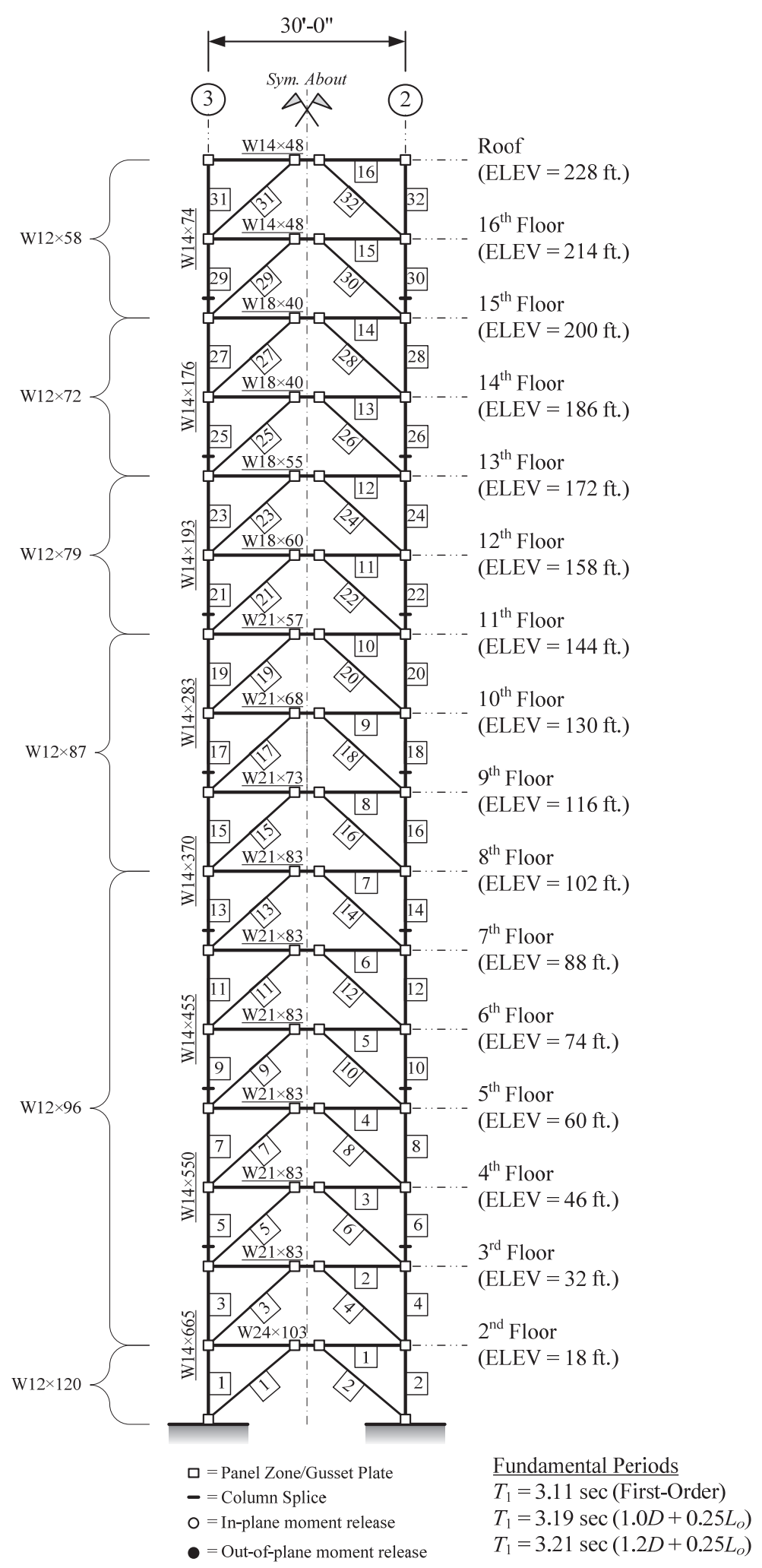

(b) ELF

Figure 2-17. 16-Story EBF Schematic, Cont'd 


\section{Chapter 3 Seismic Assessment}

This chapter presents the results of the seismic assessment of the eccentrically braced frames in accordance with ASCE 41. Section 3.1 provides general discussions on the global analysis requirements for assessment. Analysis requirements specific to eccentrically braced frames and assessment results of primary components of the frames are provided in Section 3.2.

\subsection{Assessment Overview}

A seismic performance assessment of the eccentrically braced frames is conducted using both linear and nonlinear analysis procedures prescribed in ASCE 41 \$2.4:

- Linear Static Procedure [ASCE 41 §3.3.1]

- Linear Dynamic Procedure (Response Spectrum) [ASCE $41 \S 3.3 .2]^{10}$

- Nonlinear Static Procedure [ASCE 41 §3.3.3] ${ }^{11}$

- Nonlinear Dynamic Procedure [ASCE 41 §3.3.4]

Seismic assessment analyses follow the guidelines outlined in ASCE 41 Chapters 1 through 3, and, where applicable, ASCE 41 Chapter 5. Foundations, including soil-structure interaction, and geological site hazards (ASCE 41 Chapter 4) are not considered in this study. Modeling and assessment requirements for steel structural systems follow the provisions in ASCE 41 Chapter 5. Any deviations from these ASCE 41 guidelines are explained where applicable.

The seismic performance target (i.e., 'rehabilitation objective') for this study is selected as the Basic Safety Objective (BSO) in ASCE 41 §1.4.1. This selection allows the correlation between the seismic performance objective intended by ASCE 41 and the intended design objective of ASCE 7 for an ordinary building ${ }^{12}$ to be evaluated. The BSO associated goals for Structural Performance Levels (SPLs) found in ASCE 41 §1.5.1 and Earthquake Hazard Levels (EHLs) found in ASCE $41 \S 1.6$ are given in Table 3-1. Nonstructural Performance Levels (NPLs) found in ASCE $41 \S 1.5 .2$ are not considered in this study. The target Building Performance Levels (BPLs) found in ASCE 41 §1.5.3 are given in Table 3-1.

The only explicit connection between the target structural performance objectives (i.e., SPL) of the BSO in ASCE 41 and the intended structural design performance objective of ASCE 7 is 'Collapse Prevention' given an MCE event, assuming that the BSE-2 EHL is equivalent to the MCE defined by ASCE 7-see Table 1-1 and Table 3-1. It is presumed by ASCE 7 that an appropriately designed structure using a seismic hazard of $2 / 3 \times$ MCE will achieve this structural design performance objective. ASCE 7 does not explicitly

\footnotetext{
${ }^{10}$ The user can alternatively perform a linear response history analysis. This was not done in this study, although it would bypass the limitations of using modal response spectrum analysis.

${ }^{11}$ Simplified Nonlinear Static Procedure (NSP) is not considered.

12 Structures assigned to Risk Category II or lower.
} 
identify a target structural design performance objective for 'Life Safety' at $2 / 3 \times$ MCE. Rather, ASCE 7 contains implicit life safety measures to protect against loss of life from nonstructural damage at the designlevel event, $2 / 3 \times \mathrm{MCE}$.

Table 3-1. Seismic Performance Targets (from ASCE 41-06)

\begin{tabular}{|c|c|c|c|c|}
\hline $\begin{array}{l}\text { Earthquake Hazard } \\
\text { Level }\end{array}$ & Earthquake Intensity & $\begin{array}{l}\text { Structural } \\
\text { Performance } \\
\text { Level }\end{array}$ & $\begin{array}{l}\text { Nonstructural } \\
\text { Performance Level }\end{array}$ & $\begin{array}{l}\text { Building } \\
\text { Performance Level }\end{array}$ \\
\hline BSE-1 - §1.6.1.2 & $2 / 3 \times$ BSE- 2 & Life Safety (S-3) & Not Considered (N-E) & Life Safety (3-E) \\
\hline BSE-2 - $\$ 1.6 .1 .1$ & $\begin{array}{l}\text { Maximum Considered } \\
\text { Earthquake (MCE) }\end{array}$ & $\begin{array}{l}\text { Collapse } \\
\text { Prevention (S-5) }\end{array}$ & Not Considered (N-E) & $\begin{array}{l}\text { Collapse Prevention } \\
(5-E)\end{array}$ \\
\hline
\end{tabular}

Prior to ASCE 7-10, the MCE was defined as a uniform seismic hazard associated with a two percent probability of being exceeded in 50 years, except near known faults where deterministic-based hazards controlled. ASCE 7-10 adopted a risk-targeted design philosophy that shifts from a uniform hazard design basis to a uniform risk design basis, and defines the MCE ground motion intensity (denoted as $\mathrm{MCE}_{\mathrm{R}}$ ) as ground motions having a one percent probability of causing total or partial structural collapse in 50 years. This risk has a conditional probability ('anticipated reliability') of ten percent probability of total or partial structural collapse conditioned on the occurrence of an MCE event-see ASCE 7 Table C.1.3.1b. Several reference documents are available for more information about this implementation (FEMA 2009b, commentary of ASCE 7-10 ( $3^{\text {rd }}$ printing), and NIST 2012). Therefore, the approved MCE in ASCE 41-06 $\S 1.6 .1 .1$ (see BSE-2 in Table 3-1) should be taken as the $\mathrm{MCE}_{\mathrm{R}}$ in ASCE 7-10 to maintain equivalency between the standards. ASCE 41-13 \$2.2.4 prescribes using the $\mathrm{MCE}_{\mathrm{R}}$ to define the BSE-2 EHL for the new building equivalency track. The seismic performance targets for this study are taken from ASCE 4113 and are given in Table 3-2.

Table 3-2. Seismic Performance Targets (from ASCE 41-13)

\begin{tabular}{|c|c|c|c|c|}
\hline $\begin{array}{l}\text { Earthquake Hazard } \\
\text { Level }\end{array}$ & Earthquake Intensity & $\begin{array}{l}\text { Structural Performance } \\
\text { Level }\end{array}$ & $\begin{array}{l}\text { Nonstructural } \\
\text { Performance Level }\end{array}$ & $\begin{array}{l}\text { Building Performance } \\
\text { Level }\end{array}$ \\
\hline BSE-1N ${ }^{1}-\S 2.4 .1 .2$ & $2 / 3 \times \mathrm{BSE}-2 \mathrm{~N}$ & Life Safety (S-3) & Not Considered (N-E) & Life Safety (3-E) \\
\hline $\mathrm{BSE}-2 \mathrm{~N}^{1}-\S 2.4 .1 .1$ & $\begin{array}{l}\text { Maximum Considered } \\
\text { Earthquake }\left(\mathrm{MCE}_{\mathrm{R}}\right)\end{array}$ & $\begin{array}{l}\text { Collapse Prevention } \\
(\mathrm{S}-5)\end{array}$ & Not Considered (N-E) & $\begin{array}{l}\text { Collapse Prevention } \\
(5-\mathrm{E})\end{array}$ \\
\hline
\end{tabular}

This study does not evaluate assessment results for earthquake hazard levels with return periods shorter than identified above or building performance levels below Life Safety. Future research efforts may evaluate incorporating other performance levels for design in ASCE 7-see NIST GCR 12-917-20: Tentative Framework for Development of Advanced Seismic Design Criteria for New Buildings (NIST 2012). 


\subsubsection{Seismic Hazard}

The seismic hazard is defined in ASCE $41 \S 1.6$. The spectral response parameters for the BSE-2 (三 BSE$2 \mathrm{~N}$ - see above) and BSE-1 (三 BSE-1N-see above) EHLs are given in Table 3-3. The parameters summarize the seismic hazard $^{13}$ for Site Class D, Stiff Soil, in ASCE 41 §1.6.1.4.1.

Table 3-3. Spectral Response Parameters

\begin{tabular}{|c|c|c|c|c|c|c|c|c|}
\hline EHL & $\begin{array}{c}S_{S} \\
(\mathrm{~g})\end{array}$ & $\begin{array}{l}S_{1} \\
(\mathrm{~g})\end{array}$ & $F_{a}{ }^{3}$ & $F_{v}{ }^{3}$ & $\begin{array}{l}S_{X S} \\
(\mathrm{~g})\end{array}$ & $\begin{array}{l}S_{X 1} \\
(\mathrm{~g})\end{array}$ & $T_{s}(\mathrm{sec})$ & $T_{0}(\mathrm{sec})$ \\
\hline BSE-2 & 1.5 & $0.60^{1}$ & 1.0 & 1.50 & 1.50 & 0.90 & 0.60 & 0.12 \\
\hline BSE-1 ${ }^{2}$ & 1.5 & $0.60^{1}$ & 1.0 & 1.50 & 1.00 & 0.60 & 0.60 & 0.12 \\
\hline
\end{tabular}

Figure 3-1 illustrates the generalized response spectrum for BSE-1 and BSE-2.

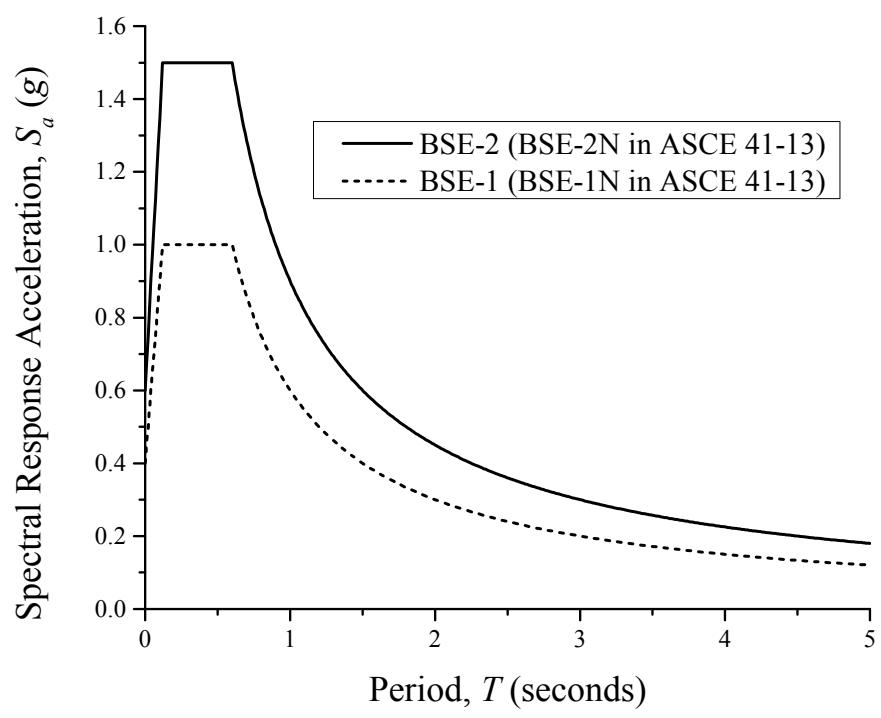

Figure 3-1. Acceleration Response Spectra

\subsubsection{General Analysis Requirements}

This section discusses the implementation of the general analysis requirements prescribed in ASCE $41 \S 3.2$ in this study.

ASCE 41 \$3.2.2-Mathematical Modeling: The archetype buildings are modeled in three-dimensions. Increased forces and displacements because of torsional demands are inherently addressed in the three-

13 The seismic hazard in ASCE 41-13 is the $\mathrm{MCE}_{\mathrm{R}}$, ground motion based on a risk-targeted design philosophy in which an appropriately designed structure will have a one percent probability of collapse (i.e., risk) in 50-years (except in areas controlled by the deterministic cap on ground motions - see ASCE 7 commentary for Chapter 11). 
dimensional analysis. Because of building symmetry, the inherent torsional moment at each floor is theoretically zero. Accidental torsional moment (i.e., five percent mass offset) at each floor is not considered in the assessment analyses because the displacement modifier, $\eta$, associated with the applied loads including accidental torsion is less than 1.1 at every floor for all buildings (see Table 3-4 and Table 3-5). The values in these tables for determining torsional irregularity are based on floor displacements relative to the base and not story drifts as used in ASCE $7^{14}$.

All members and connections of the seismic force-resisting system (SFRS) are classified as primary components in accordance with ASCE 41 \$2.4.4.2 for both linear and nonlinear assessment procedures. Gravity framing (non-SFRS members and shear tab connections) is assumed in this study to provide negligible analytical lateral stiffness and strength—see ASCE 41 §3.2.2.3. Therefore, components of the non-SFRS framing are classified as secondary components for both linear and nonlinear assessment procedures. Similar to the assumptions adopted for design, specific component stiffnesses (e.g., partially restrained shear tab connections and stairs) are not modeled explicitly in the mathematical model. This is done to minimize the influence of secondary components on the demands imposed on primary components. This will allow assessment results between linear and nonlinear analysis to be compared. Performance assessment of these secondary members is outside the scope of this study-though would need to be checked in a real retrofit design. In addition, foundation or soil flexibility is not included in the analysis. Models for anticipated component inelastic actions in nonlinear assessment procedures include both strength and stiffness degradation—-discussed subsequently in $\$ 3.2$.

ASCE 41 \$3.2.3-Configuration: Building irregularities are discussed in ASCE 41 §3.1.3.1. Building irregularities defined in ASCE 41 are used only to determine whether the linear procedures are applicable.

ASCE 41 \$3.2.4-Diaphragms: Floor diaphragms are modeled for analysis as semi-rigid membranes (i.e., stiff per ASCE 41). The same assumptions adopted in design are maintained for assessment.

ASCE $41 \S 3.2 .5-\mathrm{P}-\Delta$ Effects: Global $P$ - $\Delta$ effects are considered in the linear and nonlinear analyses, for both static and dynamic. Local $P-\delta$ effects are not addressed either explicitly or implicitly in the analyses.

ASCE 41 \$3.2.6-Soil-Structure Interaction: Soil-Structure Interaction is not considered in the seismic assessment of the archetype buildings.

ASCE 41 \$3.2.7-Multidirectional Seismic Effects: The principal axes of the archetype buildings align directly with the E-W and N-S directions (performance in the E-W direction is presented in this report). Seismic effects are determined by applying the seismic forces independently in each of the two orthogonal directions. Per ASCE 41 \$3.2.7.1, concurrent seismic effects are addressed in the assessment by combining the effects along each principal axes.

14 Amplification of the accidental torsion, if required, is consistent between ASCE 41 and ASCE 7. 
ASCE 41 \$3.2.7-Vertical Seismic Effects: Vertical seismic effects are not considered for seismic assessment of the archetype buildings. Masses are input for horizontal accelerations only for dynamic analysis.

ASCE 41 \$3.2.8 - Gravity Loads: Gravity loads for the linear assessment procedures are applied using the following two load combinations (LC). Roof live loads are considered not to be present for seismic analysis. There is no snow load acting on the buildings.

- $\quad$ LC $1=1.1 \times($ Dead $+0.25 \times$ Unreduced Floor Live $)$

- $\mathrm{LC} 2=0.9 \times$ Dead

A $P$ - $\Delta$ load combination based on LC1 above is used for the linear assessment analyses; consequently, this is conservative for analysis using LC2.

Both gravity load combinations above are used for the nonlinear static procedure. The average of the two combinations (LC1 and LC2) is applied in the nonlinear dynamic procedure.

- $\quad$ LC3 $=1.0 \times$ Dead $+0.25 \times$ Unreduced Floor Live

A $P-\Delta$ load combination based on LC3 above is used for the nonlinear assessment analyses.

ASCE 41 \$3.2.9-Verification of Design Assumptions: The following design objectives are verified with the nonlinear dynamic procedure.

- $\quad$ EBF - in-plane and out-of-plane stability of columns (AISC 360)

- $\quad \mathrm{EBF}$ - in-plane and out-of-plane stability of braces (AISC 341)

- $\mathrm{EBF}$ - plastic hinges in beams adjacent to the link beam, columns and braces (AISC 341)

ASCE 41 \$3.2.10-Overturning: Overturning is not considered for design or seismic assessment of the archetype buildings.

\subsubsection{Analysis Procedures}

This section discusses the implementation of the specific analysis procedures prescribed in ASCE $41 \S 3.3$ in this study.

\subsubsection{Linear Analysis Procedures}

The archetype buildings are modeled and analyzed in ETABS 9.7.4 (CSI 2011a) for the linear analyses. The assumptions used in the mathematical model and analysis techniques are the same as those adopted for design (see Chapter 2). Modeling and analysis considerations for the linear procedures are outlined in ASCE 41 §3.2.2 with supplemental information provided in ASCE 41 \$3.3.2.2 for the Linear Dynamic Procedure (LDP). Gravity loads and load combinations assumed present during the earthquake are computed from ASCE $41 \S 3.2 .8$ as discussed previously. The effective horizontal seismic weights, $w$, for analysis are 
computed in accordance with ASCE 41 \$3.3.1, and are the same as those used for design (see Chapter 2). Global $P$ - $\Delta$ effects (e.g., $B_{2}$ in AISC 360 Appendix 8 ) are addressed in the analysis by using a simplified algorithm — see ETABS User Manual. ETABS does not explicitly include local $P$ - $\delta$ effects (e.g., $B_{1}$ in AISC 360 Appendix 8). System specific modeling assumptions and analysis techniques are described in their respective sections.

Table 3-4. Displacement multiplier-E-W (SMF)

\begin{tabular}{ccccccc}
\hline & \multicolumn{3}{c}{$\eta=\delta_{\text {max }} / \delta_{\text {avg }}$} & \multicolumn{2}{c}{ ME16 } \\
Floor & ELF & RSA & ELF & RSA & ELF & RSA \\
\hline Roof & - & - & - & - & 1.010 & 1.008 \\
16 & - & - & - & - & 1.009 & 1.007 \\
15 & - & - & - & - & 1.009 & 1.007 \\
14 & - & - & - & - & 1.009 & 1.007 \\
13 & - & - & - & - & 1.009 & 1.007 \\
12 & - & - & - & - & 1.009 & 1.007 \\
11 & - & - & - & - & 1.008 & 1.007 \\
10 & - & - & - & - & 1.008 & 1.007 \\
9 (Roof ME8) & - & - & 1.010 & 1.007 & 1.008 & 1.007 \\
8 & - & - & 1.010 & 1.007 & 1.008 & 1.007 \\
7 & - & - & 1.009 & 1.006 & 1.008 & 1.007 \\
6 & - & - & 1.009 & 1.006 & 1.008 & 1.007 \\
5 (Roof ME4) & 1.006 & 1.006 & 1.008 & 1.005 & 1.008 & 1.007 \\
4 & 1.006 & 1.006 & 1.008 & 1.005 & 1.008 & 1.007 \\
3 & 1.005 & 1.005 & 1.007 & 1.005 & 1.008 & 1.007 \\
2 & 1.005 & 1.005 & 1.007 & 1.005 & 1.008 & 1.007 \\
\hline Values shown to four significant figures are for comparison purposes only. & & & & &
\end{tabular}

Table 3-5. Displacement Multiplier-N-S (EBF)

\begin{tabular}{ccccccc}
\hline & \multicolumn{4}{c}{$\eta=\delta_{\max } / \delta_{\text {avg }}$} \\
ME4 & \multicolumn{2}{c}{ ME8 } & \multicolumn{2}{c}{ ME16 } \\
Floor & ELF & RSA & ELF & RSA & ELF & RSA \\
\hline Roof & - & - & - & - & 1.075 & 1.072 \\
16 & - & - & - & - & 1.075 & 1.072 \\
15 & - & - & - & - & 1.075 & 1.072 \\
14 & - & - & - & - & 1.076 & 1.072 \\
13 & - & - & - & - & 1.076 & 1.073 \\
12 & - & - & - & - & 1.077 & 1.073 \\
11 & - & - & - & - & 1.077 & 1.074 \\
10 & - & - & - & - & 1.077 & 1.074 \\
9 (Roof ME8) & - & - & 1.075 & 1.078 & 1.078 & 1.075 \\
8 & - & - & 1.076 & 1.079 & 1.079 & 1.075 \\
7 & - & - & 1.076 & 1.080 & 1.079 & 1.076 \\
6 & - & - & 1.078 & 1.080 & 1.080 & 1.077 \\
5 (Roof ME4) & 1.084 & 1.088 & 1.079 & 1.081 & 1.081 & 1.078 \\
4 & 1.085 & 1.089 & 1.081 & 1.083 & 1.082 & 1.080 \\
3 & 1.086 & 1.090 & 1.083 & 1.085 & 1.082 & 1.082 \\
2 & 1.085 & 1.089 & 1.084 & 1.089 & 1.083 & 1.085 \\
\hline Values shown to four significant figures are for comparison purposes only.
\end{tabular}


ASCE $41 \S 2.4 .1 .1$ prescribes restrictions on the use of the linear procedures. First, a retrofitted ${ }^{15}$ SFRS must not contain certain types of structural irregularities where the earthquake demands on the primary components of the SFRS fail to comply with the demand capacity ratio (DCR) limitations.

$$
D C R=\frac{Q_{U D}}{Q_{C E}} \leq 2.0
$$

where $Q_{U D}$ is the demand on a component due to gravity and earthquake loads and $Q_{C E}$ is the expected strength of the component. The archetype buildings do not contain any configuration-based in-plane or outof-plane irregularities. Further, a linear analysis procedure must be performed to determine whether a building contains a weak story or torsional strength irregularity. Because of plan symmetry and regularity of the archetype buildings, there are no torsional irregularities. The required weak story irregularity verifications are discussed subsequently in the linear static procedure for each specific system type.

\subsubsection{1 ～Linear Static Procedure}

The Linear Static Procedure (LSP) is outlined in ASCE 41 §3.3.1. The provisions of the LSP closely resemble those of the ELF procedure in ASCE 7; as such, no additional analysis details are presented here. However, one place where ASCE 7 and ASCE 41 differ is in the determination of the fundamental period, $T$, in the direction being analyzed. ASCE 41 does not place an upper-bound limit on the period used for assessment as ASCE 7 does for strength design. In this study, the fundamental periods are determined by eigenvalue analysis per ASCE 41 \$3.3.1.2.1.

Further restrictions on the use of the LSP are prescribed in ASCE 41 §2.4.1.2. The LSP cannot be used if any of the following occur:

- The fundamental period of the building $T$ is greater than $3.5 \times T_{s}(=3.5 \times 0.6=2.1$ seconds in this study). This trigger is similar to that used in ASCE 7; however, like ASCE 7, which computation method for $T$ to be used in this evaluation is not clear. If the analytical method (ASCE 41 §3.3.1.2.1) is used, only the E-W component of the ELF-designed ME4 and the N-S component of the ELFand RSA-designed ME4 satisfy this requirement - see Table 3-6 (directional components that fail this criteria are shaded). If the empirical method (ASCE 41 \$3.2.1.2.2) is used, all building components satisfy this constraint except the E-W component of ME16. In this study, this analysis constraint is disregarded to allow an assessment comparison between methods - see Table 3-7 (directional components that fail this criteria are shaded).

- The building has a ratio of the horizontal dimension at any story to the corresponding dimension in an adjacent story that exceeds 1.4. In this study, this constraint is satisfied because the building plan does not change at any story.

- The building has a torsional stiffness irregularity. This limitation is satisfied as discussed previously under accidental torsion as well as in Chapter 2. In this study, this check is based on the individual story drift rather than the floor displacement relative to the base.

- The building has a vertical stiffness irregularity. In this study, this limitation is automatically satisfied by using ASCE $7 \S 12.3 .2 .2$ Exception 1 for the design of the archetype buildings.

\footnotetext{
15 This term is now used in lieu of 'rehabilitation'.
} 
- The building has a non-orthogonal SFRS. In this study, this limitation is not applicable to the archetype buildings.

Regardless of the restrictions on using the LSP, results from applying the LSP to the archetype buildings are included for the purpose of seismic performance comparison between the various assessment methods in this study.

Table 3-6. Analytical fundamental periods (seconds)

\begin{tabular}{cccccccc}
\hline & \multicolumn{2}{c}{ ME4 } & \multicolumn{2}{c}{ ME8 } & \multicolumn{2}{c}{ ME16 } \\
Direction & SFRS & ELF & RSA & ELF & RSA & ELF & RSA \\
\hline E-W & SMF & 1.83 & 2.23 & 2.93 & 3.84 & 4.39 & 5.05 \\
N-S & EBF & 1.10 & 1.09 & 2.15 & 2.46 & 3.19 & 3.88 \\
\hline
\end{tabular}

Table 3-7. Empirical fundamental periods (seconds)

\begin{tabular}{cccccccc}
\hline & & \multicolumn{2}{c}{ ME4 } & \multicolumn{2}{c}{ ME8 } & \multicolumn{2}{c}{ ME16 } \\
Direction & SFRS & ELF & RSA & ELF & RSA & ELF & RSA \\
\hline E-W & SMF & 0.93 & 0.93 & 1.57 & 1.57 & 2.69 & 2.69 \\
N-S & EBF & 0.65 & 0.65 & 1.06 & 1.06 & 1.76 & 1.76 \\
\hline
\end{tabular}

\subsection{Linear Dynamic Procedure}

The Linear Dynamic Procedure (LDP) is outlined in ASCE 41 §3.3.2. The LDP requires the use of either response spectrum analysis (RSA) or response history analysis (RHA) —only the RSA is presented in this report. Though there are significant benefits of using the RHA (e.g., maintaining sign convention on response), the RSA was selected to align with the design methodology. The provisions of the LDP closely resemble those of the RSA procedure in ASCE 7; as such, no additional analysis details are presented here, except that no base shear scaling is required by ASCE 41. Damping for analysis is taken as five percent of critical for all modes for dynamic analysis to match the response spectrum (see ASCE $41 \S 1.6 .1 .5 .3$ ). A sufficient number of modes is used in the analysis to capture at least 90 percent of the mass participation in each of the two horizontal principal directions. Masses were not modeled in the analysis to address vertical accelerations. Furthermore, the square root of the sum of the squares (SRSS) rule is used to combine the modal responses so as to obtain the maximum forces and deformations.

\subsubsection{Nonlinear Analysis Procedures}

The archetype buildings are modeled and analyzed in PERFORM-3D 5.0.0 (CSI 2011b) for the nonlinear procedures. Modeling and analysis considerations for the nonlinear procedures are outlined in ASCE 41 §3.2.2 with supplemental information provided in ASCE 41 §3.3.3.2 for the Nonlinear Static Procedure (NSP) and ASCE $41 \S 3.3 .4 .2$ for the Nonlinear Dynamic Procedure (NDP). Primary components of the SFRS expected to experience inelastic deformations are modeled using a full "backbone" curve that includes strength and stiffness (applicable only for the NDP) degradation and residual strength. For this study, all nonlinear components are modeled with the anchor points (A to E) bounding the full backbone curve as shown in ASCE 41 Figure C2-1 and Figure 5-1-see Figure 3-2. This topic will be discussed further in the respective analysis and system-specific sections. 
Gravity loads and corresponding load combination assumed to be present during the earthquake are computed from ASCE $41 \S 3.2 .8$ as discussed previously. The effective horizontal seismic weights, $w$, for analysis are computed in accordance with ASCE 41 \$3.3.1 and are the same as those used for design (see Chapter 2). Global $P$ - $\Delta$ effects (e.g., $B_{2}$ in AISC 360 Appendix 8) are addressed in the analysis by using a simplified algorithm - see PERFORM-3D User Guide (CSI 2011d). PERFORM-3D does not explicitly include local $P$ - $\delta$ effects (e.g., $B_{1}$ in AISC 360 Appendix 8). Building specific modeling assumptions and analysis techniques are described in their respective sections.

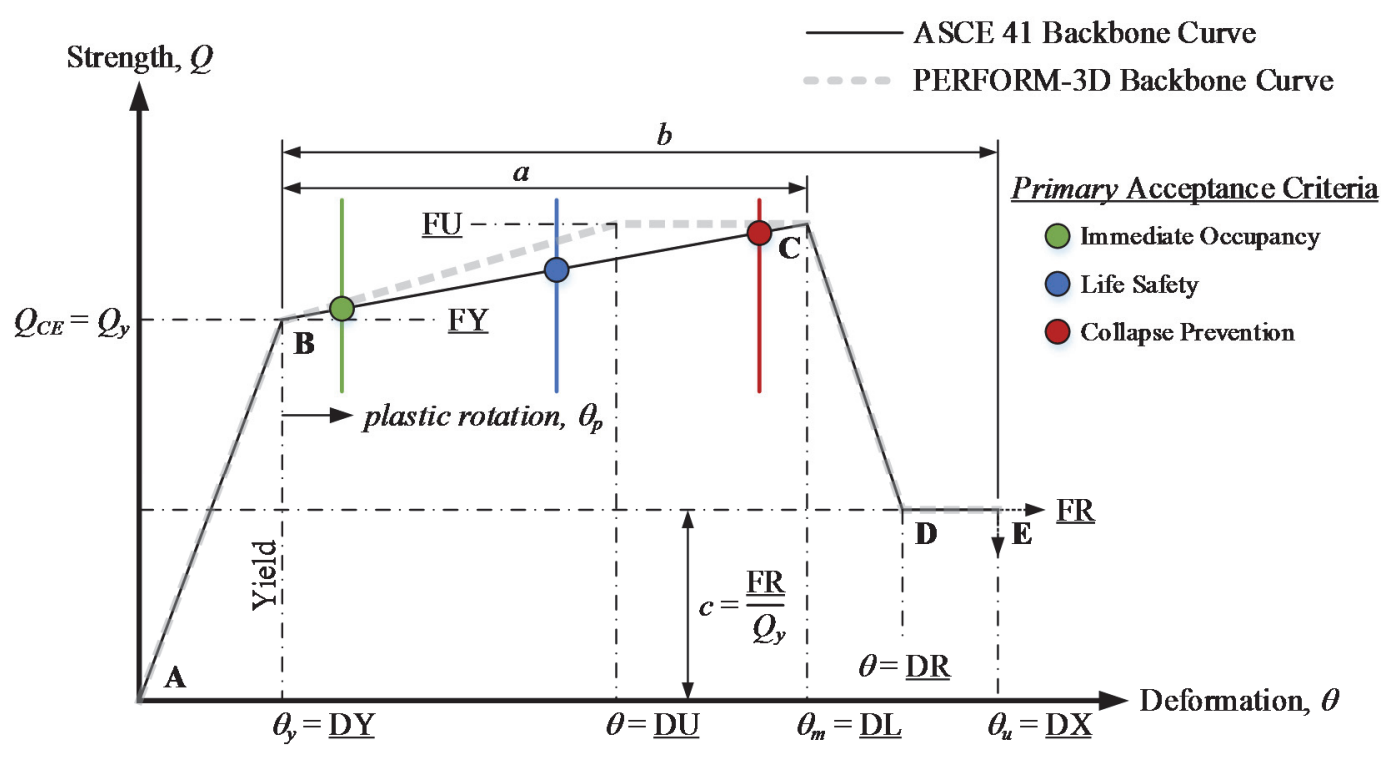

\footnotetext{
- Parameters $a, b, c$, and acceptance criteria are provided in ASCE 41.

- Underlined parameters are notation used by PERFORM-3D.

- Analysis software has the ability to maintain the strength or allow complete strength loss at Point E (for some components).
}

Figure 3-2. Generalized Component Backbone Curve (adopted from ASCE 41 Figure C2-1) 


\subsection{Nonlinear Static Procedure}

The Nonlinear Static Procedure (NSP) is outlined in ASCE 41 §3.3.3. ASCE 41 places limitations on the use of the NSP in ASCE 41 \$2.4.2. First, if higher modes are significant, the NSP is permitted with supplemental verifications required using the LDP. Higher mode effects are consider significant when the story shear computed by analysis with at least 90 percent horizontal mass participation is at least 1.3 times greater than that computed considering only response in the fundamental mode. This condition is generally triggered in multistory buildings with fundamental periods greater than 1.0 second in the direction being considered (see Table 3-8 and Table 3-9-story shear ratios that fail this criteria are shaded).

Second, if $R>R_{\max }$ (as defined in ASCE $41 \S 3.3 .3$ ), dynamic instability is a potential failure mode and the NSP is not permitted. Information regarding this ductility criterion as applied to an idealized single-degreeof-freedom (SDOF) system is provided in FEMA 440: Improvements of Nonlinear Static Seismic Analysis Procedures (FEMA 2005). However, this verification is computationally cumbersome because a nonlinear static analysis has to be conducted to determine both $R$ and $R_{\max }$, prior to knowing if the NSP is permitted. This verification is illustrated subsequently in the NSP assessment results. The NSP procedure is graphically illustrated in the flowchart of Figure 3-3. 


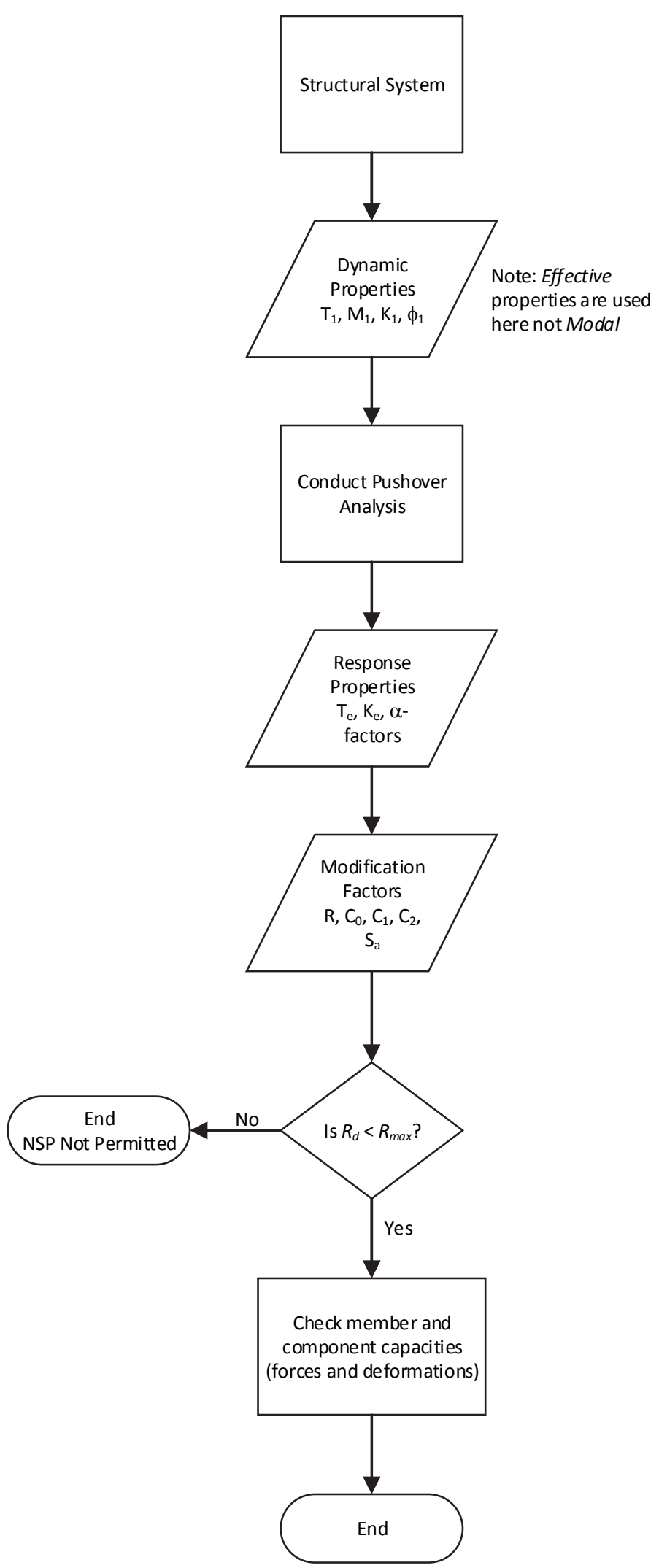

Figure 3-3. NSP Process 
Table 3-8. Story shear ratio - ELF

\begin{tabular}{cccc|ccc}
\hline & \multicolumn{7}{c}{$V_{\text {story }} / V_{\text {story, } \text { lst mode }}$} & & & \\
Story & ME4 & ME8 & ME16 & ME4 & ME8 & ME16 \\
\hline Roof & - & E-W & E-W & N-S & N-S & N-S \\
16 & - & - & 2.96 & - & - & 2.60 \\
15 & - & - & 2.03 & - & - & 2.15 \\
14 & - & - & 1.61 & - & - & 1.79 \\
13 & - & - & 1.41 & - & - & 1.52 \\
12 & - & - & 1.29 & - & - & 1.33 \\
11 & - & - & 1.14 & - & - & 1.20 \\
10 & - & - & 1.09 & - & - & 1.13 \\
9 (Roof) & - & 1.98 & 1.07 & - & 1.87 & 1.10 \\
8 & - & 1.41 & 1.07 & - & 1.48 & 1.12 \\
7 & - & 1.19 & 1.06 & - & 1.19 & 1.16 \\
6 & - & 1.10 & 1.07 & - & 1.05 & 1.22 \\
5 (Roof) & 1.45 & 1.05 & 1.08 & 1.18 & 1.04 & 1.30 \\
4 & 1.10 & 1.03 & 1.11 & 1.03 & 1.11 & 1.38 \\
3 & 1.01 & 1.05 & 1.15 & 1.01 & 1.24 & 1.46 \\
2 & 1.06 & 1.10 & 1.19 & 1.04 & 1.35 & 1.51 \\
\hline
\end{tabular}

Table 3-9. Story shear ratio - RSA

\begin{tabular}{cccc|ccc}
\hline & \multicolumn{7}{c}{$V_{\text {story }} / V_{\text {story, } \text { st mode }}$} & & & \\
Story & ME4 & ME8 & ME16 & ME4 & ME8 & ME16 \\
\hline Roof & - & E-W & E-W & N-S & N-S & N-S \\
16 & - & - & 3.10 & - & - & 3.04 \\
15 & - & - & 2.11 & - & - & 2.44 \\
14 & - & - & 1.65 & - & - & 1.96 \\
13 & - & - & 1.42 & - & - & 1.60 \\
12 & - & - & 1.30 & - & - & 1.37 \\
11 & - & - & 1.15 & - & - & 1.24 \\
10 & - & - & 1.11 & - & - & 1.18 \\
9 (Roof) & - & 2.25 & 1.09 & - & 2.02 & 1.17 \\
8 & - & 1.50 & 1.09 & - & 1.52 & 1.21 \\
7 & - & 1.22 & 1.09 & - & 1.21 & 1.26 \\
6 & - & 1.12 & 1.09 & - & 1.07 & 1.34 \\
5 (Roof) & 1.58 & 1.07 & 1.11 & 1.18 & 1.06 & 1.44 \\
4 & 1.14 & 1.05 & 1.15 & 1.03 & 1.14 & 1.56 \\
3 & 1.01 & 1.08 & 1.21 & 1.00 & 1.28 & 1.67 \\
2 & 1.08 & 1.16 & 1.26 & 1.03 & 1.40 & 1.74 \\
\hline
\end{tabular}

The mathematical model requirements for use with the NSP are outlined in ASCE $41 \S 3.2 .2$ and ASCE 41 \$3.3.3.2. Primary components of the SFRS expected to experience inelastic deformations are modeled using full backbone curves that include strength degradation and residual strength (see ASCE 41 \$3.2.2.3 and ASCE 41 \$3.3.3.2). ASCE 41 \$5.4.2.2.2 allows the generalized modeling parameters provided in ASCE 41 to model the full backbone curves of steel components for the NSP as an alternative to experimental calibration. In this study, all nonlinear components are modeled with the anchor points (A to E) bounding the full backbone curve as shown in Figure 3-2 and quantified in ASCE 41 Tables 5-6 and 57. Component strength at the ultimate deformation, point $\mathrm{E}$ on the backbone curve, retains residual strength 
and does not experience complete strength loss. SFRS-specific modeling approaches are discussed in their respective sections.

For the nonlinear static analysis algorithm in PERFORM-3D, the following apply:

- The lateral force distribution is based on a non-adaptive first-mode shape (first-order elastic) and mass distribution.

- Damping is set to zero percent for all modes with no supplemental Rayleigh damping. All elements are assigned a beta- $K$ damping stiffness reduction factor of unity (no reduction).

- Strength degradation is included in the analysis.

- Global $P-\Delta$ effects are directly included in the analysis. Local $P$ - $\delta$ effects are not addressed in the analysis. Geometric nonlinearity is assigned to all elements.

- Number of Steps is taken as 100 and Maximum Number of Events is taken as 1,000.

- Roof displacement at the center of mass relative to the base is used as the target displacement. The reference drift is therefore taken as the roof drift; the maximum allowable drift is taken as 10 percent. All story drifts are included in the list of Controlled Drifts.

- See PERFORM-3D User Guide for additional information.

\subsection{Nonlinear Dynamic Procedure}

The Nonlinear Dynamic Procedure (NDP) is outlined in ASCE 41 §3.3.4. The NDP is intended to be the most rigorous of all the assessment procedures prescribed, with no limitations placed on types of buildings allowed for the assessment because of the intent of capturing the true behavior of the building subjected to strong ground motions.

The mathematical model requirements for use with the NDP are outlined in ASCE 41 §3.2.2 and ASCE 41 $\S 3.3 .4 .2$, except that the point-in-time gravity load present during strong ground motion is taken as $(D+$ $S D)+0.25 \times L_{o, f l o o r}$, a slight but common alternative to ASCE $41 \S 3.2 .8$ (see ASCE $7 \S 16.2 .3$ ). Primary components of the SFRS expected to experience inelastic deformations are modeled using full backbone curves that include strength and stiffness degradation and residual strength (see ASCE 41 §3.2.2.3 and ASCE §3.3.4.2).

For the NSP, ASCE 41 \$5.4.2.2.2 allows using the generalized modeling parameters provided in Tables 56 and 5-7 to model the full backbone curves of steel components as an alternative to experimental calibration. However, for the NDP, ASCE 41 \$5.4.2.2.3 requires all component hysteretic behavior be based on experimental data unless permitted by the authority have jurisdiction. The benefit of calibrating component models with experimental results is that the force-deformation relationship will more accurately reflect strength and stiffness degradation, both cyclic and in-cycle - see NIST GCR 10-917-5: Nonlinear Structural Analysis for Seismic Design: A Guide for Practicing Engineers (NIST 2010a) and FEMA P440A: Effects of Strength and Stiffness Degradation on Seismic Response (FEMA 2009c). In this study, all nonlinear components are modeled with the anchor points (A to E) bounding the full backbone curve as shown in Figure 3-2 and quantified in ASCE 41 Tables 5-6 and 5-7. All nonlinear components are calibrated based on experimental results to determine cyclic and in-cycle stiffness degradation only; post-yield strength increases and strength degradation calibrations from experimental results were not included. Component strength at the ultimate deformation, point $\mathrm{E}$ on the backbone curve, retains the residual strength and does not experience complete strength loss. SFRS-specific modeling approaches are discussed in their 
respective sections. Therefore, the same PERFORM-3D model used in the NSP is also used in the NDP; analytical results from the two procedures are thus consistent and directly comparable. Future ASCE 41 revisions should unify modeling practices for the NSP and NDP, as well as provide supporting data to the authority having jurisdiction. Future research should examine the response of these systems with hysteretic models calibrated completely to test results, including performing FEMA P695 analyses.

The nonlinear dynamic analysis algorithm in PERFORM-3D used the following parameters:

- Damping is taken as three percent of critical for all modes (elastic) and 0.3 percent of critical is added as Rayleigh damping (elastic stiffness component only, beta- $K$ ) for dynamic analysis. Damping computation in PERFORM-3D is not based on the tangent stiffness matrix. All elements are assigned a beta- $K$ damping stiffness reduction factor of unity (no reduction).

- Strength and stiffness degradation are included in the analysis.

- Global $P-\Delta$ effects are directly included in the analysis. Local $P-\delta$ effects are not addressed in the analysis. Geometric nonlinearity is assigned to all elements.

- Maximum Number of Events for each time step is taken as 200.

- Time steps for analysis are taken as the time step of the input motion, ranging from 0.005 to 0.02 seconds - see FEMA P695 Appendix A.

- $\quad$ See PERFORM-3D User Guide for additional information.

A critical aspect of the NDP is the selection and scaling of input ground motions (free-field motions) which is described in ASCE 41 §1.6.2.2. The methodology adopted in this study is discussed in Appendix A.

In addition to the analysis routine terminating when a solution fails to converge, the routine was also set to terminate when an arbitrarily selected roof drift ratio of twenty percent ${ }^{16}$ is achieved (story drift ratios can be higher). While both of these methods are used to indicate and rationalize total or partial collapse of a system, the indicator of collapse used in this study is the component demands measured against the nonlinear modeling parameters and acceptance criteria. These component limits will typically be reached prior to an analysis routine failing to converge or an excessive roof drift is reached.

\subsubsection{Acceptance Criteria}

This section discusses the implementation of the acceptance criteria in ASCE 41 §3.4. Component actions are classified as force-controlled or deformation-controlled depending on the post-elastic behavior of the component (see ASCE 41 Table C2-1). Generally speaking, deformation-controlled actions are assigned to component actions capable of a ductile response (e.g., moment in a plastic hinge in a compact beam) and force-controlled actions are assigned to component actions with limited ductility (e.g., moment in a plastic hinge in a column with high axial load). Additionally, a knowledge factor, $\kappa$, is applied to account for uncertainties in the framing system and materials. Since the archetype buildings are new construction with quality control measures, $\kappa$ is taken as unity in this study to represent new component capacities or actions as discussed in ASCE 41 §2.2.2 and ASCE 41 Tables 2-1, 2-2, and 2-3.

\footnotetext{
${ }^{16}$ This value does not change the qualitative performance result of a component; however, it can influence the mean value of a performance response for a set of records. For example, mean values would be slightly larger than if ten percent was selected.
} 


\subsubsection{Linear Procedures}

Acceptance criteria of components for linear assessment procedures are provided as $m$-factors. The $m$-factor is intended to account for the ductility associated with a specific action and depends on the SPL and component type. ASCE 41 Table 5-5 provides the $m$-factors for steel components for linear assessment procedures. Adjustments to the $m$-factors for member or connection characteristics are detailed in ASCE 41 Chapter 5. In this study, actions in force-controlled components are assigned $m=1.0$ for simplicity and computational consistency in developed assessment spreadsheets. It should be noted that ASCE 41 does not assign an $m$-factor to force-controlled components.

\subsection{Calculating Component Assessment Results}

Component forces and deformations obtained by the LSP or LDP are referred to as design actions, $Q_{U}$ (e.g., flexure in a component).

Component design actions classified as deformation-controlled, $Q_{U D}$, are computed by

$$
Q_{U D}=Q_{G} \pm Q_{E} \quad(\mathrm{ASCE} 41 \text { 3 3.4.2.1.1) }
$$

Component design actions classified as force-controlled, $Q_{U F}$, are computed by

$$
Q_{U F}=Q_{G} \pm \frac{Q_{E}}{C_{1} C_{2} J} \quad(\operatorname{ASCE} 41 \S 3.4 .2 .1 .2)
$$

where $Q_{G}$ is the action due to gravity loads and $Q_{E}$ is the action due to earthquake effects. Elastic forcecontrolled demands from earthquake effects are divided by $C_{1} C_{2}$ to remove the demand amplification for short period structures from non-ductile components (see ASCE 41 §3.3.1). Similarly, the demand is divided by $J$, which is the force-delivery reduction factor and is taken as the minimum demand capacity ration (DCR) of the components in the load path delivering force to the component. Alternatively, $J$ can be taken as 2.0 when the system is located in a region of a high level of seismicity, independent of EHL. However, holding $J$ constant for multiple performance levels (disregarding the change in intensity) is not consistent with a capacity design approach, resulting in potentially overly conservative estimates of component actions in force-controlled elements at the higher EHL, or vice versa.

ASCE 41 33.4.2.2 requires that deformation-controlled and force-controlled actions in primary and secondary components satisfy:

Deformation-controlled: $\quad m \kappa Q_{C E} \geq Q_{U D} \quad$ (ASCE $\left.41 \S 3.4 .2 .2 .1\right)$

Force-controlled: $\quad(m) \kappa Q_{C L} \geq Q_{U F} \quad$ (ASCE $\left.41 \S 3.4 .2 .2 .2\right)$ 
where $m$ is the component demand modification factor (taken as unity for force-controlled actions-see above), $\kappa$ is the knowledge factor (taken as unity in this study), $Q_{C E}$ is the expected strength of the component, and $Q_{C L}$ is the lower-bound strength of the component.

The results of the linear assessment procedures are presented in this report as a normalized demand capacity ratio, $D C R_{N}$, so that the acceptance criteria verification becomes a unity check similar to that done in modern component design standards (e.g., AISC 360). $D C R_{N}$ is computed by rearranging ASCE 41 Eq. 3-20 and ASCE 41 Eq. 3-21 as required. As such, a $D C R_{N}$ value greater than unity indicates that the component does not satisfy the performance criteria for a given SPL. $D C R_{N}$ is similar to, but different than, the $D C R$ as used in ASCE 41. This approach is also a more consistent way to present results over the various types of assessment procedures used in this study. However, a slightly different interpretation is also taken in this study with regards to the $D C R_{N}$ : in lieu of $m$ and $\kappa$ adjusting the apparent strength of a component, as illustrated in ASCE $41 \S 3.4 .2 .2, m$ and $\kappa$ act to reduce the elastic demand to the expected demand given an EHL. Where required, the $D C R_{N}$ is determined from an interaction equation from the appropriate equation.

$$
\begin{gathered}
\text { Deformation-controlled: } \quad D C R_{N}=\frac{Q_{U D}}{m \kappa Q_{C E}}=\frac{D C R}{m \kappa} \\
\text { Force-controlled: } \quad D C R_{N}=\frac{Q_{U F}}{(m) \kappa Q_{C L}} \text { [with } m \text { taken as 1.0] }
\end{gathered}
$$

\subsubsection{Nonlinear Procedures}

Acceptance criteria of components for nonlinear assessment procedures are provided as plastic (inelastic) deformations dependent on the SPL and component type. ASCE 41 Tables 5-6 and 5-7 provide the plastic deformations limits for steel components for nonlinear assessment procedures. Adjustments to the acceptance criteria for member or connection characteristics are detailed in ASCE 41 Chapter 5.

Inelastic deformation parameters in ASCE 41 are provided for steel components in terms of plastic deformations rather than total deformations. The choice of whether to use plastic deformations or total deformations will depend on what nonlinear component model is adopted for each component action in the structural analysis (e.g., moment-curvature hinge or moment-rotation hinge). Consequently, yield and postyield elastic deformations may need to be added to the values given in ASCE 41 to determine the total deformation for each SPL.

In this study, demands on primary components of new buildings are measured against acceptance criteria for primary components. ASCE $41 \$ 3.4 .3 .2$ allows primary component demands to be within the acceptance criteria for secondary components for the NSP if degradation effects are explicitly modeled - a change introduced in FEMA 356. This also includes NDP, although not explicitly stated. This allowance is neglected in this study for the following reasons:

- Bypassing nonlinear acceptance criteria set for primary components suggests that acceptance criteria for primary components for linear and nonlinear assessment procedures are not calibrated. In this study, acceptance criteria for primary components are maintained for all assessment methods to provide a uniform comparison basis. 
- There is no technical justification provided in ASCE 41 as to why comprehensive component models for primary components, which are required to stabilize the structure after a large earthquake, will allow them to deform to the extent given for a secondary component and maintain the structural integrity of the system. Analytically speaking, it is debatable if collapse prevention can be reliably achieved if a concentrated few SFRS components are deformed past the deformation associated with their peak strength and lose a considerable amount of strength and stiffness. Available literature has indicated the difficulty in solution convergence in analysis when component response is following a negative stiffness branch. Still, some liberties are provided, debatably, for existing buildings, but transferring this allowance to new buildings is also of debatable validity. Engineering judgment must be applied. Broadly speaking, the function that separating existing buildings and new buildings into bins plays in developing a resilient community must be clarified.

- Reliability issues arise when adopting secondary acceptance criteria for primary components. For example, the acceptance criterion of an RBS beam-to-column connection taken as a secondary component for the Life Safety SPL is beyond the peak deformation (" $a$ " in Figure 3-2). In ASCE 41, primary acceptance criteria for the Collapse Prevention SPL generally matches the deformation associated with the peak strength of a component.

- There is little empirical evidence supporting the acceptance criteria for secondary steel components permitted in ASCE 41 \$3.4.3.2, other than for beam-to-column connections studied in the SAC project (see FEMA 350 series (FEMA 2000a through 2000d)). Experimental tests are rarely continued to achieve the peak deformations and the associated reserve strength of a component or subassembly after the required loading protocol is complete.

As a side note, ASCE 41-13 has removed all acceptance criteria for primary components for nonlinear assessment. Future research should be conducted to evaluate the systems by measuring demands against acceptance criteria for secondary components - which can be done by inspection with the analysis results presented in this study.

\subsection{Calculating Component Assessment Results}

Component forces and deformations obtained by the NSP or NDP are referred to as design actions, $Q_{U}$ (e.g., plastic rotation in a plastic hinge). Component design actions are computed as the action in the member or connection at the target displacement for the NSP and as the maximum value for a given earthquake for the NDP. Subsequently, a statistical average is computed from the maximum values from the suite of ground motions. In specific cases, the maximum value must be coupled with other actions in the component at the instant of computation of the maximum response.

Component design actions classified as deformation-controlled, $Q_{U D}$, are computed by

$$
Q_{U D}=Q_{G} \pm Q_{E} \quad(\operatorname{ASCE} 41 \S 3.4 .3 .2 .1)
$$

Component design actions classified as force-controlled, $Q_{U F}$, are computed by

$$
Q_{U F}=Q_{G} \pm Q_{E} \quad(\operatorname{ASCE} 41 \S 3.4 .3 .2 .3)
$$

where $Q_{G}$ is the action due to gravity loads (or associated deformation) and $Q_{E}$ is the action due to earthquake effects (or associated deformations). Superposition of forces or deformations is not applicable 
in a nonlinear analysis; thus, gravity loads are directly applied in the analysis. The above equations are numerical interpretations of ASCE 41 \$3.4.3.2. They are used in this study to maintain computational consistency over the various types of assessment procedures.

ASCE 41 \$3.4.3.2 requires that deformation-controlled and force-controlled actions in primary and secondary components satisfy:

$$
\begin{gathered}
\text { Deformation-controlled: } \left.\quad \kappa Q_{C E} \geq Q_{U D} \quad \text { (ASCE } 41 \S 3.4 .2 .2 .1\right) \\
\text { Force-controlled: } \quad \kappa Q_{C L} \geq Q_{U F} \quad(\text { ASCE } 41 \text { \$3.4.2.2.2) }
\end{gathered}
$$

where $Q_{C E}$ is the expected strength or deformation demand of a component, $Q_{C L}$ is the lower-bound strength of a component, and $\kappa$ is the knowledge factor (taken as unity in this study). ASCE 41 Chapter 5 does not explicitly provide a relationship between $Q_{C E}$ (or $Q_{C L}$ ) and $Q_{y}$ on the force-deformation curve.

The results of the nonlinear assessment procedures are presented in this report as a normalized demand capacity ratio, $D C R_{N}$, where the plastic or total deformation demands are normalized with respect to the plastic or total acceptance criteria, modified by $\kappa$ if required. The acceptance criteria verification then becomes a unity check similar to that done in modern component design standards (e.g., AISC 360). As such, a $D C R_{N}$ value greater than unity indicates that the component does not satisfy the performance criteria for a given SPL. This approach is a consistent way to present results over the various types of assessment procedures used in this study.

$$
\begin{aligned}
& \text { Deformation-controlled: } D C R_{N}=\frac{Q_{U D}}{\kappa Q_{C E}}= \begin{cases}\text { Total } & \frac{\theta_{\text {plastic }}+\theta_{\text {elastic }}}{\kappa\left(\theta_{y}+\theta_{p e}+\theta_{p, A C}\right)} \\
\text { Plastic } & \frac{\theta_{\text {plastic }}}{\kappa \theta_{p, A C}}\end{cases} \\
& \text { Force-controlled: } \quad D C R_{N}=\frac{Q_{U F}}{\kappa Q_{C L}}
\end{aligned}
$$

where $\theta_{\text {plastic }}$ is the plastic deformation of a component, $\theta_{\text {elastic }}$ is the elastic deformation of a component, $\theta_{y}$ is the yield deformation of a component, $\theta_{p e}$ is the post-yield elastic deformation of a component, and $\theta_{p, A C}$ is the acceptance criteria of a component based on plastic deformation. 


\subsection{Eccentrically Braced Frame}

Seismic performance assessment of steel eccentrically braced frames is performed in accordance with ASCE 41 §5.5.3. The eccentrically braced frames in the archetype buildings are designed as an EBF as identified in ASCE 7 §12.2 (item B.1 in Table 12.2-1) and AISC $341 \S$ F3.

\subsubsection{Assessment Methodology}

There are three primary characteristics of each component (i.e., member, connection, etc.) forming the structural model for each assessment method:

1. Stiffness, ASCE 41 \$5.5.3.2

2. Strength, ASCE 41 §5.5.3.3

3. Acceptance Criteria, ASCE $41 \S 5.5 .3 .4$, and whether or not the component action is forcecontrolled or deformation-controlled.

Each component characteristic is discussed in the appropriate linear and nonlinear assessment discussion.

The following component design actions are assessed in this study:

- Link shear force or deformation

- Beam, column, and brace flexural strength at potential plastic hinge zones (section strength) ${ }^{17}$

- Beam, column and brace axial-moment interaction strength (member strength)

\subsubsection{Linear Procedures}

This section discusses the three primary component characteristics listed in Section 3.2.1 and computation of the demand in the component for the linear assessment procedures.

\section{Stiffness}

The stiffnesses of all members and connections for linear assessment follow ASCE 41 §5.5.3.2. To account for the increased stiffness provided by brace-to-beam and brace-to-column (gusset plate) connections, an 18-inch prismatic section with twice the flexural stiffness, $E I$, is used to connect the beam-to-column joint (outside the panel zone) to the beams and columns. Braces are modeled with a rigid end equal to $0.05 L_{w p}$, and are 'pinned' out-of-plane and 'fixed' in-plane (see Chapter 2).

\section{$\underline{\text { Strength }}$}

The strength of all members and connections for linear assessment follow ASCE 41 §5.5.3.3.

\footnotetext{
${ }^{17}$ All components other than link beams are force-controlled per ASCE $41 \S 5.5 .3 .4$. Brace connections are not assessed in this study because they are designed to resist forces computed using the maximum strengths of the brace.
} 
The expected shear strength of a link beam, $Q_{C E}=V_{C E}\left(=Q_{y}\right)$, is computed as Equation (3-14)-ASCE 41 Equation 5-31).

$$
V_{C E}=0.6 F_{y e}\left(d-2 t_{f}\right) t_{w}
$$

For A992 steel, $F_{y e}=1.1 \times F_{y}$ (see ASCE 41 Table 5-3) which corresponds to $R_{y} F_{y}$ in AISC 341 . This strength is slightly different that $V_{n}$ in AISC 360 Chapter G and AISC $341 \S \mathrm{F} 3$. This strength is also slightly different than $V_{C E}$ of a standard beam (ASCE 41 Equation 5-7-also see below). These differences are not discussed in the commentary of ASCE 41. Having different shear strengths in AISC 360 and AISC 341, and then again in ASCE 41, results in inconsistent strength measures that can affect performance predictions of short beams. Additionally, there are no compactness requirements placed on a link beam in ASCE 41 to ensure full section yielding.

The expected flexural strength of a member, $Q_{C E}=M_{C E}\left(=Q_{y}\right)$, is computed as $M_{n}$ from AISC 360 Chapter $\mathrm{F}^{18}$ with $\phi_{b}=1.0$ and $F_{y e}$ in lieu of $F_{y}$. For ASTM A992 steel, $F_{y e}=1.1 \times F_{y}$ (see ASCE 41 Table 5-3), which corresponds to $R_{y} F_{y}$ in AISC 341. Composite action with the concrete slab is generally neglected in computing $M_{n}$ for frame beams. In so doing, it is assumed that the plastic moment strength is achievable via adequate lateral bracing, thus $M_{n}=M_{p}$. If the flexural strength is less than $M_{p}$, then the available ductility of the member is significantly reduced because of member or cross-section instability (which also affects the acceptance criteria). ASCE 41 enforces section compactness requirements through the acceptance criteria-discussed subsequently.

The lower-bound flexural strength of a member, $Q_{C L}=M_{C L}$, is computed as $M_{n}$ from AISC 360 Chapter $\mathrm{F}^{18}$ with $\phi_{b}=1.0$ and $F_{y L B}$ in lieu of $F_{y}$. For ASTM A992 steel, $F_{y L B}=1.0 \times F_{y}$ (see ASCE 41 Table 5-2).

Although not explicitly identified in ASCE $41 \S 5.4 .2 .3 .2$, the expected shear strength of a member, $Q_{C E}=$ $V_{C E}\left(=Q_{y}\right)$, is identical to that computed as $V_{n}$ from AISC 360 Chapter G with $\phi_{v}=1.0$ and $F_{y e}$ in lieu of $F_{y}$. Web slenderness, $h / t_{w}$, is critical in developing a fully yielded cross-section. As such, there are cases when

$$
\frac{418}{\sqrt{F_{y}}}=2.45 \sqrt{\frac{E}{F_{y}}}<\frac{h}{t_{w}} \leq \frac{640}{\sqrt{F_{y}}}=3.76 \sqrt{\frac{E}{F_{y}}}
$$

and the web is still capable of achieving full yield strength in shear. However, if the web slenderness approaches the upper limit (taken as the 'compact' limit in AISC 360 Table B4.1) then the beam may have difficulty achieving its plastic moment strength, $M_{p}$. There is no lower-bound shear strength, $V_{C L}$, in ASCE 41 or shear-moment interaction.

The lower-bound compression strength of a member, $Q_{C L}=P_{C L}$, is computed as $P_{n}$ from AISC 360 Chapter $\mathrm{E}^{18}$ with $\phi_{c}=1.0$ and $F_{y L B}$ in lieu of $F_{y}$. For ASTM A992 steel, $F_{y L B}=1.0 \times F_{y}$ (see ASCE 41 Table 5-2). ASCE 41 allows the effective length factor, $K$, of a brace member to be taken as 0.8 for in-plane buckling

\footnotetext{
18 ASCE 41 inadvertently states AISC 341.
} 
and 1.0 for out-of-plane buckling, and $L$ is the clear length of the brace plus the gusset plate. For the archetype buildings in this study, $K L$ is taken as $1.0 \times 0.9 L_{w p}$ for both axes, where $L_{w p}$ is the length between work points, which is a conservative estimate for in-plane buckling.

Though identified in ASCE 41 \$5.4.2.3.2-2, no guidance is provided for computing the expected flexural strength, $M_{C E}$, of a compression member if the axial load, $P$, exceeds 10 percent of the axial strength. This guidance would be useful for computing the flexural $D C R$ at a given location, such as at the column base. The term axial strength of a compression member is also not well defined (i.e., is it $P_{y e}$ or $P_{C L}$ ?). Similarly, the axial load, $P$, is not defined except in an unrelated provision for the NSP. ASCE 41 §5.4.2.2.2 states that $P$ for a linear analysis is $P_{U F}$. The flexural strength will also depend on the chosen $P-M$ interaction curve, which will use $M_{C E}$ (or $M_{C L}$ ) at $P=0$ as the anchor point. There is little need to have triggering language based on 10 percent axial load ratio because it delineates no physical phenomenon and does not also influence computing the yield chord rotation, $\theta_{y}$. In fact, it adds complexities, which will be discussed subsequently in the Acceptance Criteria section. ASCE 41 does not delineate between orthogonal buckling axes and non-flexural buckling limit states (e.g., torsional, local buckling) for its interaction verification. This can significantly affect the assessment of beam-column members where high in-plane moment is associated with a buckling limit state other than in-plane flexural buckling. Beam-columns can be further penalized in linear assessment methods where $P_{U F}$ is highly affected by selecting a value for $J$.

The expected tension strength of a member, $Q_{C E}=T_{C E}\left(=Q_{y}\right)$, is computed as $A_{c} \times F_{y e}$, where $A_{c}$ is the crosssectional area of the member. ASCE 41 does not provide other tensile strengths, potentially brittle, similar to AISC 360 Chapter D, e.g., net section fracture.

The expected shear strength of a panel zone, $Q_{C E}=V_{C E}\left(=Q_{y}\right)$, is computed as $0.55 F_{y e} \times d_{b} \times t_{p}$. (ASCE 41 Equation $5-5^{19}$ ). This strength equates to $0.92 \times V_{y}$ from AISC 360 Chapter J10.6 with $\phi_{v}=1.0$ and $F_{y e}$ in lieu of $F_{y}$. ASCE 41 does not provide additional panel zone strength to account for column flange bending.

ASCE 41 does not provide explicit guidance on computing the strength of a beam-to-column connection in an EBF other than it shall be treated as force-controlled for all actions. Consequently, the controlling mechanism for a beam-to-column connection is taken as the lower-bound flexural strength, $Q_{C L}$, measured at the face of the column. This flexural strength is determined using AISC 360 with $\phi=1.0$ and $F_{y L B}$ in lieu of $F_{y}$. Similarly, the lower-bound strength of a brace-to-beam and brace-to-beam/column connection is determined from AISC 360 and is force-controlled for all design actions.

\section{Acceptance Criteria}

The acceptance criteria of members and connections for linear assessment follow ASCE 41 \$5.5.3.4. However, SCE 41 \$5.5.3.4.1 requires that all columns, beams adjacent to link beams, braces, and all connections in an EBF to be force-controlled for axial force and flexure.

\footnotetext{
${ }^{19}$ The reason the shear yield stress for a column web (assuming it can be designated as a beam) being defined as $0.6 \times F_{y e}$ is not clear, but if the same column web is a panel zone, the shear yield stress is $0.55 \times F_{y e}$.
} 


\section{Shear Force in Link Beams}

The acceptance criteria for shear actions in link beams are provided in ASCE 41 Table 5-5 and dependent on the link length, $e$. Acceptance criteria for flexural actions (including $P-M$ interaction) in link beams are provided in ASCE 41 Table 5-5 when applicable. However, ASCE 41 does not provide significant guidance on treatment of axial-moment or shear-moment interaction within link beams except that axial compression and tension actions are designated as force-controlled. Nonetheless, the acceptance criteria for flexural actions are dependent on this axial force. Furthermore, the shear-moment interaction for intermediate links is not well explained and inconsistent for the IO SPL. ASCE 41 does not provide compactness requirements of the cross section elements to ensure full shear yielding of the section and assumes that the link beam is a wide-flange section.

\section{Axial Force in Braces and Brace Connections}

ASCE 41 §5.5.3.4.1 requires that all axial actions in a brace and brace connection in an EBF be forcecontrolled for axial actions.

\section{Flexure in Beams, Beam-to-Column Connections, and Brace Connections}

ASCE 41 \$5.5.3.4.1 requires that all flexural actions in beams (other than link beams) and all connections in an EBF be force-controlled. Though flexural action in these components is treated as force-controlled, the following discussion is presented assuming that these components are deformation-controlled for modelling and analysis purposes to align with other reports developed for this study (see NIST 2014a and 2014b). Also, this allows comparison to other braced frame systems in ASCE 41-including bucklingrestrained braced frames found in ASCE 41-13.

The acceptance criteria for flexural action at expected locations of plastic hinging in beams (members with axial load ratio less than or equal to 10 percent) are provided in ASCE 41 Table 5-5 and are dependent on web and flange slenderness. The range of flange limits match AISC 341 limits for highly and moderately ductile unstiffened compression elements. The range of web limits match AISC 341 limits for highly and moderately ductile stiffened compression elements taking at $P=0$. The flange and web slenderness limits for moderately ductile sections are taken as a 'compact' compression element in AISC 360 (i.e., $\lambda_{p}$ ). The lower-bound web slenderness limit is taken as that capable of fully section yielding in shear. These web slenderness limits were introduced in FEMA 356 whereas flange limits were introduced in FEMA 273 and subsequently modified in FEMA 356 (upper-bound limit was changed from a pure axial compression limit to a compression from flexure limit).

In cases where the expected flexural strength of an unbraced segment is governed by instability (e.g., lateral torsional buckling (LTB)) rather than full section yielding, the $m$-factors in ASCE 41 Table 5-5 shall be reduced accordingly. Again, ASCE 41 inadvertently references AISC 341 for these calculations as well as uses the notation, $M_{r}$, which is no longer used in AISC 360.

The acceptance criteria for beam-to-column connections (flexural hinge is located within the connection) are taken from Fully Restrained Moment Connections in ASCE 41 Table 5-5 and are dependent on 
connection detailing, panel zone strength in a balanced yield condition, and member and cross-section slenderness (see ASCE $41 \S 5.4 .2 .4 .2,4.1$ to 4.4). It is assumed that the acceptance criteria for FR beam-tocolumn connections ( $m$-factor) translated from results from the SAC project are applicable at the face of the column—see \$3.2.1.2. Furthermore, the $m$-factors for beams are not applicable for flexural plastic hinges within the region ${ }^{20}$ demarcating the beam-to-column connection when connection components prevent the unobstructed spread of plasticity. ASCE 41 does not provide acceptance criteria for flexural actions for a beam-to-column connection that includes a brace connection (i.e., brace-to-beam/column) or a brace-to-beam connection. ASCE 41 requires that all beams, beam-to-column connections, and brace connections in an EBF be force-controlled for flexural actions. ASCE 41 §5.5.3.3.2 introduces the expected flexural strength of beams and columns, although they are required to be force-controlled. There is limited technical justification as to the reason beams adjacent to shear links as well as the beam-to-column and brace-to-beam connections are mandatorily force-controlled for the CP BPL at the BSE-2 EHL. In so doing, an EBF is potentially overly penalized by ASCE 41 as compared to a concentrically braced frame (CBF), which does not apply force-controlled designations on all components.

\section{Flexure in Columns, Braces, and Columns-to-Base Connections}

ASCE 41 §5.5.3.4.1 requires that all flexural actions in columns, braces, and all connections in an EBF be force-controlled. Though flexural action in these components is treated as force-controlled, the following discussion is presented assuming that these components are deformation-controlled for modelling and analysis purposes to align with other reports developed for this study (see NIST 2014a and 2014b). Also, this allows comparison to other braced frame systems in ASCE 41-including buckling-restrained braced frames found in ASCE 41-13.

The acceptance criteria for flexural action at expected locations of plastic hinging in columns (members with axial load ratio greater than 10 percent) are provided in ASCE 41 Table 5-5 and are dependent on the axial load ratio, $P_{U F} / P_{C L}$, and web and flange slenderness. As discussed above, ASCE 41 does not delineate between orthogonal buckling axes and non-flexural buckling limit states (e.g., torsional, local buckling) for computing $P_{C L}$. Consequently, this can significantly affect the assessment of beam-column members where a high in-plane moment is associated with a buckling limit state other than in-plane flexural buckling. If the axial load ratio is greater than 0.5 , then flexural action is considered force-controlled and the flexural demand and strength are taken as $M_{U F}$ and $M_{C L}$, respectively. Otherwise, the $m$-factor is adjusted for $P-M$ interaction as shown in Figure 3-4 and the flexural demand and strength are taken as $M_{U D}$ and $M_{C E}$, respectively.

The flange slenderness limits for columns are the same as those for beams and are independent of axial load. The lower-bound web slenderness range is essentially ${ }^{21} 75$ percent of the slenderness limits in AISC 341-02 (AISC 2002) - taken from FEMA (2000c) — at distinct axial force ratios (0.2 and 0.5). These ratios are at the upper range of axial force ratios in ASCE 41, albeit $P_{U F} / P_{y e}$ and $P_{U F} / P_{C L}$ represent two physically different phenomena in regards to plate buckling. The upper-bound web slenderness range is essentially the slenderness limits in AISC 341-02 at distinct axial force ratios (0.1 and 0.2). These ratios are at the lower

\footnotetext{
${ }^{20}$ This region is also used in AISC 341 to define the protected zone.

${ }^{21}$ Work to develop FEMA 356 was conducted at the same time as the SAC project-see FEMA 350 series.
} 
range of axial force ratio in ASCE 41. Using fixed axial limits on slenderness can lead to excessive conservatisms because of step function triggers, as illustrated in Figure 3-5 for the LS SPL-AISC 341 web compactness limits for highly (HD) and moderately (MD) ductile compression elements are included for comparison. The spherical icons shown in the figure represent the web slenderness ratios for all wide-flange sections currently available. Essentially, 35 percent of these sections do not satisfy the lower-bound criteria in ASCE 41 whereas this value reduces to 12 percent when using the AISC 341 criteria for highly ductile elements. Ultimately, the step functions created by both the axial load ratios and the section compactness requirements result in a highly complex formulation that is difficult to implement, as illustrated in Figure 3-6 for the LS SPL (plastic rotation is shown in lieu of $m$-factor). ASCE 41 could be simplified by combining the acceptance criteria for beams and columns into one set of criteria with no 10 percent axial load ratio trigger.

In addition to the effect of $P-M$ interaction on the $m$-factors (which is a section strength issue) for checking flexural actions in a column in accordance with ASCE 41 \$3.4.2.2, member stability is also checked via global interaction equations in accordance with ASCE 41 \$5.4.2.4, as shown in Figure 3-4 - see projection of axial and moment ratios. The discontinuous curve is a result of variable $P-M$ interaction equations, with the discontinuity at $P_{U F} / P_{C L}=0.5$ being smallest when $M_{C L}$ at $P_{U F}=0$ equals $M_{p}$, and gets larger as $M_{U D} /$ $M_{U F}$ increases. Future efforts should simplify the ASCE 41 interaction curves for consistency and applicability, including eliminating $F_{y, L B}$ for a column that also uses $F_{y e}$.

Axial compression action in a column is always force-controlled due to significant reduction in ductility because of member and cross-sectional instability. Again, ASCE 41 is rather ambiguous when it comes to steel columns. First, there is no guidance on computing $M_{C E}$ for a column. Although ASCE $41 \S 5.4 .2 .4 .2-$ 2 provides some information, it is not consistent with that required to define the expected flexural strength, $M_{C E}$. Second, the $m$-factor is reduced for beams to account for LTB. Since this failure mode is also applicable to columns, the $m$-factor should similarly be reduced. However, a column that fails in LTB should be avoided, and $m=1$ adopted since LTB is not a ductile phenomenon. Also, $\kappa$ is not in the interaction equations similar to other verification procedures, and it is unclear if these equations have any physical meaning because member stability and section yielding effects are combined. Moreover, these equations can be conservative when weak-axis buckling is coupled with in-plane (strong-axis) bending.

ASCE 41 \$5.5.3.3.2 introduces the expected flexural strength of columns and beams, although they are required to be force-controlled. However, ASCE 41 does not provide guidance on how to assess a forcecontrolled column for flexural when $P \leq 0.5 P_{C L}$ similar to that given for a moment frame or concentrically braced frame. For this study, assessment of columns and braces with $P-M$ interaction is done using ASCE $41 \S 5.4 .2 .4 .2-2$ (Equations 5-10, 5-11, and 5-12), with $M_{C L}$ used in lieu of $M_{C E}$ where applicable and the $m$ factor taken as unity.

Columns are designed in accordance with capacity design provisions in AISC 341. ASCE 41 does similarly adopt a capacity design approach for the assessment of EBF columns and beams (see ASCE 41 §5.5.3.4.1). 


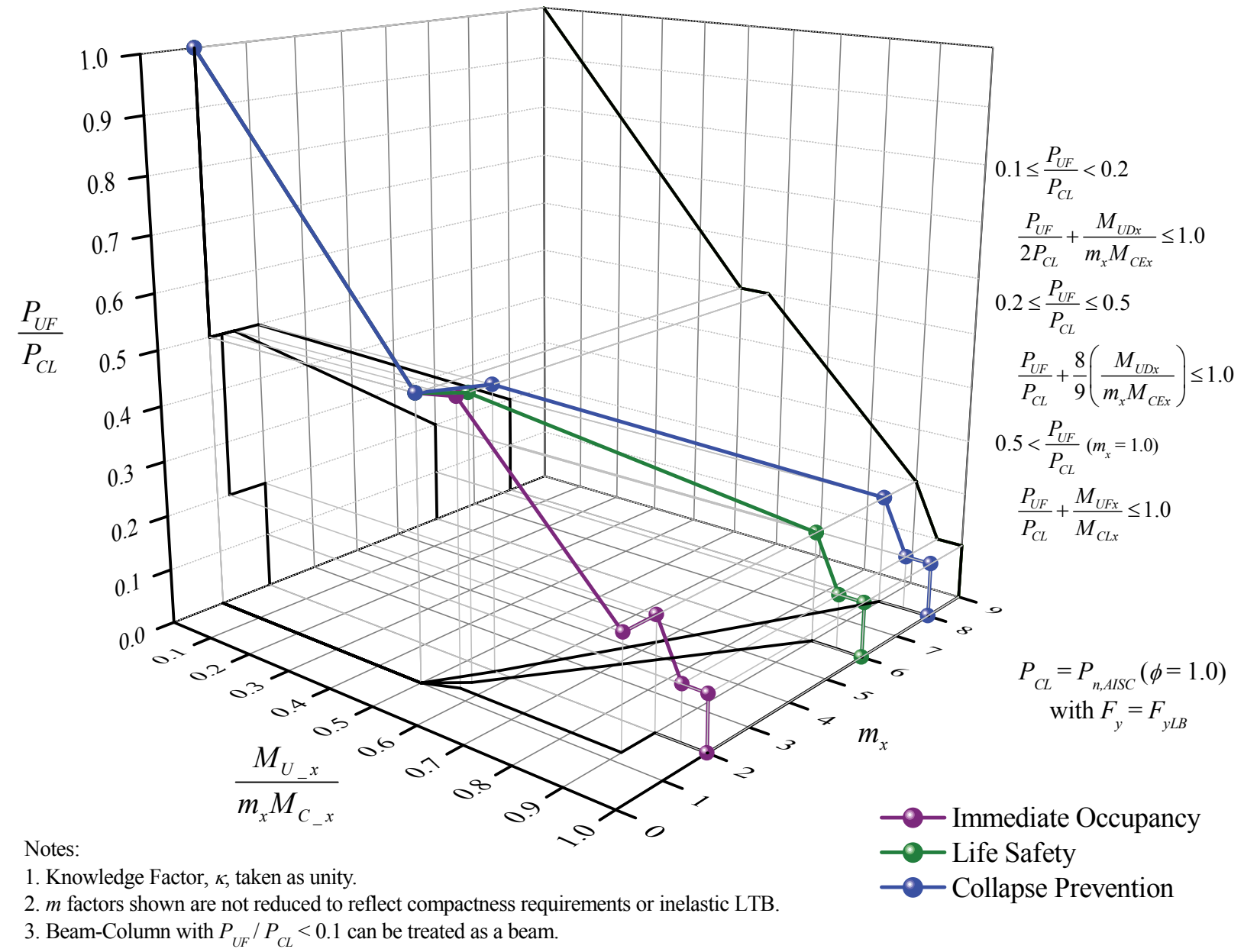

Figure 3-4. $P$ - $M$ Interaction on Section $m$-factor (in-plane) and Member Instability (Primary Component) 


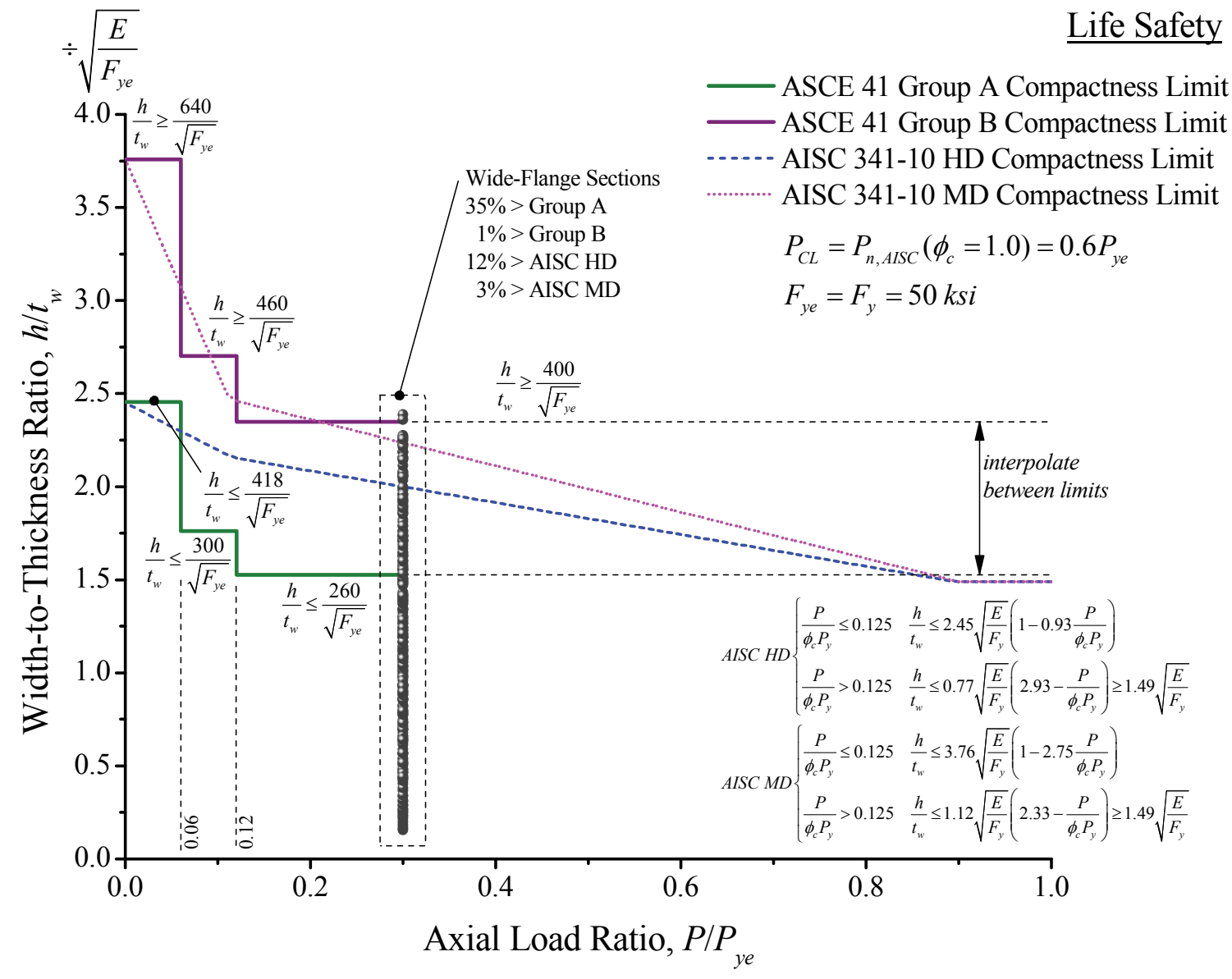

Figure 3-5. Compactness Requirements as a Function of Axial Load Ratio, LS Acceptance Criteria 


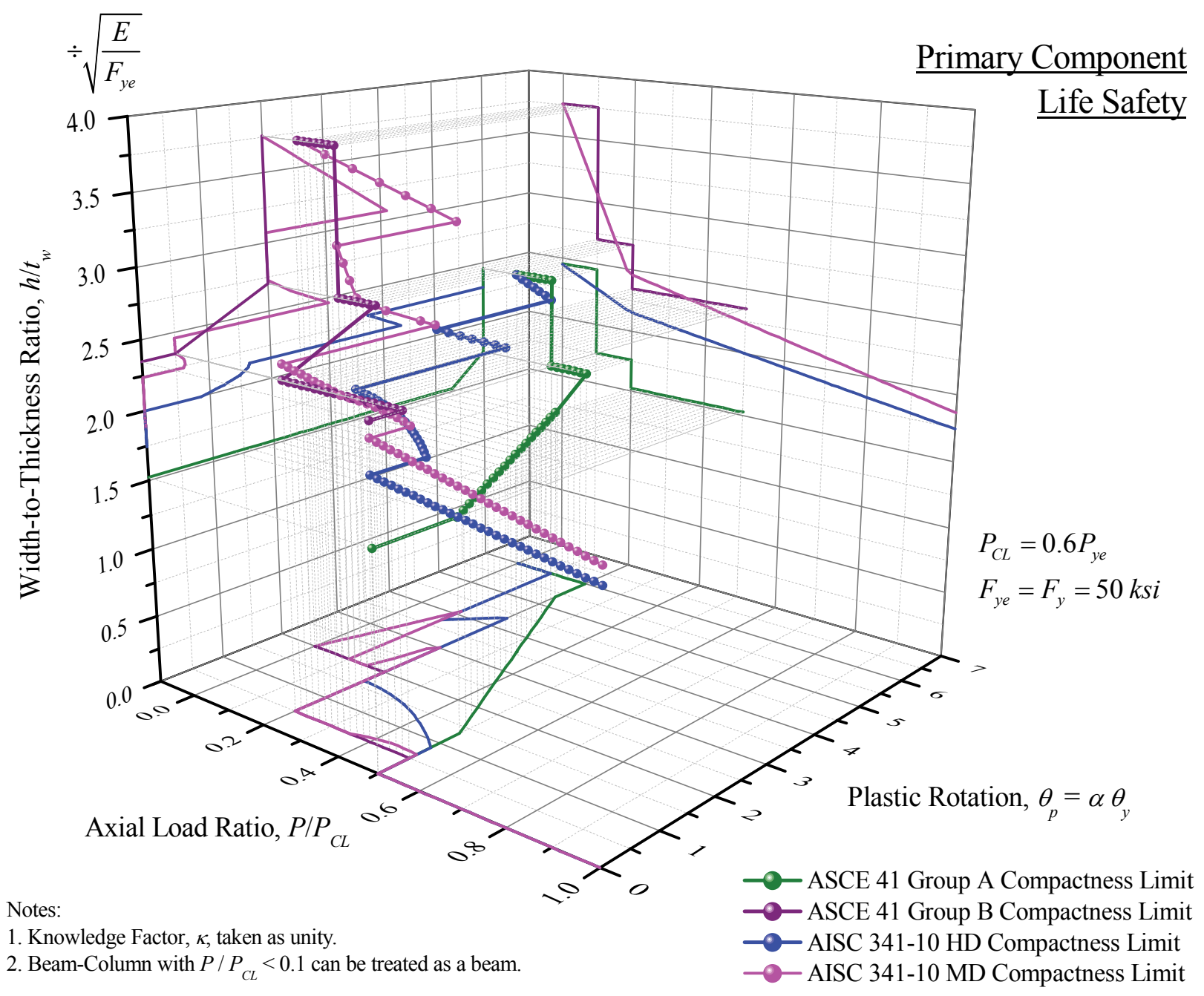

Figure 3-6. Acceptance Criteria as a Function of Axial Load Ratio and Section Compactness, LS Acceptance Criteria

\section{Shear in Panel Zones}

ASCE 41 requires that all panel zones in an EBF be force-controlled.

\section{Additional Commentary on the Forces in all EBF Components other than Link Beams}

As discussed previously, ASCE 41 \$5.5.3.4.1 requires all component actions other than link beams to be force-controlled. However, ASCE 41 does not provide explicit guidance with regards to computing forces in force-controlled components in an EBF for linear assessment procedures. First, force-controlled actions are computed in accordance with ASCE 41 \$3.4.2.1.2 — either Method 1 or 2. Second, ASCE 41 $\$$ 5.5.3.4.2 requires components to be assessed for a maximum developed link force of 1.25 times the lesser of the expected flexural or shear strengths of the link beams. It is assumed in this study that this guidance is intended to outline ASCE 41 33.4.2.1.2-1 (Method 1) for computing force-controlled component actions 
and that this guidance is not also applicable for ASCE 41 \$3.4.2.1.2-2 (Method 2). Therefore, assessment of this framing system is done twice, using force-controlled actions computed (1) according to ASCE 41 $\S 3.4 .2 .1 .2-2$ and (2) according to ASCE 41 \$3.4.2.1.2-1 assuming a maximum developed link force of 1.25 times the lesser of the expected flexural or shear expected strengths of the link beams. The maximum value of the two will govern assessment procedures.

The capacity design procedure described above matches that prescribed in AISC $341 \S \mathrm{F} 2.3$ for design of an EBF, except for negligible variation in the gravity load contribution and that qualified columns can be designed for 88 percent of the seismic demand. In regards to ASCE 41 assessment, setting $\phi_{c}$ equal to unity to compute $P_{C L}$ effectively compensates for the 12 percent reduction provided for new EBF columns. Because the EBF in each archetype building is designed in accordance with AISC 341, the force-controlled components of each EBF satisfy ASCE 41 \$3.4.2.1.2-1 by default and no further assessment is required. Consequently, assessment of the force-controlled components in this study is performed in accordance with ASCE 41 §3.4.2.1.2-2 for comparison purposes between the two methods.

\section{Demand}

The flexural demand, $M_{U D}$ or $M_{U F}$, and axial force, $P_{U F}$, for the columns and braces are taken as the moment and axial force at the face of the each beam (top and bottom) or brace connection, respectively. The flexural demand, $M_{U D}$ or $M_{U F}$, for the beams is taken as the moment at the face of the brace connection. The flexural demand, $M_{U D}$ or $M_{U F}$, for the beam-to-column connections is taken as the moment at the face of the column or edge of the brace connection.

Table 3-10 summarizes the basic $m$-factors for the components of the EBF for the linear procedures. Figure 3-7 through Figure 3-9 provide the load-independent $m$-factors - taking in to account force-controlled and deformation-controlled classifications (force-controlled component actions are assigned an $m$-factor of unity, see §3.1.4.1). These figures are referred to herein as "Frame Capacity Schematics."

Table 3-10. Basic $m$-factors for Linear Procedures, EBF

\begin{tabular}{llc}
\hline \multicolumn{1}{c}{ Component - Action } & \multicolumn{2}{c}{ Performance Level } \\
\hline EBF Link Beam & $\mathrm{LS}$ & $\mathrm{CP}$ \\
a) $e \leq 1.6 M_{C E} / V_{C E}$ & 9 & 13 \\
b) $e \geq 2.6 M_{C E} / V_{C E}$ & Same as for beams \\
b) $1.6 M_{C E} / V_{C E}<e<2.6 M_{C E} / V_{C E}$ & linear interpolation \\
\hline
\end{tabular}




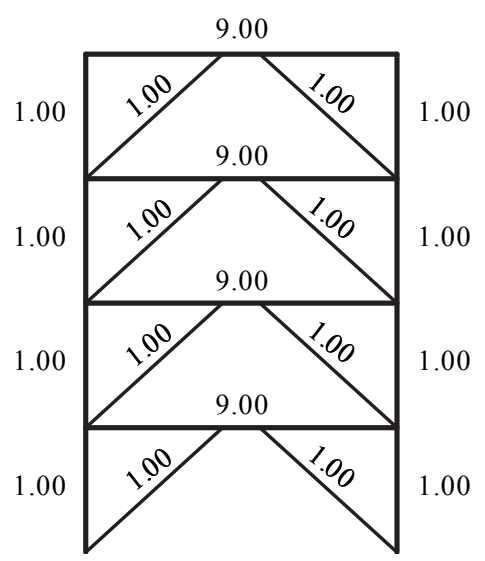

(a) Life Safety

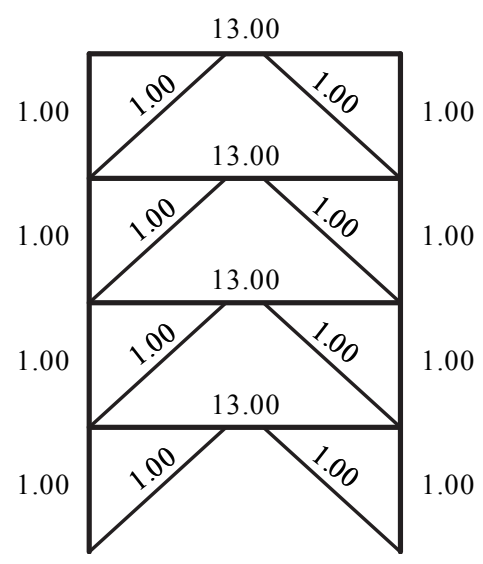

(b) Collapse Prevention

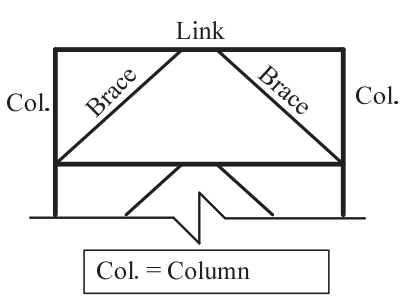

(c) Key

Figure 3-7. Frame Capacity Schematic ( $m$-factor), LS and CP, 4-Story EBF ELF and RSA

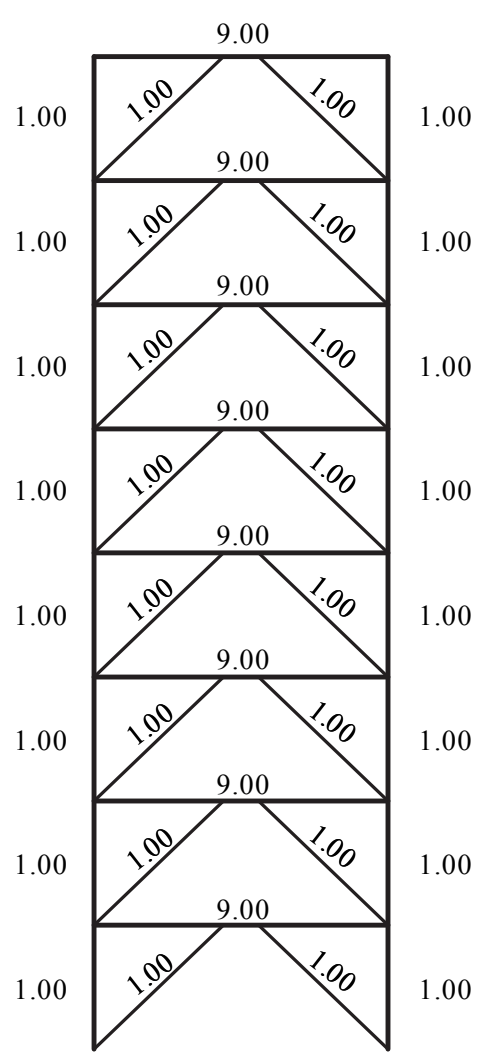

(a) Life Safety

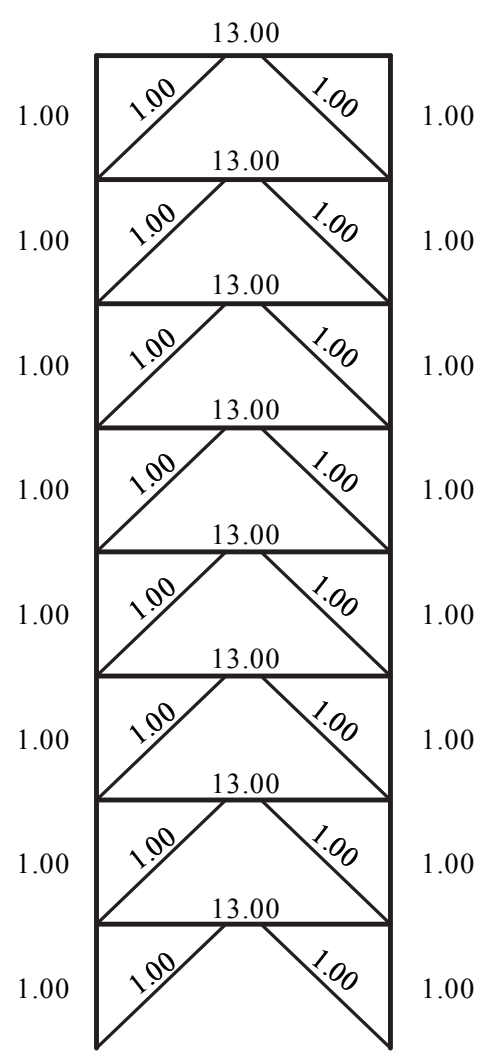

(b) Collapse Prevention

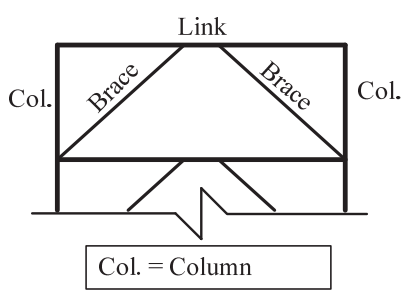

Figure 3-8. Frame Capacity Schematic ( $m$-factor), LS and CP, 8-Story EBF ELF and RSA 


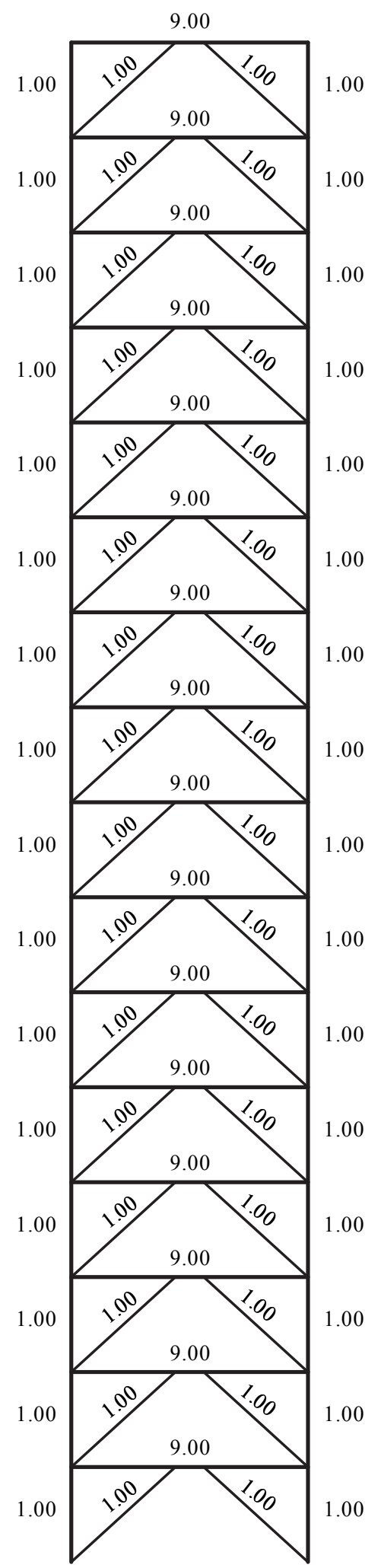

(a) Life Safety

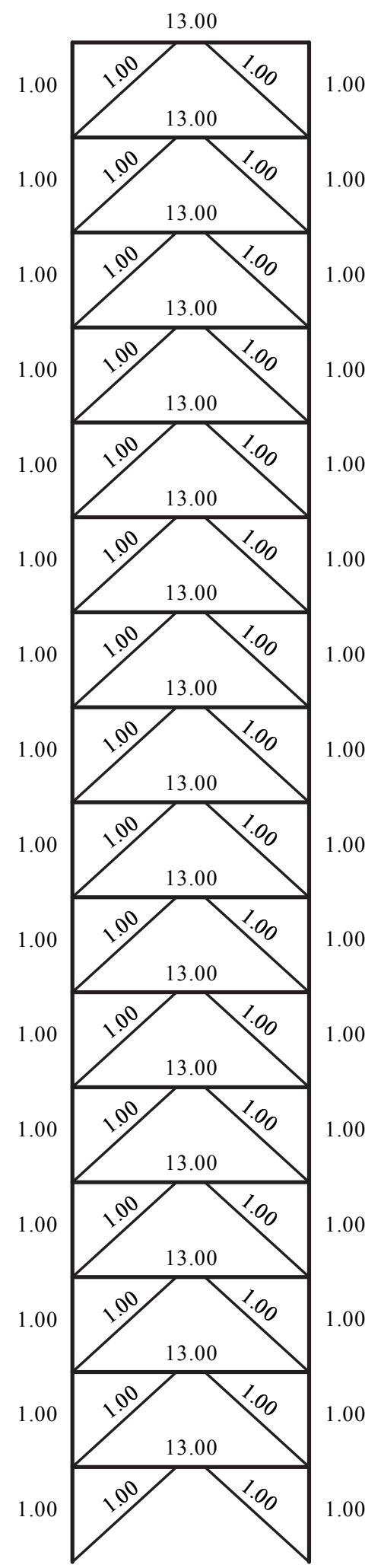

(b) Collapse Prevention

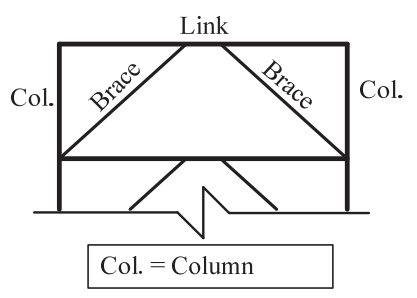

Figure 3-9. Frame Capacity Schematic ( $m$-factor), LS and CP, 16-Story EBF ELF and RSA 


\subsubsection{Nonlinear Procedures}

This section discusses the three primary component characteristics listed in Section 3.2.1 and computation of the demand in the component for the nonlinear assessment procedures.

\section{Stiffness, Strength, Acceptance Criteria, and Demand}

Component characteristics follow that outlined previously for the linear procedures. Although component stiffness is the primary characteristic in linear procedures, component strength is of equal importance in nonlinear procedures.

As noted previously, ASCE 41 §5.5.3.4.1 requires that all components in an EBF other than the link beams be force-controlled. Though component actions are classified as force-controlled, the complete nonlinear response of these components is modeled for the NSP and NDP in this study. That is, the following discussion is presented assuming that these components are deformation-controlled for modelling and analysis purposes to align with other reports developed for this study (see NIST 2014a and 2014b). Also, this allows comparison to other braced frame systems in ASCE 41-including buckling-restrained braced frames found in ASCE 41-13.

The stiffnesses of all members and connections for nonlinear assessment follow ASCE 41 §5.5.3.2.2 for the NSP and ASCE $41 \S 5.5 .3 .2 .3$ for the NDP. The strength of all members and connections for nonlinear assessment follow ASCE 41 \$5.5.3.3.3 for the NSP and ASCE 41 §5.5.3.3.4 for the NDP. The same analytical model is used for both the NSP and NDP—see §3.1.3.2.

Compound elements with elastic and inelastic components are used for constructing all members in PERFORM-3D. Elastic stiffness and strength characteristics for each component follow that outlined for the linear procedures. Nonlinear components include the column and brace flexural hinge modeled with axial-moment $(P-M)$ interaction and the panel zone modeled using the Krawinkler model (Krawinkler 1978). Gusset plate connections for braces are not explicitly modeled. The flexural rigidity, EI, of the portion of a member adjacent to a gusset plate is increased to approximate the effect of gusset plate rigidity in the plane of the frame. Figure 3-10 illustrates the analytical model of a brace-to-beam / column subassembly for nonlinear assessment procedures. In the figure, Default End Zones are modeled with a flexural rigidity factor of two; a higher end zone factor is potentially too rigid to capture flexural deformations within the joint region. Detailed information concerning all aspects of the analytical model for nonlinear analysis can be found in PERFORM-3D Components and Elements (CSI 2011c).

Shear plasticity in a link beam is represented by a plastic shear strain hinge with a plastic hinge length equal to the link length, $e$. Inelastic strain parameters are taken from ASCE 41 Table 5-7. Shear strength and stiffness degradation are modeled based on calibrating the PERFORM-3D MODEL with experimental test data (see Figure 3-11-PERFORM-3D response is presented as "Analysis"). The shear hinge model in PERFROM-3D gives the response in terms of plastic strain. In this study, the shear yield force, $V_{y}$, is taken as $1.15 \times V_{C E}$ as defined in ASCE 41 to account for the influence of the flanges at yield and the ultimate shear force is taken as $1.25 \times V_{y}\left(=1.4375 \times V_{C E}\right)$ - see Figure 3-11. As will be graphically illustrated subsequently in the analysis results, this adopted link beam strength will increase the (axial) forces in the adjacent members beyond that determined from the capacity analysis prescribed in ASCE 41 §5.5.3.4.2. 


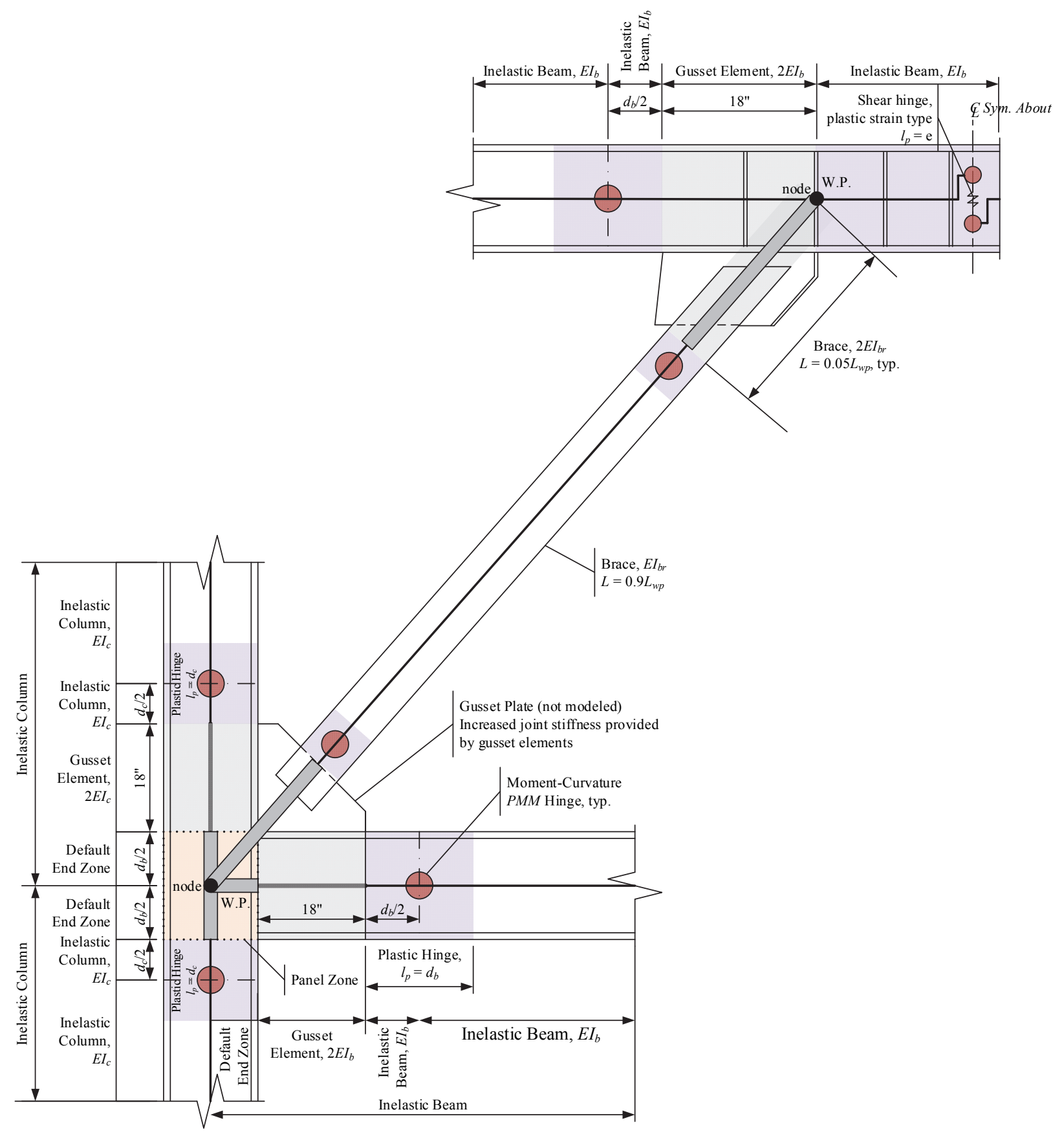

Figure 3-10. EBF Analytical Modeling Schematic

Flexural plasticity in beams and beam-columns is represented by nonlinear moment-curvature (MC) relationships which in turn are based on moment-chord rotation (MR) relationships provided by ASCE 41 Table 5-6. This shift in basis highlights a discrepancy between ASCE 41 \$5.4.2.2.2-2 and the prescribed acceptance criteria. Conversion between plastic rotation and plastic curvature is done using a defined plastic hinge length, $l_{p}$. An MC hinge is preferred in lieu of an MR hinge, as yield rotation, $\theta_{y}$, specified in ASCE 41 can lead to inconsistencies when beam models include rigid end offsets and when hinges are modeled away from the column faces. Further, ASCE 41 does not provide guidance on an appropriate beam length, 
$l_{b}$ (ASCE 41 Equation 5-1). Theoretically, there is no difference between an MC hinge and an MR hinge model as long as the conversion procedure for all nonlinear model parameters between the two is maintained. $P-M$ interaction effects on a moment-curvature hinge are included in the analytical model (discussed subsequently).

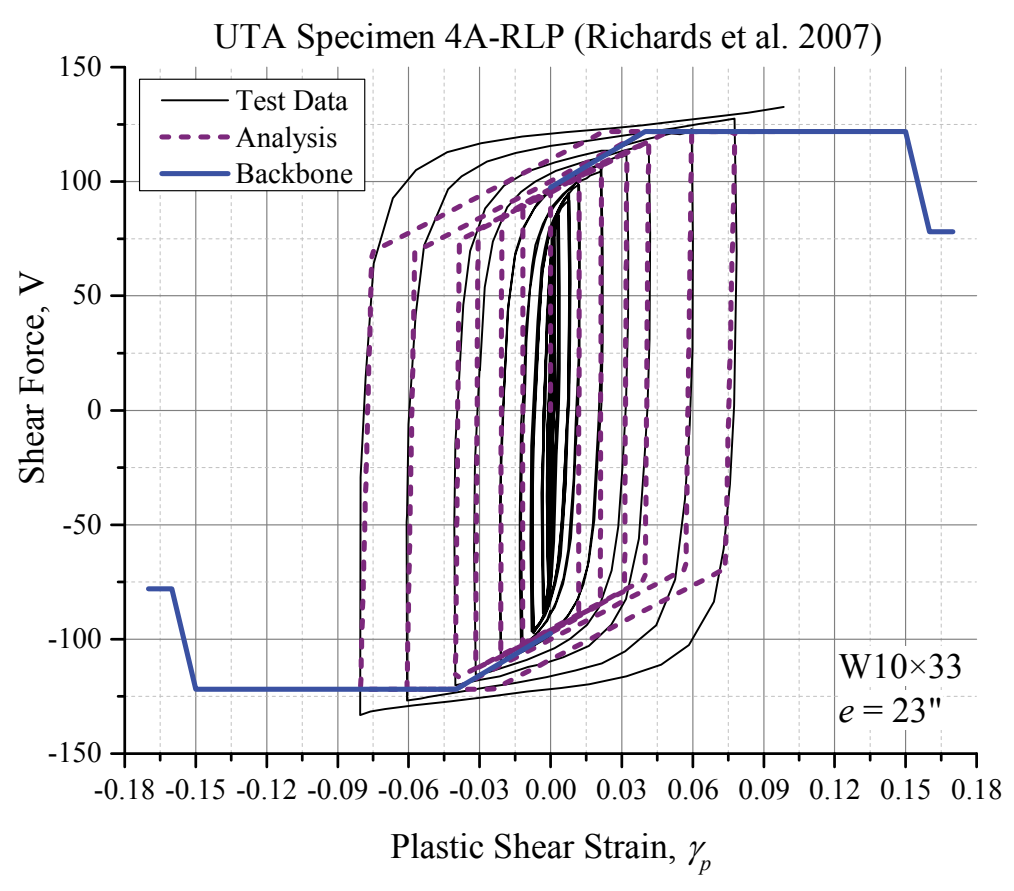

Figure 3-11. PERFORM-3D Link Beam Shear Hinge Calibration

Where needed to model a flexural hinge within a beam span or a flexural hinge within (or adjacent to) a connection (e.g., next to the brace-to-beam connection in the chevron frame), an MC hinge is placed at the center of the hinge with a plastic hinge length equal to the depth of the beam, $d$. Plastic rotation parameters modeling the backbone curve of the beam or connection hinge are taken from ASCE 41 Table 5-6. These values are converted to plastic curvature and, if needed, adjusted from application at the column face to the center of the hinge (no adjustment is needed for a conventional beam hinge) and other connection adjustments discussed next. The residual strength ratio ( $c$ in ASCE 41 Table 5-6) is normalized to the yield strength, $M_{C E}$, not the ultimate strength as required in PERFORM-3D.

Where applicable, the acceptance criteria for beam-to-column connections (flexural hinge is located within the connection) are taken from Fully Restrained Moment Connections in ASCE 41 Table 5-6 and are dependent on connection detailing, panel zone strength in a balanced yield condition, and member and cross-section slenderness (see ASCE $41 \S 5.4 .2 .4 .3,4.1$ to 4.4). These reduction factors are also applied to the plastic rotation values defining the backbone curve. This is done because the $\mathrm{CP}$ acceptance criteria for a primary FR connection was taken to match $a$ - see Figure 3-2, and therefore any connection configuration that affects the value for $a$ also affects the acceptance criteria. Similarly, the acceptance criteria are converted to plastic curvature and the application point is adjusted to the center of the hinge. Expanded commentary on beam-to-column connection versus beam hinge acceptance criteria is needed in ASCE 41, as well as a discussion clarifying the reductions to the plastic rotation values defining the backbone curve 
and acceptance criteria. ASCE 41 does not provide acceptance criteria for flexural actions for a beam-tocolumn connection that includes a brace connection (i.e., brace-to-beam/column) or a brace-to-beam connection. ASCE 41 requires that connections in an EBF be force-controlled for all actions.

Frame columns (i.e., beam-columns) are modeled similar to beams discussed previously, except that flexural PMM MC hinges that capture the combined effects of axial force and biaxial moments are placed near the joint region boundaries (see Figure 3-10). The plastic hinge length is assumed to be equal to the depth of the column, $d_{c}$. Out-of-plane moments are small relative to the in-plane moments in the SFRS members because each SFRS is an isolated planar frame in the direction of loading. Plastic rotation parameters of the flexural PMM hinges are taken from "columns" in ASCE 41 Table 5-6 and converted to plastic curvature (no adjustment for hinge location is needed). Criteria for flexural hinges based on member buckling strengths can be complex and problematic in capturing the in-plane nonlinear flexure behavior. For example, yield rotation, $\theta_{y}$, for a column is based on section strength, $P_{y e}$, while modeling parameters and acceptance criteria (function of $\theta_{y}$ ) are based on member strength, $P_{C L}$, without regard to the plane of buckling. ASCE 41-13 took the first step in resolving some issues concerning steel columns by permitting the modeling parameters to be determined using $P / P_{C L}$ in the plane of buckling. Still, $P-M$ interaction curves still require some clarification and guidance. In terms of assessment, ASCE 41 does not explicitly address column hinges near the column-to-base connections of a frame (similar to a beam-to-column connection).

In this study, modeling and acceptance criteria for the beam-column flexural hinges are taken as those provided for columns in ASCE 41 Table 5-6. The $P-M$ interaction effect on the in-plane flexural strength of a column hinge, $M_{C E x}$, where $x$ denotes the in-plane bending axis, is modeled by the section strength of the member (i.e., yield surface) using $P / P_{y e}$ in ASCE 41 Equation 5-4 (repeated below as Equation (3-16); see Figure 3-12, Figure 3-14, and Figure 3-15). $P$ - $M$ interaction relationships provided in PERFORM-3D for the yield surfaces of MC hinges in beam-columns are calibrated to approximate this curve as illustrated in Figure 3-15.

$$
M_{C E x}=1.18 M_{p e, x}\left(1-\frac{P}{P_{y e}}\right) \leq M_{p e, x}(\operatorname{ASCE} 41, \text { Equation 5-4) }
$$

ASCE 41 Equation 5-4 is based on plastic design theory and applicable for the in-plane section strength (strong-axis bending) of a wide flange section. AISC 360 Equation H1-1 can also be applied for computing the in-plane section strength by using $P / P_{y e}$ in lieu of $P_{r} / P_{c}$ as defined in AISC 360. Plastic design theory also gives the out-of-plane section strength (weak-axis bending, with $y$ denoting the out-of-plane bending axis) of a wide flange section as Equation (3-17).

$$
M_{C E y}=1.19 M_{p e, y}\left(1-\left(\frac{P}{P_{y e}}\right)^{2}\right) \leq M_{p e, y}
$$


$P-M$ interaction effect on the plastic rotation parameters and acceptance criteria of a column hinge is modeled by member strength using $P_{C L}$, computed for buckling about any axis or failure mode independent of the effect it may have on the in-plane flexure response of the column hinge (see Figure 3-12).

First, ASCE 41 requires a column (i.e., flexural hinge in the column) to be force-controlled for flexure when $P / P_{C L}>0.5$ for the nonlinear procedures and references the same equation used for linear assessment. This can be extremely problematic as separate strengths and interaction equations create discontinuities that cannot be effectively addressed in analysis software (see Figure 3-12). This elastic interaction equation is neglected in this study for nonlinear assessment and the hinge model obeys ASCE 41 Equation 5-4 independent of $P_{C L}$. ASCE 41 Equation 5-12 is more applicable for checking member stability than defining the section yield surface; Equation (3-18) rearranges ASCE 41 Equation 5-12 in terms of the moment strength.

$$
M_{C E}=M_{C L}\left(1-\frac{P}{P_{C L}}\right)(\text { from ASCE 41, Equation 5-12) }
$$

Second, flexural hinge model parameters and associated acceptance criteria are a function of the axial load ratio $P$ / $P_{C L}$. Provisions for this interaction in ASCE 41, however, create a discontinuity in the curve, as shown in Figure 3-13., which cannot be effectively addressed in analysis software. PERFORM-3D provides a simplified curve to model the variation in acceptance criteria with axial force, also shown in Figure 3-13. The adopted curves in this study are conservative for $P / P_{C L} \leq 0.2$. Further, because of constraints on $P$ - $M$ hinge models in PERFORM-3D, the model parameters ( $a$ in ASCE 41 Table 5-6 plus the elastic component as shown in Figure 3-12, $a$ plus elastic $=$ DL in PERFORM-3D - see Figure 3-2 for $a$ and DL) do not coincide for $P / P_{C L}>0.2$. A column hinge with high axial load can reach its peak strength ( $a$ plus elastic $=\mathrm{CP}$ in this case) in regards to the provisions of ASCE 41 yet still be able to maintain its flexural strength. In general, column hinges are not expected to be significantly strained; however, demands may affect the performance of base hinges, which can have detrimental effects on the system upon loss of strength. More detailed information can be found in the PERFORM-3D User Guide (CSI 2011d) and PERFORM-3D Components and Elements (CSI 2011c). Lastly, it is of questionable validity to allow a primary column component in a SFRS to have acceptance criteria based on a secondary component when based on a function of $P / P_{C L}$ as was mentioned earlier.

The ultimate flexural strength of the $\mathrm{MC}$ hinge is taken as 1.1 times $M_{C E}$ at $P=0$. This flexural strength increase is held constant for all values of axial force in PERFORM-3D. The residual strength ratio ( $c$ in ASCE 41 Table 5-6) is normalized to the yield strength, $M_{C E}$, at distinct $P / P_{C L}$ values (not $P / P_{y e}$, which is used to compute $M_{C E}$ ) and not the ultimate strength as required in PERFORM-3D.

Stability of a beam-column needs to be addressed in addition to capturing flexural plasticity. However, ASCE 41 does not provide explicit provisions to check member stability when $P / P_{C L} \leq 0.5$ for nonlinear procedures. When $P / P_{C L}>0.5$, ASCE 41 Equation 5-12 (primarily used for the linear procedures) can be used, but is not recommended as discussed previously.

In this study, section strength flags are applied to the frame columns as an indicator of member instability. For in-plane buckling and strong axis bending, a $P M$ strength flag is placed at the ends of the column using 
AISC $360 \S \mathrm{H} 1.3$ (a) for the interaction curve using $P_{n, x}$ as $P_{c}$. This approach closely aligns with ASCE 41 Equations 5-10, 5-11, and 5-12. The in-plane effective length of the column is taken as that computed for design (i.e., adjusted $K$ factor-see Chapter 2). This is considered a conservative practice as the analysis adjusts the stiffness matrix (only for material nonlinearity) at every time step and the leaning column effect is explicitly included. However, geometric imperfections (system and member), residual stresses, and epistemic uncertainties (i.e., $\phi$ factor) are not included in the mathematical model. Consequently, member strengths based on the actual unbraced length (i.e., $K L_{x}=L_{x}$ ) is of questionable validity, but so is using the design value based on a vertical load-only load combination. Research is needed concerning in-plane dynamic instability of inelastic members.

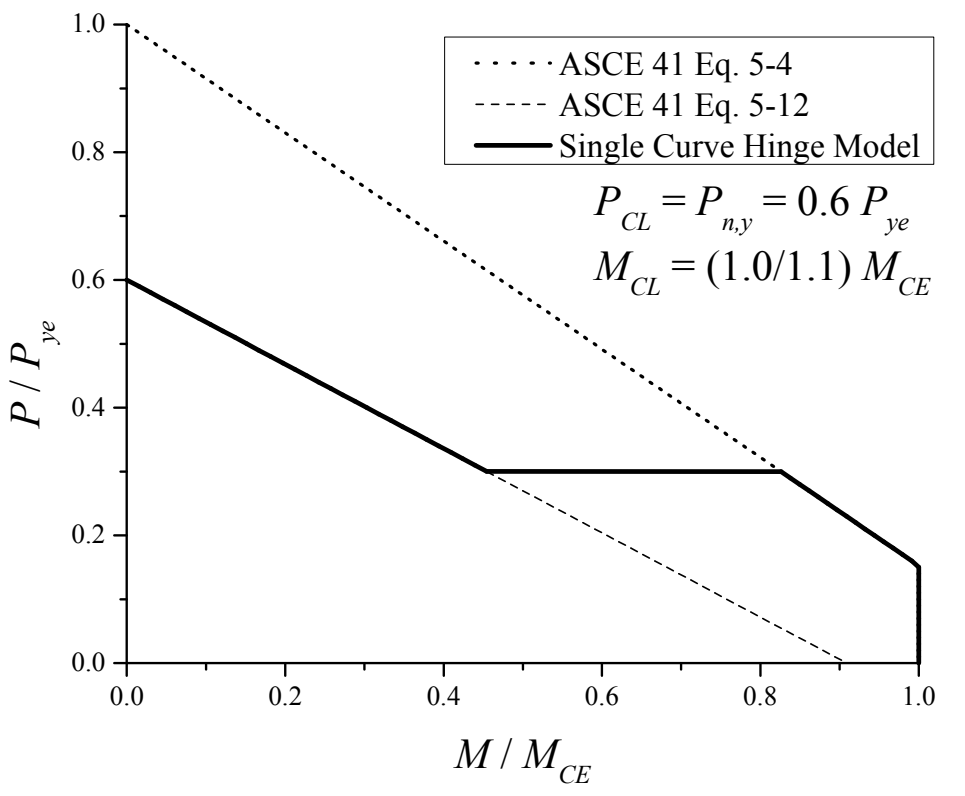

Figure 3-12. In-plane Flexural Hinge Yield Surface Model (Including Force-Controlled Response)

For out-of-plane buckling and strong axis bending, a $P M$ strength flag is placed at the ends of the column using AISC $360 \S \mathrm{H} 1.3(\mathrm{~b})$ for the interaction curve and the effective length is taken as the actual unbraced length (i.e., $K L_{y}=L_{y}$ ). Adopting ASCE 41 Equations 5-10, 5-11, and 5-12 as an indicator of weak-axis instability coupled with in-plane bending can be highly conservative. PERFORM-3D uses a single continuous interaction curve as shown in Figure 3-16 which illustrates several interactions curves together with the approximations from PERFORM-3D. Similar to beams, section strengths are modified as needed based on cross-section geometry, member strengths (commonly controls columns in compression), and design-assessment criteria prescribed in ASCE 41.

ASCE 41 requires that columns and braces in an EBF be force-controlled for flexure. However, ASCE 41 does not provide guidance on how to assess the component when $P \leq 0.5 P_{C L}$, other than referencing provisions given for a concentrically braced frame, which in turn references provisions applicable for the expected strength of components in a moment frame. Further, the use of $F_{y L B}$ needs clarification in terms of determination of section compactness and member slenderness requirements for force-controlled components. 


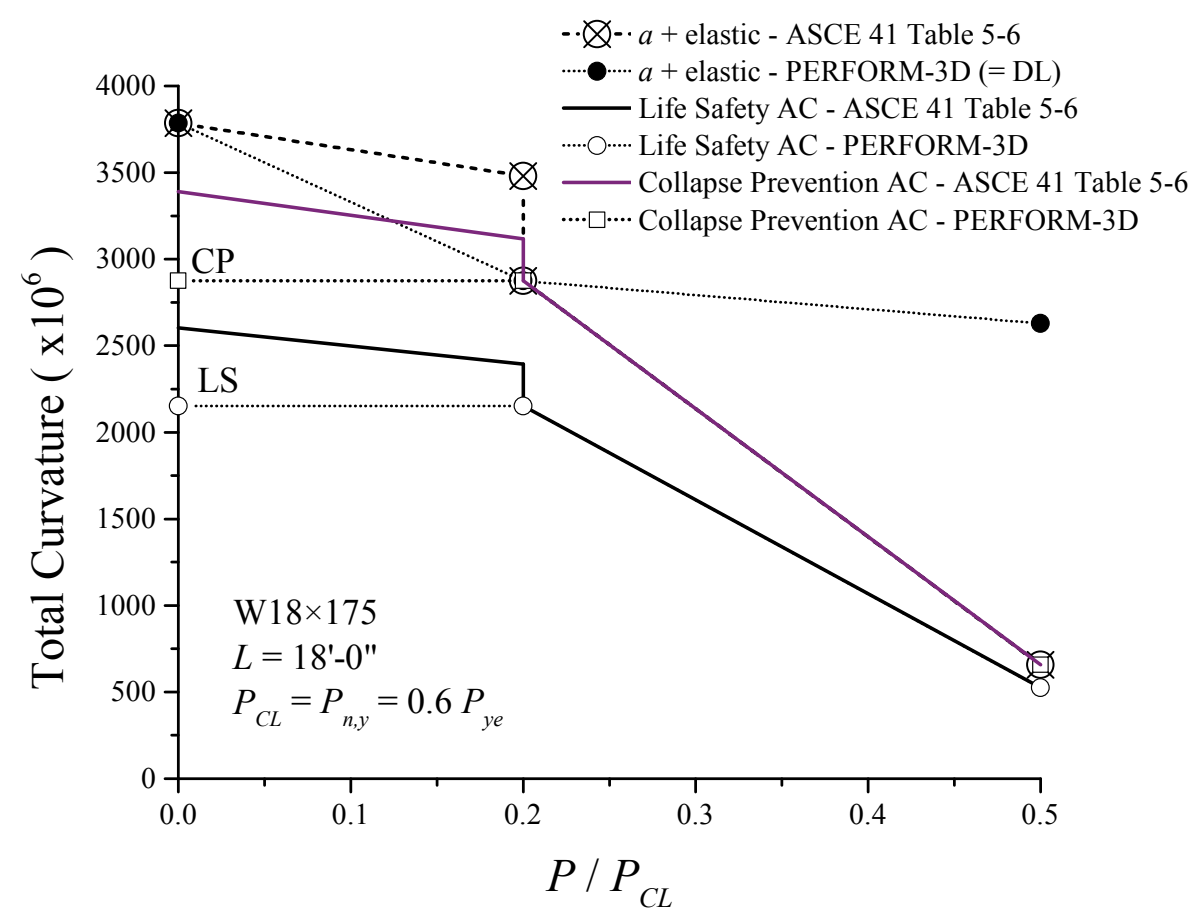

Figure 3-13. Variation in Acceptance Criteria and Hinge Model for Axial Force

For this study, force-controlled flexural hinges in columns and braces are modeled as discussed above, which is the same as a deformation-controlled flexural hinge in a moment frame and concentrically braced frame. The acceptance criterion for these force-controlled hinges and compression members is therefore based on (1) the expected yield strength of the section and whether or not the section develops a plastic hinge and (2) member instability using the expected $P M$ interaction strength, respectively. Modeling and assessment of force-controlled components in an EBF for nonlinear assessment methods is a topic that needs further clarification in ASCE 41.

Figure 3-17 through Figure 3-22provide the load-independent acceptance criteria-with consideration of force-controlled and deformation-controlled classifications. These figures are referred to herein as "Frame Capacity Schematics." The two values given for a column represent the cases when $P_{U F} / P_{C L}=0.2$ and 0.5 , adjusted for section compactness requirements. As a side note, the LS acceptance criterion for the link rotation angle in ASCE 41 is 1.375 times the allowable rotation angle prescribed in AISC $341(=0.08$ radians) at the BSE-1 EHL (when taken as $2 / 3 \times \mathrm{MCE}_{\mathrm{R}}$ ) - the CP acceptance criterion is 1.625 times this rotation angle at the BSE-1 EHL. 


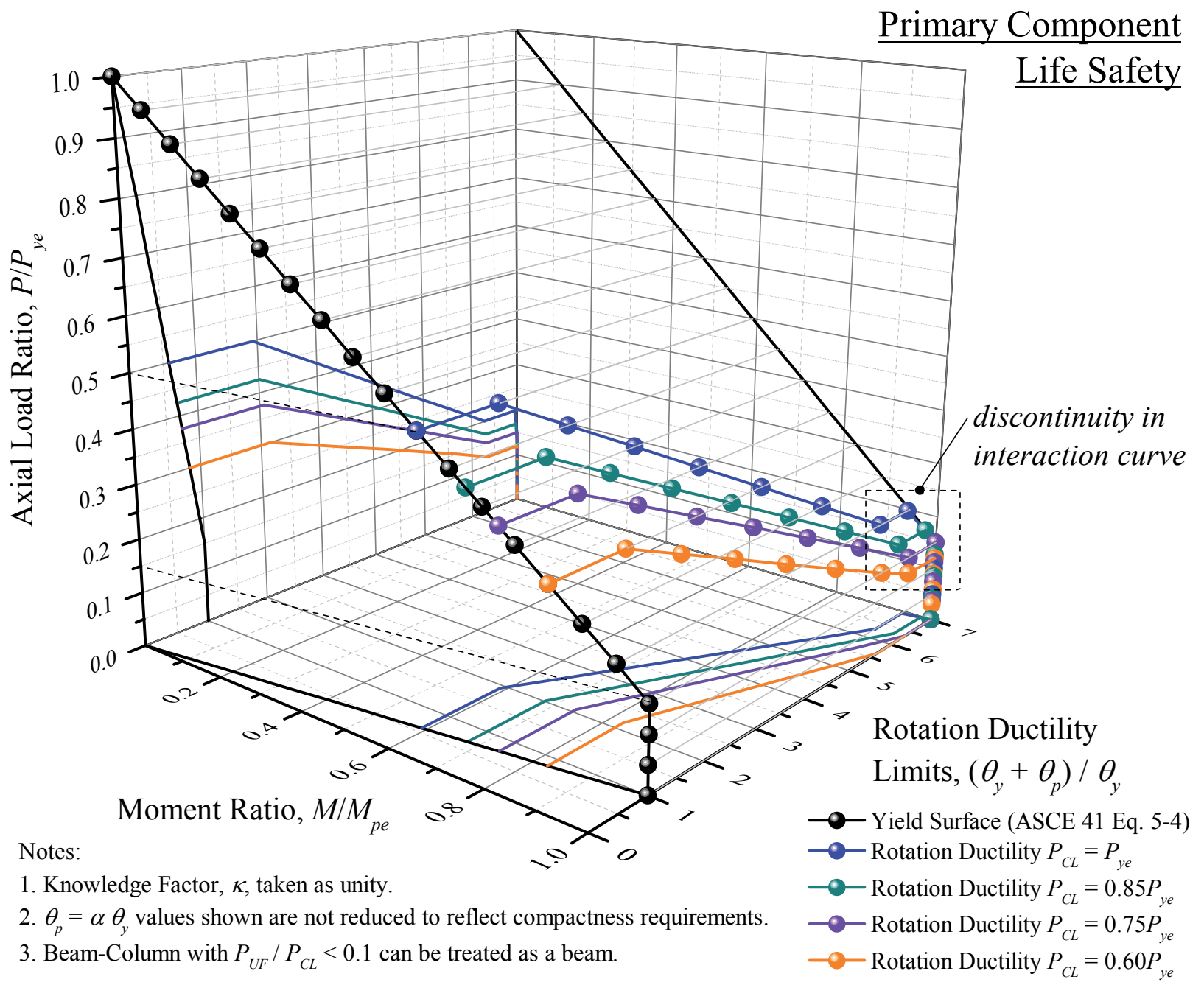

Figure 3-14. $P$-M Interaction on Plastic Rotation, LS Acceptance Criteria (Primary Component) 


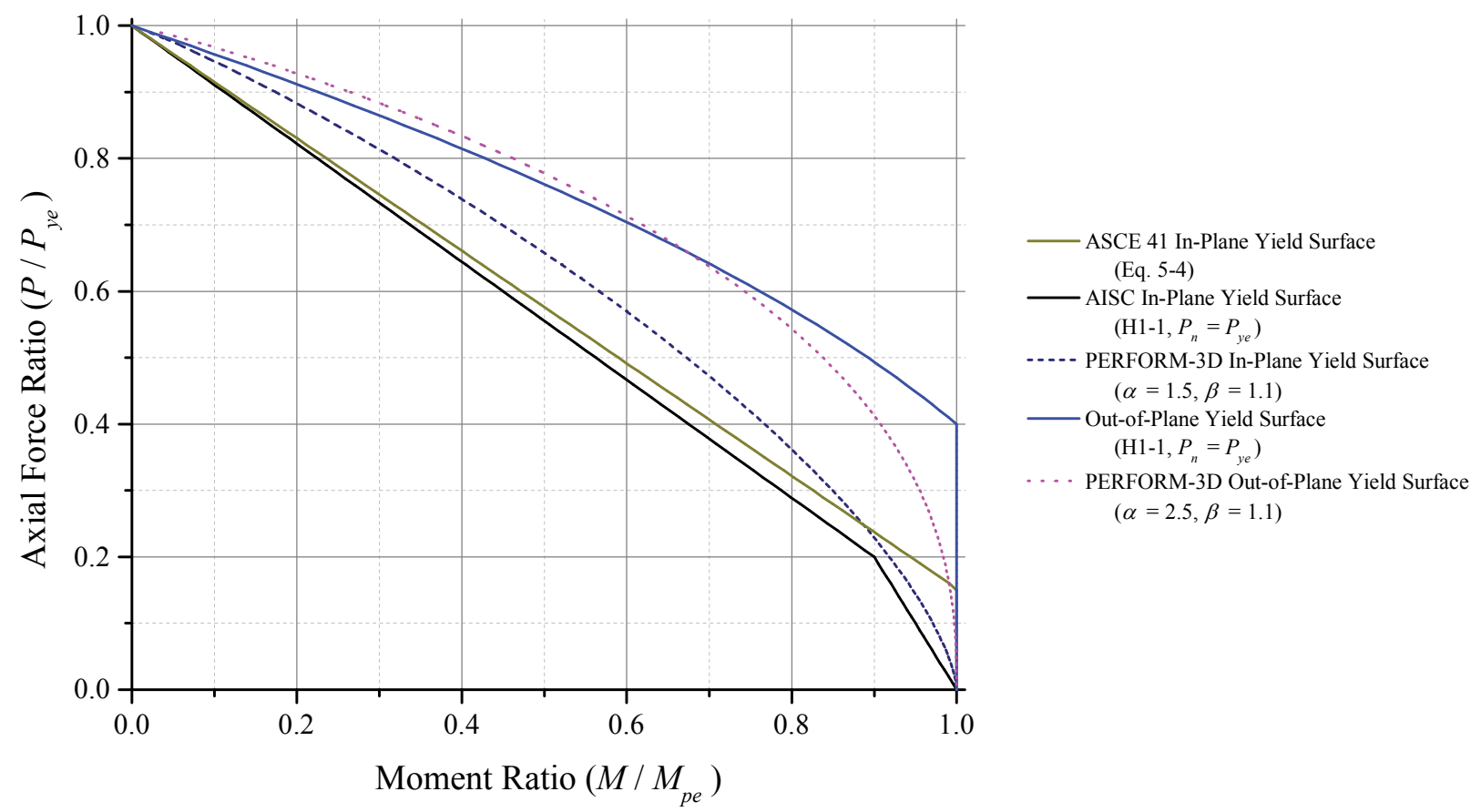

Figure 3-15. $P$ - $M$ Interaction Curve (Section Yield Surface)

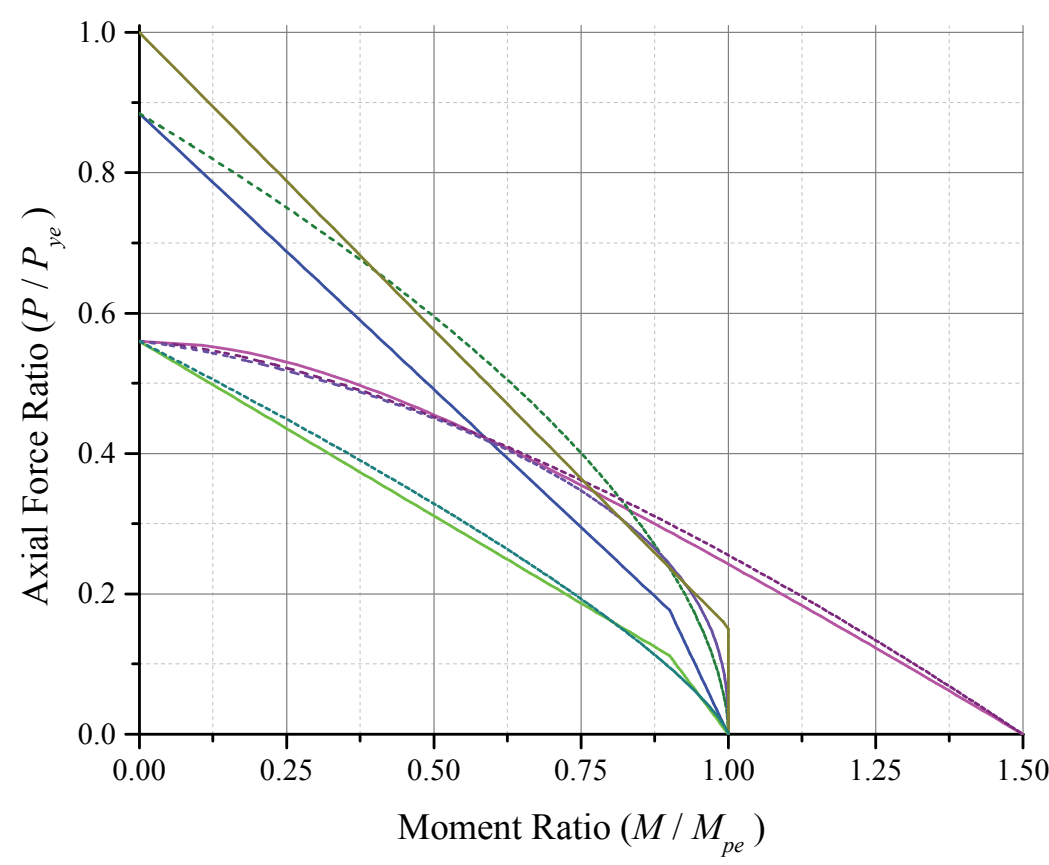

ASCE 41 In-Plane Yield Surface (Eq. 5-4)

- AISC In-Plane Stability $\left(C_{b}>1\right)$

$\left(\mathrm{H} 1-1, P_{c}=P_{n e, x}\right)$

.... PERFORM-3D In-Plane Stability (Truncated)

$(\alpha=1.75, \beta=1.0)$

AISC Out-of-Plane Stability $\left(C_{b}>1\right)$

$\left(\mathrm{H} 1-1, P_{c}=P_{n e, y}\right)$

-... PERFORM-3D Out-of-Plane Stability

$(\alpha=1.3, \beta=1.0)$

- AISC Out-of-Plane Stability $\left(C_{b}>1\right)$

$\left(\mathrm{H} 1-2, P_{c y}=P_{n e, y}\right)$

.... PERFORM-3D Out-of-Plane Stability

$(\alpha=1.0, \beta=1.5)$

.... PERFORM-3D Out-of-Plane Stability (Truncated)

$(\alpha=2.5, \beta=1.25)$

Figure 3-16. $P$ - $M$ Interaction Curve (Member Instability) 


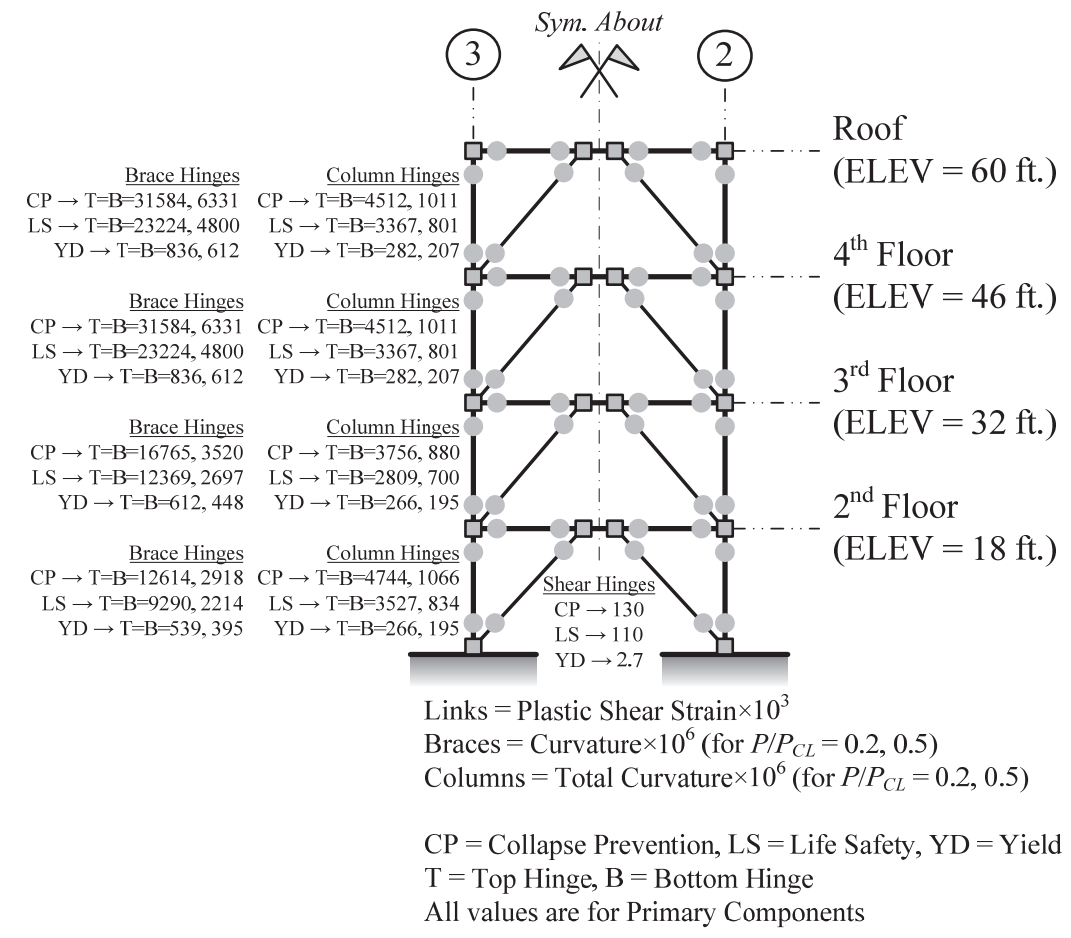

Figure 3-17. Frame Capacity Schematic (Inelastic), LS and CP, 4-Story EBF - ELF

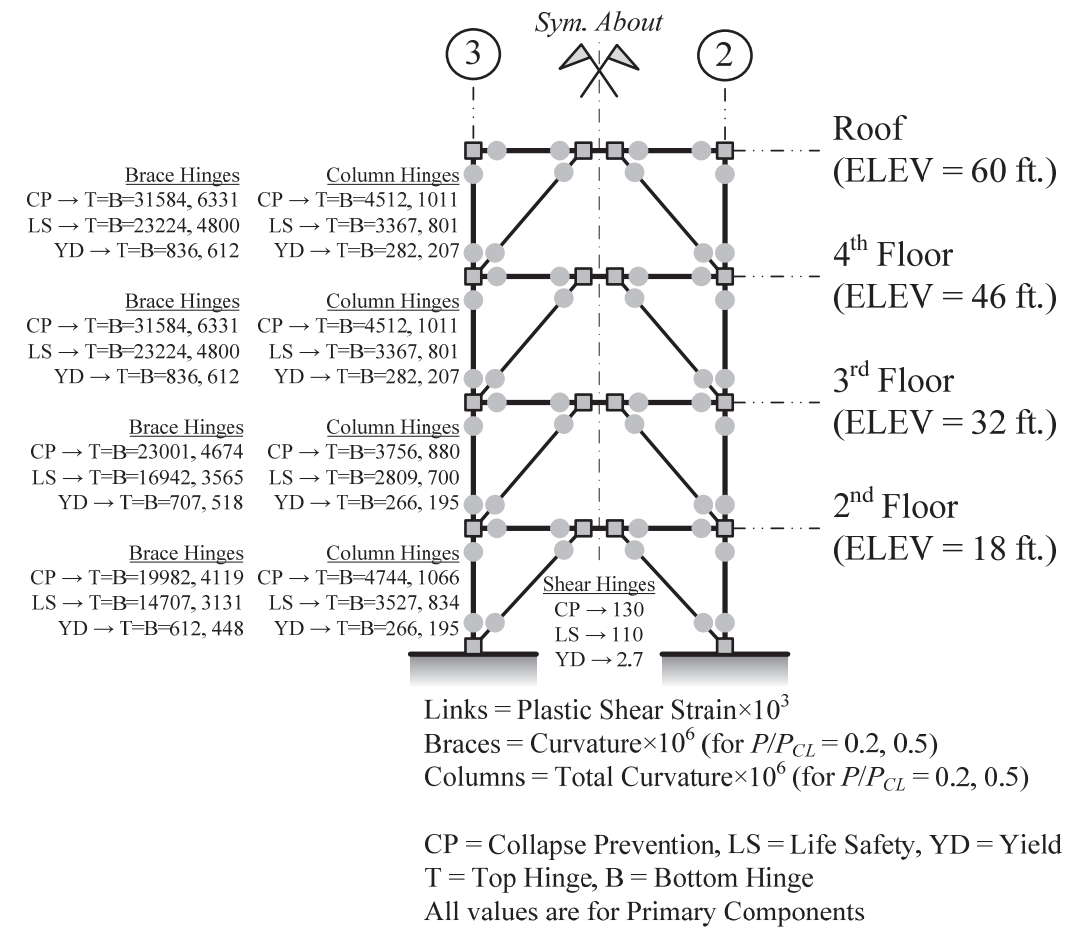

Figure 3-18. Frame Capacity Schematic (Inelastic), LS and CP, 4-Story EBF - RSA 


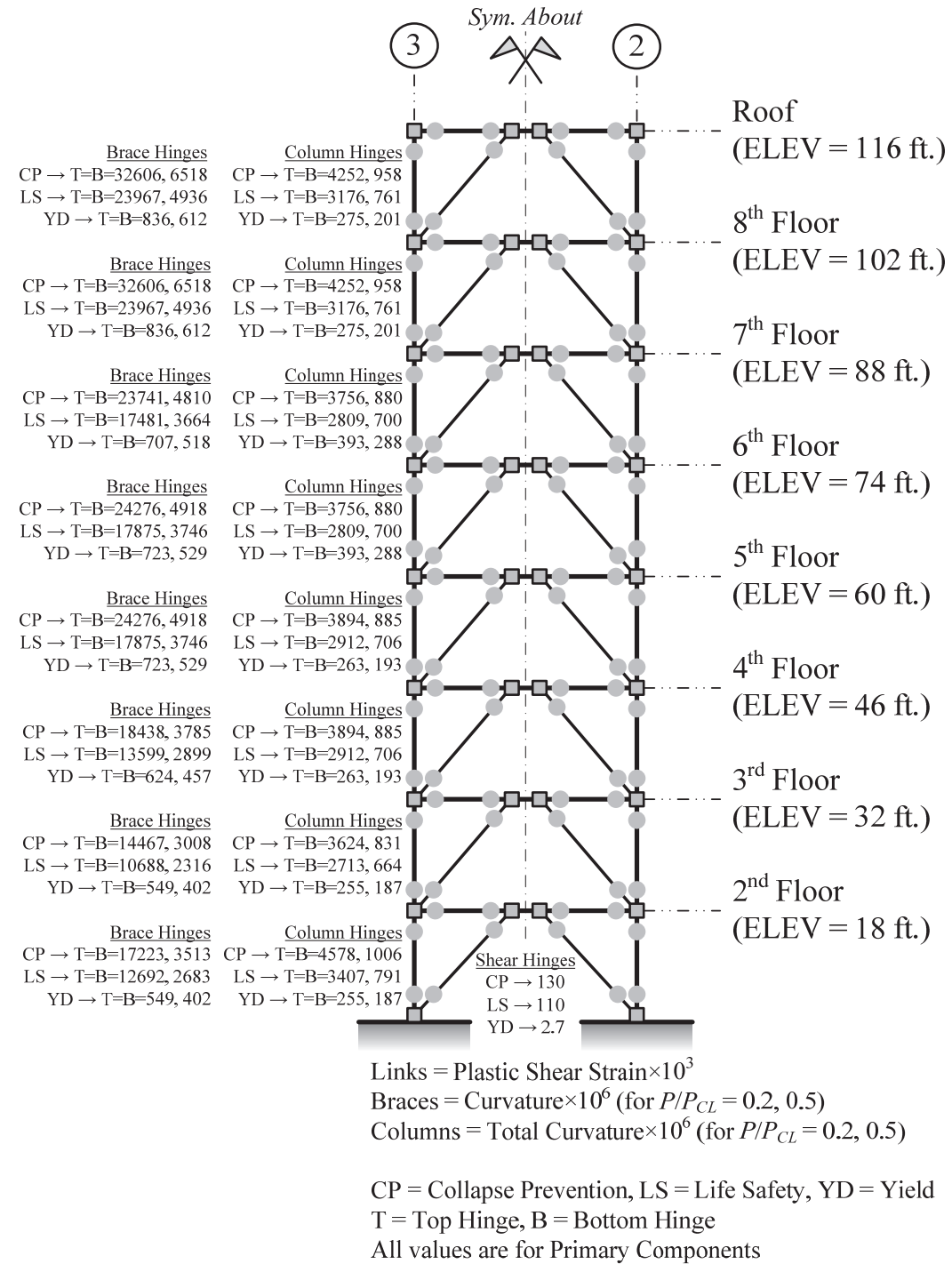

Figure 3-19. Frame Capacity Schematic (Inelastic), LS and CP, 8-Story EBF - ELF 


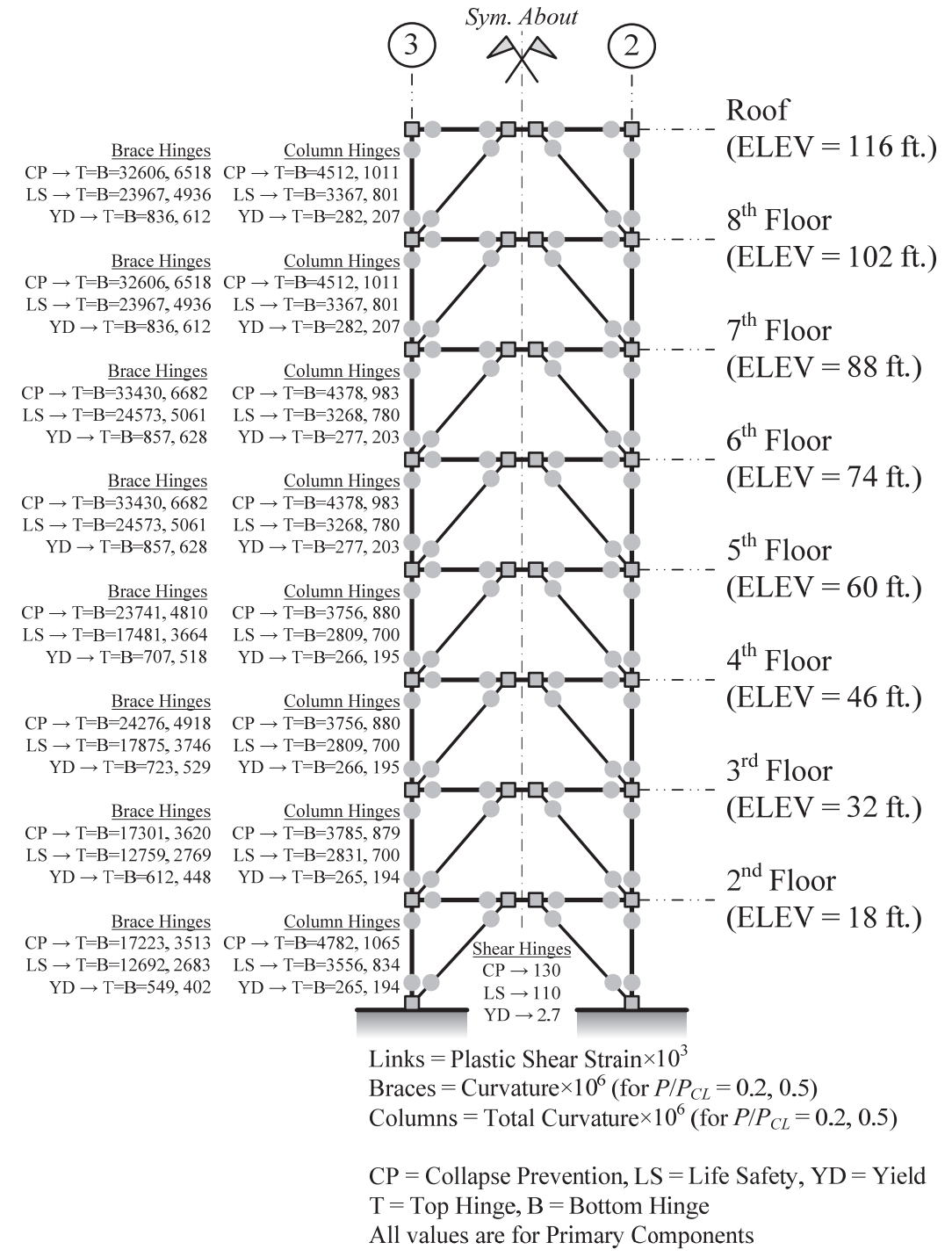

Figure 3-20. Frame Capacity Schematic (Inelastic), LS and CP, 8-Story EBF - ELF 


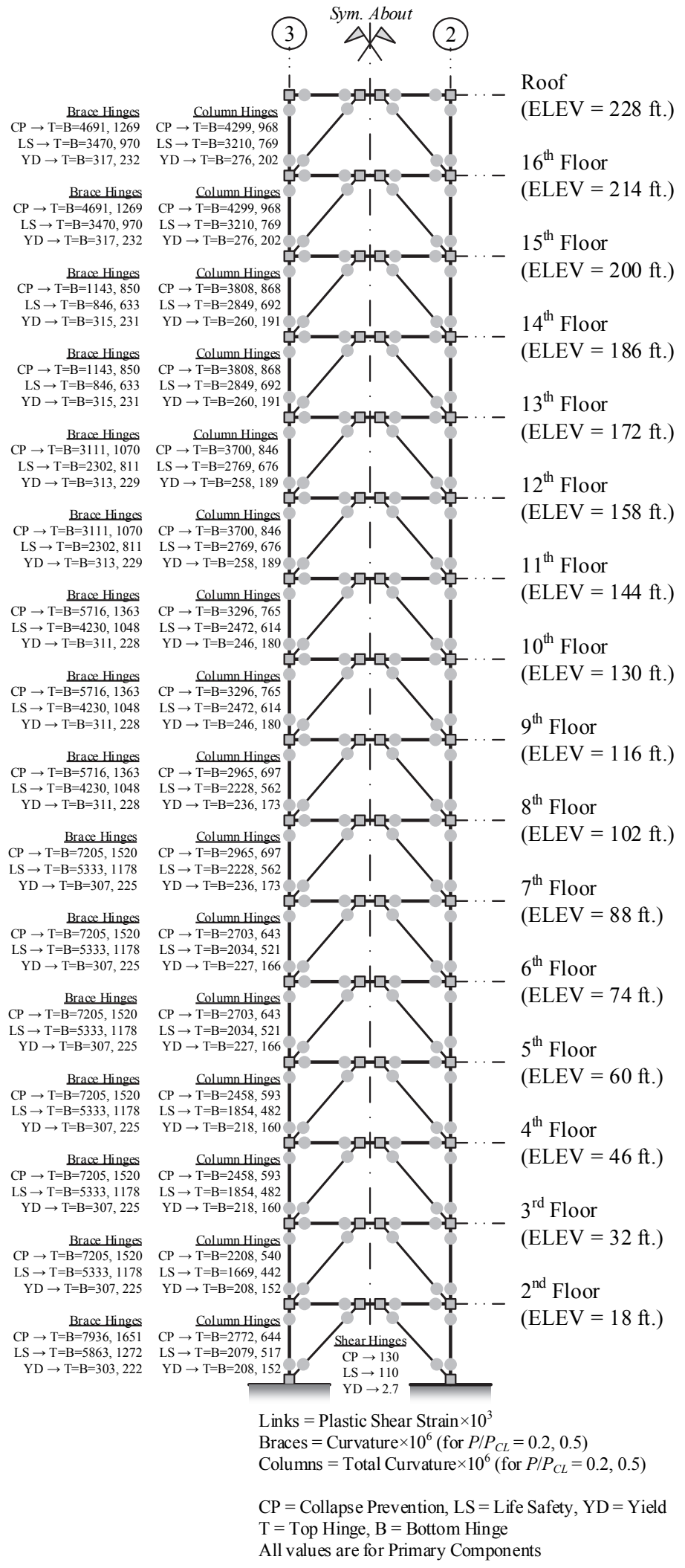

Figure 3-21. Frame Capacity Schematic (Inelastic), LS and CP, 16-Story EBF - ELF 


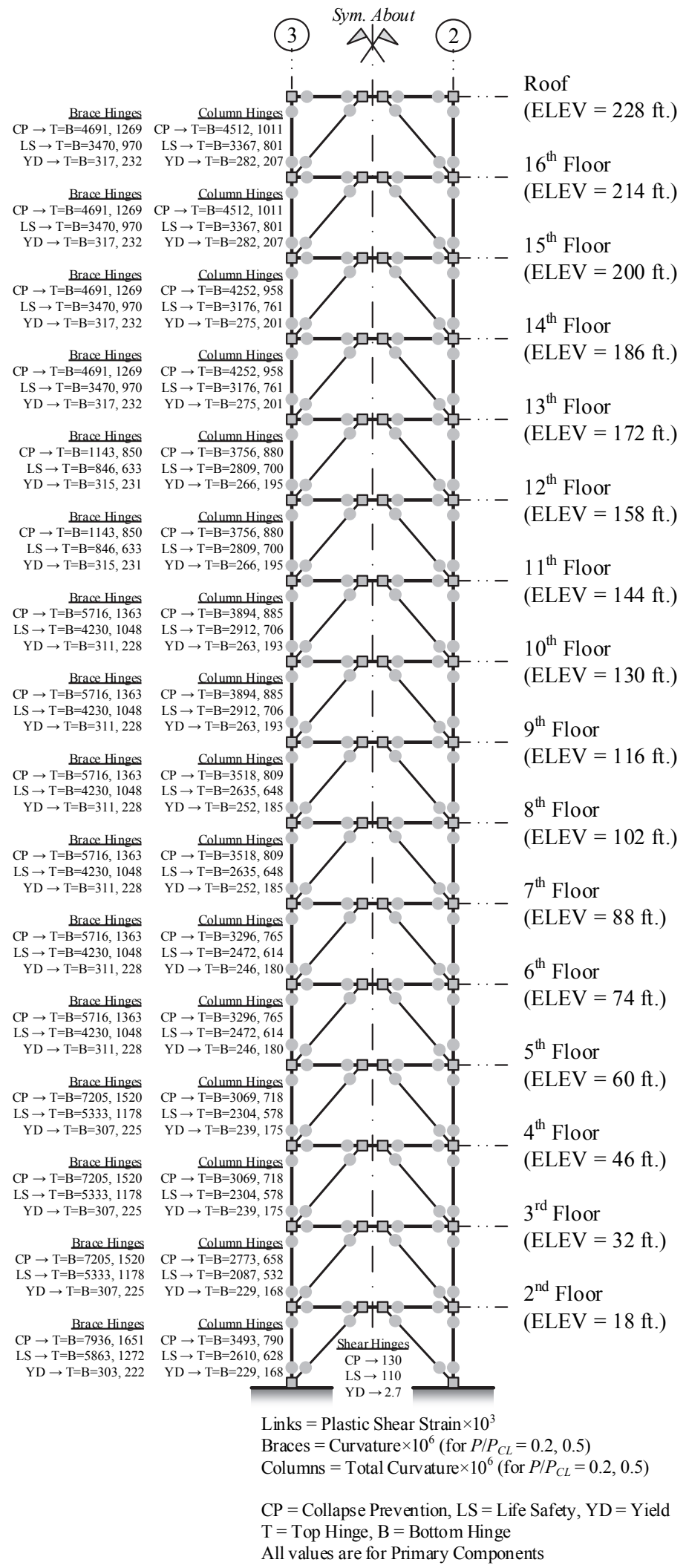

Figure 3-22. Frame Capacity Schematic (Inelastic), LS and CP, 16-Story EBF - RSA 


\subsubsection{Seismic Assessment Results}

The results for the EBFs assessed using each of the four analysis procedures are presented in this section. The following section highlights any failure to satisfy the acceptance criteria.

For the LSP and LDP, force-controlled column compression demands, $P_{U F}$, are computed by taking $J$ in ASCE 41 \$3.4.2.1.2-2 as the minimum DCR — as defined in ASCE 41-of the component(s) (i.e., link beams) delivering force to the member, but not less than 2.0. In this case, this method generally provides the least conservative axial force demand estimates than that determined from a fully yielded capacity design analysis per ASCE 41 \$3.4.2.1.2-1 following ASCE 41 \$5.5.3.4.2. The capacity design analysis ${ }^{22}$ used in this study to determine the maximum axial load demands to satisfy ASCE $41 \S 3.4 .2 .1 .2-1$ assumes that all yielding components have achieved their maximum strength simultaneously (i.e., link beams achieving their maximum shear strength as defined by ASCE 41 §5.5.3.4.2). This axial load will be roughly ten percent larger than that computed using AISC 341 provisions for design of columns in new EBFs. Further, axial force demand estimates from the capacity design procedure using the system overstrength factor, $\Omega_{o}$, in ASCE 7 are included with those determined from ASCE 41 in the respective analysis sections below. Force-controlled component actions are assigned an $m$-factor of unity, see $§ 3.1 .4 .1$.

Analysis results (e.g., $D C R_{N}$ ) for the NDP using the ground motion record set are statistically summarized by the median, mean, $84^{\text {th }}$ percentile, and mean plus one standard deviation response for both the LS BPL (given the BSE-1 EHL) and the CP BPL (given the BSE-2 EHL). Mean and median response are presented because (1) ASCE 41 does not discuss how to process the results in the event of a structural collapse under a given record in the set, (2) new provisions under investigation for inclusion in the 2015 NEHRP Provisions are being debated as to how to process results from eleven records (an increase from seven in ASCE 7-10), and (3) the difference is small for well-performing systems that remain stable for all motions in a set. It is the goal here that showing the mean and median will assist in developing future code provisions.

For comparison purposes only, the $D C R_{N}$ values obtained from the LSP, LDP, and NSP are superimposed on the figures showing the results obtained from the NDP. Results from the NSP and NDP can be directly compared because the basis of measurement is identical. However, caution should be used when comparing linear and nonlinear results by inspection because the nature of the analysis is fundamentally different; presenting them together here is not intended to imply they are equivalent. Results from the two are not always directly comparable as linear results would first need to be converted to total demand where applicable, and in certain cases not based on an interaction equation. Results from the linear assessment procedures are presented alongside results from the nonlinear assessment procedures primarily to highlight the distribution of performance predictions between the analytical methods.

For comparison purposes only, the base shears computed using the LSP and LDP are provided in §3.2.3.1.3.

${ }^{22}$ ASCE $41 \S 3.4 \cdot 2.1 .2-1$ refers to this type of analysis as a "limit state" analysis. 


\subsubsection{Four-Story Eccentrically Braced Frame}

\subsection{Linear Static Procedure}

\subsection{BSE-1 Earthquake Hazard Level (LS BPL)}

In this section, the following apply:

- Figure 3-23 and Figure 3-24 provide the $D C R_{N}$ and load-dependent $m$-factor values for the ELF and RSA designs, respectively, for the LSP at the BSE-1 EHL. In these figures, $D C R_{N}$ values greater than unity are highlighted in red and underlined. $D C R$ values, as defined by ASCE 41, can be obtained by multiplying $D C R_{N}$ by $m$ and $\kappa$, see Eq. 3-6.

- Figure 3-25 provides the maximum axial compression demands, $P_{U F}$, in the exterior column lines for various analysis methods and the column capacity, $P_{C L}$.

All component actions satisfy the LS BPL acceptance criteria.

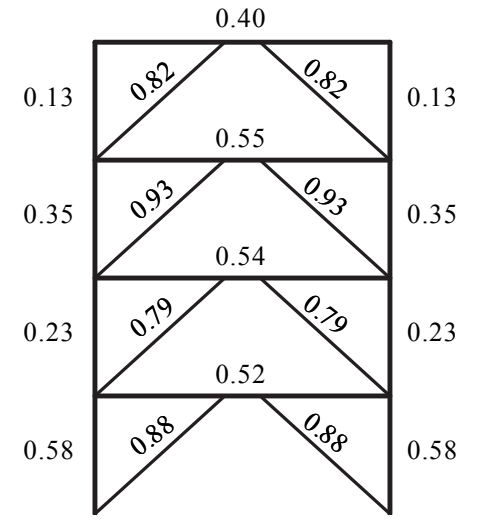

(a) $D C R_{N}$

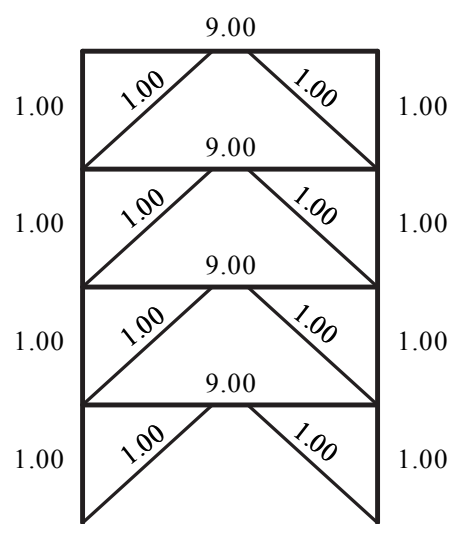

(b) $m$-factors

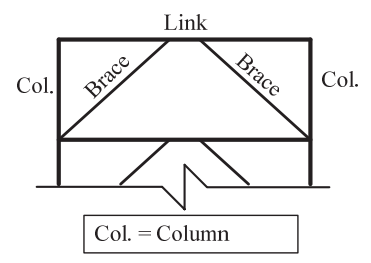

(c) Key

Figure 3-23. LSP Assessment Results, 4-Story EBF ELF, BSE-1 LS

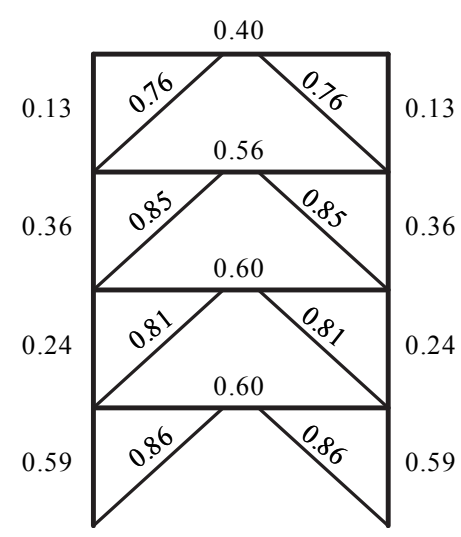

(a) $D C R_{N}$

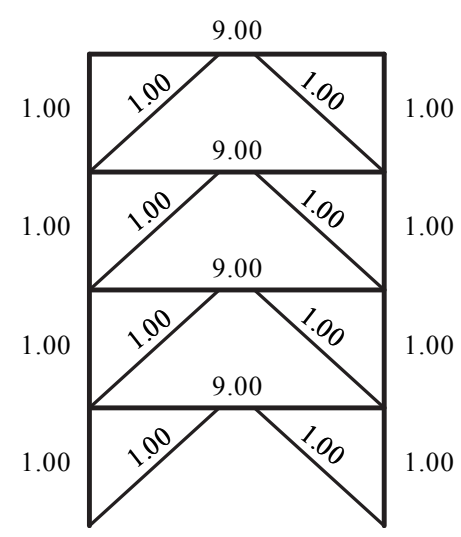

(b) $m$-factors

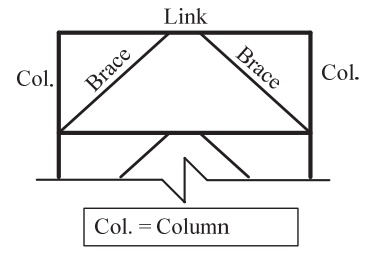

(c) Key

Figure 3-24. LSP Assessment Results, 4-Story EBF RSA, BSE-1 LS 


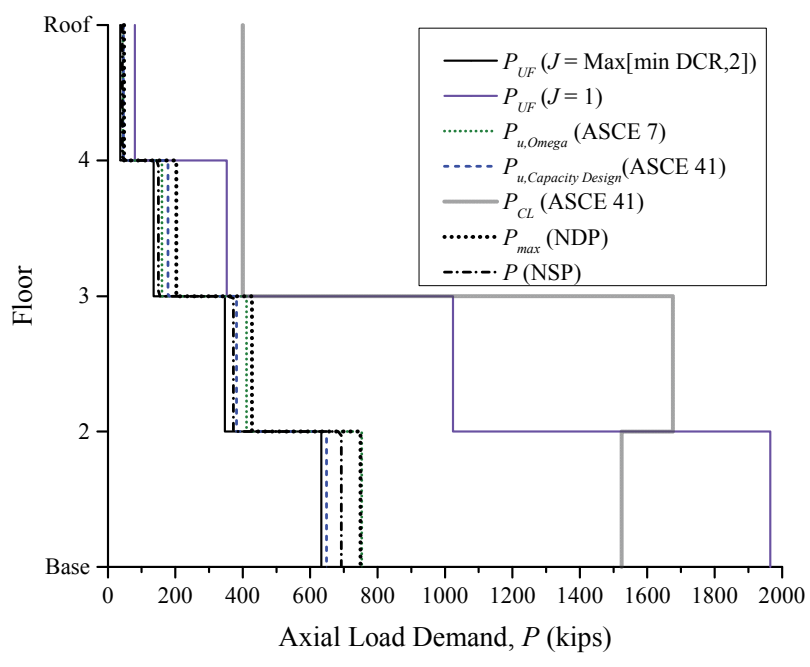

(a) ELF

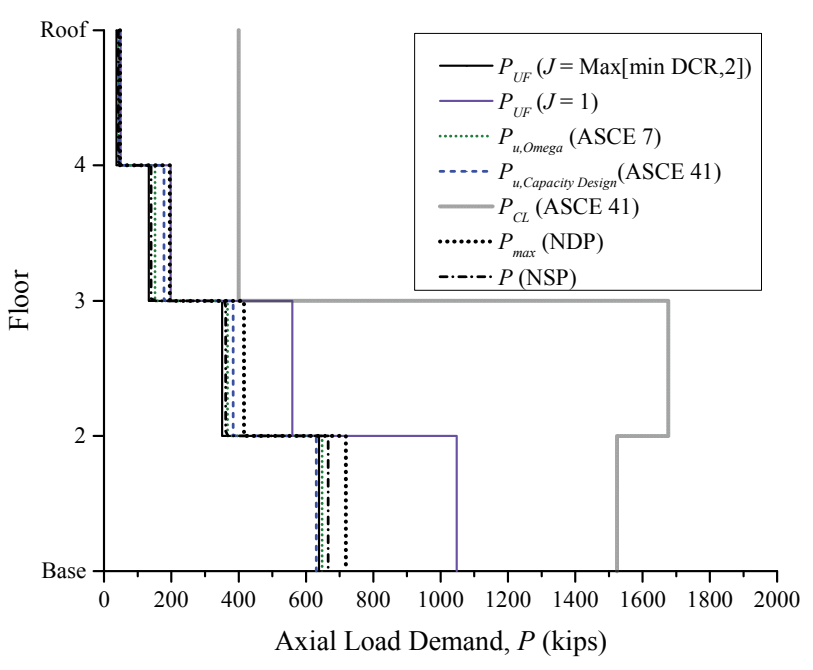

(b) RSA

Figure 3-25. LSP Assessment Results, Compression in Exterior Columns, 4-Story EBF, BSE-1

\subsection{BSE-2 Earthquake Hazard Level (CP BPL)}

In this section, the following apply:

- $\quad$ Figure 3-26 and Figure 3-27 provide the $D C R_{N}$ and load-dependent $m$-factor values for the ELF and RSA designs, respectively, at the LSP at the BSE-2 EHL. In these figures, $D C R_{N}$ values greater than unity are highlighted in red and underlined. $D C R$ values, as defined by ASCE 41, can be obtained by multiplying $D C R_{N}$ by $m$ and $\kappa$, see Eq. 3-6.

- Figure 3-28 provides the maximum axial compression demands, $P_{U F}$, in the exterior column lines for various analysis methods and the column capacity, $P_{C L}$.

All component actions satisfy the CP BPL acceptance criteria. The $D C R_{N}$ results for the force-controlled components (columns and braces) are the same for both LSP LS and LSP CP because all link beams have yielded, capping the force delivered to the components based on the minimum DCR. In the case of the columns, the minimum DCR is computed at the top story link beam, and is greater than 2.0. If $J$ were set to the default of 2.0, then the $D C R_{N}$ values would differ, which would not be consistent with the actual plastic mechanism (see 16-story LSP results). The $D C R_{N}$ results for the link beams differ for LSP LS and LSP CP by approximately (13/9)/1.5 $=0.963$ times the $D C R_{N}$, approximate because of second-order effects. 


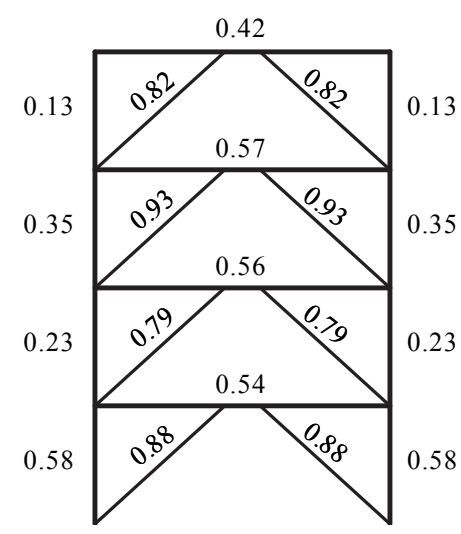

(a) $D C R_{N}$

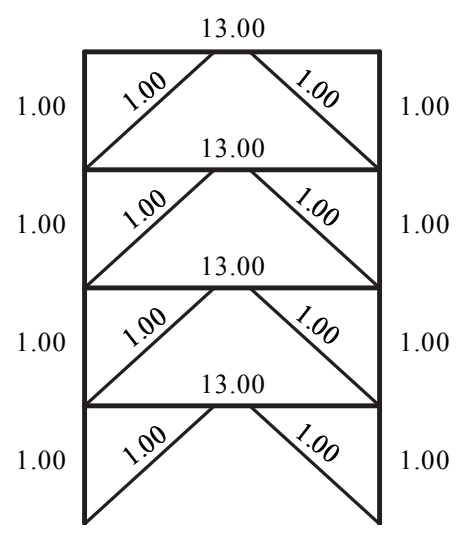

(b) $m$-factors

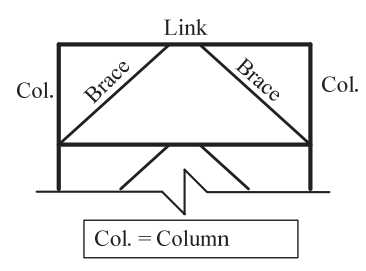

(c) Key

Figure 3-26. LSP Assessment Results, 4-Story EBF ELF, BSE-2 CP

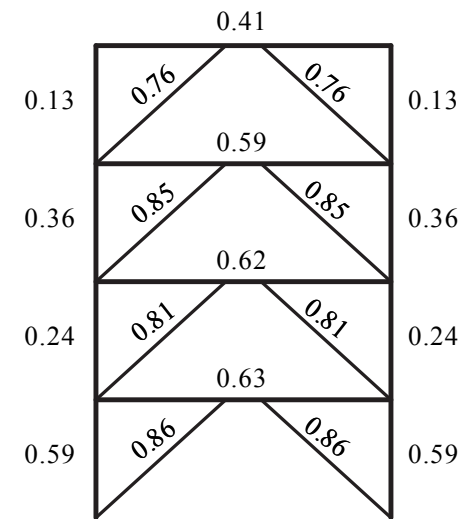

(a) $D C R_{N}$

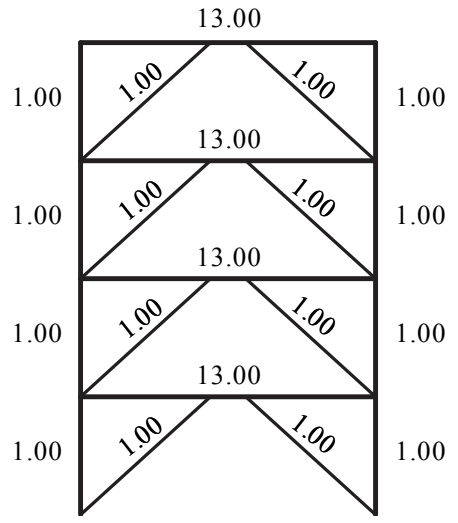

(b) $m$-factors

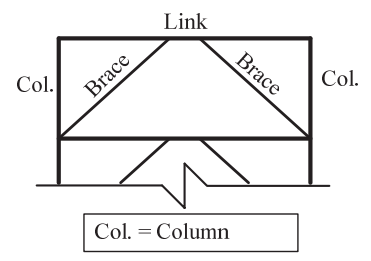

(c) Key

Figure 3-27. LSP Assessment Results, 4-Story EBF RSA, BSE-2 CP

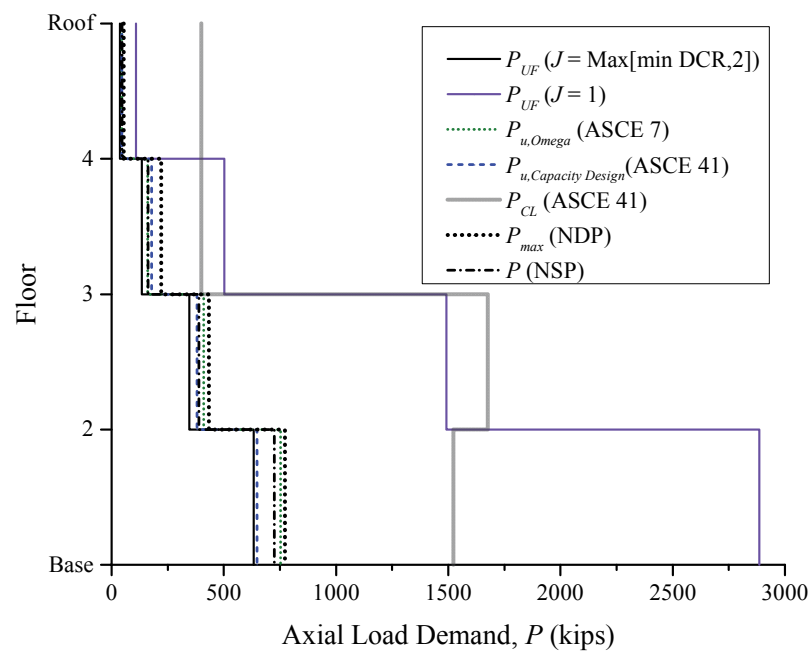

(a) ELF

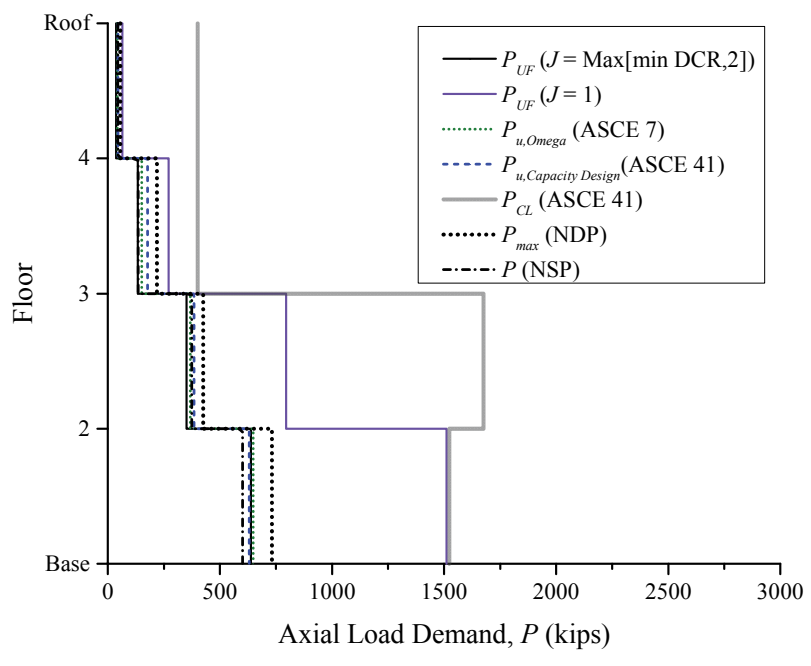

(b) RSA

Figure 3-28. LSP Assessment Results, Compression in Exterior Columns, 4-Story EBF, BSE-2 


\subsection{Linear Dynamic Procedure}

\subsection{BSE-1 Earthquake Hazard Level (LS BPL)}

In this section, the following apply:

- Figure 3-29 and Figure 3-30 provide the $D C R_{N}$ and load-dependent $m$-factor values for the ELF and RSA designs, respectively, for the LDP at the BSE-1 EHL. In these figures, $D C R_{N}$ values greater than unity are highlighted in red and underlined. $D C R$ values, as defined by ASCE 41, can be obtained by multiplying $D C R_{N}$ by $m$ and $\kappa$, see Eq. 3-6.

- Figure 3-31 provides the maximum axial compression demands, $P_{U F}$, in the exterior column lines for various analysis methods and the column capacity, $P_{C L}$.

All component actions satisfy the LS BPL acceptance criteria.

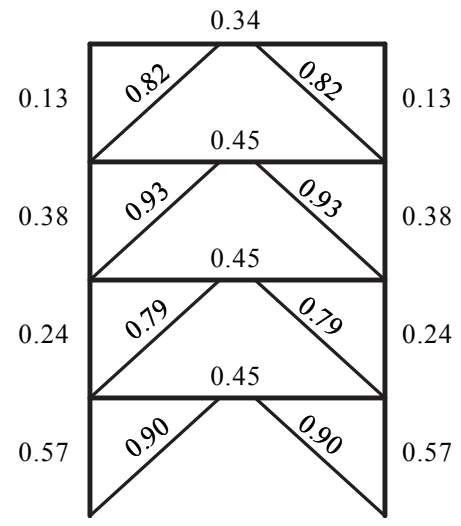

(a) $D C R_{N}$

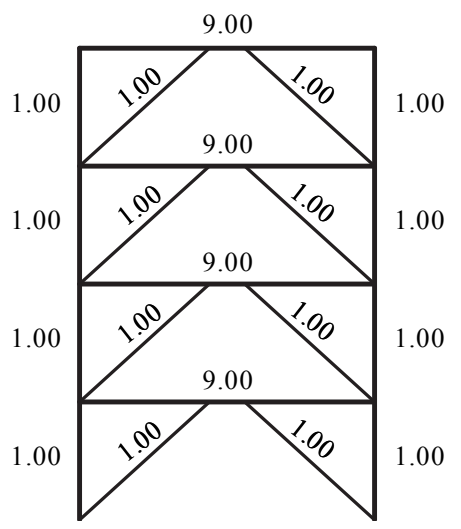

(b) $m$-factors

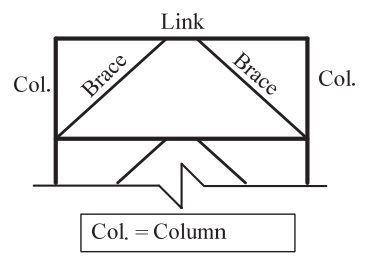

(c) Key

Figure 3-29. LDP Assessment Results, 4-Story EBF ELF, BSE-1 LS

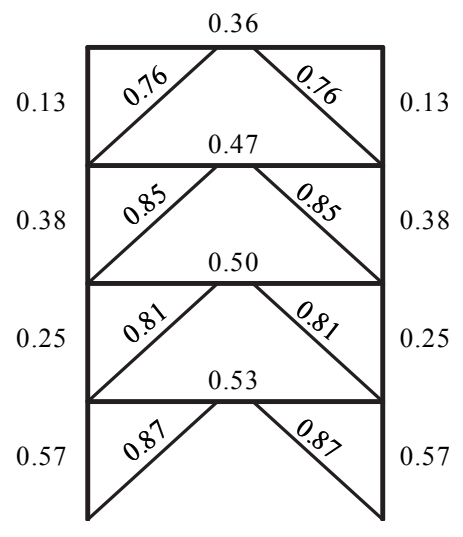

(a) $D C R_{N}$

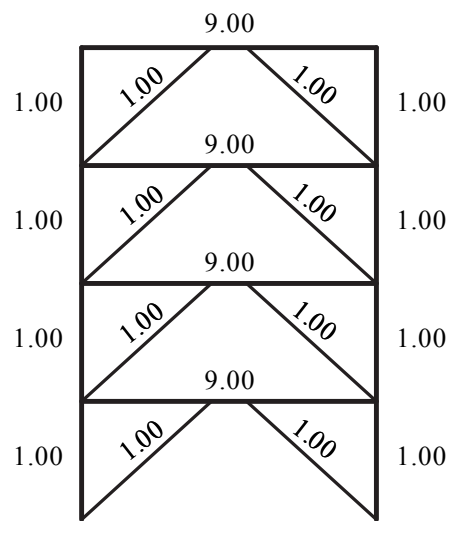

(b) $m$-factors

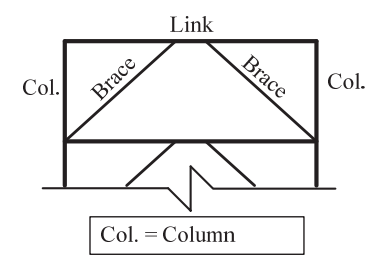

(c) Key

Figure 3-30. LDP Assessment Results, 4-Story EBF RSA, BSE-1 LS 


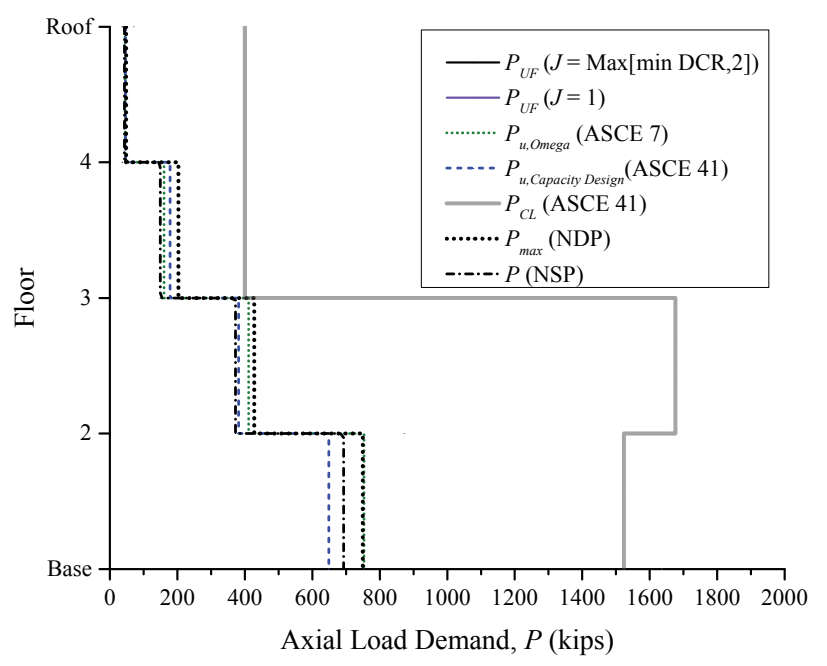

(a) ELF

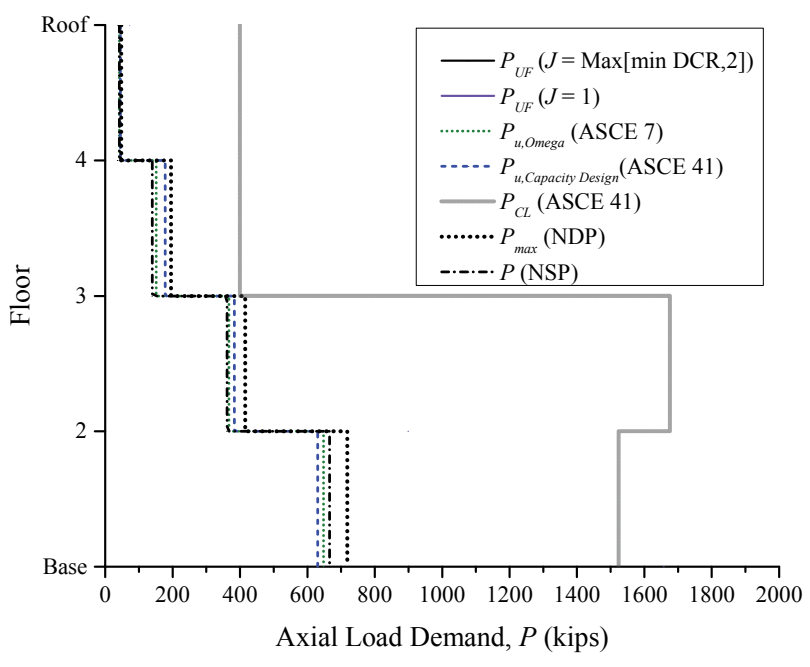

(b) RSA

Figure 3-31. LDP Assessment Results, Compression in Exterior Columns, 4-Story EBF, BSE-1

\subsection{BSE-2 Earthquake Hazard Level (CP BPL)}

In this section, the following apply:

- Figure 3-32 and Figure 3-33 provide the $D C R_{N}$ and load-dependent $m$-factor values for the ELF and RSA designs, respectively, for the LDP at the BSE-2 EHL. In these figures, $D C R_{N}$ values greater than unity are highlighted in red and underlined. $D C R$ values, as defined by ASCE 41, can be obtained by multiplying $D C R_{N}$ by $m$ and $\kappa$, see Eq. 3-6.

- Figure 3-34 provides the maximum axial compression demands, $P_{U F}$, in the exterior column lines for various analysis methods and the column capacity, $P_{C L}$.

All component actions satisfy the CP BPL acceptance criteria. The $D C R_{N}$ results for the force-controlled components (columns and braces) are the same for both LDP LS and LDP CP because all link beams have yielded—see $\$ 3.2 .2 .1 .1 .1$ for additional information. 


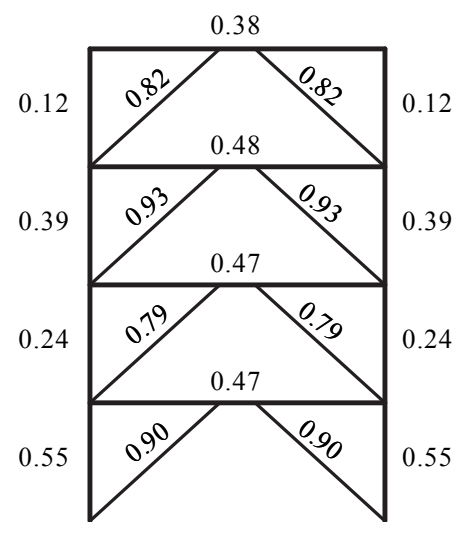

(a) $D C R_{N}$

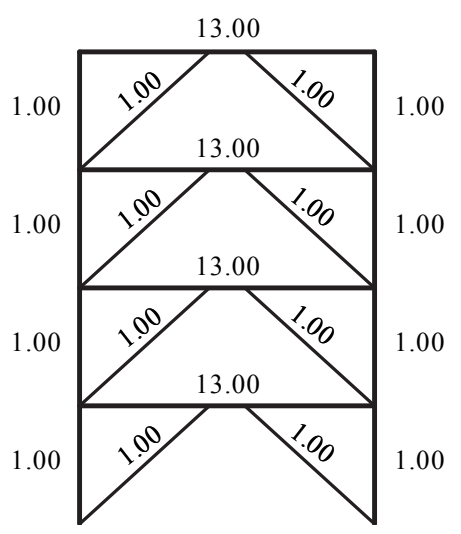

(b) $m$-factors

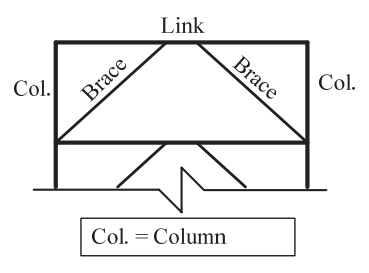

(c) Key

Figure 3-32. LDP Assessment Results, 4-Story EBF ELF, BSE-2 CP

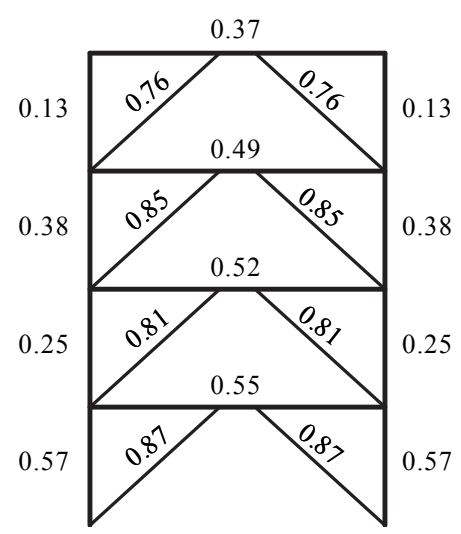

(a) $D C R_{N}$

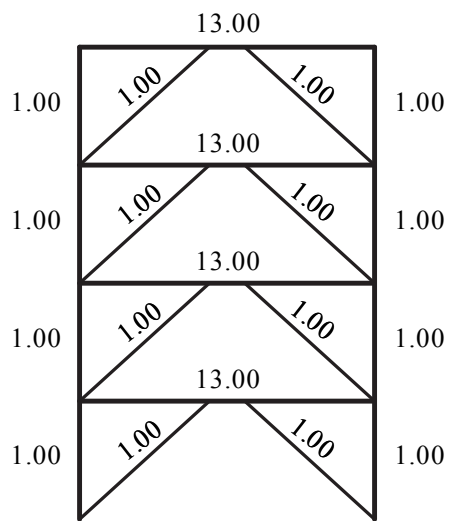

(b) $m$-factors

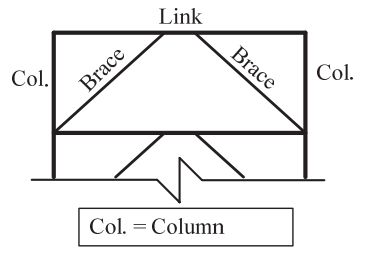

(c) Key

Figure 3-33. LDP Assessment Results, 4-Story EBF RSA, BSE-2 CP

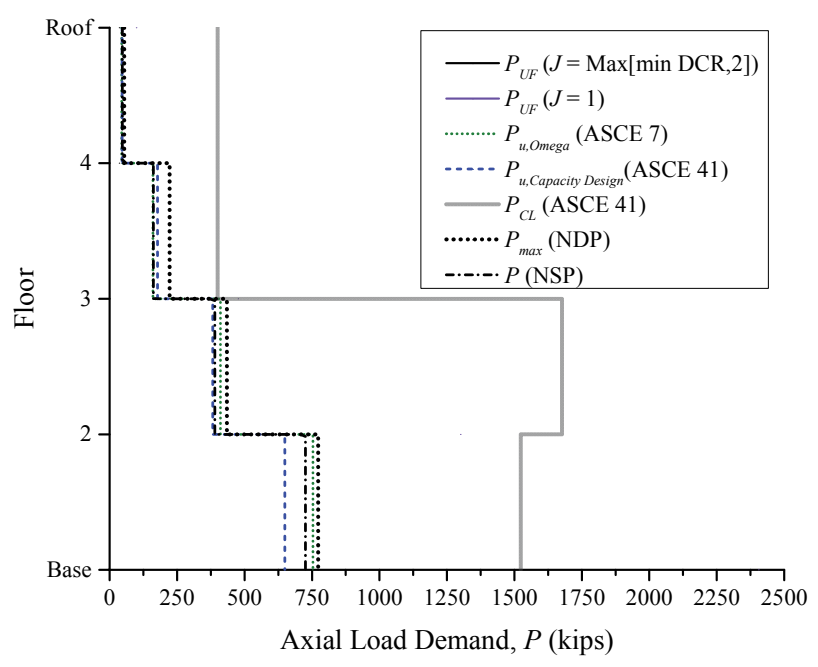

(a) ELF

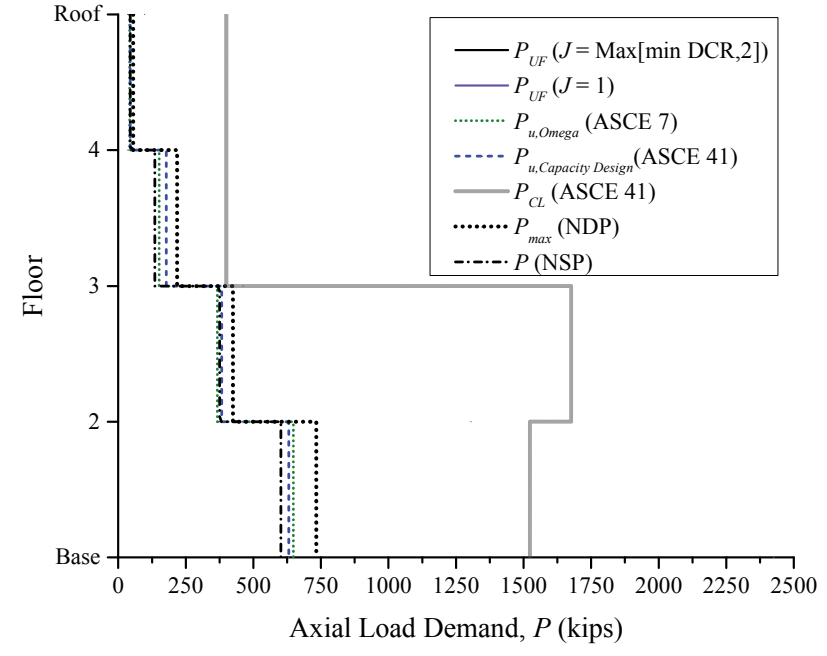

(b) RSA

Figure 3-34. LDP Assessment Results, Compression in Exterior Columns, 4-Story EBF, BSE-2 


\subsection{Nonlinear Static Procedure}

In this section, the following apply:

- Table 3-11 through Table 3-13 provide the computed NSP analysis and assessment parameters in accordance with ASCE 41 \$3.3.3.

- Figure 3-35 and Figure 3-36 illustrate the monotonic pushover curves for the ELF- and RSAdesigned frames, respectively, and the associated pushover parameters from ASCE 41 at the BSE2 EHL. Roof displacement is measured at the Center of Mass (CoM). A significant change in base shear is due to component strength loss of components (e.g., link beams), notated in the figures. First-order and second-order responses, shown in these figures, aids in computing a physically meaningful value for $\alpha_{P-\Delta}$ used in ASCE 41 Equation 3-17.

- Figure 3-37 and Figure 3-38 illustrate the story drift ratios in terms of the roof drift ratio.

As discussed in $\$ 3.1 .3 .2 .1$, the NSP is permitted, but requires supplemental verification using the LDPsee 3.2.2.1.2. In this case, the target displacement governs $\Delta_{d}$ for the ELF-designed frame at both the BSE1 and BSE-2 EHLs and the RSA-designed frame at the BSE-1 EHL, whereas the displacement at the maximum base shear governs the RSA-designed frame at the BSE-2 EHL. The change in $\Delta_{d}$ between BSE1 and BSE-2 adds complexity to NSP process by changing the pushover variables. Further, when $\Delta_{d}$ is governed by the target displacement, the system can have an increased $R_{\max }$. Axial compression force in the exterior columns at the target displacement are shown previously in the linear assessment sections.

Table 3-11. NSP General Information, 4-Story EBF (kip, inch)

\begin{tabular}{ccccccccccccc}
\hline Design & $T_{1}$ & $K_{1}$ & $\Delta_{y}$ & $V_{y}$ & $K_{e}$ & $T_{e}$ & $h$ & $\Delta_{\text {peak }}$ & $V_{\text {peak }}$ & $W$ & $C_{m}$ & $C_{0}$ \\
\hline ELF & 1.03 & 303.0 & 2.7 & 813 & 303.0 & 1.03 & 1.00 & 12.1 & 919.6 & 5132 & 1.00 & 1.30 \\
RSA & 1.08 & 286.1 & 2.6 & 743 & 286.1 & 1.08 & 1.01 & 11.1 & 851.6 & 5103 & 1.00 & 1.30 \\
\hline
\end{tabular}

Table 3-12. CP NSP Analysis Parameters, 4-Story EBF BSE-2 CP (kip, inch)

\begin{tabular}{cccccccccccccc}
\hline Design & $S_{a}$ & $R$ & $C_{1}$ & $C_{2}$ & $\Delta_{t}$ & $V_{t}$ & $\Delta_{d}$ & $\alpha_{1}$ & $\alpha_{2}$ & $\alpha_{P-\Delta}$ & $\alpha_{e}$ & $R_{\max }$ & $R_{\max }$ \\
\hline ELF & 0.90 & 5.68 & 1.00 & 1.00 & 12.0 & 919.6 & 12.0 & 0.04 & -0.08 & -0.01 & -0.03 & 14.60 & OK \\
RSA & 0.90 & 6.19 & 1.00 & 1.00 & 13.3 & 745.0 & 11.1 & 0.05 & -0.07 & -0.01 & -0.02 & 14.89 & OK \\
\hline
\end{tabular}

Table 3-13. LS NSP Analysis Parameters, 4-Story EBF BSE-1 LS (kip, inch)

\begin{tabular}{ccccccccccccccc}
\hline \multirow{2}{*}{ Design } & \multirow{2}{*}{$S_{a}$} & $R$ & $C_{1}$ & $C_{2}$ & $\Delta_{t}$ & $V_{t}$ & $\Delta_{d}$ & $\alpha_{1}$ & $\alpha_{2}$ & $\alpha_{P-\Delta}$ & $\alpha_{e}$ & $R_{\max }$ & $\begin{array}{c}R \leq \\
R_{\max }\end{array}$ \\
\hline ELF & 0.60 & 3.79 & 1.00 & 1.00 & 8.0 & 897.3 & 8.0 & 0.05 & -0.06 & -0.01 & -0.02 & 14.75 & OK \\
RSA & 0.60 & 4.12 & 1.00 & 1.00 & 8.9 & 840.8 & 8.9 & 0.05 & -0.06 & -0.01 & -0.02 & 14.91 & OK \\
\hline
\end{tabular}


Roof Drift Ratio at CoM (\%)

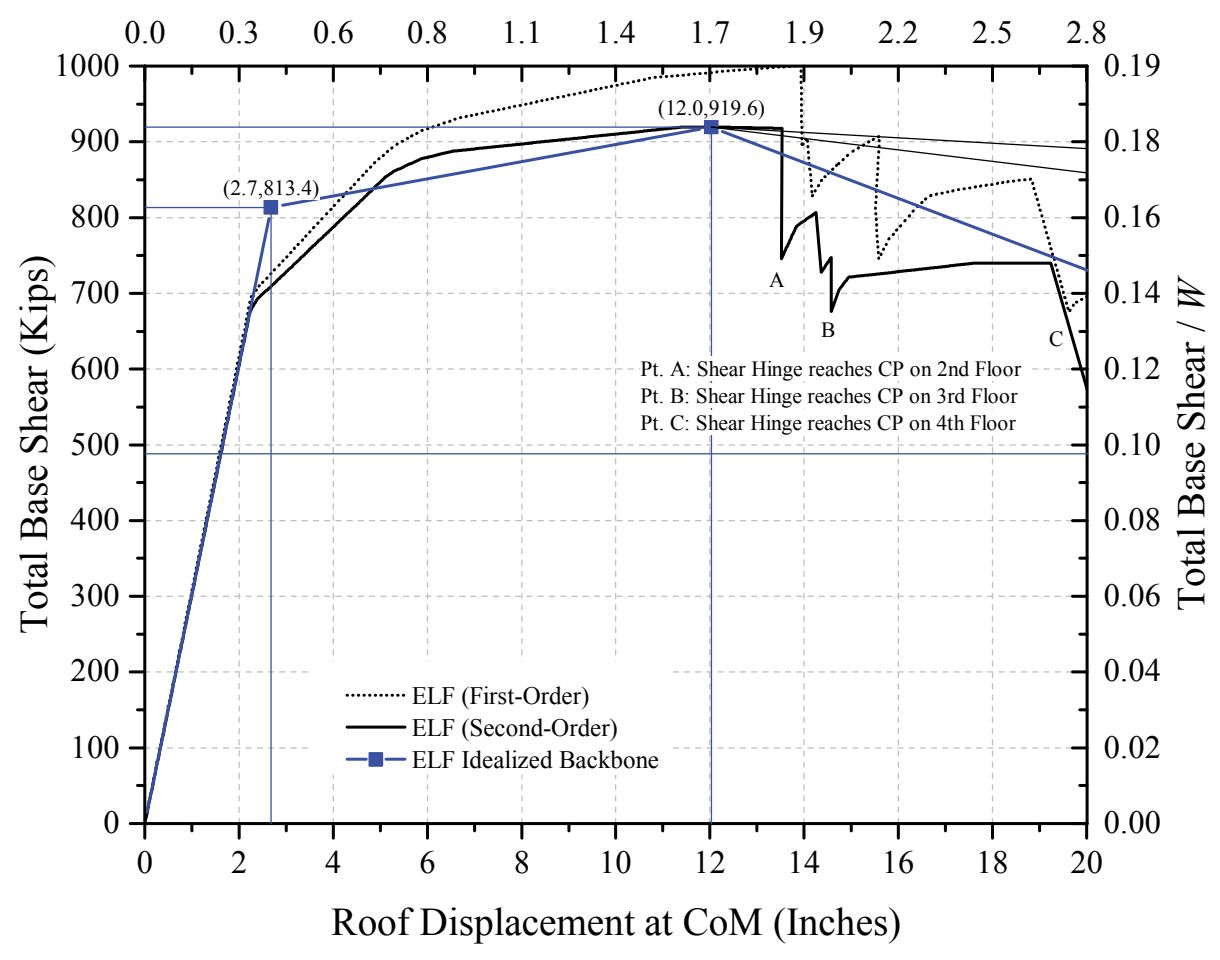

Figure 3-35. 4-Story EBF ELF Pushover, BSE-2

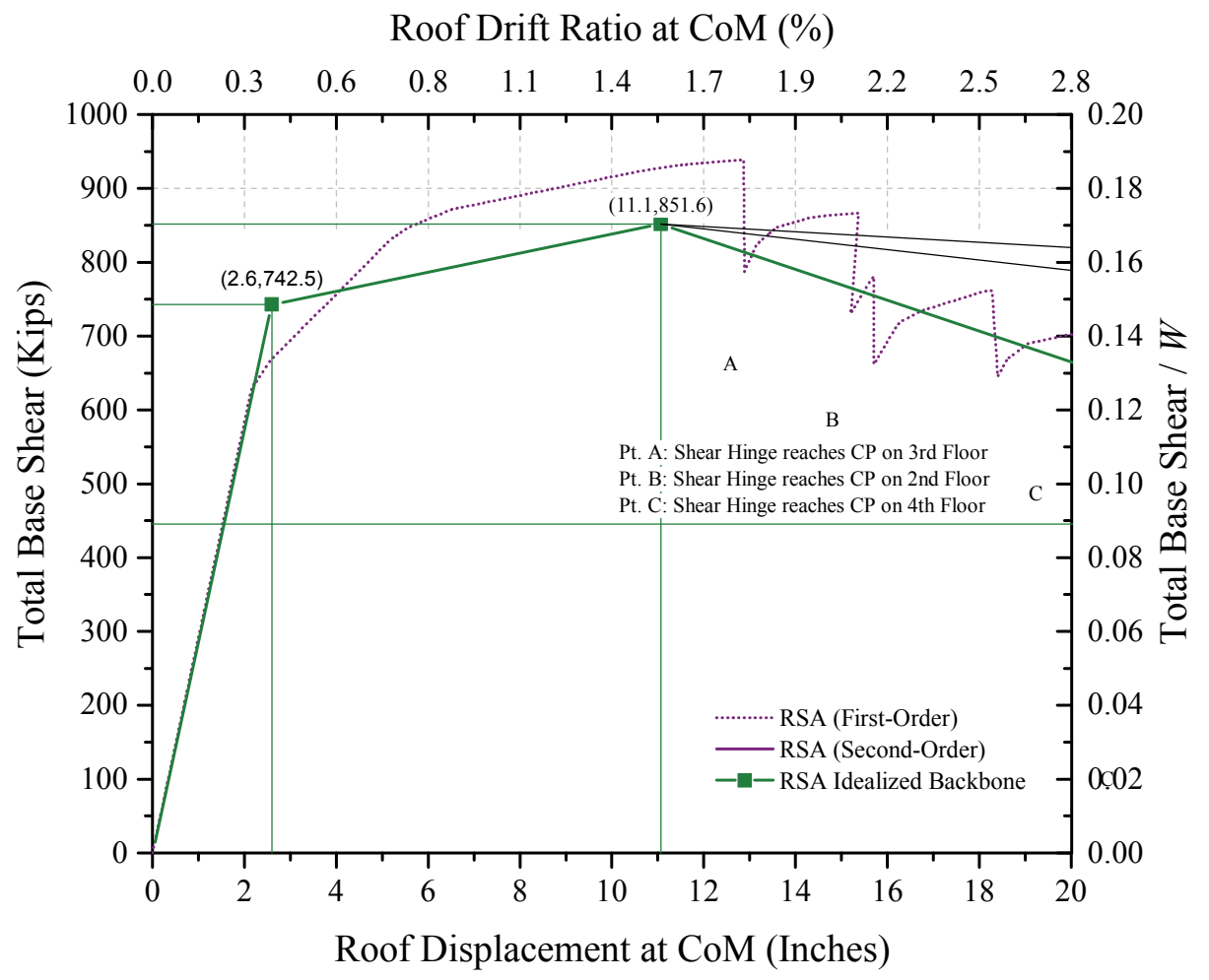

Figure 3-36. 4-Story EBF RSA Pushover, BSE-2 


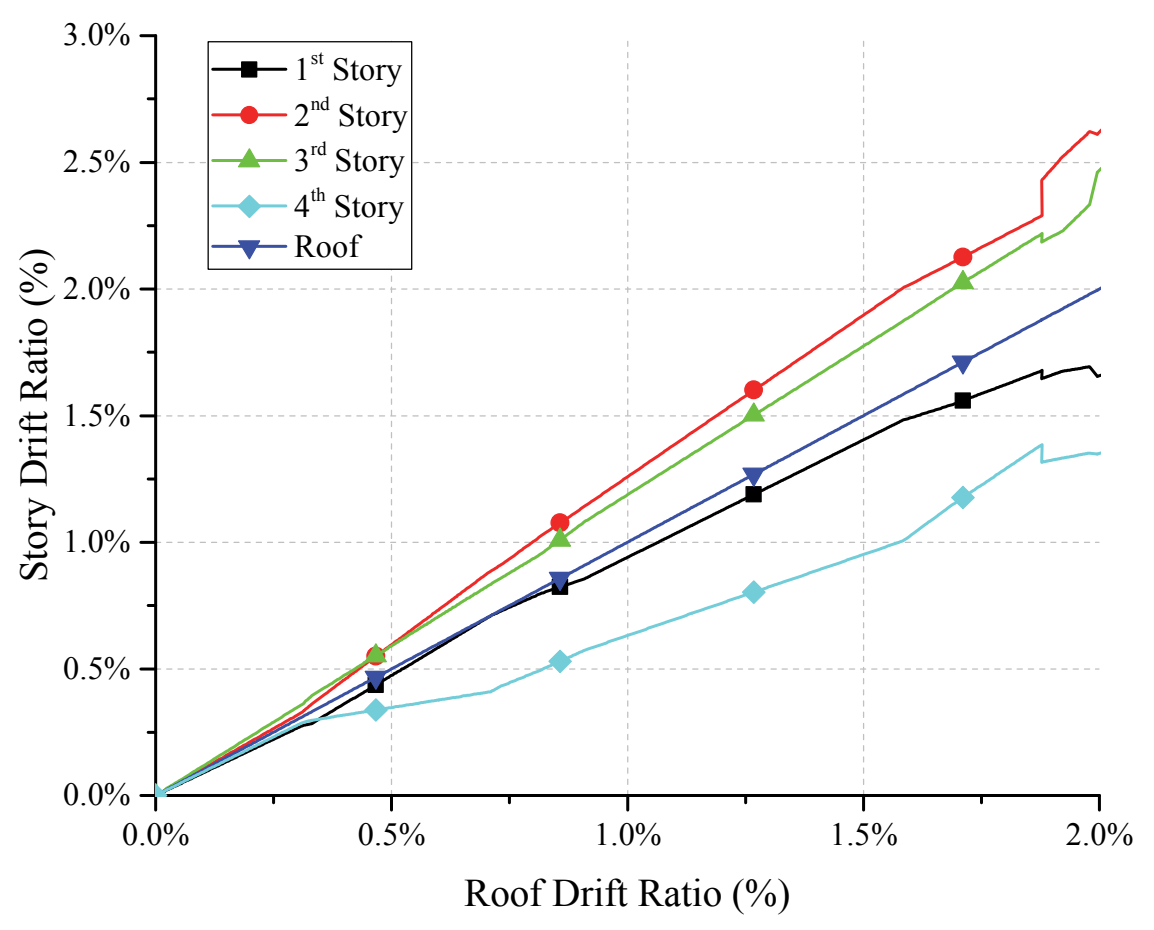

Figure 3-37. 4-Story EBF ELF Pushover - Story Drift Ratios - BSE-2

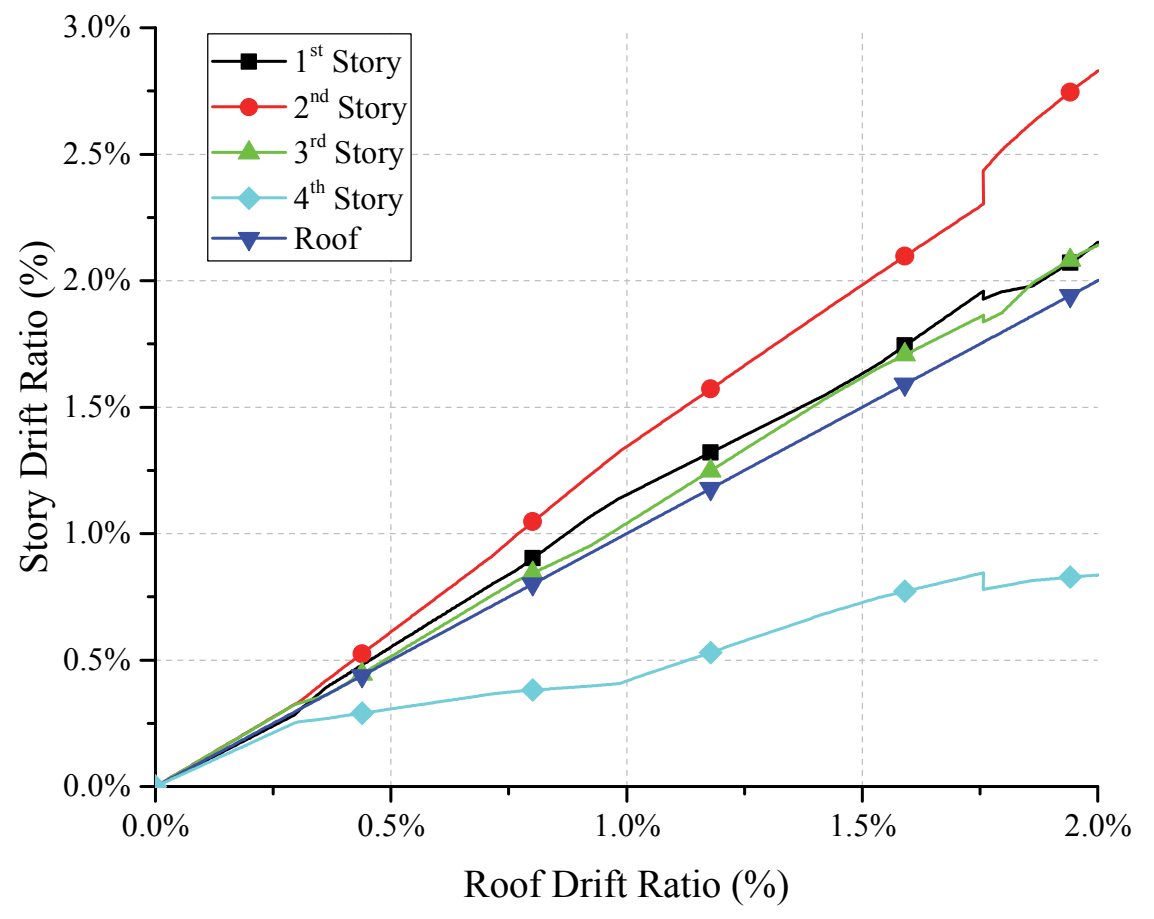

Figure 3-38. 4-Story EBF RSA Pushover - Story Drift Ratios - BSE-2 
Figure 3-39 illustrates which frame components are force-controlled for flexure for both the NSP and NDP; red circles indicate anticipated plastic hinge locations that are force-controlled for flexure at the target displacement. Figure 3-40 and Figure 3-41 illustrate the $D C R_{N}$ values if greater than unity for the ELF- and RSA-designed frames at the target displacement for the LS BPL at the BSE-1 EHL and CP BPL at the BSE2 EHL. The figures illustrate demands when the system is loaded to the left. Nearly all shear actions in the link beams satisfy the LS and CP BPL acceptance criteria except for the third floor link beam in the RSAdesigned frame for the CP BPL.

All other force-controlled component actions satisfy the LS BPL acceptance criteria. However, base column hinges do not satisfy the criteria for the CP BPL for both the ELF- and RSA-designed frames. This effect is two-fold: a consequence of modeling rotationally restrained base connections, which more closely characterizes how the base connection is detailed in these buildings than assuming rotationally unrestrained connections as is commonly done in practice, and, to a lesser extent, the minor increase in $P_{E}$ because the shear strength, $V_{C E}$, of the link beams was increased by 15 percent for nonlinear analysis - see \$3.2.1.2. As discussed in $\$ 3.2 .1 .2$, the flexural hinge model for force-controlled components obeys ASCE 41 Equation 5-4 (yield surface for the section). As such, unsatisfactory performance is demarcated by section yielding.

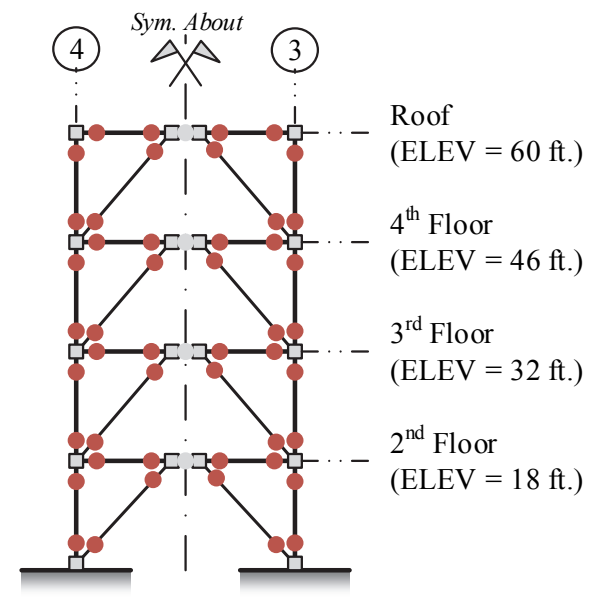

All columns, braces, and beams adjacent to link beams are force-controlled (FC) for flexure

Figure 3-39. Schematic of Flexural Actions in Components, 4-Story EBF (NSP and NDP) 


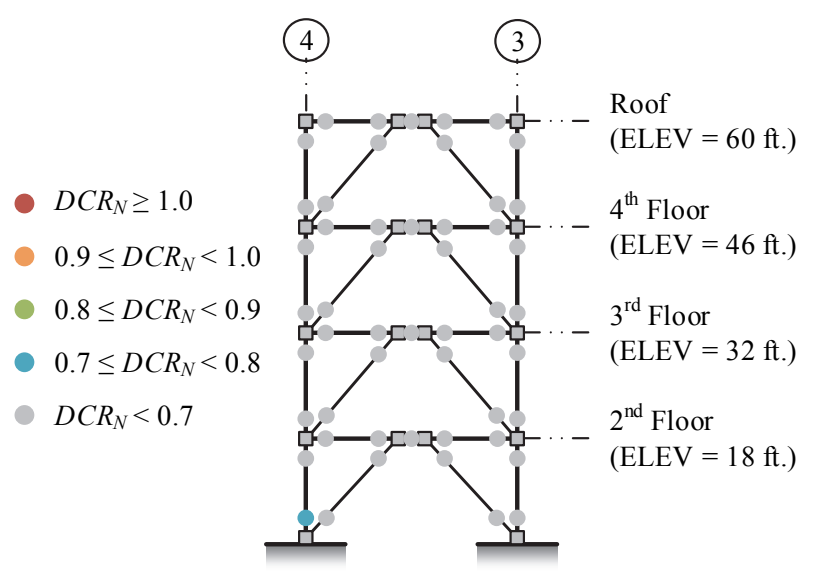

(a) ELF

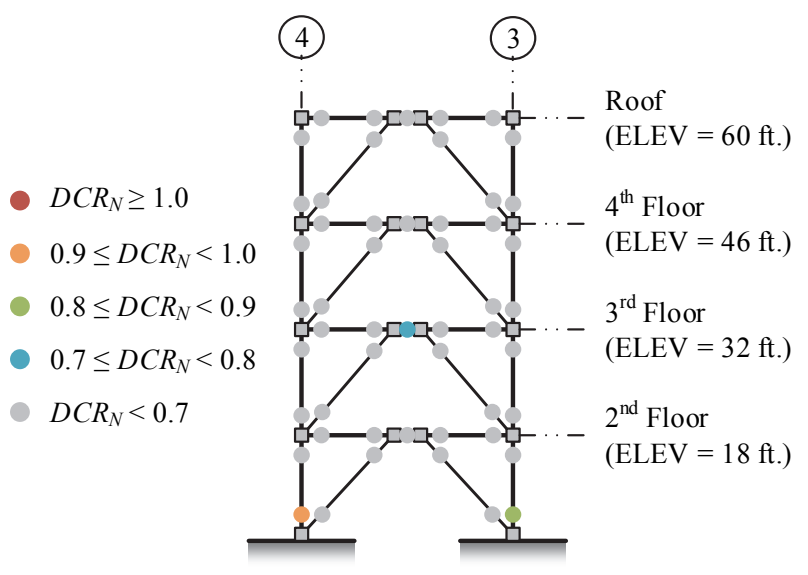

(b) RSA

Figure 3-40. NSP Assessment Results, 4-Story EBF, BSE-1 LS (+push to left)

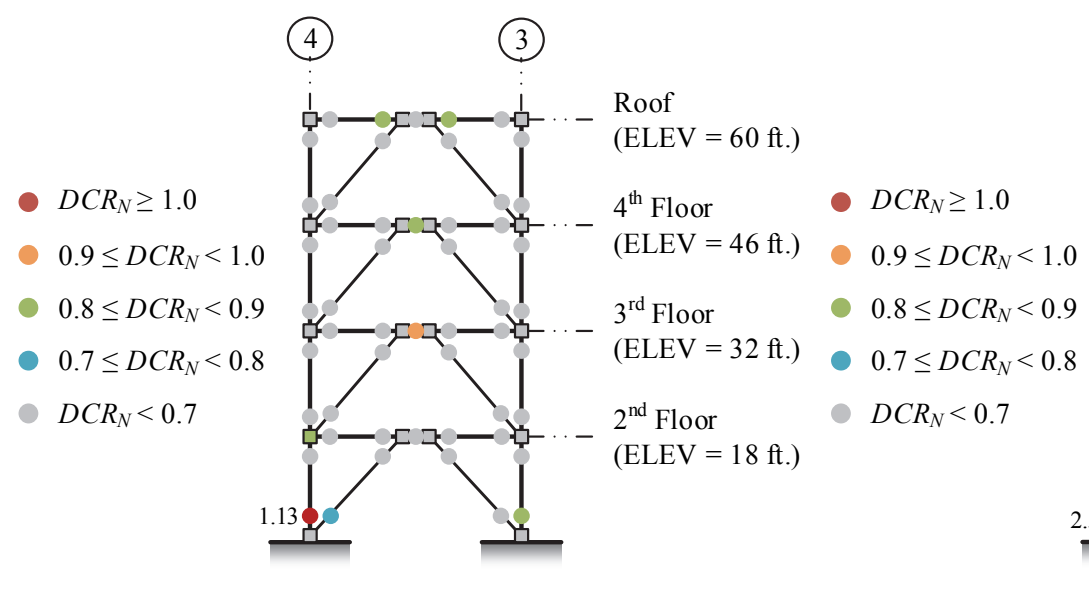

(a) ELF

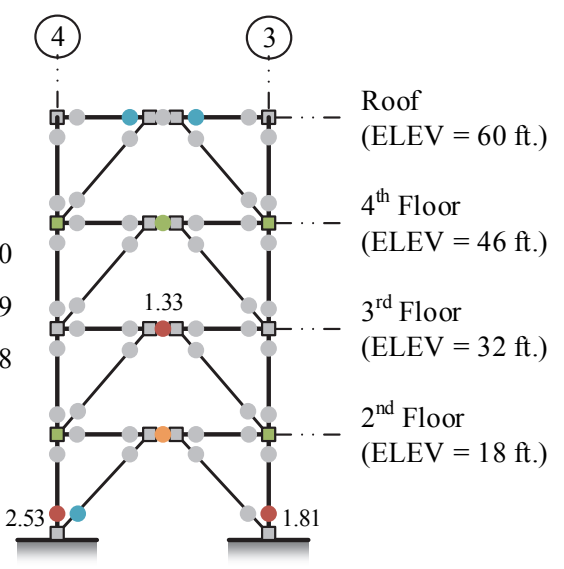

(b) RSA

Figure 3-41. NSP Assessment Results, 4-Story EBF, BSE-2 CP (+push to left)

\subsection{Nonlinear Dynamic Procedure}

The earthquake record set used to assess the N-S direction of MC4 is shown in Appendix A. For the ELF and RSA design, the analysis successfully completed for all 14 records at the BSE-1 and BSE-2 EHL. Maximum axial compression force in the exterior column lines from the record set are shown previously in the linear assessment sections.

Figure 3-42 and Figure 3-43 show the performance of the link beams at the BSE-1 (LS BPL) and BSE-2 (CP BPL) for the ELF- and RSA-designed frames, respectively. The results from the LSP, LDP, and NSP (loaded to the left) are included in the figures. Comparison discussions between the various procedures are addressed subsequently. As is evident in the figures, the ELF-designed frame performs slightly better than the RSA-designed frame - a corollary of the two frame designs being nearly the same. The results indicate that several link beams in both designs do not satisfy the CP BPL acceptance criteria using the mean 
response. In contrast to the mean response, the median response indicates better performance because it is less influenced by large deformations resulting from component strength loss potentially resulting in collapse of the system. Consequently, the median is potentially a more stable performance metric when analyzing a large number of ground motion records, but should be restrained relative to a mean value. The average ratio of secondary to primary component acceptance criteria for a wide-flange section with the configuration in this frame is 1.14 for the CP SPL (1.27 for the LS SPL). A $D C R_{N}$ value of 0.73 equates to a plastic rotation angle of 0.08 radians at the BSE-1 EHL (0.62 at the BSE-2 EHL).

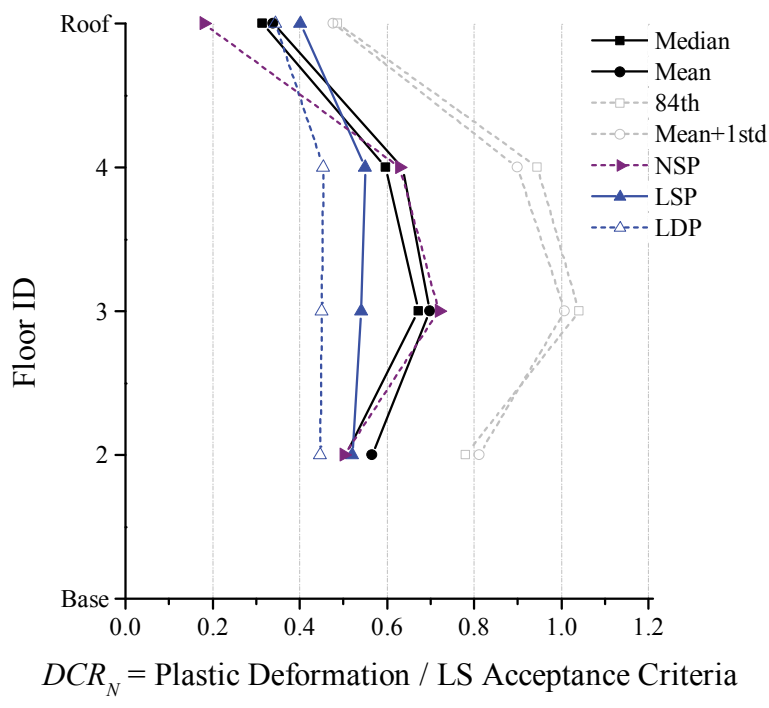

(a) ELF

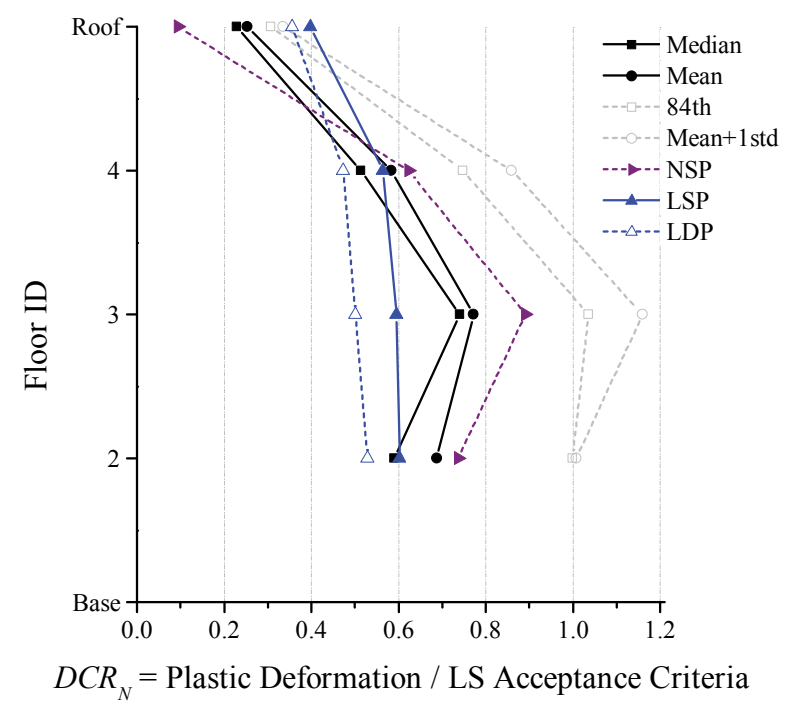

(b) RSA

Figure 3-42. NDP Assessment Results, Shear Hinges, 4-Story EBF BSE-1 LS

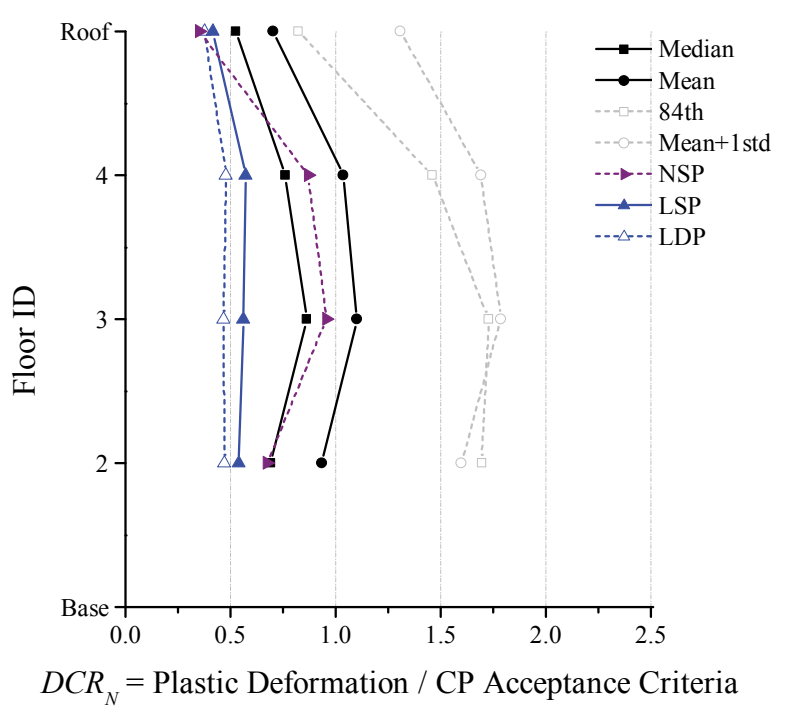

(a) ELF

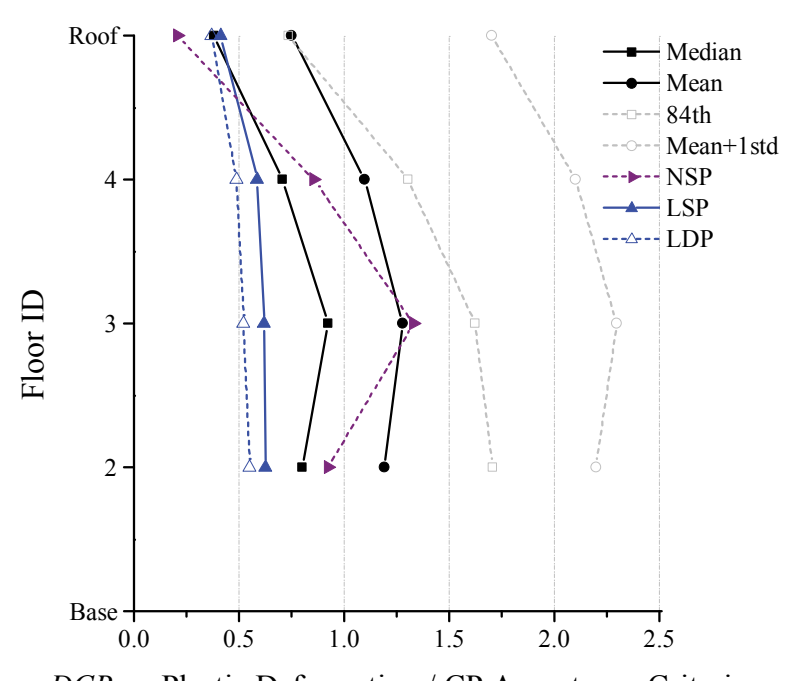

$D C R_{N}=$ Plastic Deformation / CP Acceptance Criteria

(b) RSA

Figure 3-43. NDP Assessment Results, Shear Hinges, 4-Story EBF BSE-2 CP 
Figure 3-44 and Figure 3-45 show the curvature ductility demand of the column hinges (i.e., section strength) at the BSE-2 EHL— see discussion on column hinge modeling in \$3.2.1.2. Figure 3-46 and Figure 3-47 show the elastic member strength interaction results at the BSE-2 EHL-see discussion on column strength modeling in \$3.2.1.2. Column hinges at the base above the brace connection experience inelastic strain demands (yield corresponds to a $D C R_{N}=1$ in the figures). The deformation demands are considerably lower than the primary CP acceptance criteria for a Column. Still, the base column hinges do not satisfy the criteria for the CP BPL for both the ELF- and RSA-designed frames. This effect is two-fold: a consequence of modeling rotationally restrained base connections, which more closely characterizes how the base connection is detailed in these buildings than assuming rotationally unrestrained connections as is commonly done in practice, and, to a lesser extent, the minor increase in $P_{E}$ because the shear strength, $V_{C E}$, of the link beams was increased by 15 percent for nonlinear analysis—see §3.2.1.2 and §3.2.2.1.3.

The $D C R_{N}$ results for the LSP and LDP are based on an interaction equation and not from $M_{U D} / m \times M_{C E}$, or $M_{U F} / M_{C L}$, which would be a more physically consistent metric for comparison against the results from the nonlinear assessment procedures. Nonetheless, the linear results are generally applicable here because the columns are force-controlled for flexure in the linear assessment procedures. Though there is a fundamental difference in how the $D C R_{N}$ is computed for the linear and nonlinear procedures, the linear assessment results show similar distributions of demands and location of potential performance concerns.

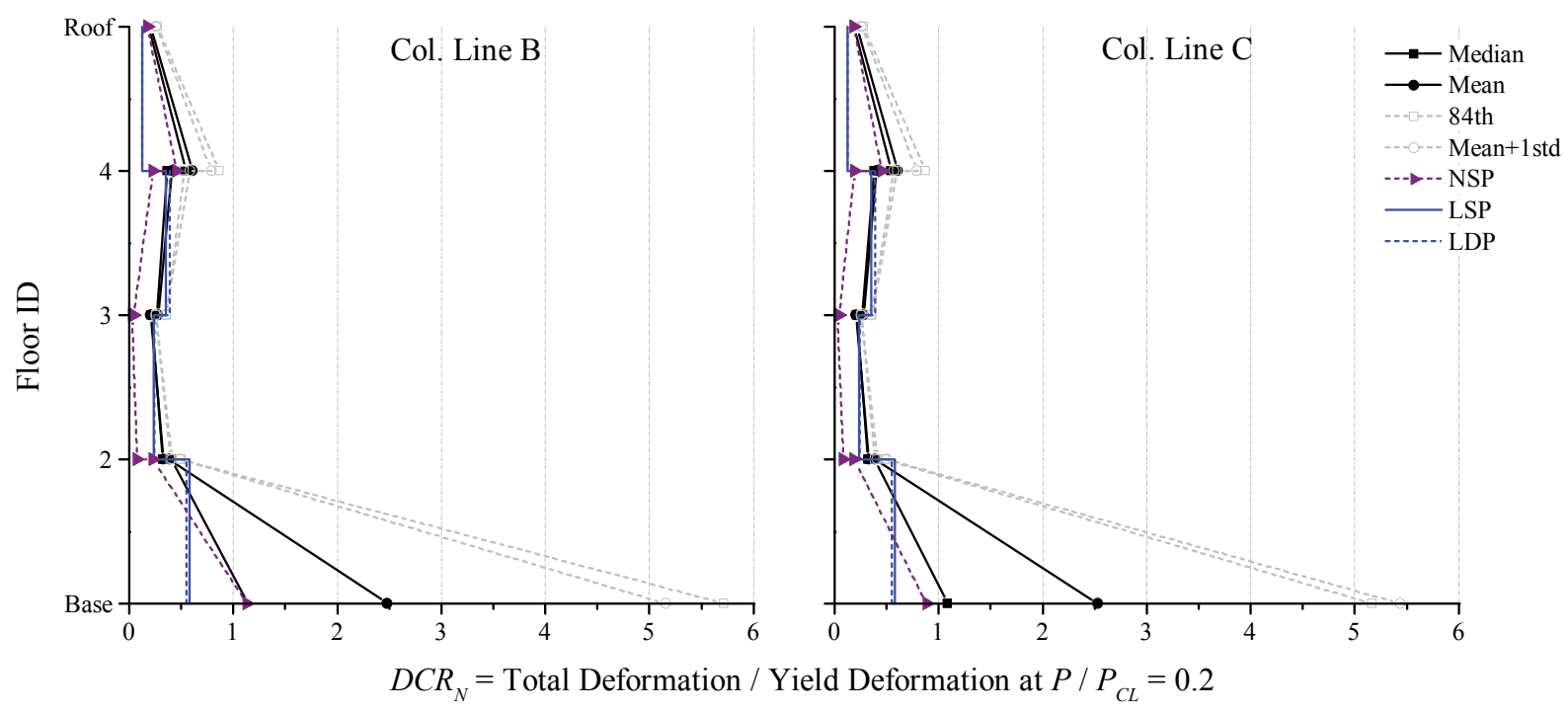

Figure 3-44. NDP Assessment Results, Column Hinges, 4-Story EBF ELF, BSE-2 Yield 


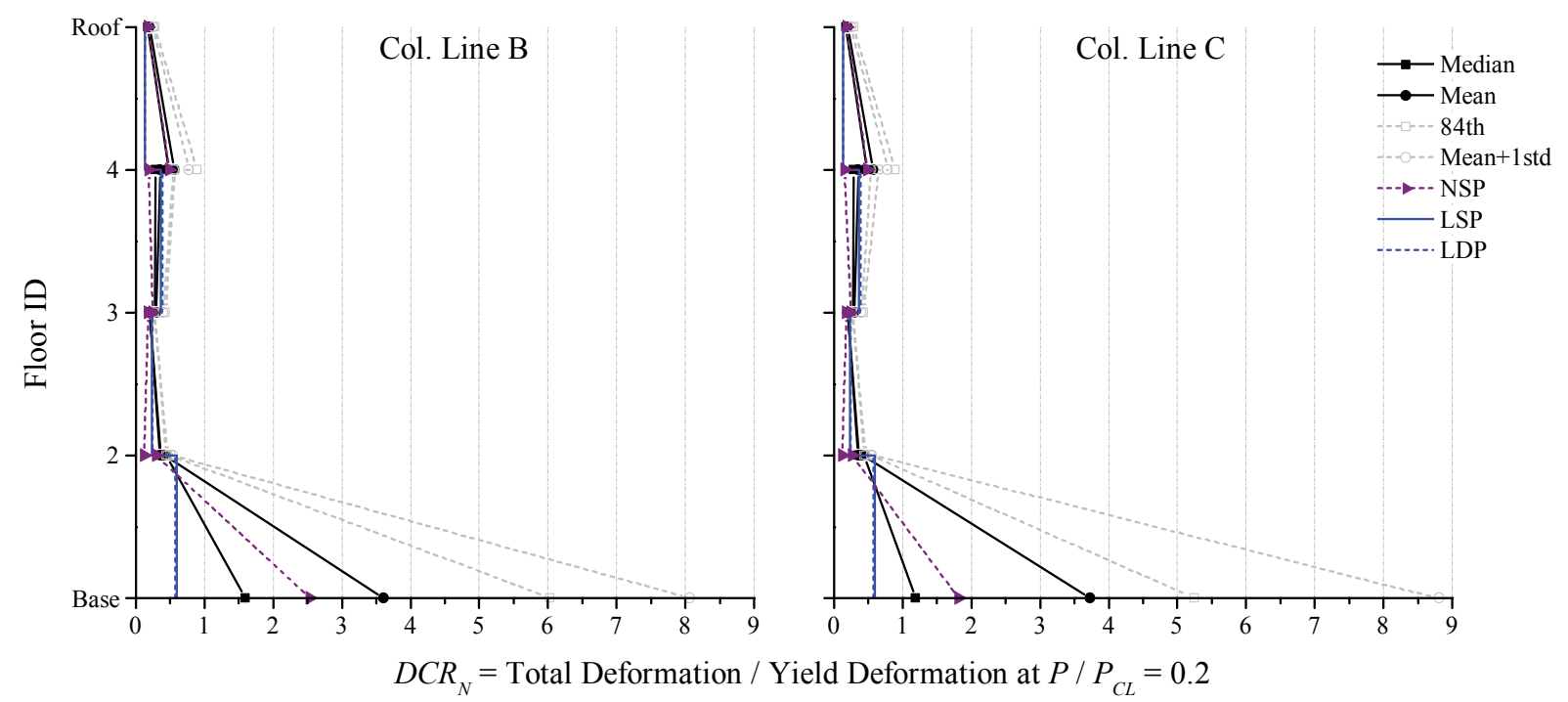

Figure 3-45. NDP Assessment Results, Column Hinges, 4-Story EBF RSA, BSE-2 Yield

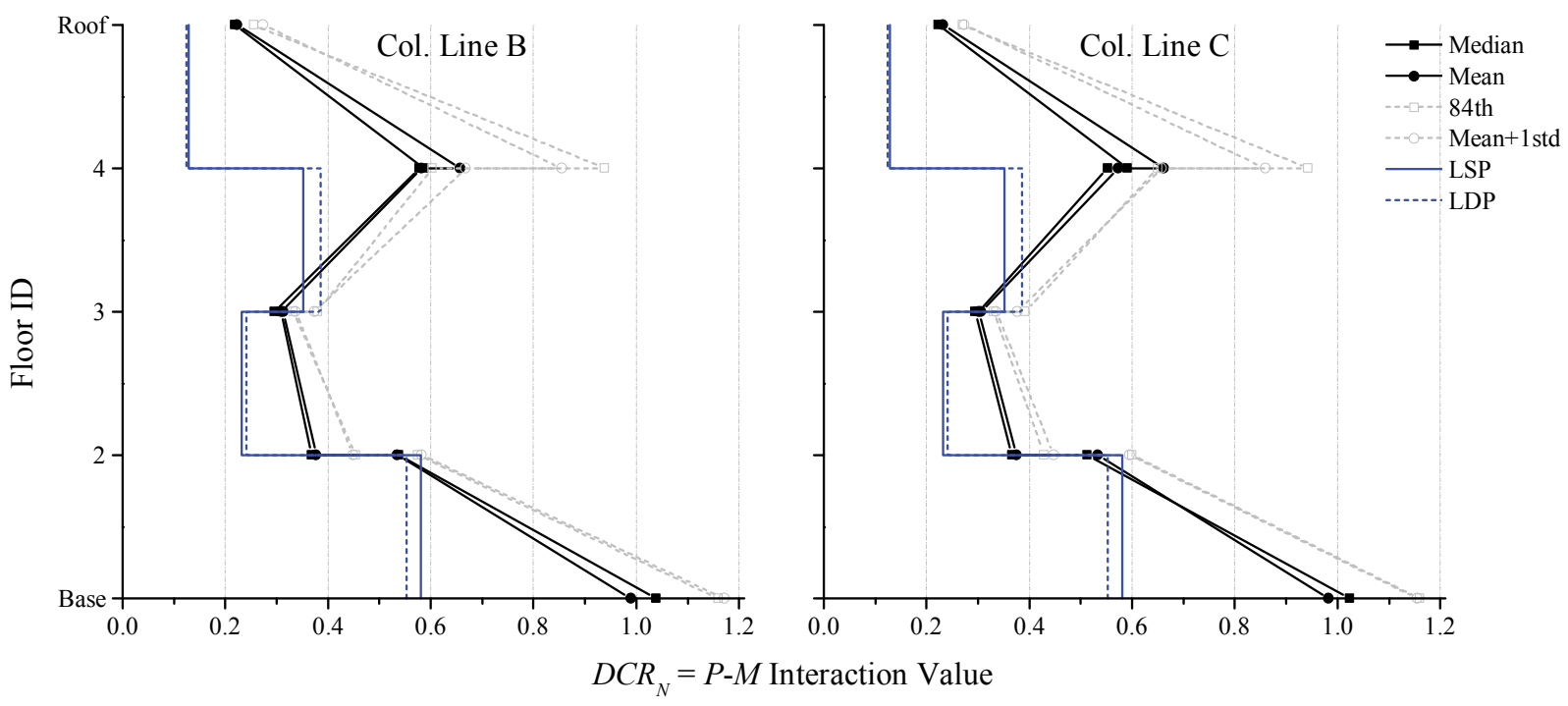

Figure 3-46. NDP Assessment Results, Column Members, 4-Story EBF ELF, BSE-2 


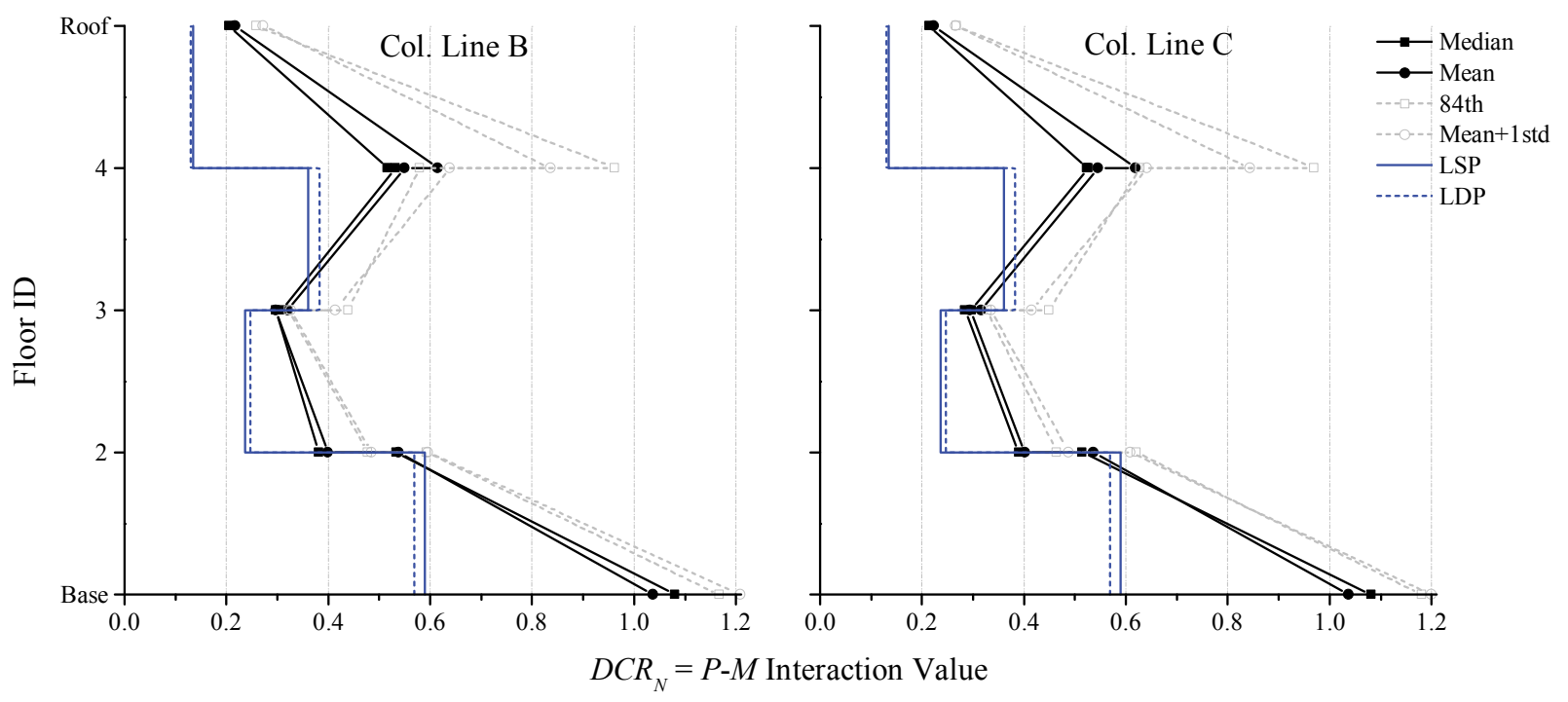

Figure 3-47. NDP Assessment Results, Column Members, 4-Story EBF RSA, BSE-2

Figure 3-48 and Figure 3-49 show the curvature ductility demand of the brace hinges (i.e., section strength) at the BSE-2 EHL - see discussion on column hinge modeling in $\$ 3.2 .1 .2$. Figure 3-50 and Figure 3-51 show the elastic member strength interaction results at the BSE-2 EHL - see discussion on column strength modeling in — see discussion on column strength modeling in \$3.2.1.2. Brace hinges at the base above the brace connection experience inelastic strain demands (yield corresponds to a $D C R_{N}=1$ in the figures). The deformation demands are considerably lower than the primary CP acceptance criteria for a Column. Still, the brace hinges do not satisfy the criteria for the CP BPL for both the ELF- and RSA-designed framessee previous discussion on column results for additional information.

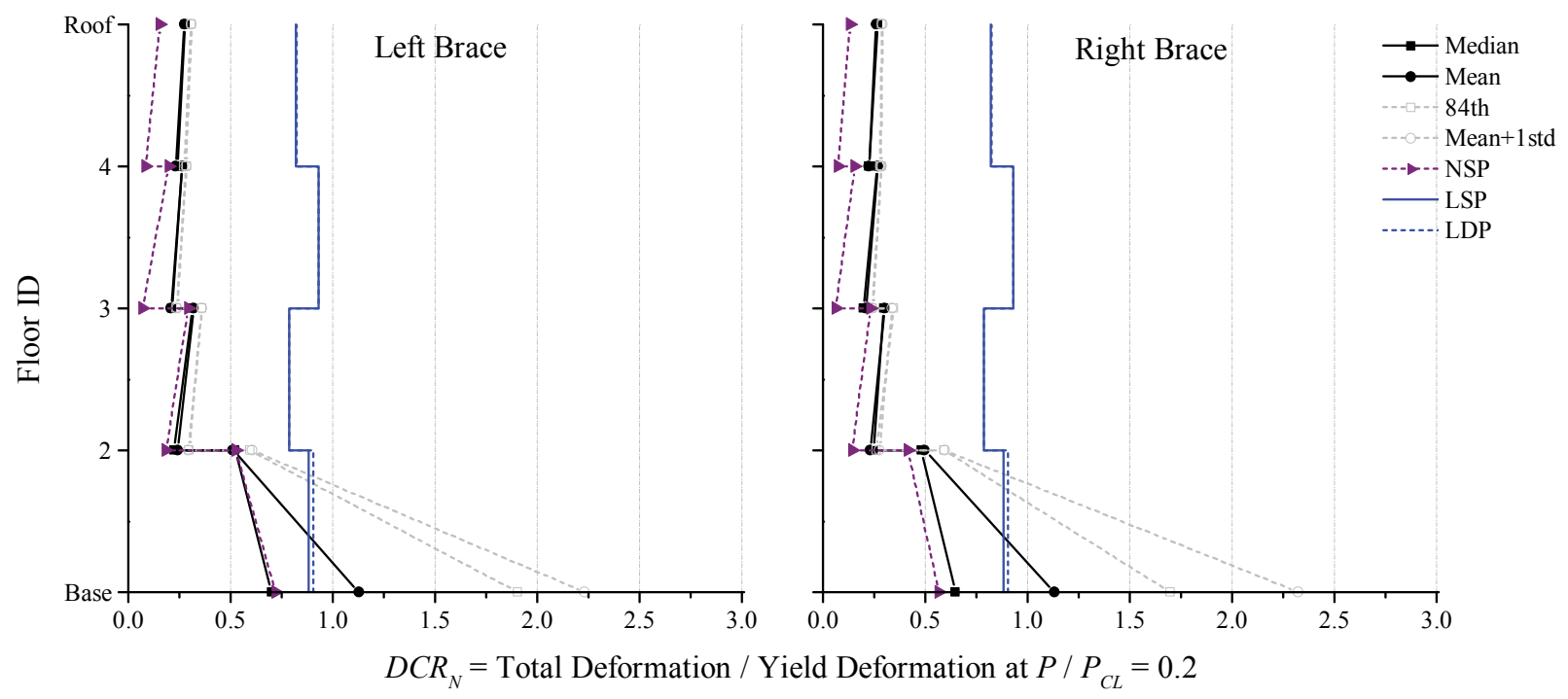

Figure 3-48. NDP Assessment Results, Brace Hinges, 4-Story EBF ELF, BSE-2 Yield 


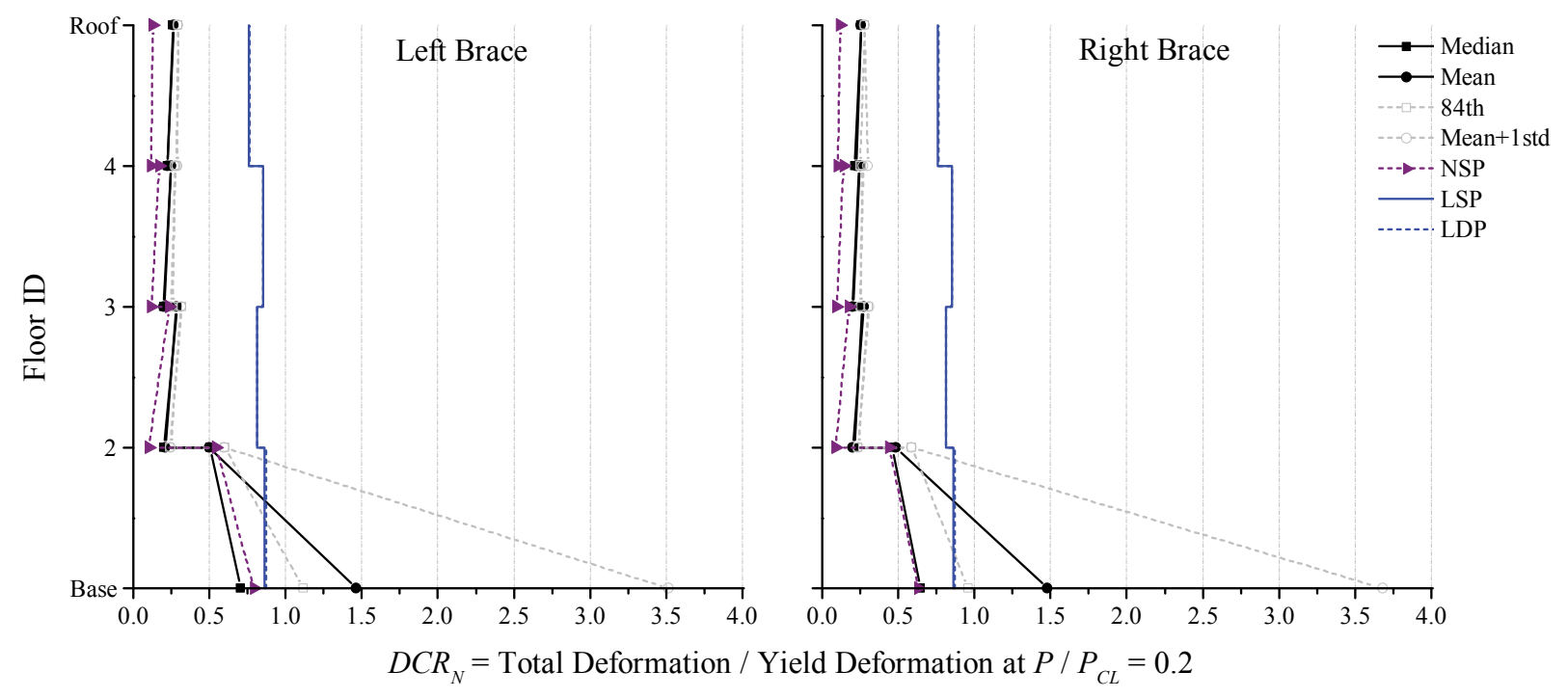

Figure 3-49. NDP Assessment Results, Brace Hinges, 4-Story EBF RSA, BSE-2 Yield

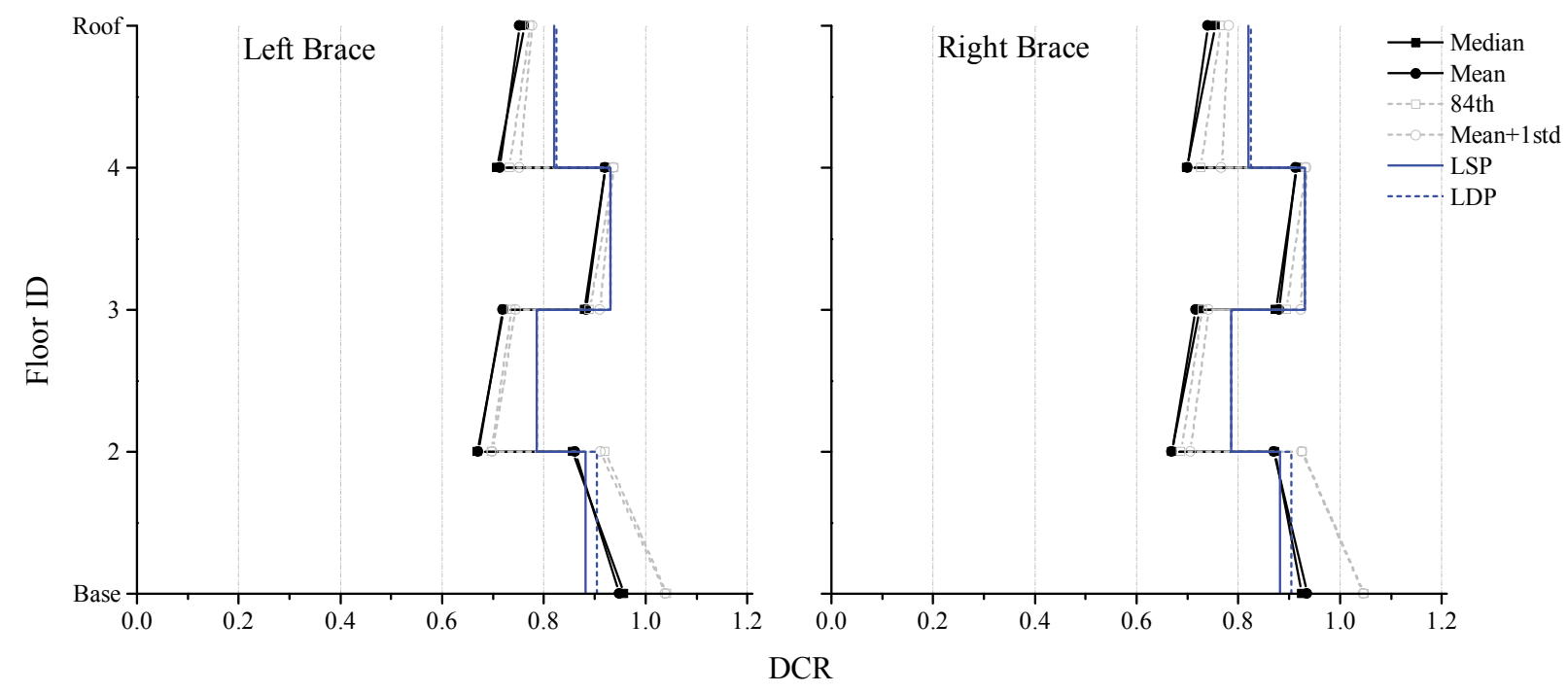

Figure 3-50. NDP Assessment Results, Brace Members, 4-Story EBF ELF, BSE-2 


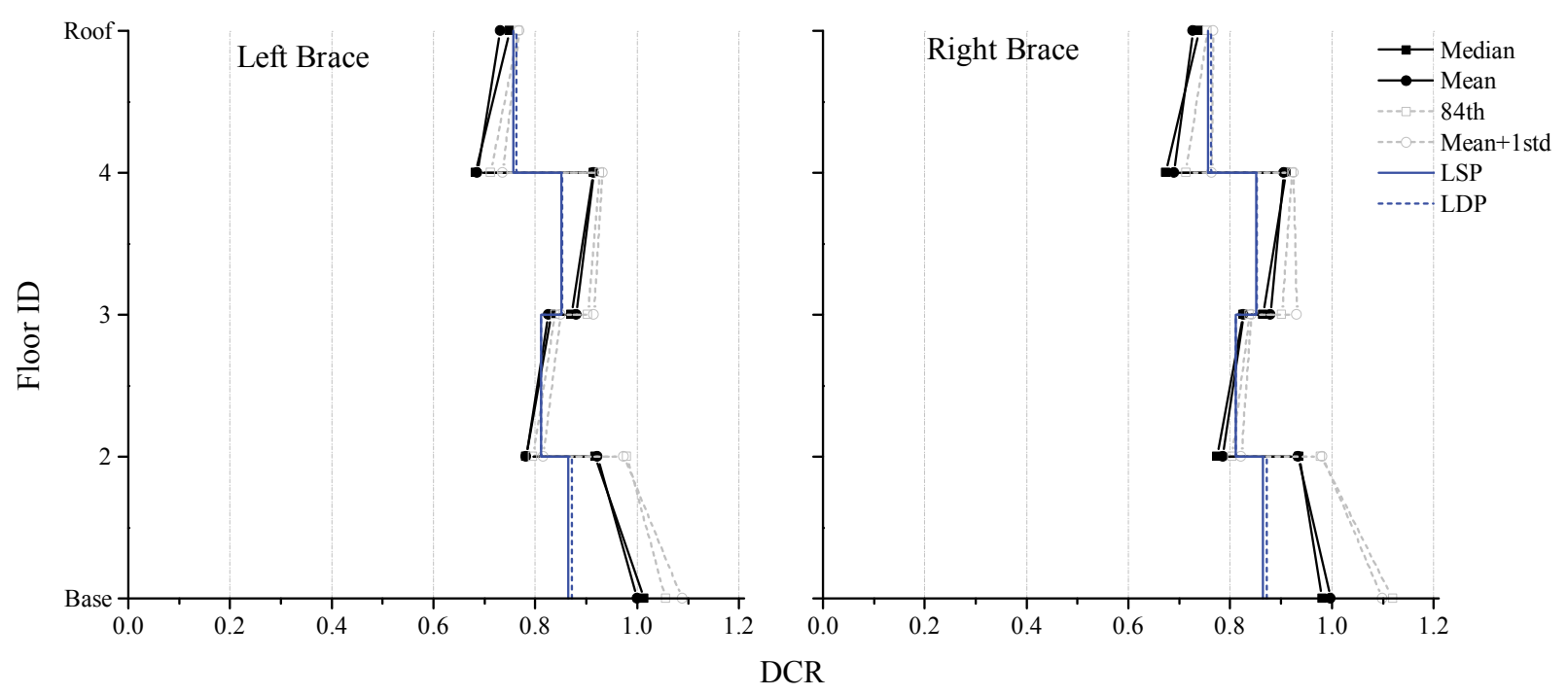

Figure 3-51. NDP Assessment Results, Brace Members, 4-Story EBF RSA, BSE-2

\subsubsection{Eight-Story Eccentric Braced Frame}

\subsection{Linear Static Procedure}

\subsection{BSE-1 Earthquake Hazard Level (LS BPL)}

In this section, the following apply:

- Figure 3-52 and Figure 3-53 provide the $D C R_{N}$ and load-dependent $m$-factor values for the ELF and RSA designs, respectively, for the LSP at the BSE-1 EHL. In these figures, $D C R_{N}$ values greater than unity are highlighted in red and underlined. $D C R$ values, as defined by ASCE 41, can be obtained by multiplying $D C R_{N}$ by $m$ and $\kappa$, see Eq. 3-6.

- Figure 3-54 provides the maximum axial compression demands, $P_{U F}$, in the exterior column lines for various analysis methods and the column capacity, $P_{C L}$.

All link beam and brace actions satisfy the LS BPL acceptance criteria.

Base columns do not satisfy the LS BPL acceptance criteria (force-controlled) using the interaction equation because of the combination of high axial force and moment. High flexural demand at the base of the frame is a consequence of modeling rotationally restrained base connections, which more closely characterizes how the base connection is detailed in these buildings than assuming rotationally unrestrained connections as is commonly done in practice. As discussed previously in $\$ 3.2 .2, P_{U F}$ is determined by taking $J$ (ASCE $41 \S 3.4 .2 .1 .2-2$ ) as the minimum $D C R$ of the component(s) delivering force to the column, but not less than 2.0. This approach produces an upper-bound estimate for $P_{U F}$ for the LS SPL as compared to ASCE 41 $\S 3.4 .2 .1 .2-1$ (based on ASCE 41 \$5.5.3.4.2), as shown in Figure 3-54. 


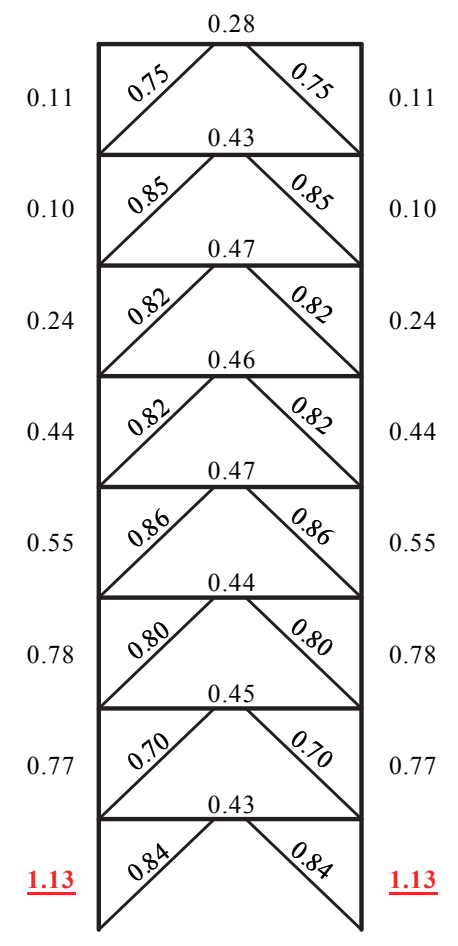

(a) $D C R_{N}$

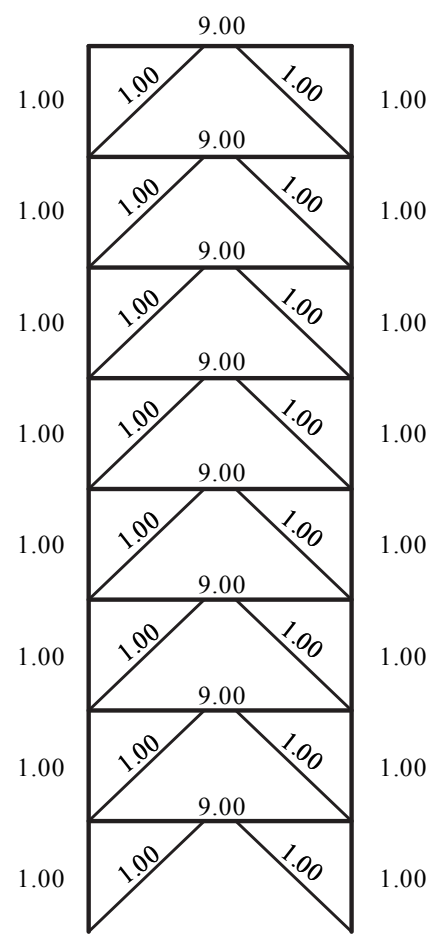

(b) $m$-factors

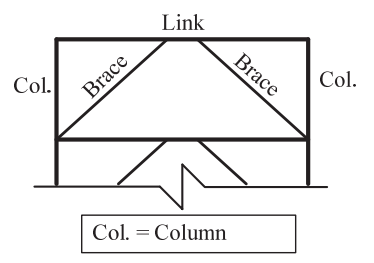

(c) Key

Figure 3-52. LSP Assessment Results, 8-Story EBF ELF, BSE-1 LS

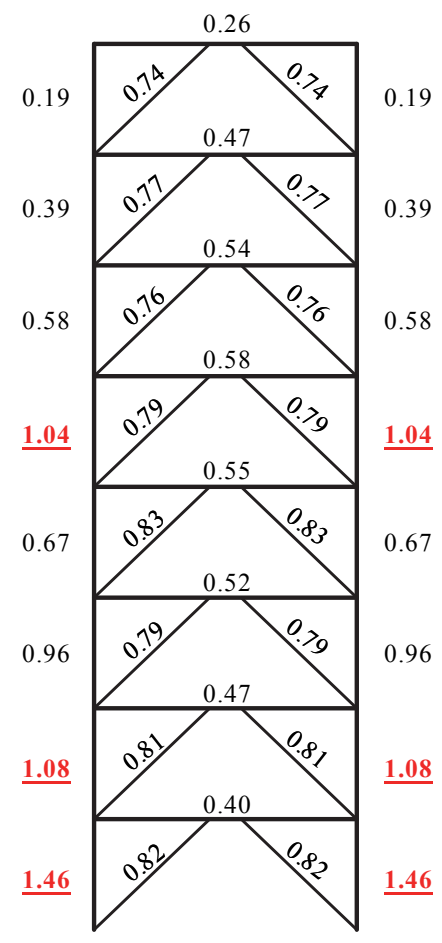

(a) $D C R_{N}$

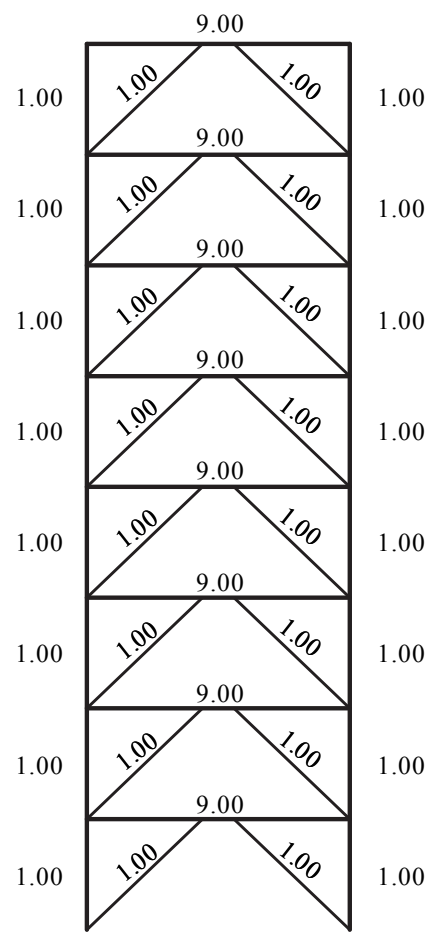

(b) $m$-factors

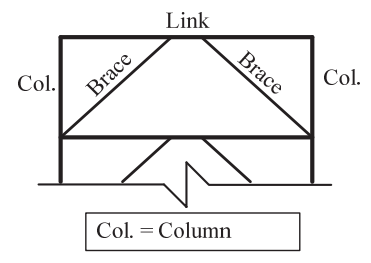

(c) Key

Figure 3-53. LSP Assessment Results, 8-Story EBF RSA, BSE-1 LS 


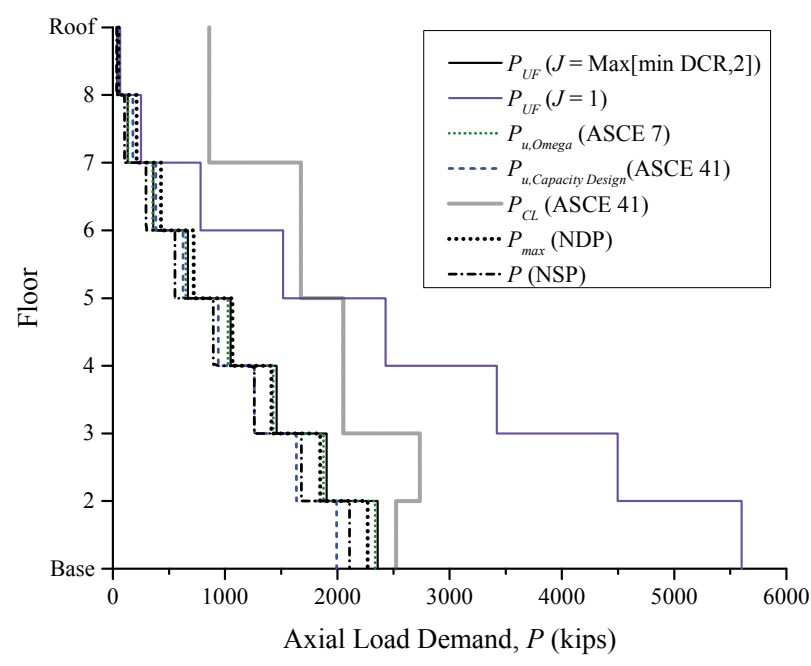

(a) ELF

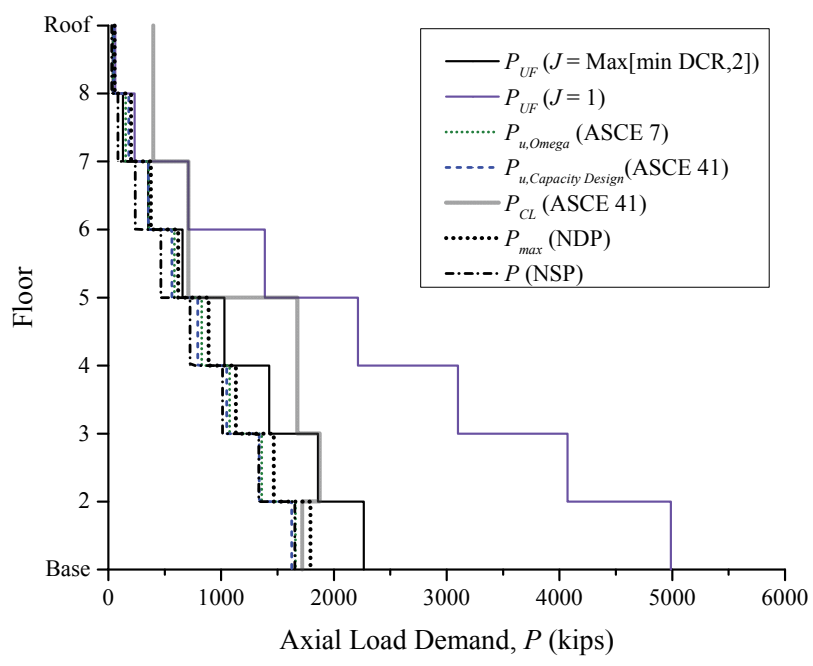

(b) RSA

Figure 3-54. LSP Assessment Results, Compression in Exterior Columns, 8-Story EBF, BSE-1

One could initially argue that columns in an EBF frame (greater than three stories) designed per AISC 341 would satisfy ASCE 41 criteria by default. Following ASCE $41 \S 5.5 .3 .4 .2$ results in the following for the base column (RSA-designed frame is used here):

○ $P_{C L}=1721$ kips

○ $\quad P_{\text {Gravity }}=259$ kips

○ $\quad P_{U F}=2265$ kips (determined using $J$ equal to the minimum $\mathrm{DCR}=2.36$ )

- $P_{U F} / P_{C L}=1.32\left(M_{U F} / M_{C L}=0.16\right) \rightarrow$ interaction result $=1.46$ (fails)

○ $\quad P_{U F}=1626$ kips (determined from a fully yielded frame per ASCE 41§5.5.3.4.2)

- $P_{U F} / P_{C L}=0.95\left(M_{U F} / M_{C L}=0.16\right) \rightarrow$ interaction result $=1.09$ (fails)

○ $\quad P_{U F}=1469$ kips (determined from a fully yielded frame per AISC $341 \S$ F3.3)

- $P_{U F} / P_{C L}=0.85\left(M_{U F} / M_{C L}=0\right) \rightarrow$ interaction result $=0.85$ (passes)

$P_{U F}$ computed per ASCE $41 \S 5.5 .3 .4 .2$ is slightly larger than that computed from AISC $341 \S$ F3.3 because of the 12 percent reduction in $P_{E Q}$ allowed by AISC 341. Furthermore, ASCE 41 does not provide guidance on how to compute $M_{U F}$ in a column-AISC 341 states the flexural forces due to seismic drift can be neglected. Consequently, the $P-M$ interaction equation used for assessment can be a combination of ASCE 41 3 3.4.2.1.2-1 $\left(P_{U F}\right)$ and ASCE 41 33.4.2.1.2-2 $\left(M_{U F}\right)$. This combination can be inconsistent and, as shown above, ASCE 41 \$3.4.2.1.2-2 can overestimate the force demands in the column (almost 40 percent in this example). Still, the question remains whether ASCE 41 should adopt AISC $341 \S$ F3.3 in its entirety in ASCE 41 §5.5.3.4.2. 


\subsection{BSE-2 Earthquake Hazard Level (CP BPL)}

In this section, the following apply:

- Figure 3-55 and Figure 3-56 provide the $D C R_{N}$ and load-dependent $m$-factor values for the ELF and RSA designs, respectively, for the LSP at the BSE-1 EHL. In these figures, $D C R_{N}$ values greater than unity are highlighted in red and underlined. $D C R$ values, as defined by ASCE 41, can be obtained by multiplying $D C R_{N}$ by $m$ and $\kappa$, see Eq. 3-6.

- Figure 3-57 provides the maximum axial compression demands, $P_{U F}$, in the exterior column lines for various analysis methods and the column capacity, $P_{C L}$.

All link beam and brace actions satisfy the CP BPL acceptance criteria.

Similar to the LSP LS results, base columns do not satisfy the CP BPL assessment criteria (force-controlled) using the interaction equation because of the combination of high axial force and moment. High flexural demand at the base of the frame is a consequence of modeling rotationally restrained base connections, which more closely characterizes how the base connection is detailed in these buildings than assuming rotationally unrestrained connections as is commonly done in practice.

The $D C R_{N}$ interaction results for force-controlled components are the same between LSP LS and LSP CP because all links have yielded, capping the force delivered to the columns based on the minimum DCR of all links. In this case, the minimum DCR of the top story link is greater than 2.0. If $J$ were set to the default of 2.0 then the interaction values would differ, which would not be consistent with the actual plastic mechanism (see 16-story LSP results). The results for the links differ by approximately (13/9)/1.5 = 0.963 times the $D C R_{N}$, approximate because of second-order effects. 


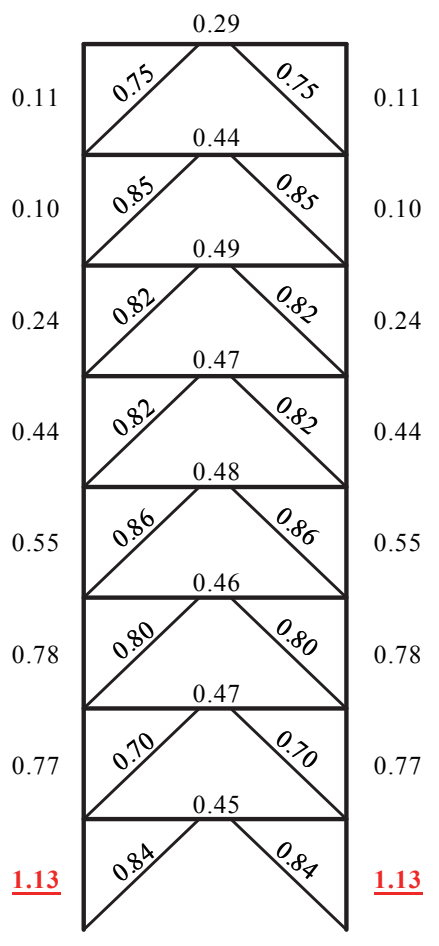

(a) $D C R_{N}$

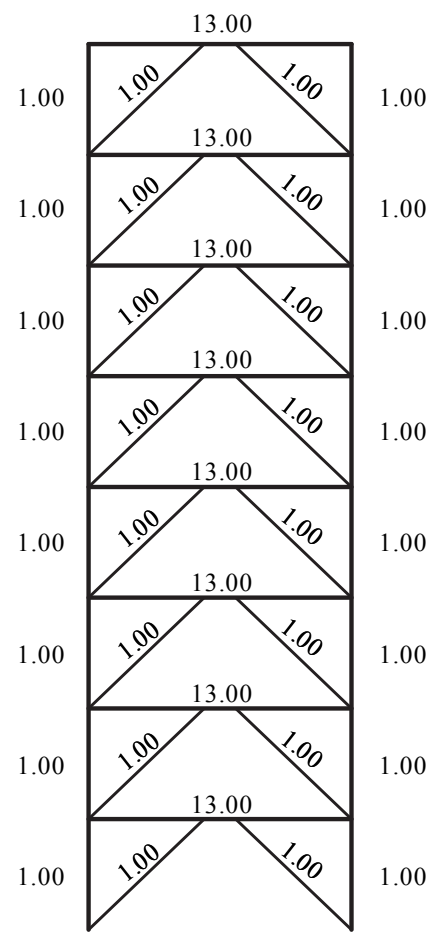

(b) $m$-factors

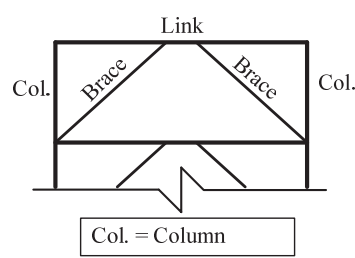

(c) Key

Figure 3-55. LSP Assessment Results, 8-Story EBF ELF, BSE-2 CP

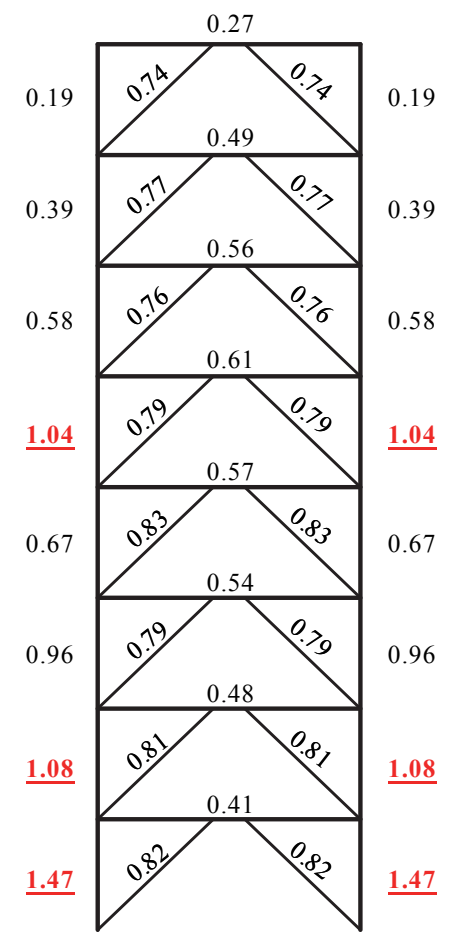

(a) $D C R_{N}$

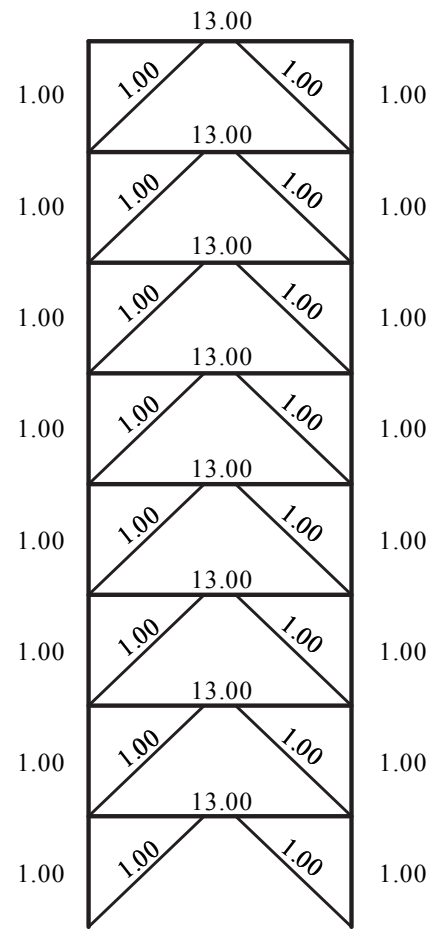

(b) $m$-factors

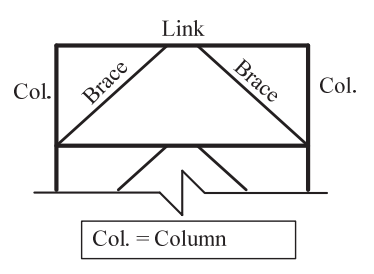

(c) Key

Figure 3-56. LSP Assessment Results, 8-Story EBF RSA, BSE-2 CP 


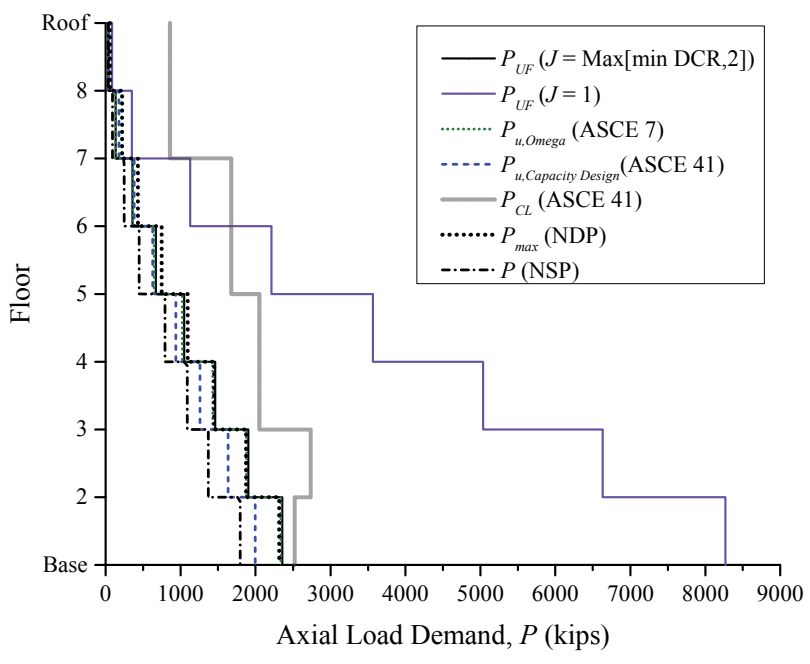

(a) ELF

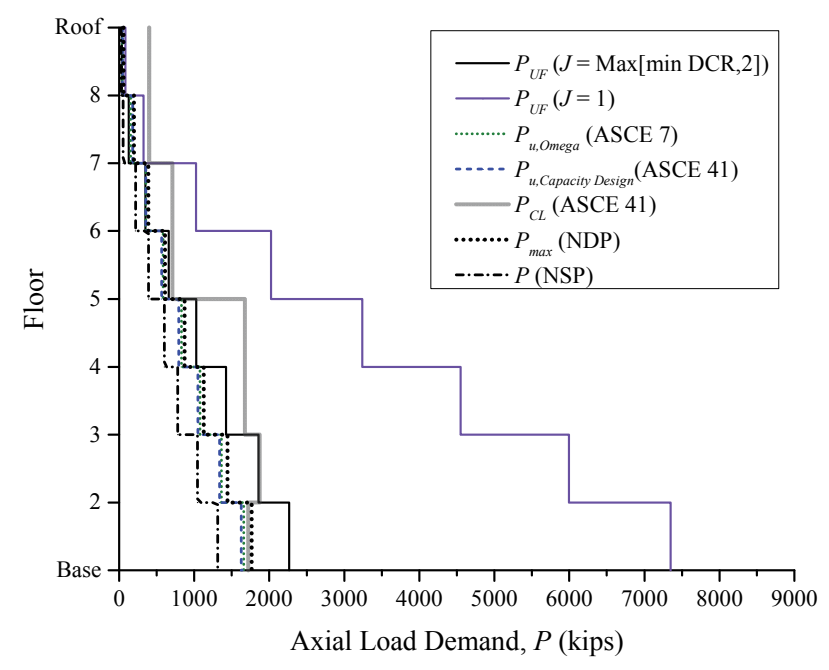

(b) RSA

Figure 3-57. LSP Assessment Results, Compression in Exterior Columns, 8-Story EBF, BSE-2

\subsection{Linear Dynamic Procedure}

\subsection{BSE-1 Earthquake Hazard Level (LS BPL)}

In this section, the following apply:

- Figure 3-58 and Figure 3-59 provide the $D C R_{N}$ and load-dependent $m$-factor values for the ELF and RSA designs, respectively, for the LSP at the BSE-2 EHL. In these figures, $D C R_{N}$ values greater than unity are highlighted in red and underlined. $D C R$ values, as defined by ASCE 41, can be obtained by multiplying $D C R_{N}$ by $m$ and $\kappa$, see Eq. 3-6.

- Figure 3-60 provides the maximum axial compression demands, $P_{U F}$, in the exterior column lines for various analysis methods and the column capacity, $P_{C L}$.

All link beam and brace actions satisfy the LS BPL acceptance criteria.

Base columns do not satisfy the LS BPL acceptance criteria (force-controlled) using the interaction equation because of the combination of high axial force and moment—see \$3.2.2.2.1 for more information. As discussed previously in $\S 3.2 .2, P_{U F}$ is determined by taking $J$ (ASCE $41 \S 3.4 .2 .1 .2-2$ ) as the minimum $D C R$ of the component(s) delivering force to the column, but not less than 2.0. This approach produces a lowerbound estimate for $P_{U F}$ for the LS SPL as compared to ASCE 41 \$3.4.2.1.2-1 (based on ASCE 41 §5.5.3.4.2), as shown in Figure 3-60. 


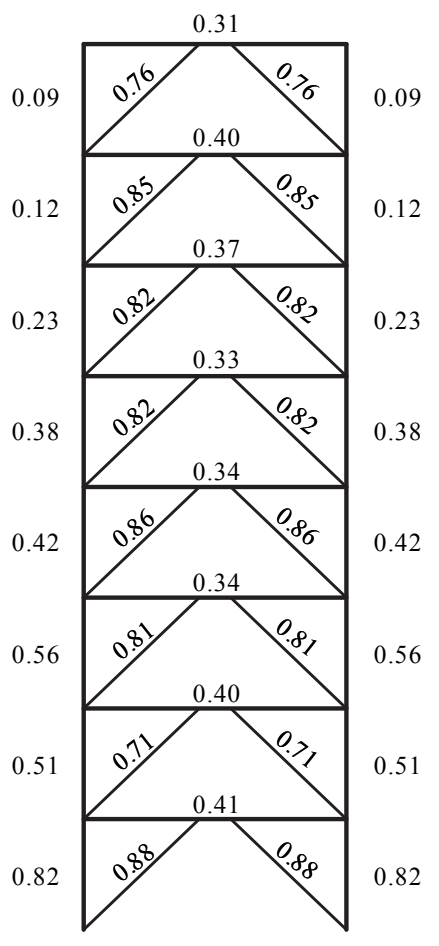

(a) $D C R_{N}$

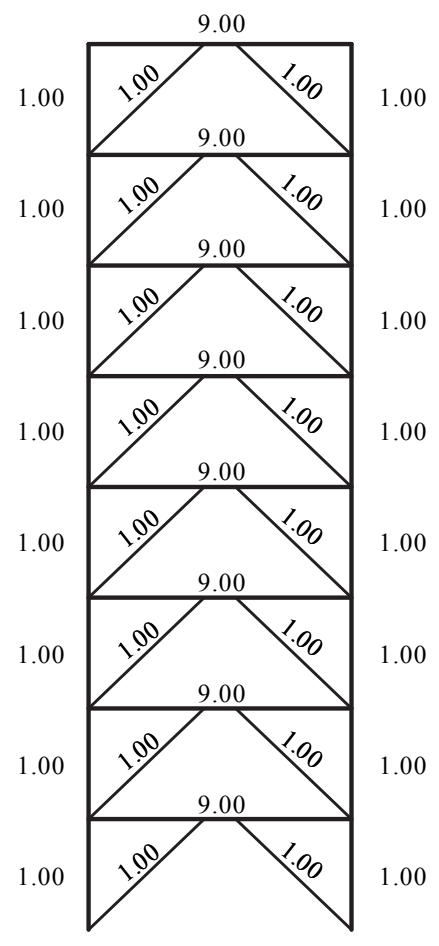

(b) $m$-factors

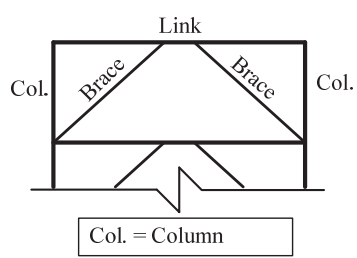

(c) Key

Figure 3-58. LDP Assessment Results, 8-Story EBF ELF, BSE-1 LS

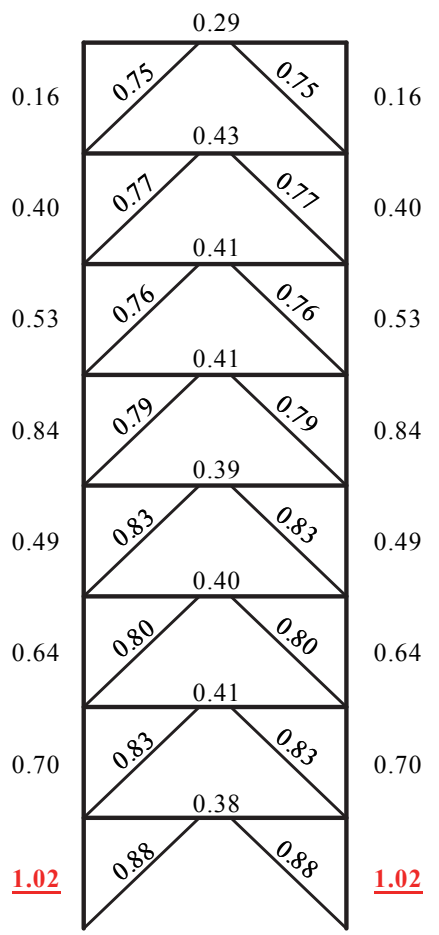

(a) $D C R_{N}$

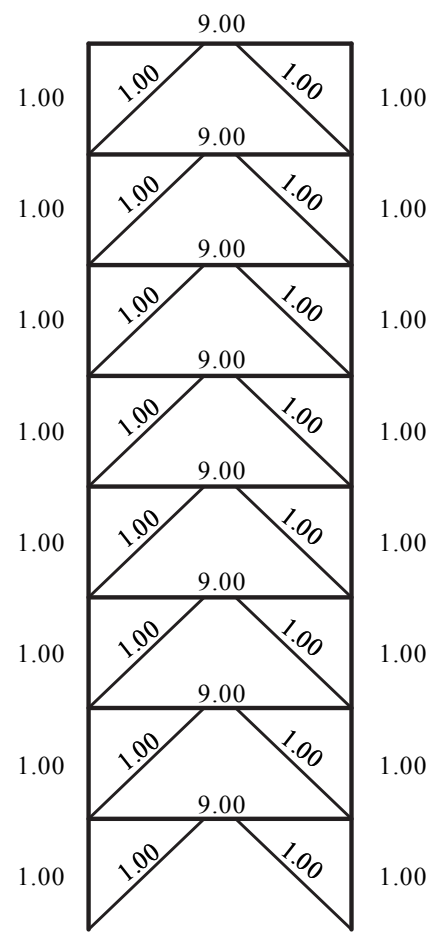

(b) $m$-factors

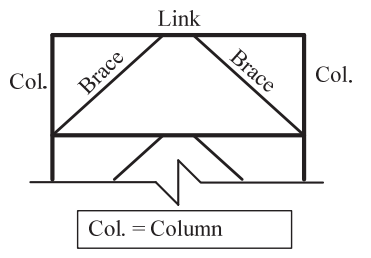

(c) Key

Figure 3-59. LDP Assessment Results, 8-Story EBF RSA, BSE-1 LS 


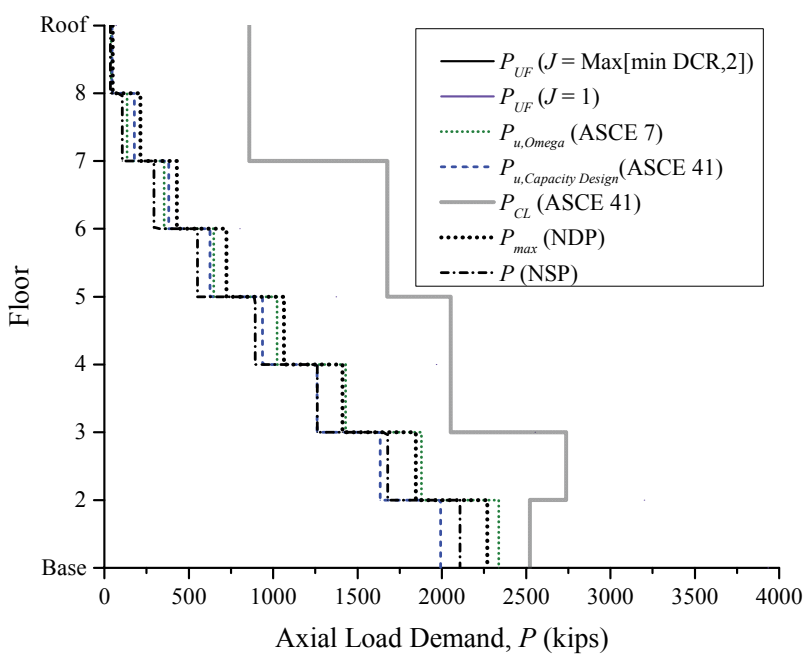

(a) ELF

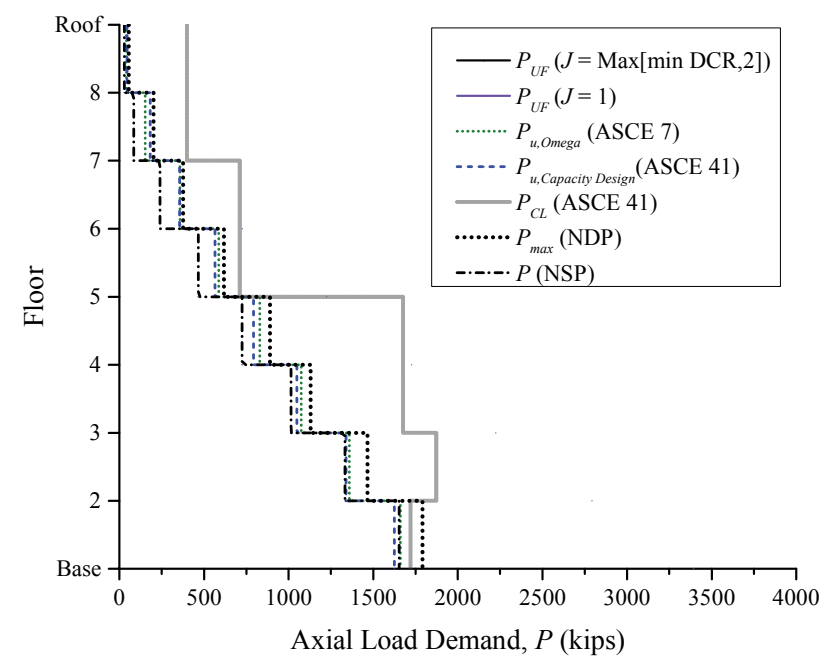

(b) RSA

Figure 3-60. LDP Assessment Results, Compression in Exterior Columns, 8-Story EBF, BSE-1

\subsection{BSE-2 Earthquake Hazard Level (CP BPL)}

In this section, the following apply:

- Figure 3-61 and Figure 3-62 provide the $D C R_{N}$ and load-dependent $m$-factor values for the ELF and RSA designs, respectively, for the LSP at the BSE-1 EHL. In these figures, $D C R_{N}$ values greater than unity are highlighted in red and underlined. $D C R$ values, as defined by ASCE 41, can be obtained by multiplying $D C R_{N}$ by $m$ and $\kappa$, see Eq. 3-6.

- Figure 3-63 provides the maximum axial compression demands, $P_{U F}$, in the exterior column lines for various analysis methods and the column capacity, $P_{C L}$.

All link beam and brace actions satisfy the CP BPL acceptance criteria. For all intents and purpose, all column actions also satisfy the CP BPL.

As discussed previously in $\S 3.2 .2, P_{U F}$ is determined by taking $J$ (ASCE $41 \S 3.4 .2 .1 .2-2$ ) as the minimum $D C R$ of the component(s) delivering force to the column, but not less than 2.0. This approach produces a lower-bound estimate for $P_{U F}$ for the LS SPL as compared to ASCE $41 \S 3.4 .2 .1 .2-1$ (based on ASCE 41 $\S 5.5 .3 .4 .2)$, as shown in Figure 3-63. Further, the $D C R_{N}$ interaction results for force-controlled components are the same between LDP LS and LSP CP because all links have yielded capping the force delivered to the columns based on the minimum DCR of all links—see $\$ 3.2 .2 .2 .1 .2$ for additional information. 


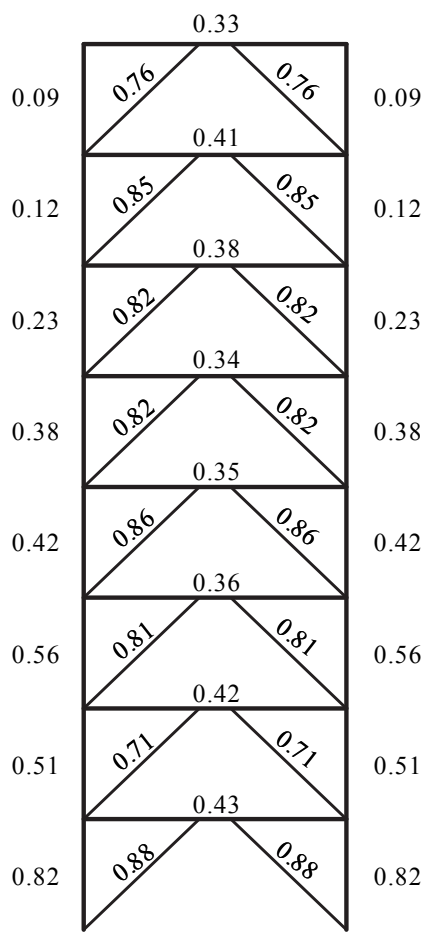

(a) $D C R_{N}$

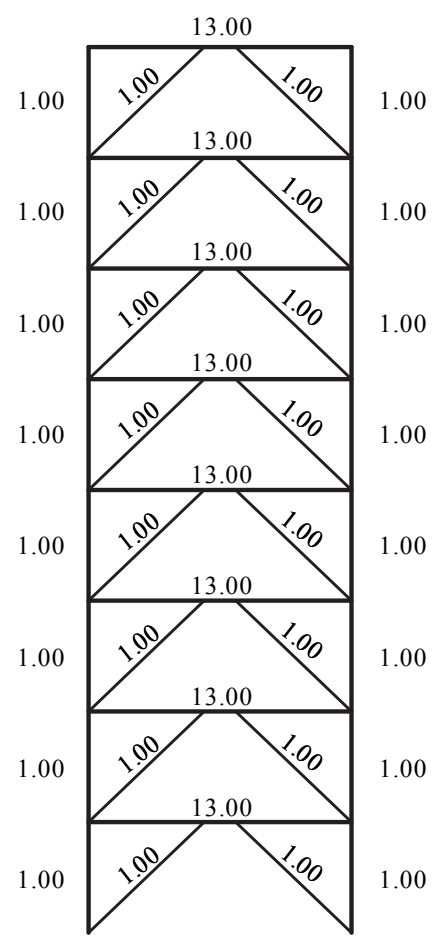

(b) $m$-factors

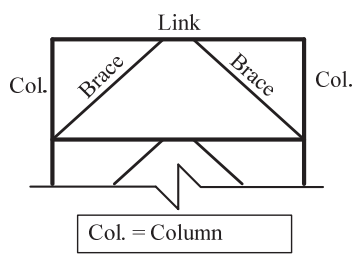

(c) Key

Figure 3-61. LDP Assessment Results, 8-Story EBF ELF, BSE-2 CP

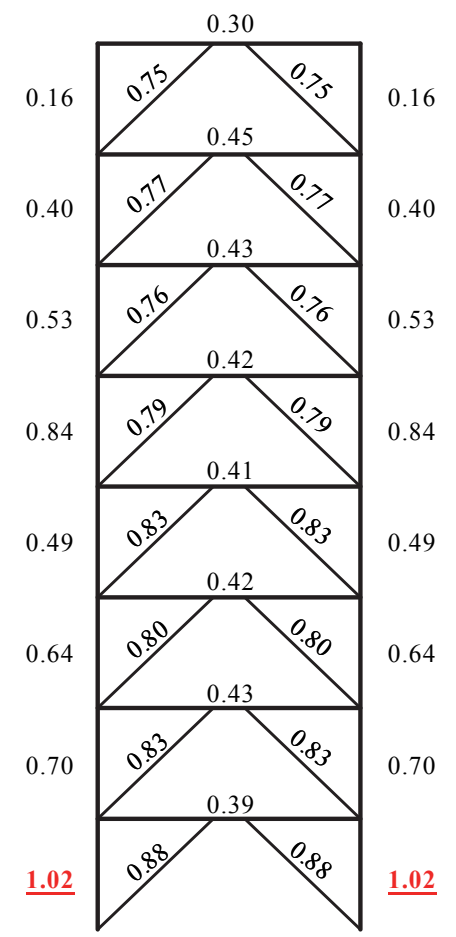

(a) $D C R_{N}$

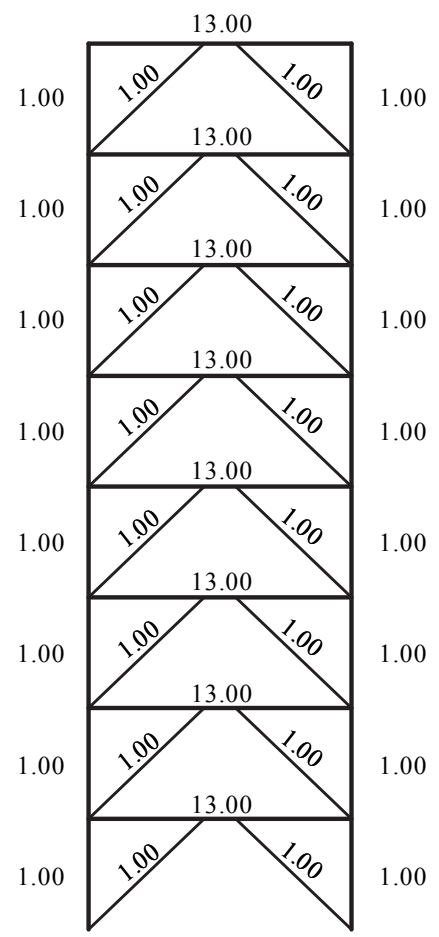

(b) $m$-factors

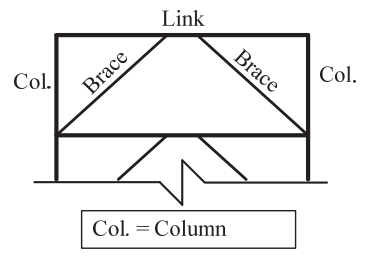

(c) Key

Figure 3-62. LDP Assessment Results, 8-Story EBF RSA, BSE-2 CP 


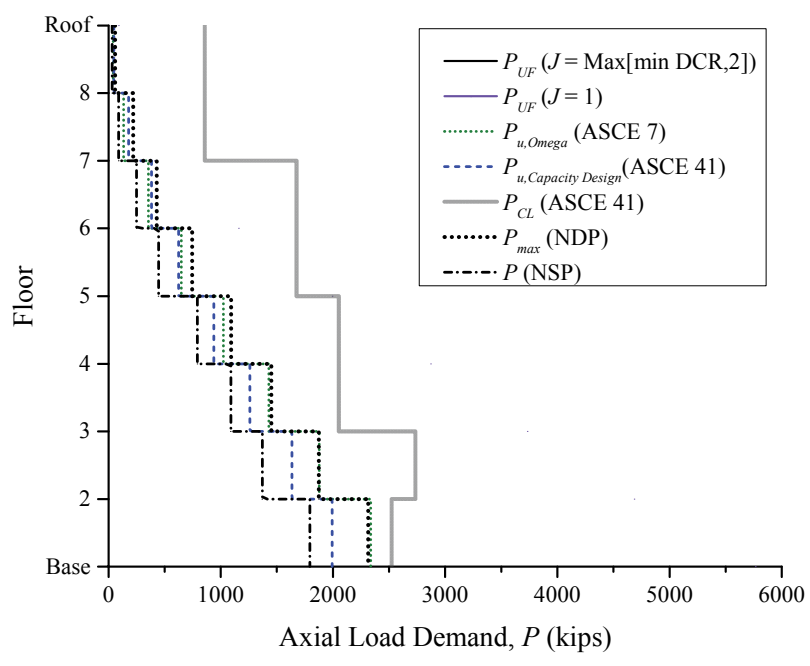

(a) ELF

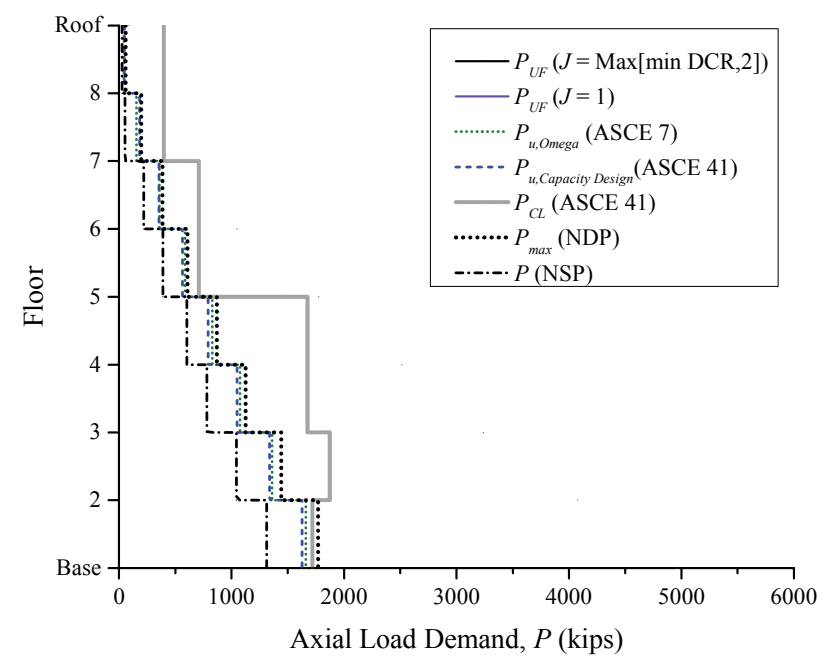

(b) RSA

Figure 3-63. LDP Assessment Results, Compression in Exterior Columns, 8-Story EBF, BSE-2

\subsection{Nonlinear Static Procedure}

In this section, the following apply:

- Table 3-14 through Table 3-16 provide the computed NSP analysis and assessment parameters in accordance with ASCE 41 §3.3.3.

- Figure 3-64 and Figure 3-65 illustrate the monotonic pushover curves for the ELF- and RSAdesigned frames, respectively, and the associated pushover parameters from ASCE 41 at the BSE2 EHL. Roof displacement is measured at the Center of Mass (CoM). A significant change in base shear is due to component strength loss of components (e.g., link beams), notated in the figures. First-order and second-order responses, shown in these figures, aids in computing a physically meaningful value for $\alpha_{P-\Delta}$ used in ASCE 41 Equation 3-17.

- Figure 3-66 and Figure 3-67 illustrate the story drift ratios in terms of the roof drift ratio.

As discussed in $\$ 3.1 .3 .2 .1$, the NSP is permitted, but requires supplemental verification using the LDPsee $\$ 3.2 .2 .2 .2$. In this case, the displacement at the maximum base shear governs $\Delta_{d}$ for both the ELF- and RSA-designed frame for both the BSE-1 and BSE-2 EHLs. Axial compression force in the exterior columns at the target displacement are shown previously in the linear assessment sections. Results indicate that the NSP generally results in a lower estimate of the axial force demands compared to the other methods used in this study. This is partly because the fundamental mode-based lateral force distribution does not adequately capture higher mode effects. Also, the target displacement at the roof computed based on fundamental mode properties may underestimate the story demands in the upper stories.

Table 3-14. NSP General Information, 8-Story EBF (kip, inch)

\begin{tabular}{|c|c|c|c|c|c|c|c|c|c|c|c|c|}
\hline Design & $T_{1}$ & $K_{1}$ & $\Delta_{y}$ & $V_{y}$ & $K_{e}$ & $T_{e}$ & $h$ & $\Delta_{\text {peak }}$ & $V_{\text {peak }}$ & $W$ & $C_{m}$ & $C_{0}$ \\
\hline ELF & 2.05 & 123.6 & 7.7 & 946 & 123.1 & 2.06 & 1.11 & 16.4 & 1068.2 & 10566 & 1.00 & 1.36 \\
\hline RSA & 2.34 & 92.1 & 7.7 & 706 & 91.9 & 2.34 & 1.13 & 15.0 & 787.7 & 10460 & 1.00 & 1.35 \\
\hline
\end{tabular}


Table 3-15. CP NSP Analysis Parameters, 8-Story EBF BSE-2 CP (kip, inch)

\begin{tabular}{cccccccccccccc}
\hline \multirow{2}{*}{ Design } & $S_{a}$ & $R$ & $C_{l}$ & $C_{2}$ & $\Delta_{t}$ & $V_{t}$ & $\Delta_{d}$ & $\alpha_{1}$ & $\alpha_{2}$ & $\alpha_{P-\Delta}$ & $\alpha_{e}$ & $R_{\max }$ & $R_{\max }$ \\
\hline ELF & 0.45 & 5.03 & 1.00 & 1.00 & 25.2 & 809.6 & 16.4 & 0.11 & -0.13 & -0.02 & -0.05 & 9.66 & OK \\
RSA & 0.39 & 5.80 & 1.00 & 1.00 & 28.2 & 501.5 & 15.0 & 0.12 & -0.11 & -0.02 & -0.04 & 12.66 & OK \\
\hline
\end{tabular}

Table 3-16. LS NSP Analysis Parameters, 8-Story EBF BSE-1 LS (kip, inch)

\begin{tabular}{cccccccccccccccc}
\hline \multirow{2}{*}{ Design } & \multirow{2}{*}{$S_{a}$} & $R$ & $C_{1}$ & $C_{2}$ & $\Delta_{t}$ & $V_{t}$ & $\Delta_{d}$ & $\alpha_{1}$ & $\alpha_{2}$ & $\alpha_{P-\Delta}$ & $\alpha_{e}$ & $R_{\max }$ & $\begin{array}{c}R \leq \\
R_{\max }\end{array}$ \\
\hline ELF & 0.30 & 3.35 & 1.00 & 1.00 & 16.8 & 1068.2 & 16.4 & 0.11 & -0.13 & -0.02 & -0.05 & 9.66 & OK & \\
RSA & 0.26 & 3.87 & 1.00 & 1.00 & 18.8 & 781.5 & 15.0 & 0.12 & -0.11 & -0.02 & -0.04 & 12.66 & OK \\
\hline
\end{tabular}

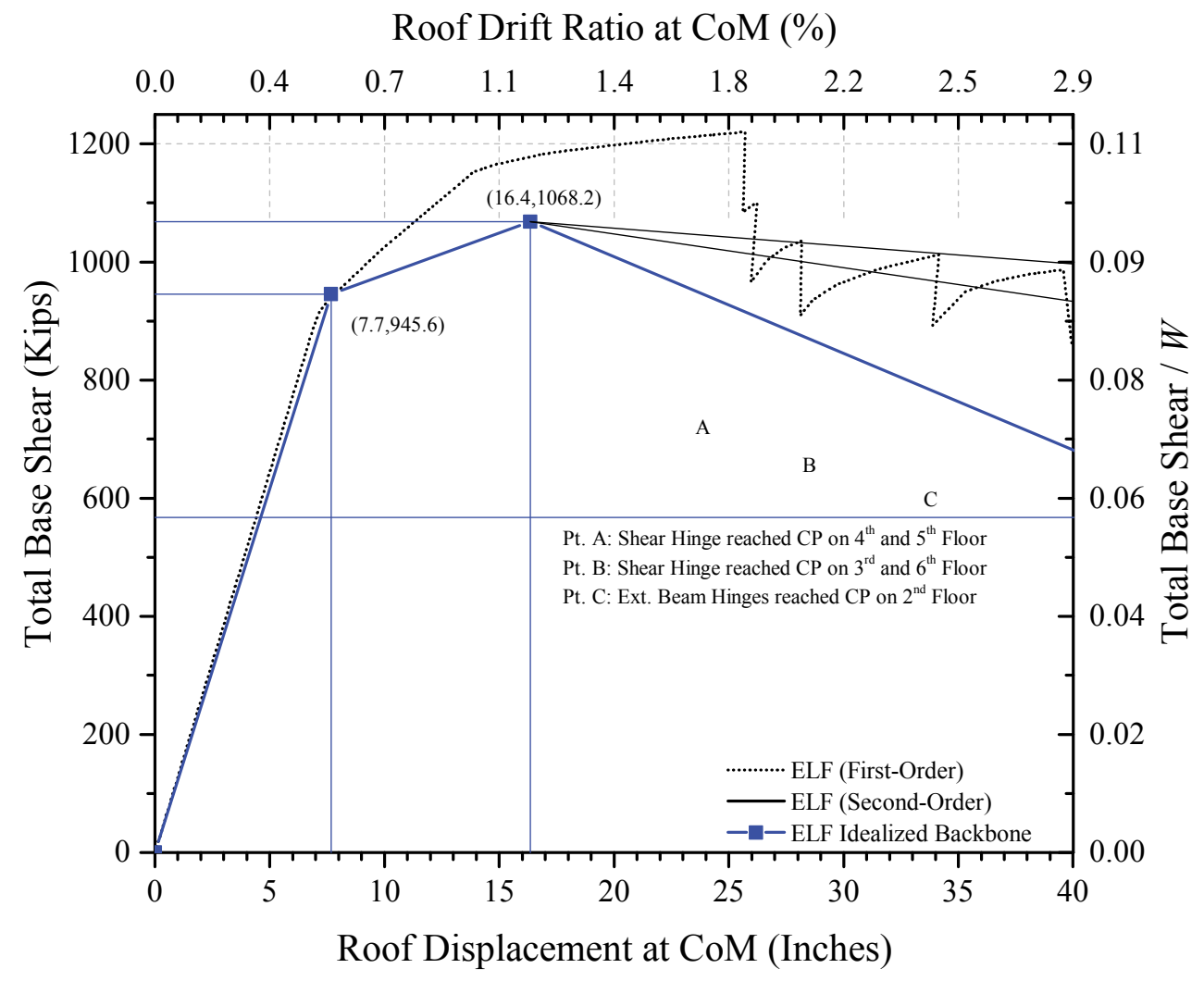

Figure 3-64. 8-Story EBF ELF Pushover, BSE-2 


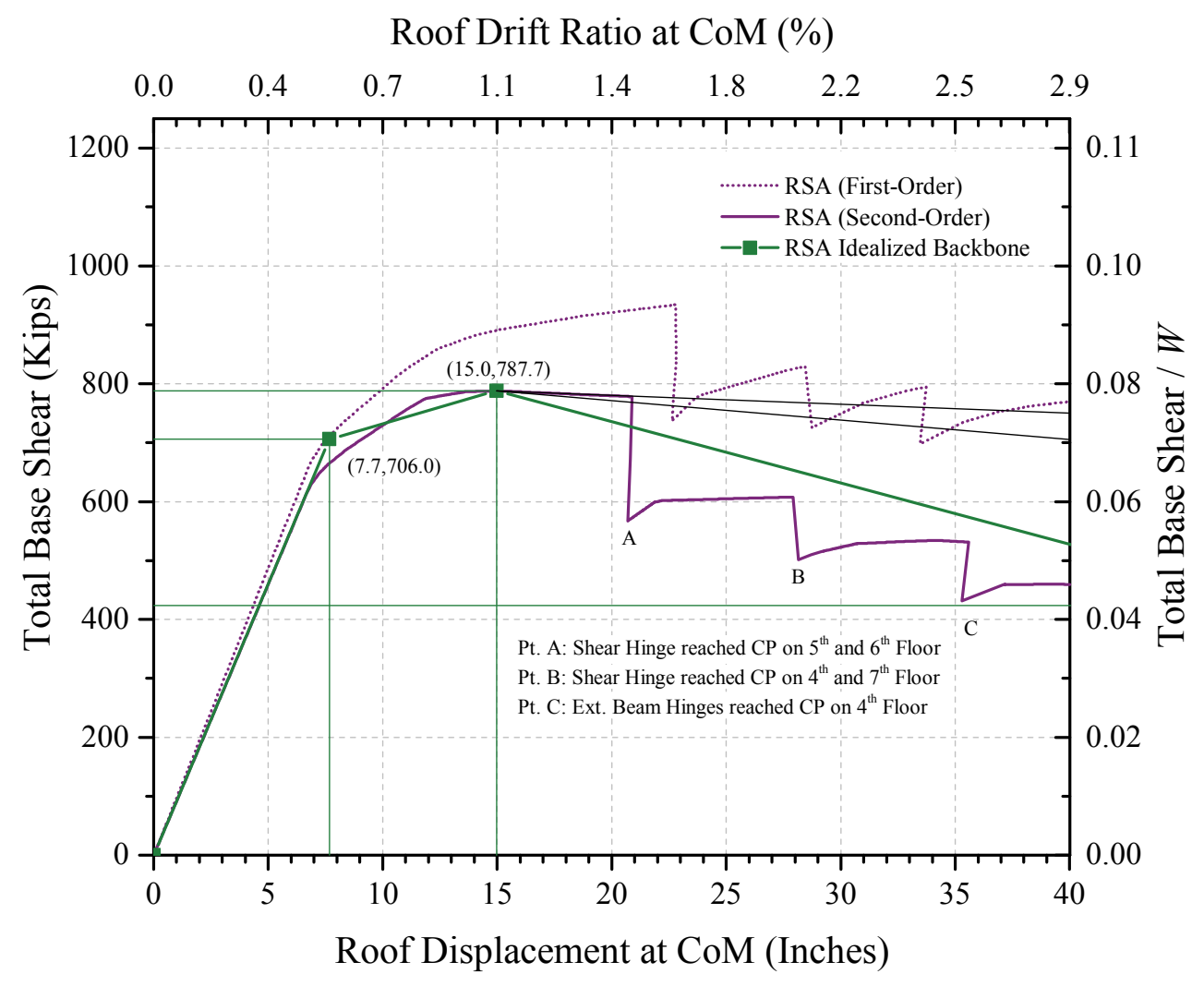

Figure 3-65. 8-Story RSA ELF Pushover, BSE-2

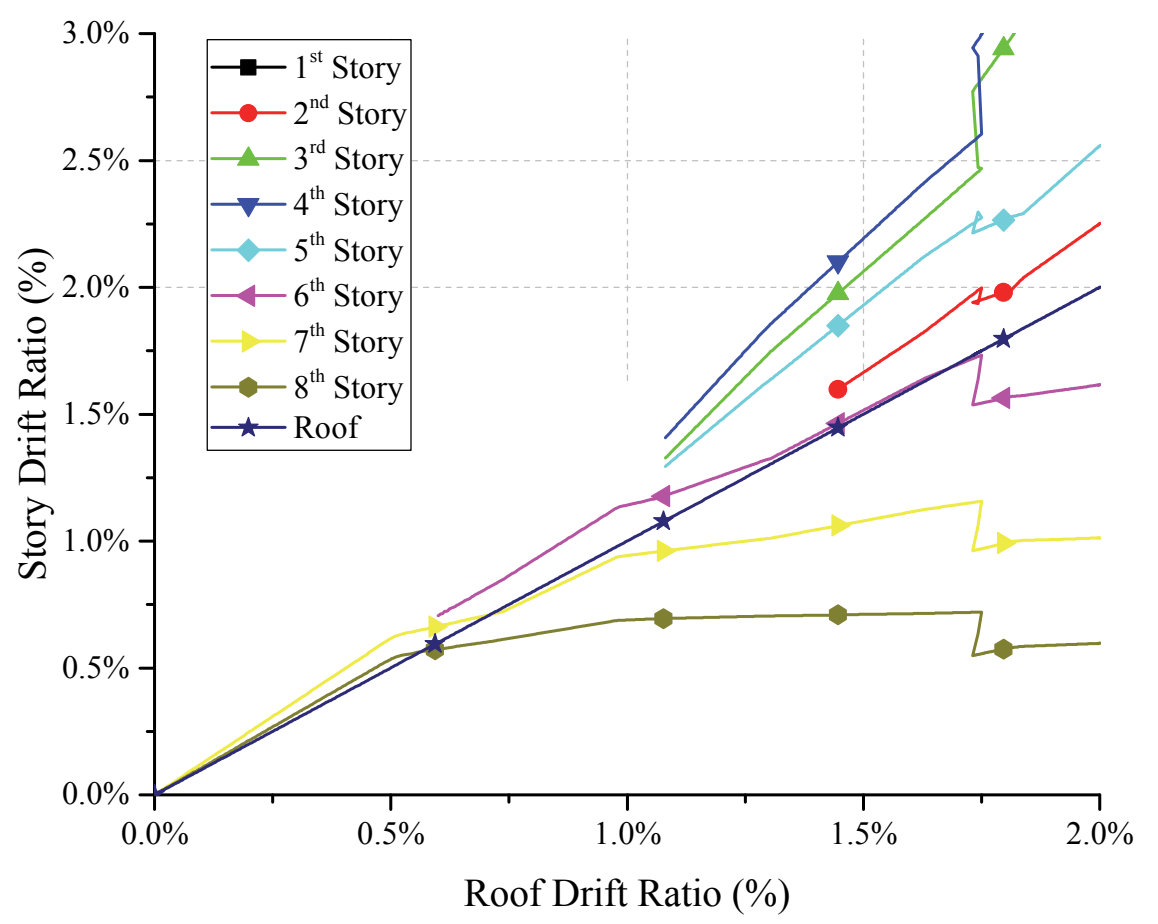

Figure 3-66. 8-Story EBF ELF Pushover - Story Drift Ratios - BSE-2 


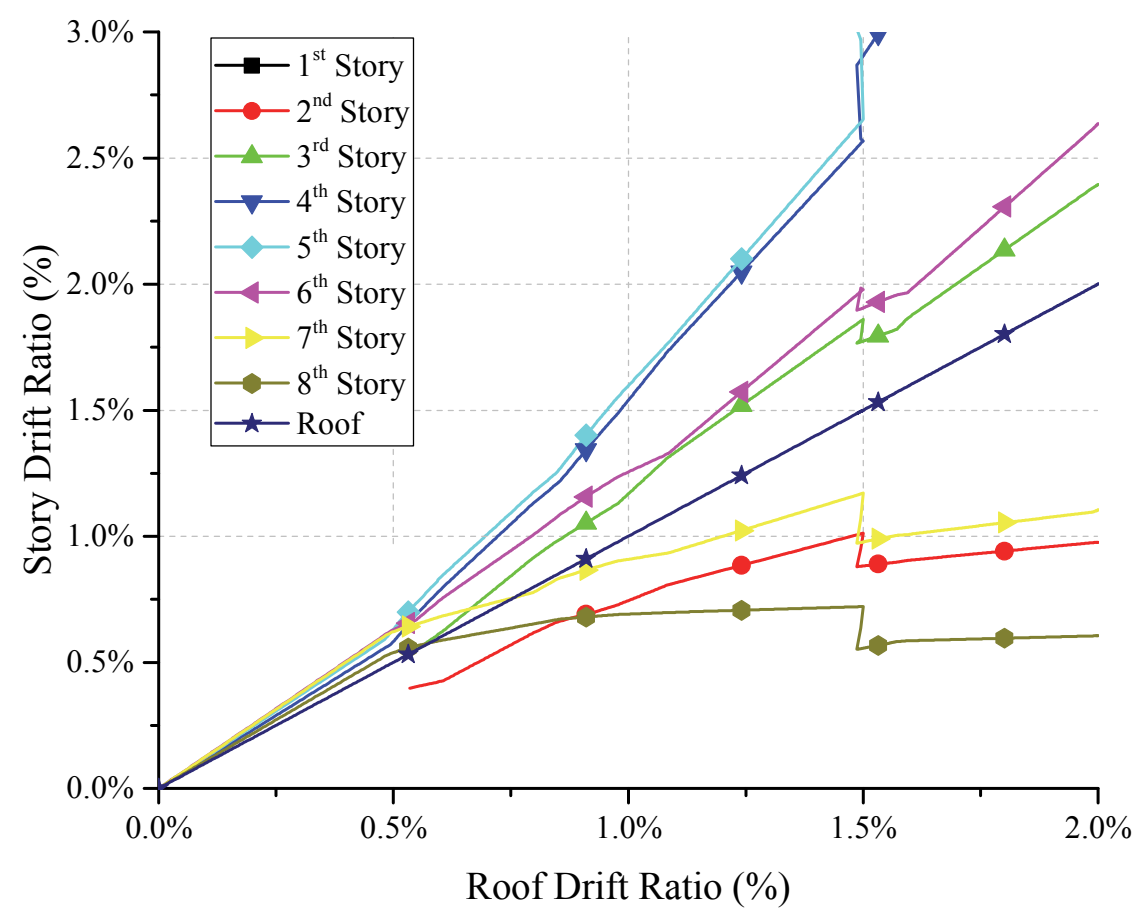

Figure 3-67. 8-Story EBF RSA Pushover - Story Drift Ratios - BSE-2

Figure 3-68 illustrates which frame components are force-controlled for flexure for both the NSP and NDP; red circles indicate anticipated plastic hinge locations that are force-controlled for flexure at the target displacement. Figure 3-69 and Figure 3-70 illustrate the $D C R_{N}$ values if greater than unity for the ELF- and RSA-designed frames at the target displacement for the LS BPL at the BSE-1 EHL and CP BPL at the BSE2 EHL. The figures illustrate demands when the system is loaded to the left. All shear actions satisfy the LS BPL acceptance criteria for both designs. However, several link beams near the mid-height of the frame do not satisfy the CP BPL acceptance criteria for both designs - RSA-designed frame performs markedly poorer than the ELF-designed frame.

All other force-controlled component actions satisfy the LS BPL acceptance criteria. However, base column hinges do not satisfy the criteria for the CP BPL for the ELF-designed frames. This effect is two-fold: a consequence of modeling rotationally restrained base connections, which more closely characterizes how the base connection is detailed in these buildings than assuming rotationally unrestrained connections and, to a lesser extent, the minor increase in $P_{E}$ because the shear strength, $V_{C E}$, of the link beams was increased by 15 percent for nonlinear analysis - see $\$ 3.2 .1 .2$. As discussed in $\$ 3.2 .1 .2$, the flexural hinge model for force-controlled components obeys ASCE 41 Equation 5-4 (yield surface for the section). As such, unsatisfactory performance is characterized by section yielding. 


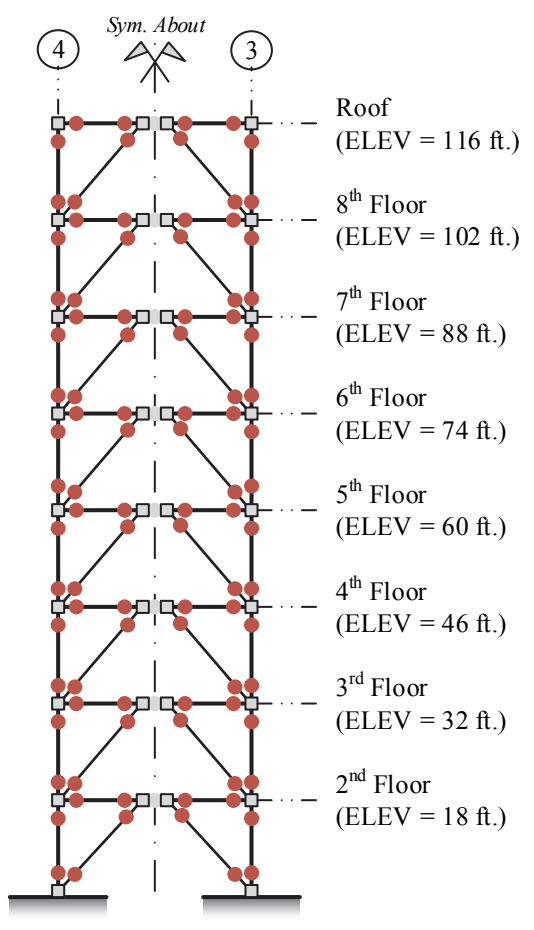

All columns, braces, and beams adjacent to link beams are force-controlled (FC) for flexure

Figure 3-68. Schematic of Flexural Actions in Components, 8-Story EBF (NSP and NDP)

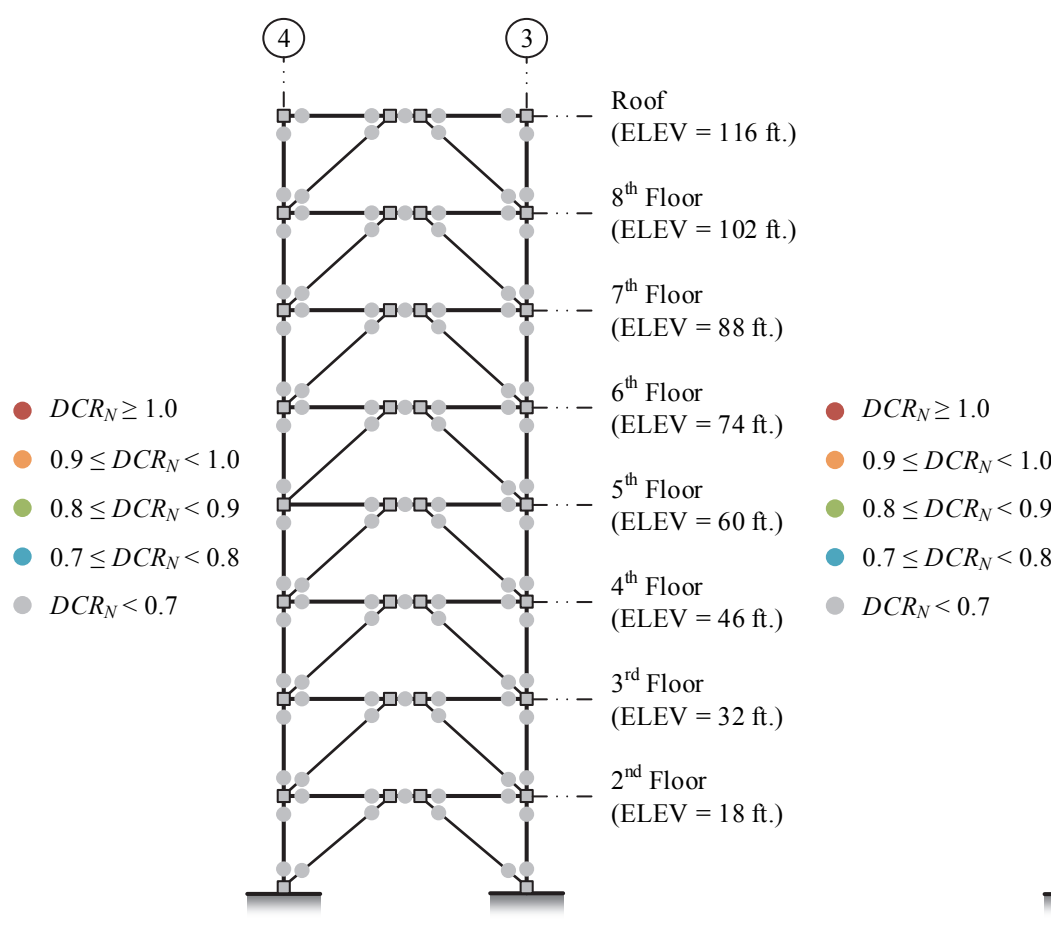

(a) ELF
(4)

(3)

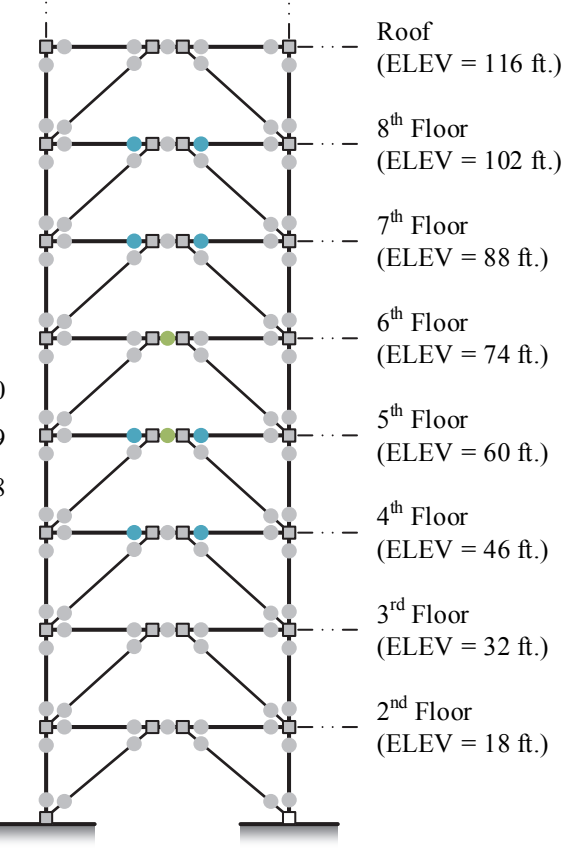

(b) RSA

Figure 3-69. NSP Assessment Results, 8-Story EBF, BSE-1 LS (+push to left) 


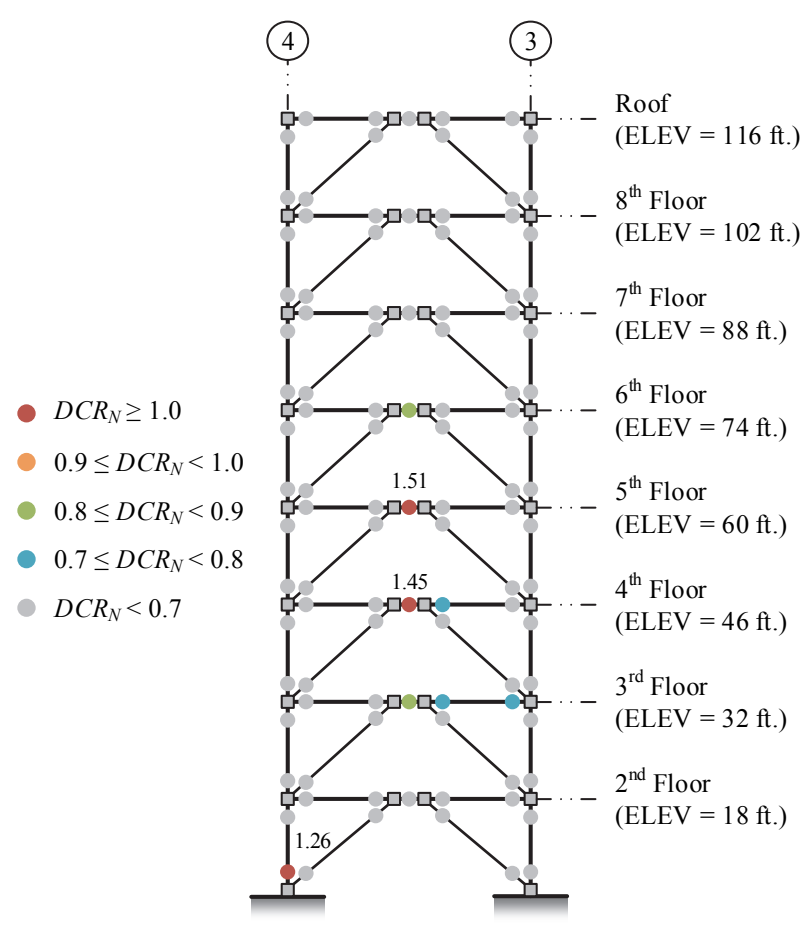

(a) ELF

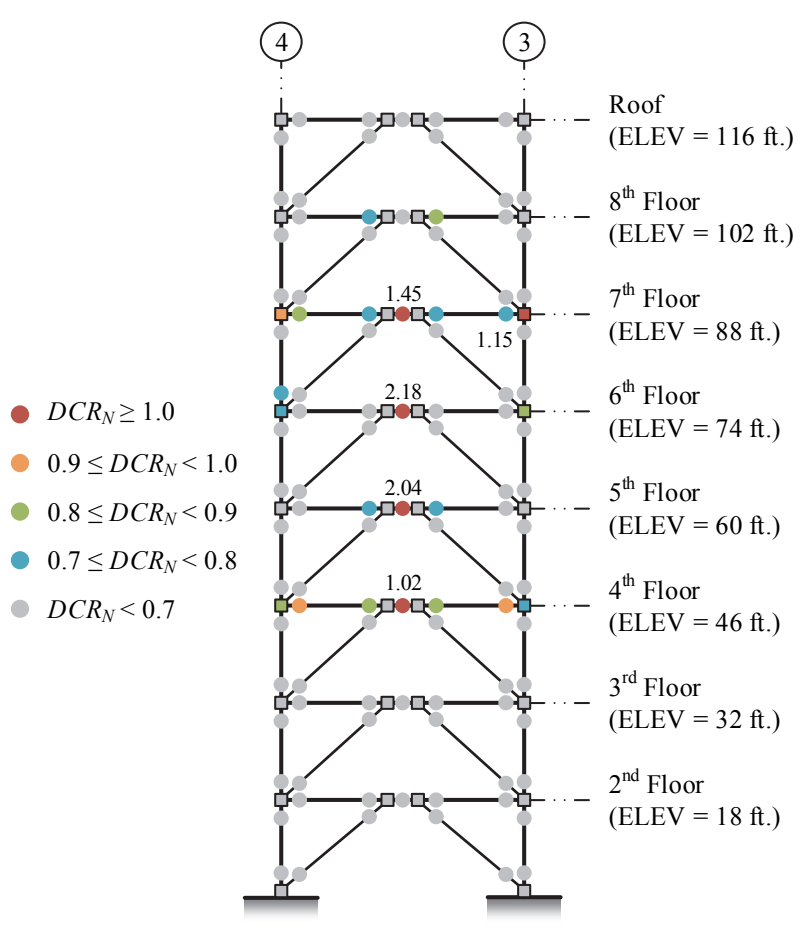

(b) RSA

Figure 3-70. NSP Assessment Results, 8-Story EBF, BSE-2 CP (+push to left)

\subsection{Nonlinear Dynamic Procedure}

The earthquake record set used to assess the N-S direction of ME8 is shown in Appendix A. For the ELF design, the analysis successfully completed for all 14 records at the BSE-1 EHL whereas four did not complete successfully at the BSE-2 EHL because of excessive lateral drifts. For the RSA design, the analysis successfully completed for all 14 records at the BSE-1 EHL whereas five did not complete successfully at the BSE-2 EHL because of excessive lateral drifts. Maximum axial compression force in the exterior column lines from the record set are shown previously in the linear assessment sections.

Figure 3-71 and Figure 3-72 show the performance of the link beams at the BSE-1 (LS BPL) and BSE-2 (CP BPL) for the ELF and RSA design, respectively, in terms of a $D C R_{N}$. The $D C R_{N}$ results from the LSP, LDP, and NSP (loaded to the left) are included in the figures. Comparison discussions of the various procedures are addressed subsequently. As is evident in the figures, the ELF-designed frame performs noticeably better than the RSA-designed frame. The results indicate that several links in the RSA-designed frame do not satisfy the LS BPL acceptance criteria. Both the ELF- and RSA-designed frames perform markedly poorer at the BSE-2 EHL, with nearly all links not satisfying the CP BPL acceptance criteria using both median and mean metric. A $D C R_{N}$ value of 0.73 equates to a plastic rotation angle of 0.08 radians at the BSE-1 EHL (0.62 at the BSE-2 EHL).

The average ratio of secondary to primary component acceptance criteria for a wide-flange section with the configuration in this frame is 1.14 for the CP SPL (1.27 for the LS SPL). The figures for the ELF- and RSA-designed frame illustrate that this value is exceeded in a number of stories at the BSE-1 EHL and 
BSE-2 EHL. This highlights the rapid analytical progression towards a collapsed state when several components are strained past the deformation associated with their peak strength—see §3.1.4.2.

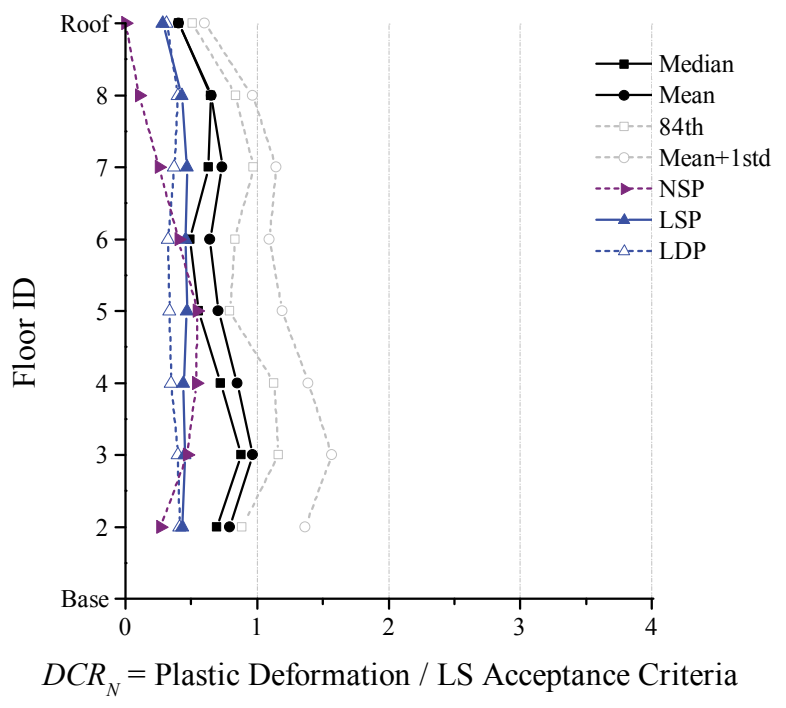

(a) ELF

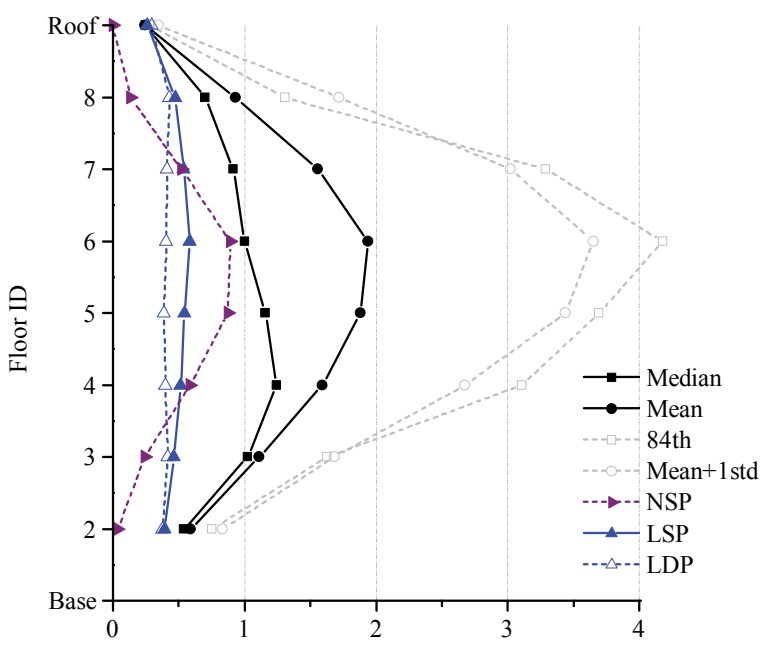

$D C R_{N}=$ Plastic Deformation / LS Acceptance Criteria

(b) RSA

Figure 3-71. NDP Assessment Results, Shear Hinges, 8-Story EBF, BSE-1 LS

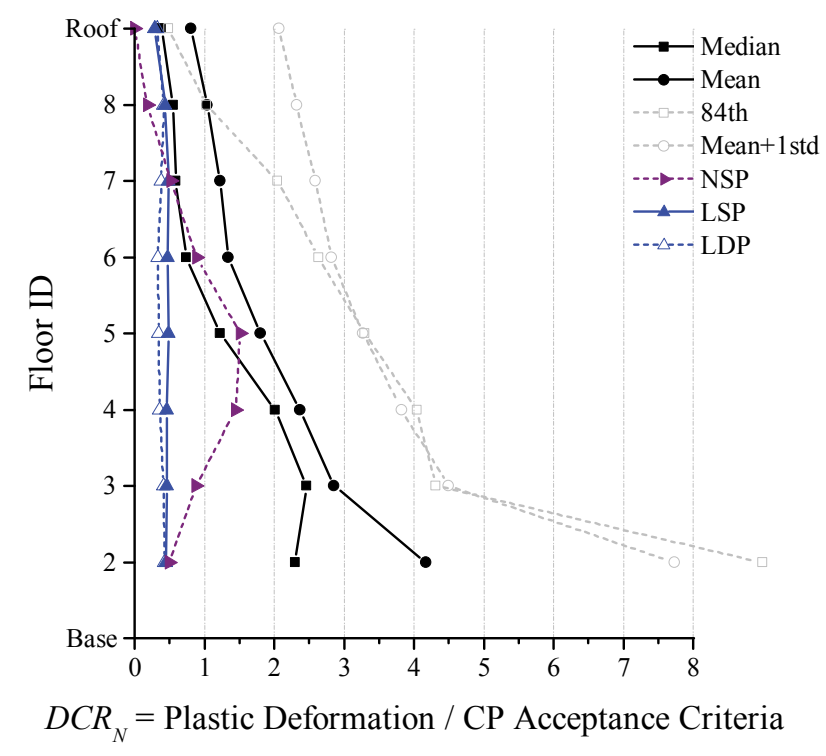

(a) ELF

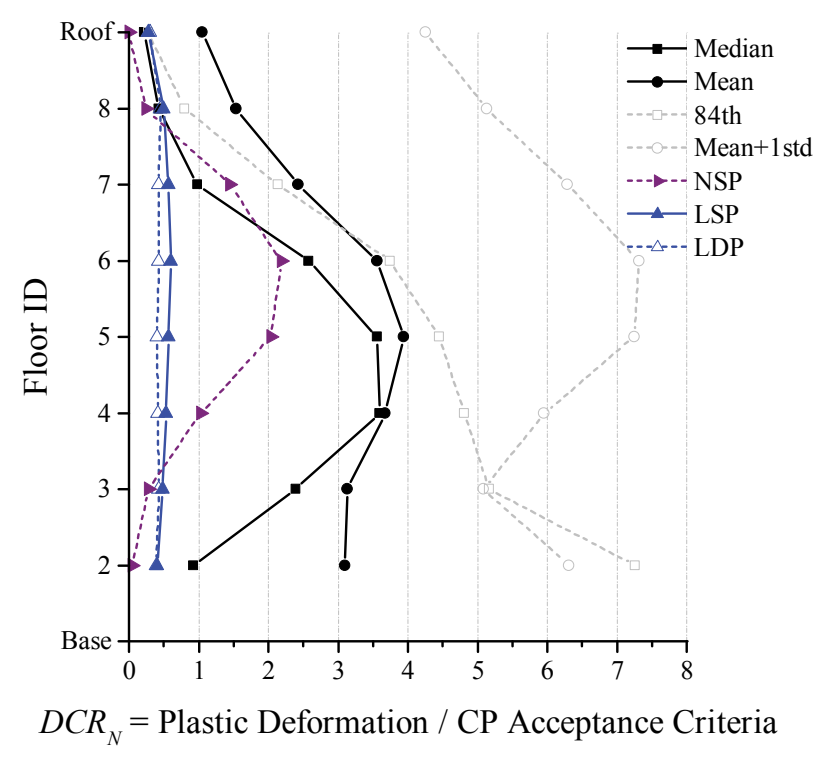

(b) RSA

Figure 3-72. NDP Assessment Results, Shear Hinges, 8-Story EBF, BSE-2 CP

Figure 3-73 and Figure 3-74 show the curvature ductility demand of the column hinges (i.e., section strength) at the BSE-2 EHL— see discussion on column hinge modeling in \$3.2.1.2. Figure 3-75 and Figure 
3-76 show the elastic member strength interaction results at the BSE-2 EHL - see discussion on column strength modeling in $\$ 3.2 .1 .2$. Column hinges at the base above the brace connection experience inelastic strain demands (yield corresponds to a $D C R_{N}=1$ in the figures). The deformation demands are considerably lower than the primary $\mathrm{CP}$ acceptance criteria for a Column. Still, the base column hinges do not satisfy the criteria for the CP BPL for both the ELF- and RSA-designed frames. This effect is two-fold: a consequence of modeling rotationally restrained base connections, which more closely characterizes how the base connection is detailed in these buildings than assuming rotationally unrestrained connections and, to a lesser extent, the minor increase in $P_{E}$ because the shear strength, $V_{C E}$, of the link beams was increased by 15 percent for nonlinear analysis—-see $\$ 3.2 .1 .2$ and $\S 3.2 .2 .1 .3$.

The $D C R_{N}$ results for the LSP and LDP are based on an interaction equation and not from $M_{U D} / m \times M_{C E}$, or $M_{U F} / M_{C L}$, which would be a more physically consistent metric for comparison against the results from the nonlinear assessment procedures. Nonetheless, the linear results are generally applicable here because the exterior frame columns are force-controlled for flexure in the linear assessment procedures. Though there is a fundamental difference in how the $D C R_{N}$ is computed for the linear and nonlinear procedures, the linear assessment results show similar distributions of demands and location of potential performance concerns.

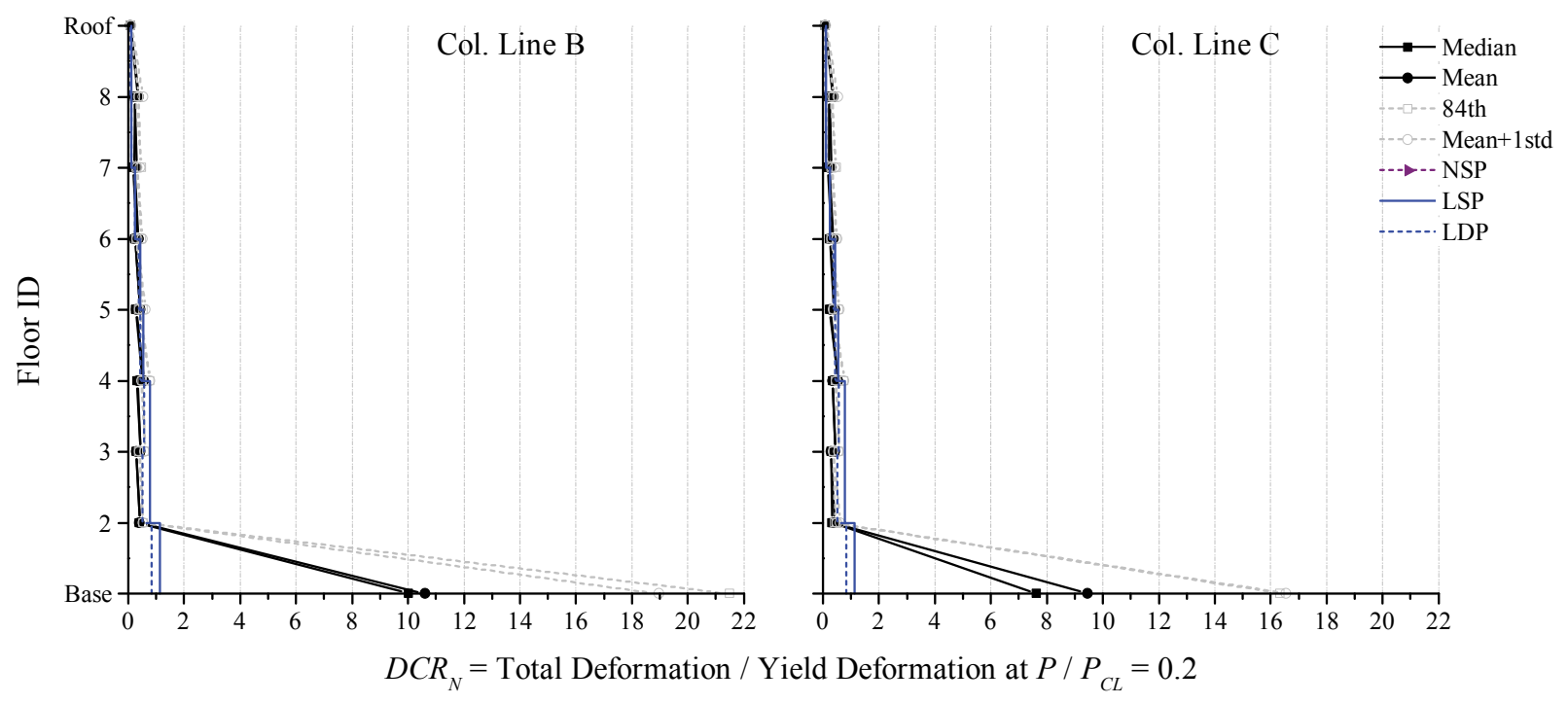

Figure 3-73. NDP Assessment Results, Column Hinges, 8-Story EBF ELF, BSE-2 Yield 


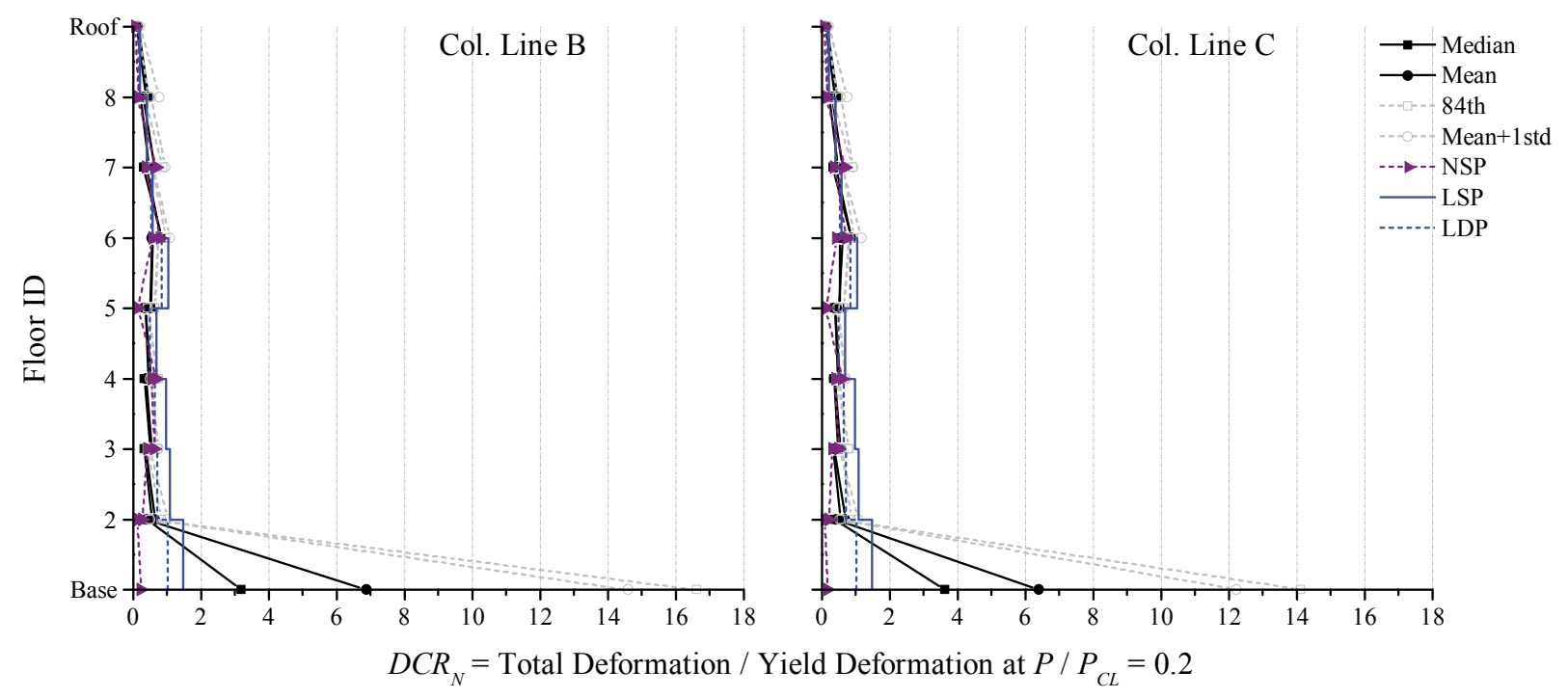

Figure 3-74. NDP Assessment Results, Column Hinges, 8-Story EBF RSA, BSE-2 Yield

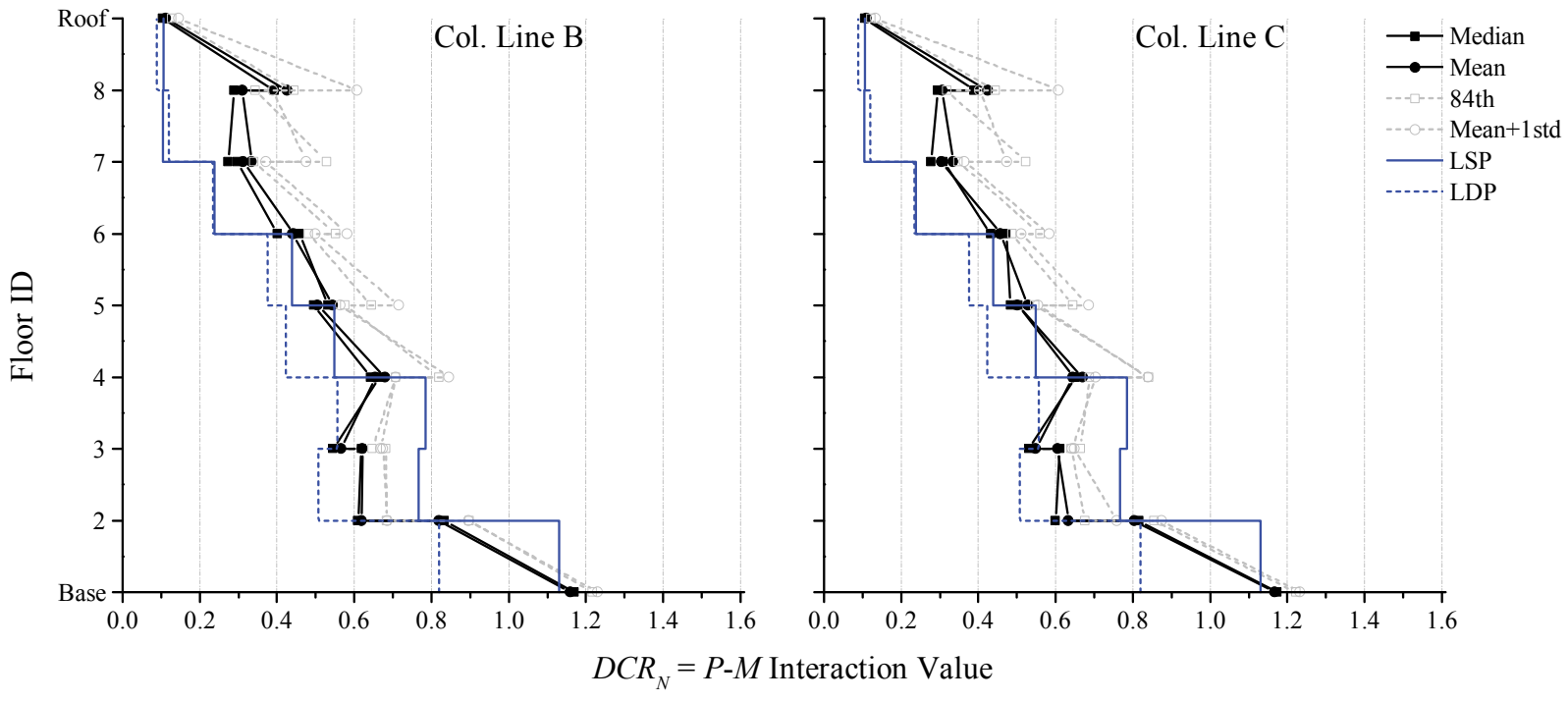

Figure 3-75. NDP Assessment Results, Column Members, 8-Story EBF ELF, BSE-2 


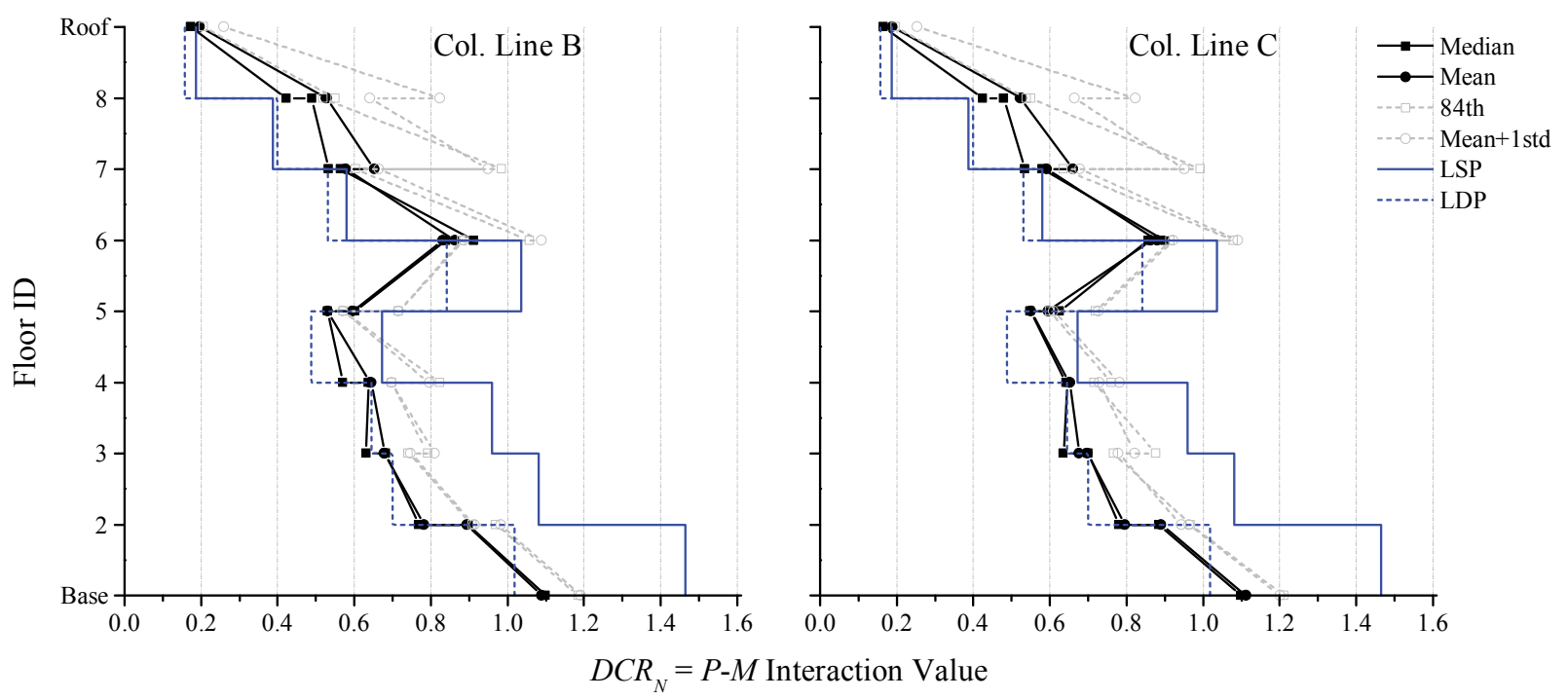

Figure 3-76. NDP Assessment Results, Column Members, 8-Story EBF RSA, BSE-2

Figure 3-77 and Figure 3-78 show the curvature ductility demand of the brace hinges (i.e., section strength) at the BSE-2 EHL-see discussion on column hinge modeling in $\$ 3.2 .1 .2$. Figure 3-79 and Figure 3-80 show the elastic member strength interaction results at the BSE-2 EHL - see discussion on column strength modeling in - see discussion on column strength modeling in \$3.2.1.2. Brace hinges at the base above the brace connection experience inelastic strain demands (yield corresponds to a $D C R_{N}=1$ in the figures). The deformation demands are considerably lower than the primary CP acceptance criteria for a Column. Still, the brace hinges do not satisfy the criteria for the CP BPL for both the ELF- and RSA-designed framessee previous discussion on column results for additional information.

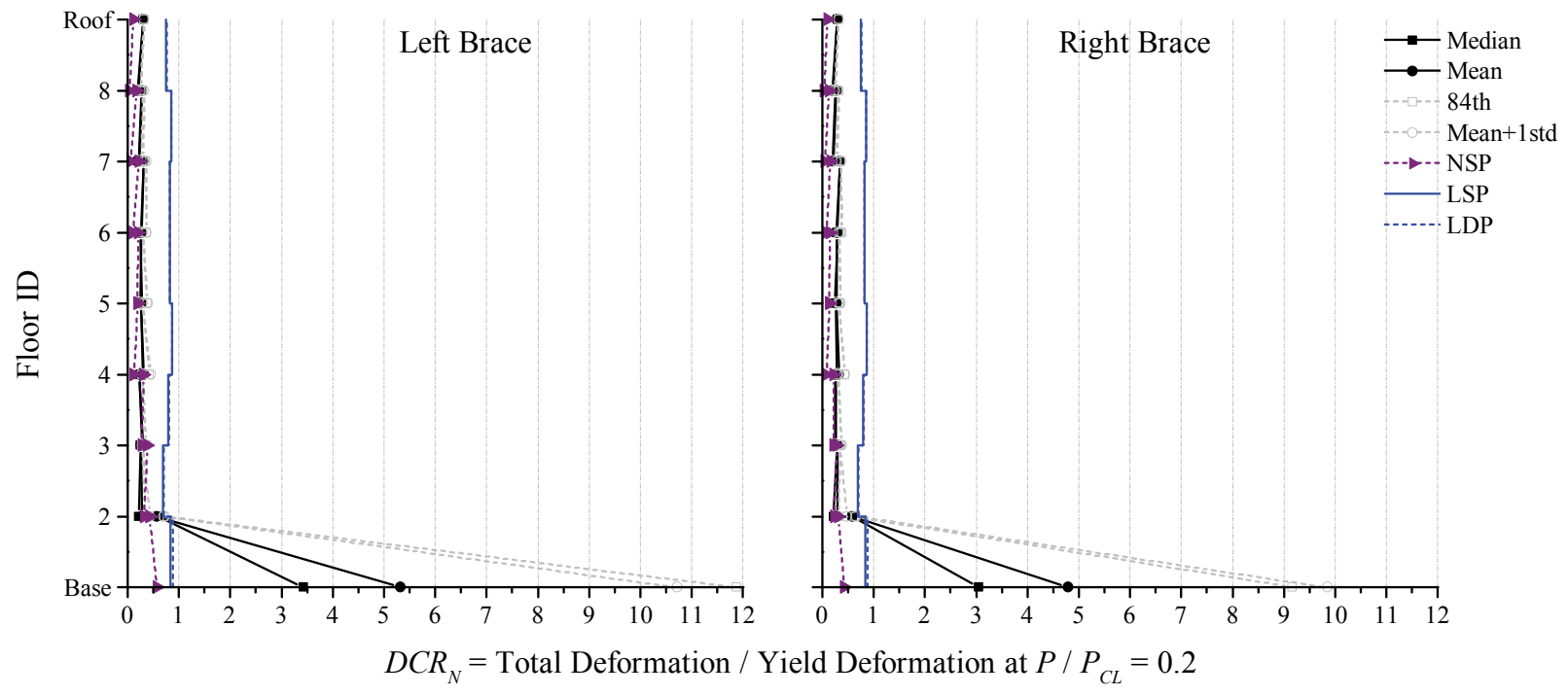

Figure 3-77. NDP Assessment Results, Brace Hinges, 8-Story EBF ELF, BSE-2 Yield 


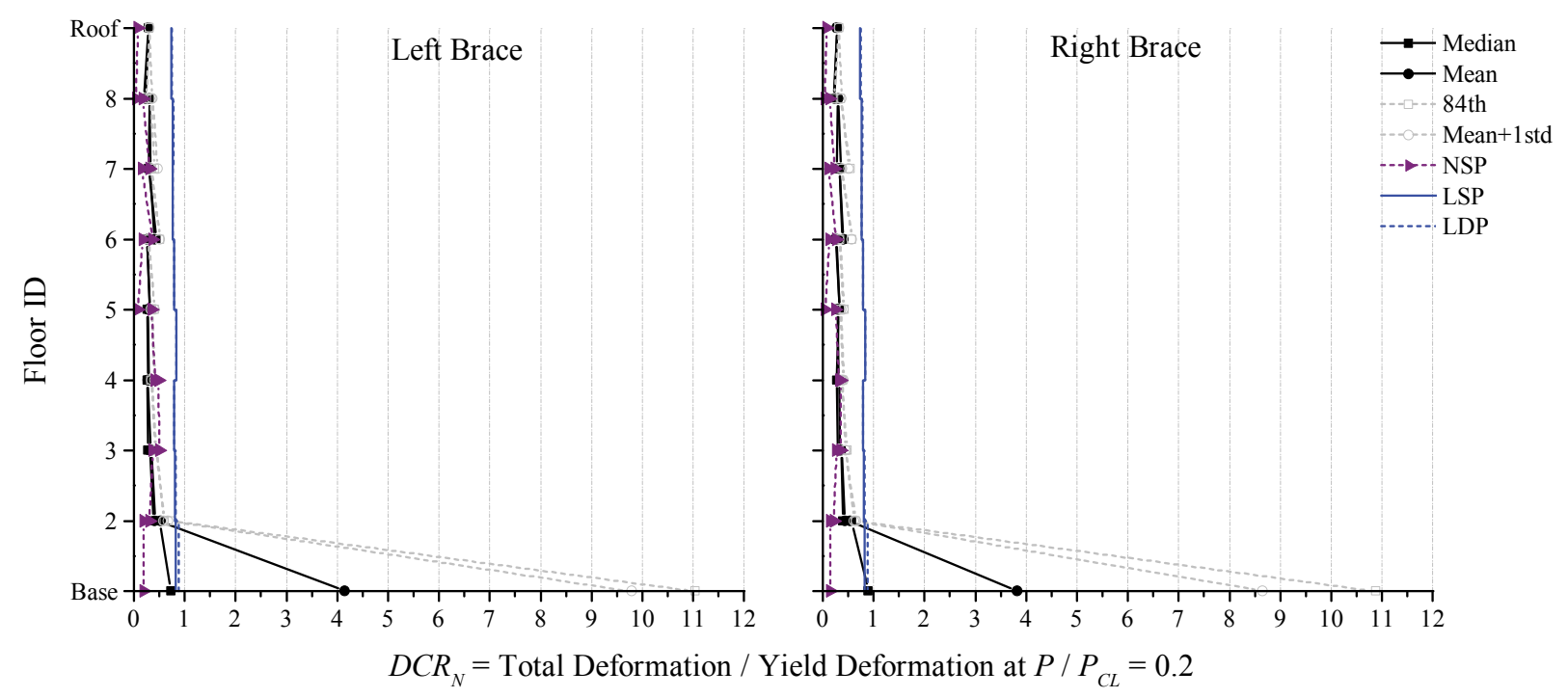

Figure 3-78. NDP Assessment Results, Brace Hinges, 8-Story EBF RSA, BSE-2 Yield

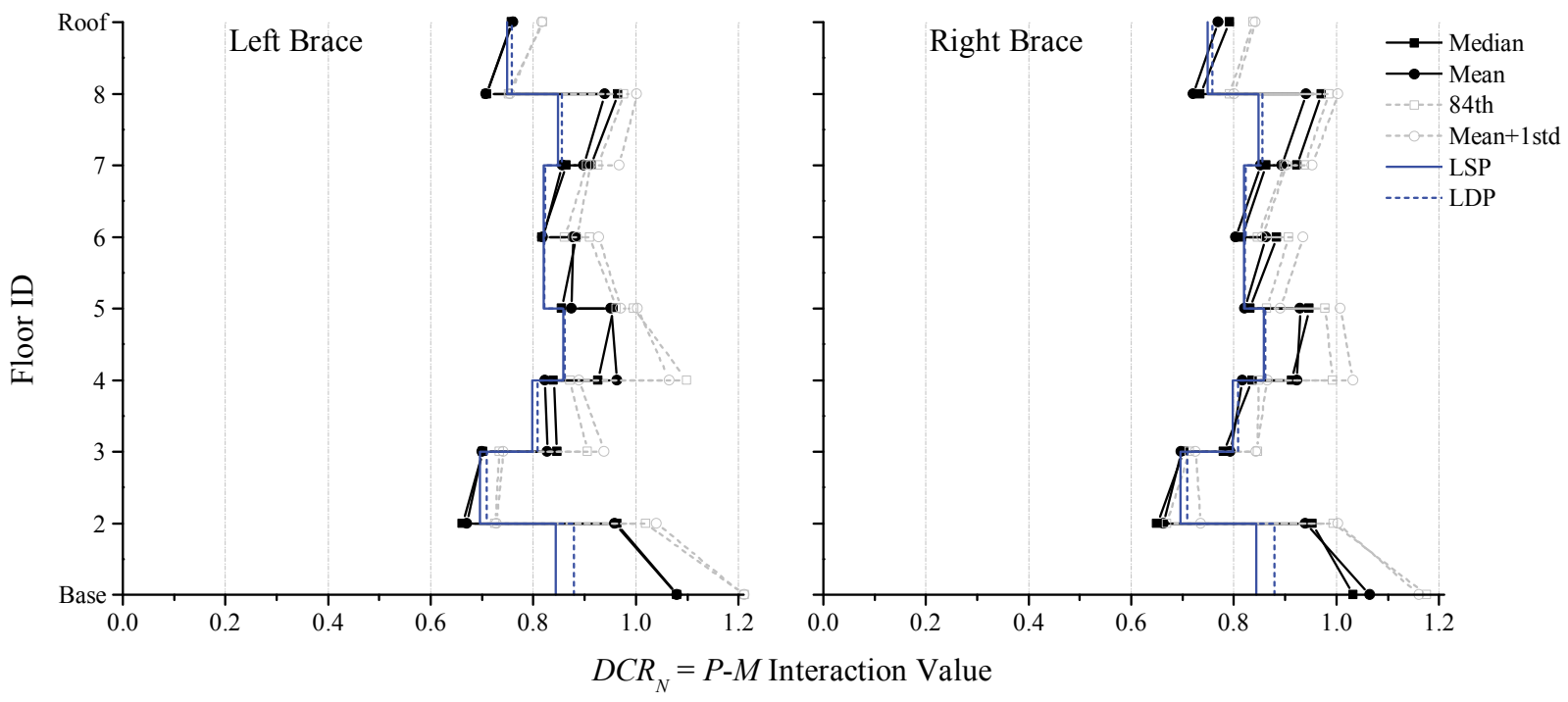

Figure 3-79. NDP Assessment Results, Brace Members, 8-Story EBF ELF, BSE-2 


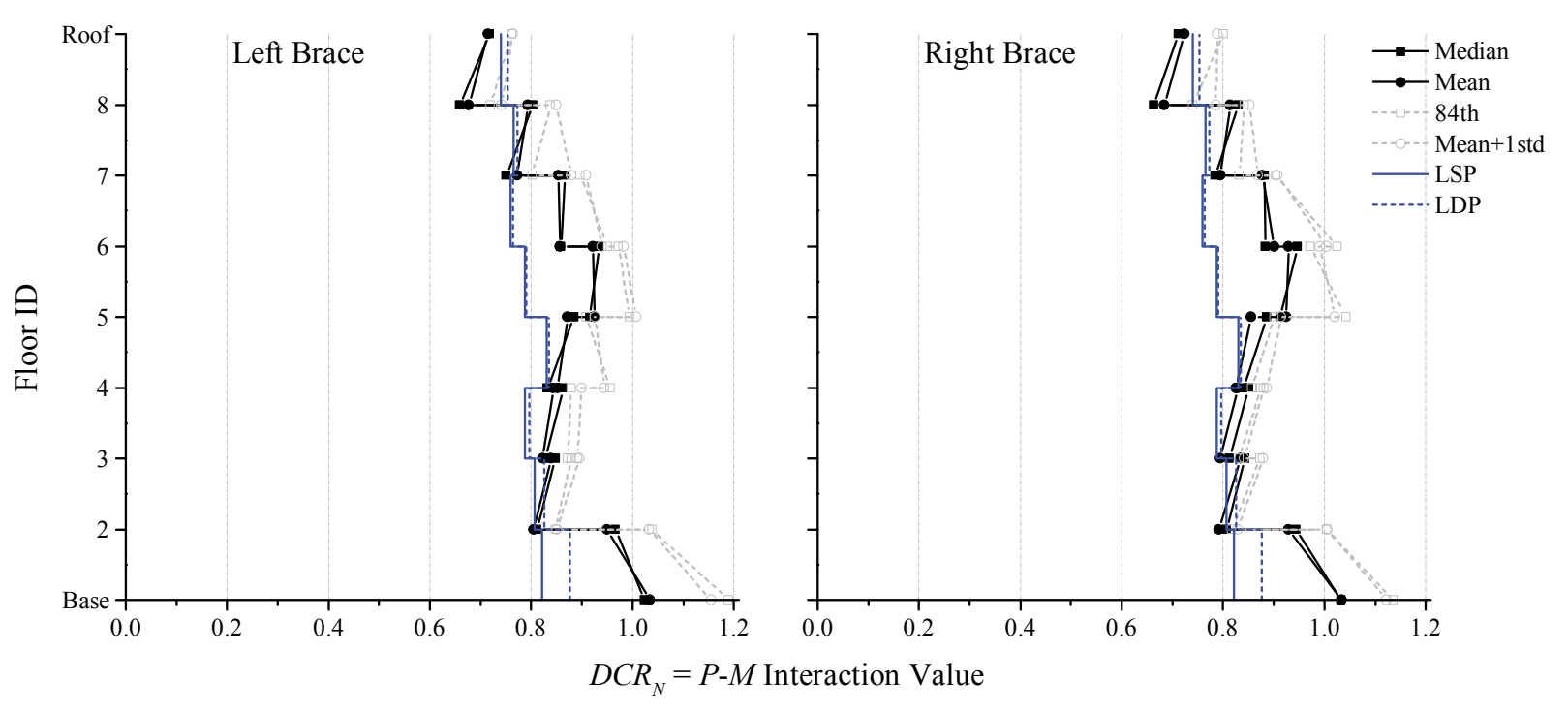

Figure 3-80. NDP Assessment Results, Brace Members, 8-Story EBF RSA, BSE-2

\subsubsection{Sixteen-Story Eccentrically Braced Frame}

\subsection{Linear Static Procedure}

\subsection{BSE-1 Earthquake Hazard Level (LS BPL)}

In this section, the following apply:

- Figure 3-81 and Figure 3-82 provide the $D C R_{N}$ and load-dependent $m$-factor values for the ELF and RSA designs, respectively, for the LSP at the BSE-1 EHL. In these figures, $D C R_{N}$ values greater than unity are highlighted in red and underlined. $D C R$ values, as defined by ASCE 41, can be obtained by multiplying $D C R_{N}$ by $m$ and $\kappa$, see Eq. 3-6.

- Figure 3-83 provides the maximum axial compression demands, $P_{U F}$, in the exterior column lines for various analysis methods and the column capacity, $P_{C L}$.

All link beam and brace actions satisfy the LS BPL acceptance criteria.

Base columns in the RSA-designed frame do not satisfy the LS BPL acceptance criteria (force-controlled) using the interaction equation because of the combination of high axial force and moment. High flexural demand at the base of the frame is a consequence of modeling rotationally restrained base connections, which more closely characterizes how the base connection is detailed in these buildings than assuming rotationally unrestrained connections. As discussed previously in $\$ 3.2 .2, P_{U F}$ is determined by taking $J$ (ASCE 41 \$3.4.2.1.2-2) as the minimum DCR of the component(s) delivering force to the column, but not less than 2.0. This approach produces an upper-bound estimate for $P_{U F}$ for the LS SPL as compared to ASCE 41 §3.4.2.1.2-1 (based on ASCE 41 §5.5.3.4.2), as shown in Figure 3-83. 
Similar to the discussion in $\$ 3.2 .2 .2 .1 .1$, one could initially argue that columns in an EBF frame (greater than three stories) designed per AISC 341 would satisfy ASCE 41 criteria by default. Following ASCE 41 \$5.5.3.4.2 results in the following for the base column (RSA-designed frame is used here):

$$
\begin{aligned}
\circ & P_{C L}=5215 \mathrm{kips} \\
\circ & P_{\text {Gravity }}=655 \mathrm{kips} \\
\circ & P_{U F}=5399 \mathrm{kips}(\text { determined using } J \text { equal to } 2.0) \\
\text { - } & P_{U F} / P_{C L}=1.03\left(M_{U F} / M_{C L}=0.11\right) \rightarrow \text { interaction result }=1.13 \text { (fails) } \\
\circ & P_{U F}=4742 \mathrm{kips}(\text { determined from a fully yielded frame per ASCE } 41 \S 5.5 .3 .4 .2) \\
& \text { - } \left.\quad P_{U F} / P_{C L}=0.91\left(M_{U F} / M_{C L}=0.11\right) \rightarrow \text { interaction result }=1.01 \text { (fails }-?\right) \\
\circ & P_{U F}=4269 \mathrm{kips}(\text { determined from a fully yielded frame per AISC } 341 \S \mathrm{F} 3.3) \\
\text { - } & P_{U F} / P_{C L}=0.82\left(M_{U F} / M_{C L}=0\right) \rightarrow \text { interaction result }=0.82 \text { (passes) }
\end{aligned}
$$

Adopting a full frame plastic mechanism to represent the peak dynamic demands in the columns can penalize force-controlled columns in an EBF. As an alternative, ASCE 41 could allow EBF columns to be deformation-controlled similar to a CBF, ASCE 41 §5.5.2, and follow AISC 341 provisions to reduce the axial load demands by 12 percent. The maximum axial load demand from the NDP is $4528 \mathrm{kips}$, a 5 percent reduction, including the effect of increasing $V_{C E}$ for nonlinear analysis—see §3.2.1.2 and \$3.2.2.1.3. 


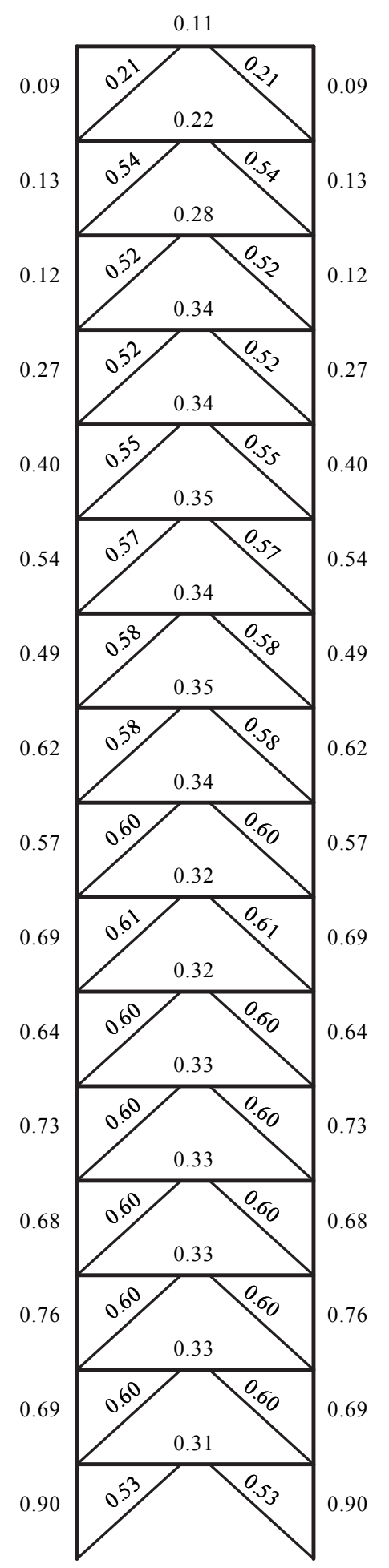

(a) $D C R_{N}$
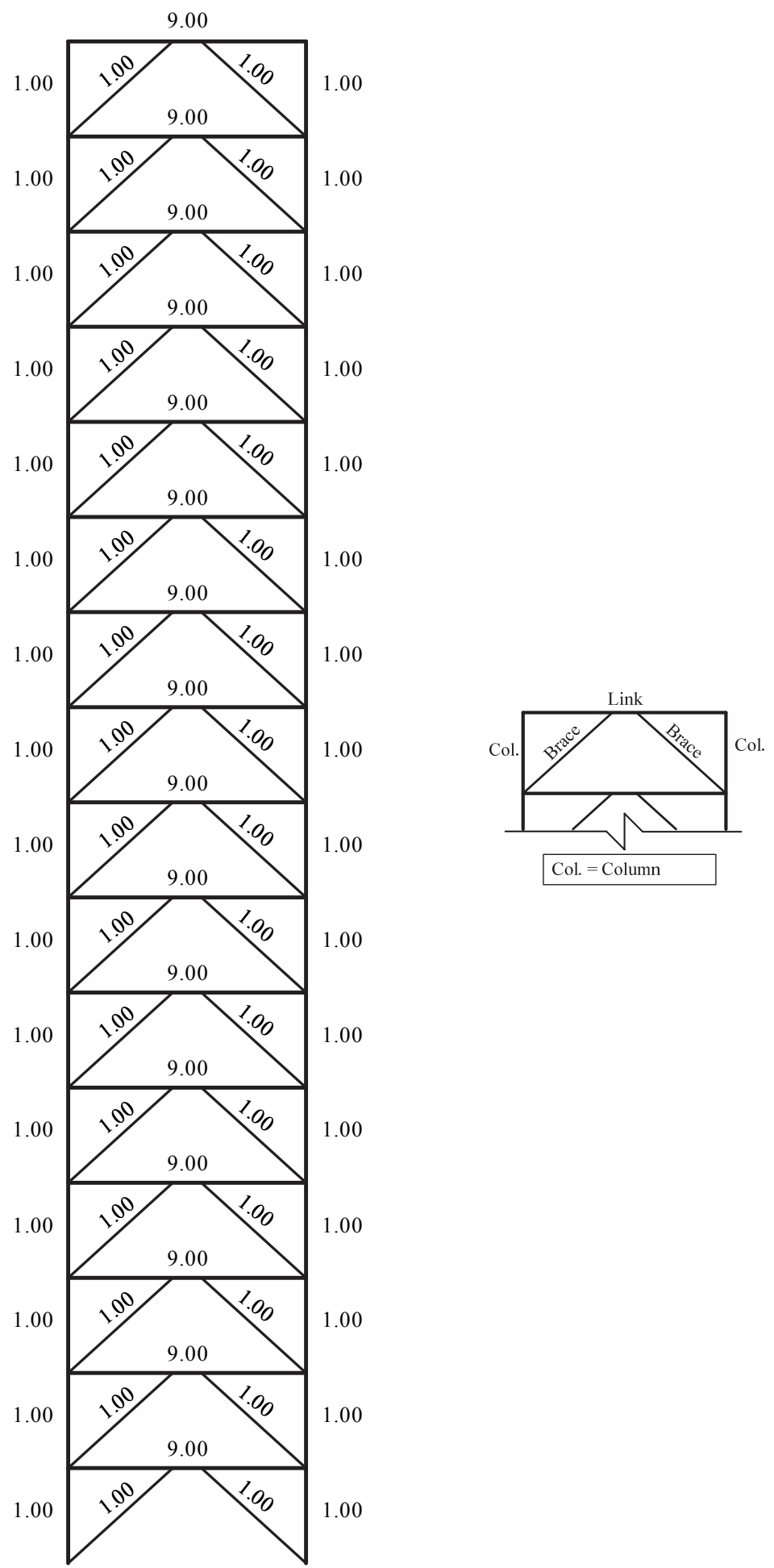

(b) $m$-factors (c) Key

Figure 3-81. LSP Assessment Results, 16-Story EBF ELF, BSE-1 LS 


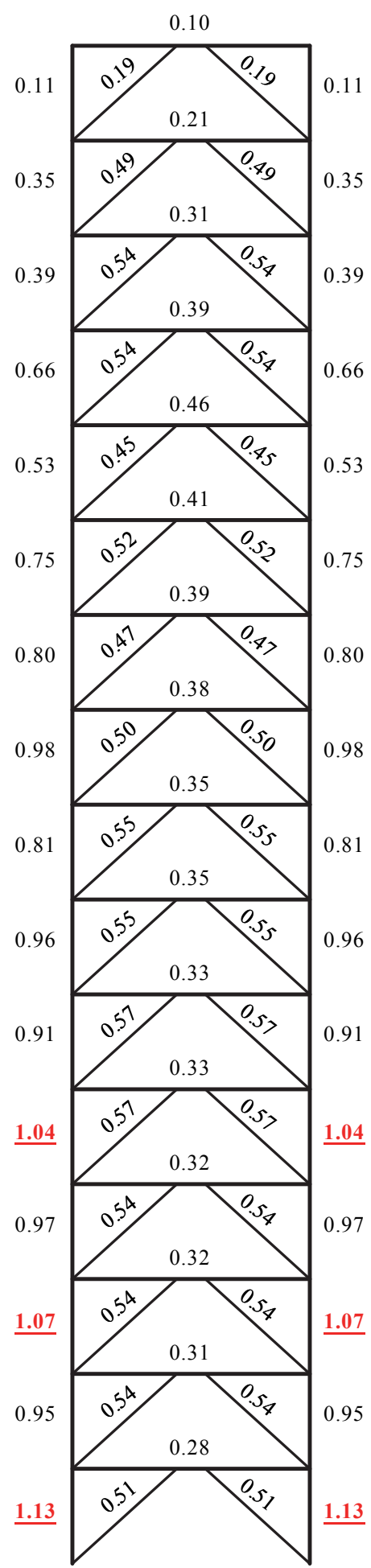

(a) $D C R_{N}$
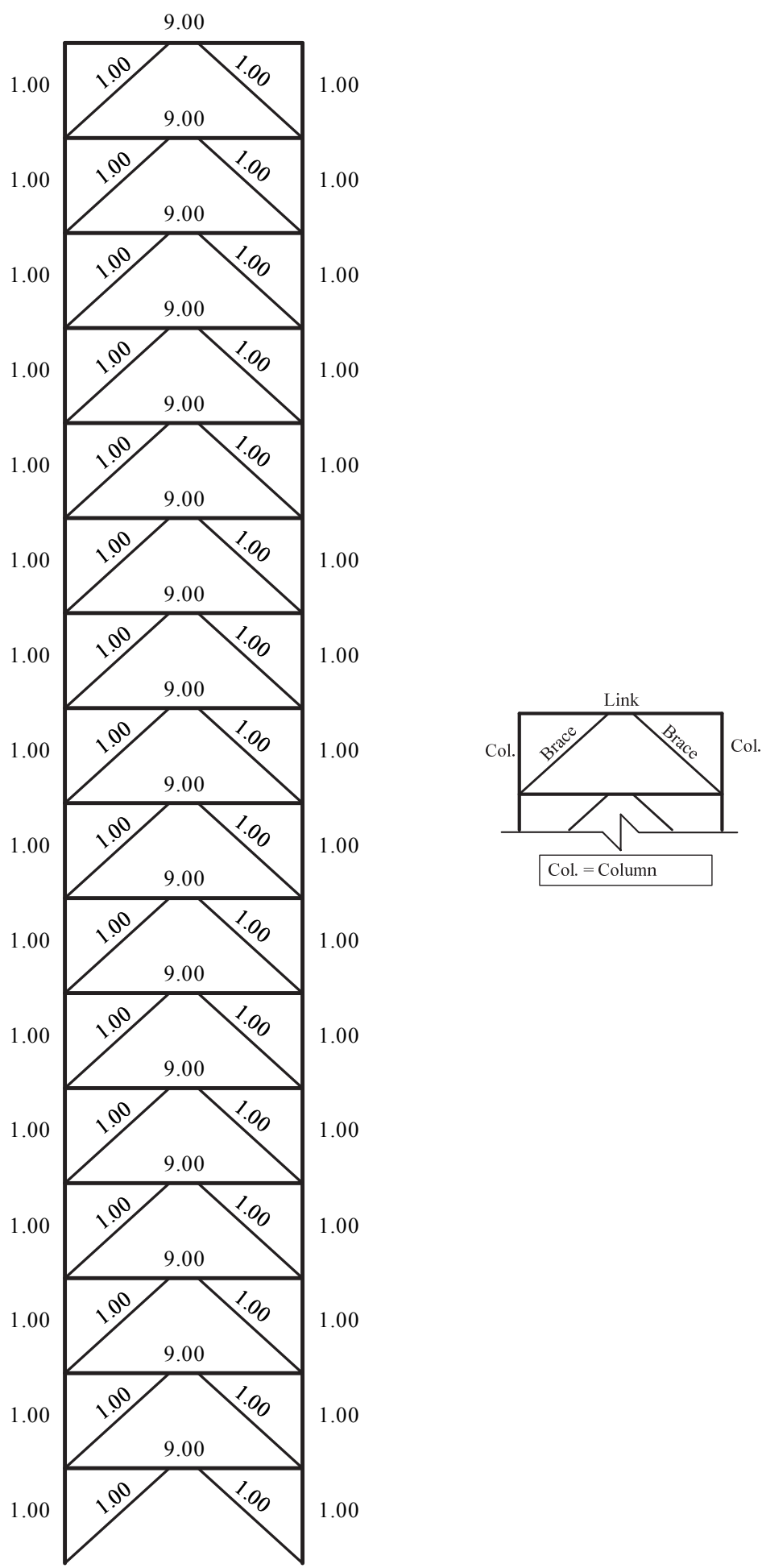

(b) $m$-factors (c) Key

Figure 3-82. LSP Assessment Results, 16-Story EBF RSA, BSE-1 LS 


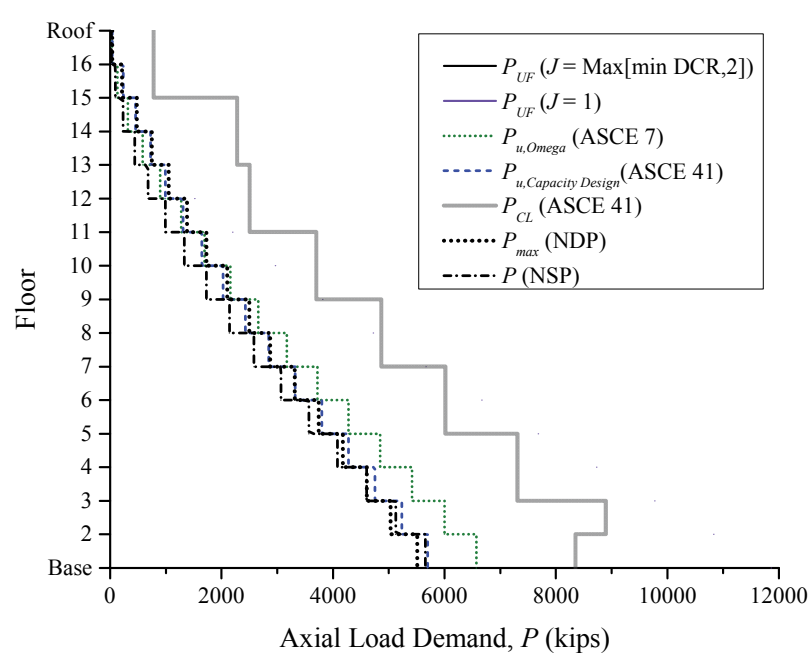

(a) ELF

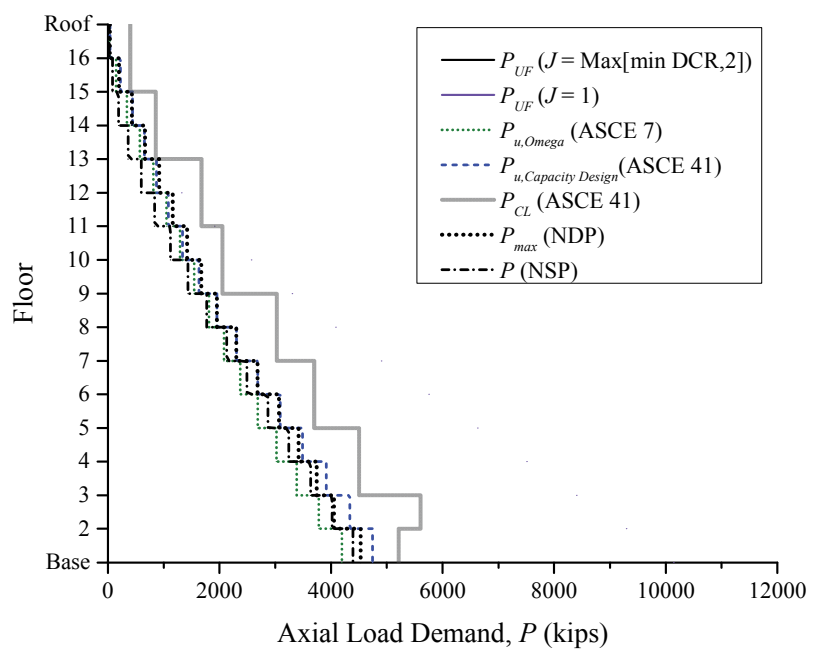

(b) RSA

Figure 3-83. LSP Assessment Results, Compression in Exterior Columns, 16-Story EBF, BSE-1

\subsection{BSE-2 Earthquake Hazard Level (CP BPL)}

In this section, the following apply:

- Figure 3-84 and Figure 3-85 provide the $D C R_{N}$ and load-dependent $m$-factor values for the ELF and RSA designs, respectively, for the LSP at the BSE-1 EHL. In these figures, $D C R_{N}$ values greater than unity are highlighted in red and underlined. $D C R$ values, as defined by ASCE 41, can be obtained by multiplying $D C R_{N}$ by $m$ and $\kappa$, see Eq. 3-6.

- Figure 3-86 provides the maximum axial compression demands, $P_{U F}$, in the exterior column lines for various analysis methods and the column capacity, $P_{C L}$.

All link beam and brace actions satisfy the CP BPL acceptance criteria.

Similar to the LSP LS results, several frame columns in the ELF- and RSA-designed frames do not satisfy the CP BPL assessment criteria using the force-controlled interaction equation because of the combination of high axial force and moment. As discussed previously in $\S 3.2 .2, P_{U F}$ is determined by taking $J$ (ASCE $41 \S 3.4 .2 .1 .2-2$ ) as the minimum $D C R$ of the component(s) delivering force to the column, but not less than 2.0. This approach produces a high estimate for $P_{U F}$ for the LS SPL as compared to ASCE $41 \S 3.4 .2 .1 .2-1$ (based on ASCE 41 §5.5.3.4.2), as shown in Figure 3-86.

The $D C R_{N}$ interaction results for force-controlled columns (and a few braces) are not the same between LSP LS and LSP CP because $J$ was set to the default of 2.0 in this frame. This default value is not consistent with the actual plastic mechanism, $J$ for the CP SPL should be $2 / 2 / 3=3$ assuming all links yield (or $J=2$ $\times 2 / 3$ for the LS SPL). The effect on axial load demand by maintaining $J$ can be seen in Figure 3-86. The results for the links differ by approximately $(13 / 9) / 1.5=0.963$ times the $D C R_{N}$, approximate because of second-order effects. 


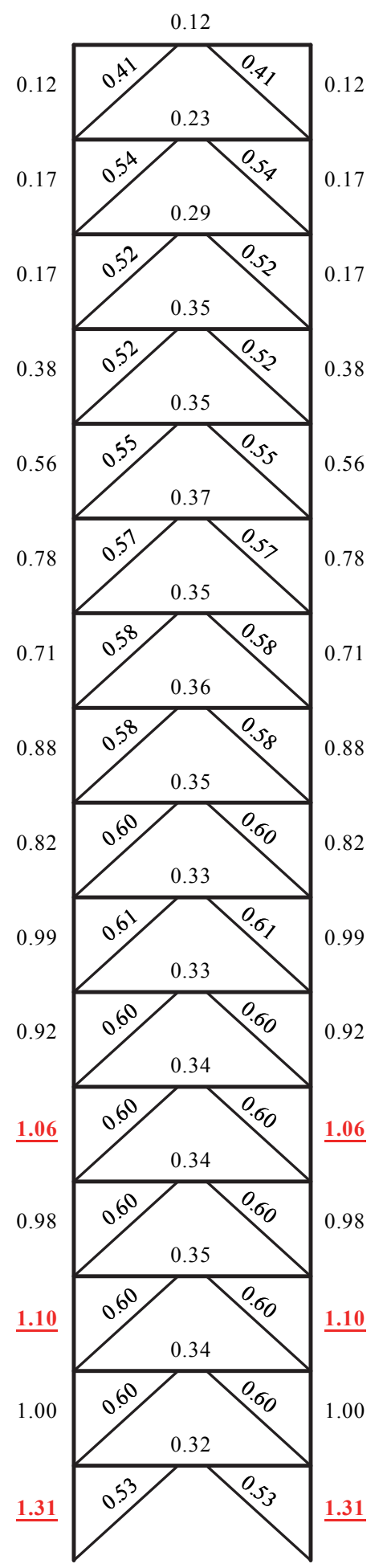

(a) $D C R_{N}$
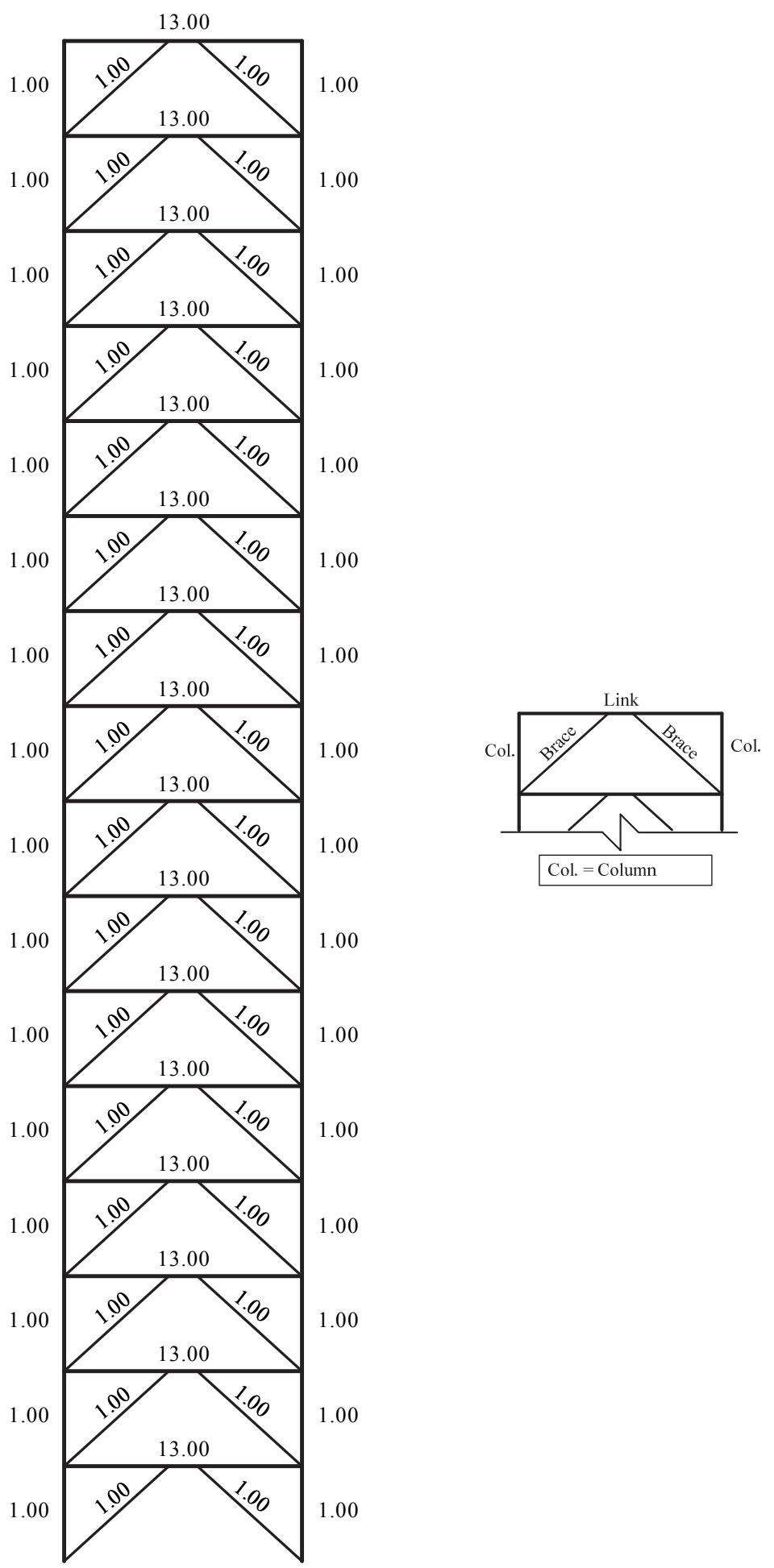

(b) $m$-factors (c) Key

Figure 3-84. LSP Assessment Results, 16-Story EBF ELF, BSE-2 CP 


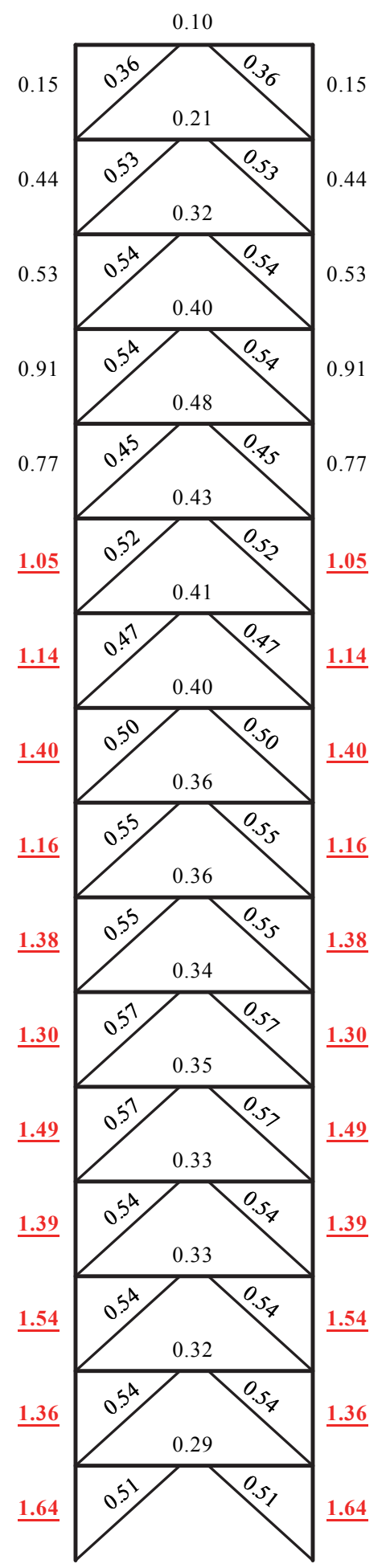

(a) $D C R_{N}$

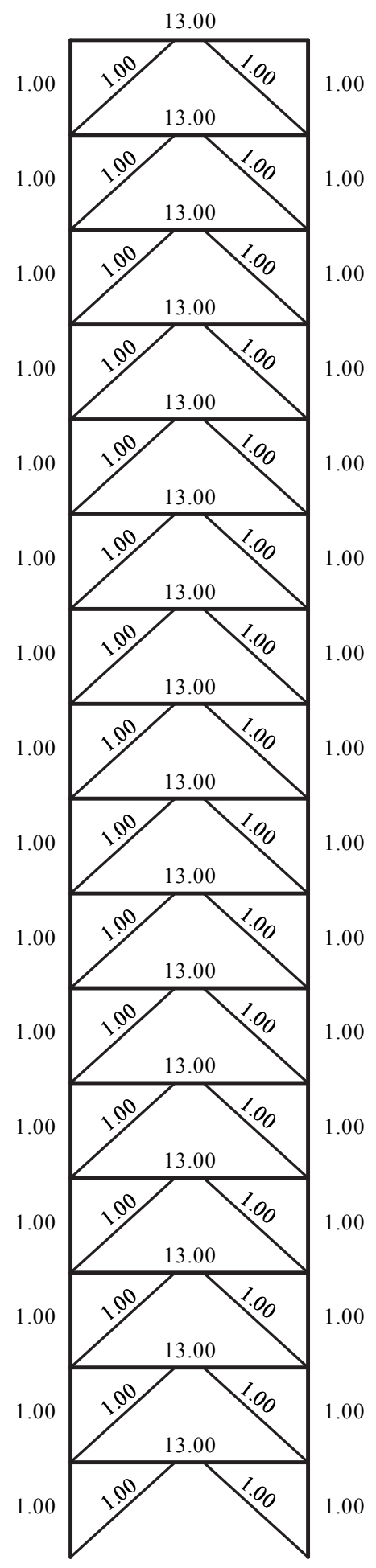

(b) $m$-factors

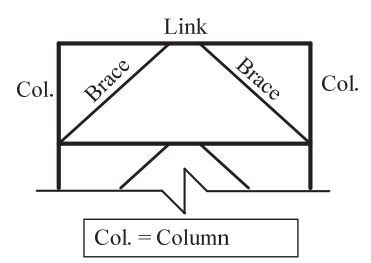

(c) Key

Figure 3-85. LSP Assessment Results, 16-Story EBF RSA, BSE-2 CP 


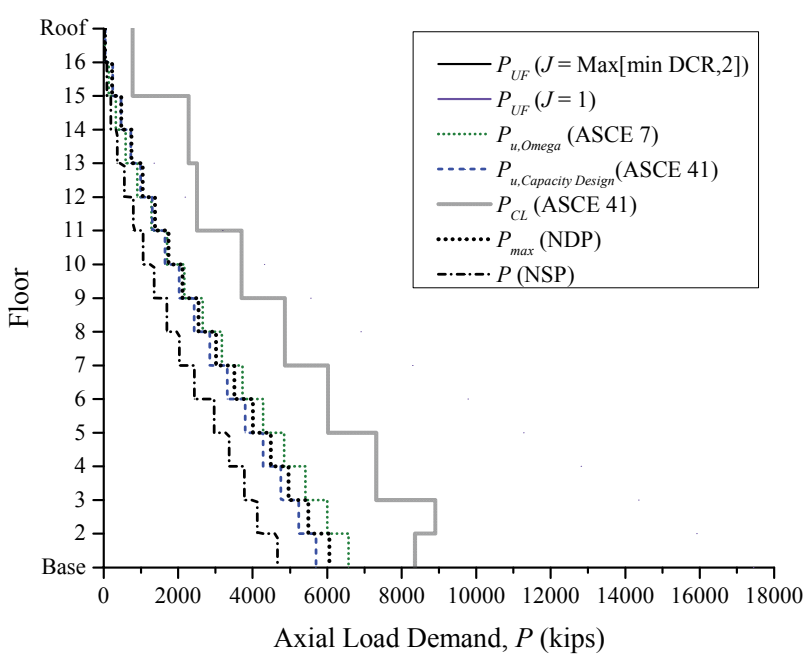

(a) ELF

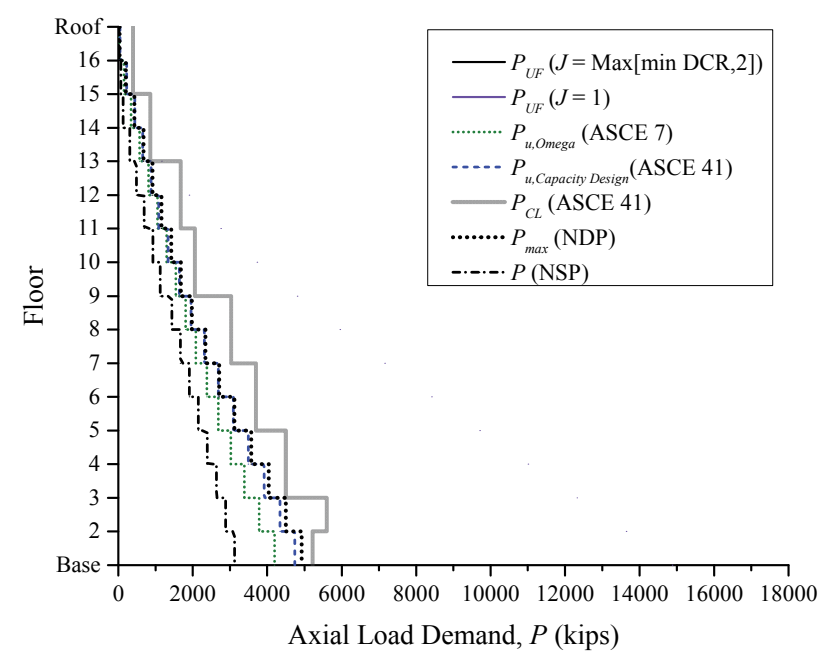

(b) RSA

Figure 3-86. LSP Assessment Results, Compression in Exterior Columns, 16-Story EBF, BSE-2

3.2.2.3.2 Linear Dynamic Procedure

\subsection{BSE-1 Earthquake Hazard Level (LS BPL)}

In this section, the following apply:

- $\quad$ Figure 3-87 and Figure 3-88 provide the $D C R_{N}$ and load-dependent $m$-factor values for the ELF and RSA designs, respectively, for the LDP at the BSE-1 EHL. In these figures, $D C R_{N}$ values greater than unity are highlighted in red and underlined. $D C R$ values, as defined by ASCE 41, can be obtained by multiplying $D C R_{N}$ by $m$ and $\kappa$, see Eq. 3-6.

- Figure 3-89 provides the maximum axial compression demands, $P_{U F}$, in the exterior column lines for various analysis methods and the column capacity, $P_{C L}$.

All component actions satisfy the LS BPL acceptance criteria. As discussed previously in $\S 3.2 .2, P_{U F}$ is determined by taking $J$ (ASCE $41 \S 3.4 .2 .1 .2-2$ ) as the minimum $D C R$ of the component(s) delivering force to the column, but not less than 2.0. This approach produces a lower-bound estimate for $P_{U F}$ for the LS SPL as compared to ASCE 41 §3.4.2.1.2-1 (based on ASCE 41 §5.5.3.4.2), as shown in Figure 3-89. 


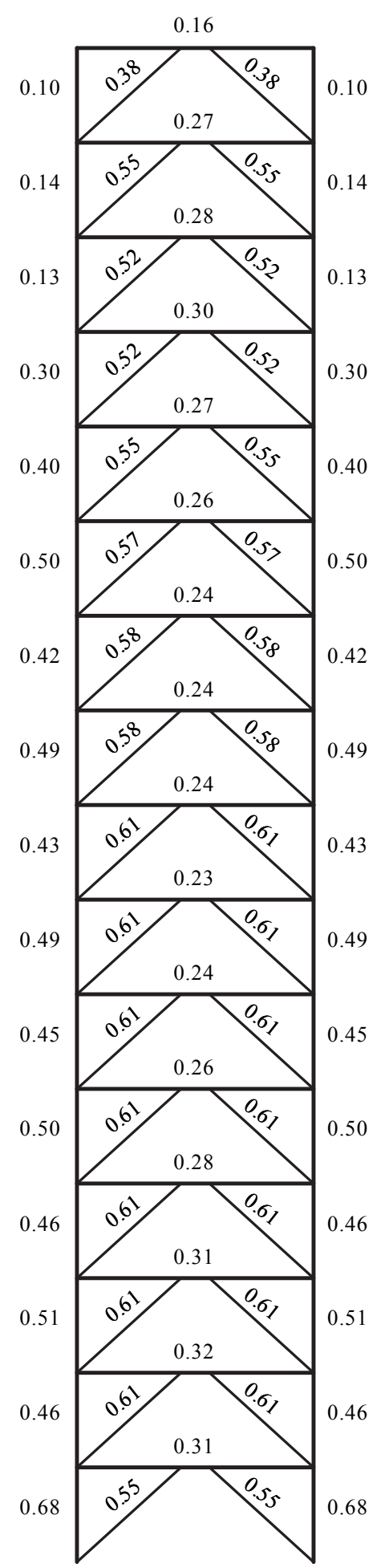

(a) $D C R_{N}$

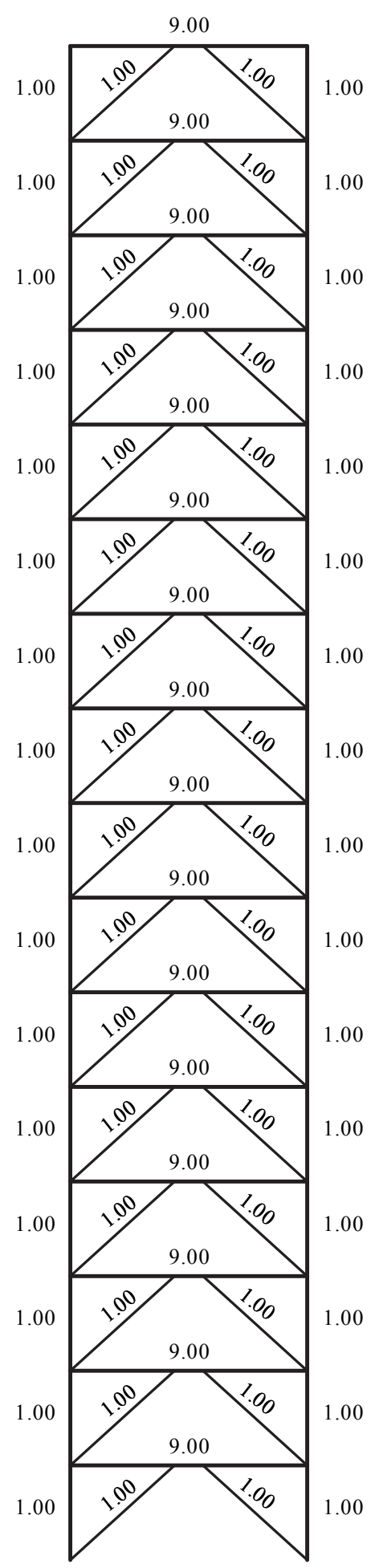

(b) $m$-factors

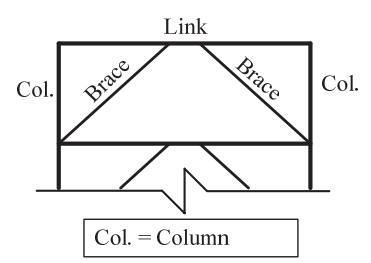

(c) Key

Figure 3-87. LDP Assessment Results, 16-Story EBF ELF, BSE-1 LS 


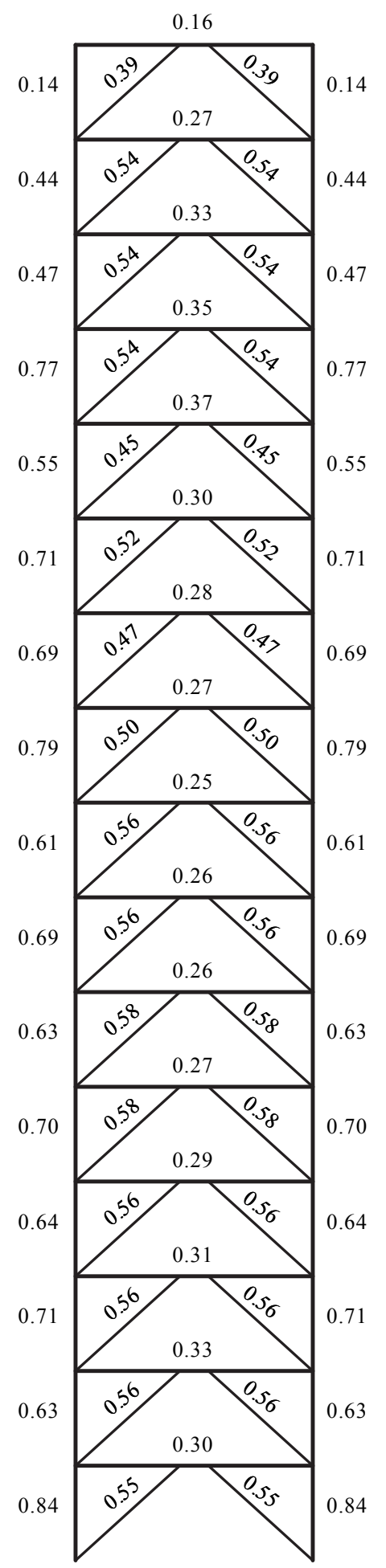

(a) $D C R_{N}$
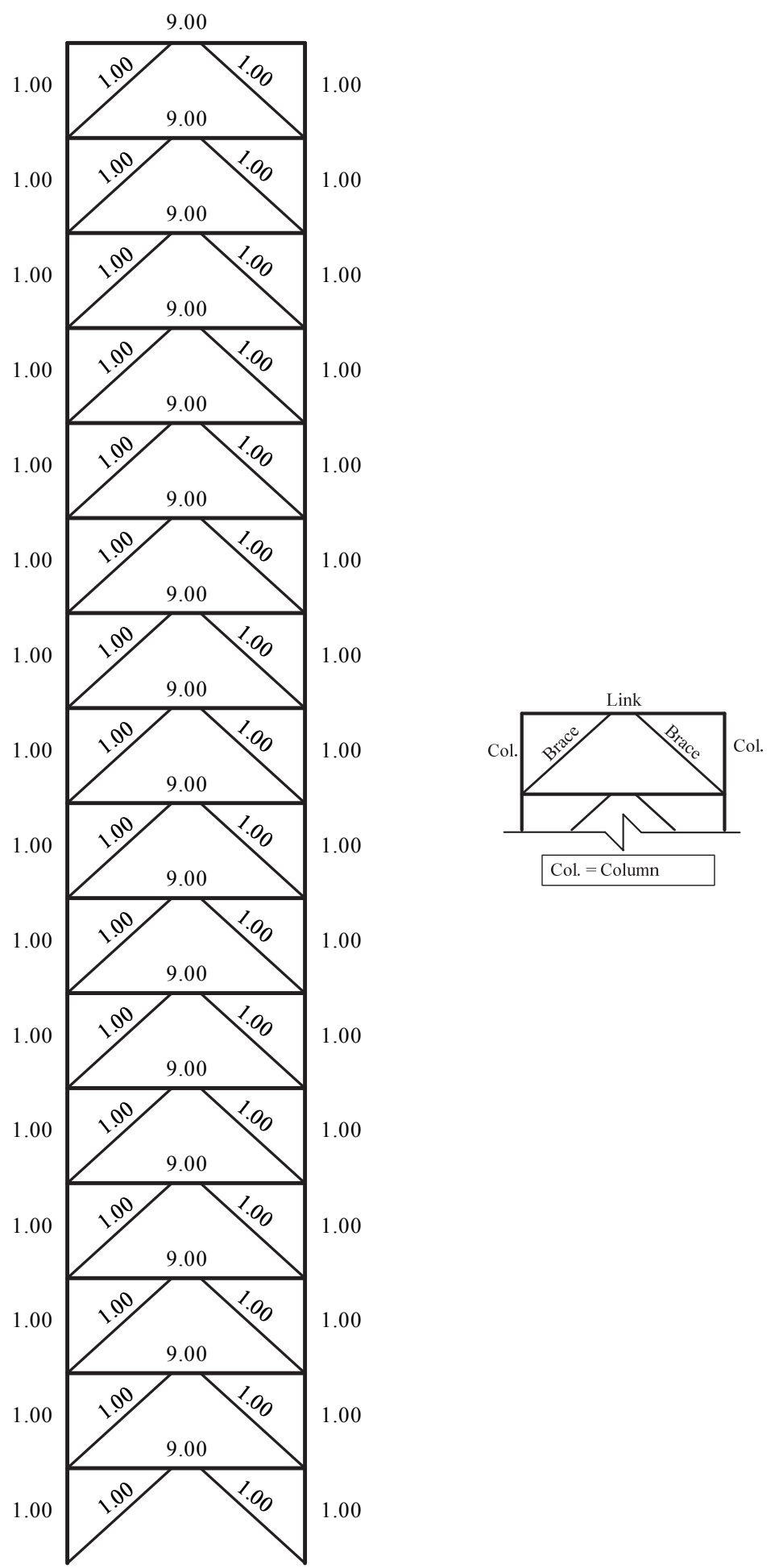

(b) $m$-factors (c) Key

Figure 3-88. LDP Assessment Results, 16-Story EBF RSA, BSE-1 LS 


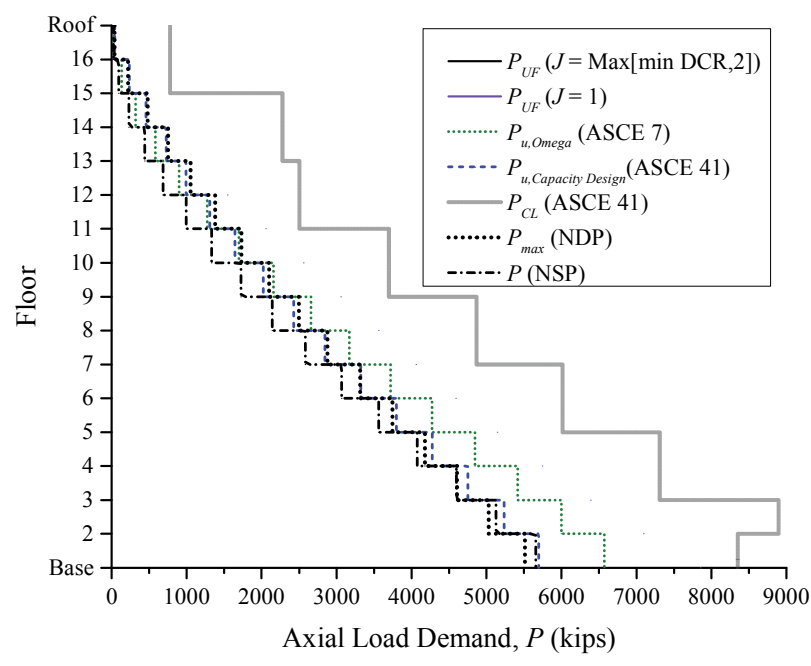

(a) ELF

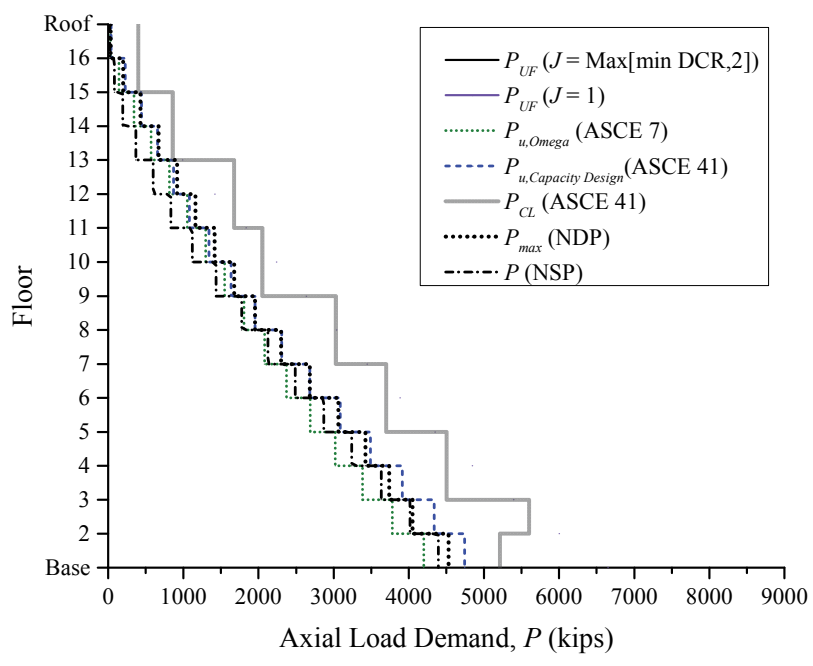

(b) RSA

Figure 3-89. LDP Assessment Results, Compression in Exterior Columns, 16-Story EBF, BSE-1

\subsection{BSE-2 Earthquake Hazard Level (CP BPL)}

In this section, the following apply:

- Figure 3-90 and Figure 3-91 provide the $D C R_{N}$ and load-dependent $m$-factor values for the ELF and RSA designs, respectively, for the LDP at the BSE-1 EHL. In these figures, $D C R_{N}$ values greater than unity are highlighted in red and underlined. $D C R$ values, as defined by ASCE 41, can be obtained by multiplying $D C R_{N}$ by $m$ and $\kappa$, see Eq. 3-6.

- Figure 3-92 provides the maximum axial compression demands, $P_{U F}$, in the exterior column lines for various analysis methods and the column capacity, $P_{C L}$.

All link beam and brace actions satisfy the CP BPL acceptance criteria.

Base columns in the RSA-designed frame do not satisfy the LS BPL acceptance criteria using the forcecontrolled interaction equation because of the combination of high axial force and moment. High flexural demand at the base of the frame is a consequence of modeling rotationally restrained base connections, which more closely characterizes how the base connection is detailed in these buildings than assuming rotationally unrestrained connections. As discussed previously in $\S 3.2 .2, P_{U F}$ is determined by taking (ASCE 41 \$3.4.2.1.2-2) as the minimum DCR of the component(s) delivering force to the column, but not less than 2.0. This approach produces a lower-bound estimate for $P_{U F}$ for the LS SPL as compared to ASCE 41 §3.4.2.1.2-1 (based on ASCE 41 §5.5.3.4.2), as shown in Figure 3-92.

The $D C R_{N}$ interaction results for force-controlled columns (and a few braces) are not the same between LSP LS and LSP CP because $J$ was set to the default of 2.0 in this frame. This default value is not consistent with the actual plastic mechanism—see $§ 3.2 .2 .3 .1 .2$ for more information. The results for the links differ by approximately (13/9)/1.5 $=0.963$ times the $D C R_{N}$, approximate because of second-order effects. 


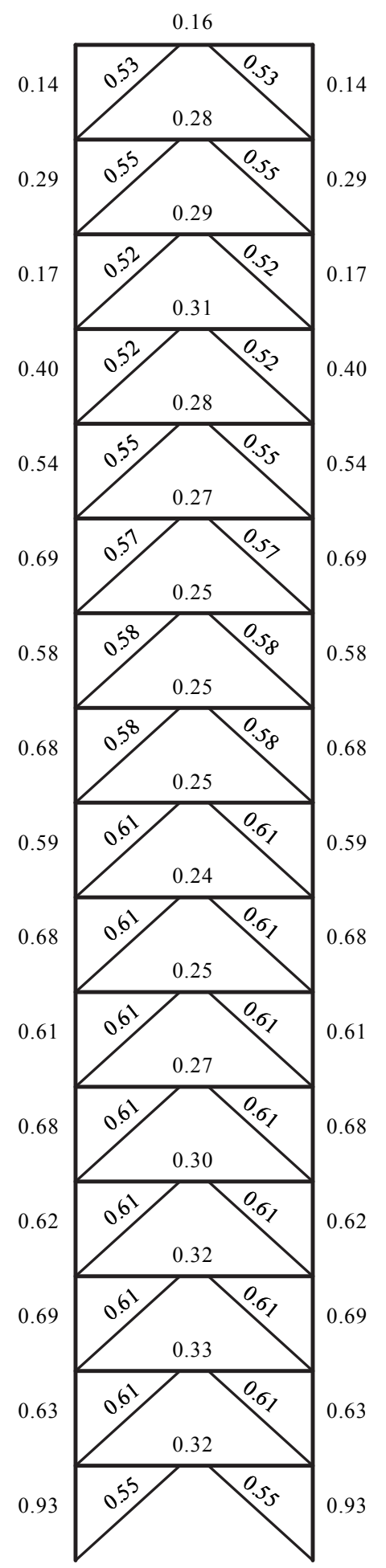

(a) $D C R_{N}$
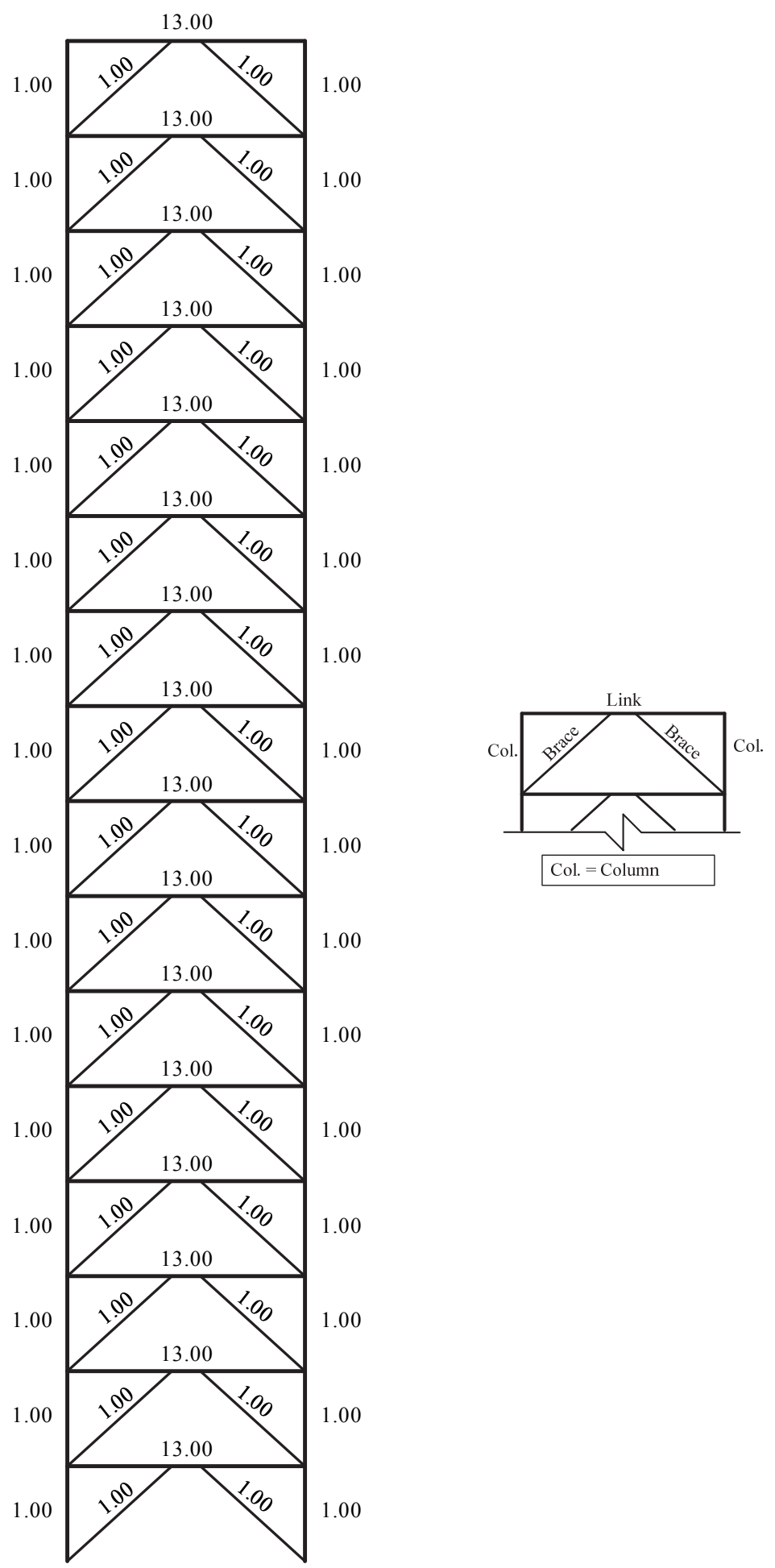

(b) $m$-factors (c) Key

Figure 3-90. LDP Assessment Results, 16-Story EBF ELF, BSE-2 CP 


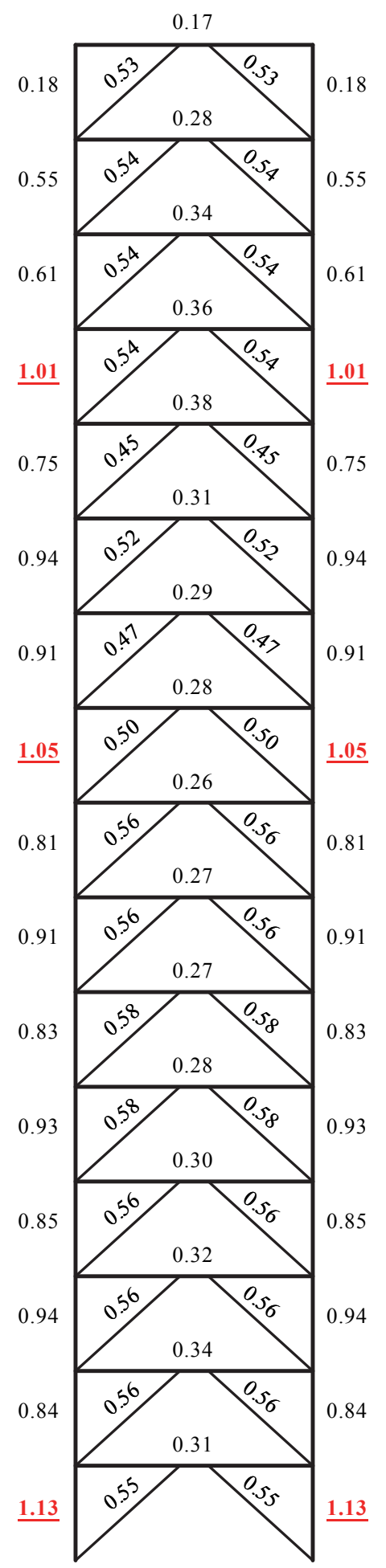

(a) $D C R_{N}$

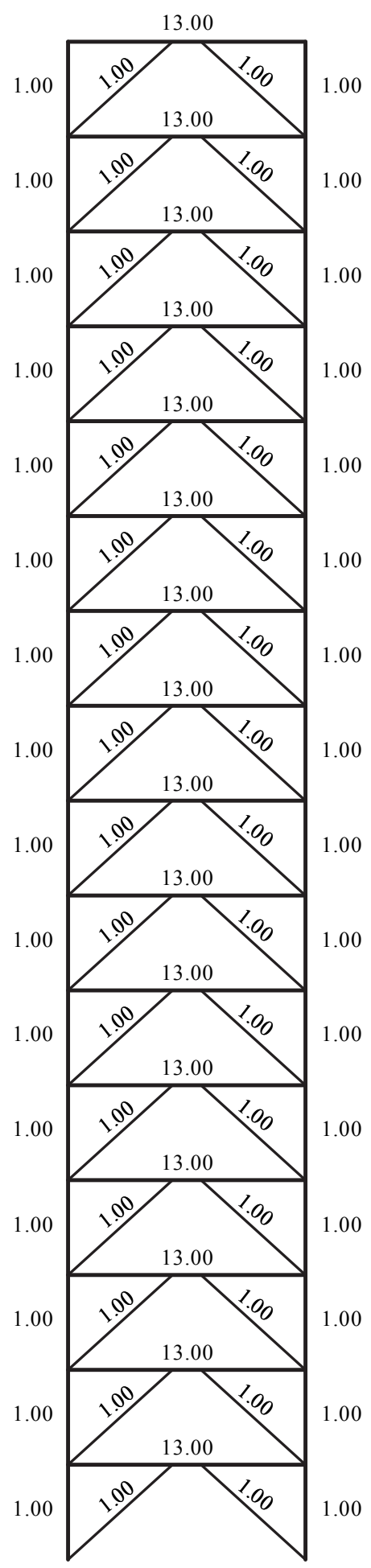

(b) $m$-factors

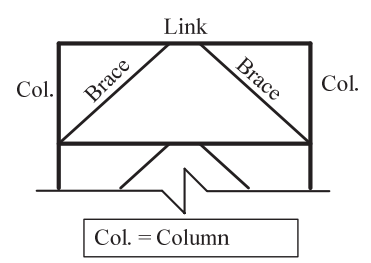

(c) Key

Figure 3-91. LDP Assessment Results, 16-Story EBF RSA, BSE-2 CP 


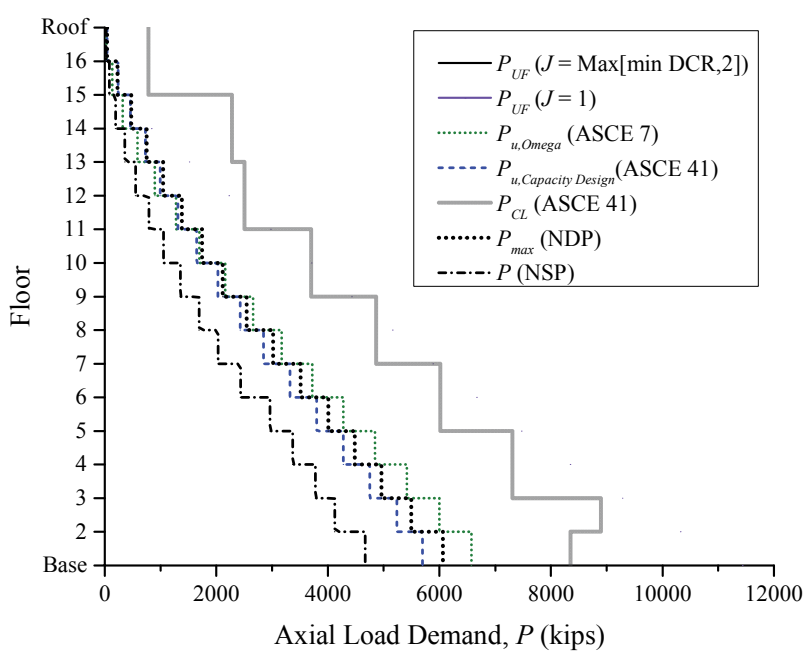

(a) ELF

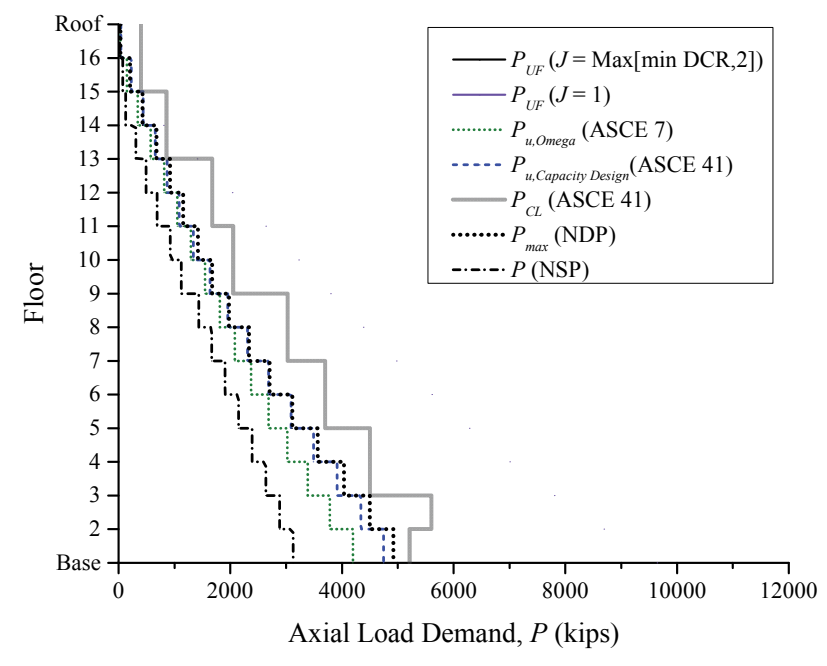

(b) RSA

Figure 3-92. LDP Assessment Results, Compression in Exterior Columns, 16-Story EBF, BSE-2

\subsection{Nonlinear Static Procedure}

- Table 3-17 to Table 3-19 provide the computed NSP analysis and assessment parameters in accordance with ASCE 41 §3.3.3.

- Figure 3-93 and Figure 3-94 illustrate the monotonic pushover curves for the ELF- and RSAdesigned frames, respectively, and the associated pushover parameters from ASCE 41 at the BSE2 EHL. Roof displacement is measured at the Center of Mass (CoM). A significant change in base shear is due to component strength loss of components (e.g., link beams), notated in the figures. First-order and second-order responses, shown in these figures, aids in computing a physically meaningful value for $\alpha_{P-\Delta}$ used in ASCE 41 Equation 3-17.

- Figure 3-95and Figure 3-96 illustrate the story drift ratios in terms of the roof drift ratio.

As discussed in $\S 3.1 .3 .2 .1$, the NSP is permitted, but requires supplemental verification using the LDPsee $\$ 3.2 .2 .3$.2. In this case, the displacement at the maximum base shear governs $\Delta_{d}$ for the RSA-designed frame for both BSE-1 and BSE-2 EHLs and ELF frame for BSE-2 EHL whereas the target displacement governs the ELF-designed frame for BSE-1 EHL. The change in $\Delta_{d}$ between BSE-1 and BSE-2 adds complexity to NSP process by changing the pushover variables. Further, when $\Delta_{d}$ is governed by the target displacement, the system can have an increased $R_{\max }$. Axial compression force in the exterior columns at the target displacement are shown previously in the linear assessment sections. Results indicate that the NSP generally results in a lower estimate of the axial force demands compared to the other methods used in this study. This is partly because the fundamental mode-based lateral force distribution does not adequately capture higher mode effects. Also, the target displacement at the roof computed based on fundamental mode properties may underestimate the story demands in the upper stories. 
Table 3-17. NSP General Information, 16-Story EBF (kip, inch)

\begin{tabular}{ccccccccccccc}
\hline Design & $T_{1}$ & $K_{1}$ & $\Delta_{y}$ & $V_{y}$ & $K_{e}$ & $T_{e}$ & $h$ & $\Delta_{\text {peak }}$ & $V_{\text {peak }}$ & $W$ & $C_{m}$ & $C_{0}$ \\
\hline ELF & 3.02 & 106.2 & 16.5 & 1748 & 105.8 & 3.03 & 1.17 & 28.8 & 2008.8 & 21900 & 1.00 & 1.43 \\
RSA & 3.55 & 70.9 & 18.5 & 1300 & 70.4 & 3.57 & 1.19 & 28.1 & 1429.7 & 21659 & 1.00 & 1.46 \\
\hline
\end{tabular}

Table 3-18. CP NSP Analysis Parameters, 16-Story EBF BSE-2 CP (kip, inch)

\begin{tabular}{cccccccccccccc}
\hline Design & $S_{a}$ & $R$ & $C_{1}$ & $C_{2}$ & $\Delta_{t}$ & $V_{t}$ & $\Delta_{d}$ & $\alpha_{1}$ & $\alpha_{2}$ & $\alpha_{P-\Delta}$ & $\alpha_{e}$ & $R_{\max }$ & $R \leq$ \\
$R_{\max }$ \\
\hline ELF & 0.30 & 3.76 & 1.00 & 1.00 & 38.4 & 1457.0 & 28.8 & 0.20 & -0.23 & -0.05 & -0.08 & 6.32 & OK \\
RSA & 0.26 & 4.28 & 1.00 & 1.00 & 46.8 & 844.5 & 28.1 & 0.19 & -0.34 & -0.05 & -0.11 & 5.01 & OK \\
\hline
\end{tabular}

Table 3-19. LS NSP Analysis Parameters, 16-Story EBF BSE-1 LS (kip, inch)

\begin{tabular}{cccccccccccccc}
\hline \multirow{2}{*}{ Design } & $S_{a}$ & $R$ & $C_{1}$ & $C_{2}$ & $\Delta_{t}$ & $V_{t}$ & $\Delta_{d}$ & $\alpha_{1}$ & $\alpha_{2}$ & $\alpha_{P-\Delta}$ & $\alpha_{e}$ & $R_{\max }$ & $R \leq$ \\
$R_{\max }$ \\
\hline ELF & 0.20 & 2.51 & 1.00 & 1.00 & 25.6 & 1967.8 & 25.6 & 0.23 & -0.20 & -0.05 & -0.08 & 6.50 & OK \\
RSA & 0.17 & 2.86 & 1.00 & 1.00 & 31.2 & 1421.0 & 28.1 & 0.19 & -0.34 & -0.05 & -0.11 & 5.01 & OK \\
\hline
\end{tabular}

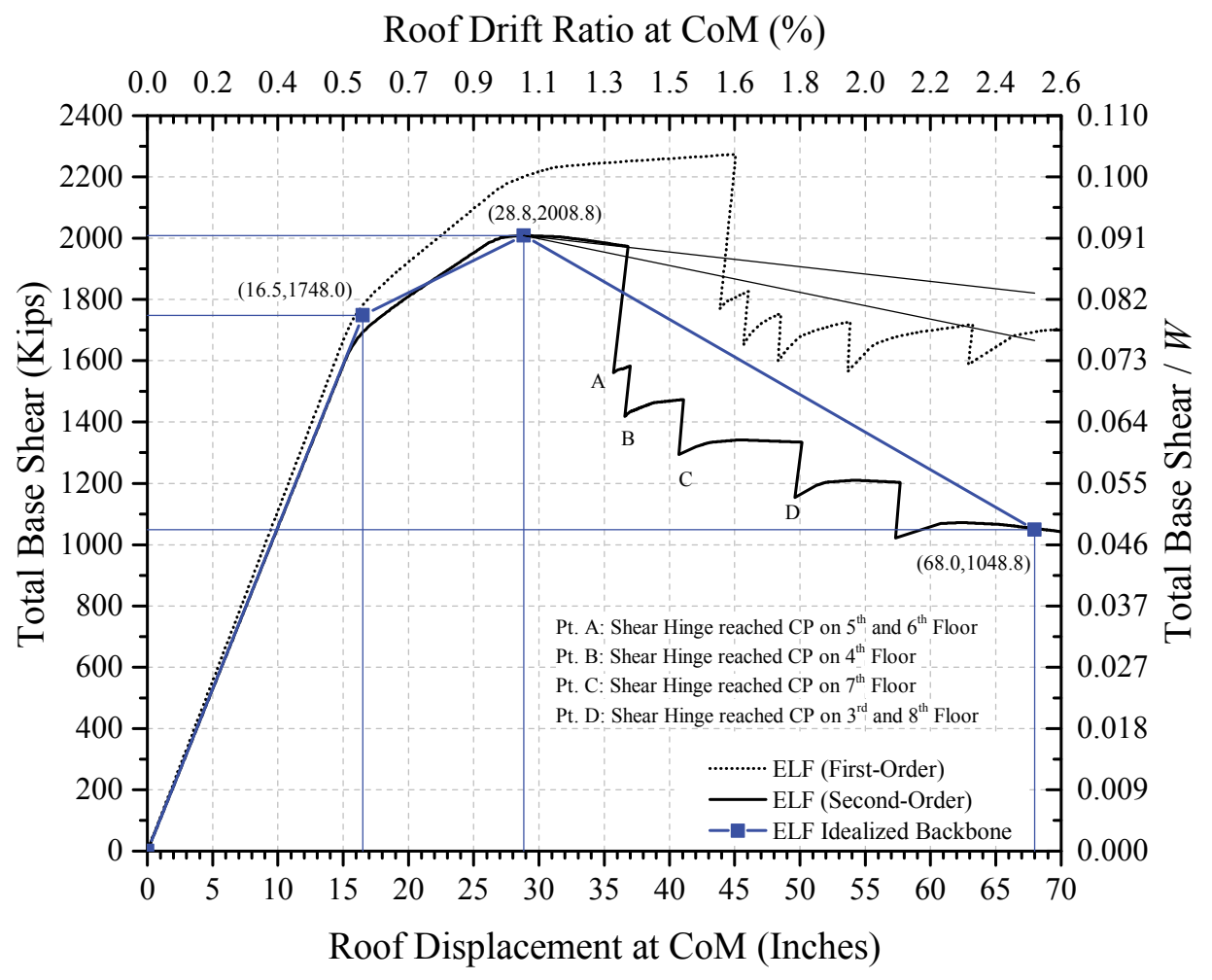

Figure 3-93. 16-Story EBF ELF Pushover, BSE-2 


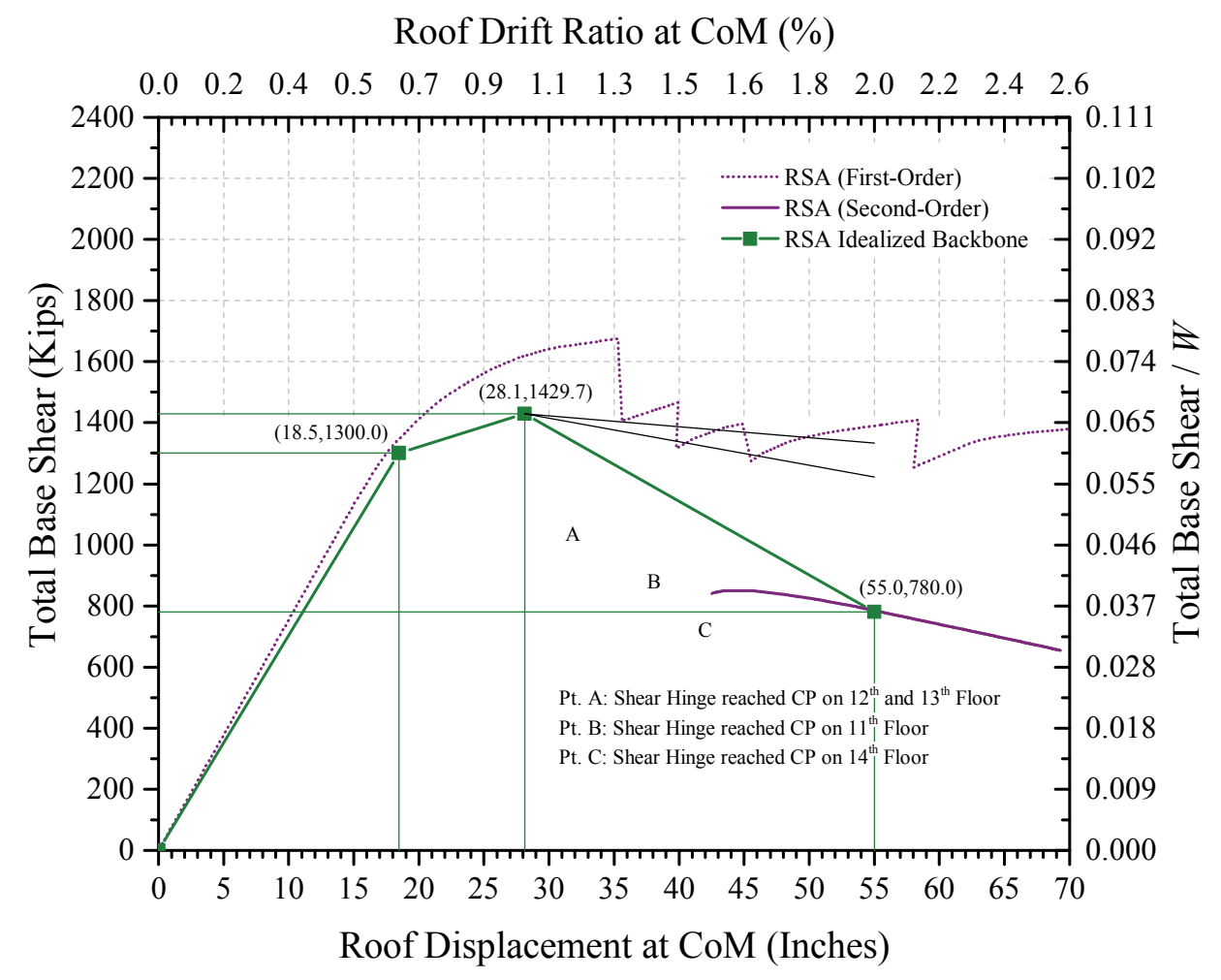

Figure 3-94. 16-Story EBF RSA Pushover, BSE-2

Figure 3-97 illustrates which frame components are force-controlled for flexure for both the NSP and NDP; red circles indicate anticipated plastic hinge locations that are force-controlled for flexure at the target displacement.

Figure 3-98 and Figure 3-99 illustrate the $D C R_{N}$ values if greater than unity for the ELF- and RSA-designed frames at the target displacement for the LS BPL at the BSE-1 EHL and CP BPL at the BSE-2 EHL. The figures illustrate demands when the system is loaded to the left. All shear actions for the ELF-designed frame satisfy the LS BPL acceptance criteria; however, one link beam in the RSA-designed frame does not satisfy the same criteria. Several link beams in both designs do not satisfy the CP BPL acceptance criteria. These failures occur at different locations within the frame, illustrating the variation in the distribution of link beam strengths from the ELF and RSA design procedures.

All other force-controlled component actions adjacent to the link beams do satisfy the LS BPL acceptance criteria. Although the same observation is evident for the ELF-designed frame for the CP BPL acceptance criteria, several hinges in columns and beams in the RSA-designed frame do not satisfy the criteria for the CP BPL. This result is a consequence of the target displacement being much greater than the displacement at the peak base shear. There are several frame column hinges (not at the base) in the RSA-designed frame that do not satisfy the criteria for the CP BPL. This effect is primarily because of the minor increase in $P_{E}$ because the shear strength, $V_{C E}$, of the link beams was increased by 15 percent for nonlinear analysis - see $\S 3.2 .1 .2$. As discussed in $\$ 3.2 .1 .2$, the flexural hinge model for force-controlled components obeys ASCE 
41 Equation 5-4 (yield surface for the section). As such, unsatisfactory performance is characterized by section yielding.

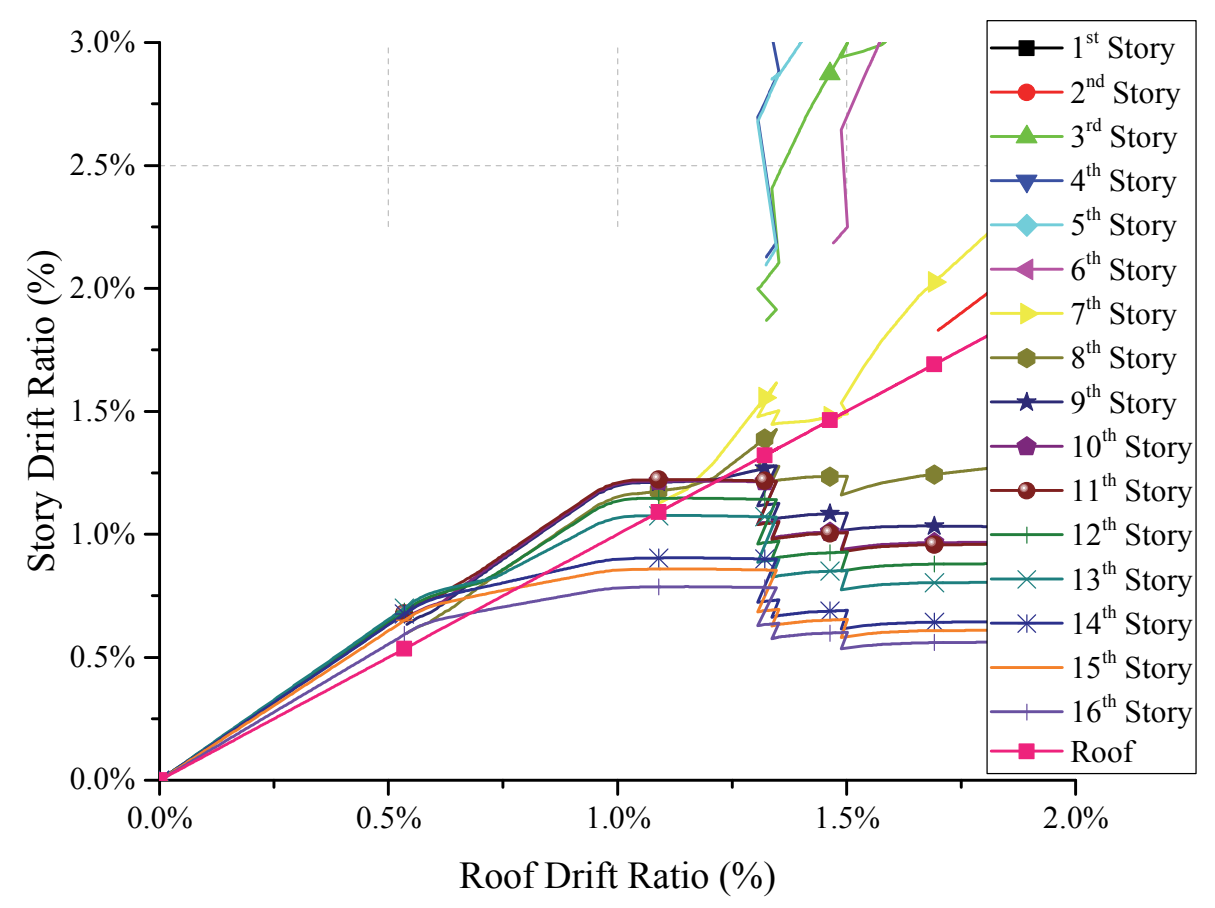

Figure 3-95. 16-Story EBF ELF Pushover - Story Drift Ratios - BSE-2

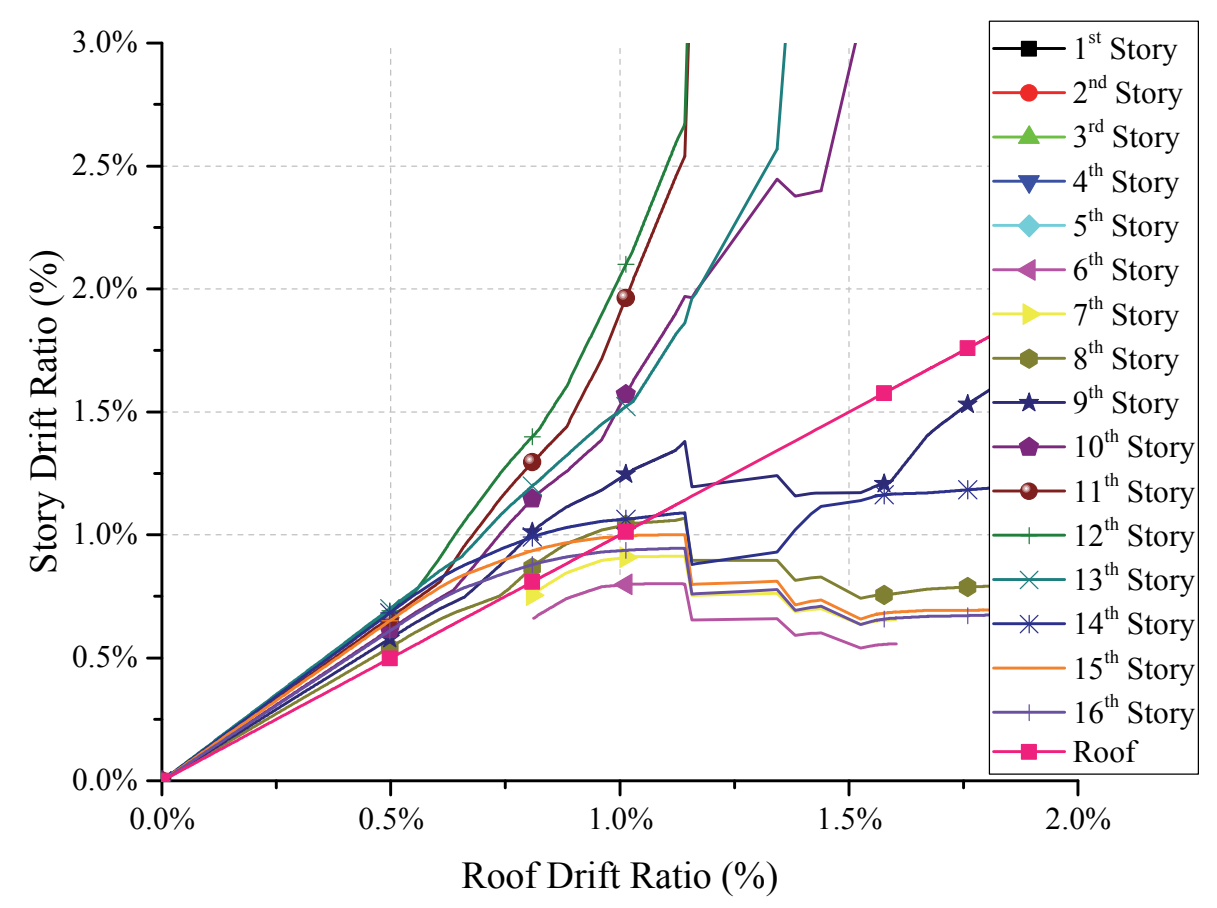

Figure 3-96. 16-Story EBF RSA Pushover - Story Drift Ratios - BSE-2 


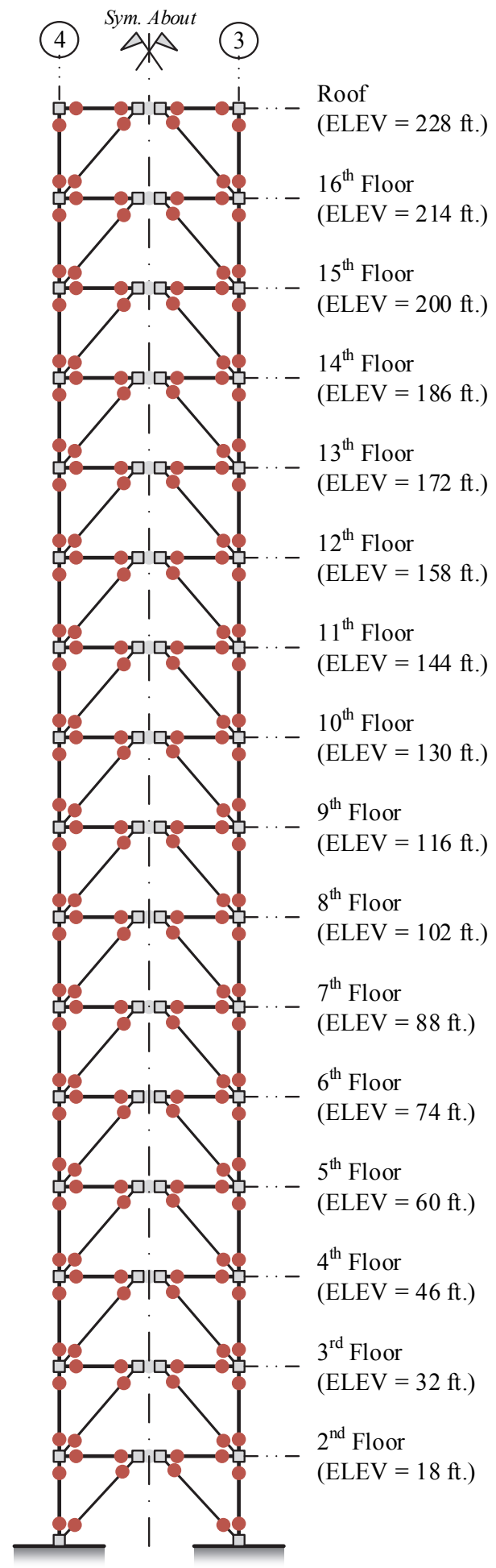

All columns, braces, and beams adjacent to link beams are force-controlled (FC) for flexure

Figure 3-97. Schematic of Flexural Actions in Components, 16-Story EBF (NSP and NDP) 


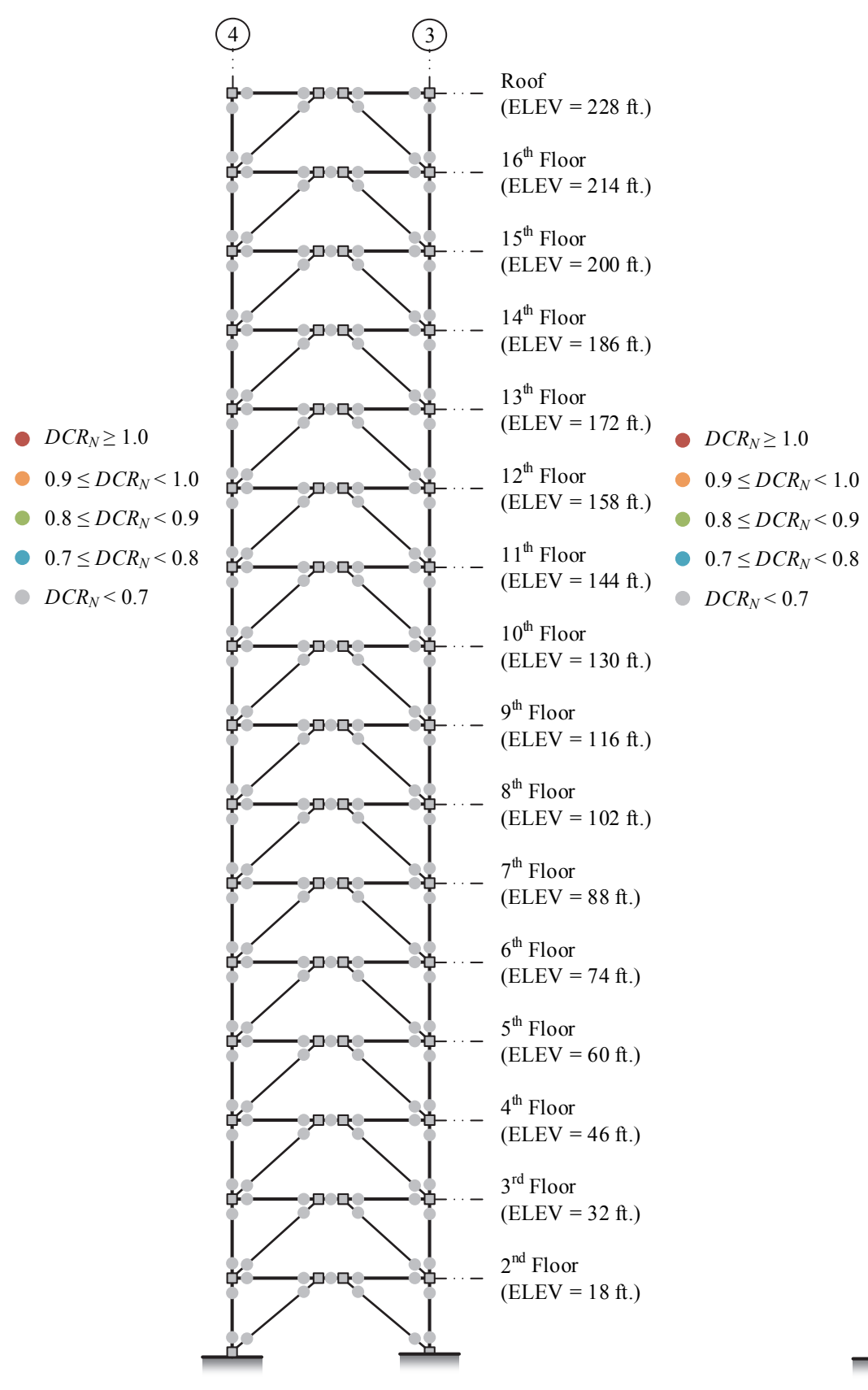

(a) ELF

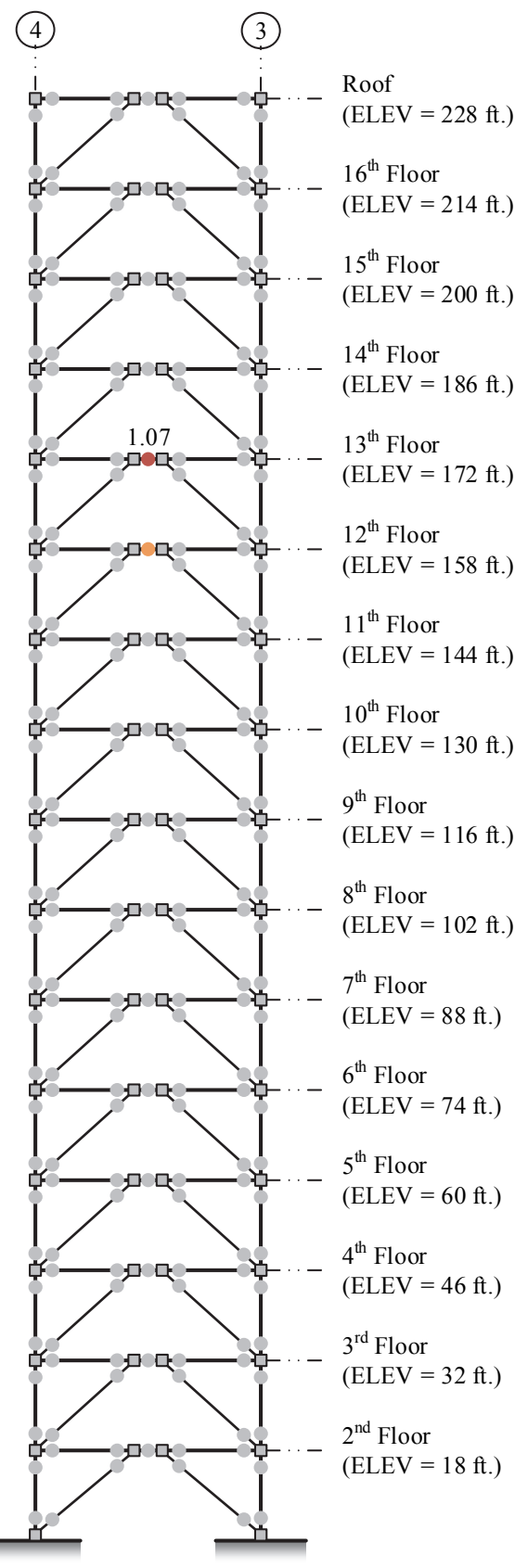

(b) RSA

Figure 3-98. NSP Assessment Results, 16-Story EBF ELF, BSE-1 LS (+push to left) 


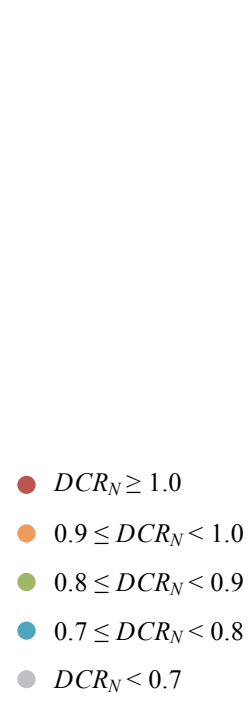

(4)

(3)

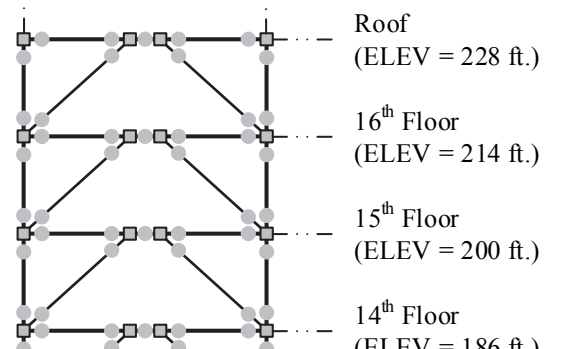

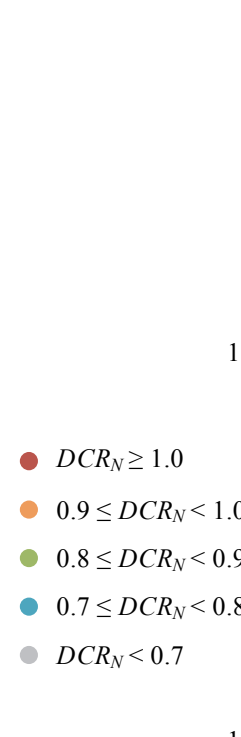

(ELEV = $130 \mathrm{ft}$.)

$9^{\text {th }}$ Floor

$(\mathrm{ELEV}=116 \mathrm{ft}$.)

$8^{\text {th }}$ Floor

(ELEV $=102 \mathrm{ft}$.)

$7^{\text {th }}$ Floor

$(\mathrm{ELEV}=88 \mathrm{ft}$.)

$6^{\text {th }}$ Floor

$(\mathrm{ELEV}=74 \mathrm{ft}$.)

$-5^{\text {th }}$ Floor

$(\mathrm{ELEV}=60 \mathrm{ft}$.)

$-4^{\text {th }}$ Floor

$(\mathrm{ELEV}=46 \mathrm{ft}$.)

$-3^{\text {rd }}$ Floor

$(\mathrm{ELEV}=32 \mathrm{ft}$.)

$2^{\text {nd }}$ Floor

$(\mathrm{ELEV}=18 \mathrm{ft}$.)

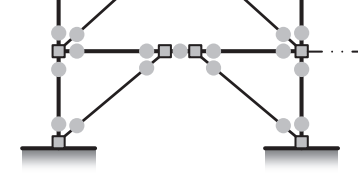

(a) ELF
(4)

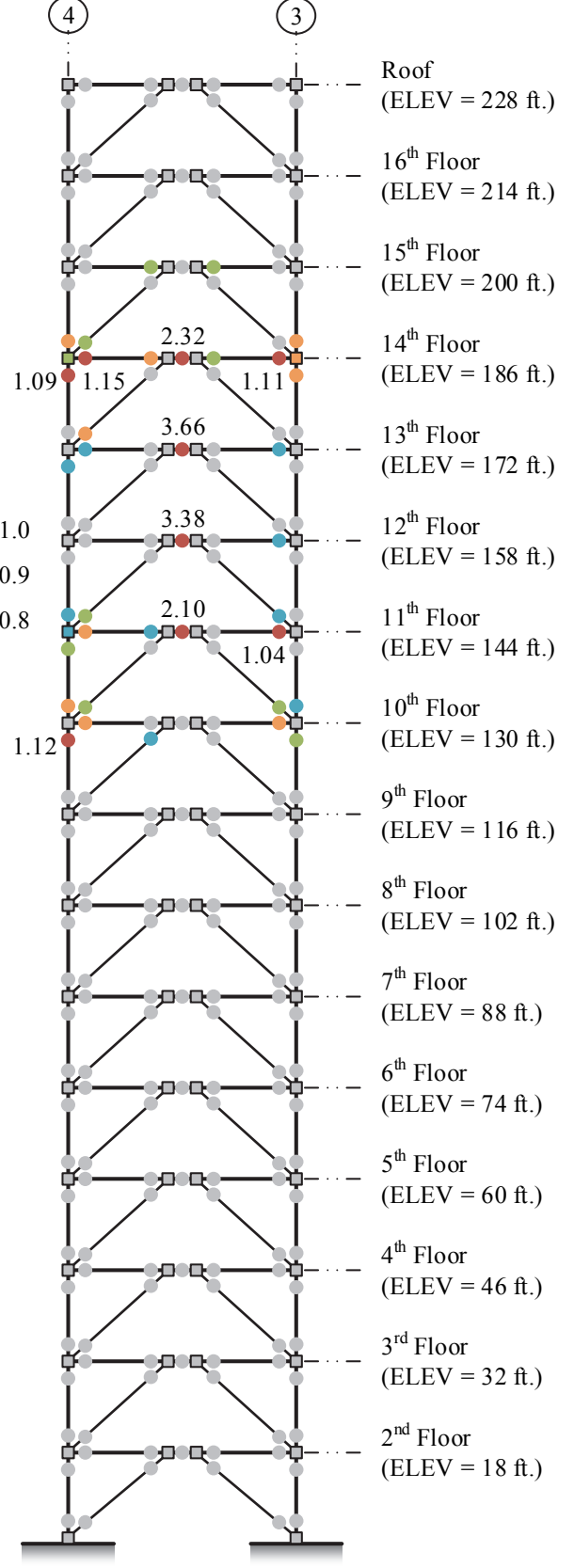

(b) RSA

Figure 3-99. NSP Assessment Results, 16-Story EBF RSA, BSE-2 CP (+push to left)

\subsection{Nonlinear Dynamic Procedure}

The earthquake record set used to assess the N-S direction of ME16 is shown in Appendix A. For the ELF design, the analysis successfully completed for all 14 records at the BSE-1 EHL whereas three did not complete successfully at the BSE-2 EHL because of excessive lateral drifts. For the RSA design, the 
analysis successfully completed for all 14 records at the BSE-1 EHL whereas four did not complete successfully at the BSE-2 EHL because of excessive lateral drifts. Maximum axial compression force in the exterior column lines from the record set are shown previously in the linear assessment sections.

Figure 3-100 and Figure 3-101 show the performance of the link beams at the BSE-1 (LS BPL) and BSE2 (CP BPL) for the ELF and RSA design, respectively, in terms of a $D C R_{N}$. The $D C R_{N}$ results from the LSP, LDP, and NSP (loaded to the left) are included in the figures. Comparison discussions of the various procedures are addressed subsequently. As is evident in the figures, the ELF-designed frame performs noticeably better than the RSA-designed frame. The results indicate that several links in the RSA-designed frame do not satisfy the LS BPL acceptance criteria. Both the ELF- and RSA-designed frames perform markedly poorer at the BSE-2 EHL, with several links not satisfying the CP BPL acceptance criteria using both median and mean metric. The distribution of demands is somewhat similar between the two designs illustrating the effect of higher modes on the response. A $D C R_{N}$ value of 0.73 equates to a plastic rotation angle of 0.08 radians at the BSE-1 EHL (0.62 at the BSE-2 EHL).

The average ratio of secondary to primary component acceptance criteria for a wide-flange section with the configuration in this frame is 1.14 for the CP SPL (1.27 for the LS SPL). The figures for the ELF- and RSA-designed frame illustrate that this value is exceeded in a number of stories at the BSE-1 EHL and BSE-2 EHL. This highlights the rapid analytical progression towards a collapsed state when several components are strained past the deformation associated with their peak strength—see §3.1.4.2.

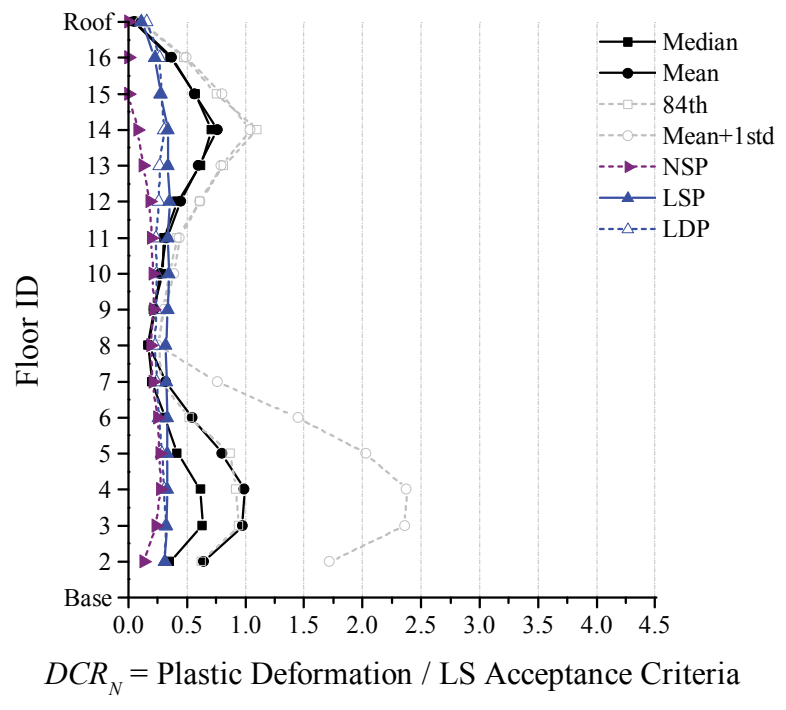

(a) ELF

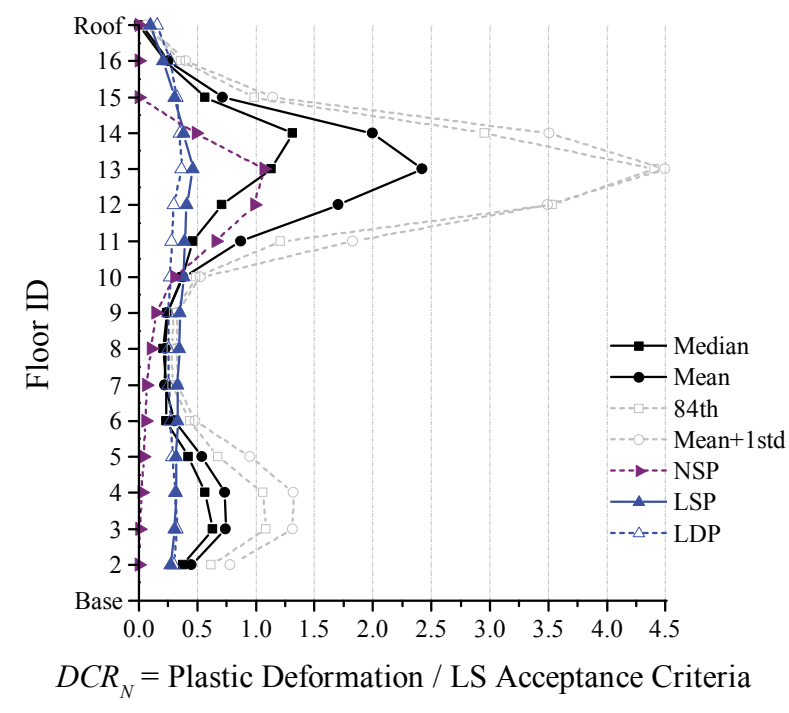

(b) RSA

Figure 3-100. NDP Assessment Results, Shear Hinges, 16-Story EBF, BSE-1 LS 


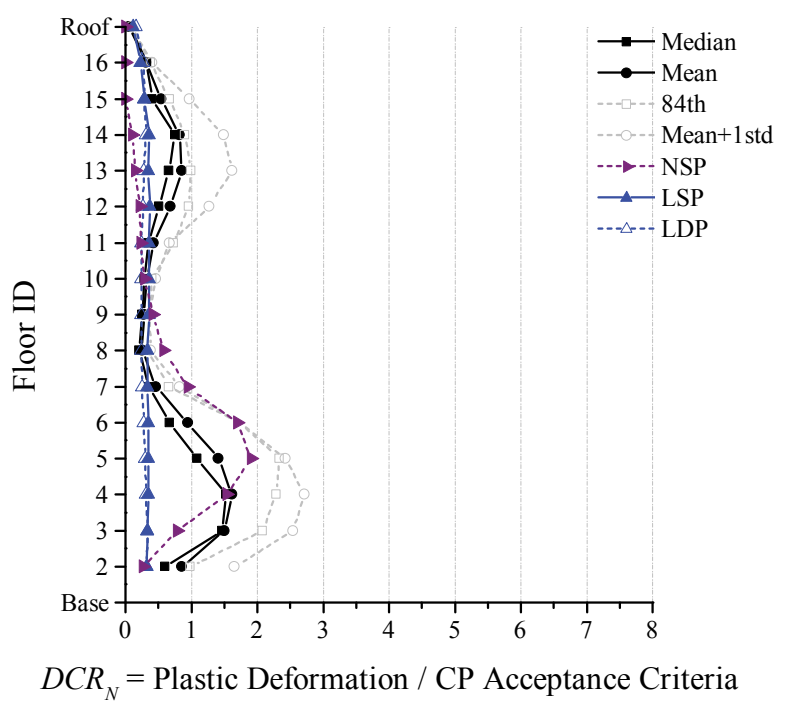

(a) ELF

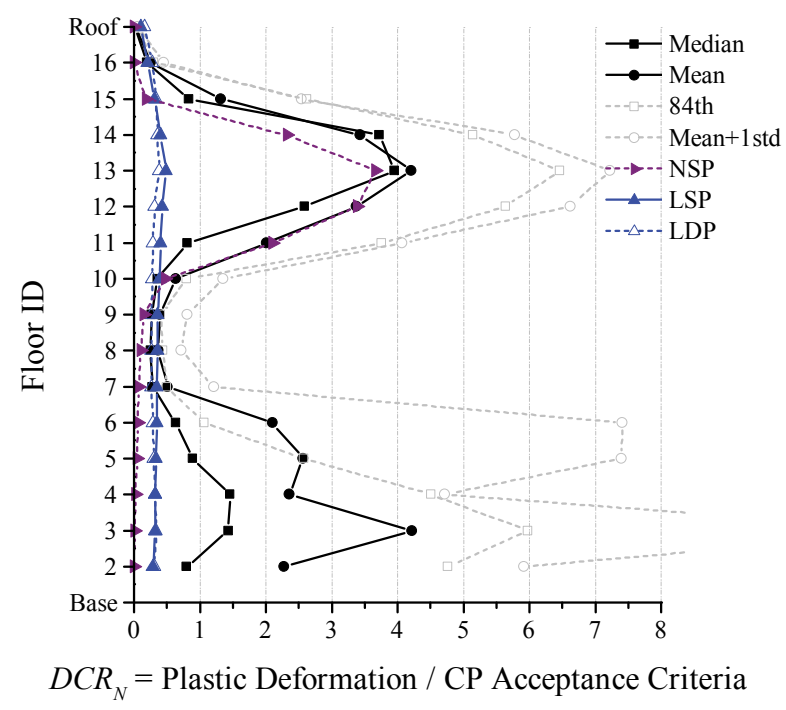

(b) RSA

Figure 3-101. NDP Assessment Results, Shear Hinges, 16-Story EBF, BSE-2 CP

Figure 3-102 and Figure 3-103 show the curvature ductility demand of the column hinges (i.e., section strength) at the BSE-2 EHL—see discussion on column hinge modeling in \$3.2.1.2. Figure 3-104 and Figure 3-105 show the elastic member strength interaction results at the BSE-2 EHL - see discussion on column strength modeling in $\$ 3.2 .1 .2$. Column hinges at the base above the brace connection experience inelastic strain demands (yield corresponds to a $D C R_{N}=1$ in the figures). The deformation demands are considerably lower than the primary CP acceptance criteria for a 'column'. Still, the base column hinges do not satisfy the criteria for the CP BPL for both the ELF- and RSA-designed frames. This effect is twofold: a consequence of modeling rotationally restrained base connections, which more closely characterizes how the base connection is detailed in these buildings than assuming rotationally unrestrained connections and, to a lesser extent, the minor increase in $P_{E}$ because the shear strength, $V_{C E}$, of the link beams was increased by 15 percent for nonlinear analysis - see $\$ 3.2 .1 .2$ and $\$ 3.2 .2 .1 .3$. There is also evidence of column hinging in stories other than at the base at the BSE-2 EHL, which is additionally a consequence of the strength loss experienced by the links in those stories.

The $D C R_{N}$ results for the LSP and LDP are based on an interaction equation and not from $M_{U D} / m \times M_{C E}$, or $M_{U F} / M_{C L}$, which would be a more physically consistent metric for comparison against the results from the nonlinear assessment procedures. Nonetheless, the linear results are generally applicable here because the exterior frame columns are force-controlled for flexure in the linear assessment procedures. Though there is a fundamental difference in how the $D C R_{N}$ is computed for the linear and nonlinear procedures, the linear assessment results show similar distributions of demands and location of potential performance concerns. 


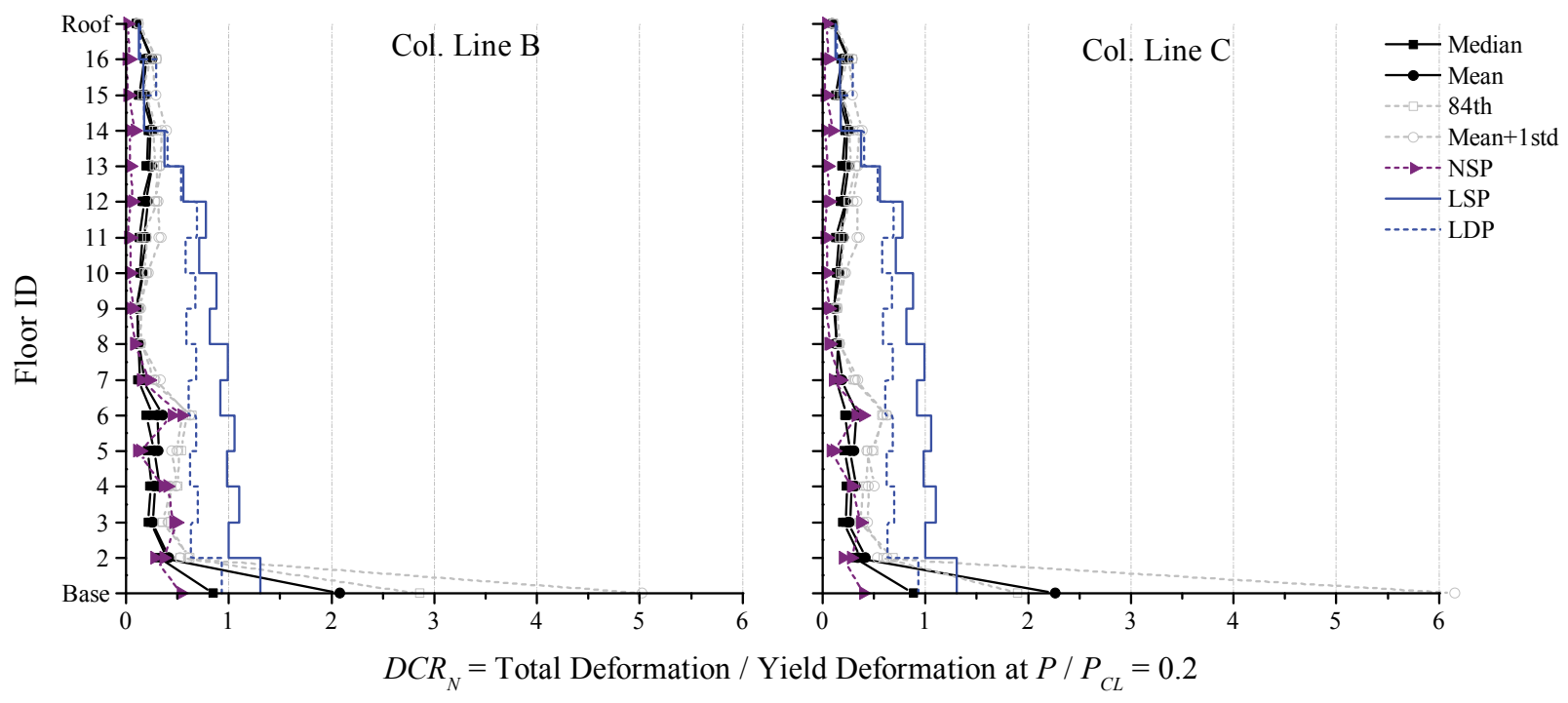

Figure 3-102. NDP Assessment Results, Column Hinges, 16-Story EBF ELF, BSE-2 Yield

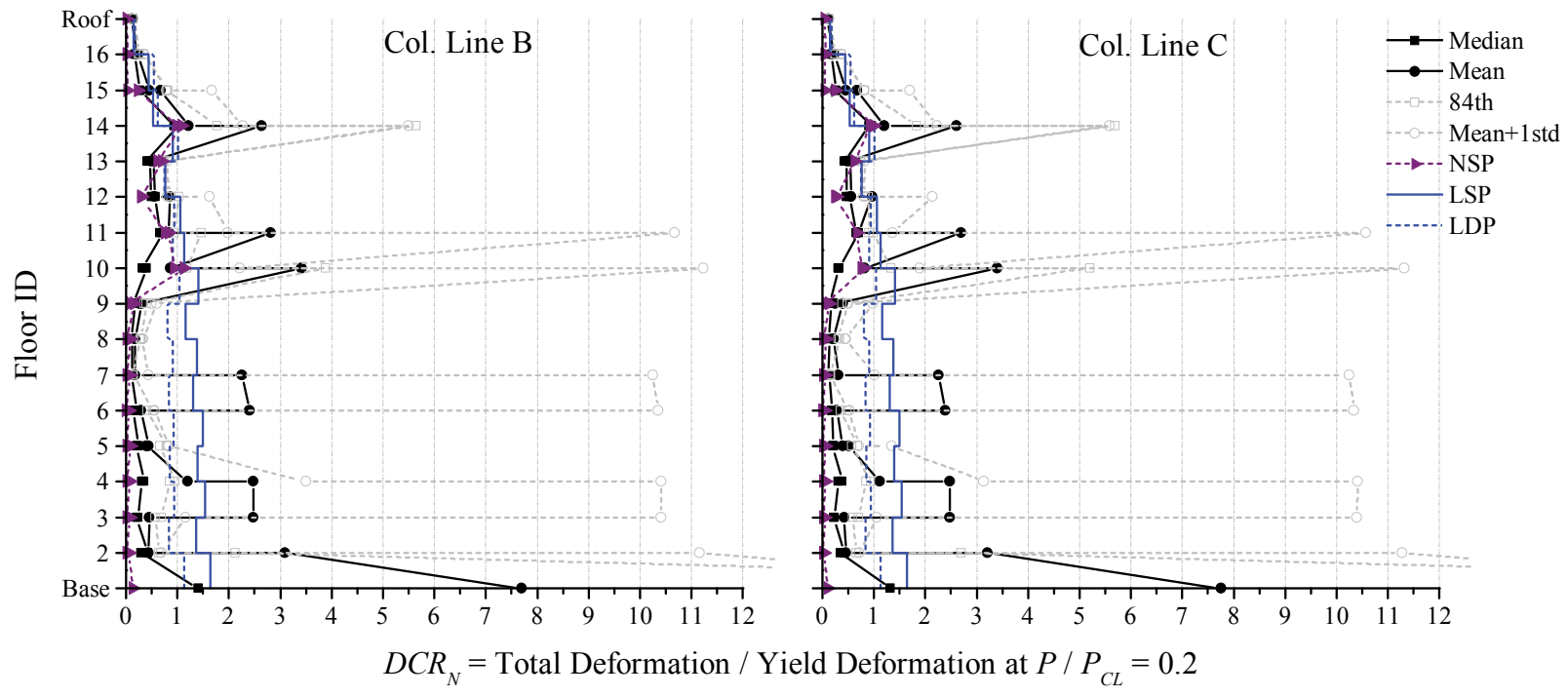

Figure 3-103. NDP Assessment Results, Column Hinges, 16-Story EBF RSA, BSE-2 Yield 


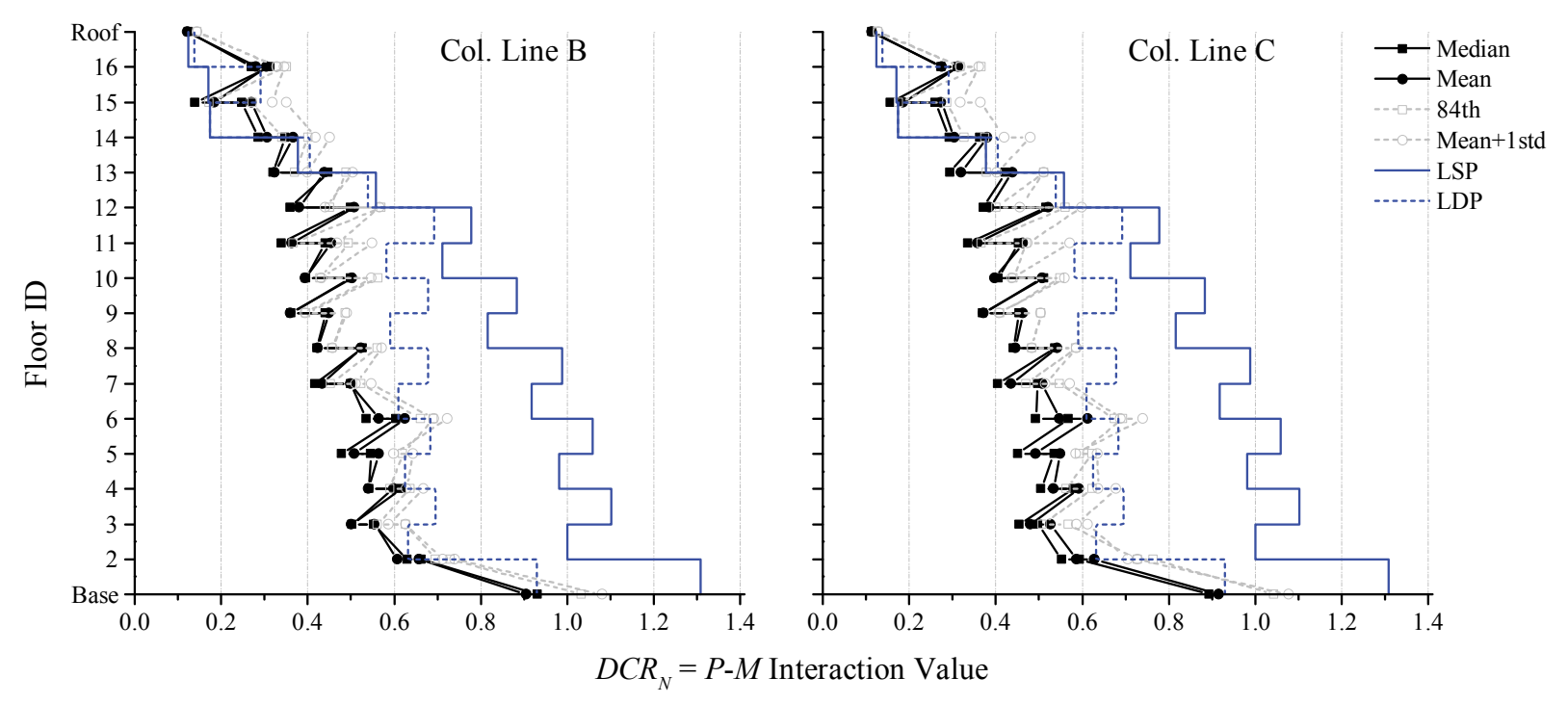

Figure 3-104. NDP Assessment Results, Column Members, 16-Story EBF ELF, BSE-2

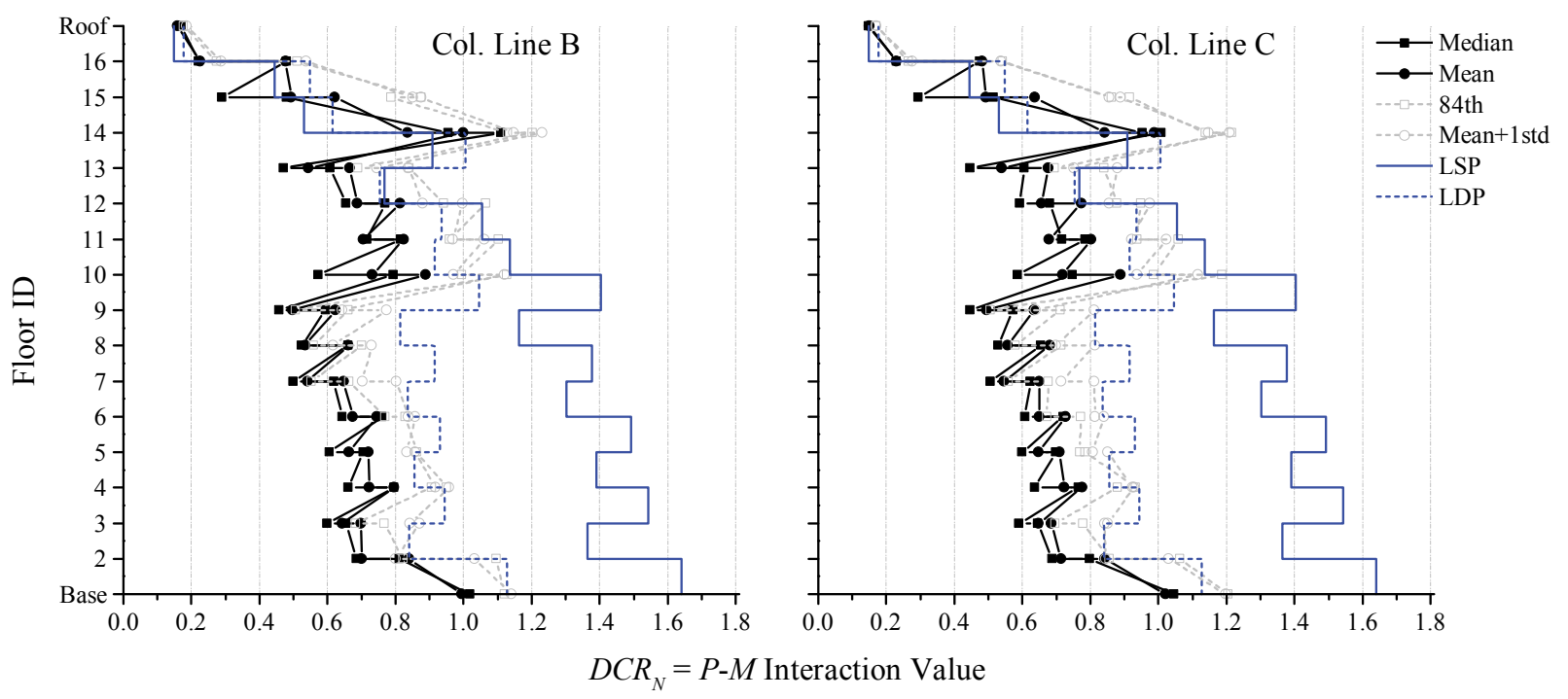

Figure 3-105. NDP Assessment Results, Column Members, 16-Story EBF RSA, BSE-2

Figure 3-106 and Figure 3-107 show the curvature ductility demand of the brace hinges (i.e., section strength) at the BSE-2 EHL— see discussion on column hinge modeling in \$3.2.1.2. Figure 3-108 and Figure 3-109 show the elastic member strength interaction results at the BSE-2 EHL- see discussion on column strength modeling in — see discussion on column strength modeling in §3.2.1.2. Brace hinges at the base above the brace connection experience inelastic strain demands (yield corresponds to a $D C R_{N}=1$ in the figures). The deformation demands are considerably lower than the primary CP acceptance criteria for a Column. Still, the brace hinges do not satisfy the criteria for the CP BPL for both the ELF- and RSAdesigned frames - see previous discussion on column results for additional information. 


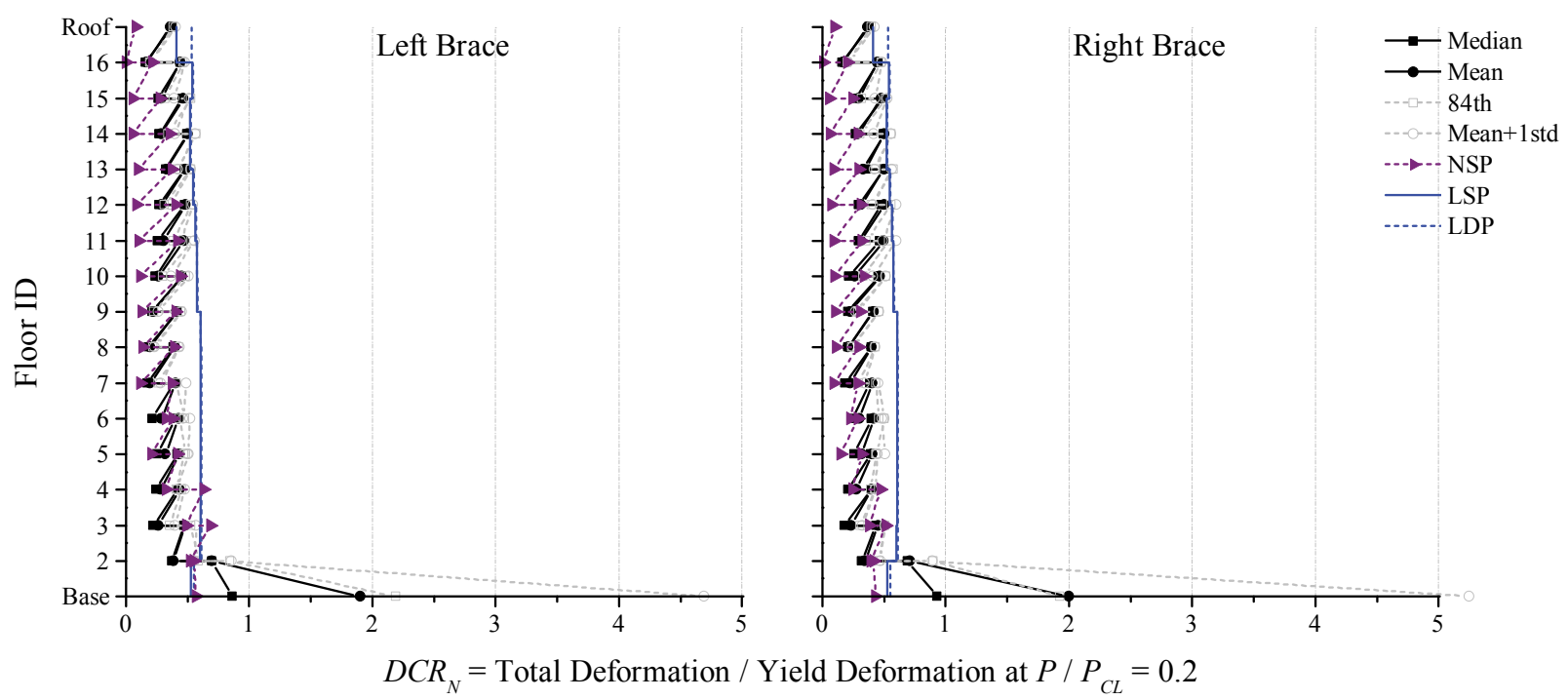

Figure 3-106. NDP Assessment Results, Brace Hinges, 16-Story EBF ELF, BSE-2 Yield

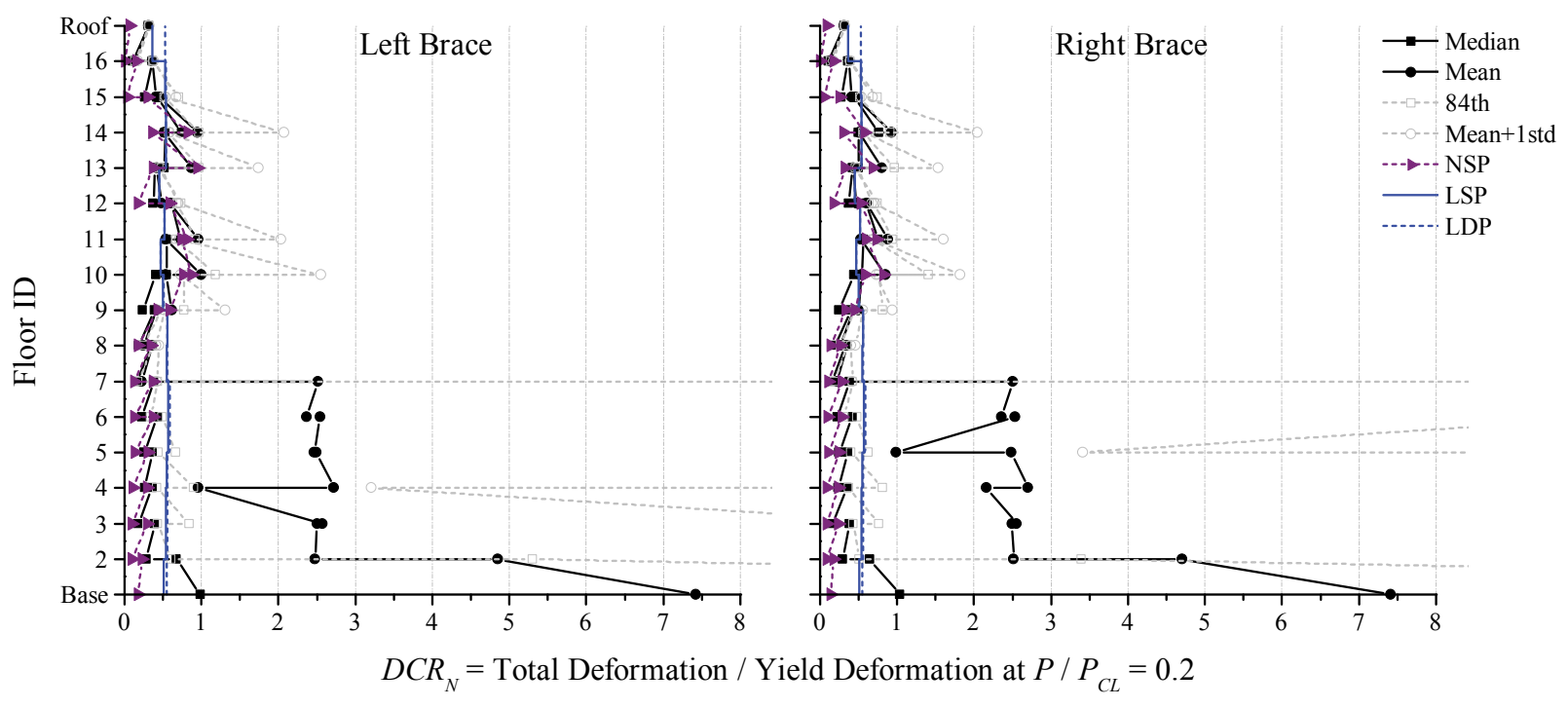

Figure 3-107. NDP Assessment Results, Brace Hinges, 16-Story EBF RSA, BSE-2 Yield 


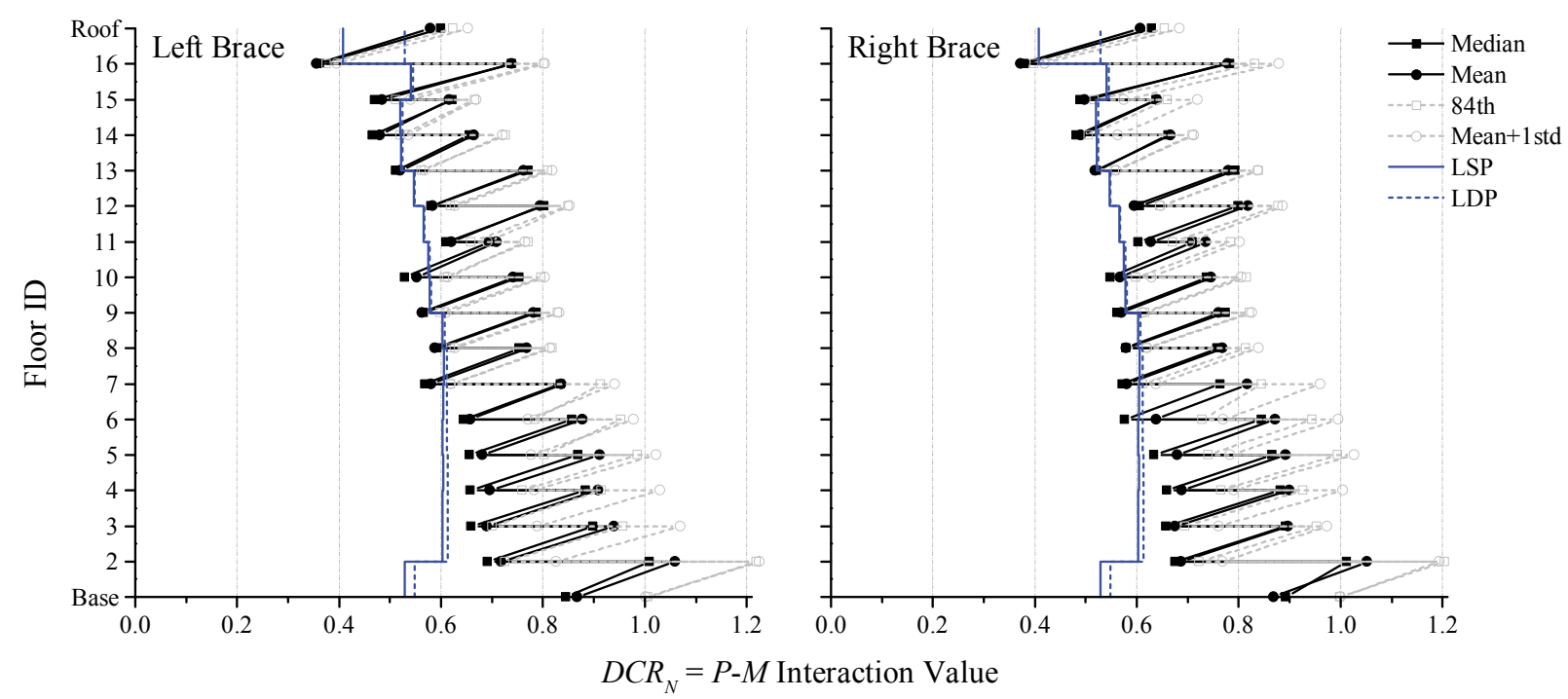

Figure 3-108. NDP Assessment Results, Brace Members, 16-Story EBF ELF, BSE-2

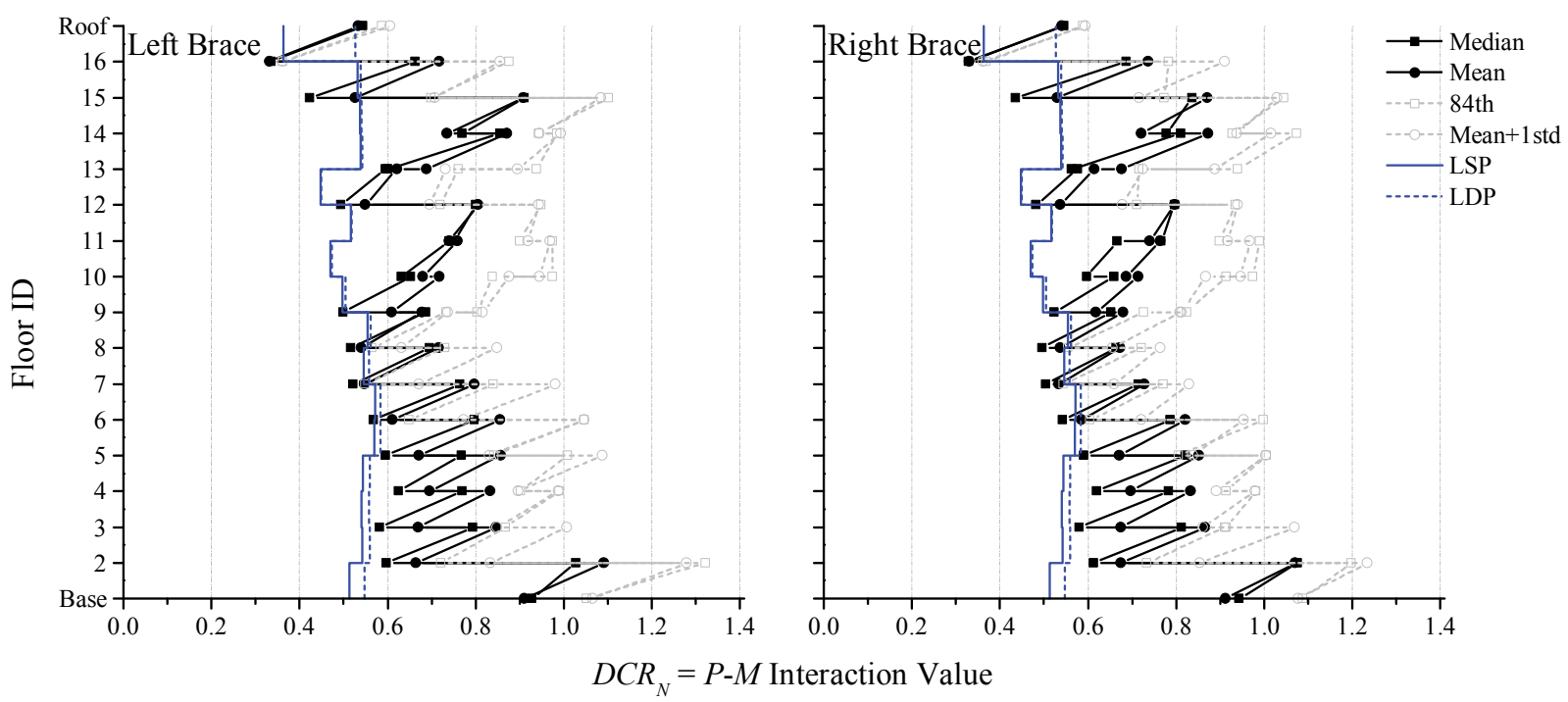

Figure 3-109. NDP Assessment Results, Brace Members, 16-Story EBF RSA, BSE-2 


\subsubsection{Seismic Assessment Discussion}

The discussion in this section focuses on the following component design actions:

- Link shear force

- Beam, column, and brace flexure within anticipated plastic hinge zones (section strength) ${ }^{23}$

- Column and brace axial-moment interaction strength (member strength)

ASCE 41 requires all frame components that do not satisfy the acceptance criteria to be retrofitted or replaced, even if only a small percentage of the total components fail the criteria (see ASCE $41 \S 5.5 .3 .5$ ). Therefore, a building can only satisfy a selected BPL when all structural components satisfy the corresponding SPL. Building behavior is rarely governed by the response of a single component, with the one notable exception being collapse resulting from failure of a column. It can be argued that a shortcoming of ASCE 41 is the focus on component performance to ensure that all elements pass the evaluation, when failures of individual elements may not lead to catastrophic failure. Further, ASCE 41 requires that all brace connections are force-controlled and does not provide generalized modeling parameters of a brace connection for nonlinear analysis as done for a "ductile" beam-to-column connection in a moment frame. As such, the performance of a brace-to-beam connection adjacent to a link beam, as well as a brace-tocolumn connection, is not considered in the assessment.

Design choices, constructability considerations, code requirements, nonseismic loads, analytical modeling assumptions, and other project specific requirements may add strength to critical components in a frame. Further, allocation of component strengths within the frame because of the lateral force distribution adopted in design can cause deviations of the component strengths from one story to another. Thus, capturing all possible permutations, in essence, would create an infinitely large design space. Nonetheless, the change in component strengths can significantly influence the $D C R_{N}$ values obtained from the ASCE 41 assessments.

\subsubsection{Linear Procedures}

The following discusses the analytical results for the noted components from the linear procedures for each archetype building.

\subsection{Link Beams}

Table 3-20 provides a summary of the performance of the shear links for each linear assessment procedure and each BPL. The link beams consistently satisfy the performance criteria for the LS and CP BPL for both linear procedures. The $D C R_{N}$ values all remained relatively low, $0.2-0.6$, indicating potentially that either (1) the design criteria for new EBFs introduces significant member overstrength in reference to the acceptance criteria, (2) the acceptance criteria in ASCE 41 is overly generous for short link beams, or (3) a combination thereof.

\footnotetext{
${ }^{23}$ All components other than link beams are force-controlled per ASCE 41 §5.5.3.4. Brace connections are not assessed in this study because they are designed to resist forces computed using the maximum strengths of the brace.
} 
Table 3-20. Performance Summary of Shear Links (SL) per Frame, Linear Procedures

\begin{tabular}{|c|c|c|c|c|c|}
\hline \multirow{2}{*}{ Archetype } & \multirow{2}{*}{ Design } & \multicolumn{2}{|c|}{ LSP } & \multicolumn{2}{|c|}{ LDP } \\
\hline & & LS & $\mathrm{CP}$ & LS & $\mathrm{CP}$ \\
\hline \multirow{2}{*}{ 4-Story } & ELF & All SL Pass & All SL Pass & All SL Pass & All SL Pass \\
\hline & RSA & All SL Pass & All SL Pass & All SL Pass & All SL Pass \\
\hline \multirow{2}{*}{ 8-Story } & ELF & All SL Pass & All SL Pass & All SL Pass & All SL Pass \\
\hline & RSA & All SL Pass & All SL Pass & All SL Pass & All SL Pass \\
\hline \multirow{2}{*}{ 16-Story } & ELF & All SL Pass & All SL Pass & All SL Pass & All SL Pass \\
\hline & RSA & All SL Pass & All SL Pass & All SL Pass & All SL Pass \\
\hline
\end{tabular}

As a side note, Table 3-21 summarizes the effects of design (AISC 341) and assessment (ASCE 41) provisions on the $D C R_{N}$ for a link beam at $2 / 3 \times \mathrm{MCE}_{\mathrm{R}}$ for the LS BPL. On the component capacity side, the shear yield strength prescribed in ASCE 41, $V_{C E}$, and AISC 341, $V_{n}$, have the same underlying equation; differences arise when using nominal versus expected material properties and a strength reduction factor, $\phi_{v}=0.9$, resulting in a capacity ratio (ASCE $41 /$ AISC 341) of 1.22. On the component demand side, assuming an eigenvalue analysis is conducted, ASCE 41 allows the use of the period determined from analysis, $T_{\text {analysis }}$, whereas ASCE 7 will limit this period to $C_{u} T_{a}$ when greater than. If this limit is triggered, the demand in the link beams will generally increase for design resulting in a demand ratio (ASCE $41 /$ ASCE 7) less than unity. Additionally, ASCE 41 effectively divides the demand by $m$ ( $=9$ for a link beam) while ASCE 7 divides the demand by $R(=8$ for an EBF). Thus, for a link beam designed per ASCE 7 and its referenced standards whose design strength exactly equals the demand, the corresponding $D C R_{N}$ for the linear assessment will be less than or equal to 0.72 . This value increases to 0.76 for the CP SPL at the BSE2. Conversely, a link beam that exactly satisfies the ASCE 41 assessment criteria will have a demand equal to 1.4 times the design strength-larger than what would be comfortably accepted in practice. This indicates an area where ASCE 41 linear assessment procedures are potentially unconservative.

Table 3-21. Effect of Design and Assessment Provisions on $D C R_{N}$ for LS at BSE-1

\begin{tabular}{lcccc}
\hline & \multicolumn{2}{c}{ Demand } & Capacity & Effect on $D C R_{N}$ \\
\hline a) ASCE 41 & $S_{a}$ at $T=T_{\text {analysis }}$ & $1 / m=1 / 9$ & $V_{C E}=0.6\left(1.1 F_{y}\right) A_{w}$ & \\
b) ASCE7 and AISC 341 & $\begin{array}{c}S_{a} \text { at } T= \\
\min \left[T_{\text {analysis }}, C_{u} T_{a}\right]\end{array}$ & $1 / R=1 / 8$ & $\phi_{v} V_{n}=0.9\left(0.6 F_{y} A_{w}\right)$ & $(\# \leq 1.0)(0.88)$ \\
& $a / b \leq 1.0$ & $a / b=0.8 \overline{8}$ & $a / b=1.1 / 0.9=1.2 \overline{2}$ & $\therefore D C R_{N} \leq 0.72$ \\
Ratio (a/b) & & & \\
\hline
\end{tabular}

In addition to the above performance observations, the effects of additional strengthening of link beams in design should be recognized. For instance, specific link beams may require additional strength above that required for seismic demands when wind effects govern the elastic design. AISC 360 prescribes a slightly different shear strength, $\phi_{v} V_{n}$, than AISC 341. This situation occurs in the lower stories of the 16-story frames (see Appendix B). Also, link rotation limits as well as section compactness limits played a role in member selection. The cumulative effects of these requirements resulted in additional strength in specific link beams, which can further reduce the $D C R_{N}$ values obtained from an ASCE 41 assessment. ASCE 41 requires modification of the modelling parameters and acceptance criteria when link beams do not satisfy the AISC 341 criteria - see footnotes for ASCE 41 Table 5-6. More comprehensive guidance for assessment of these link beams would allow for a greater range of retrofit options. The linear assessment procedures also address neither the shear-moment interaction as the link length varies from long to short nor the axialmoment interaction, both of which could further impact the $D C R_{N}$. 


\subsection{Member Cross-section Strength (flexural hinge) and Global Strength (member stability)}

Table 3-22 provides a summary of the performance of the column members for each linear assessment procedure and each BPL. The number listed in parentheses denotes the number of failed components in the frame-shown as bolded text. Also shown in the table is the percentage of failed components compared to the total number of similar components. Recall that section strength and member strength of a column is combined into a single $P$ - $M$ interaction equation for linear assessment procedures in ASCE 41 (see ASCE 41 Equations 5-10, 5-11, and 5-12.) Consequently, identifying an efficient retrofit option for a column can be challenging because understanding and isolating the failure mechanism of the column can be difficult. Recall from previous discussions that all members other than the link beam in an EBF are force-controlled per ASCE 41. In applying ASCE $41 \S 3.4 .2$ to compute $P_{U F}$ and $M_{U F}, J$ was taken as the minimum DCR (as defined by ASCE 41) from all link beams at or above the column under investigation.

Table 3-22. Performance Summary of Column Members (CM) per Frame, Linear Procedures

\begin{tabular}{|c|c|c|c|c|c|}
\hline \multirow{2}{*}{ Archetype } & \multirow{2}{*}{ Design } & \multicolumn{2}{|c|}{ LSP } & \multicolumn{2}{|c|}{ LDP } \\
\hline & & LS & $\mathrm{CP}$ & LS & $\mathrm{CP}$ \\
\hline \multirow{2}{*}{ 4-Story } & ELF & All CM Pass & All CM Pass & All CM Pass & All CM Pass \\
\hline & RSA & All CM Pass & All CM Pass & All CM Pass & All CM Pass \\
\hline \multirow{2}{*}{ 8-Story } & ELF & $\begin{array}{c}\text { Ext CM Fail (2) } \\
13 \%\end{array}$ & $\begin{array}{c}\text { Ext CM Fail (2) } \\
13 \%\end{array}$ & All CM Pass & All CM Pass \\
\hline & RSA & $\begin{array}{c}\text { Ext CM Fail (6) } \\
38 \%\end{array}$ & $\begin{array}{c}\text { Ext CM Fail (6) } \\
38 \%\end{array}$ & $\begin{array}{c}\text { Ext CM Fail (2) } \\
13 \%\end{array}$ & $\begin{array}{c}\text { Ext CM Fail (2) } \\
13 \%\end{array}$ \\
\hline \multirow{2}{*}{ 16-Story } & ELF & All CM Pass & Ext CM Fail (6) & All CM Pass & All CM Pass \\
\hline & RSA & $\begin{array}{c}\text { Ext CM Fail (6) } \\
19 \%\end{array}$ & $\begin{array}{c}\text { Ext CM Fail (22) } \\
69 \%\end{array}$ & All CM Pass & $\begin{array}{c}\text { Ext CM Fail (6) } \\
19 \%\end{array}$ \\
\hline
\end{tabular}

Several columns do not satisfy the assessment criteria using the interaction equation because of high axial force and moment in the 8-story and 16-story archetype buildings. The first floor columns consistently have the most difficulty satisfying the assessment criteria because of high axial load combined with significant flexural demand because of the fixity assigned to the column-to-base connection. ASCE 41 does not provide guidance on how to assess column-to-base connections similar to beam-to-column connections in a moment frame-albeit they are force-controlled. In reality, it is likely that the flexural demand will not be as great (as that computed in the analysis) at large deformations because some of the fixity would be relieved because of bolt elongation, weld fracture, and/or gusset plate damage. This relief would effectively reduce some of the moment demand, reducing the likelihood of a flexural hinge being formed. However, the alternative is to model the base connection as pinned, which would not capture the rigidity of the columnto-base connection assumed in design. In this study the base connections are maintained as fixed throughout the analysis, thus being conservative on the treatment of the flexural demand.

As noted previously, the force distribution prescribed in the LSP is one of the main factors in producing high column forces. However, the estimated axial load demands in the columns in many cases are the least conservative approximations, as compared to more rigorous analysis procedures (see axial load figures (e.g., Figure 3-60)). Regardless, there are many columns that pass the assessment criteria. It is unclear whether certain upper story columns fail by instability or section hinging due to the adopted interaction 
equation. Additionally, it is difficult to assess column failures indicated by interaction results barely over 1.0 due to the uncertainties in the analysis procedures and conservatisms in the assessment provisions. ASCE 41 would benefit from decoupling the single interaction curve for member stability and section strength into two separate interaction equations as done in AISC ASD Chapter N, Plastic Design (AISC 1989). Decoupling the stability and strength would provide a clearer picture of potential retrofit schemes for frame columns. Nonetheless, some member stability equations were derived from beam-columns test results where column ends did not translate relative to each other. Future research is required to justify updated interaction equations for assessment of beam-columns with ASCE 41, as well as a critical examination of the acceptance criteria in regard to experimental test results.

In addition to the above performance observations, the effects of additional strengthening of columns in design should be recognized. Several link beams in the 8- and 16-story ELF-designed frames were controlled by link rotation requirements prescribed in AISC 341. Because the link rotations are tied to the design story drift in ASCE 7 that was in turn driven by a global flexural deformation mode, the lower-level frame columns were upsized to limit these rotations and satisfy the requirements. The RSA-designed frames were not affected by this as significantly as the ELF-designed frames were, because of differences in the lateral load distributions resulting in less global flexural deformations. Regardless, this effort still did not result in all base columns passing the acceptance criteria.

Recall that section strength and member strength of a column is combined into a single $P-M$ interaction equation for linear assessment procedures in ASCE 41 (see ASCE 41 Equations 5-10, 5-11, and 5-12.) Consequently, identifying an efficient retrofit option for a column can be challenging because understanding and isolating the failure mechanism of the column can be difficult. Recall from previous discussions that all members other than the link beam in an EBF are force-controlled per ASCE 41. In applying ASCE $41 \S 3.4 .2$ to compute $P_{U F}$ and $M_{U F}, J$ was taken as the minimum DCR (as defined by ASCE 41) from all link beams at or above the column under investigation.

Table 3-23 provides a summary of the performance of the brace members for each linear assessment procedure and each BPL. The number listed in parentheses denotes the number of failed components in the frame - shown as bolded text. Recall that section strength and member strength of a column is combined into a single $P-M$ interaction equation for linear assessment procedures in ASCE 41 (see ASCE 41 Equations 5-10, 5-11, and 5-12.) Consequently, identifying an efficient retrofit option for a column can be challenging because understanding and isolating the failure mechanism of the column can be difficult. Recall from previous discussions that all members other than the link beam in an EBF are force-controlled per ASCE 41. In applying ASCE $41 \S 3.4 .2$ to compute $P_{U F}$ and $M_{U F}, J$ was taken as the minimum DCR (as defined by ASCE 41) from the adjacent link in the story under investigation. Brace members consistently satisfy the performance criteria for the LS and CP BPL for both linear procedures.

ASCE 41 \$5.5.3.4.2 requires design actions in EBF columns and braces to be computed in accordance with ASCE 41 \$3.4.2.1.2. This assessment criterion is further expounded in ASCE 41 \$5.5.3.4.2, which states that the columns and braces should be assessed assuming that every link has fully yielded and strainhardened, resulting in a force of $1.25 \times V_{C E}$. This requirement originated from an earlier edition of the AISC seismic provisions (now AISC 341) for design of new buildings. Consequently, the columns and braces in the archetype buildings have been capacity-designed. Therefore, these members (by default) should pass 
the force-controlled assessment criteria in ASCE 41 and thus should yield essentially equivalent results as design (and not require an ancillary assessment). However, this does not occur because of variations between ASCE 41 and AISC 341 provisions—-see §3.2.2.2.1.1.

Table 3-23. Performance Summary of Brace Members (BM) per Frame, Linear Procedures

\begin{tabular}{|c|c|c|c|c|c|}
\hline \multirow{2}{*}{ Archetype } & \multirow{2}{*}{ Design } & \multicolumn{2}{|c|}{$\overline{\mathrm{LSP}}$} & \multicolumn{2}{|c|}{$\overline{\mathrm{LDP}}$} \\
\hline & & LS & $\mathrm{CP}$ & LS & $\mathrm{CP}$ \\
\hline \multirow{2}{*}{ 4-Story } & ELF & All BM Pass & All BM Pass & All BM Pass & All BM Pass \\
\hline & RSA & All BM Pass & All BM Pass & All BM Pass & All BM Pass \\
\hline \multirow{2}{*}{ 8-Story } & ELF & All BM Pass & All BM Pass & All BM Pass & All BM Pass \\
\hline & RSA & All BM Pass & All BM Pass & All BM Pass & All BM Pass \\
\hline \multirow{2}{*}{ 16-Story } & ELF & All BM Pass & All BM Pass & All BM Pass & All BM Pass \\
\hline & RSA & All BM Pass & All BM Pass & All BM Pass & All BM Pass \\
\hline
\end{tabular}

ASCE 41 does not provide specific guidance on how to perform the analysis prescribed in ASCE 41 $\$ 5.5 .3 .4 .2$. It is assumed that this provision is intended to provide guidance on implementing ASCE 41 $\S 3.4 .2 .1 .2-1$. As such, the method outlined in AISC $341 \S \mathrm{F} 3.3$ is adopted in this study. Accordingly, it is assumed that the flexural forces from seismic drift resulting in fully yielded links can be ignored, per AISC $341 \S$ F3.3 exception (2). This exception is interpreted to mean that the flexural forces in the columns and braces caused by the application of $1.25 \times V_{C E}$ at each link can be neglected. As such, the acceptance check on the braces and columns is based primarily on the axial load demand. Presumably, a capacity analysis as outlined in AISC 341 gives results that match closely with the maximum expected demands, although the conservatism of assuming simultaneous yielding of all links may be too stringent. Regardless of the approach, it is recommended that additional guidance be provided to ensure proper application of the provisions.

In contrast to using the capacity design approach discussed above, ASCE 41 \$3.4.2.1.2-2 permits an alternative method to compute force-controlled design actions (i.e., $P_{U F}$ and $M_{U F}$ ) by dividing the earthquake demand by the force-delivery reduction factor, $J$. In so doing, $J$ can be taken as the minimum $D C R$ of the components (i.e., link beams) delivering force to the columns and braces. It is assumed that this method does not remove the capacity design requirement described above. However, ASCE 41 is not clear in this regard and needs improvement in the analysis guidance for force-controlled demands. Consequently, assessment of the force-controlled components in this study is performed in accordance with this method (ASCE 41 33.4.2.1.2-2) for comparison purposes between the two methods.

The 8-story ELF-designed EBF presents a good example. Figure 3-110 illustrates the variation in $D C R_{N}$ values for the columns and braces using three different approaches. The easiest and most conservative approach is to adopt $J=2.0$ (assuming high seismicity), with the corresponding $D C R_{N}$ values shown in (a). Alternatively, $J$ can be set to the minimum DCR, as discussed previously, in an attempt to compute a more realistic demand and embody a capacity-design philosophy. The corresponding $D C R_{N}$ values are shown in (b). Finally, in lieu of (a) and (b), a capacity-design approach is used and the corresponding $D C R_{N}$ values are shown in (c) - flexural forces form seismic drift is neglected. Furthermore, it is interesting to note that (b) and (c) in this case give reasonably similar results for the braces and lower-level columns $-P_{U F}$ is slightly greater in (b) than in (c), see Figure 3-54. 


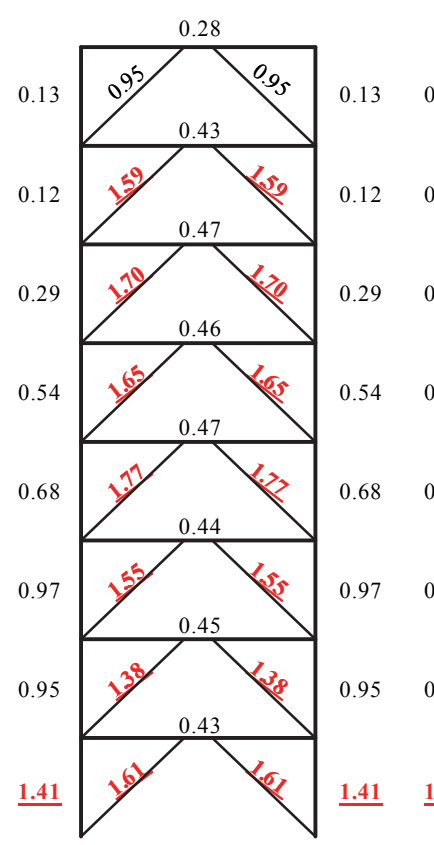

(a) $J=2$

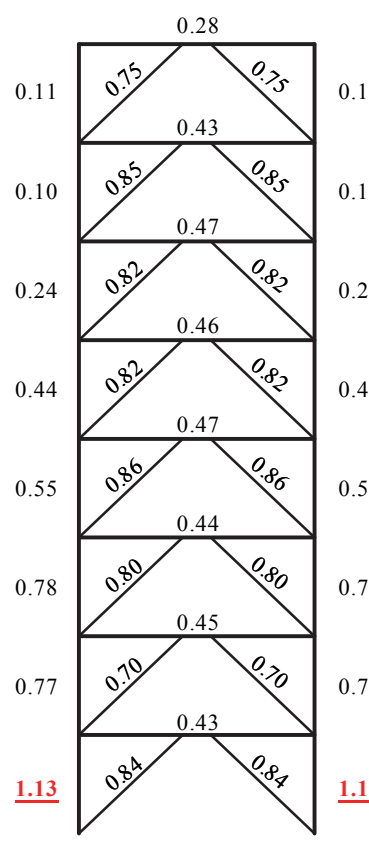

(b) $J=\min (\mathrm{DCR})$

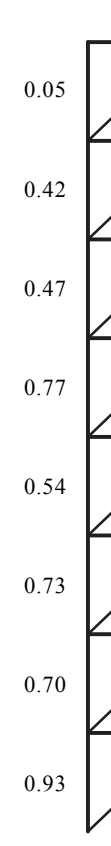

(c) capacity analysis

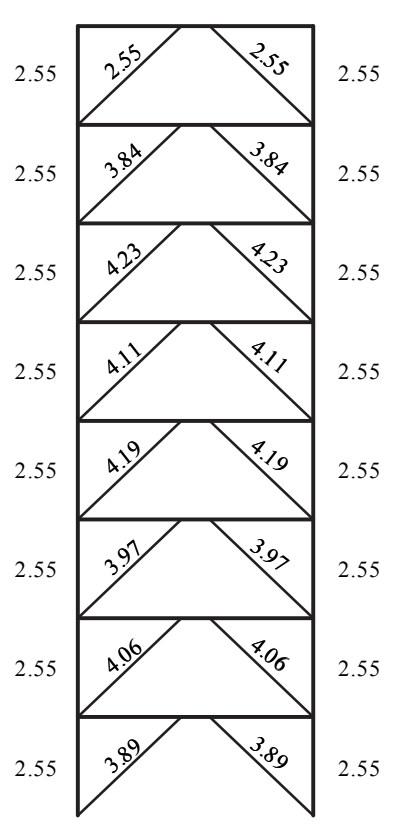

(d) $J$-factor for (b)

Figure 3-110. $D C R_{N}$ values for EBF columns and braces using LSP, BSE-1, LS BPL

\subsection{Summary}

Table 3-24 summarizes the performance of the archetype buildings in reference to the BSO for both linear procedures. The LDP consistently results in lower $D C R_{N}$ values than the LSP for both the ELF- and RSAdesigned frames for all archetype buildings, an indication that a more accurate distribution of seismic demands (based on elastic modes) is better captured in taller frames. However, assessment of the RSAdesigned frame consistently illustrates inferior performance using the LSP compared to the LDP because of the variation between the distribution of seismic demands and the allocation of component strengths within the frame. This variation is not as substantial when assessing the ELF-designed frame with the LDP. Moreover, the lateral force distribution in the LSP does not capture higher modes well, leading to conservative estimates of column forces in the taller frames. This can be problematic for beam-columns due to the lower-bound estimate of compressive strength, $P_{C L}$.

Table 3-25 provides the base shears computed with the linear assessment procedures. Column performance (primarily at the base) from both assessment procedures controls the overall assessment of the frames. However, in this analytical context, base column failure is not detrimental to the overall structural performance unless total strength loss of the column or adjacent braces occurs. As noted above, additional research is needed on assessment criteria for columns. The qualitative ratings are assigned primarily based on the performance of the column members. In the end, the 8- and 16-story ELF-designed frames satisfy the seismic performance objective using only the LDP (recall from Chapter 2 that the ELF procedure is not 
permitted for design of the 16-story EBF), with the 4-story frames satisfying the criteria using both linear procedures - a corollary of the two frame designs being nearly the same.

The assessment results from the LSP and LDP illustrate that, on average, the ELF-designed EBF performs better than the RSA-designed EBF for all archetype buildings. This result can be attributed to the increased strength and stiffness provided to the ELF-designed frames (see Table 2-5) by differences in the ELF and RSA procedures, including associated scaling provisions, in ASCE 7.

Table 3-24. BSO Performance Summary of Archetype Buildings, Linear Procedures

\begin{tabular}{|c|c|c|c|c|c|c|c|c|}
\hline \multirow{2}{*}{ Archetype } & \multirow{2}{*}{ Design } & \multicolumn{3}{|c|}{ LSP } & \multirow{2}{*}{ Design } & \multicolumn{3}{|c|}{ LDP } \\
\hline & & Link & $\mathrm{CM}$ & BM & & Link & $\mathrm{CM}$ & BM \\
\hline \multirow{2}{*}{ 4-Story } & ELF & Pass & Pass & Pass & ELF & Pass & Pass & Pass \\
\hline & RSA & Pass & Pass & Pass & RSA & Pass & Pass & Pass \\
\hline \multirow{2}{*}{ 8-Story } & ELF & Pass & Fail & Pass & ELF & Pass & Pass & Pass \\
\hline & RSA & Pass & Fail & Pass & RSA & Pass & Fail & Pass \\
\hline \multirow{2}{*}{ 16-Story } & ELF & Pass & Fail & Pass & ELF & Pass & Pass & Pass \\
\hline & RSA & Pass & Fail & Pass & RSA & Pass & Fail & Pass \\
\hline
\end{tabular}

The LDP consistently results in lower $D C R_{N}$ values than the LSP for both the ELF- and RSA-designed frames for all archetype buildings, an indication that a more accurate distribution of seismic demands (based on elastic modes) is better captured in taller frames. However, assessment of the RSA-designed frame consistently illustrates inferior performance using the LSP compared to the LDP because of the variation between the distribution of seismic demands and the allocation of component strengths within the frame. This variation is not as substantial when assessing the ELF-designed frame with the LDP. Moreover, the lateral force distribution in the LSP does not capture higher modes well, leading to conservative estimates of column forces in the taller frames. This can be problematic for beam-columns due to the lower-bound estimate of compressive strength, $P_{C L}$.

Table 3-25. Summary of Base Shears, Linear Procedures (kips)

\begin{tabular}{cccccccc}
\hline \multirow{2}{*}{ EHL } & Routine & ELF & RSA & ELF & RSA & ELF & RSA \\
\hline \multirow{3}{*}{ BSE-1 } & LSP & 2809 & 2819 & 2953 & 2548 & 4117 & 3351 \\
& LDP & 2433 & 2498 & 2893 & 2512 & 4195 & 3765 \\
& Ratio & 0.87 & 0.89 & 0.98 & 0.99 & 1.02 & 1.12 \\
& LSP & 4213 & 4229 & 4430 & 3821 & 6176 & 5027 \\
BSE-2 & LDP & 3708 & 3748 & 4339 & 3769 & 6292 & 5647 \\
& Ratio & 0.88 & 0.89 & 0.98 & 0.99 & 1.02 & 1.12 \\
\hline
\end{tabular}

Analytical results based on component-level performances obtained from the LSP and LDP suggest that some long-period eccentrically braced frames designed in accordance with ASCE 7 and its referenced standards have difficulty achieving the selected seismic performance objective of an existing building intended to be equivalent to a new building. This notion is driven by the performance of the columns. Conversely, results from the linear procedures can be enhanced by more mechanistically consistent column provisions and analytical modeling parameters. 


\subsubsection{Nonlinear Procedures}

The following discusses the analytical results for the noted components from the nonlinear procedures for each archetype building.

As noted previously, the NSP is permitted for the 8- and 16-story frames but requires supplemental verification using the LDP. The following summaries for the NSP reflect results only from the NSP (see previous for the linear verification using the LDP). For the NDP, results are mainly discussed in reference to the mean response from the set of records except where noted otherwise.

\subsection{Link Beams}

Table 3-26 provides a summary of the performance of the shear links for each nonlinear assessment procedure and each BPL. The number listed in parentheses denotes the number of failed components in the frame - shown as bolded text. Also shown in the table is the percentage of failed components compared to the total number of similar components. Contrary to the results from the linear procedures, the link beams consistently do not satisfy the performance criteria for the LS and CP BPL for both nonlinear procedures. Although the median response is less biased by a collapsed state of a given frame than the mean response, the results are, in general, marginally improved for the NDP. Additionally, the NDP consistently indicates poorer performance of the link beams than that given by the NSP.

Table 3-26. Performance Summary of Shear Links (SL) per Frame, Nonlinear Procedures

\begin{tabular}{|c|c|c|c|c|c|}
\hline \multirow[t]{2}{*}{ Archetype } & \multirow[t]{2}{*}{ Design } & \multicolumn{2}{|c|}{ NSP } & \multicolumn{2}{|c|}{$\begin{array}{c}\mathrm{NDP} \\
\text { (based on mean response of record set) }\end{array}$} \\
\hline & & LS & $\mathrm{CP}$ & LS & $\mathrm{CP}$ \\
\hline \multirow{2}{*}{ 4-Story } & ELF & All SL Pass & All SL Pass & All SL Pass & $\begin{array}{l}\text { Link Fail (2) } \\
50 \%\end{array}$ \\
\hline & RSA & All SL Pass & $\begin{array}{c}\text { Link Fail (1) } \\
25 \%\end{array}$ & All SL Pass & $\begin{array}{c}\text { Link Fail (3) } \\
\quad 75 \%\end{array}$ \\
\hline \multirow{2}{*}{ 8-Story } & ELF & All SL Pass & $\begin{array}{l}\text { Link Fail (3) } \\
\quad 38 \%\end{array}$ & All SL Pass & $\begin{array}{l}\text { Link Fail (5) } \\
\quad 63 \%\end{array}$ \\
\hline & RSA & $\begin{array}{c}\text { Link Fail (2) } \\
\quad 25 \%\end{array}$ & $\begin{array}{l}\text { Link Fail (4) } \\
\quad 50 \%\end{array}$ & $\begin{array}{l}\text { Link Fail (5) } \\
\quad 63 \%\end{array}$ & $\begin{array}{l}\text { Link Fail (6) } \\
\quad 75 \%\end{array}$ \\
\hline \multirow{2}{*}{ 16-Story } & ELF & All SL Pass & $\begin{array}{c}\text { Link Fail (3) } \\
19 \%\end{array}$ & All SL Pass & $\begin{array}{c}\text { Link Fail (5) } \\
\quad 31 \%\end{array}$ \\
\hline & RSA & $\begin{array}{c}\text { Link Fail (2) } \\
13 \%\end{array}$ & $\begin{array}{c}\text { Link Fail (4) } \\
25 \%\end{array}$ & $\begin{array}{c}\text { Link Fail (3) } \\
19 \%\end{array}$ & $\begin{array}{l}\text { Link Fail (10) } \\
\quad 63 \%\end{array}$ \\
\hline
\end{tabular}

The distribution of link beam failures in the NDP illustrates the effect of higher modes on the frames. Some of the higher mode periods for these archetype buildings fall directly in the constant acceleration region of the spectrum where input energy is the highest - see Appendix A. In fact, the second mode produced the highest modal base shear in design of the 8- and 16-story frames. In reference to design, the design force distribution in the ELF and RSA procedure is potentially inadequate when frames exhibit increased higher mode participation and a high level of nonlinearity. Additional discussion regarding the NSP and NDP is provided subsequently in the summary section. 


\subsection{Member Cross-section Strength (flexural hinge) and Global Strength (member stability)}

Table 3-27 provides a summary of the performance of the column hinges for each nonlinear assessment procedure for the CP BPL. The number listed in parentheses denotes the number of failed components in the frame - shown as bolded text. Also shown in the table is the percentage of failed components compared to the total number of similar components. In general, column hinges satisfy the performance criteria for the LS and CP BPL for both procedures. However, base column hinges at the exterior of both 8-story frames consistently fail the performance criteria using the NDP. These failures are a corollary of the column-tobase connection assumptions adopted for analysis, the modeling parameters for $P$ - $M$ hinges in ASCE 41 (see linear discussion), and the slight increase in $V_{C E}$ of the link beams for nonlinear analyses. As discussed above in the linear assessment discussion, changes in (i.e., up sizing) column sizes to limit design link rotations appear to have contributed to the satisfactory performance of the column hinges (except at the base).

Table 3-27. Performance Summary of Column Hinges (CH) per Frame, Nonlinear Procedures

\begin{tabular}{|c|c|c|c|c|c|}
\hline \multirow[t]{2}{*}{ Archetype } & \multirow[t]{2}{*}{ Design } & \multicolumn{2}{|c|}{ NSP } & \multicolumn{2}{|c|}{$\begin{array}{c}\text { NDP } \\
\text { (based on mean response of record set) }\end{array}$} \\
\hline & & LS & $\mathrm{CP}$ & LS & $\mathrm{CP}$ \\
\hline \multirow{2}{*}{ 4-Story } & ELF & --- & $\begin{array}{c}\text { Ext CH Fail (2) } \\
13 \%\end{array}$ & --- & $\begin{array}{c}\text { Ext CH Fail (2) } \\
13 \%\end{array}$ \\
\hline & RSA & --- & $\begin{array}{c}\text { Ext CH Fail (2) } \\
13 \%\end{array}$ & --- & $\begin{array}{c}\text { Ext CH Fail (2) } \\
13 \%\end{array}$ \\
\hline \multirow{2}{*}{ 8-Story } & ELF & --- & $\begin{array}{c}\text { Ext CH Fail (2) } \\
6 \%\end{array}$ & -- & $\underset{6 \%}{\text { Ext CH Fail (2) }}$ \\
\hline & RSA & --- & All CH Pass & --- & $\begin{array}{c}\text { Ext CH Fail (2) } \\
\qquad \%\end{array}$ \\
\hline \multirow{2}{*}{ 16-Story } & ELF & --- & All CH Pass & --- & $\begin{array}{c}\text { Ext CH Fail (2) } \\
3 \%\end{array}$ \\
\hline & RSA & --- & All CH Pass & --- & $\begin{array}{c}\text { Ext CH Fail (22) } \\
34 \%\end{array}$ \\
\hline
\end{tabular}

As detailed in previous discussions, all members other than the link beam in an EBF are force-controlled per ASCE 41. Further, the 16-story RSA-designed frame indicates significantly poorer column hinge performance partly because four of the ground motion records triggered "collapse". Still, the axial force demand, $P_{\max }$, is from an individual record and is, therefore, biased by the behavior of the frame to that record. As such, it is difficult to capture record-to-record variability on force- and deformation-controlled response directly in the analysis for a set of ground motion records. Recall that the linear procedures also identified concerns with these columns.

Establishing the in-plane column hinge model and performance metrics as a function of the out-of-plane flexural buckling strength, when governs $P_{C L}$, can be problematic for wide-flange columns. More so, when the gravity load alone produces an axial force greater than $0.2 \times P_{C L}$. Consequently, modeling the section flexural strength of a column hinge using the section axial strength of that section would not result in a force-controlled condition. This was the approach taken in FEMA 273, the predecessor of ASCE 41. It is theoretically inconsistent to model the component strengths within the same column as a function of both $P_{y e}$ and $P_{C L}$. 
Table 3-28 summarizes the performance of the column member strength for each nonlinear assessment procedure for the CP BPL. ASCE 41 does not provide guidance on checking column member stability when using the nonlinear procedures unless the column is designated as force-controlled (as is the case with an EBF). Except for the 8- and 16-story frames, which indicate column hinges developing in the base columns using the NDP, analytical results of the member strength interaction curves indicate that column members satisfy the performance criteria. Nonetheless, the in-plane stability of a column with plastic hinges from inplane flexure is highly complex and is a topic that is not well understood in the literature or implicitly or explicitly addressed in ASCE 41 for the nonlinear procedures. Experimental testing on shallow wideflanged steel beam-columns has illustrated that the weak-axis buckling strength of a wide-flange is not affected by plastic hinges from in-plane flexure.

Table 3-28. Performance Summary of Column Members (CM) per Frame, Nonlinear Procedures

\begin{tabular}{cccccc}
\hline \multirow{2}{*}{ Archetype } & Design & \multicolumn{2}{c}{ NSP } & \multicolumn{2}{c}{ NDP } \\
& & LS & CP & LS & CP \\
\hline \multirow{2}{*}{ 4-Story } & ELF & --- & All CM Pass & --- & All CM Pass \\
& RSA & -- & All CM Pass & --- & All CM Pass \\
\multirow{2}{*}{ 8-Story } & ELF & --- & All CM Pass & --- & All CM Pass \\
& RSA & --- & All CM Pass & --- & All CM Pass \\
\multirow{2}{*}{$16-$ Story } & ELF & --- & All CM Pass & --- & All CM Pass \\
& RSA & --- & All CM Pass & --- & All CM Pass \\
\hline
\end{tabular}

The EBF force-controlled columns consistently satisfy the performance objective, indicating that the capacity design provisions in AISC 341 result in adequate column sizes. These results are somewhat contrary to that suggested by the analytical results using the linear procedures. Still, the linear procedures have limitations in capturing a realistic force demands in the base columns and braces, thus contributing to the cause of this unanticipated discrepancy.

Table 3-29 provides a summary of the performance of the brace hinges for each nonlinear assessment procedure for the CP BPL. The number listed in parentheses denotes the number of failed components in the frame- shown as bolded text. Also shown in the table is the percentage of failed components compared to the total number of similar components. In general, brace hinges consistently satisfy the performance criteria using the NSP. However, braces at the base of the frame consistently fail the performance criteria using the NDP. These failures are a corollary of the column-to-base connection assumptions adopted for analysis, the modeling parameters for $P$ - $M$ hinges in ASCE 41 (see linear discussion), and the slight increase in $V_{C E}$ of the link beams for nonlinear analyses. Similarly, brace hinge performance is strongly biased by a collapsed state of a given frame (e.g., 16-story RSA).

Table 3-30 summarizes the performance of the brace member strength for each nonlinear assessment procedure for the CP BPL. ASCE 41 does not provide guidance on checking brace member stability when using the nonlinear procedures unless the brace is designated as force-controlled (as is the case with an EBF). Analytical results of the member strength interaction curves indicate that brace members satisfy the performance criteria. This result is similar to that indicated by the linear procedures.

The EBF force-controlled braces consistently satisfy the performance objective, indicating that the capacity design provisions in AISC 341 result in adequate brace sizes. 
Table 3-29. Performance Summary of Brace Hinges (BH) per Frame, Nonlinear Procedures

\begin{tabular}{|c|c|c|c|c|c|}
\hline \multirow[t]{2}{*}{ Archetype } & \multirow[t]{2}{*}{ Design } & \multicolumn{2}{|c|}{ NSP } & \multicolumn{2}{|c|}{$\begin{array}{c}\mathrm{NDP} \\
\text { (based on mean response of record set) }\end{array}$} \\
\hline & & LS & $\mathrm{CP}$ & LS & $\mathrm{CP}$ \\
\hline \multirow{2}{*}{ 4-Story } & ELF & --- & All BH Pass & --- & $\begin{array}{c}\text { Ext CH Fail (2) } \\
13 \%\end{array}$ \\
\hline & RSA & --- & All BH Pass & --- & $\begin{array}{c}\text { Ext CH Fail (2) } \\
13 \%\end{array}$ \\
\hline \multirow{2}{*}{ 8-Story } & ELF & --- & All BH Pass & --- & $\begin{array}{c}\text { Ext CH Fail (2) } \\
6 \%\end{array}$ \\
\hline & RSA & --- & All BH Pass & --- & $\begin{array}{c}\text { Ext CH Fail (2) } \\
6 \%\end{array}$ \\
\hline \multirow{2}{*}{ 16-Story } & ELF & --- & All BH Pass & --- & $\underset{3 \%}{\text { Ext CH Fail (2) }}$ \\
\hline & RSA & --- & All BH Pass & --- & $\begin{array}{c}\text { Ext BH Fail (24) } \\
38 \%\end{array}$ \\
\hline
\end{tabular}

Table 3-30. Performance Summary of Brace Members (BM) per frame, Nonlinear Procedures

\begin{tabular}{cccccc}
\hline \multirow{2}{*}{ Archetype } & \multicolumn{2}{c}{ NSP } & \multicolumn{2}{c}{ NDP } \\
& Design & LS & CP & LS & CP \\
\hline \multirow{2}{*}{ 4-Story } & ELF & --- & All BM Pass & --- & All BM Pass \\
& RSA & --- & All BM Pass & -- & All BM Pass \\
\multirow{3}{*}{ 8-Story } & ELF & --- & All BM Pass & -- & All BM Pass \\
& RSA & --- & All BM Pass & --- & All BM Pass \\
\multirow{2}{*}{$16-$ Story } & ELF & --- & All BM Pass & --- & All BM Pass \\
\hline
\end{tabular}

\subsection{Summary}

Table 3-31 summarizes the performance of the archetype buildings in reference to the BSO for both nonlinear procedures. Link beam performance from both assessment procedures controls the overall assessment of the frames. Similar to the linear assessments, column-to-base connection assumptions led to performance issues for the force-controlled base columns. In the end, none of the designed frames satisfy the seismic performance objective using the NSP or NDP (recall from Chapter 2 that the ELF procedure is not permitted for design of the 16-story EBF). Diagonal brace members consistently satisfy the performance criteria using the NSP, but consistently fail the criteria using the NDP.

Table 3-31. BSO Performance Summary of Archetype Buildings, Nonlinear Procedures

\begin{tabular}{|c|c|c|c|c|c|c|c|c|c|c|c|c|}
\hline \multirow[t]{2}{*}{ Archetype } & \multirow[t]{2}{*}{ Design } & \multicolumn{5}{|c|}{ NSP } & \multirow[t]{2}{*}{ Design } & \multicolumn{5}{|c|}{$\begin{array}{c}\text { NDP } \\
\text { (based on mean response of record set) }\end{array}$} \\
\hline & & Link & $\mathrm{CH}$ & $\mathrm{CM}$ & $\mathrm{BH}$ & $\mathrm{BM}$ & & Link & $\mathrm{CH}$ & $\mathrm{CM}$ & $\mathrm{BH}$ & $\mathrm{BM}$ \\
\hline \multirow{2}{*}{ 4-Story } & ELF & Pass & Fail & Pass & Pass & Pass & ELF & Fail & Fail & Pass & Fail & Pass \\
\hline & RSA & Fail & Fail & Pass & Pass & Pass & RSA & Fail & Fail & Pass & Fail & Pass \\
\hline \multirow{2}{*}{ 8-Story } & ELF & Fail & Fail & Pass & Pass & Pass & ELF & Fail & Fail & Pass & Fail & Pass \\
\hline & RSA & Fail & Pass & Pass & Pass & Pass & RSA & Fail & Fail & Pass & Fail & Pass \\
\hline \multirow{2}{*}{ 16-Story } & ELF & Fail & Pass & Pass & Pass & Pass & ELF & Fail & Fail & Pass & Fail & Pass \\
\hline & RSA & Fail & Pass & Pass & Pass & Pass & RSA & Fail & Fail & Pass & Fail & Pass \\
\hline
\end{tabular}


The assessment results from the NSP and NDP illustrate that, on average, the ELF-designed EBF performs better than the RSA-designed EBF for all archetype buildings. This result can be attributed to the increased strength and stiffness provided to the ELF-designed frames (see Table 2-5) by differences in the ELF and RSA procedures, including associated scaling provisions, in ASCE 7.

The NSP (without supplemental verification) consistently results in lower $D C R_{N}$ values than the NDP for both the ELF- and RSA-designed frames for all archetype buildings, an indication that a more accurate distribution of seismic demands is not well captured in taller frames using the NSP (LDP results are consistently greater than NSP, albeit a direct comparison is problematic as discussed previously). Nonlinear results indicate that the NSP has a tendency to underestimate the demands in the upper stories. This occurs primarily because of the differences in the distribution of seismic demands and the lack of modal representation other than the fundamental mode in the NSP. This effect was also noticed in NIST GCR 10917-9: Applicability of Nonlinear Multi-Degree-of-Freedom Modeling for Design (NIST 2010c).

The results from the NDP are sensitive to excitation input, analysis parameters, and component modeling. In this study, generalized component models were incorporated with degradation effects calibrated to an experimental test. Future research should critically examine the applicability of the generalized modeling parameters for steel components in ASCE 41. Experimental research has shown that subassembly tests can have large scatter in acceptable performance given the stochastic variations in the type of loading, that being cyclical, near-fault, random, etc. Future research should investigate the influence of the loading protocol adopted to establish the deterministic acceptance criteria for short link beams.

Analytical results based on component-level performances obtained from the NSP and NDP suggest that long-period eccentrically braced frames designed in accordance with ASCE 7 and its referenced standards have difficulty achieving the selected seismic performance objective of an existing building intended to be equivalent to a new building. This notion is driven by the performance of the link beams and, to a lesser extent, the columns and braces. The results for the columns can be enhanced by more mechanistically consistent column provisions and analytical modeling parameters. The results for the link beams provide a contrary inference to that provided for the linear procedures. Although link beam performance is based on high fidelity analytical modeling parameters, enhanced modeling practices could be investigated.

\subsubsection{Comparison between Linear and Nonlinear Assessment Results}

Table 3-32 summarizes the performance of the archetype buildings for each analysis procedure. The results indicate that the linear procedures do not consistently provide $D C R_{N}$ values greater than that given by the nonlinear procedures. As discussed previously, direct comparison of results between linear and nonlinear procedures can be problematic, except for direct comparison of the distribution of results. Some consistency is evident in the global performance rating of the 8-story EBF as well as frames designed per the MRSA procedure among the various assessment procedures. However, not all component performance failures align between the procedures, as discussed previously (e.g., link beams). The conservatism of the linear procedures is also apparent, as expected.

The nonlinear procedures provide a more rigorous assessment approach as compared to the linear

procedures. However, the results from the nonlinear procedures indicate more performance failures in the 
link beams than identified using the linear procedures, which is contrary to the assumption that the linear procedures are more conservative. Several factors contribute to this outcome, but it is difficult to narrow down any single primary contributor. Certainly for the NDP, the effects of ground motion selection and scaling can be significant, including the number of records adopted to achieve a reasonable level of statistical confidence and the method by which the records were chosen with a bias to achieve an unfairly beneficial binary outcome. Moreover, some of the higher mode periods fall directly in localized high energy regions of the response spectrum resulting in increased axial demands that cannot be captured efficiently in a linear analysis using a smooth generalized spectrum. Furthermore for the NSP, the force distribution is potentially inadequate for frames that exhibit increased higher mode participation, either elastically or triggered by nonlinearity.

Table 3-32. BSO Performance Summary of Archetype Buildings

\begin{tabular}{cccccc}
\hline Archetype & Design & LSP & LDP & NSP & $\begin{array}{c}\text { NDP } \\
\text { (based on mean } \\
\text { response of } \\
\text { record set) }\end{array}$ \\
\hline 4-Story & ELF & Pass & Pass & Fail & Fail \\
& RSA & Pass & Pass & Fail & Fail \\
8-Story & ELF & Fail & Pass & Fail & Fail \\
& RSA & Fail & Fail & Fail & Fail \\
16-Story & ELF & Fail & Pass & Fail & Fail \\
& RSA & Fail & Fail & Fail & Fail \\
\hline
\end{tabular}

In contrast to the nonlinear procedures, the linear analysis model and assessment is implied to be less rigorous and more conservative. As already discussed, the linear procedures yielded less conservative results for the deformation-controlled link beams. In contrast, the force-controlled members (i.e., columns and braces) were seemingly overconservative when using the simplified " $J$-factor" approach. The linear procedures can also illustrate the trend in demands but may fail to highlight critical performance zones within a given frame. A case in point is the acceptable $D C R_{N}$ values of the link beams while the nonlinear procedures illustrate that the link beams fail the acceptance criteria.

The failure of the EBF using the NDP exposes the need for more sophisticated assessment guidance. A building with seemingly reasonable strength distribution and ductility is seen to be prone to high concentration of demands in lower-to-middle stories, which went undetected during the (linear) design process and the ensuing linear assessment. High demands resulted in several link beams losing their load carrying ability and the analysis ultimately terminating prior to record completion because of set limits to represent a collapsed state of the frame. Problems with an EBF have been documented in a few other studies, although many questions still remain (Koboevic et al., 2012). At the component level, link beams in an EBF are well understood and have been thoroughly tested. However, at the system level, analysis and experience (e.g., Christchurch, NZ 2010 earthquake) have shown that an EBF may behave in a manner that is undesirable, or at least as not predicted. 


\section{Chapter 4 Summary, Conclusions, and Recommendations}

This report presents the results of a study investigating the correlation between the seismic performance of an ASCE 7 code-compliant building and its performance as quantified using ASCE 41 analysis procedures and structural performance metrics. This investigation is performed by evaluating a suite of structural steel buildings in a high seismicity region that are designed using ASCE 7 and evaluated using ASCE 41. The basic question is whether the standards for designing new steel buildings and assessing existing steel buildings provide consistent levels of performance. An additional outcome of this research is to advance the state-of-knowledge in PBSD and assessment of buildings using ASCE 41. Further, results provide the technical background for provisions that target equivalent seismic performance between a new building and an existing building that is required to meet the seismic performance objective of a new building.

This chapter highlights significant observations and conclusions from the seismic assessment of the archetype buildings using four assessment procedures in ASCE 41. General findings and recommendations are based on the collective results for the seismic force-resisting system. More in-depth findings specific to the system are in the relevant subsections in this chapter, as well as in relevant sections of the assessment discussion in Chapter 3. Although the primary emphasis of this study is on benchmarking ASCE 41 assessment procedures, questions arise that may be more applicable to the design criteria used rather than to the assessment results - these items are identified in the section about future research.

\subsection{Summary of Project Work}

This report presents the results of a structural seismic performance assessment using ASCE 41 procedures and performance measures of buildings utilizing steel eccentrically braced frames (EBF) as the lateral forceresisting system (LFRS).

A suite of archetype buildings that incorporate EBFs along one principal direction of the buildings is designed in accordance with ASCE 7. The suite consists of 4-, 8-, and 16-story buildings designed using both the Equivalent Lateral Force (ELF) Procedure and Modal Response Spectrum Analysis (RSA). Both analysis procedures are used to provide a generally applicable range of LFRS strength within the selected seismic intensity region. As such, an LFRS may include significant overstrength to resist nonseismic loads or to satisfy other design criteria. A design space is created to investigate the effects of design methodology, building height and other LFRS-specific geometric modifications on seismic performance. In reality, the design space is infinitely large and many design choices made in this study can also have different configurations to evaluate the variation in performance specific to a design choice.

The seismic performance assessment of the building suite is conducted using both linear and nonlinear analysis procedures prescribed in ASCE 41: 
- Linear Static Procedure (LSP)

- Linear Dynamic Procedure (Response Spectrum) (LDP)

- Nonlinear Static Procedure (NSP)

- Nonlinear Dynamic Procedure (NDP)

For this study, the performance assessment targets the Basic Safety Objective (BSO) prescribed in ASCE 41. This objective includes the interrelated goals of Life Safety (LS) Building Performance Level (BPL) at the Basic Safety Earthquake-1 (BSE-1) earthquake hazard level (EHL) and Collapse Prevention (CP) BPL at the BSE-2 EHL. This performance objective is chosen to align with the intended structural performance objective of an ordinary building in ASCE 7, which is qualitatively defined here as "life safety" provided by collapse prevention of the building, given a maximum considered earthquake (MCE) event.

To evaluate seismic assessment criteria, each component of the EBFs is designated as a primary member (or component) in accordance with ASCE 41 §2.2.5 (and ASCE 41 §2.4.4.2). Similarly, quantitative performance measures (i.e., acceptance criteria) for primary components are used for all assessment procedures, although performance measures for secondary components are permitted by ASCE 41 for some primary components. The consistent use of primary acceptance criteria keeps all components and associated assessment results correlated among the assessment procedures for this study.

The goals of this research are as follows:

- Assess new structural steel buildings utilizing EBFs designed per ASCE 7 requirements and, in turn, evaluated using ASCE 41,

- Develop a qualitative link between the performance implied in ASCE 7 in light of the performance identified by ASCE 41 procedures and performance measures,

- Provide guidance or technical support for improved or new provisions in ASCE 41 (and to a lesser extent, ASCE 7),

- Reduce uncertainty in first-generation PBSD procedures for performance-based seismic assessment, and

- Identify any inconsistencies, ambiguities, and confusing provisions in ASCE 41.

In reference to developing a link between ASCE 7 and ASCE 41, the primary difficulty in equating the two standards is rooted in their disjointed performance objectives. That is, acceptance criteria for a component in ASCE 41 are not directly calibrated to the seismic performance objective of ASCE 7, which is a 10 percent probability of partial or total collapse given an MCE event - that is $\mathrm{MCE}_{R}$ (or one percent probability of partial or total collapse in 50 years). Equating the two objectives of the standards would imply that only one structural performance level with an associated earthquake hazard level can be coupled: that being, $\mathrm{CP}$ at the $\mathrm{MCE}_{\mathrm{R}}$. However, this would be difficult based on a member-level binary performance solution. Consequently, the question becomes what percentage of components needs to fail the associated CP SPL to achieve a 10 percent probability of total or partial collapse given an $M_{C} E_{R}$ event? Future research should assess the archetype buildings in FEMA P695 analysis to ascertain the collapse probability in relation to the ASCE 7 performance objective. Results from that study can be used to probabilistically relate the $R$-factor in ASCE 7 to the $m$-factors and inelastic deformations using story drift. Clearly, the study presented in this report presumes that the $R$-factor used for design has been derived to provide the intended 
collapse performance objective. As such, the analysis results do not necessarily reflect satisfactory or unsatisfactory performance in relation to the seismic performance objective of ASCE 7.

A consequence of a deterministic-type component evaluation (i.e., pass or fail) is that analytical results, depending on the accuracy of the model and analysis algorithms, can be independent of the behavior of the system. Individual member performance and the potential need to retrofit or replace it are therefore based on an analysis output rather than the influence of the component performance on the system performance. This is a challenging issue to overcome, and only recently has there been some progress made (e.g., FEMA P695 and FEMA P-58 (FEMA 2012)) toward having the ability to probabilistically correlate member performance to system performance. However, these efforts are not without their limitations and debatable performance metrics. It is still yet to be determined whether practitioners will accept these developing methods because of the time and resources needed to successfully apply their recommendations. However, ASCE 41 is available and being used for performance-based seismic engineering of building systems and components. In many cases, the acceptance criteria in ASCE 41 are being used to justify computed seismic performance to buildings officials as being satisfactory. The question is what seismic performance is being justified: the objective defined in ASCE 41 or that intended in ASCE 7? If satisfying ASCE 7, then this would infer that the CP SPL associated with the $\mathrm{MCE}_{\mathrm{R}}$ (taken as the BSE-2) defined in ASCE 41 matches the intended collapse performance of ASCE 7. A significant effort is still needed to bring ASCE 41 to the state-of-the-art and equivalent to ASCE 7. In this regard, assessment provisions are meaningless without the technical support provided by experimental research and subsequent case studies that evaluate how the research findings affect component and system performance.

\subsection{Assumptions and Limitations of this Study}

The following discussion summarizes notable assumptions employed in this study and other limitations of the work that could impact the results, which form the basis for the conclusions and observations.

\section{Building System and Component Characteristics for Design and Assessment}

- The archetype buildings are representative of a specific type of building, which uses a seismically designed system to resist lateral loads and deformations. The selected system in this study represents one design option out of the many available for steel framed buildings. In designing the SFRS, there are many specific design assumptions made that play an important role in resisting lateral loads and deformations. Different selections for frame configuration, plan layout, bay spacing, height, connection details, and magnitude of non-seismic loads all could affect the assessment results.

- The buildings are regular in layout and configuration as defined in both ASCE 7 and ASCE 41 . Irregular building configurations can affect seismic performance and are not addressed in this study, as they could complicate the comparisons that are being made.

- The archetype buildings are simple in concept and do not contain stairwells, elevator cores, architectural setbacks, atriums or other features found in typical buildings. The goal here is to study the basic performance of the SFRS in resisting lateral loads and deformations without the complexity posed by other attributes found in buildings today. 
- Strength and stiffness of specific secondary components, as defined in ASCE 41, were not fully represented in the mathematical model for linear and nonlinear analyses (e.g., shear tab connection for gravity framing, façade, stairs, etc.). This assumption, while reasonable from an analysis standpoint, highlights a difference in requirements between ASCE 7 provisions for design and ASCE 41 provisions for assessment (ASCE 7 §12.7 and ASCE 41 §3.2.2).

- Composite action developed between primary and secondary structural components and the portion of slab they support was not included in the mathematical model for seismic design or assessment. This approach is consistent with that used by many practitioners and provides presumably conservative results because floor slabs are not active in providing composite action and added moment capacity. Composite action was included for the moment frame beams for verifying elastic story drifts under service-level wind loading.

- The column-to-base connections of the SFRS and the seismic base of the buildings were assumed to be horizontally, vertically, and rotationally restrained, resulting in a "fixed" connection to the ground. The base of non-SFRS columns were rotationally unrestrained. Soil-structure interaction effects, modeling the flexibility of the soil and / or the foundation components, and modelling partially-restrained column-to-base connections were not included in this study. Inclusion of these effects would likely affect the assessment results. However, inclusion of the effects of the soilfoundation flexibility into the analysis is complex and not well established at the present time. Moreover, current design practice commonly does not include soil-foundation effects; column-tobase connections to the building foundations are often idealized models, as is done in this study.

- No formal investigation was included in this study to evaluate the accuracy of the quantitative modeling parameters for nonlinear analysis or acceptance criteria for linear and nonlinear analysis provided in ASCE 41 for primary or secondary component models. There is a project currently ongoing with ATC (ATC-114: Development of Accurate Models and Efficient Simulation Capabilities for Collapse Analysis to Support Implementation of Performance Based Seismic Engineering) that will examine the component modelling parameters and acceptance criteria for specific components.

\section{Structural Analysis}

- No formal investigation was included in this study to evaluate the accuracy of the analysis algorithms in the software packages used for structural analysis. These software packages are the same as those used by practitioners. The stability of solution algorithms when the stiffness and strength of the component models have significantly degraded can vary between software packages. Therefore, any software accuracy limitations encountered in this study are consistent with those present in design offices.

- The methodology used in this study for ground motion selection and scaling resulted in a set of earthquake records that may not be applicable or suitable for a specific site. A different record setselected by engineering judgment, selected by revising the parameters of the methodology, or developed from an alternative methodology - could affect the assessment results. However, the process employed here is consistent with that used in practice, representing a typical building site in an area with a high level of seismicity. 
No formal investigation was included in this study to evaluate all potential sources of uncertainty or error, or whether multiple sources of error are correlated. The question of uncertainties in the analytical models, solution algorithms, material properties and even potential as-built final dimensions and positions of members are all beyond the scope of this study. The load and resistance factor design (LRFD) philosophy in use for structural design today are based on pioneering work on uncertainties in material and load characterizations performed starting in the 1950's. Whether a new similar large national effort to that conducted for LRFD is required today is not clear. Quantifying the effect of any source of uncertainty or error, as it relates to the design or assessment of buildings to resist earthquake motions, is a significant issue and would require its own research program to study all of the aspects.

\subsection{Conclusions and Observations}

This section highlights significant observations and conclusions from the seismic assessment of the archetype buildings. Topics are categorized as general or system specific. Additionally, more in-depth discussions of the observations and conclusions specific to the SFRS are in the relevant subsections in this chapter as well as in relevant summary sections of the assessment discussion.

\subsubsection{ASCE 41}

The following observations and conclusions can be drawn from this study. Topics are grouped by either general applicability to ASCE 41 assessment procedures or specific to the structural system.

\subsubsection{General}

The following general topics focus on observations identified by the assessment provisions for the selected assessment methods:

- The LSP generally results in more conservative normalized demand to capacity ratios, $D C R_{N}$, values than that of the LDP, because of the differences in the distribution of seismic demands and the lack of modal representation other than the fundamental mode in the LSP.

- The NSP generally results in less conservative $D C R_{N}$ values than that of the NDP, contrary to what would be expected with increasing the analytical complexity, because of the differences in the distribution of seismic demands and the lack of modal representation other than the fundamental mode in the NSP.

- The nonlinear procedures provide a more rigorous assessment approach as compared to the linear procedures. The results from the LSP, and to a lesser extent the LDP, indicate more performance failures in force-controlled components than identified using the nonlinear procedures. The results presented emphasize the inherent conservatism in the linear procedures. However, this conservatism is accompanied by a reduction in required analytical resources and proficiency of the analyst.

- The linear procedures can illustrate the trend in demands but may fail to highlight critical performance zones within a given frame.

- No clear consistency exists between linear assessment results and nonlinear assessment results for link beams. Contrary performance results indicate acceptance criteria that need further calibration. 


\subsubsection{Eccentrically Braced Frame}

The following significant observations and conclusions are based on the collective results obtained from the assessment of the EBFs. More details about the specific items are in the relevant sections of the assessment discussion in Chapter 3.

- Analytical results based on component-level performances indicate that new long-period EBFs designed in accordance with ASCE 7, and its referenced standards, have difficulty achieving the ASCE 41 BSO for an existing building intended to be equivalent to a new building. This observation is driven by the performance of the link beams and, to a lesser extent, the columns and braces. Assessment results for the link beams from the nonlinear procedures provide a conclusion opposite to that provided for the linear procedures in that nonlinear assessment reveals higher $D C R_{N}$ values than the linear assessment.

- Assuming the archetype buildings meet the collapse performance objective of ASCE 7, the results of the assessment procedures indicate that ASCE 41 is generally conservative for EBFs. ASCE 41 analysis would require retrofit or replacement of specific components of a code-compliant SFRS to satisfy the CP BPL, given an MCE event. The results highlight that columns (i.e., beam-columns) with high axial and flexural demands and link beams have difficulty in satisfying the performance criteria in ASCE 41. Future research is needed to couple the collapse performance objectives of the two standards, as well as other performance objectives associated with a seismic hazard with a lower return period.

- Analysis results indicate that the linear procedures generally give smaller $D C R_{N}$ values than that obtained from nonlinear procedures, contrary to what would be expected with increasing the analytical complexity. Given a properly designed new link beam, the linear procedures will result in a maximum $D C R_{N}$ value for a primary component of 0.72 for the BSE-1 EHL $(0.76$ for the BSE2 EHL).

- A significant number of link beams did not satisfy the acceptance criteria for the nonlinear procedures. This result was particularly problematic for EBFs as they quickly progressed towards a collapse state after the ultimate link capacity in the second story was reached. Though link beam performance is based on high fidelity analytical modeling parameters, enhanced modeling practices should be investigated.

- A significant number of columns, primarily at the base of the frame, did not satisfy the ASCE 41 acceptance criteria (force-controlled). The results for columns can be enhanced by more mechanistically consistent assessment provisions and analytical modeling parameters for columns (e.g., column/brace-to-base connection modeling).

- Components of the EBFs that do not satisfy the CP acceptance criteria would need to be strengthened to achieve the performance required by ASCE 41. However, the results from the various assessment procedures were seen to be inconsistent in some cases for a given design routine (i.e., LSP vs. NDP) or the same assessment procedure was inconsistent between design routines (i.e., ELF and RSA). This makes it difficult to definitively suggest that using ASCE 41 to design a new EBF would produce a system capable of achieving the seismic performance objective of ASCE 7. Future research is needed to evaluate the collapse probability of a new system strengthened by ASCE 41 relative to the seismic performance objective of ASCE 7. The same is required for a new system that has component strengths reduced from that required by ASCE 7 to meet an ASCE 41 
performance objective. Further, the adequacy of the components of the enhanced frame (those required to satisfy ASCE 41) would be dependent upon which analysis procedure is used to iterate between design and assessment, and therefore the fidelity of the analytical model and analysis parameters.

- Results from this study indicate that for ASCE 41 to be used as a seismic design procedure for new steel buildings, as a performance-based alternative to ASCE 7 (see ASCE 7 §1.3.1.3), acceptance criteria for the various analysis methods must be calibrated to each other to consistently result in a uniform collapse risk. Additionally, ASCE 41 would need to reference material-specific design standards (e.g., AISC 341) for their seismic design requirements, as well as consistent requirements for defining acceptance criteria for a component (e.g., plastic rotation).

\subsection{Recommendations for Future Research}

The following sections identify items for future research. The recommendations are grouped by the applicable standard: ASCE 41, ASCE 7, and AISC 341 / 360 / 358.

\subsubsection{ASCE 41}

\subsubsection{General}

The following items are general considerations for future studies to enhance ASCE 41 assessment provisions:

- $\quad$ The archetype buildings should be analyzed using the methodology formulated in FEMA P695. This will provide the requisite data to identify the collapse probability of the systems (or frames) in relation to the intended collapse objective of ASCE 7. However, the same seismic performance factors as used in design should be used in the analysis. Results from this study can be used to probabilistically relate the $R$-factor in ASCE 7 to $m$-factors and inelastic deformations using story drift.

- Research should investigate the implementation of risk-targeted collapse assessment criteria into ASCE 41 similar to the design philosophy introduced in ASCE 7-10. As such, comparison of system fragility curves should be done to correlate the risk-target of ASCE 7 and the risk-target of an existing building intended to be equivalent to a new building.

- Research should evaluate the influence of gravity framing (e.g., partially restrained shear tab connections) on assessment results of the primary components of the SFRS.

- Research should investigate alternative lateral force distributions for taller systems for the NSP, including comparison between adaptive and non-adaptive loading.

- Research should be conducted to determine the number of components that do not need to satisfy the ASCE 41 component acceptance criteria while still permit the building to be classified as meeting a performance objective.

- Research should evaluate the systems used in this study by measuring demands against acceptance criteria for secondary components to quantify variations in performance results; for example, the link beams in the ME8 buildings. Even if secondary component criteria were implemented, the $D C R_{N}$ values still indicate unsatisfactory performance in the several floors. The primary acceptance 
criteria for the nonlinear procedures has been removed in ASCE 41-13. Based on some trends seen in this study, this should be done only if the acceptance criteria for linear and nonlinear procedures have been correlated and calibrated.

- Enhanced commentary is needed in ASCE 41, similar to the effort used to develop FEMA 274. Commentary can be used to explain differences in component strengths between ASCE 41 and ASCE 7 and its reference standards (e.g., AISC 341 and AISC 360). This effort would include cleaning up incorrect references (e.g., AISC 341 or AISC 360, FEMA 355F or FEMA 355D). Similarly, the commentary can detail the experimental tests used to derive the acceptance criteria.

- Consideration should be given to reorganize Chapter 5 (Chapter 9 in ASCE 41-13) to remove system-to-system references, most notably when they are not applicable. For example, a forcecontrolled column in an EBF cannot reference provisions for a column in a moment frame. This chapter would benefit with an outline similar to AISC 341, where the section on member strength is outlined similar to AISC 360. Therefore, the individual systems would reference a member strength in lieu of another system that may or may not be applicable.

\subsubsection{Eccentrically Braced Frames}

The following items are considerations for future studies to enhance ASCE 41 assessment provisions for eccentrically braced frames:

- Research should critically examine the applicability of the generalized modeling parameters for shear hinges used in link beams for use in the nonlinear procedures.

- Research should investigate the correlation between acceptance criteria for the linear and nonlinear procedures. This effort can include the influence of shear-moment interaction and axial-moment interaction on the acceptance criteria.

- Research should investigate the influence of the loading protocol adopted to establish the deterministic acceptance criteria for shear hinges in link beams.

- Research is needed to develop acceptance criteria and modeling parameters for column / brace-tobase connections, including embedded connections.

- Research should investigate the use of acceptance criteria and modeling parameters for brace connections. Similarly, guidance is needed concerning adjusting acceptance criteria and modeling parameters to simulate the change in response of a component due to damage of a brace connection.

- Removing the force-controlled requirement for all members except the link (or conversely apply this philosophy to a $\mathrm{CBF}$ ) should be considered. If so, research is required to justify updated interaction equations for assessment of beam-columns using ASCE 41, as well as a critical examination of the acceptance criteria in regard to experimental test results. Decoupling interaction equations into specific failure mechanisms and referencing highly vetted design standards should be considered. Removing $P_{C L}$ as the basis for force-controlled response and acceptance criteria for a column hinges (i.e., revert back to FEMA 273) and using $P_{C E}$ when $F_{y e}$ is used to assess a flexure hinge in the same column should also be considered.

\subsubsection{ASCE 7}

The following items are considerations for future studies to enhance ASCE 7 provisions: 
- The assessment results illustrate that on average the ELF-designed frames perform better than the RSA-designed frames for all archetype buildings. However, the ELF procedure is not permitted in some cases. Research should investigate the applicability of the analysis limitations in terms of the intended collapse objective of ASCE 7.

- Research should investigate the lateral design force distributions in ASCE 7 and modal scaling provisions, and their influence on the allocation of component strengths within a frame. Research has indicated that higher modal base shear scaling may be warranted (NIST 2010b and NIST 2012). Potential modifications to the MRSA procedure could also include scaling provisions to additionally account for higher mode effects resulting from nonlinear response. Provisions can be directly transferred to the linear procedures in ASCE 41.

- Research efforts should evaluate incorporating other performance levels for design into ASCE 7 (NIST 2012).

\subsubsection{AISC $341 / 360 / 358$}

\subsubsection{Eccentrically Braced Frames}

The following items are considerations for future studies to enhance AISC 341 provisions of eccentrically braced frames:

- Research should investigate the Response Modification Factor, $R$, assigned to an EBF. Results from the assessment suggest that an assigned value of 8 (equal to that for an SMF) may be high. A side study examined an EBF designed with $R=6$. Preliminary results indicate similar performance to the results presented here. Adjustments to $R$ would require extensive nonlinear analysis that could impact the assessment provisions of ASCE 41. This system has not been evaluated in a FEMA P695-type analysis.

- Research should investigate the Displacement Amplification Factor, $C_{d}$, assigned to an EBF, and its influence on separation of flexural and shear deformation modes of a story to determine a representative plastic link rotation. Adjustments to $C_{d}$ would require extensive nonlinear analysis that could impact the assessment provisions of ASCE 41.

- Research should investigate the approximate period formulation, $C_{u} T_{a}$, assigned to an EBF. Adjustments to $C_{u} T_{a}$ would require extensive nonlinear analysis that could impact the assessment provisions of ASCE 41.

- Research should investigate the applicability of the maximum shear strength of a link beam and correlation with the loading sequence on the plastic link rotation.

- The provisions should revisit the methodology on determining the plastic link rotation angle when using an elastic analysis. As other researchers have shown, basing the link rotation limits off of total story drift can be excessively conservative for taller frames. Similarly, the allowable link rotation angle in AISC 341 is not a function of the Risk Category, $I_{e}$, assigned to the building. As such, frame in buildings assigned to higher risk categories than Class II-Ordinary, see ASCE 7will always satisfy AISC 341 due to stricter story drift requirements in ASCE 7.

- Analysis results are dependent on link beam calibration with experimental test results that dictated a higher overstrength than $1.25 \times V_{p}$ as used in design. As such, the axial force demand in the columns from the capacity analysis is consistently lower than the results from the NDP. This trend 
is also true for the braces. Still, the columns and braces consistently satisfied the stability criteria using the NDP; this may be partly due to the columns having been upsized to limit plastic link rotations in the ELF-design frames. Research should data mine existing research to explore the possibility of using a larger overstrength on short link beams. Further, ASCE 41 can provide more guidance on analytical modeling techniques for this capacity analysis.

- Research should unify the nominal shear strength equations in AISC 341 and AISC 360 for compact wide-flange sections. 


\section{Appendix A Ground Motions for Response History Analysis}

\section{A.1 Ground Motion Record Set}

The far-field record set (22 records, each with two horizontal components) from FEMA P695 (FEMA 2009a) is selected as the input motion database for the NDP; 14 of the 44 horizontal component records are selected as the ground motion set for each archetype building, with no two records coming from the same station. The records are normalized for magnitude, distance, and source conditions as discussed in FEMA P695.

The scaled record set (see Ground Motion Selection and Scaling section below) for each archetype building is taken directly as the Basic Safety Earthquake-2 (BSE-2) earthquake hazard level (EHL). Although this EHL is not strictly the same as having a two percent probability of exceedance in 50 years, it is comparable, and anticipated future changes in ASCE 41-13 will likely match the seismic hazard defined in ASCE 7-10 (see Chapter 3). The scaled record set is factored by two-thirds to represent the BSE-1 EHL in lieu of explicitly determining the ground motion parameters with a ten percent probability of exceedance in 50 years. No spectral shape modifier, $\varepsilon$, is used to adjust the seismic demands for either BSEs (FEMA 2009a; Haselton et al. 2009), this is a topic of needed research.

As a side note, the goal initially was to analyze the archetype buildings using seven scaled pairs of ground motion records along each principal axis of the structure (14 records in each direction). However, because of complications in scaling orthogonal pairs for the maximum direction of response when a structure has significantly different periods along the principal axes, it was decided to analyze 14 unique records independently along each principal axis - see above. This is permitted by ASCE 41 since requirements for considering multidirectional seismic effects are not triggered in this study-see ASCE 41 §3.2.7. Further, there is no guidance regarding the application of scaled ground motion pairs for the maximum direction of response when a structure has significantly different periods along the principal axes. Future research is needed to provide provisions on scaling and application of ground motion pairs.

\section{A.2 Ground Motion Selection and Scaling}

The ground motion selection and scaling procedure for each archetype building is described below. This procedure was developed in consultation with select members of the peer review team. Each set of records (14 total) is used for both the equivalent lateral force (ELF) and response spectrum analysis (RSA) designs to gauge performance between the two. The process is as follows:

1. Determine the fundamental lateral mode period, $T_{1}$, of the building in the direction being considered not including gravity load effects (i.e., first-order period) for both the ELF and RSA 
designs. Second-order periods may also be computed with expected point-in-time gravity loads rather than factored loads. Determine the average (arithmetic mean) of the periods for the ELF and RSA designs, $T_{1, a v g}$. This will keep the scaling of the two designs consistent.

2. For each of the 44 far-field component records (not the records computed from the square root of the sum of the squares (SRSS) of the two horizontal components of an event), compute the error between $S_{a}$ from the recorded spectrum and $S_{a}$ from the maximum considered earthquake (MCE $)$ design spectrum at each period between $0.2 \times T_{1, \text { avg }}$ and $1.5 \times T_{1, \text { avg }}$. The period step used here is 0.01 second. The error at each period ranges from 0 to 1 , with 0 being an identical match.

3. Sum the error values over the periods between $0.2 \times T_{1, \text { avg }}$ and $1.5 \times T_{1, \text { avg }}$ to get a single composite error value for each record.

4. Scale each record to minimize the total error from step 3.

5. Select the 14 records with least total error. If both horizontal components of a specific station are in the set, then remove the record with the larger error of the pair and select the next unique record from the remaining record set. This step is repeated as needed until all records selected are from different stations.

6. Compute the average spectrum from the record set (14 total) from step 5 .

7. Scale the average spectrum from step 6 so that no value between $0.2 \times T_{1, \text { avg }}$ and $1.5 \times T_{1, \text { avg }}$ is less than the $\mathrm{MCE}_{\mathrm{R}}$ spectrum. The 1.3 factor in ASCE $41 \S 1.6 .2$ is not included here, so as to address the change of spectrum parameters from geomean to maximum direction response in ASCE 7 (this factor will be removed in ASCE 41-13).

8. Scale the record set from step 5 by the value computed in step 7. Therefore, there are two scaling factors: step 4 and step 7.

9. Apply the total scaling factor (step 4 times step 7) to each component record in the set from step 5 and perform analysis.

For comparison purposes, the process in ASCE $41 \S 1.6 .2 .2$ is summarized as follows:

1. Select a minimum of three recorded events (each event is a data set), each with two horizontal components.

2. Take the SRSS of the two horizontal components of each selected data set from step 1.

3. Select a scaling factor for each SRSS from step 2. Note that application of a scaling factor to the unscaled SRSS is equivalent to taking the SRSS of the similarly scaled components.

4. Compute the average of the scaled SRSS spectra from step 3 for all selected events.

5. Scale the average spectrum from step 4 so that no value between $0.2 \times T_{1, \text { avg }}$ and $1.5 \times T_{1, \text { avg }}$ is less than 1.3 times the design spectrum.

6. Apply the total scaling factor (step 3 times step 5) to each component record in an event and perform analysis.

The selection and scaling procedure in this study differs slightly from that found in ASCE 41. Because this study investigates a generalized SDC $\mathrm{D}_{\max }$ analysis without a specific location, it is difficult to select a site and apply common selection and scaling processes typically performed by a geotechnical engineer. In lieu of taking the SRSS of the two horizontal components of an event and having 22 SRSS spectra and in turn computing the error of the SRSS records and associated scaling factor for the event, the error and scaling factor were computed for each component (44 spectra). 14 unique records were selected per principal 
direction and the average of this set scaled to meet the target spectrum. In summary, steps 1 to 5 in the process identify the "best fit" to the ASCE 41 code spectrum (which matches ASCE 7). The average of this set is computed and scaled similarly to that in ASCE 41 - without the 1.3 factor.

Other ground motion selection and scaling methods are discussed in NIST GCR 11-917-15: Selecting and Scaling Earthquake Ground Motions for Performing Response-History Analyses (NIST 2011d).

\section{A.3 Four-Story Archetype Building}

\section{A.3.1 4-Story Eccentrically Braced Frame}

Table A-1 summarizes the 14 strong motion records used for the nonlinear dynamic procedure (NDP) for the N-S direction of ME4 eccentrically braced frame (EBF). Figure A-1 illustrates the set of acceleration response spectra, original and scaled, and the scaled average spectrum. Figure A-2 illustrates the acceleration response spectra, original and scaled, for each select record. For comparison, the ASCE 41 code spectrum is shown in the figures. All analyses completed for the BSE-1 and BSE-2 for the ELF and RSA design.

Table A-1. Ground Motion Records for N-S Direction of ME4

\begin{tabular}{|c|c|c|c|c|c|c|c|c|c|}
\hline ID & EQ No. & Event Name & Station & Comp. ${ }^{1}$ & Error $^{2}$ & $\begin{array}{c}\text { BSE-2 } \\
\text { Scaling }^{3}\end{array}$ & $\begin{array}{c}\text { BSE-1 } \\
\text { Scaling }^{3}\end{array}$ & Step $^{4}$ & Time $^{5}$ \\
\hline 1 & 35 & Cape Mendocino & Rio Dell Overpass & 1 & 15 & 1.879 & 1.253 & 0.020 & 36 \\
\hline 2 & 34 & Superstition Hills & Poe Road (temp) & 2 & 17 & 3.083 & 2.055 & 0.010 & 23 \\
\hline 3 & 17 & Kocaeli, Turkey & Duzce & 1 & 17 & 2.524 & 1.683 & 0.005 & 28 \\
\hline 4 & 31 & Superstition Hills & El Centro Imp. Co. & 1 & 18 & 2.308 & 1.539 & 0.005 & 40 \\
\hline 5 & 19 & Kocaeli, Turkey & Arcelik & 1 & 19 & 8.166 & 5.444 & 0.005 & 30 \\
\hline 6 & 2 & Northridge & Beverly Hills - Mulhol & 2 & 20 & 1.093 & 0.729 & 0.010 & 30 \\
\hline 7 & 10 & Imperial Valley & Delta & 2 & 21 & 2.293 & 1.529 & 0.010 & 100 \\
\hline 8 & 16 & Kobe, Japan & Shin-Osaka & 2 & 22 & 2.668 & 1.778 & 0.010 & 41 \\
\hline 9 & 4 & Northridge & Canyon Country-WLC & 2 & 26 & 1.622 & 1.082 & 0.010 & 20 \\
\hline 10 & 43 & Friuli, Italy & Tolmezzo & 1 & 26 & 4.163 & 2.775 & 0.005 & 37 \\
\hline 11 & 28 & Loma Prieta & Gilroy Array \#3 & 2 & 27 & 2.285 & 1.523 & 0.005 & 40 \\
\hline 12 & 24 & Landers & Coolwater & 2 & 27 & 1.832 & 1.221 & 0.003 & 28 \\
\hline 13 & 30 & Manjil, Iran & Abbar & 2 & 28 & 1.834 & 1.222 & 0.020 & 54 \\
\hline 14 & 6 & Duzce, Turkey & Bolu & 2 & 28 & 1.192 & 0.795 & 0.010 & 56 \\
\hline \multicolumn{10}{|c|}{$\begin{array}{cc}\text { Notes: } \\
\text { 1. } \mathrm{Co} \\
\text { 2. } \mathrm{Si} \\
\text { 3. } \mathrm{Sc} \\
\text { 4. } \mathrm{Ti} \\
\text { 5. } \mathrm{To}\end{array}$} \\
\hline
\end{tabular}



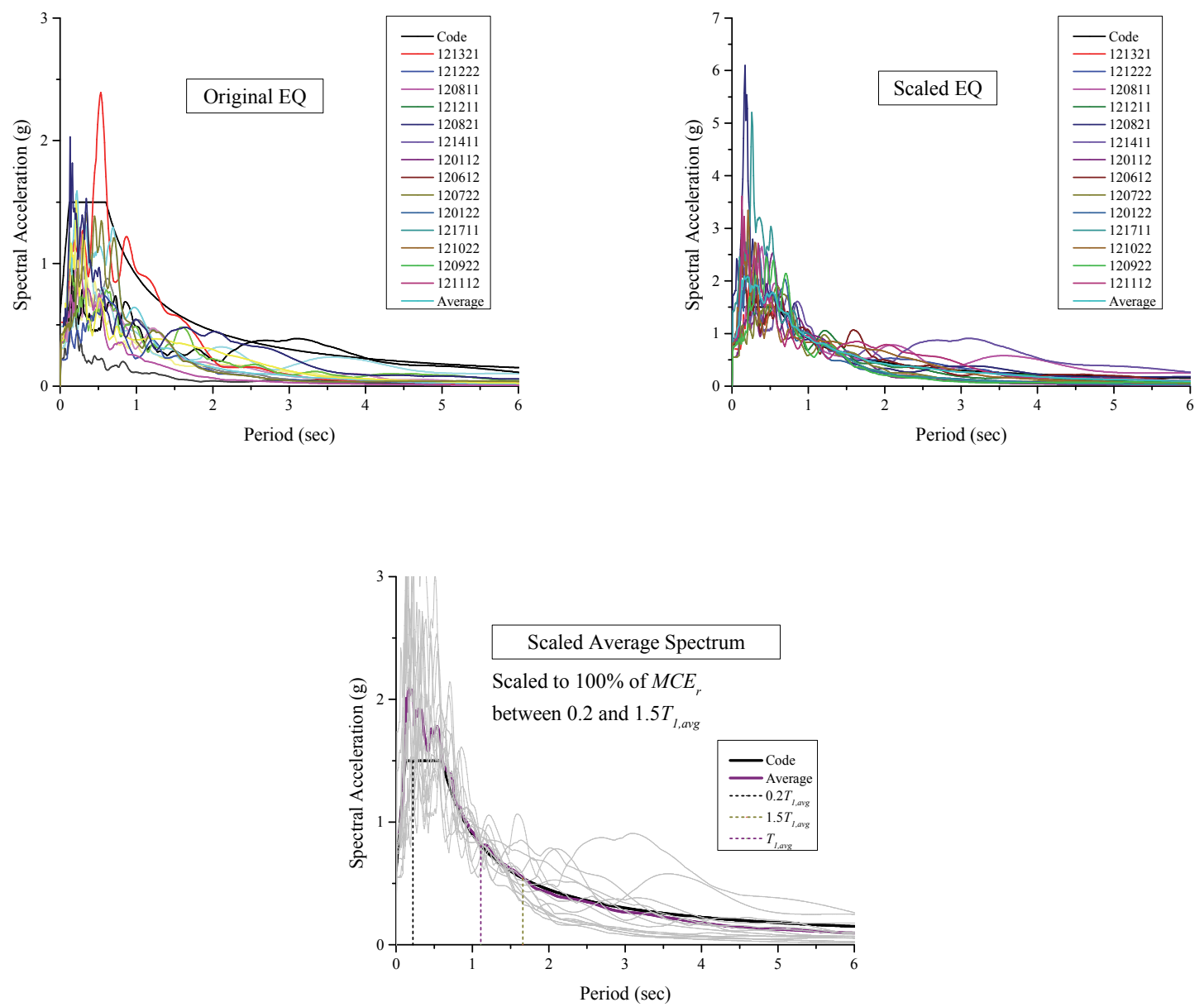

Figure A-1. Acceleration Response Spectra: Original, Scaled, and Scaled Average Spectrum for N-S Direction of ME4 

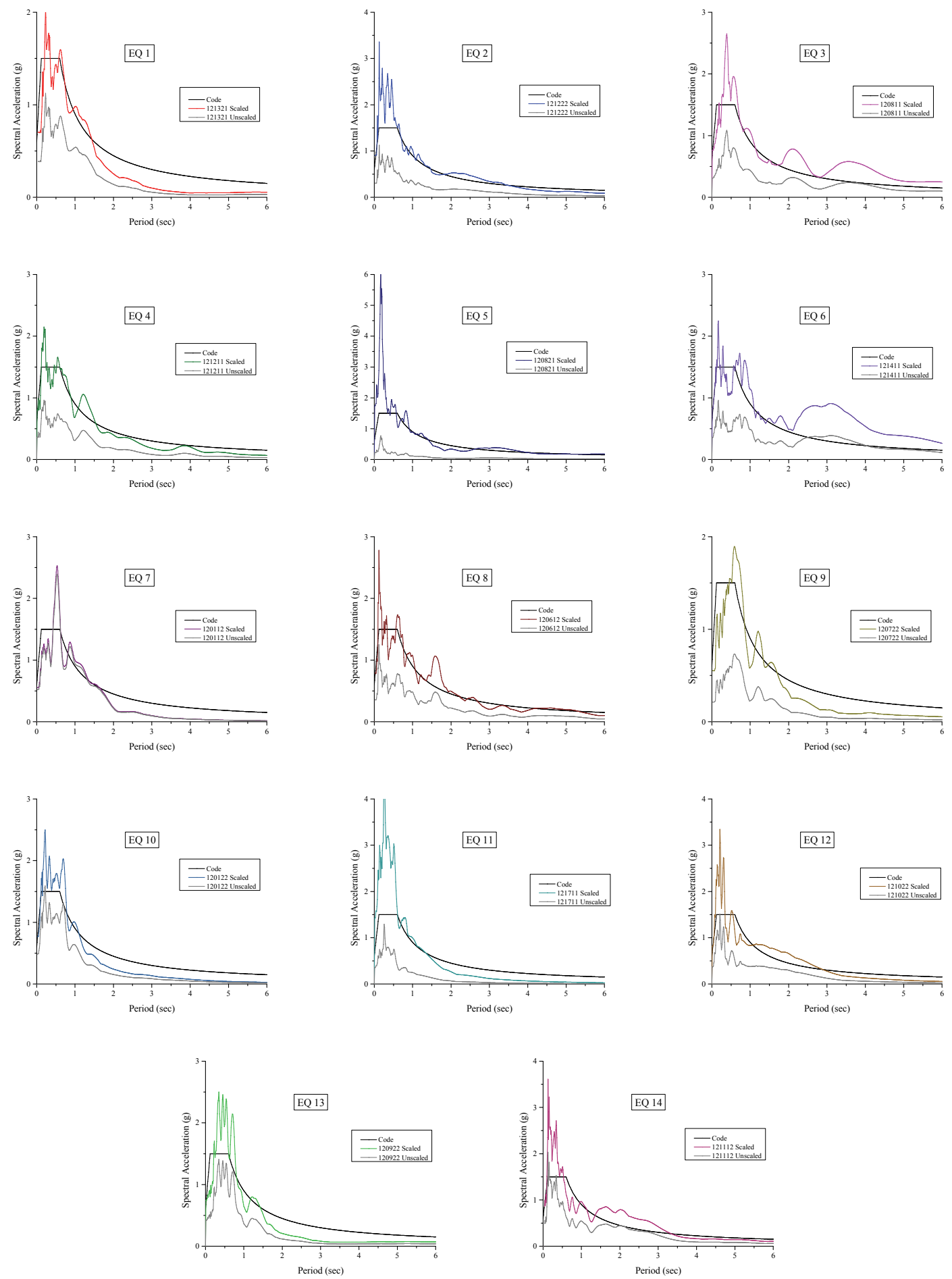

Figure A-2. Acceleration Response Spectra: Original and Scaled for Each Selected Record for N-S Direction of ME4 


\section{A.4 Eight-Story Archetype Building}

\section{A.4.1 8-Story Eccentrically Braced Frame}

Table A-2 summarizes the 14 strong motion records used for the NDP for the N-S direction of ME8 (EBF). Figure A-3 illustrates the set of acceleration response spectra, original and scaled, and the scaled average spectrum. Figure A-4 illustrates the acceleration response spectra, original and scaled, for each select record. For comparison, the ASCE 41 code spectrum is shown in the figures. All analyses completed for the BSE-1 for the ELF and RSA design. All analyses except those using records 1, 4, 5, and 6 completed for the BSE-2 for the ELF design. All analyses except those using records 1, 4, 5, 6, and 10 completed for the BSE-2 for the RSA design.

Table A-2. Ground Motion Records for N-S Direction of ME8

\begin{tabular}{|c|c|c|c|c|c|c|c|c|c|}
\hline $\begin{array}{l}\mathrm{I} \\
\mathrm{D}\end{array}$ & EQ No. & Event Name & Station & Comp. ${ }^{1}$ & Error $^{2}$ & $\begin{array}{c}\text { BSE-2 } \\
\text { Scaling }^{3}\end{array}$ & $\begin{array}{c}\text { BSE-1 } \\
\text { Scaling }^{3}\end{array}$ & Step $^{4}$ & Time $^{5}$ \\
\hline 1 & 12 & Imperial Valley & E1 Centro Array \#11 & 2 & 28 & 4.120 & 2.747 & 0.005 & 40 \\
\hline 2 & 34 & $\begin{array}{c}\text { Superstition } \\
\text { Hills }\end{array}$ & Poe Road (temp) & 2 & 28 & 3.000 & 2.000 & 0.010 & 23 \\
\hline 3 & 5 & Duzce, Turkey & Bolu & 1 & 35 & 2.055 & 1.370 & 0.010 & 56 \\
\hline 4 & 10 & Imperial Valley & Delta & 2 & 40 & 2.464 & 1.643 & 0.010 & 100 \\
\hline 5 & 20 & Kocaeli, Turkey & Arcelik & 2 & 42 & 5.781 & 3.854 & 0.005 & 30 \\
\hline 6 & 21 & Landers & Yermo Fire Station & 1 & 48 & 2.928 & 1.952 & 0.020 & 44 \\
\hline 7 & 31 & $\begin{array}{c}\text { Superstition } \\
\text { Hills }\end{array}$ & El Centro Imp. Co. & 1 & 44 & 2.849 & 1.899 & 0.005 & 40 \\
\hline 8 & 38 & Chi-Chi, Taiwan & CHY101 & 2 & 52 & 1.182 & 0.788 & 0.005 & 90 \\
\hline 9 & 42 & San Fernando & LA - Hollywood Stor & 2 & 55 & 8.024 & 5.350 & 0.010 & 28 \\
\hline 10 & 29 & Manjil, Iran & Abbar & 1 & 57 & 3.127 & 2.085 & 0.020 & 54 \\
\hline 11 & 15 & Kobe, Japan & Shin-Osaka & 1 & 46 & 3.067 & 2.045 & 0.010 & 41 \\
\hline 12 & 28 & Loma Prieta & Gilroy Array \#3 & 2 & 48 & 2.098 & 1.399 & 0.005 & 40 \\
\hline 13 & 7 & Hector Mine & Hector & 1 & 58 & 4.731 & 3.154 & 0.010 & 46 \\
\hline 14 & 3 & Northridge & Canyon Country-WLC & 1 & 57 & 2.235 & 1.490 & 0.010 & 20 \\
\hline $\begin{array}{r}\text { Notes } \\
1 . \\
2 . \\
3 . \\
4 . \\
5 .\end{array}$ & $\begin{array}{l}\text { Single compc } \\
\text { Scaling facto } \\
\text { Time step in }\end{array}$ & $\begin{array}{l}\text { nber. See FEMA P- } 695 \text { A } \\
\text { e error value computed ir } \\
\text { r the component for the } \\
\text { onds. } \\
\text { ord in seconds. }\end{array}$ & $\begin{array}{l}\text { endix A for additional paramete } \\
\text { ep } 3 \text {. } \\
-2 \text { or BSE-1 EHL (BSE-1 = } 2 / 3\end{array}$ & sociated & pon & & & & \\
\hline
\end{tabular}



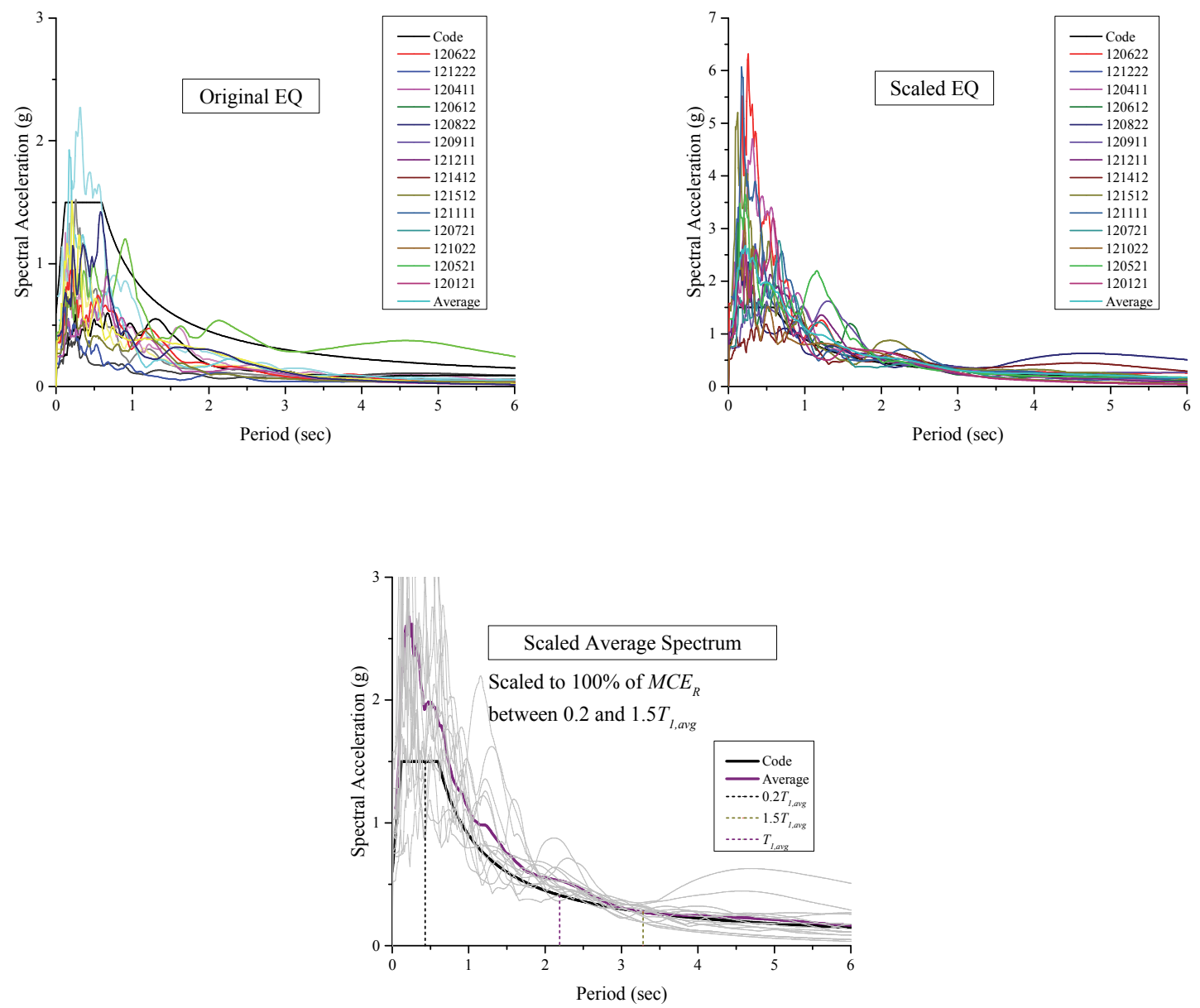

Figure A-3. Acceleration Response Spectra: Original, Scaled, and Scaled Average Spectrum for N-S Direction of ME8 

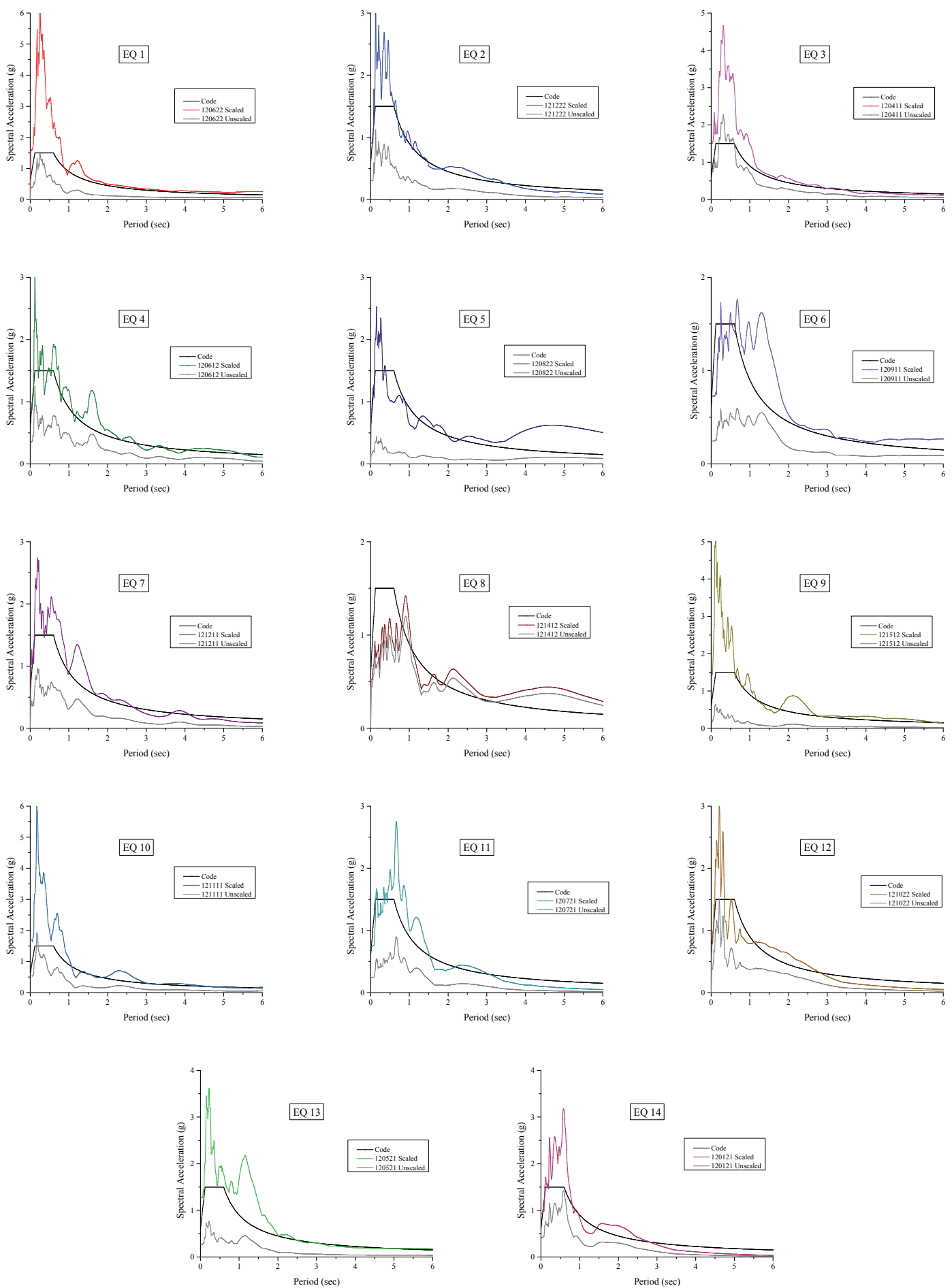

Figure A-4. Acceleration Response Spectra: Original and Scaled for Each Selected Record for N-S Direction of ME8 


\section{A.5 Sixteen-Story Archetype Building}

\section{A.5.1 16-Story Eccentrically Braced Frame}

Table A-3 summarizes the 14 strong motion records used for the NDP for the N-S direction of ME16 (EBF). Figure A-5 illustrates the set of acceleration response spectra, original and scaled, and the scaled average spectrum. Figure A-6 illustrates the acceleration response spectra, original and scaled, for each select record. For comparison, the ASCE 41 code spectrum is shown in the figures. All analyses completed for the BSE-1 and BSE-2 for the ELF design. All analyses completed for the BSE-1 for the RSA design. All analyses except those using records 1, 2, 6, and 11 completed for the BSE-2 for the RSA design.

Table A-3. Ground Motion Records for N-S Direction of ME16

\begin{tabular}{|c|c|c|c|c|c|c|c|c|c|}
\hline ID & EQ No. & Event Name & Station & Comp. & Error $^{2}$ & $\begin{array}{c}\text { BSE-2 } \\
\text { Scaling }^{3}\end{array}$ & $\begin{array}{c}\text { BSE-1 } \\
\text { Scaling }^{3}\end{array}$ & Step $^{4}$ & Time $^{5}$ \\
\hline 1 & 12 & Imperial Valley & El Centro Array \#11 & 2 & 31 & 3.845 & 2.563 & 0.005 & 40 \\
\hline 2 & 10 & Imperial Valley & Delta & 2 & 59 & 2.330 & 1.554 & 0.01 & 100 \\
\hline 3 & 19 & Kocaeli, Turkey & Arcelik & 1 & 67 & 8.791 & 5.861 & 0.005 & 30 \\
\hline 4 & 42 & San Fernando & LA - Hollywood Stor & 2 & 67 & 6.744 & 4.496 & 0.01 & 28 \\
\hline 5 & 29 & Manjil, Iran & Abbar & 1 & 64 & 3.072 & 2.048 & 0.02 & 54 \\
\hline 6 & 21 & Landers & Yermo Fire Station & 1 & 59 & 2.799 & 1.866 & 0.02 & 44 \\
\hline 7 & 27 & Loma Prieta & Gilroy Array \#3 & 1 & 61 & 5.487 & 3.658 & 0.005 & 40 \\
\hline 8 & 7 & Hector Mine & Hector & 1 & 74 & 5.273 & 3.515 & 0.01 & 46 \\
\hline 9 & 5 & Duzce, Turkey & Bolu & 1 & 65 & 2.043 & 1.362 & 0.01 & 56 \\
\hline 10 & 34 & Superstition Hills & Poe Road (temp) & 2 & 68 & 3.154 & 2.103 & 0.01 & 23 \\
\hline 11 & 44 & Friuli, Italy & Tolmezzo & 2 & 82 & 9.497 & 6.331 & 0.005 & 37 \\
\hline 12 & 14 & Kobe, Japan & Nishi-Akashi & 2 & 103 & 4.017 & 2.678 & 0.01 & 41 \\
\hline 13 & 17 & Kocaeli, Turkey & Duzce & 1 & 100 & 1.688 & 1.125 & 0.005 & 28 \\
\hline 14 & 31 & Superstition Hills & E1 Centro Imp. Co. & 1 & 78 & 2.864 & 1.909 & 0.005 & 40 \\
\hline $\begin{array}{c}\text { Notes: } \\
1 . \\
2 . \\
3 . \\
4 . \\
5 .\end{array}$ & $\begin{array}{l}\text { Component Nur } \\
\text { Single composit } \\
\text { Scaling factor fc } \\
\text { Time step in sec } \\
\text { Total time of re }\end{array}$ & $\begin{array}{l}\text { See FEMA P-695 Appen } \\
\text { or value computed in Step } \\
\text { component for the BSE- } 2 \\
\text { n seconds. }\end{array}$ & $\begin{array}{l}\text { for additional parameters assoc } \\
\text { SE-1 EHL (BSE-1 }=2 / 3 \times \text { BSE-2 }\end{array}$ & & & & & & \\
\hline
\end{tabular}



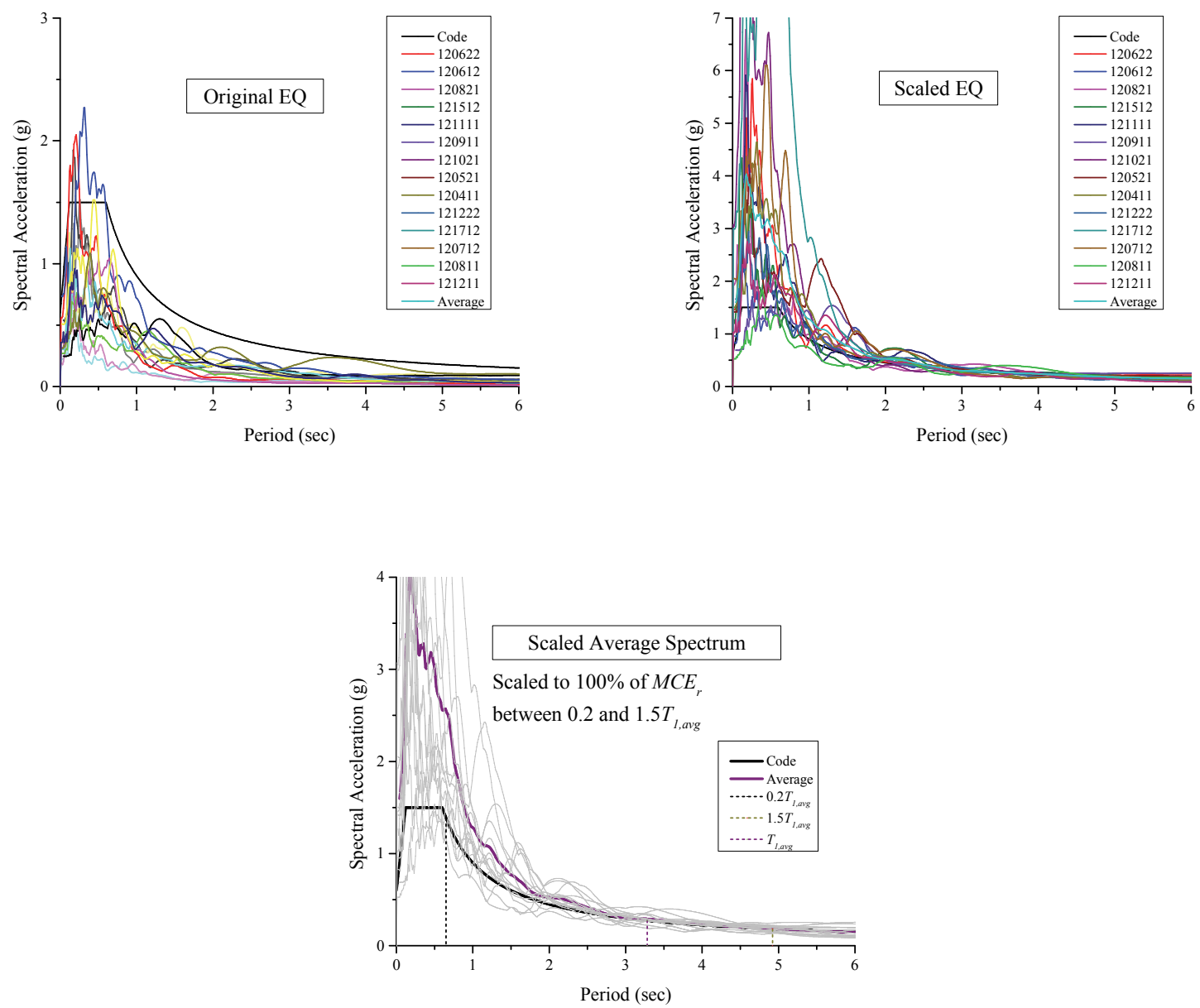

Figure A-5. Acceleration Response Spectra: Original, Scaled, and Scaled Average Spectrum for N-S Direction of ME16 

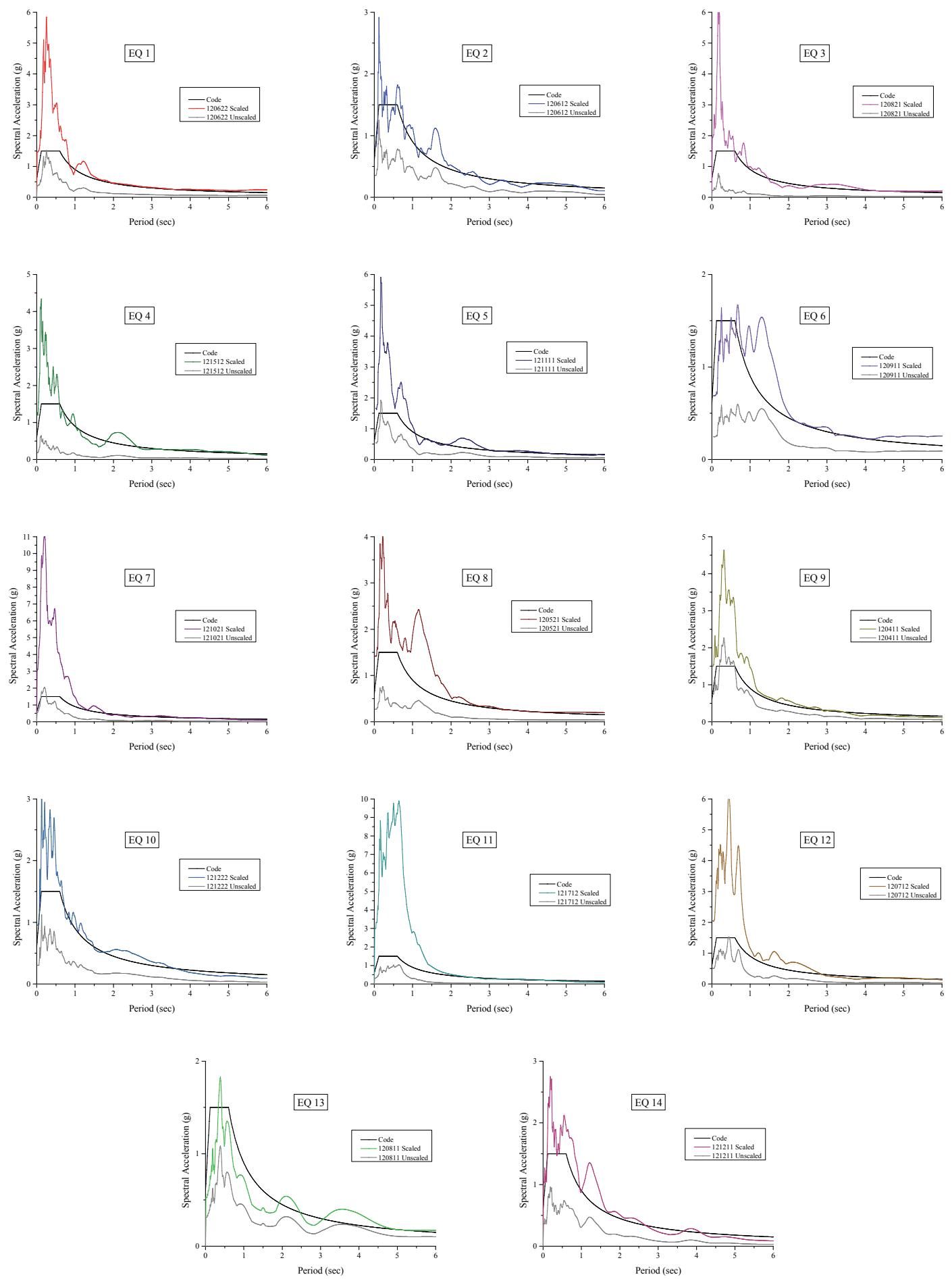

Figure A-6. Acceleration Response Spectra: Original and Scaled for Each Selected Record for N-S Direction of ME16 


\section{A.6 FEMA P-695 Far-Field Record Set}

Figure A-7 through Figure A-50 illustrate the recorded ground motion, Fourier amplitude (frequency and period), and the five percent damped response spectra (displacement, velocity, acceleration) for each component.
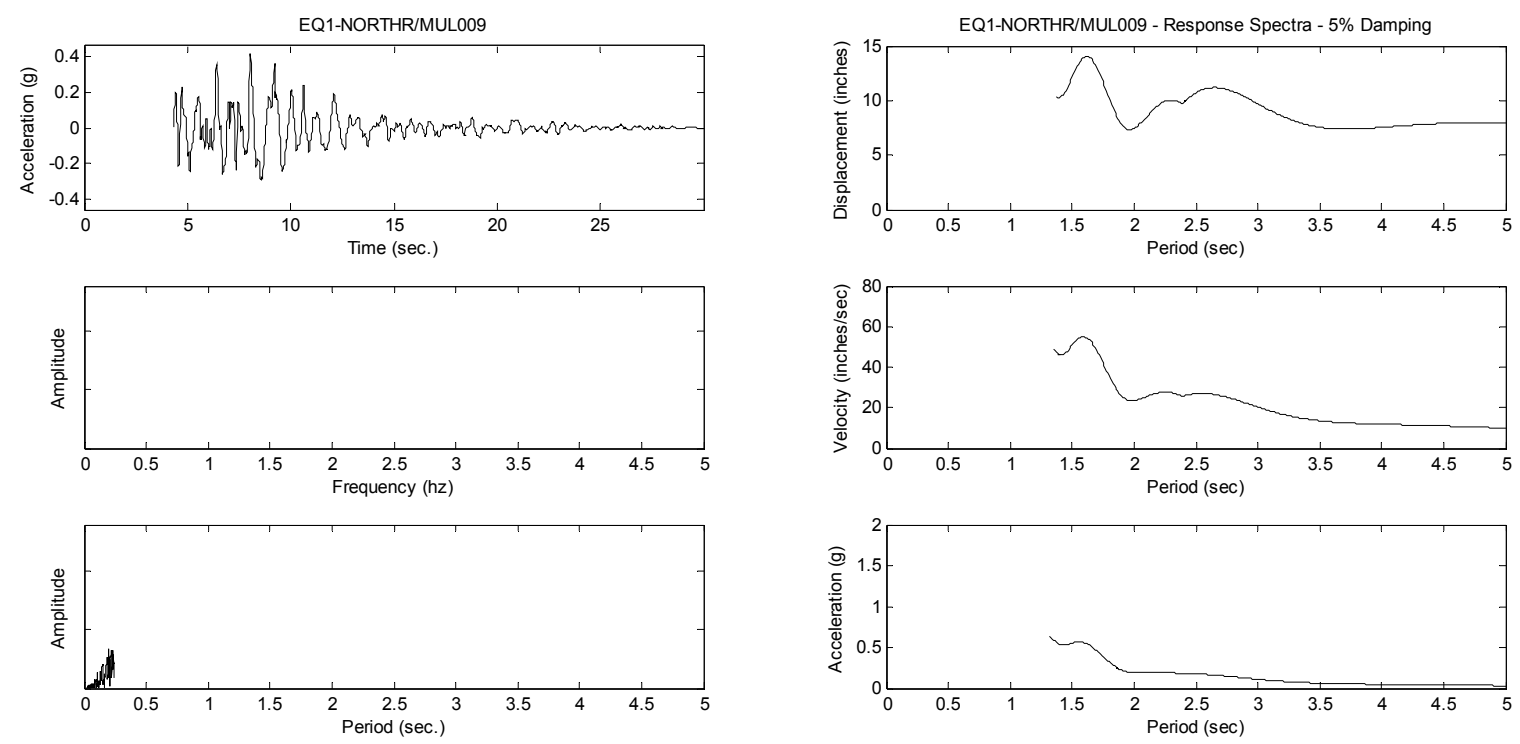

Figure A-7. 1994 Northridge Earthquake at Beverly Hills, Mulholland Drive Station, Comp. 009
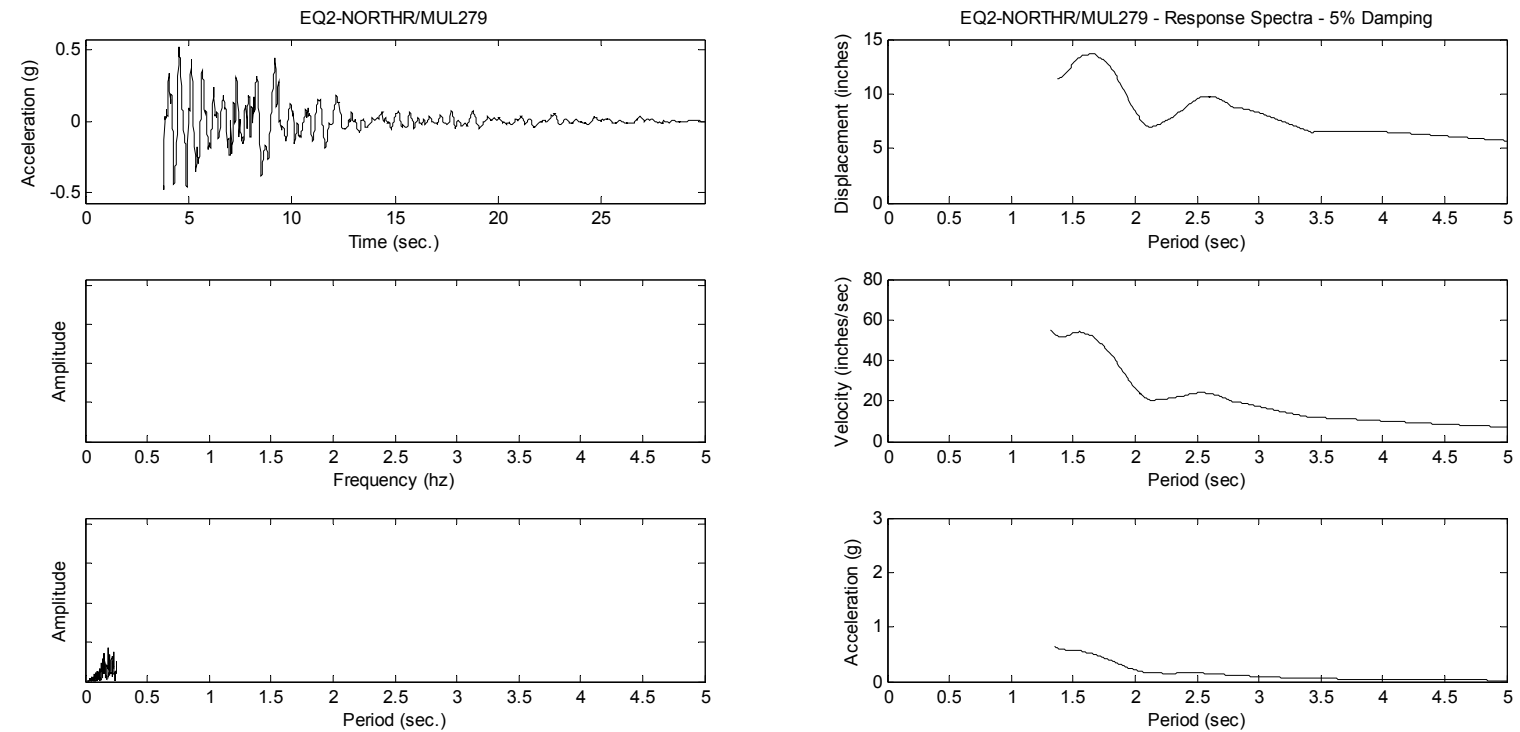

Figure A-8. 1994 Northridge Earthquake at Beverly Hills, Mulholland Drive Station, Comp. 279 

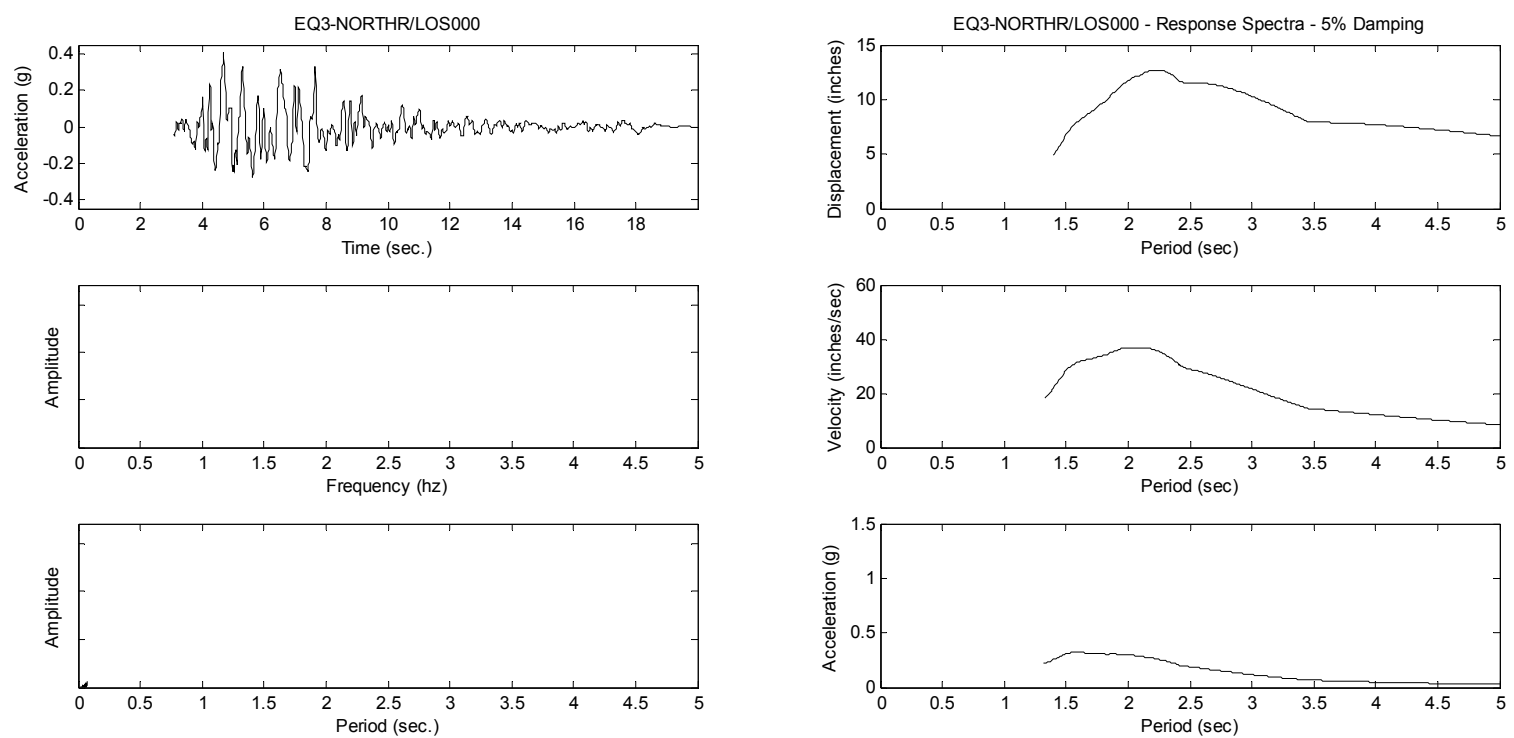

Figure A-9. 1994 Northridge Earthquake at Canyon Country WLC Station, Comp. 000
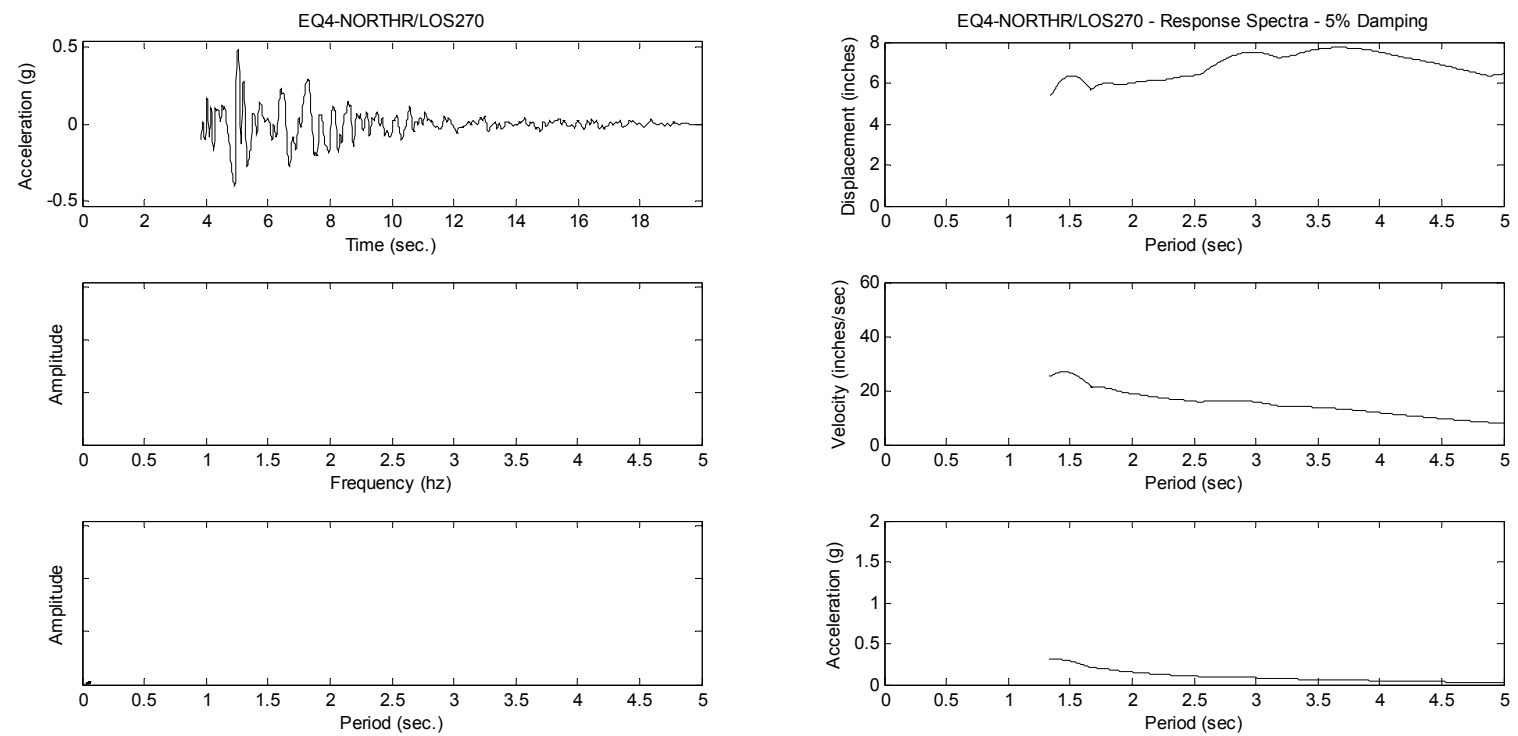

Figure A-10. 1994 Northridge Earthquake at Canyon Country WLC Station, Comp. 270 

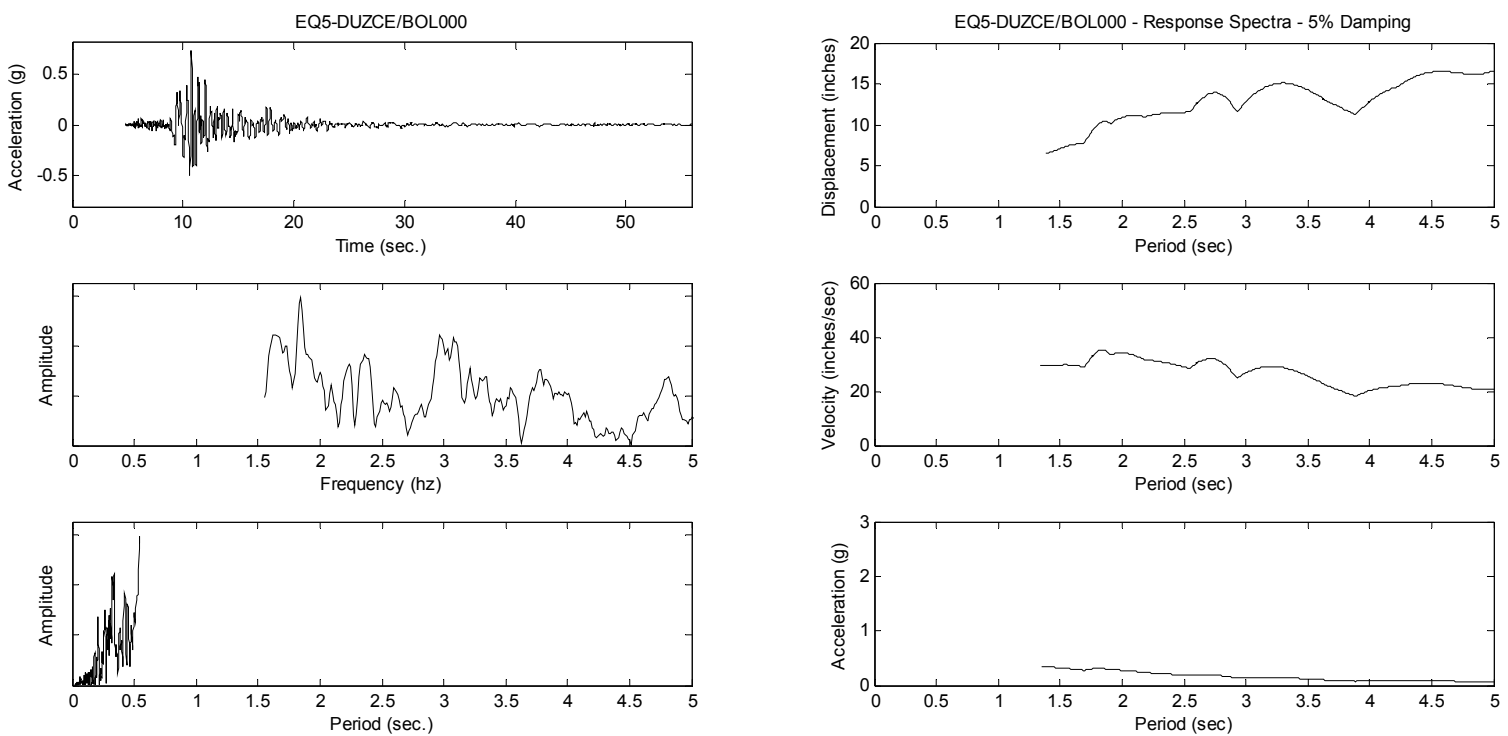

Figure A-11. 1999 Duzce, Turkey Earthquake at Bolu Station, Comp. 000
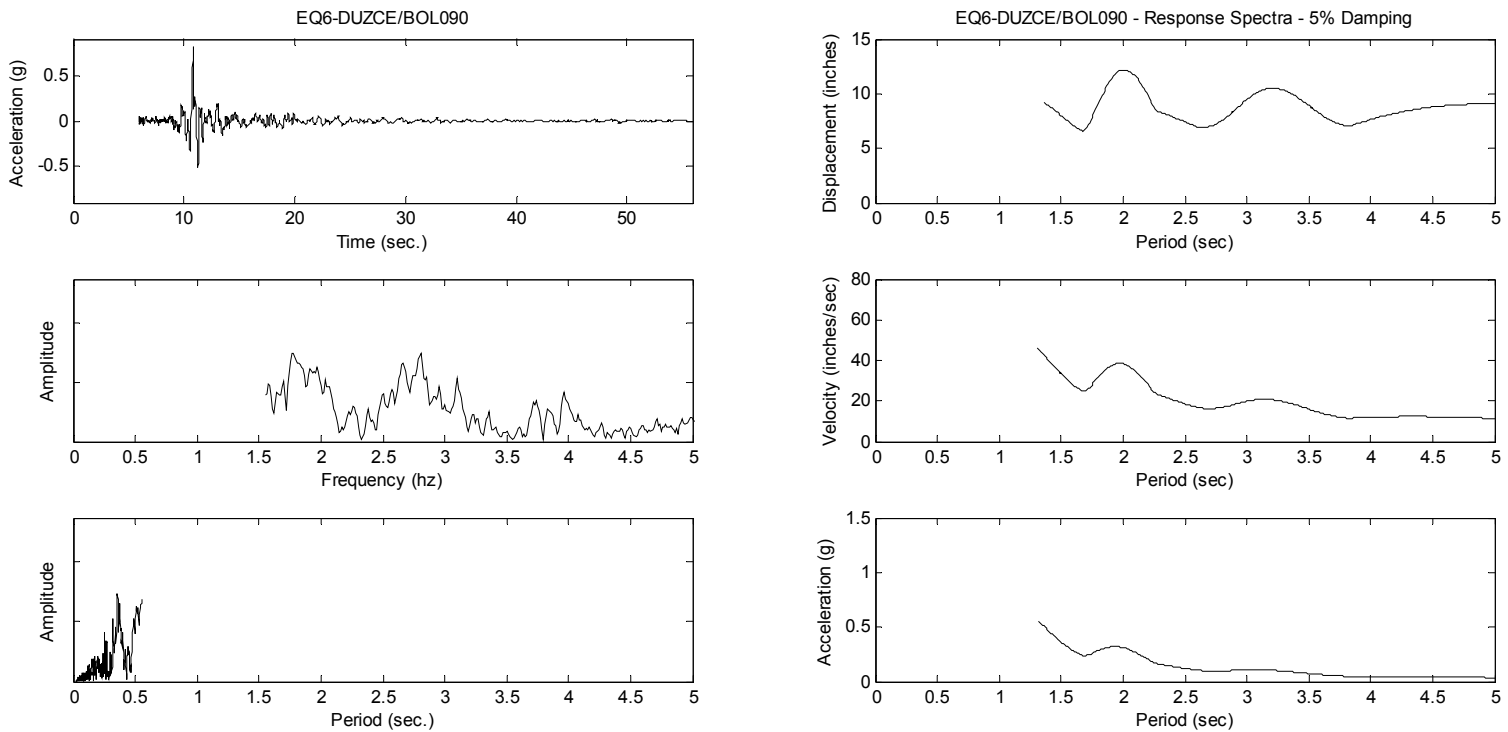

Figure A-12. 1999 Duzce, Turkey Earthquake at Bolu Station, Comp. 090 

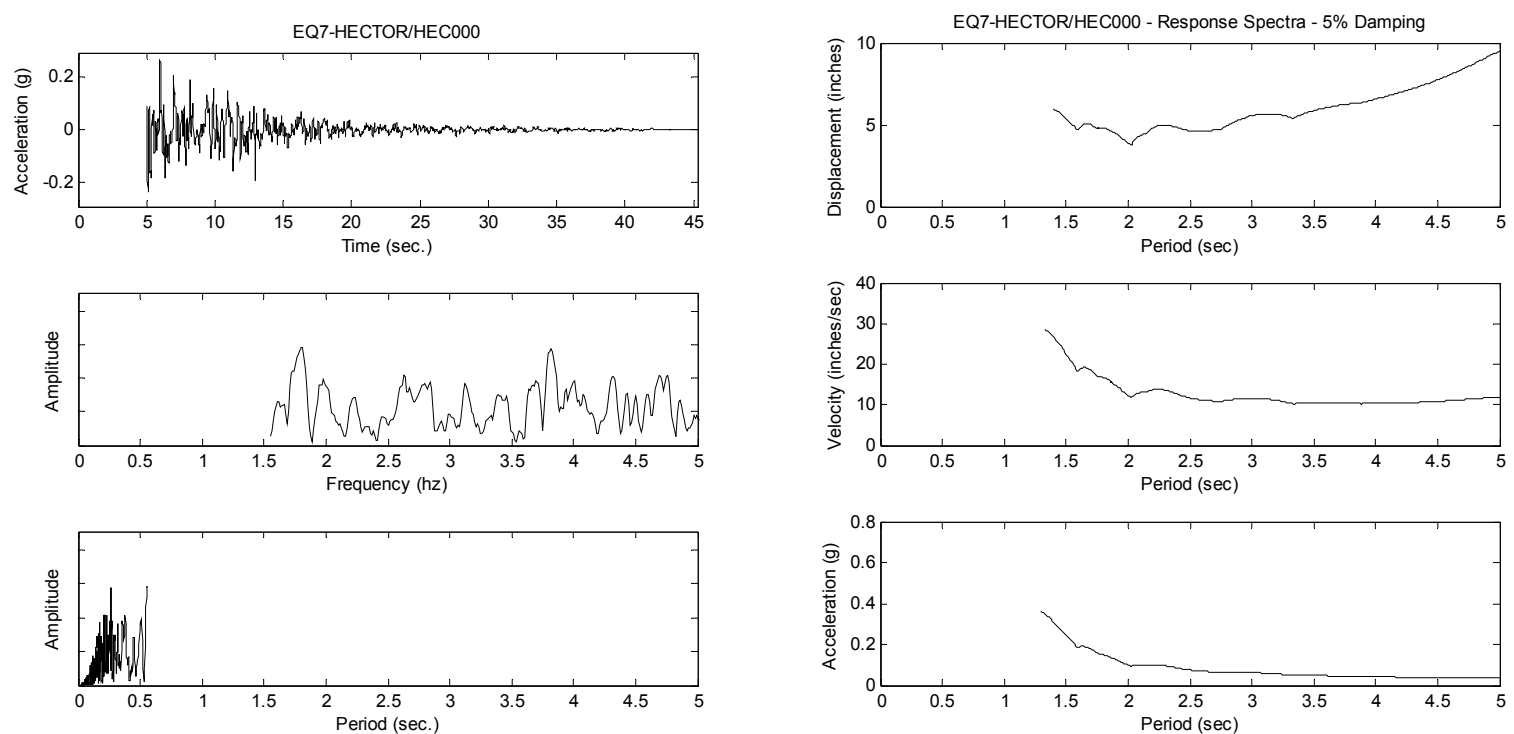

Figure A-13. 1999 Hector Mine Earthquake at Hector Station, Comp. 000
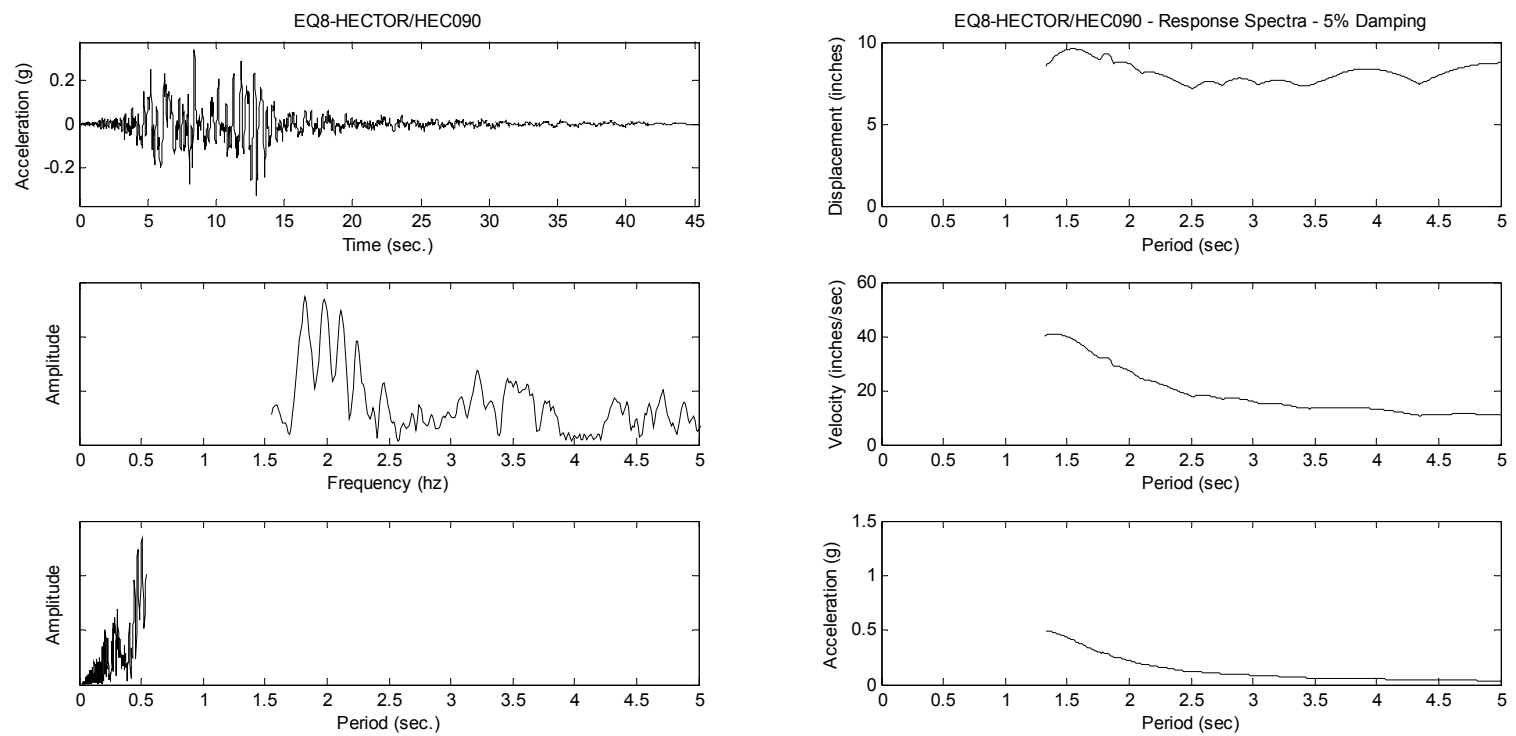

Figure A-14. 1999 Hector Mine Earthquake at Hector Station, Comp. 090 

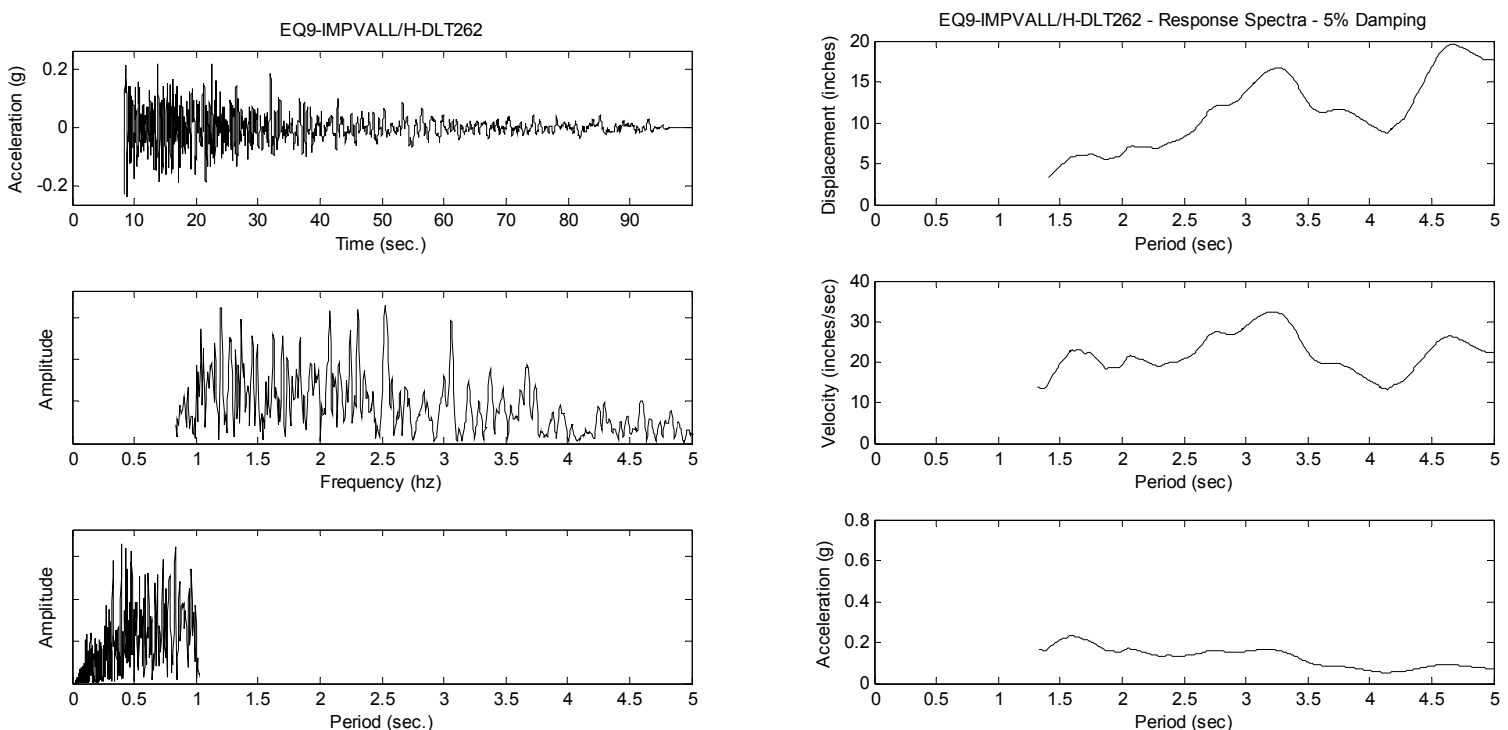

Figure A-15. 1979 Imperial Valley Earthquake at Delta Station, Comp. 262
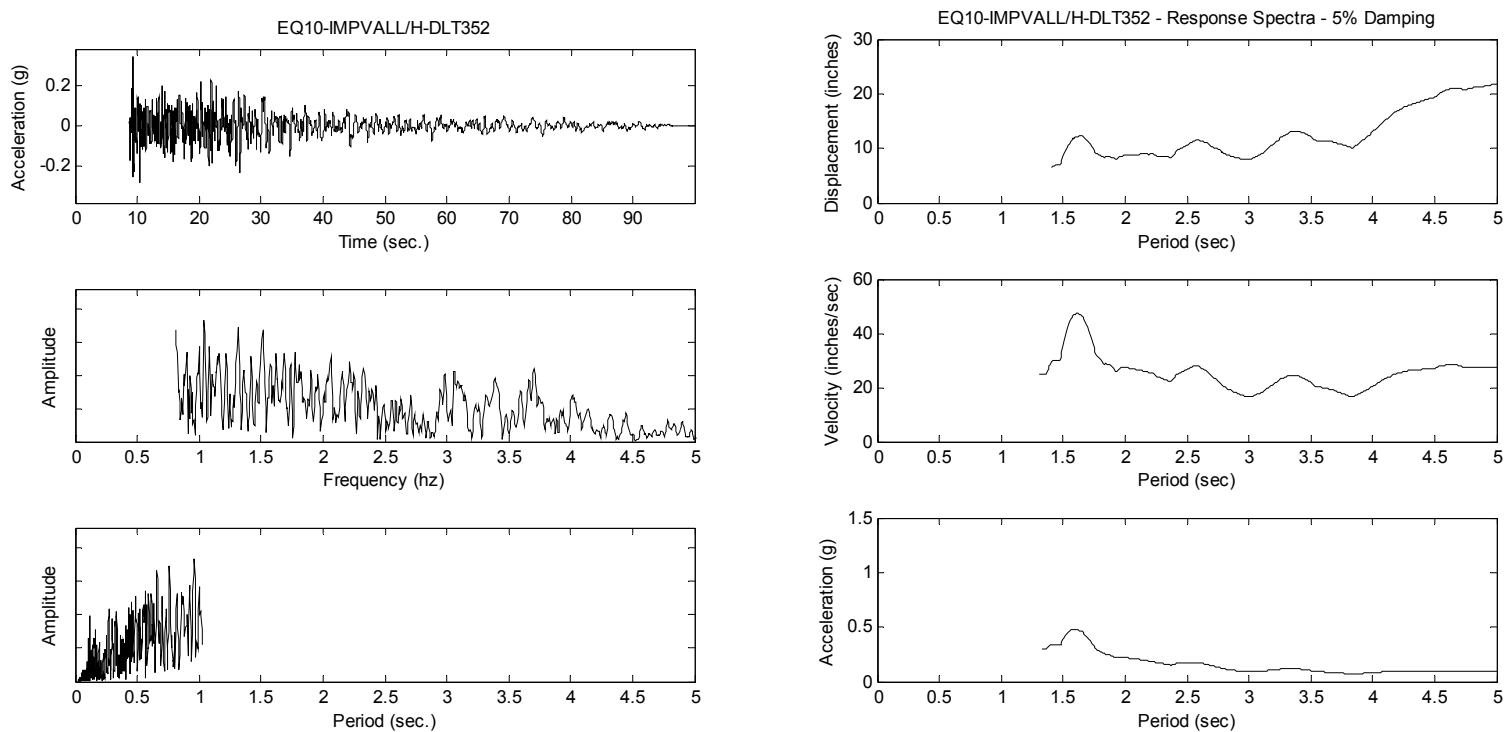

Figure A-16. 1979 Imperial Valley Earthquake at Delta Station, Comp. 352 

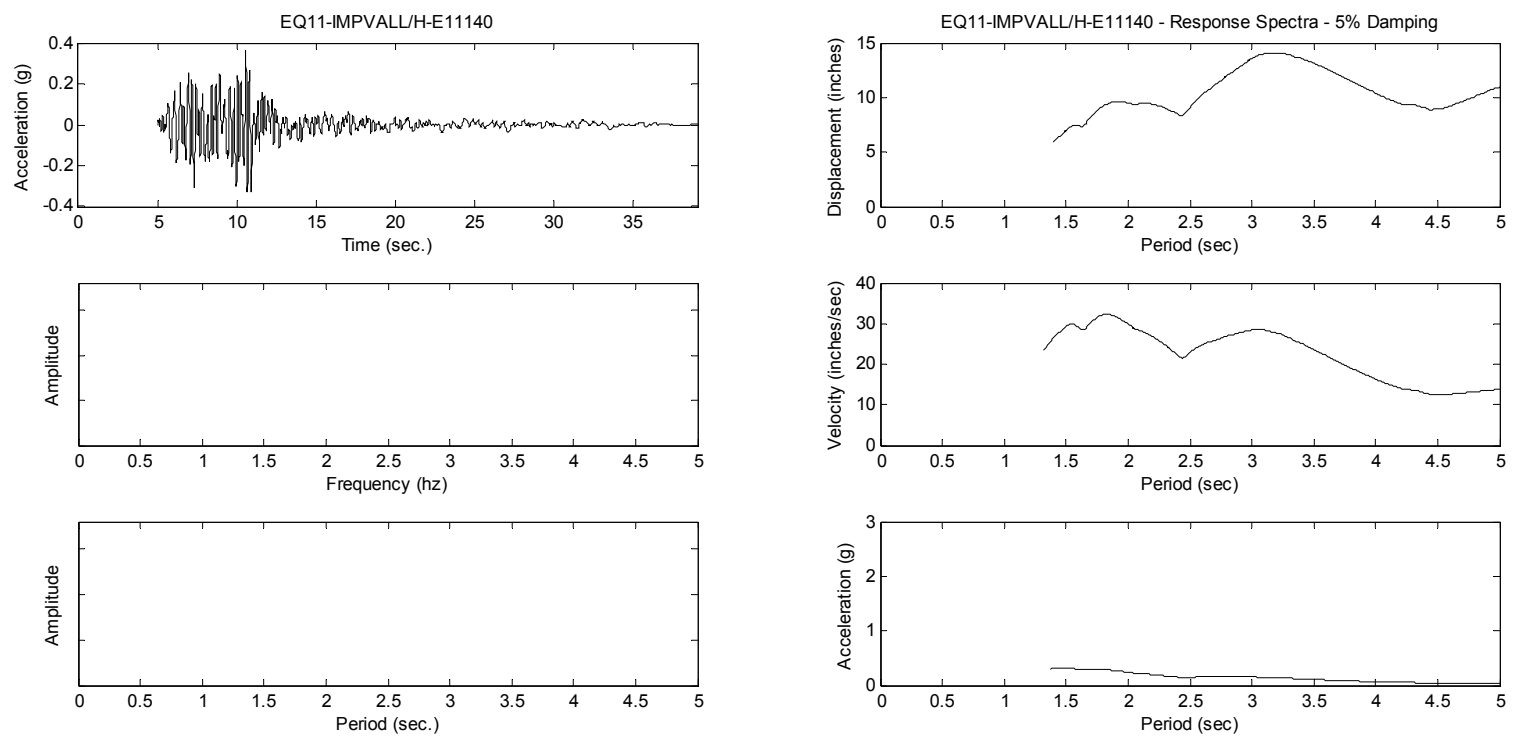

Figure A-17. 1979 Imperial Valley Earthquake at El Centro Array Station \#11, Comp. 140
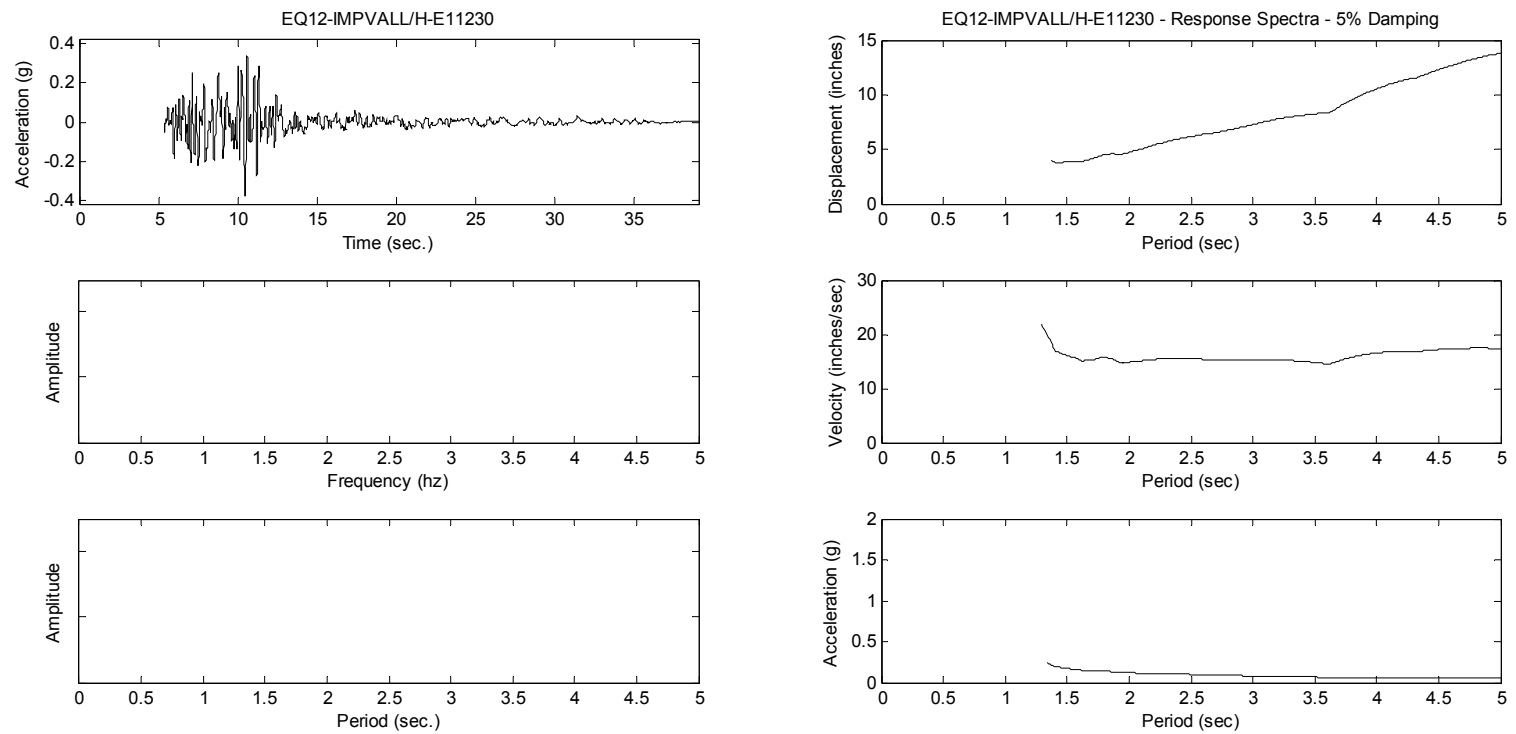

Figure A-18. 1979 Imperial Valley Earthquake at El Centro Array Station \#11, Comp. 230 

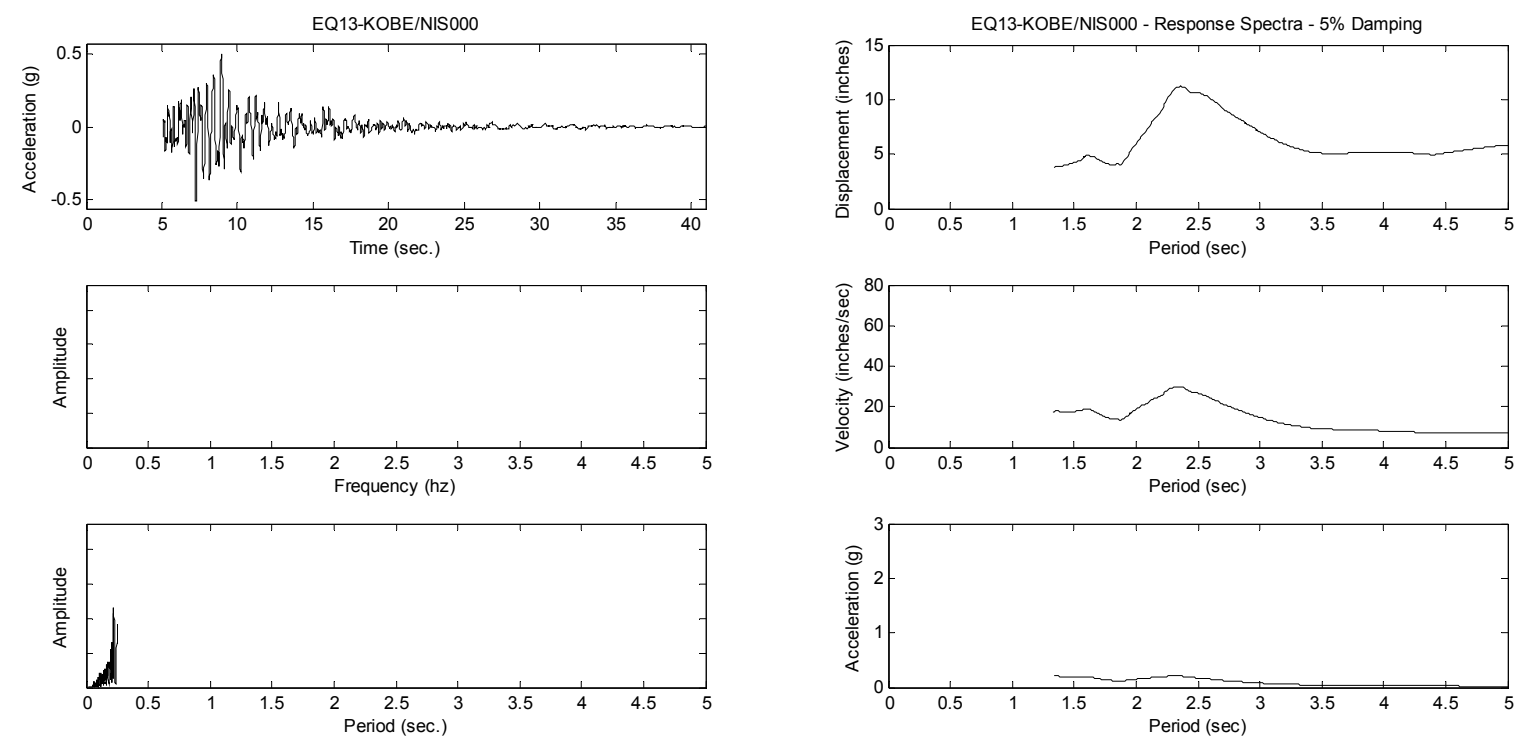

Figure A-19. 1995 Kobe, Japan Earthquake at Nishi-Akashi Station, Comp. 000
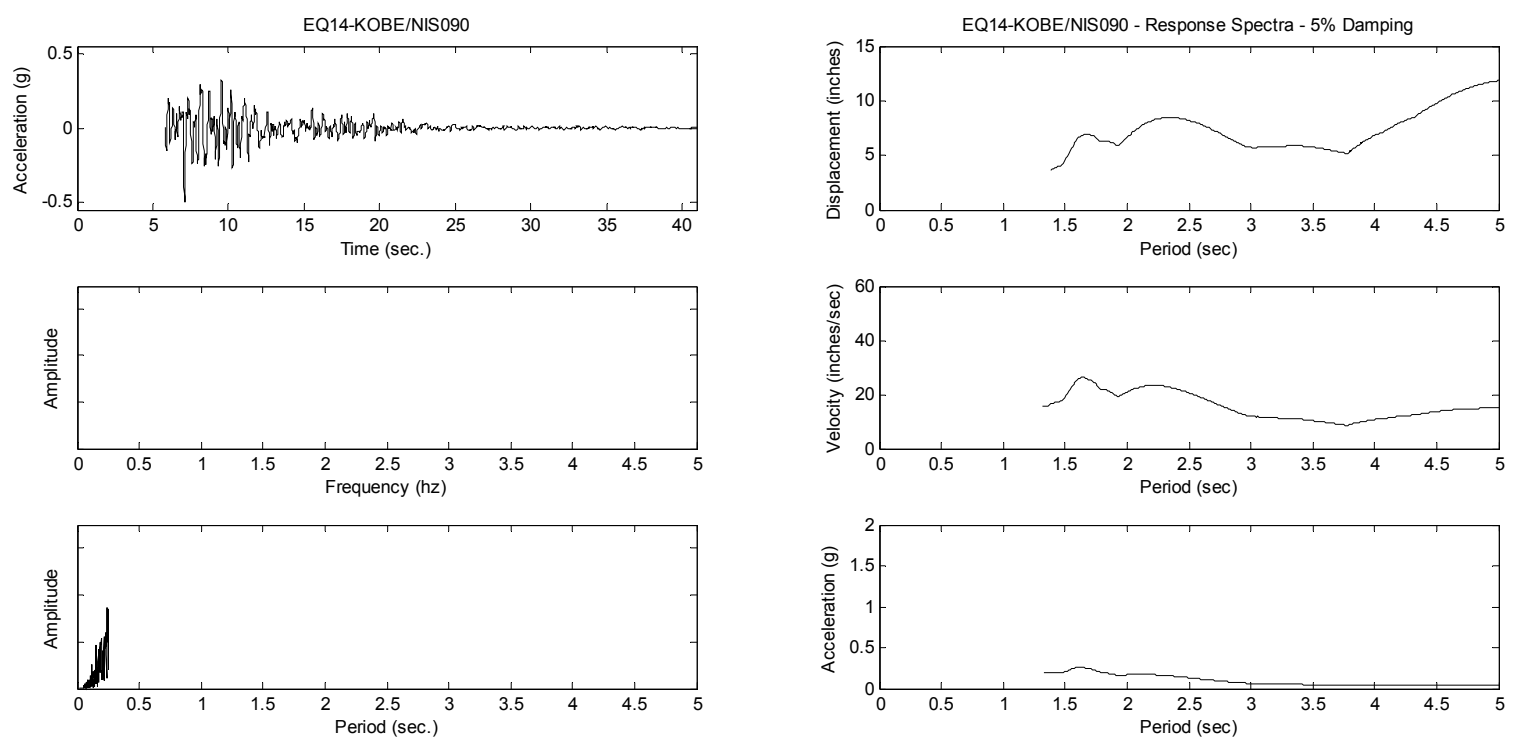

Figure A-20. 1995 Kobe, Japan Earthquake at Nishi-Akashi Station, Comp. 090 

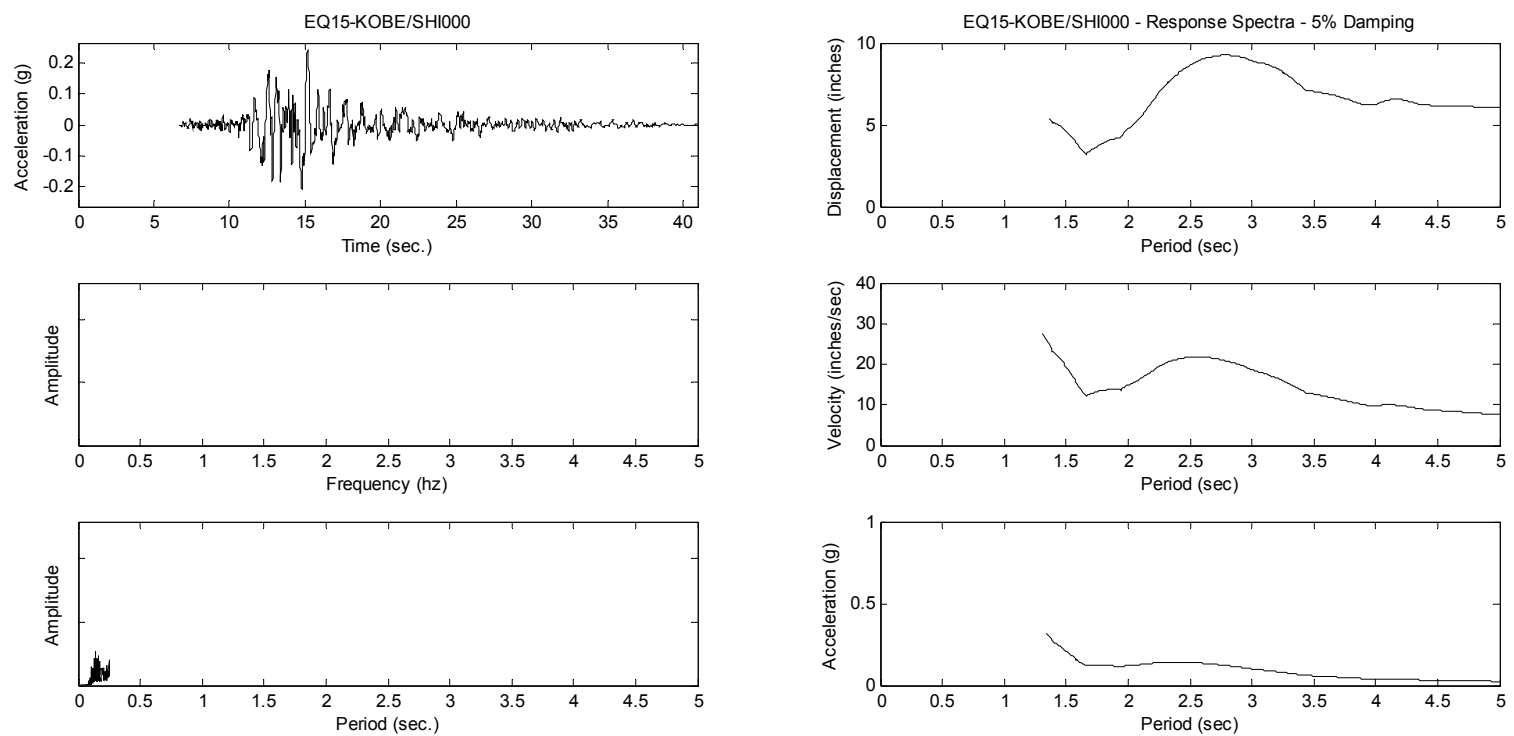

Figure A-21. 1995 Kobe, Japan Earthquake at Shin-Osaka Station, Comp. 000
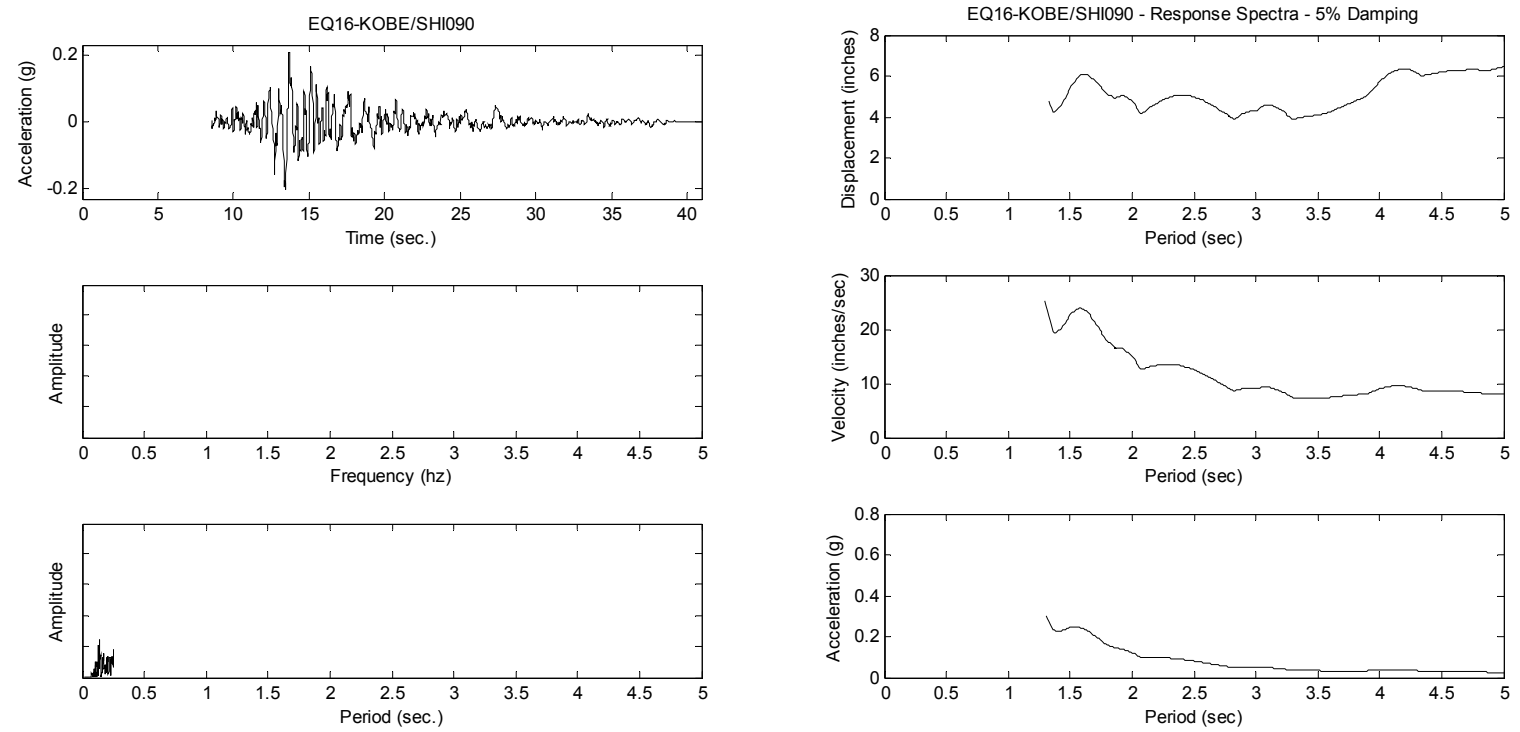

Figure A-22. 1995 Kobe, Japan Earthquake at Shin-Osaka Station, Comp. 090 

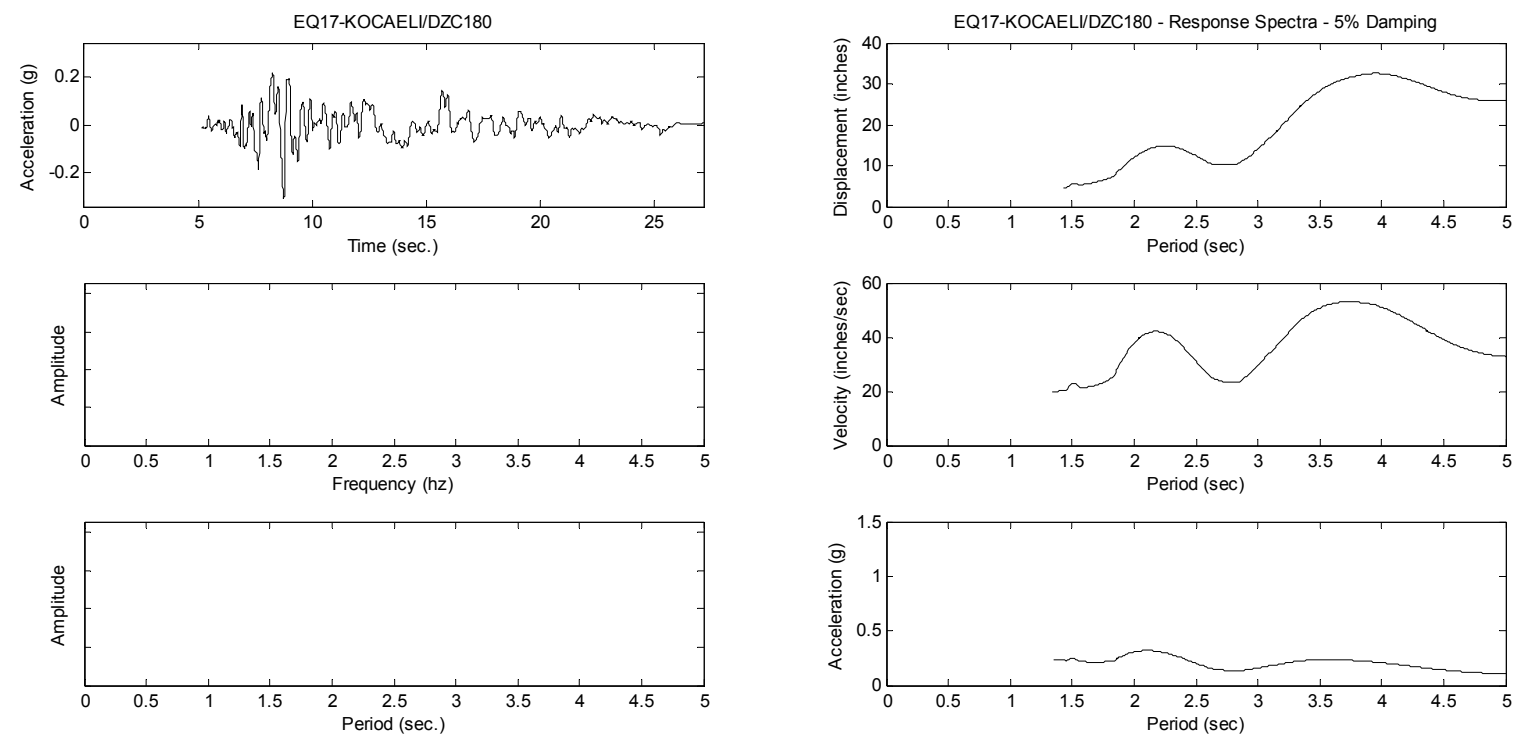

Figure A-23. 1999 Kocaeli, Turkey Earthquake at Duzce Station, Comp. 180
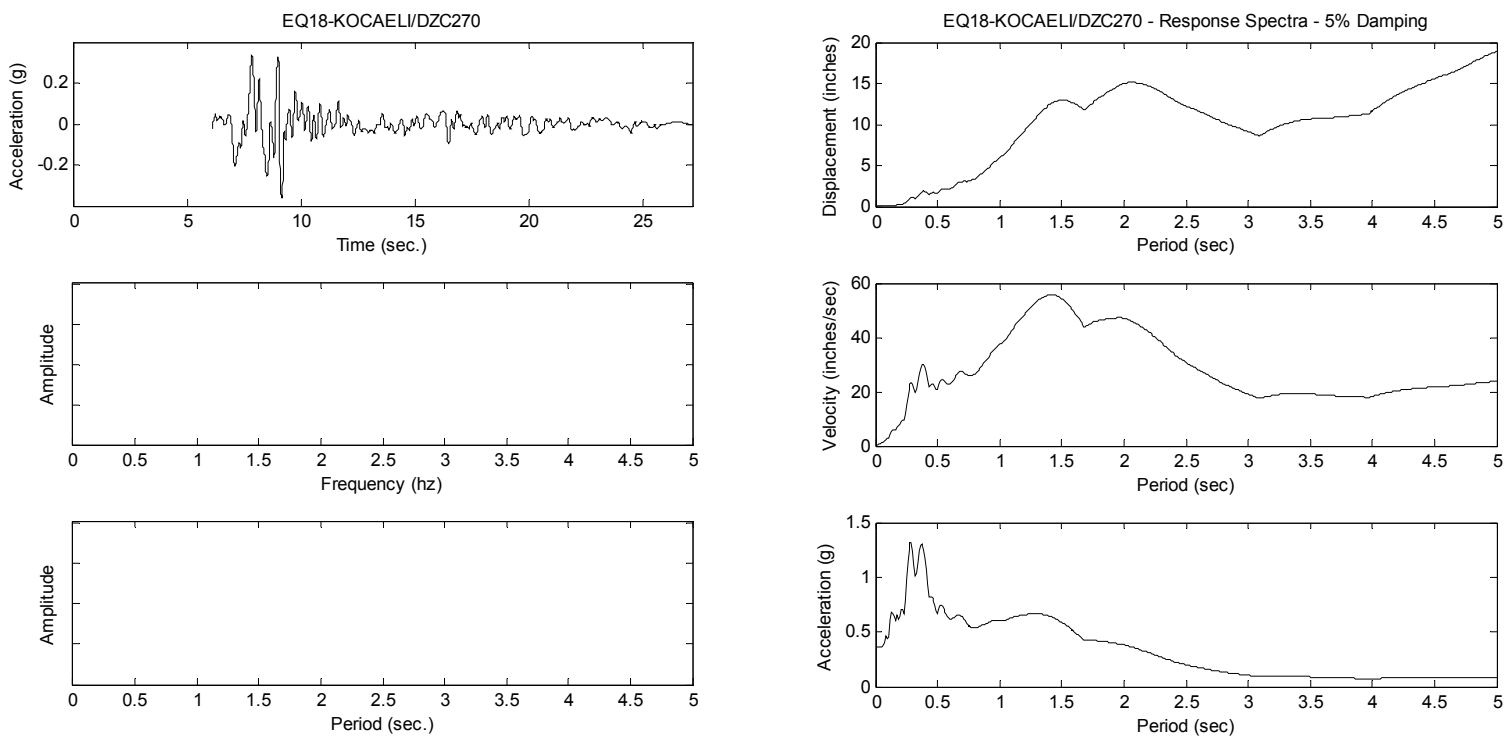

Figure A-24. 1999 Kocaeli, Turkey Earthquake at Duzce Station, Comp. 270 

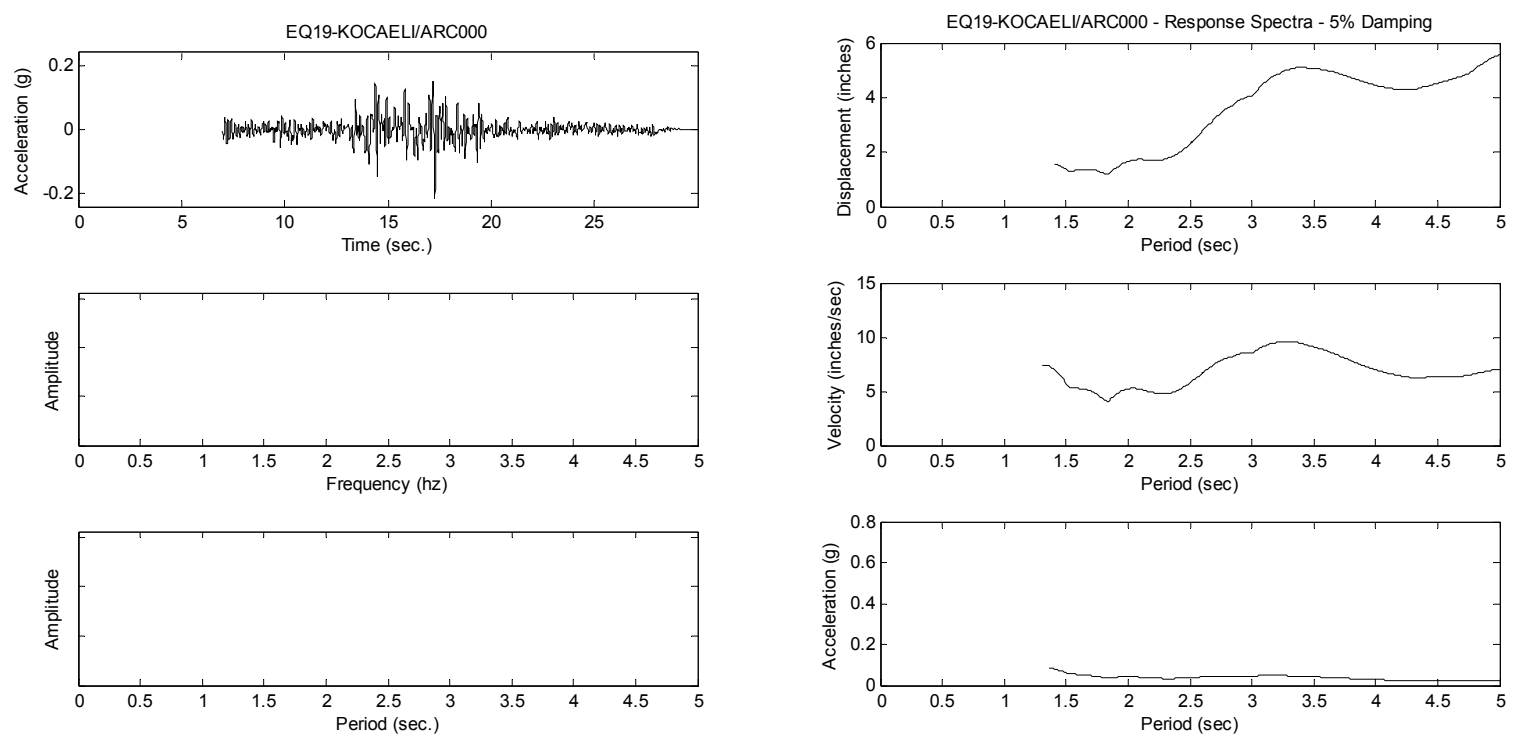

Figure A-25. 1999 Kocaeli, Turkey Earthquake at Arcelik Station, Comp. 000
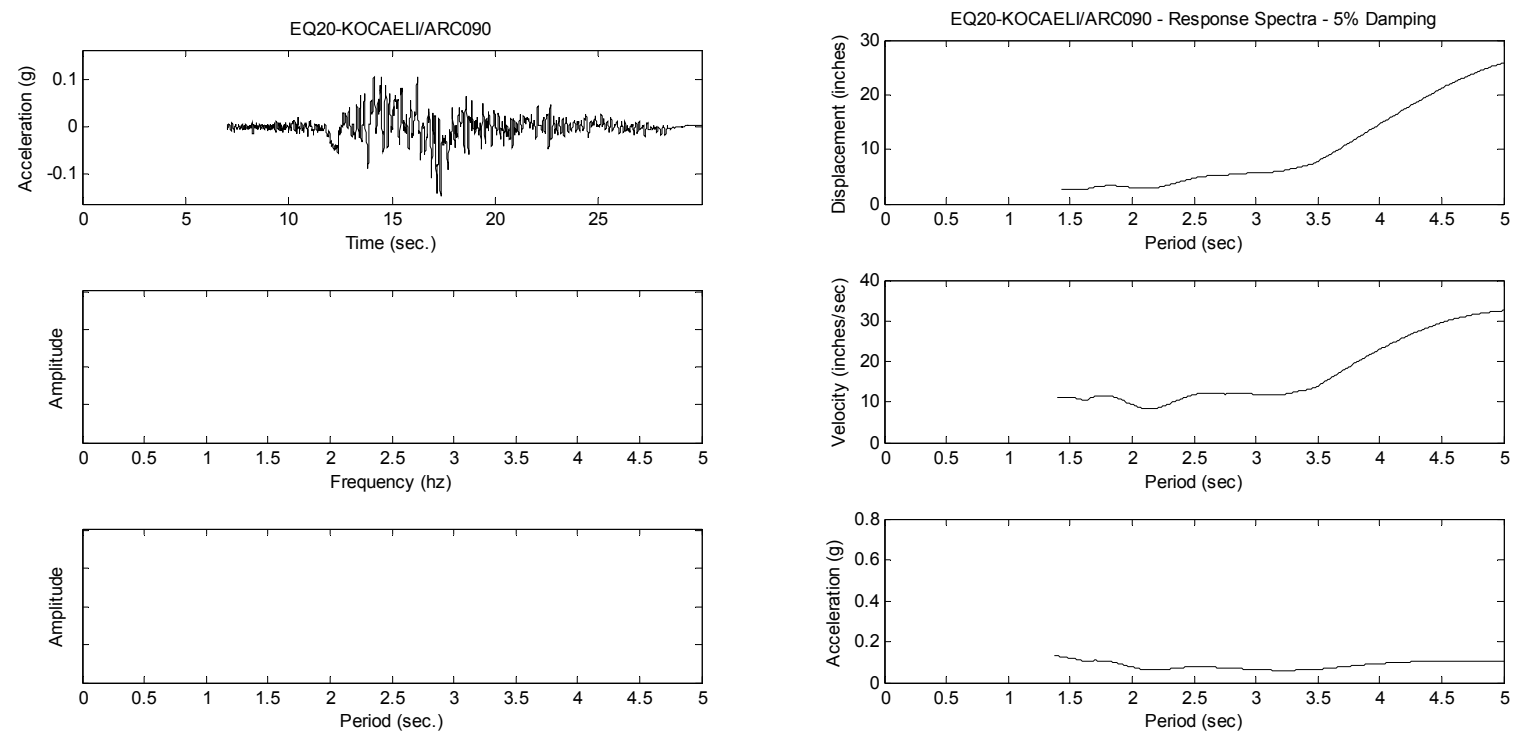

Figure A-26. 1999 Kocaeli, Turkey Earthquake at Arcelik Station, Comp. 090 

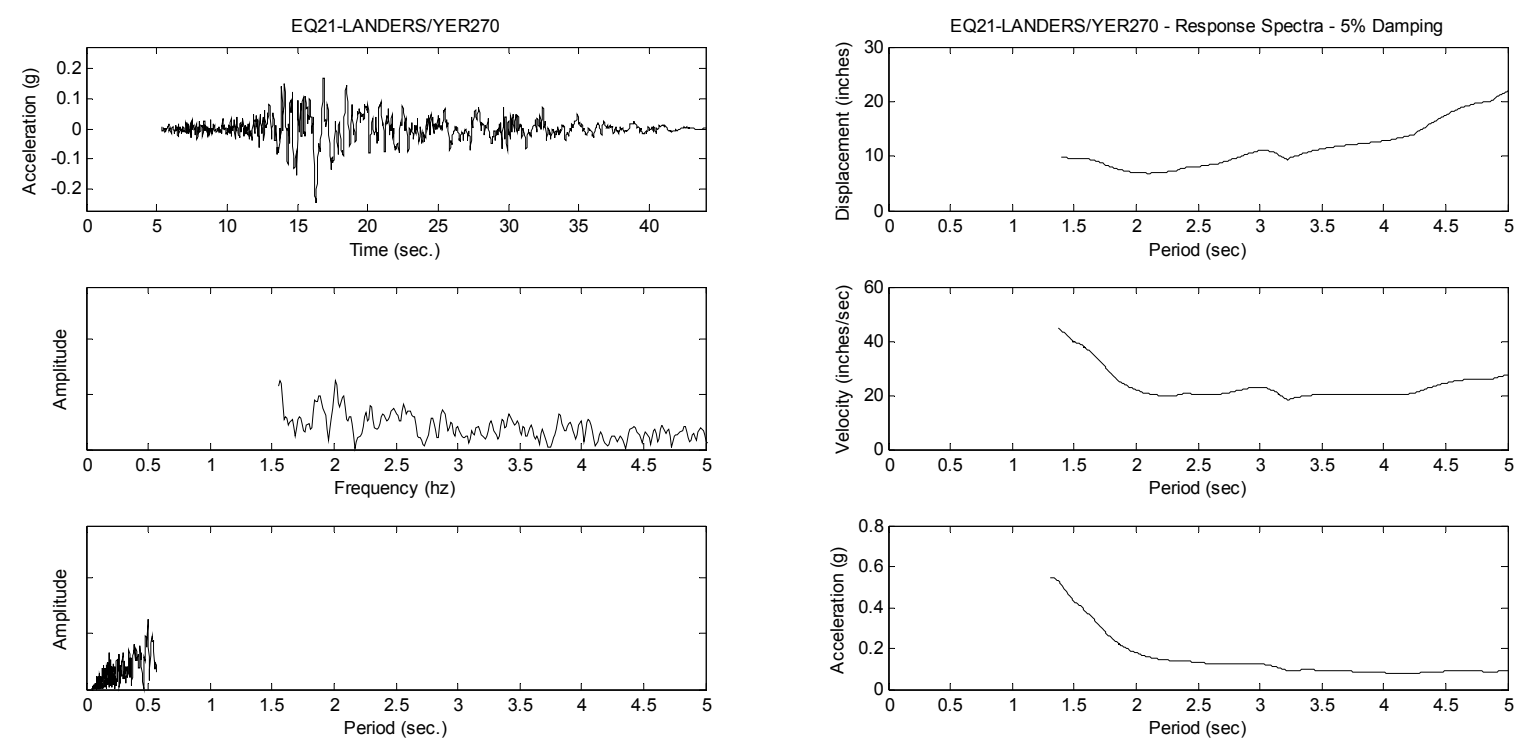

Figure A-27. 1992 Landers Earthquake at Yermo Fire Station, Comp. 270
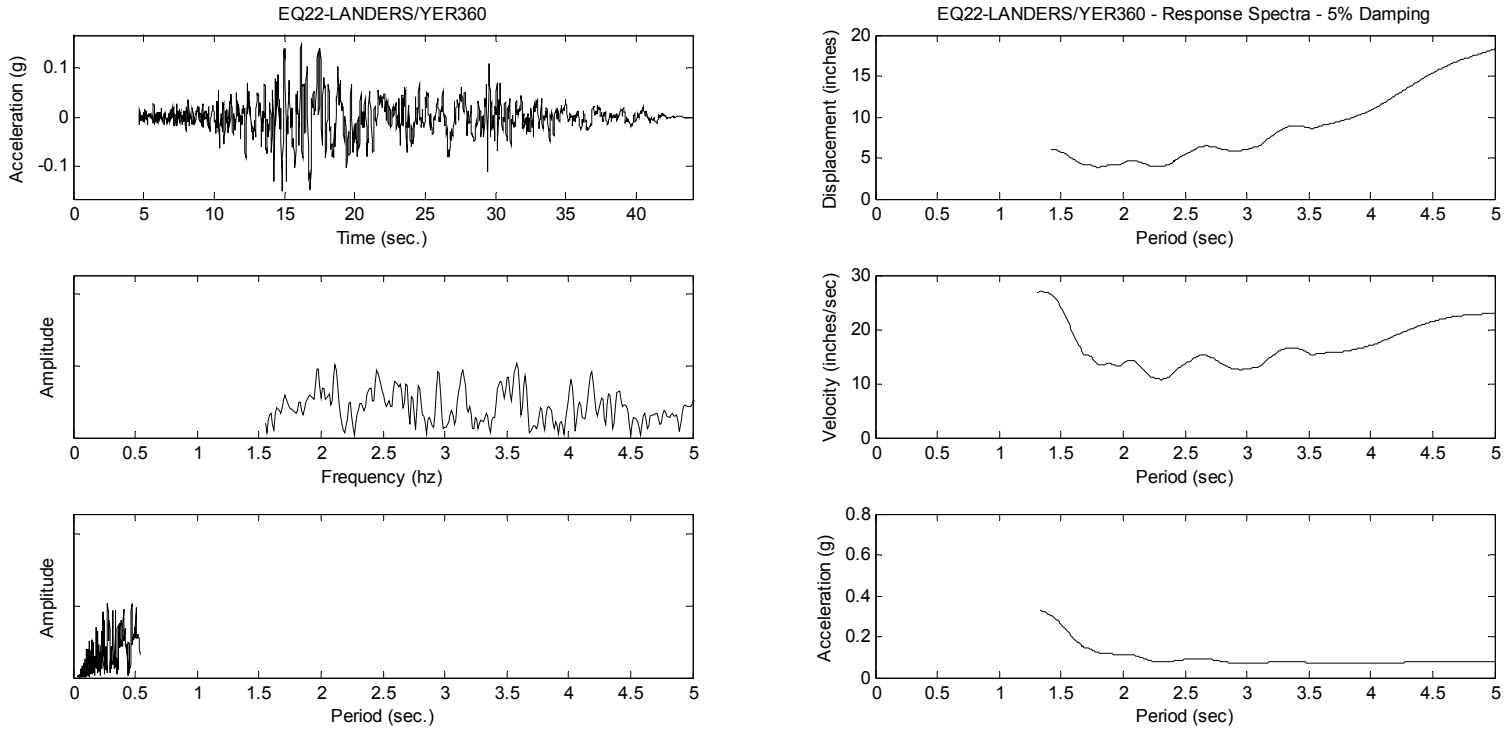

Figure A-28. 1992 Landers Earthquake at Yermo Fire Station, Comp. 360 

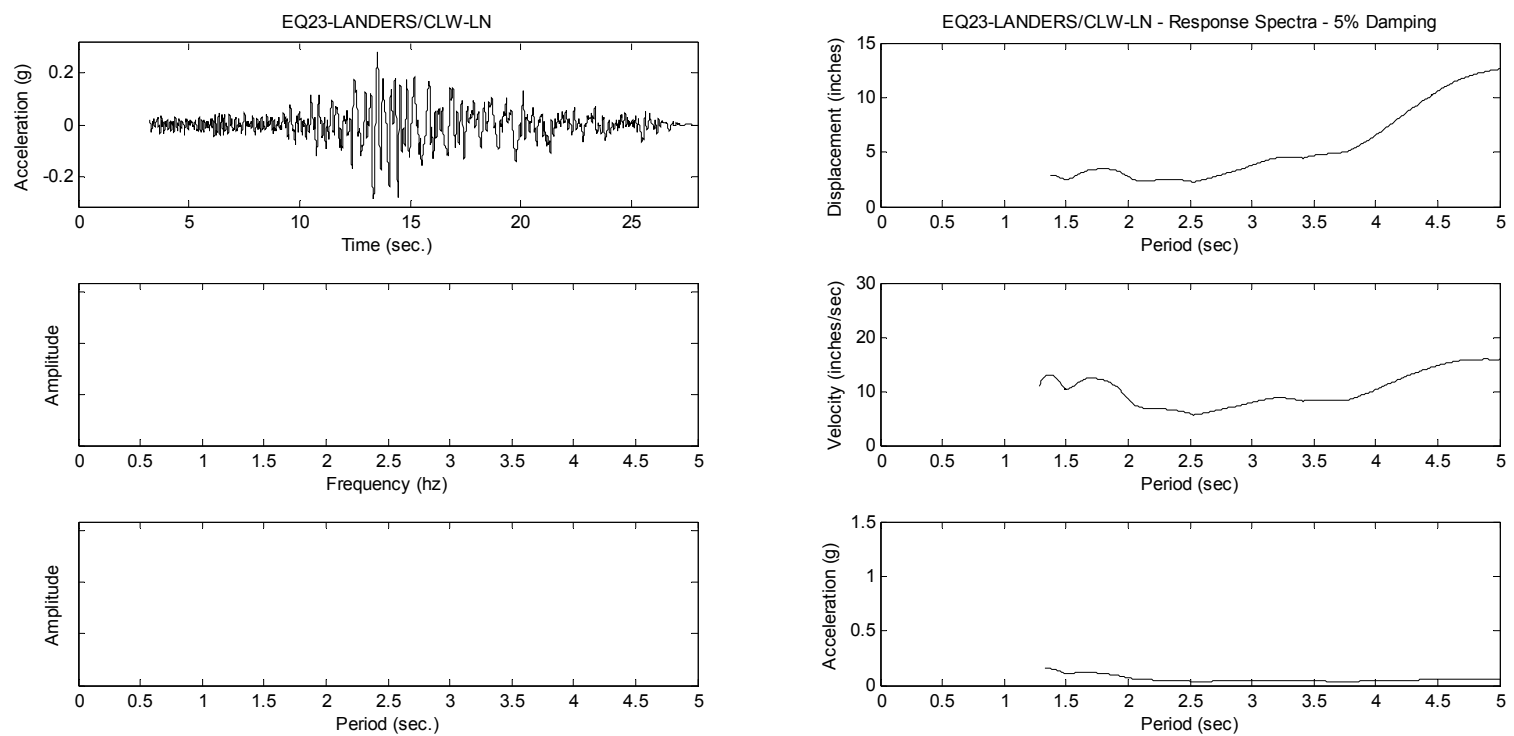

Figure A-29. 1992 Landers Earthquake at Coolwater Station, Longitudinal Direction
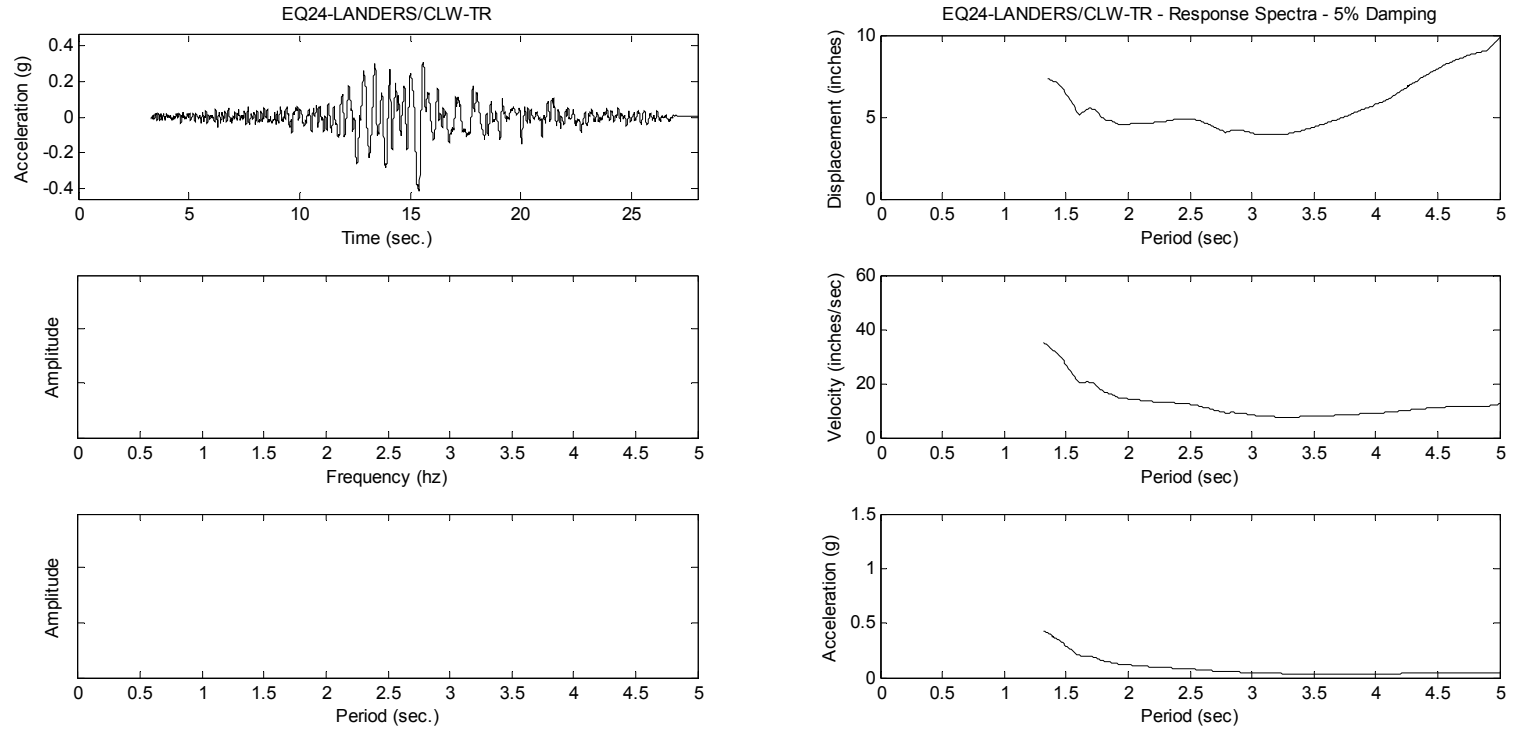

Figure A-30. 1992 Landers Earthquake at Coolwater Station, Transverse Direction 

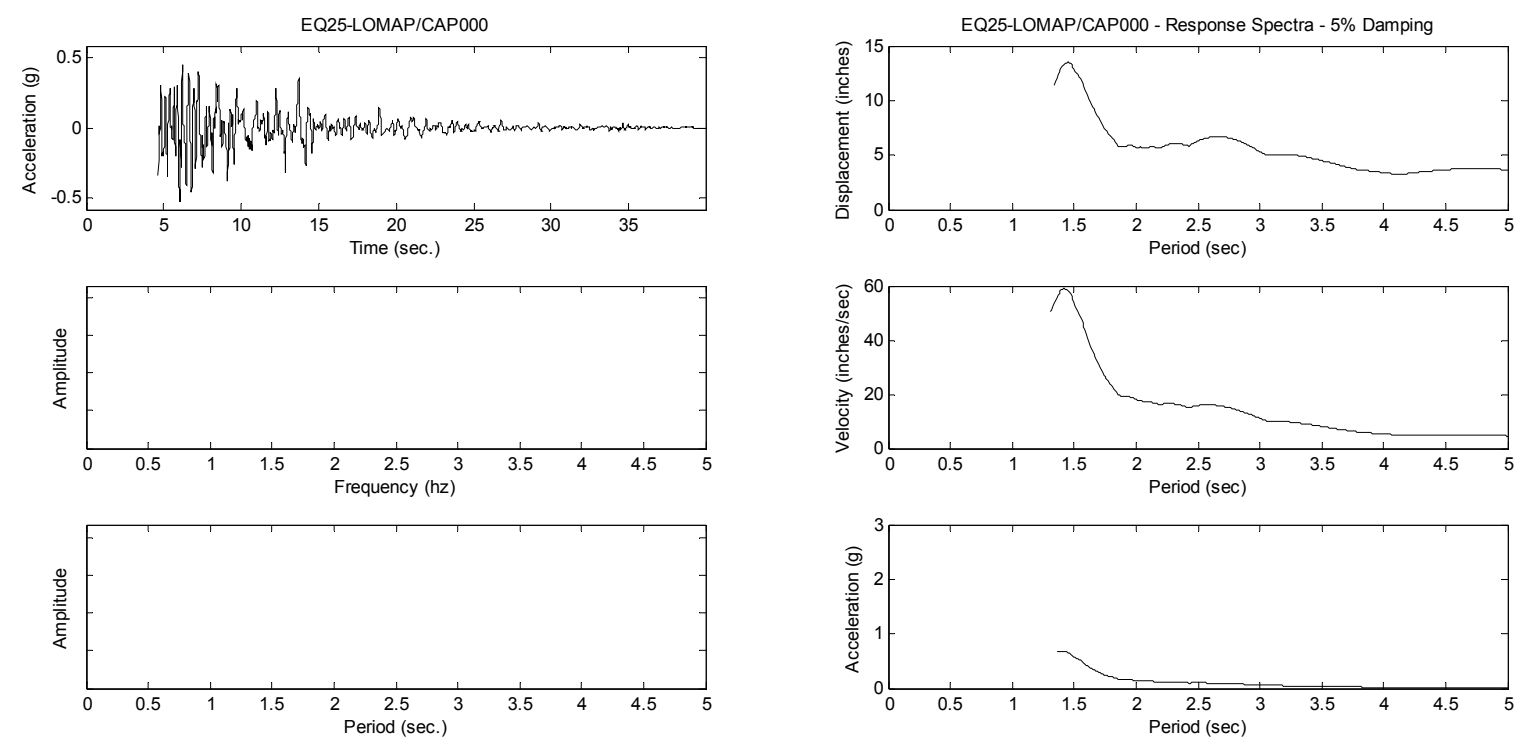

Figure A-31. 1989 Loma Prieta Earthquake at Capitola Station, Comp. 000
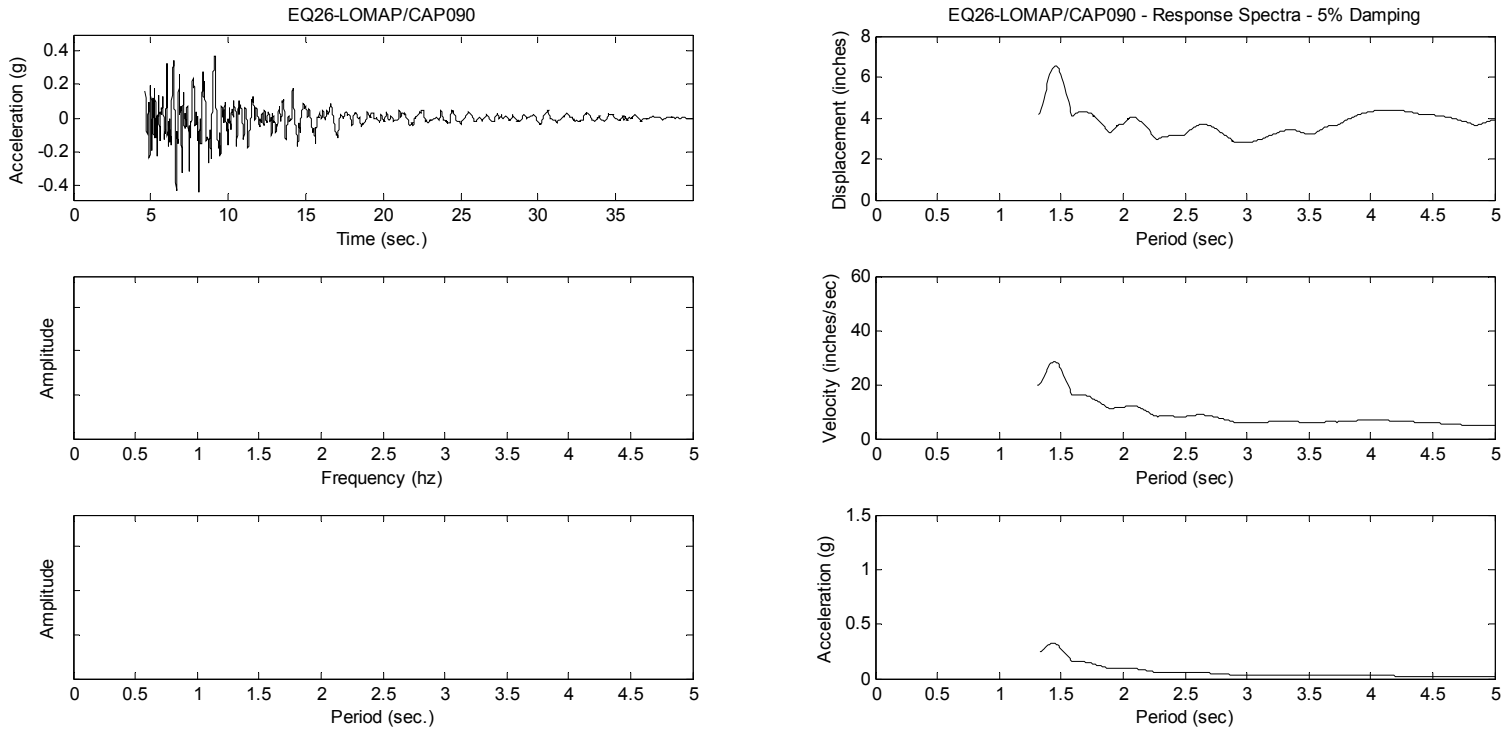

Figure A-32. 1989 Loma Prieta Earthquake at Capitola Station, Comp. 090 

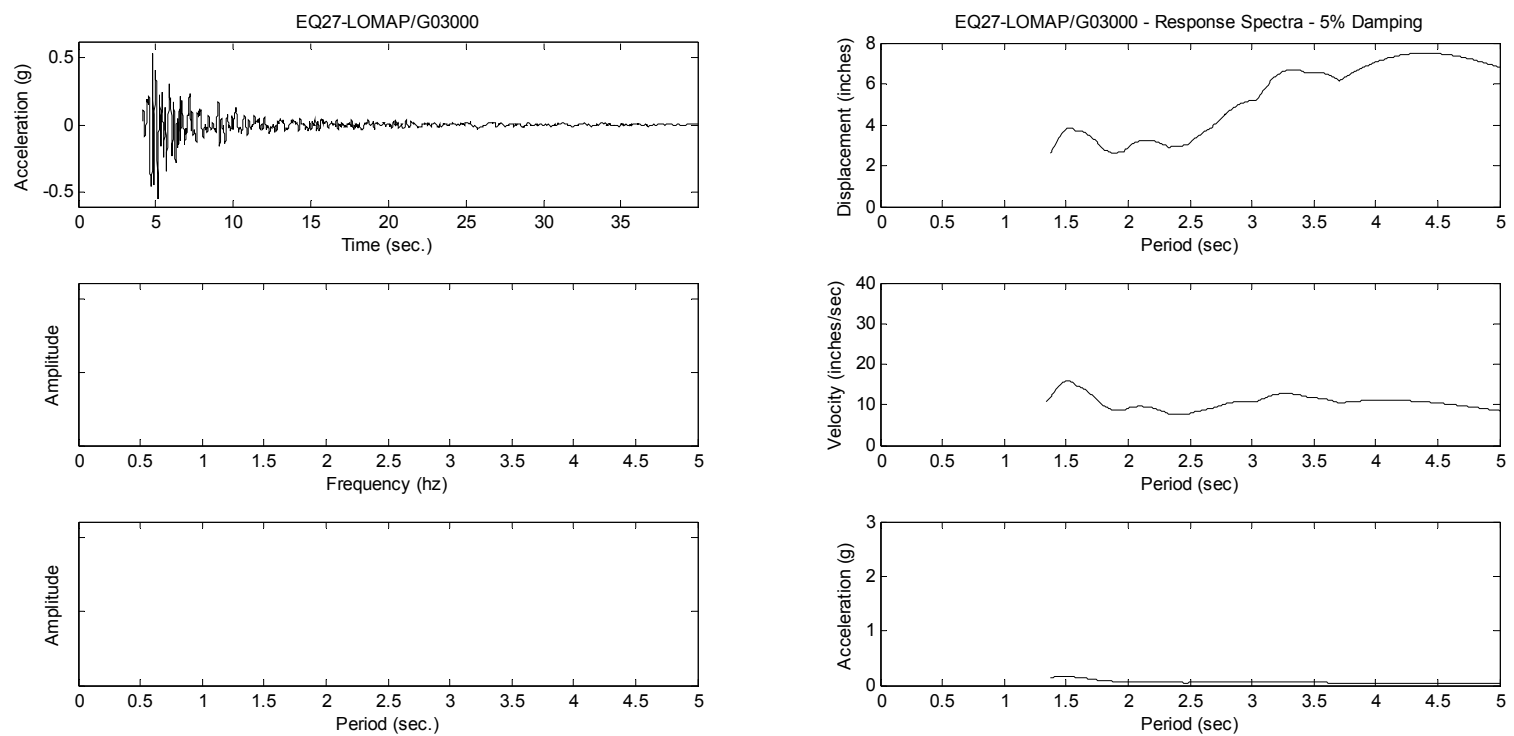

Figure A-33. 1989 Loma Prieta Earthquake at Gilroy Array Station \#3, Comp. 000
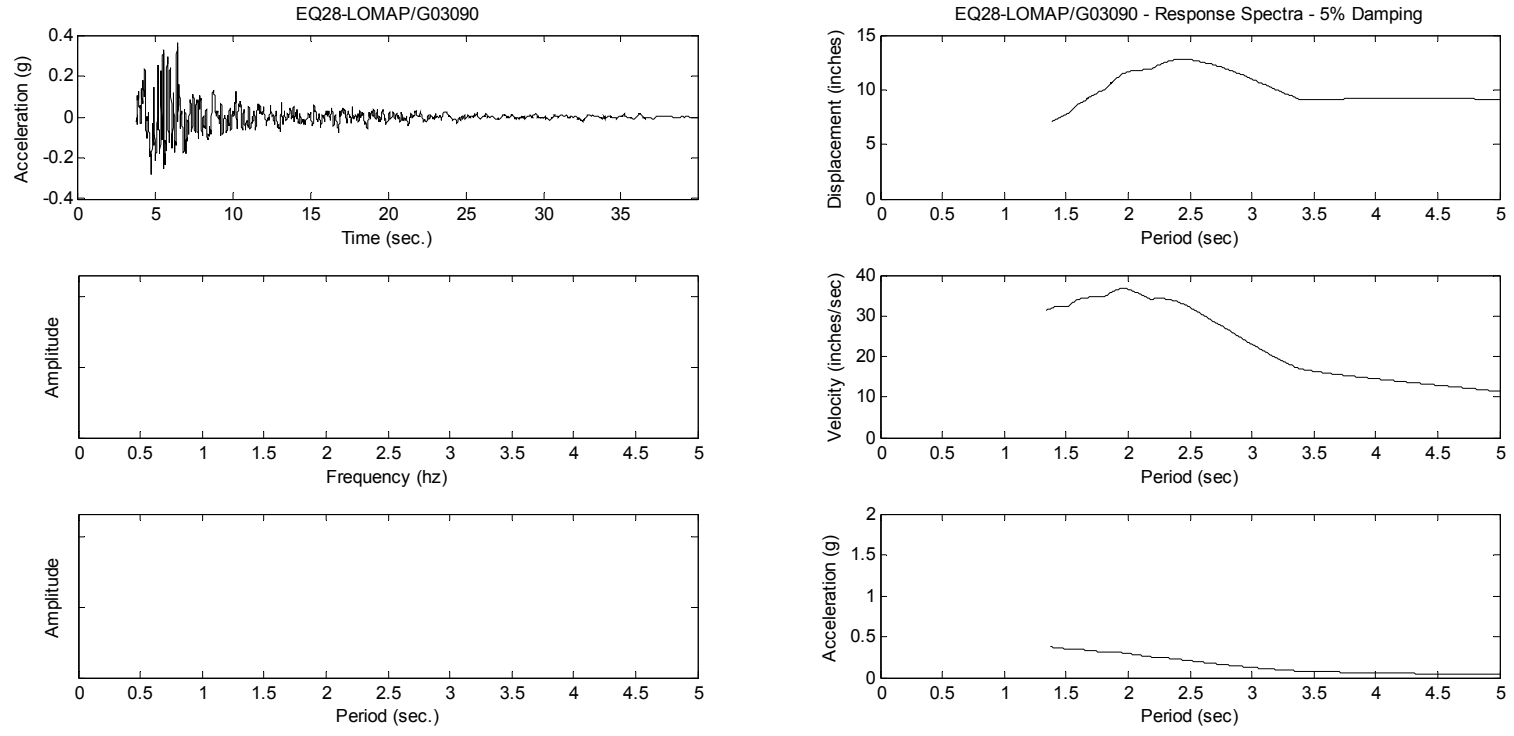

Figure A-34. 1989 Loma Prieta Earthquake at Gilroy Array Station \#3, Comp. 090 

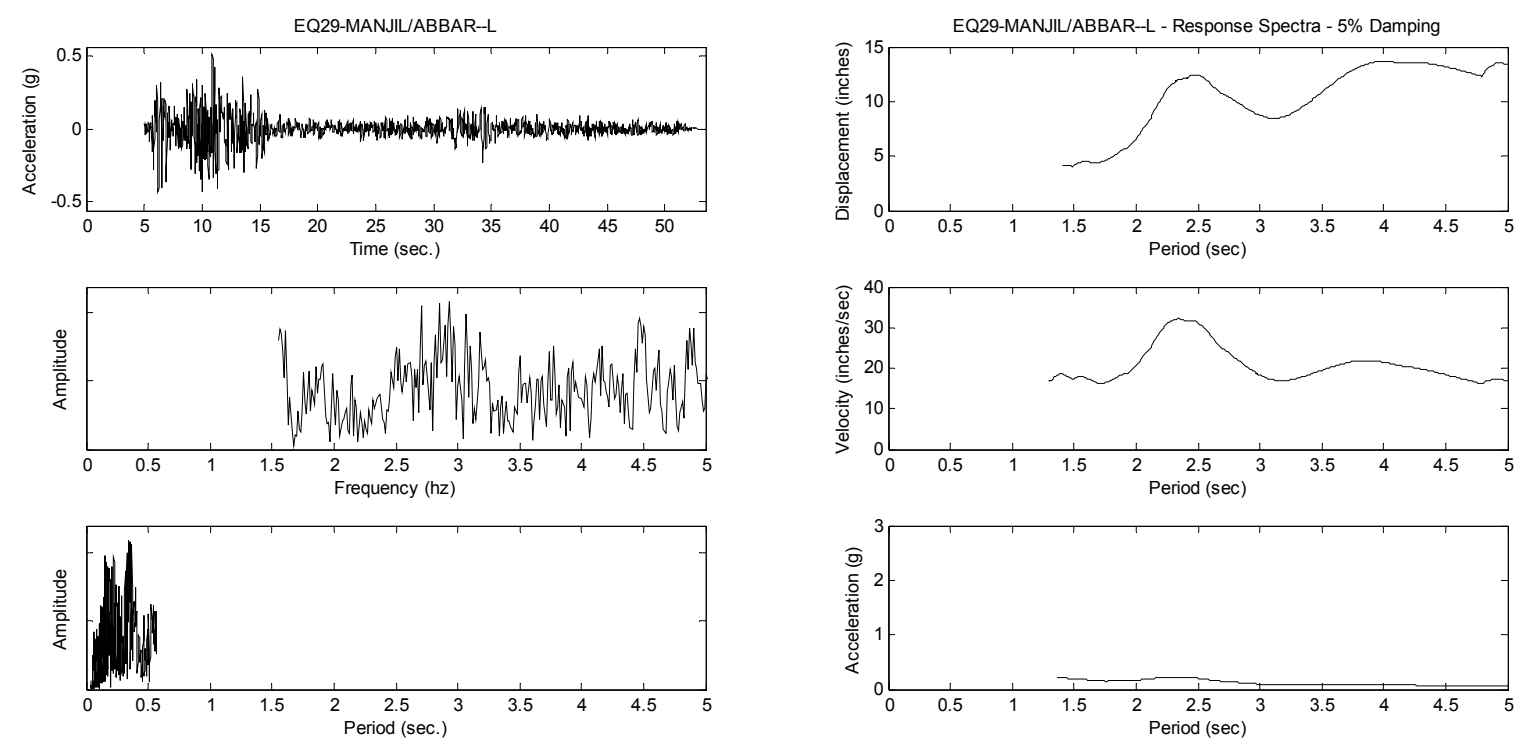

Figure A-35. 1990 Manjil, Iran Earthquake at Abbar Station, Longitudinal Direction
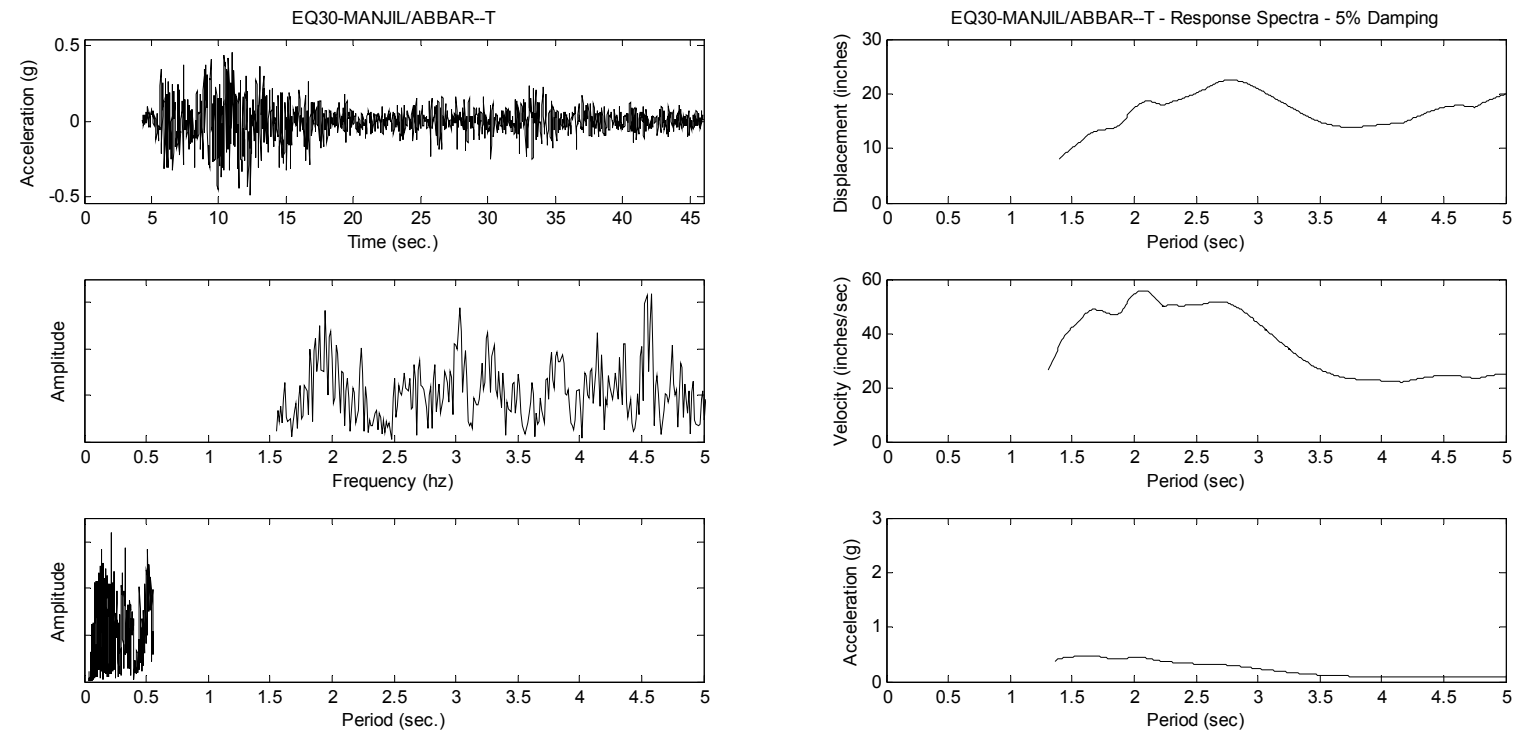

Figure A-36. 1990 Manjil, Iran Earthquake at Abbar Station, Transverse Direction 

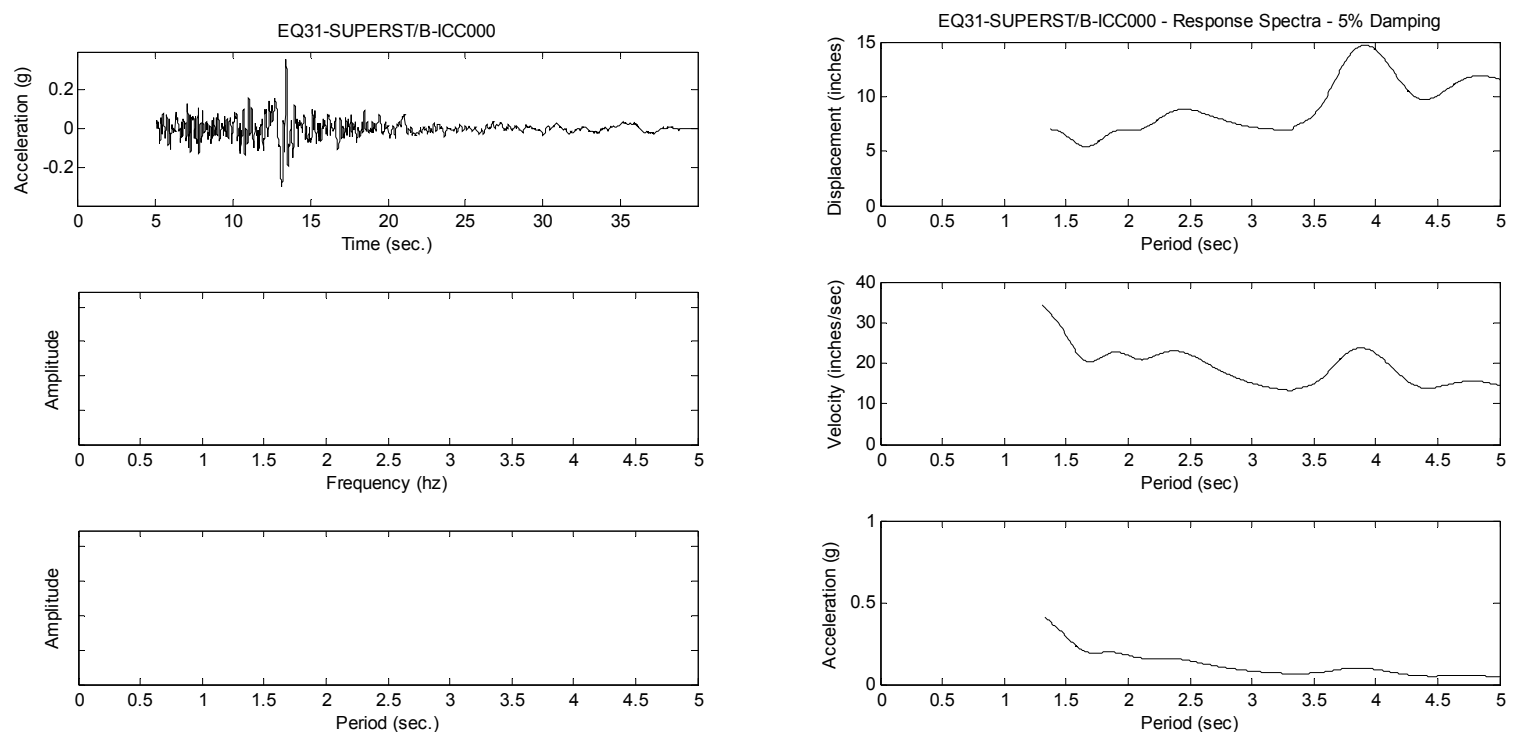

Figure A-37. 1987 Superstition Hills Earthquake at El Centro, Imperial County, Comp. 000
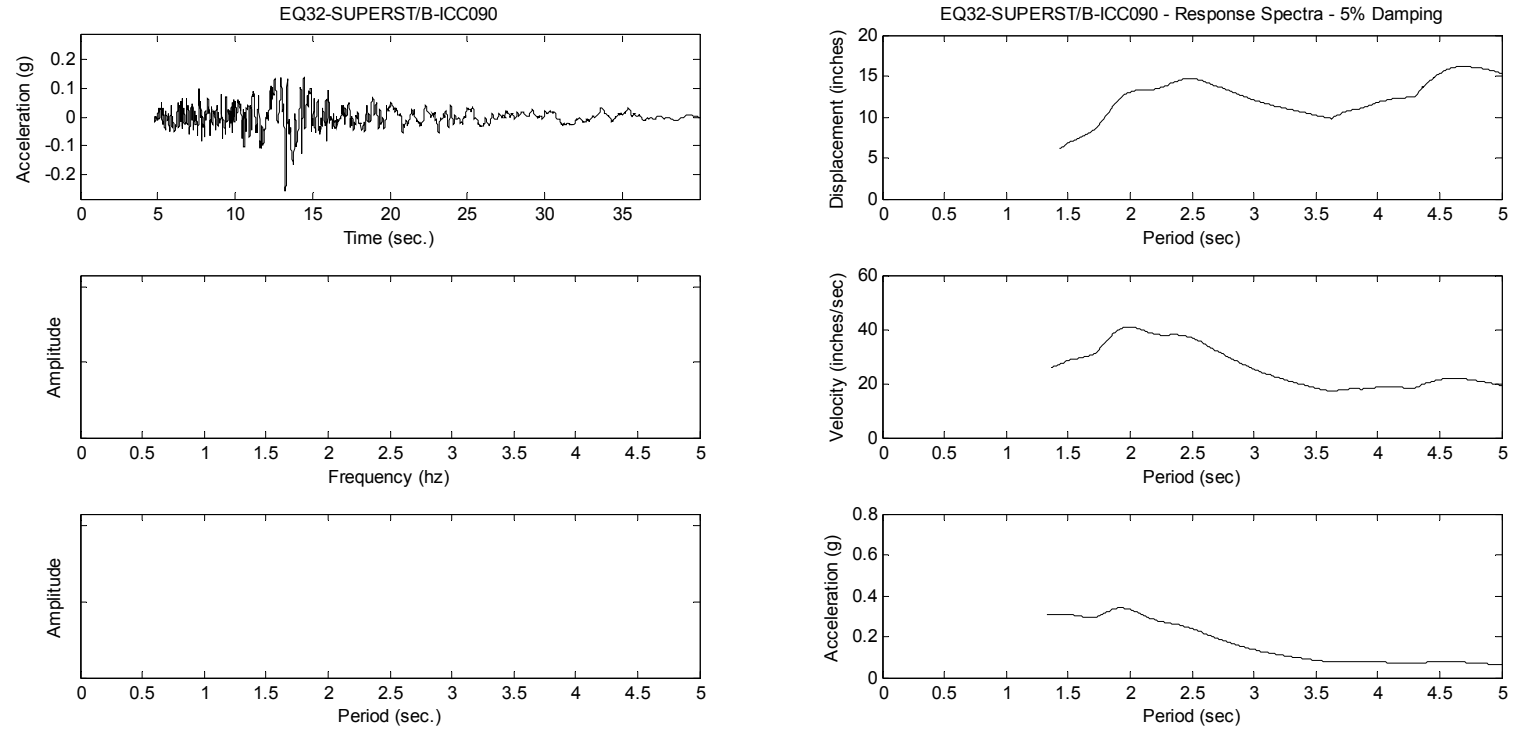

Figure A-38. 1987 Superstition Hills Earthquake at El Centro, Imperial County, Comp. 090 

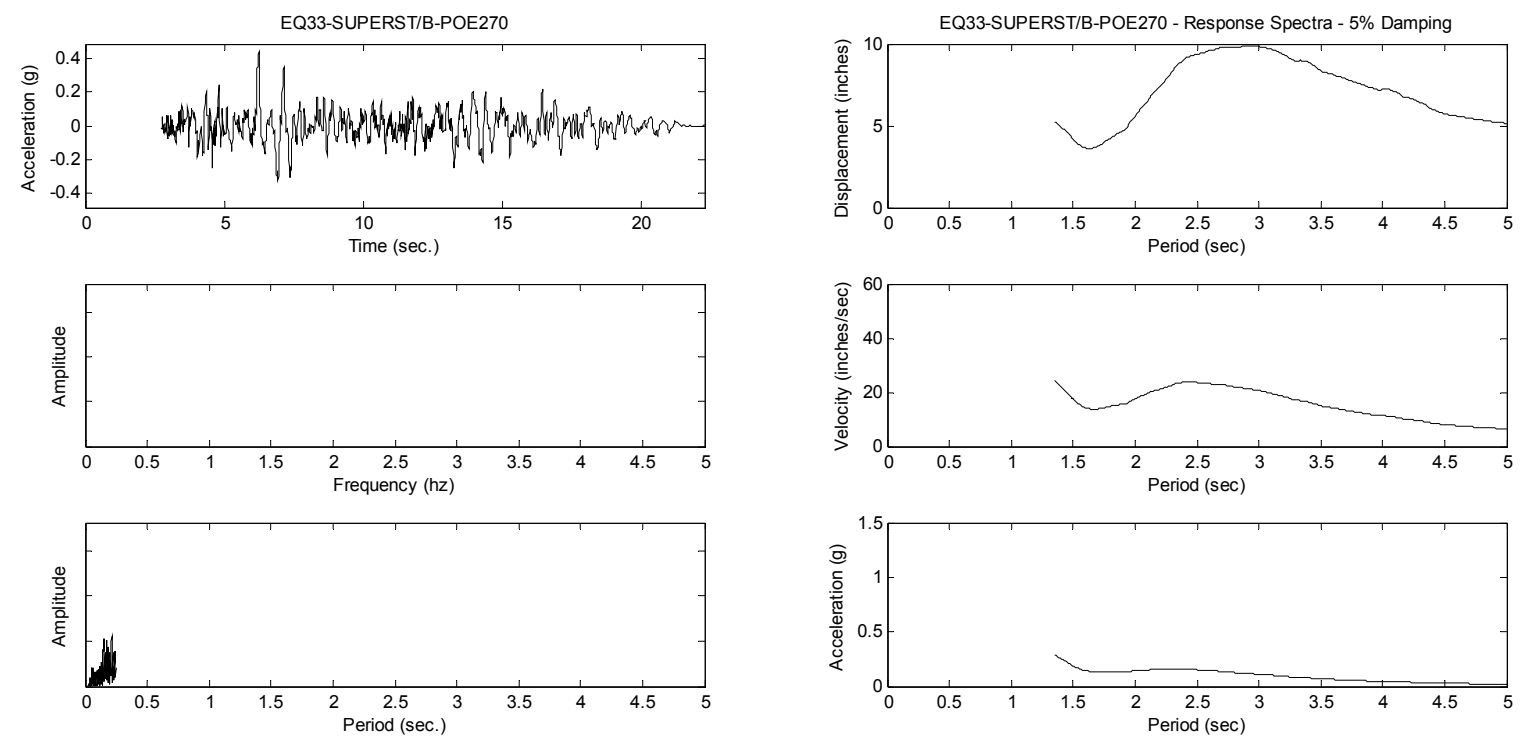

Figure A-39. 1987 Superstition Hills Earthquake at Poe Road, Comp. 270
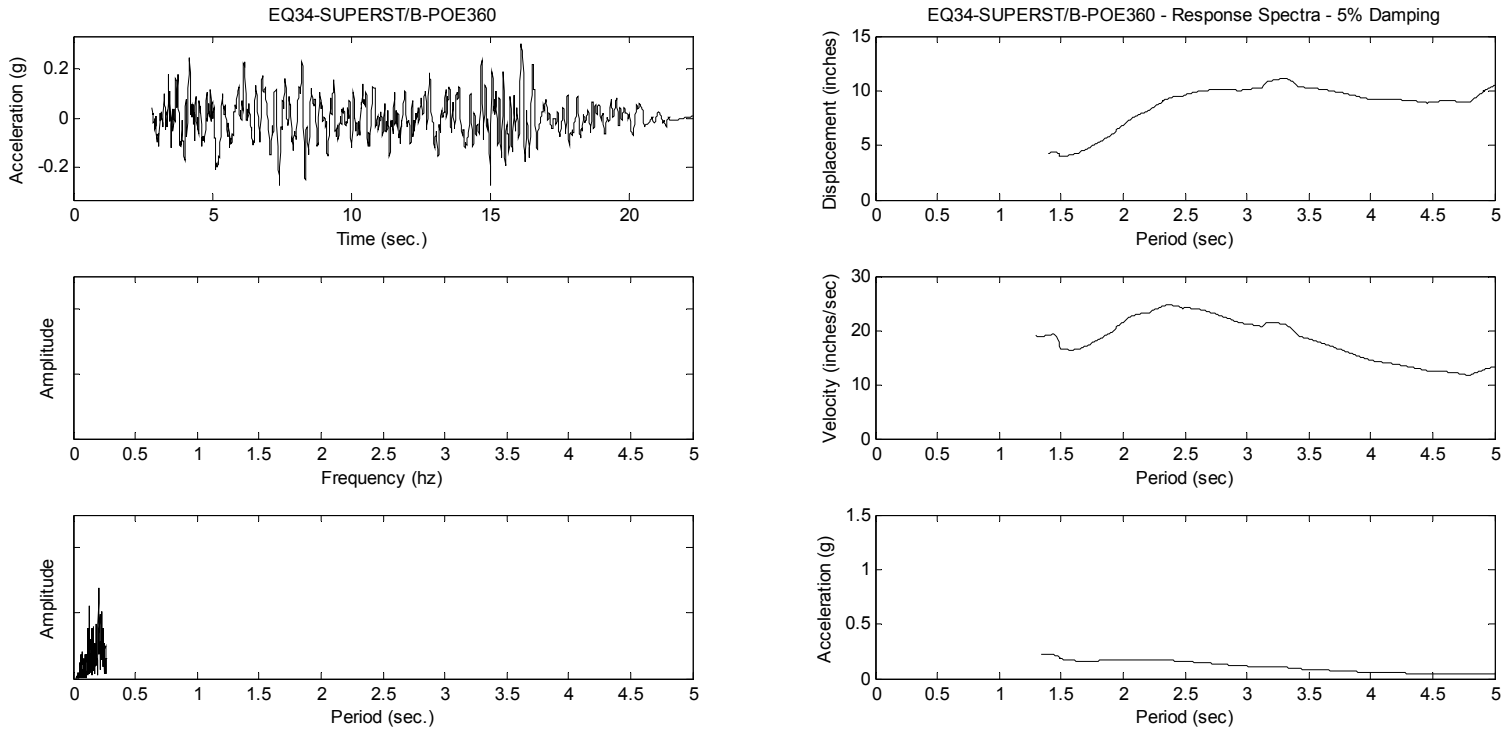

Figure A-40. 1987 Superstition Hills Earthquake at Poe Road, Comp. 360 

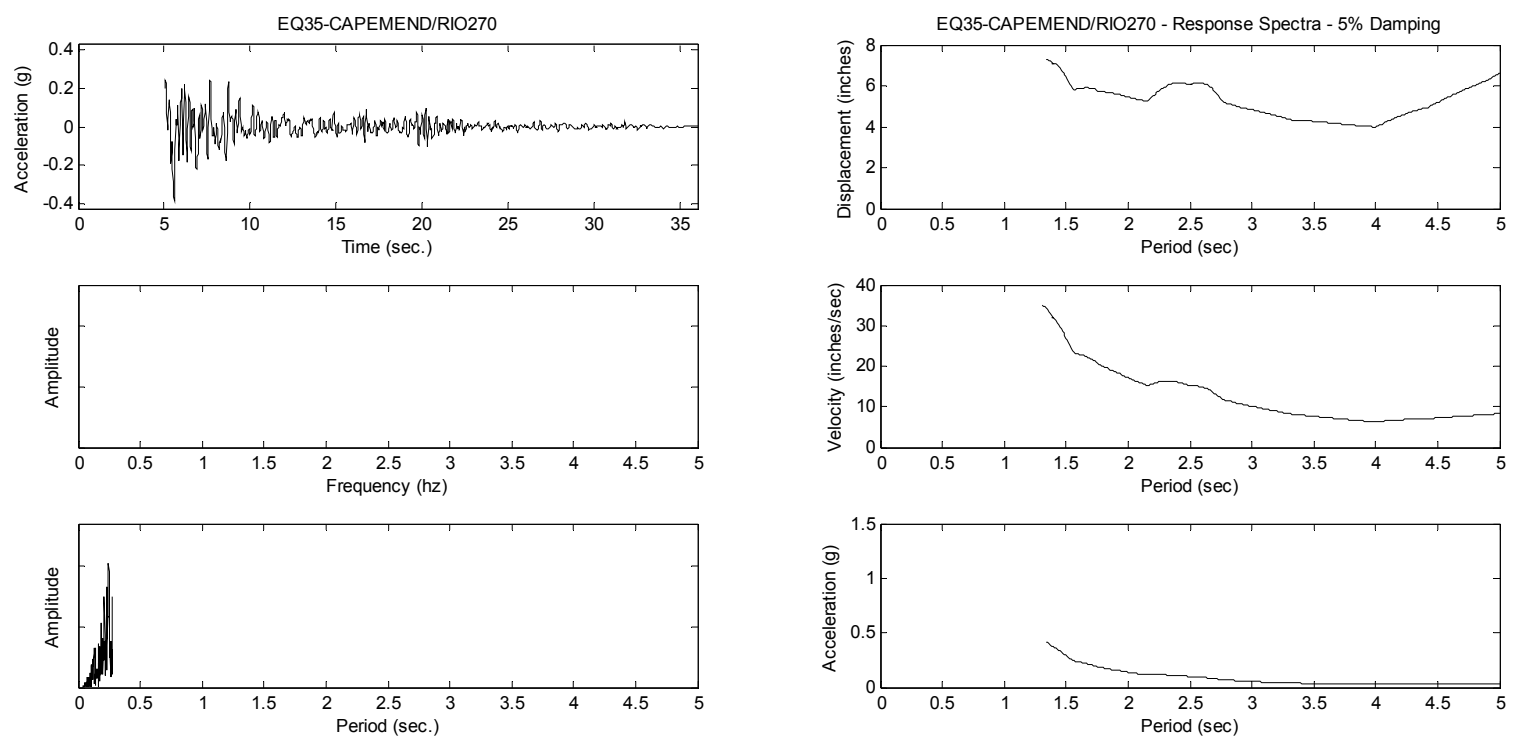

Figure A-41. 1992 Cape Mendocino Earthquake at Rio Dell Overpass, Comp. 270
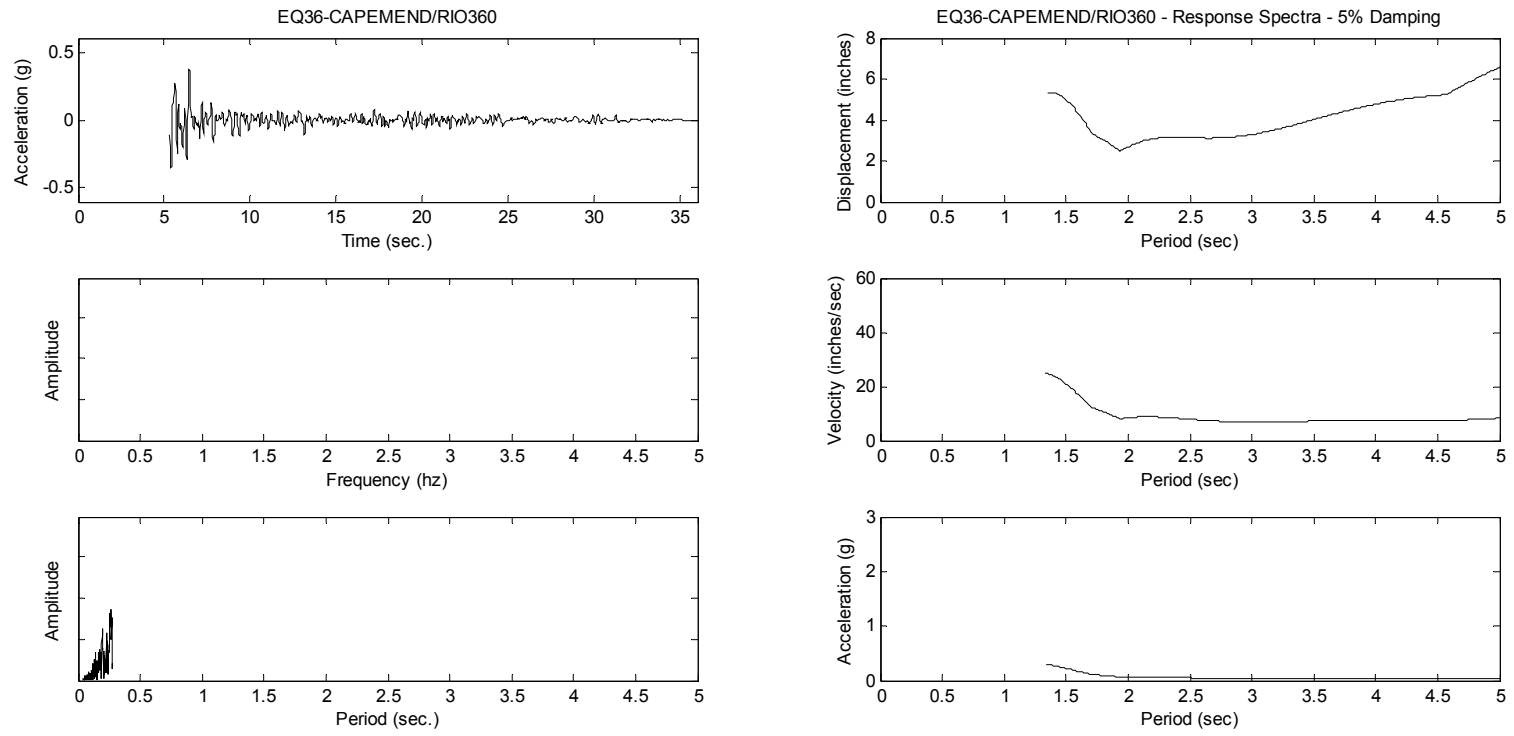

Figure A-42. 1992 Cape Mendocino Earthquake at Rio Dell Overpass, Comp. 360 

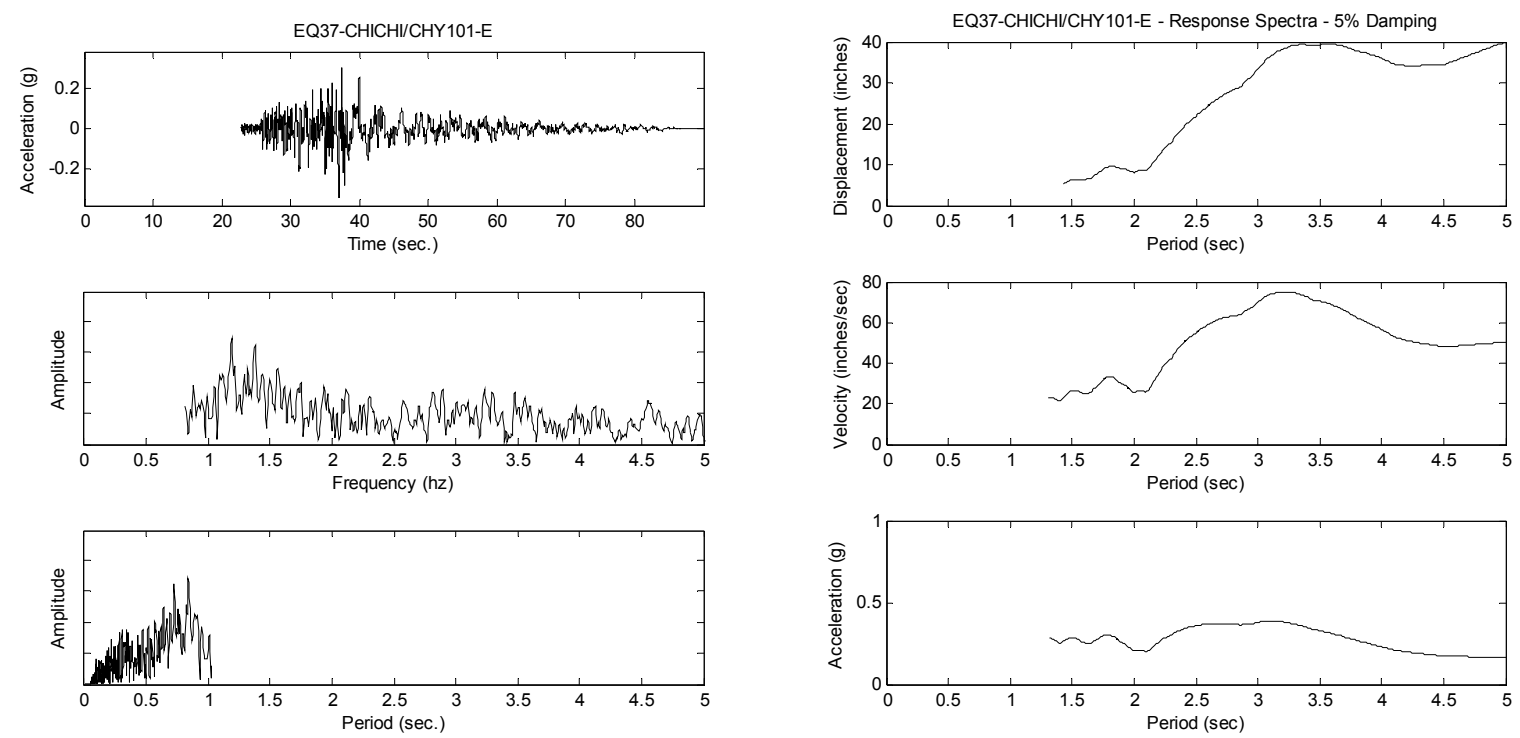

Figure A-43. 1999 Chi-Chi, Taiwan Earthquake at CHY101 Station, E-W Component
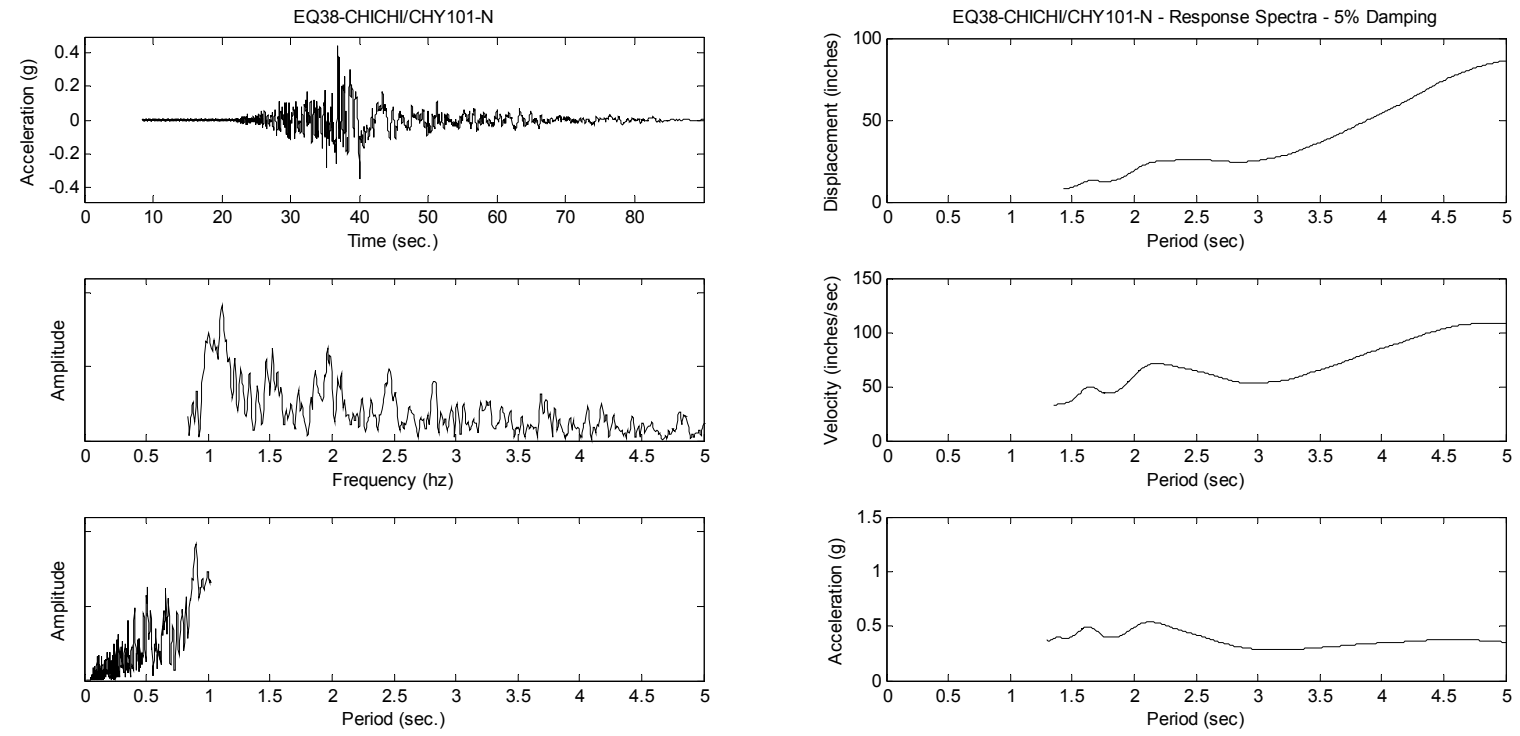

Figure A-44. 1999 Chi-Chi, Taiwan Earthquake at CHY101 Station, N-S Component 

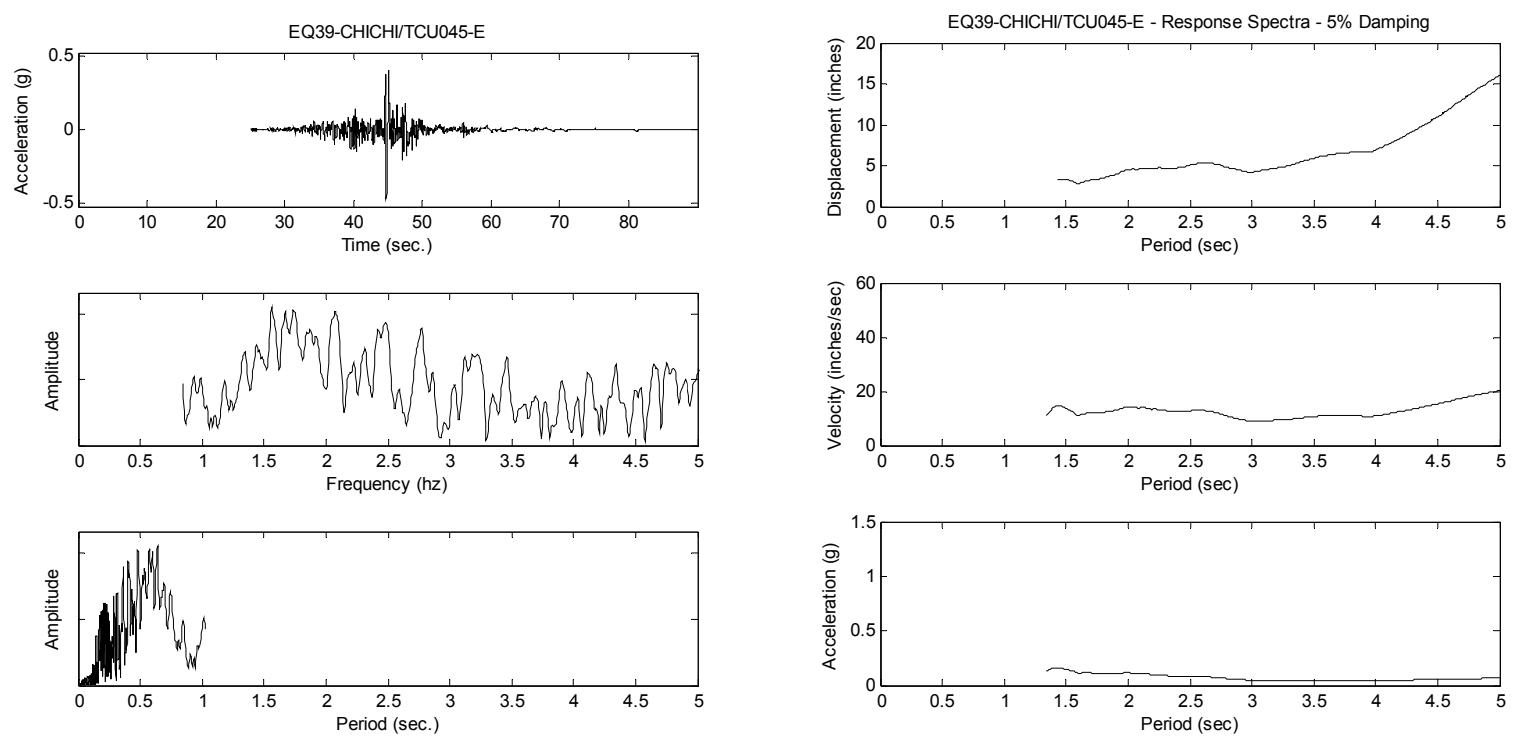

Figure A-45. 1999 Chi-Chi, Taiwan Earthquake at TCU045 Station, E-W Component
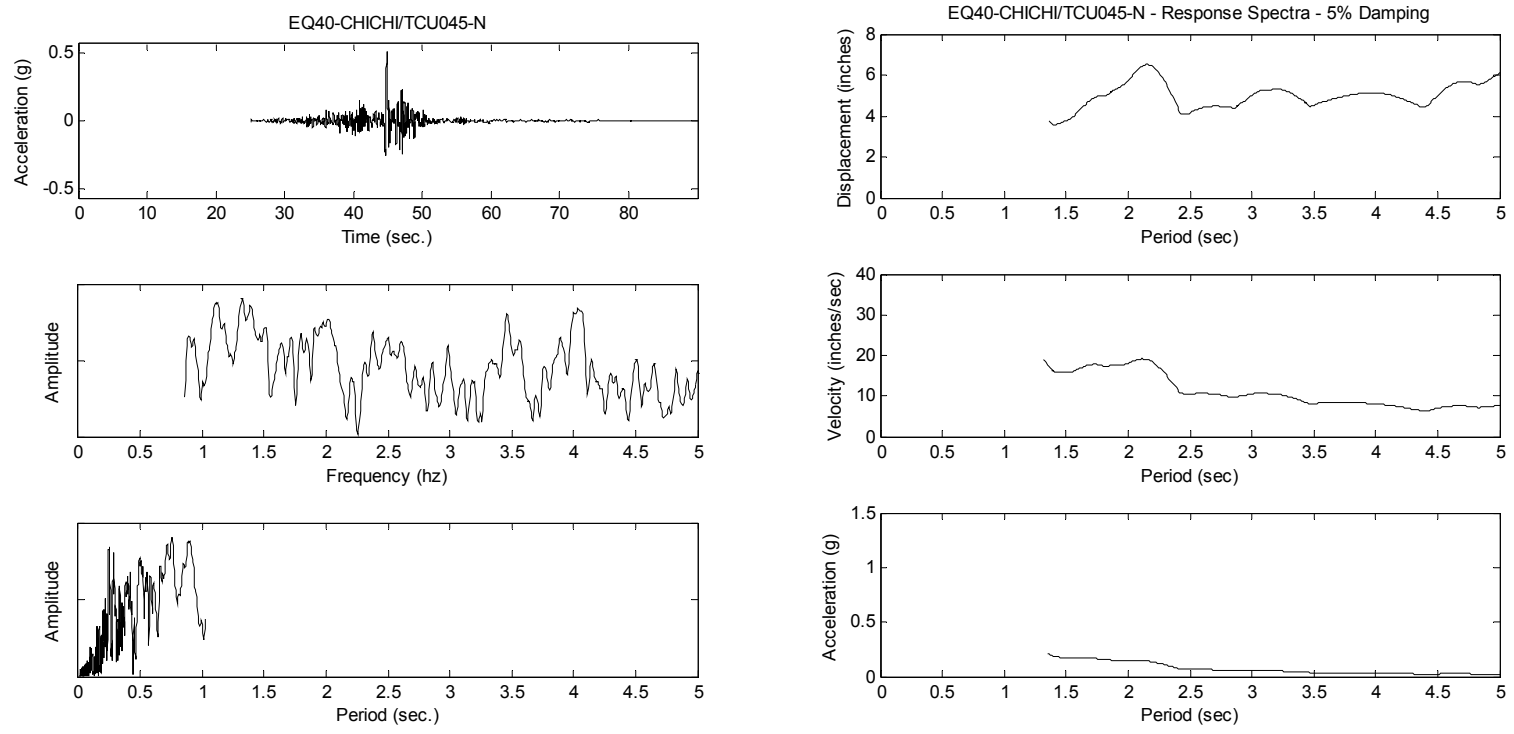

Figure A-46. 1999 Chi-Chi, Taiwan Earthquake at TCU045 Station, N-S Component 

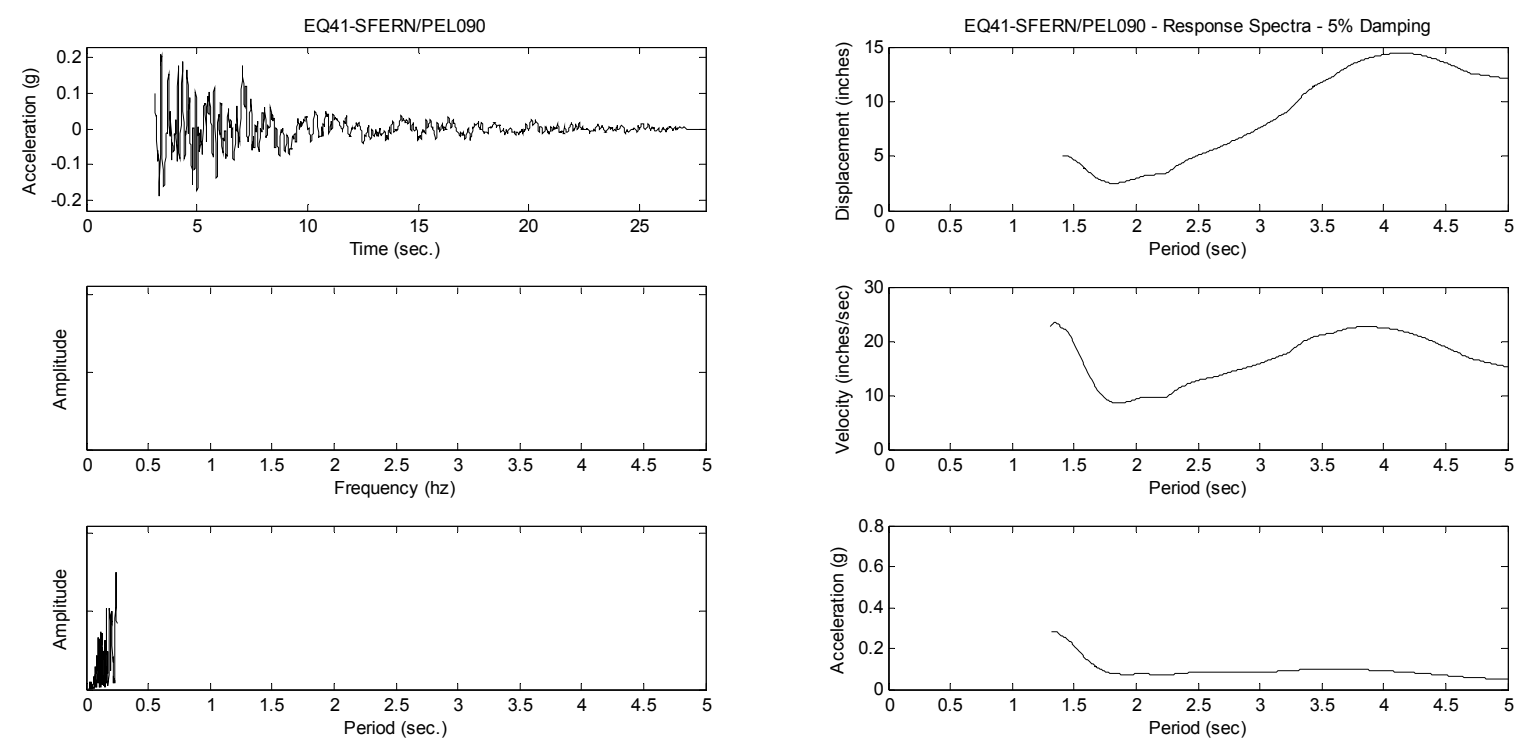

Figure A-47. 1971 San Fernando Earthquake at Los Angeles Hollywood Store Station, Comp. 090
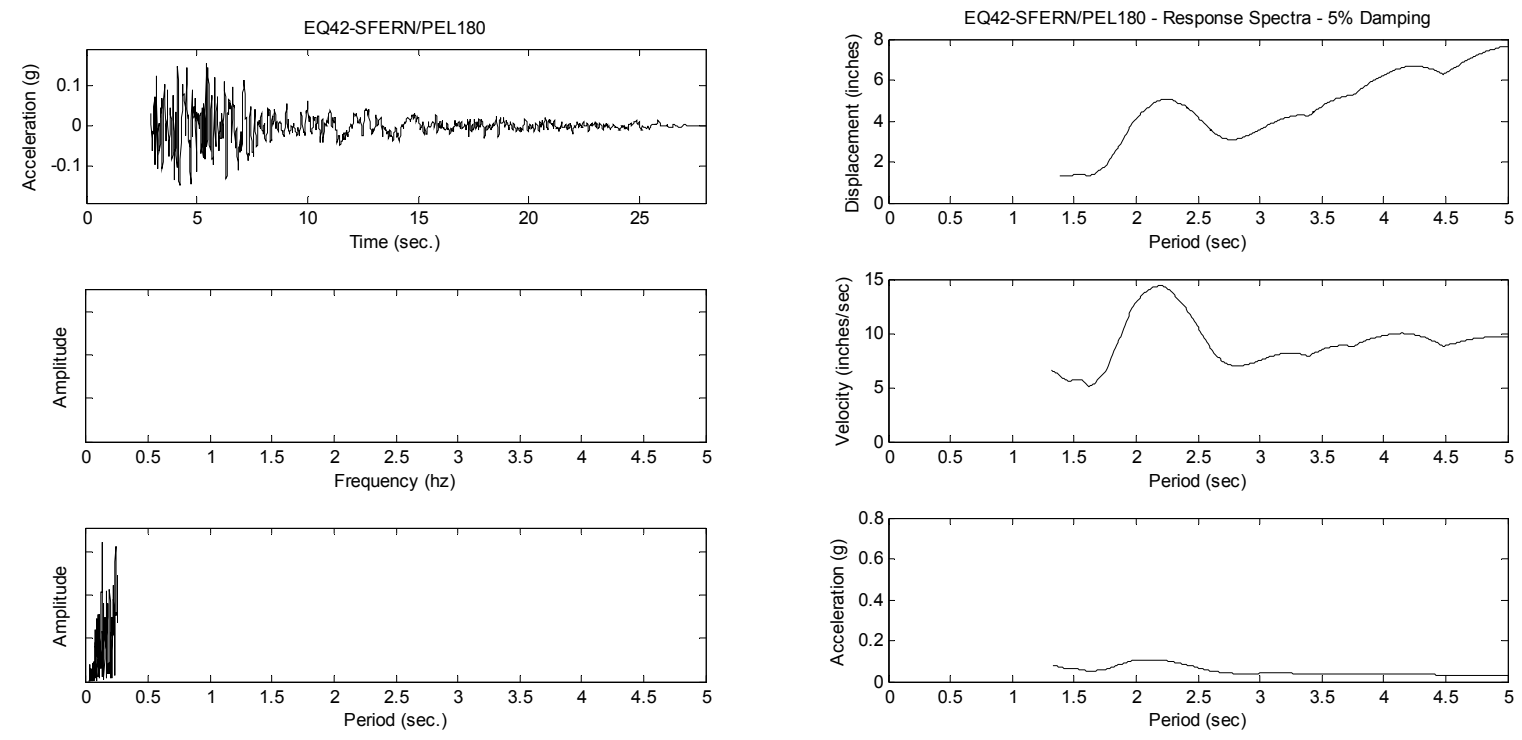

Figure A-48. 1971 San Fernando Earthquake at Los Angeles Hollywood Store Station, Comp. 180 

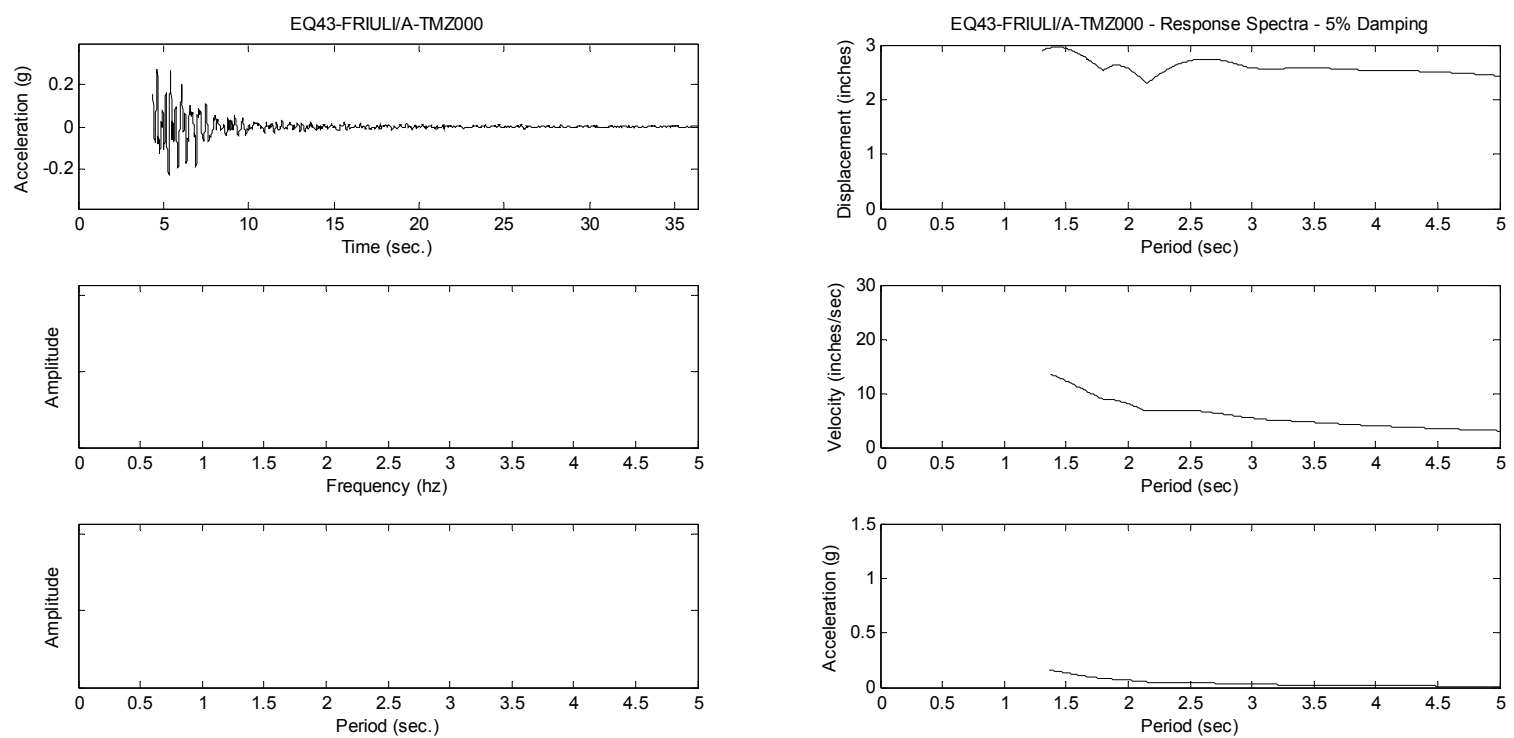

Figure A-49. 1976 Fruili, Italy Earthquake at Tolmezzo Station, Comp. 000
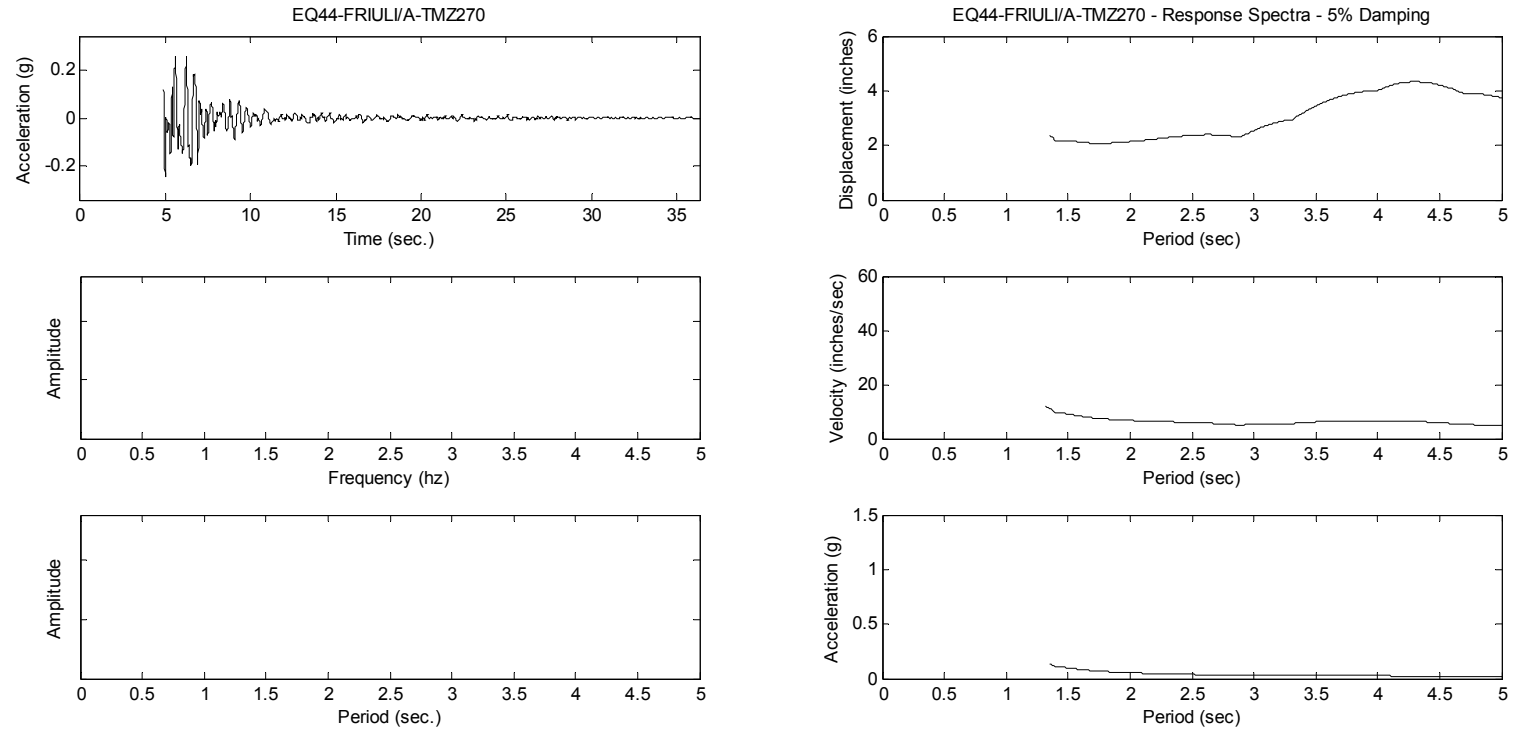

Figure A-50. 1976 Fruili, Italy Earthquake at Tolmezzo Station, Comp. 270 
A-34 


\section{Appendix B Supplemental Design Information and Design Examples}

This appendix presents supplemental information and details on the design of each archetype building.

Section B.1 provides information about the following:

- Wind forces both for strength and drift analyses

- Effective seismic weights and story gravity forces

- Seismic forces both for strength and drift analyses

- Lateral story drifts and displacements (ASCE 7 \$2.8.6)

- $\quad$ ASCE 7 stability verification (ASCE 7 12.8.7)

Section B.2 provides information regarding horizontal and vertical irregularities of the SFRS.

Section B. 3 provides information regarding the AISC 360 frame stability calculation $\left(B_{2}\right.$ for the effective length method) for the moment frame designs.

Section B.4 provides information regarding the AISC 341 plastic rotation requirement of the link beams in the eccentric braced frame designs.

Section B.5 provides example calculations for the strength design of a few specific members and components:

- EBF link

- EBF brace

- EBF column

\section{B.1 Horizontal Design Forces}

\section{B.1.1 Wind Forces}

Table B-1 through Table B-3 provide the horizontal wind forces for the 700-year wind for strength design of the members and components of the MWFRS and wind forces for the 10-year wind for verification of serviceability criteria for each archetype building. In these tables, $F_{x}$ represents wind forces in the E-W direction and $F_{y}$ represents wind in the N-S direction. Slight differences in applied wind forces are due to stiffness variations between the ELF and RSA designs. 
Table B-1. Wind Forces on ME4 (kips)

\begin{tabular}{|c|c|c|c|c|c|c|c|c|c|}
\hline \multirow{4}{*}{ Level } & \multirow{4}{*}{$\begin{array}{c}\text { Elevation } \\
\text { (ft) }\end{array}$} & \multicolumn{8}{|c|}{ Diaphragm Forces (kips), Applied at Geometric Center } \\
\hline & & \multicolumn{4}{|c|}{ 700-Year Wind (Design) } & \multicolumn{4}{|c|}{ 10-Year Wind (Drift) } \\
\hline & & \multicolumn{2}{|c|}{ RSA } & \multicolumn{2}{|c|}{ ELF } & \multicolumn{2}{|c|}{ RSA } & \multicolumn{2}{|c|}{ ELF } \\
\hline & & $F_{x}$ & $F_{y}$ & $F_{x}$ & $F_{y}$ & $F_{x}$ & $F_{y}$ & $F_{x}$ & $F_{y}$ \\
\hline Roof & 60 & 36.09 & 52.43 & 35.30 & 52.43 & 14.82 & 22.46 & 14.67 & 22.46 \\
\hline 4 & 46 & 35.48 & 50.50 & 33.98 & 50.50 & 13.99 & 21.63 & 13.69 & 21.63 \\
\hline 3 & 32 & 33.21 & 47.53 & 31.81 & 47.53 & 13.09 & 20.36 & 12.82 & 20.36 \\
\hline 2 & 18 & 34.36 & 49.60 & 32.91 & 49.60 & 13.55 & 21.25 & 13.26 & 21.25 \\
\hline Base & 0 & 139.14 & 200.06 & 134.01 & 200.06 & 55.46 & 85.71 & 54.44 & 85.71 \\
\hline
\end{tabular}

Table B-2. Wind Forces on ME8 (kips)

\begin{tabular}{|c|c|c|c|c|c|c|c|c|c|}
\hline \multirow{4}{*}{ Level } & \multirow{4}{*}{$\begin{array}{c}\text { Elevation } \\
(\mathrm{ft})\end{array}$} & \multicolumn{8}{|c|}{ Diaphragm Forces (kips), Applied at Geometric Center } \\
\hline & & \multicolumn{4}{|c|}{ 700-Year Wind (Design) } & \multicolumn{4}{|c|}{ 10-Year Wind (Drift) } \\
\hline & & \multicolumn{2}{|c|}{ RSA } & \multicolumn{2}{|c|}{ ELF } & \multicolumn{2}{|c|}{ RSA } & \multicolumn{2}{|c|}{ ELF } \\
\hline & & $F_{x}$ & $F_{y}$ & $F_{x}$ & $F_{y}$ & $F_{x}$ & $F_{y}$ & $F_{x}$ & $F_{y}$ \\
\hline Roof & 116 & 46.18 & 65.78 & 44.19 & 65.22 & 18.47 & 27.23 & 18.00 & 27.13 \\
\hline 8 & 102 & 49.32 & 67.67 & 45.43 & 66.58 & 18.55 & 27.14 & 17.64 & 26.94 \\
\hline 7 & 88 & 47.98 & 65.97 & 44.20 & 64.91 & 18.05 & 26.46 & 17.16 & 26.26 \\
\hline 6 & 74 & 46.48 & 64.07 & 42.81 & 63.04 & 17.49 & 25.70 & 16.62 & 25.51 \\
\hline 5 & 60 & 44.76 & 61.89 & 41.23 & 60.90 & 16.84 & 24.82 & 16.00 & 24.64 \\
\hline 4 & 46 & 42.72 & 59.31 & 39.35 & 58.36 & 16.07 & 23.79 & 15.28 & 23.61 \\
\hline 3 & 32 & 40.18 & 56.09 & 37.01 & 55.19 & 15.12 & 22.50 & 14.37 & 22.33 \\
\hline 2 & 18 & 41.88 & 59.00 & 38.58 & 58.05 & 15.76 & 23.66 & 14.98 & 23.49 \\
\hline Base & 0 & 359.49 & 499.77 & 332.81 & 492.24 & 136.35 & 201.31 & 130.04 & 199.90 \\
\hline
\end{tabular}

Table B-3. Wind Forces on ME16 (kips)

\begin{tabular}{|c|c|c|c|c|c|c|c|c|c|}
\hline \multirow{4}{*}{ Level } & \multirow{4}{*}{$\begin{array}{l}\text { Elevation } \\
(\mathrm{ft})\end{array}$} & \multicolumn{8}{|c|}{ Diaphragm Forces (kips), Applied at Geometric Center } \\
\hline & & \multicolumn{4}{|c|}{ 700-Year Wind (Design) } & \multicolumn{4}{|c|}{ 10-Year Wind (Drift) } \\
\hline & & \multicolumn{2}{|c|}{ RSA } & \multicolumn{2}{|c|}{ ELF } & \multicolumn{2}{|c|}{ RSA } & \multicolumn{2}{|c|}{ ELF } \\
\hline & & $F_{x}$ & $F_{y}$ & $F_{x}$ & $F_{y}$ & $F_{x}$ & $F_{y}$ & $F_{x}$ & $F_{y}$ \\
\hline Roof & 228 & 56.78 & 82.70 & 55.35 & 82.17 & 22.58 & 33.58 & 22.22 & 33.46 \\
\hline 16 & 214 & 62.23 & 89.05 & 59.41 & 88.00 & 23.22 & 34.48 & 22.50 & 34.26 \\
\hline 15 & 200 & 61.44 & 88.01 & 58.66 & 86.97 & 22.93 & 34.08 & 22.22 & 33.86 \\
\hline 14 & 186 & 60.61 & 86.92 & 57.86 & 85.89 & 22.62 & 33.66 & 21.92 & 33.44 \\
\hline 13 & 172 & 59.74 & 85.76 & 57.03 & 84.75 & 22.29 & 33.21 & 21.60 & 32.99 \\
\hline 12 & 158 & 58.81 & 84.54 & 56.14 & 83.54 & 21.95 & 32.73 & 21.27 & 32.52 \\
\hline 11 & 144 & 57.82 & 83.23 & 55.20 & 82.25 & 21.58 & 32.23 & 20.91 & 32.02 \\
\hline 10 & 130 & 56.76 & 81.83 & 54.19 & 80.86 & 21.18 & 31.69 & 20.53 & 31.48 \\
\hline 9 & 116 & 55.62 & 80.32 & 53.10 & 79.37 & 20.76 & 31.10 & 20.11 & 30.90 \\
\hline 8 & 102 & 54.37 & 78.67 & 51.91 & 77.74 & 20.29 & 30.46 & 19.66 & 30.26 \\
\hline 7 & 88 & 53.00 & 76.85 & 50.60 & 75.94 & 19.78 & 29.76 & 19.16 & 29.56 \\
\hline 6 & 74 & 51.46 & 74.81 & 49.12 & 73.93 & 19.20 & 28.97 & 18.61 & 28.78 \\
\hline 5 & 60 & 49.69 & 72.48 & 47.44 & 71.62 & 18.54 & 28.06 & 17.97 & 27.88 \\
\hline 4 & 46 & 47.60 & 69.71 & 45.44 & 68.89 & 17.76 & 26.99 & 17.21 & 26.82 \\
\hline 3 & 32 & 44.98 & 66.25 & 42.94 & 65.47 & 16.79 & 25.66 & 16.26 & 25.49 \\
\hline 2 & 18 & 47.26 & 70.24 & 45.12 & 69.41 & 17.64 & 27.20 & 17.09 & 27.02 \\
\hline Base & 0 & 878.18 & 1271.37 & 839.50 & 1256.80 & 329.11 & 493.87 & 319.23 & 490.74 \\
\hline
\end{tabular}




\section{B.1.2 Seismic Forces}

\section{B.1.2.1 Effective Seismic Weights and Story Gravity Forces}

Table B-4 through Table B-6 provide the effective seismic weights lumped at each story as well as the lumped gravity force acting on each story for each building design. The gravity force is computed using the two load combinations as discussed in Chapter 2.

Table B-4. Effect Seismic Weights and Story Gravity Forces, ME4 (kips)

\begin{tabular}{|c|c|c|c|c|c|c|}
\hline \multirow[b]{2}{*}{ Level, $x$} & \multicolumn{3}{|c|}{ RSA } & \multicolumn{3}{|c|}{ ELF } \\
\hline & $w_{x}{ }^{1}$ & $P_{\text {story }}{ }^{2}$ & $P_{x}^{3}$ & $w_{x}{ }^{1}$ & $P_{\text {story }}{ }^{2}$ & $P_{x}{ }^{3}$ \\
\hline Roof & 1085 & 1302 & 1085 & 1086 & 1304 & 1086 \\
\hline 4 & 1333 & 2909 & 2457 & 1339 & 2919 & 2465 \\
\hline 3 & 1338 & 4523 & 3834 & 1348 & 4544 & 3851 \\
\hline 2 & 1351 & 6152 & 5223 & 1363 & 6187 & 5253 \\
\hline Total & 5107 & - & - & 5137 & - & - \\
\hline
\end{tabular}

Table B-5. Effect Seismic Weights and Story Gravity Forces, ME8 (kips)

\begin{tabular}{|c|c|c|c|c|c|c|}
\hline \multirow[b]{2}{*}{ Level, $x$} & \multicolumn{3}{|c|}{ RSA } & \multicolumn{3}{|c|}{ ELF } \\
\hline & $w_{x}{ }^{1}$ & $P_{\text {story }}{ }^{2}$ & $P_{x}{ }^{3}$ & $w_{x}^{1}$ & $P_{\text {story }}{ }^{2}$ & $P_{x}{ }^{3}$ \\
\hline Roof & 1078 & 1294 & 1078 & 1082 & 1299 & 1082 \\
\hline 8 & 1321 & 2887 & 2439 & 1328 & 2900 & 2449 \\
\hline 7 & 1327 & 4488 & 3805 & 1343 & 4519 & 3830 \\
\hline 6 & 1332 & 6094 & 5175 & 1351 & 6148 & 5220 \\
\hline 5 & 1341 & 7711 & 6555 & 1358 & 7786 & 6617 \\
\hline 4 & 1347 & 9335 & 7941 & 1363 & 9429 & 8019 \\
\hline 3 & 1353 & 10967 & 9333 & 1367 & 11077 & 9425 \\
\hline 2 & 1368 & 12617 & 10740 & 1382 & 12743 & 10845 \\
\hline Total & 10469 & - & - & 10574 & - & - \\
\hline
\end{tabular}


Table B-6. Effect Seismic Weights and Story Gravity Forces, ME16 (kips)

\begin{tabular}{|c|c|c|c|c|c|c|}
\hline \multirow[b]{2}{*}{ Level, $x$} & \multicolumn{3}{|c|}{ RSA } & \multicolumn{3}{|c|}{ ELF } \\
\hline & $w_{x}^{1}$ & $P_{\text {story }}{ }^{2}$ & $P_{x}{ }^{3}$ & $w_{x}^{1}$ & $P_{\text {story }}{ }^{2}$ & $P_{x}^{3}$ \\
\hline Roof & 1084 & 1301 & 1084 & 1086 & 1303 & 1086 \\
\hline 16 & 1329 & 2903 & 2452 & 1331 & 2907 & 2455 \\
\hline 15 & 1332 & 4510 & 3823 & 1346 & 4530 & 3840 \\
\hline 14 & 1336 & 6120 & 5197 & 1355 & 6164 & 5233 \\
\hline 13 & 1349 & 7747 & 6585 & 1362 & 7806 & 6634 \\
\hline 12 & 1356 & 9382 & 7980 & 1366 & 9453 & 8039 \\
\hline 11 & 1360 & 11022 & 9379 & 1371 & 11106 & 9449 \\
\hline 10 & 1364 & 12666 & 10781 & 1377 & 12767 & 10865 \\
\hline 9 & 1370 & 14318 & 12190 & 1387 & 14438 & 12290 \\
\hline 8 & 1376 & 15977 & 13605 & 1393 & 16118 & 13723 \\
\hline 7 & 1380 & 17641 & 15024 & 1399 & 17805 & 15160 \\
\hline 6 & 1385 & 19310 & 16447 & 1405 & 19498 & 16604 \\
\hline 5 & 1397 & 20995 & 17883 & 1414 & 21202 & 18056 \\
\hline 4 & 1405 & 22688 & 19327 & 1423 & 22917 & 19518 \\
\hline 3 & 1413 & 24392 & 20779 & 1435 & 24646 & 20991 \\
\hline 2 & 1440 & 26128 & 22258 & 1469 & 26416 & 22498 \\
\hline Total & 21677 & - & - & 21917 & - & - \\
\hline
\end{tabular}

\section{B.1.2.2 Horizontal Seismic Forces, E-W Direction}

\section{B.1.2.2.1 ME4 (Special Moment Frame)}

Table B-7 and Table B-8 provide the horizontal seismic forces and story shears for each building design for the seismic strength analysis and the drift analysis. The data are also graphically illustrated in Figure B-1 through Figure B-3. In these tables, $F_{x}$ represents the seismic forces acting in the E-W direction only. The equivalent story forces for the RSA design are backed out from the story shears computed via a modal combination procedure. Although not theoretically correct, the forces provide a comparison basis for evaluating variations in the vertical distribution.

Table B-7. Seismic Strength Design Forces, E-W ME4

\begin{tabular}{ccc|ccc|cc}
\hline & \multicolumn{2}{c|}{ RSA } & \multicolumn{3}{c|}{ ELF } & \multicolumn{2}{c}{ Wind (700-Year) } \\
Level, $x$ & $F_{x}$ & $V_{i}$ & $F_{x}$ & $V_{i}$ & $V_{E L F} /$ & $F_{x}$ & $V_{i}$ \\
& (kips) & (kips) & (kips) & (kips) & $V_{R S A}$ & (kips) & (kips) \\
\hline Roof & 145 & 145 & 138 & 138 & 1.05 & 36 & 36 \\
4 & 68 & 213 & 121 & 259 & 0.82 & 35 & 72 \\
3 & 46 & 259 & 77 & 336 & 0.77 & 33 & 105 \\
2 & 56 & 316 & 38 & 374 & 0.84 & 34 & 139 \\
\hline
\end{tabular}




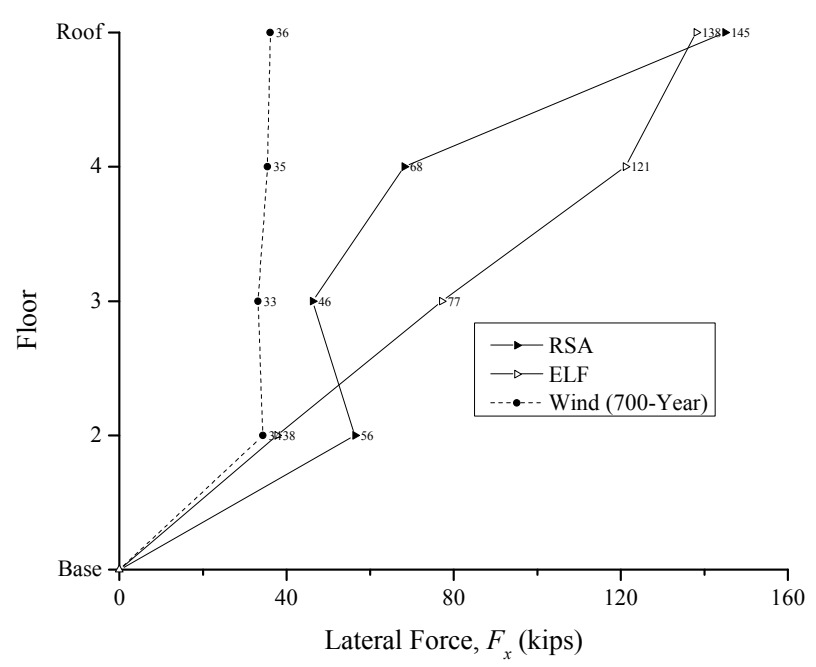

(a) Lateral Forces

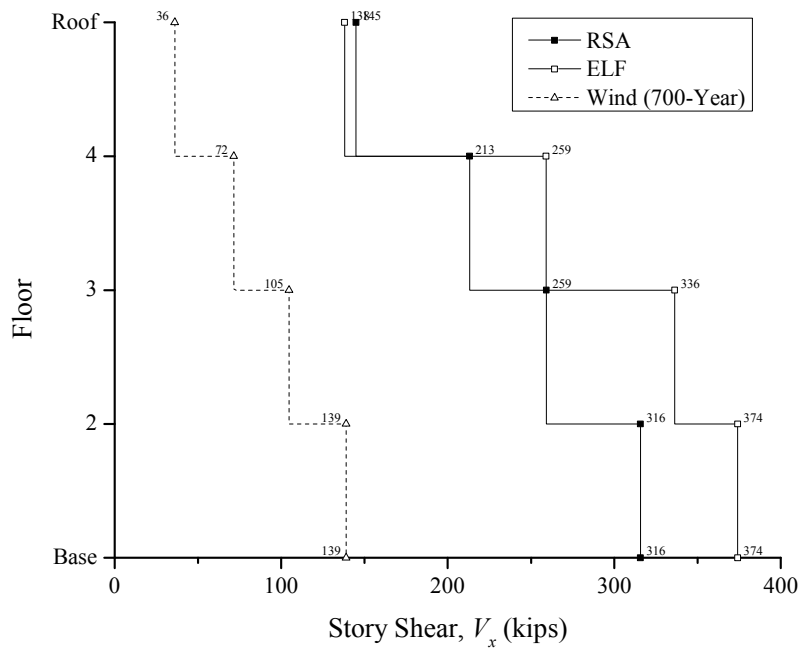

(b) Story Shears

Figure B-1. Strength Design Lateral Forces and Story Shears

Table B-8. Seismic Drift Forces, E-W ME4

\begin{tabular}{|c|c|c|c|c|c|c|c|c|c|c|c|c|}
\hline \multirow[b]{2}{*}{ Level, $x$} & \multicolumn{5}{|c|}{ RSA } & \multicolumn{5}{|c|}{ ELF } & \multicolumn{2}{|c|}{ Wind (10-Year) } \\
\hline & $\begin{array}{c}F_{x} \\
\text { (kips) }\end{array}$ & $\begin{array}{c}V_{i} \\
\text { (kips) }\end{array}$ & $\begin{array}{c}\delta_{x} \\
\text { (inch) }\end{array}$ & $\begin{array}{c}\Delta_{i} \\
\text { (inch) }\end{array}$ & $\Delta_{i} / h_{s x}$ & $\begin{array}{c}F_{x} \\
\text { (kips) }\end{array}$ & $\begin{array}{c}V_{i} \\
\text { (kips) }\end{array}$ & $\begin{array}{c}\delta_{x} \\
\text { (inch) }\end{array}$ & $\begin{array}{c}\Delta_{i} \\
\text { (inch) }\end{array}$ & $\Delta_{i} / h_{s x}$ & $\begin{array}{c}F_{x} \\
\text { (kips) }\end{array}$ & $\begin{array}{c}V_{i} \\
\text { (kips) }\end{array}$ \\
\hline Roof & 76 & 76 & 11.82 & 1.88 & 0.011 & 89 & 89 & 12.13 & 2.28 & 0.014 & 15 & 15 \\
\hline 4 & 36 & 112 & 9.94 & 2.73 & 0.016 & 70 & 159 & 9.85 & 3.00 & 0.018 & 14 & 29 \\
\hline 3 & 24 & 136 & 7.21 & 3.39 & 0.020 & 39 & 198 & 6.85 & 3.33 & 0.020 & 13 & 42 \\
\hline 2 & 30 & 166 & 3.82 & 3.82 & 0.018 & 15 & 213 & 3.51 & 3.51 & 0.016 & 14 & 55 \\
\hline
\end{tabular}

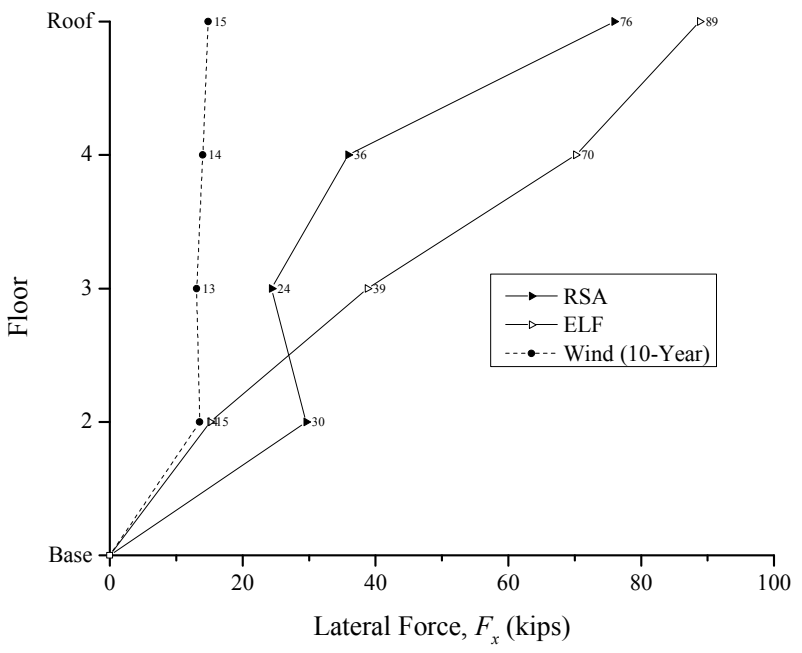

(a) Lateral Forces

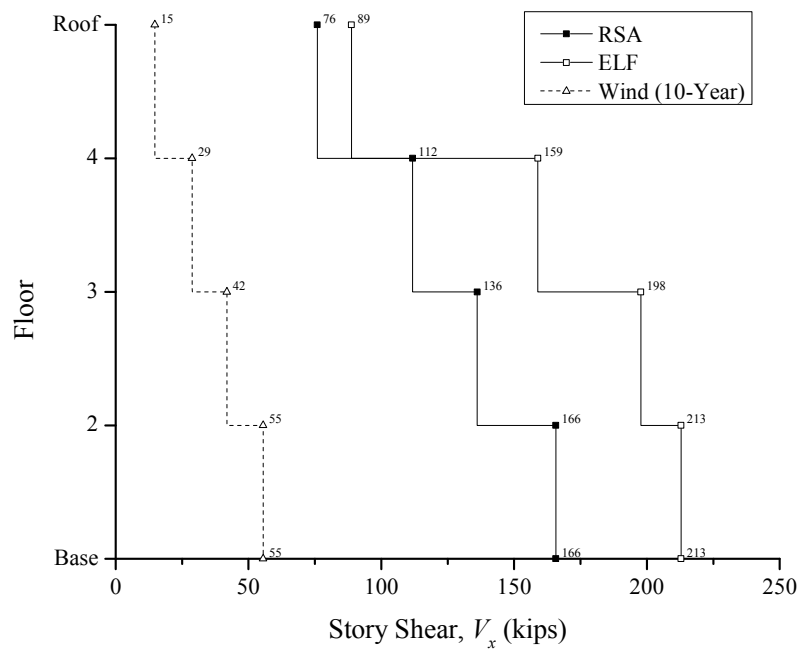

(b) Story Shears

Figure B-2. Drift Design Lateral Forces and Story Shears 


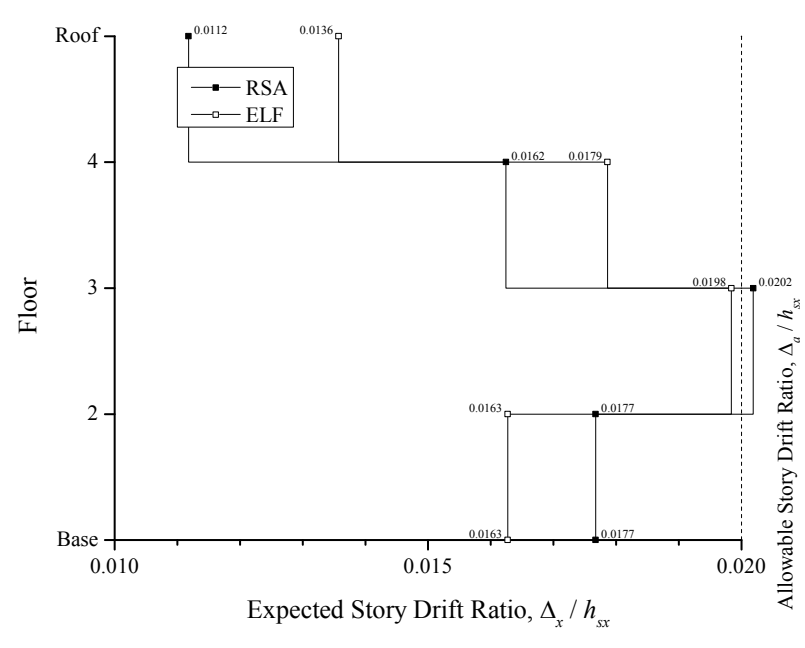

(a) Story Drift Ratios at Center of Mass

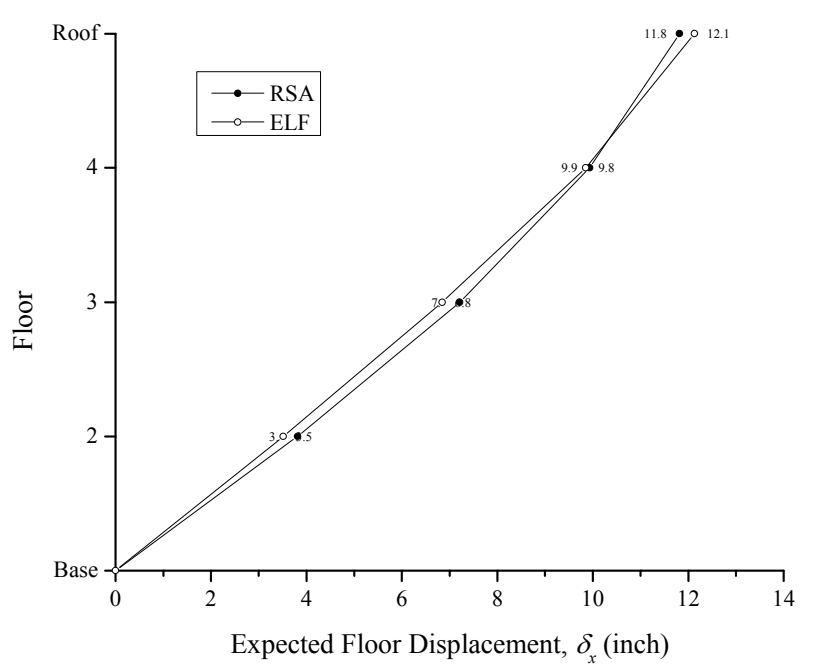

(b) Floor Displacements at Center of Mass

Figure B-3. Story Drift Ratios and Deflected Shape

Table B-9 and Table B-10 provide the tabulated data for verifying the allowable story drifts and the seismic stability coefficient per ASCE $7 \S 12.8 .6$ and ASCE $\$ 12.8 .7$ for each archetype building design. The following formulas were used in these calculations - see ASCE 7, where $\delta$ is the story drift and $\Delta$ is the story drift ratio as defined in ASCE 7.

$$
\begin{gathered}
\delta_{x}=\frac{C_{d} \delta_{x e}}{I_{e}} \\
\Delta_{i}=\delta_{x}-\delta_{x-1}=\frac{C_{d}}{I_{e}}\left(\delta_{x e}-\delta_{(x-1) e}\right) \\
\theta_{2 i}=\frac{P_{x} \Delta_{i} I_{e}}{V_{i} h_{s x} C_{d}}=\frac{P_{x}\left(\delta_{x e}-\delta_{(x-1) e}\right)}{V_{i} h_{s x}} \\
\theta_{1 i}=\frac{\theta_{2 i}}{1+\theta_{2 i}}
\end{gathered}
$$

Table B-9. ASCE 7 Allowable Drift and Stability Verification, E-W ME4 RSA

\begin{tabular}{cccccccccc}
\hline Level, $x$ & $\begin{array}{c}V_{i} \times C_{d} \\
\text { (kips) }\end{array}$ & $\begin{array}{c}P_{x} \\
\text { (kips) }\end{array}$ & $\begin{array}{c}\Delta_{i} \\
\text { (inch) }\end{array}$ & $\begin{array}{c}h_{s x} \\
\text { (inch) }\end{array}$ & $\Delta_{i} / h_{s x}$ & $\theta_{2 i}$ & $\theta_{1 i}$ & $\beta_{i}$ & $\theta_{\max , i}$ \\
\hline ROOF & 418 & 1091 & 1.88 & 168 & 0.011 & 0.029 & 0.028 & 0.39 & 0.232 \\
4 & 615 & 2470 & 2.73 & 168 & 0.016 & 0.065 & 0.061 & 0.54 & 0.168 \\
3 & 749 & 3854 & 3.39 & 168 & 0.020 & 0.104 & 0.094 & 0.63 & 0.143 \\
2 & 911 & 5257 & 3.82 & 216 & 0.018 & 0.102 & 0.093 & 0.70 & 0.129 \\
\hline
\end{tabular}


Table B-10. ASCE 7 Allowable Drift and Stability Verification, E-W ME4 ELF

\begin{tabular}{cccccccccc}
\hline Level, $x$ & $\begin{array}{c}V_{i} \times C_{d} \\
\text { (kips) }\end{array}$ & $\begin{array}{c}P_{x} \\
\text { (kips) }\end{array}$ & $\begin{array}{c}\Delta_{i} \\
\text { (inch) }\end{array}$ & $\begin{array}{c}h_{s x} \\
\text { (inch) }\end{array}$ & $\Delta_{i} / h_{s x}$ & $\theta_{2 i}$ & $\theta_{1 i}$ & $\beta_{i}$ & $\theta_{\max , i}$ \\
\hline ROOF & 488 & 1095 & 2.28 & 168 & 0.014 & 0.030 & 0.030 & 0.39 & 0.231 \\
4 & 874 & 2479 & 3.00 & 168 & 0.018 & 0.051 & 0.048 & 0.44 & 0.208 \\
3 & 1088 & 3877 & 3.33 & 168 & 0.020 & 0.071 & 0.066 & 0.50 & 0.183 \\
2 & 1171 & 5293 & 3.51 & 216 & 0.016 & 0.074 & 0.069 & 0.53 & 0.172 \\
\hline
\end{tabular}

\section{B.1.2.2.2 ME8 (Special Moment Frame)}

Table B-11 and Table B-12 provide the horizontal seismic forces and story shears for each archetype building design for the seismic strength analysis and the drift analysis. The data are also graphically illustrated in Figure B-4 through Figure B-6. In these tables, $F_{x}$ represents the seismic forces acting in the E-W direction only. The equivalent story forces for the RSA design are backed out from the story shears computed via a modal combination procedure. Although not theoretically correct, the forces provide a comparison basis for evaluating variations in the vertical distribution.

Table B-11. Seismic Strength Design Forces, E-W ME8

\begin{tabular}{ccc|ccc|cc}
\hline & \multicolumn{2}{c|}{ RSA } & \multicolumn{3}{c|}{ ELF } & \multicolumn{2}{c}{ Wind (700-Year) } \\
Level, $x$ & $F_{x}$ & $V_{i}$ & $F_{x}$ & $V_{i}$ & $V_{E L F} /$ & $F_{x}$ & $V_{i}$ \\
& (kips) & (kips) & (kips) & (kips) & $V_{R S A}$ & (kips) & (kips) \\
\hline Roof & 135 & 135 & 108 & 108 & 1.25 & 46 & 46 \\
8 & 57 & 192 & 108 & 216 & 0.89 & 49 & 96 \\
7 & 39 & 231 & 86 & 302 & 0.77 & 48 & 143 \\
6 & 37 & 268 & 65 & 367 & 0.73 & 46 & 190 \\
5 & 31 & 299 & 47 & 413 & 0.72 & 45 & 235 \\
4 & 26 & 326 & 30 & 444 & 0.73 & 43 & 277 \\
3 & 31 & 357 & 17 & 460 & 0.77 & 40 & 318 \\
2 & 37 & 394 & 7 & 467 & 0.84 & 42 & 359 \\
\hline
\end{tabular}

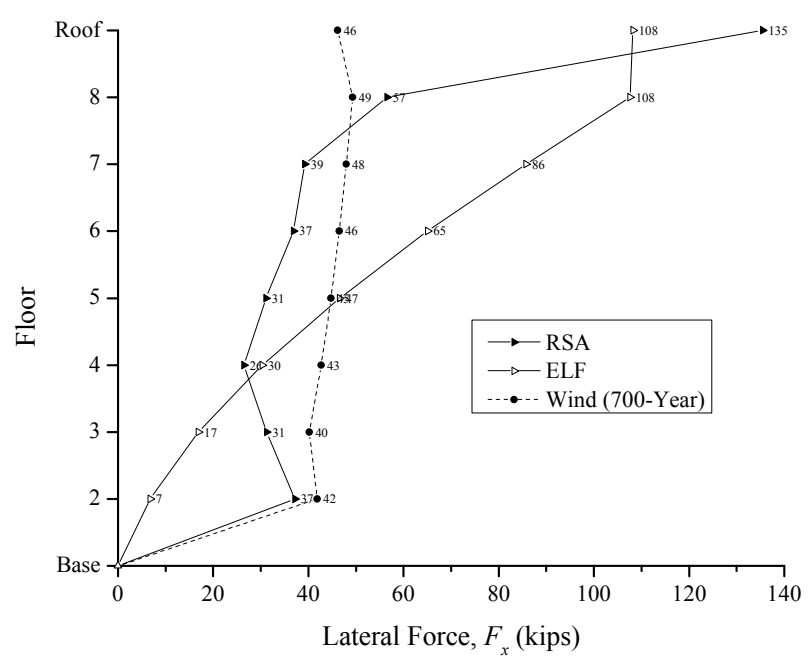

(a) Lateral Forces

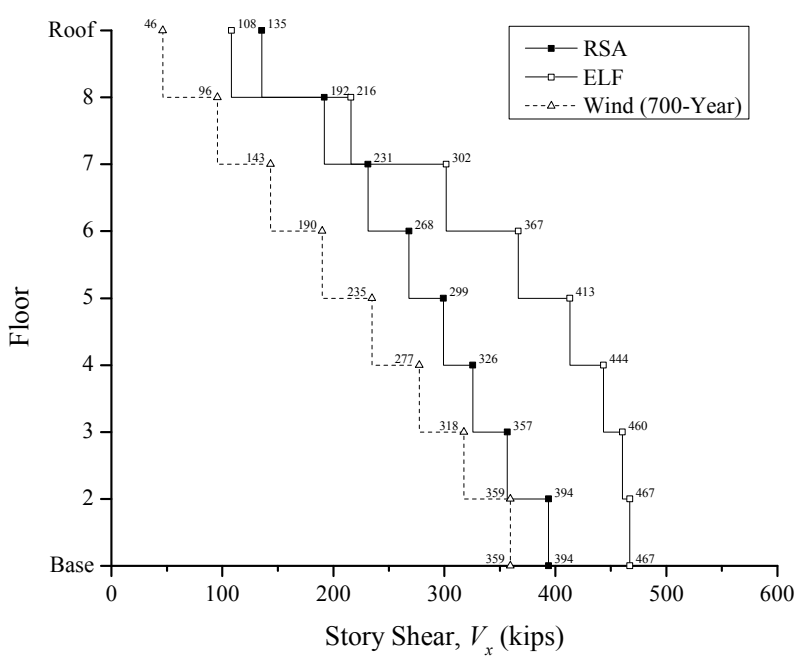

(b) Story Shears

Figure B-4. Strength Design Lateral Forces and Story Shears 
Table B-12. Seismic Drift Forces, E-W ME8

\begin{tabular}{|c|c|c|c|c|c|c|c|c|c|c|c|c|}
\hline \multirow{3}{*}{ Level, $x$} & \multicolumn{5}{|c|}{ RSA } & \multicolumn{5}{|c|}{ ELF } & \multicolumn{2}{|c|}{ Wind (10-Year) } \\
\hline & $F_{x}$ & $V_{i}$ & $\delta_{x}$ & $\Delta_{i}$ & $\Delta_{i} / h_{s x}$ & $F_{x}$ & $V_{i}$ & $\delta_{x}$ & $\Delta_{i}$ & $\Delta_{i} / h_{s x}$ & $F_{x}$ & $V_{i}$ \\
\hline & (kips) & (kips) & (inch) & (inch) & & (kips) & (kips) & (inch) & (inch) & & (kips) & (kips) \\
\hline Roof & 66 & 66 & 21.48 & 1.84 & 0.011 & 71 & 71 & 22.95 & 2.25 & 0.013 & 18 & 18 \\
\hline 8 & 28 & 93 & 19.64 & 2.38 & 0.014 & 68 & 139 & 20.70 & 2.83 & 0.017 & 19 & 37 \\
\hline 7 & 19 & 112 & 17.26 & 2.75 & 0.016 & 51 & 190 & 17.87 & 2.81 & 0.017 & 18 & 55 \\
\hline 6 & 18 & 131 & 14.52 & 2.92 & 0.017 & 36 & 226 & 15.06 & 3.00 & 0.018 & 17 & 73 \\
\hline 5 & 15 & 146 & 11.59 & 2.90 & 0.017 & 24 & 250 & 12.06 & 3.06 & 0.018 & 17 & 89 \\
\hline 4 & 13 & 159 & 8.69 & 2.99 & 0.018 & 14 & 264 & 9.00 & 3.11 & 0.019 & 16 & 105 \\
\hline 3 & 15 & 174 & 5.70 & 2.86 & 0.017 & 7 & 271 & 5.89 & 2.96 & 0.018 & 15 & 121 \\
\hline 2 & 18 & 192 & 2.84 & 2.84 & 0.013 & 2 & 273 & 2.93 & 2.93 & 0.014 & 16 & 136 \\
\hline
\end{tabular}

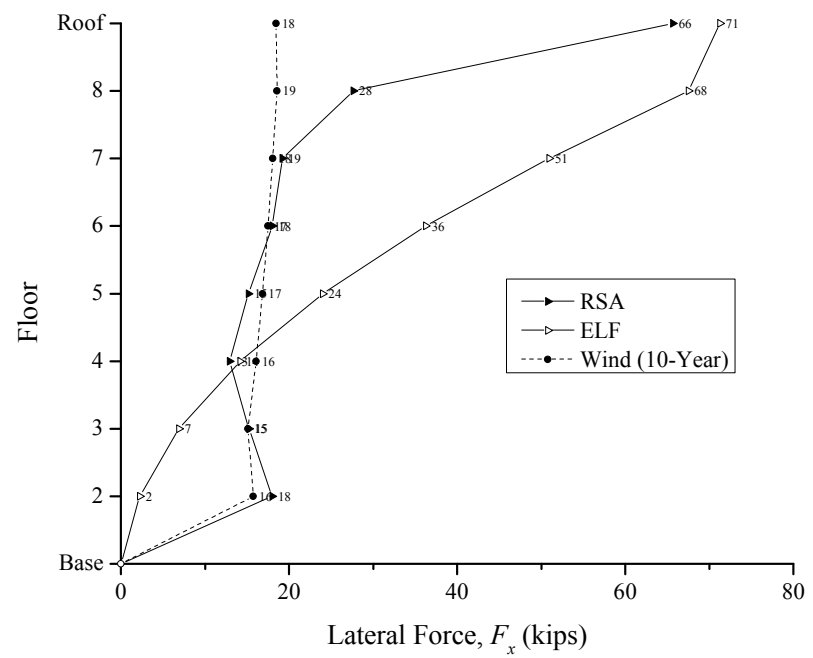

(a) Lateral forces

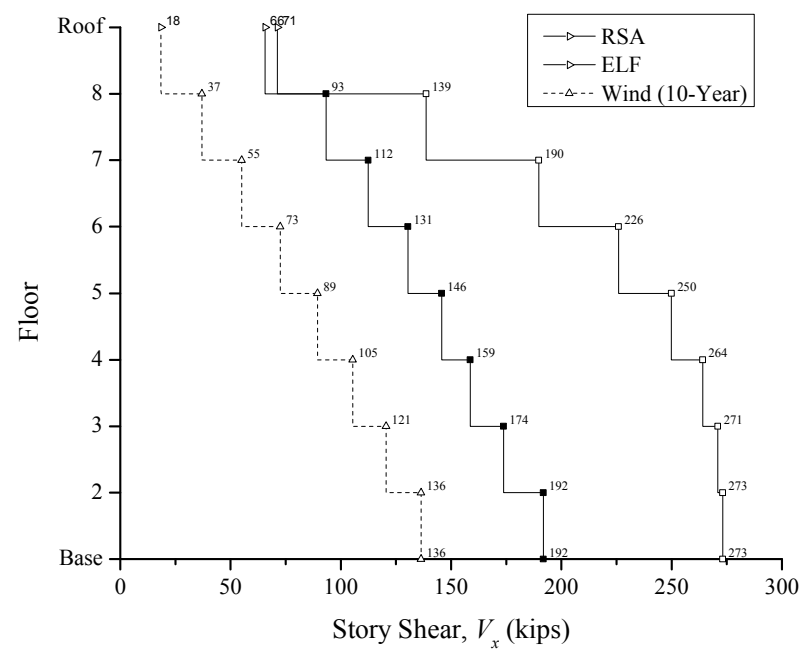

(b) Story Shears

Figure B-5. Drift Design Lateral Forces and Story Shears 


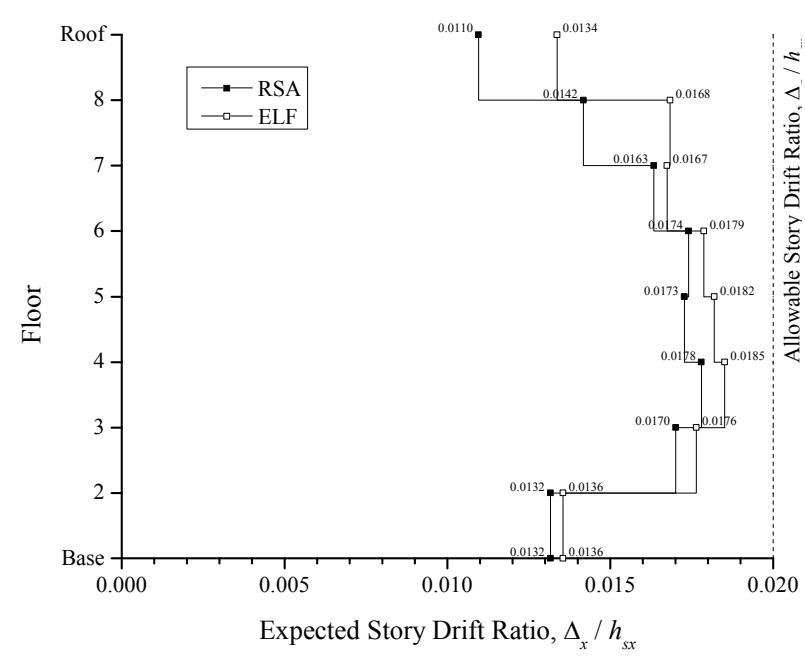

(a) Story Drift ratios at Center of Mass

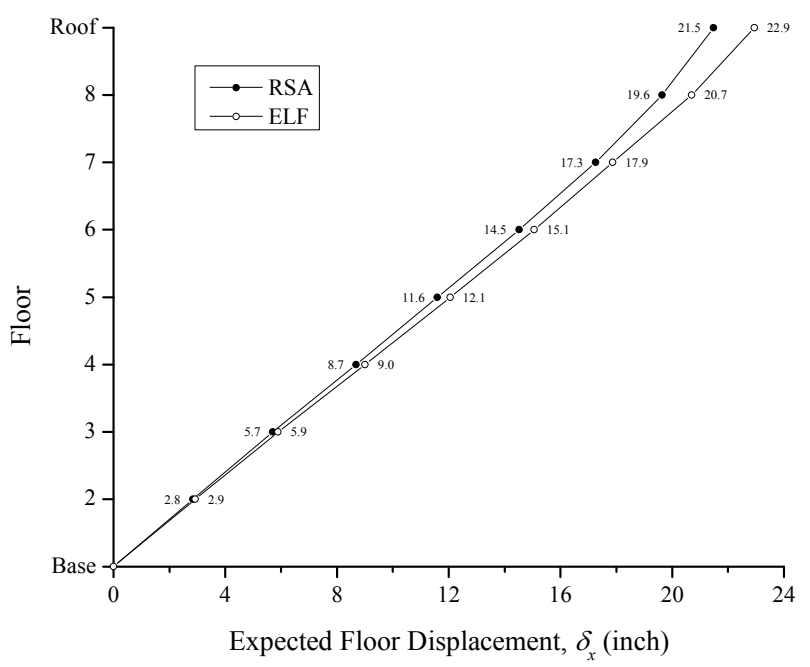

(b) Floor Displacements at Center of Mass

Figure B-6. Story Drift Ratios and Deflected Shape

Table B-13 and Table B-14 provide the tabulated data for verifying the allowable story drifts and the seismic stability coefficient per ASCE $7 \S 12.8 .6$ and $\$ 12.8 .7$ for each archetype building design.

Table B-13. ASCE 7 Allowable Drift and Stability Verification, E-W ME8 RSA

\begin{tabular}{cccccccccc}
\hline Level, $x$ & $\begin{array}{c}V_{i} \times C_{d} \\
\text { (kips) }\end{array}$ & $\begin{array}{c}P_{x} \\
\text { (kips) }\end{array}$ & $\begin{array}{c}\Delta_{i} \\
\text { (inch) }\end{array}$ & $\begin{array}{c}h_{s x} \\
\text { (inch) }\end{array}$ & $\Delta_{i} / h_{s x}$ & $\theta_{2 i}$ & $\theta_{1 i}$ & $\beta_{i}$ & $\theta_{\text {max }, i}$ \\
\hline ROOF & 361 & 1081 & 1.84 & 168 & 0.011 & 0.033 & 0.032 & 0.53 & 0.172 \\
8 & 513 & 2443 & 2.38 & 168 & 0.014 & 0.067 & 0.063 & 0.68 & 0.134 \\
7 & 619 & 3815 & 2.75 & 168 & 0.016 & 0.101 & 0.092 & 0.67 & 0.136 \\
6 & 718 & 5192 & 2.92 & 168 & 0.017 & 0.126 & 0.112 & 0.71 & 0.128 \\
5 & 801 & 6580 & 2.90 & 168 & 0.017 & 0.142 & 0.124 & 0.62 & 0.147 \\
4 & 872 & 7973 & 2.99 & 168 & 0.018 & 0.163 & 0.140 & 0.63 & 0.145 \\
3 & 956 & 9378 & 2.86 & 168 & 0.017 & 0.167 & 0.143 & 0.60 & 0.151 \\
2 & 1055 & 10807 & 2.84 & 216 & 0.013 & 0.135 & 0.119 & 0.60 & 0.152 \\
\hline
\end{tabular}

Table B-14. ASCE 7 Allowable Drift and Stability Verification, E-W ME8 ELF

\begin{tabular}{cccccccccc}
\hline Level, $x$ & $\begin{array}{c}V_{i} \times C_{d} \\
\text { (kips) }\end{array}$ & $\begin{array}{c}P_{x} \\
\text { (kips) }\end{array}$ & $\begin{array}{c}\Delta_{i} \\
\text { (inch) }\end{array}$ & $\begin{array}{c}h_{s x} \\
\text { (inch) }\end{array}$ & $\Delta_{i} / h_{s x}$ & $\theta_{2 i}$ & $\theta_{1 i}$ & $\beta_{i}$ & $\theta_{\text {max }, i}$ \\
\hline ROOF & 392 & 1083 & 2.25 & 168 & 0.013 & 0.037 & 0.036 & 0.37 & 0.246 \\
8 & 763 & 2450 & 2.83 & 168 & 0.017 & 0.054 & 0.051 & 0.51 & 0.180 \\
7 & 1043 & 3835 & 2.81 & 168 & 0.017 & 0.062 & 0.058 & 0.43 & 0.210 \\
6 & 1243 & 5229 & 3.00 & 168 & 0.018 & 0.075 & 0.070 & 0.48 & 0.190 \\
5 & 1375 & 6631 & 3.06 & 168 & 0.018 & 0.088 & 0.081 & 0.46 & 0.199 \\
4 & 1453 & 8038 & 3.11 & 168 & 0.019 & 0.102 & 0.093 & 0.48 & 0.190 \\
3 & 1491 & 9457 & 2.96 & 168 & 0.018 & 0.112 & 0.101 & 0.47 & 0.192 \\
2 & 1503 & 10898 & 2.93 & 216 & 0.014 & 0.098 & 0.089 & 0.45 & 0.200 \\
\hline
\end{tabular}




\section{B.1.2.2.3 ME16 (Special Moment Frame)}

Table B-15 and Table B-16 provide the horizontal seismic forces and story shears for each archetype building design for the seismic strength analysis and the drift analysis. The data are also graphically illustrated in Figure B-7 through Figure B-9. In these tables, $F_{x}$ represents the seismic forces acting in the E-W direction only. The equivalent story forces for the RSA design are backed out from the story shears computed via a modal combination procedure. Although not theoretically correct, the forces provide a comparison basis for evaluating variations in the vertical distribution.

Table B-15. Summary of Seismic Strength Design Forces, E-W ME16

\begin{tabular}{ccc|ccc|cc}
\hline & \multicolumn{2}{c|}{ RSA } & \multicolumn{3}{c|}{ ELF } & \multicolumn{2}{c}{ Wind (700-Year) } \\
Level, $x$ & $F_{x}$ & $V_{i}$ & $F_{x}$ & $V_{i}$ & $V_{E L F} / V_{R S A}$ & $F_{x}$ & $V_{i}$ \\
& (kips) & (kips) & (kips) & (kips) & & 57 & (kips) \\
\hline Roof & 196 & 196 & 133 & 133 & 1.47 & 57 & 119 \\
16 & 95 & 292 & 144 & 278 & 1.05 & 62 & 180 \\
15 & 54 & 346 & 127 & 405 & 0.85 & 61 & 241 \\
14 & 46 & 391 & 111 & 515 & 0.76 & 61 & 301 \\
13 & 45 & 436 & 95 & 611 & 0.71 & 60 & 360 \\
12 & 41 & 477 & 80 & 691 & 0.69 & 59 & 417 \\
11 & 33 & 510 & 67 & 758 & 0.67 & 58 & 474 \\
10 & 31 & 541 & 55 & 813 & 0.67 & 57 & 530 \\
9 & 33 & 573 & 44 & 857 & 0.67 & 56 & 584 \\
8 & 33 & 607 & 34 & 891 & 0.68 & 54 & 637 \\
7 & 29 & 636 & 26 & 917 & 0.69 & 53 & 689 \\
6 & 26 & 662 & 18 & 935 & 0.71 & 51 & 738 \\
5 & 29 & 691 & 12 & 947 & 0.73 & 50 & 831 \\
4 & 38 & 729 & 7 & 954 & 0.76 & 48 & 878 \\
3 & 44 & 773 & 3 & 957 & 0.81 & 45 & 47 \\
2 & 37 & 810 & 1 & 958 & 0.85 & &
\end{tabular}

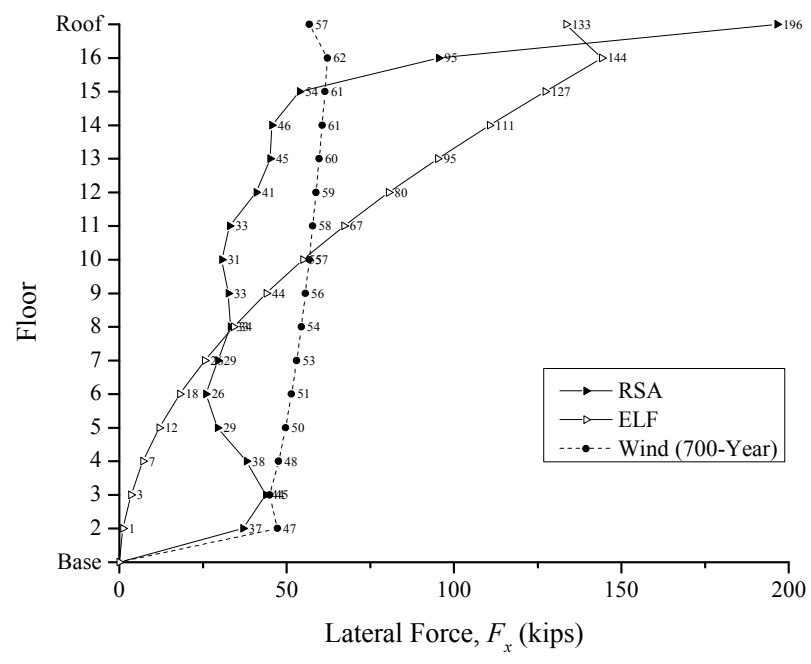

(a) Lateral forces

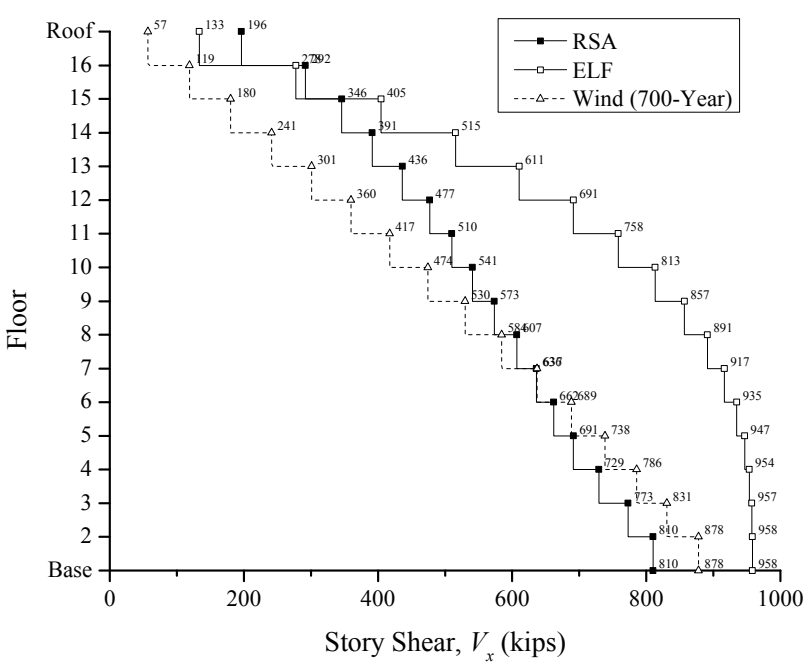

(b) Story Shears

Figure B-7. Strength Design Lateral Forces and Story Shears 
Table B-16. Summary of Seismic Drift Forces, E-W ME16

\begin{tabular}{|c|c|c|c|c|c|c|c|c|c|c|c|c|}
\hline \multirow{3}{*}{ Level, $x$} & \multicolumn{5}{|c|}{ RSA } & \multicolumn{5}{|c|}{ ELF } & \multicolumn{2}{|c|}{ Wind (10-Year) } \\
\hline & $F_{x}$ & $V_{i}$ & $\delta_{x}$ & $\Delta_{i}$ & $\Delta_{i} / h_{s x}$ & $F_{x}$ & $V_{i}$ & $\delta_{x}$ & $\Delta_{i}$ & $\Delta_{i} / h_{s x}$ & $F_{x}$ & $V_{i}$ \\
\hline & (kips) & (kips) & (inch) & (inch) & & (kips) & (kips) & (inch) & (inch) & & (kips) & (kips) \\
\hline Roof & 71 & 71 & 29.39 & 1.28 & 0.008 & 52 & 52 & 38.12 & 1.76 & 0.010 & 23 & 23 \\
\hline 16 & 35 & 106 & 28.11 & 1.69 & 0.010 & 56 & 109 & 36.36 & 2.16 & 0.013 & 23 & 46 \\
\hline 15 & 20 & 126 & 26.42 & 2.00 & 0.012 & 50 & 158 & 34.20 & 2.30 & 0.014 & 23 & 69 \\
\hline 14 & 17 & 142 & 24.42 & 2.02 & 0.012 & 43 & 202 & 31.89 & 2.51 & 0.015 & 23 & 91 \\
\hline 13 & 16 & 159 & 22.41 & 1.89 & 0.011 & 37 & 239 & 29.39 & 2.62 & 0.016 & 22 & 114 \\
\hline 12 & 15 & 174 & 20.51 & 1.99 & 0.012 & 31 & 270 & 26.77 & 2.81 & 0.017 & 22 & 136 \\
\hline 11 & 12 & 186 & 18.52 & 2.09 & 0.012 & 26 & 296 & 23.96 & 2.92 & 0.017 & 22 & 157 \\
\hline 10 & 11 & 197 & 16.43 & 2.10 & 0.012 & 21 & 318 & 21.05 & 2.84 & 0.017 & 21 & 178 \\
\hline 9 & 12 & 209 & 14.33 & 2.04 & 0.012 & 17 & 335 & 18.21 & 2.64 & 0.016 & 21 & 199 \\
\hline 8 & 12 & 221 & 12.29 & 2.07 & 0.012 & 13 & 348 & 15.57 & 2.60 & 0.015 & 20 & 219 \\
\hline 7 & 11 & 232 & 10.22 & 2.07 & 0.012 & 10 & 358 & 12.97 & 2.57 & 0.015 & 20 & 239 \\
\hline 6 & 9 & 241 & 8.15 & 1.94 & 0.012 & 7 & 365 & 10.40 & 2.51 & 0.015 & 19 & 258 \\
\hline 5 & 11 & 252 & 6.21 & 1.75 & 0.010 & 5 & 370 & 7.89 & 2.37 & 0.014 & 19 & 277 \\
\hline 4 & 14 & 266 & 4.47 & 1.67 & 0.010 & 3 & 373 & 5.52 & 2.20 & 0.013 & 18 & 295 \\
\hline 3 & 16 & 282 & 2.80 & 1.49 & 0.009 & 1 & 374 & 3.32 & 1.83 & 0.011 & 17 & 311 \\
\hline 2 & 13 & 295 & 1.31 & 1.31 & 0.006 & 0 & 375 & 1.49 & 1.49 & 0.007 & 18 & 329 \\
\hline
\end{tabular}

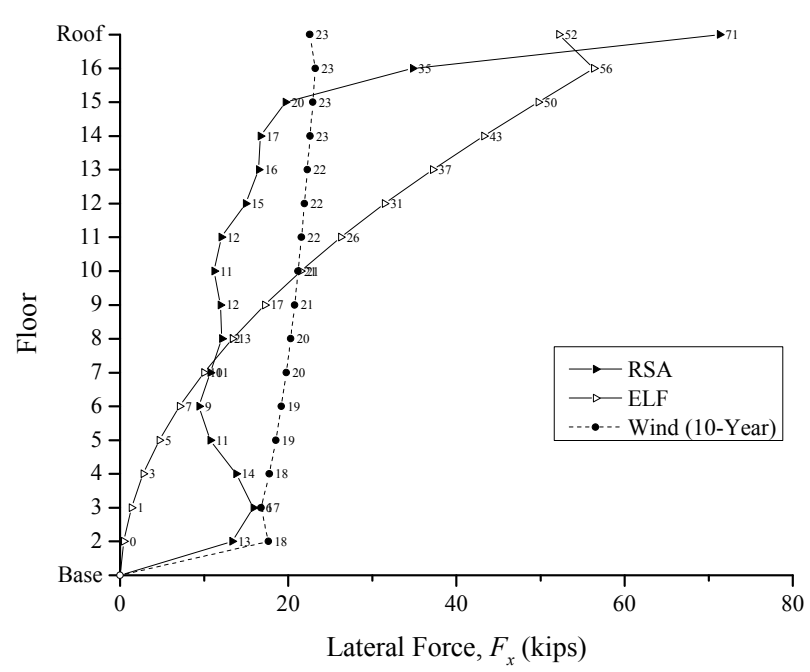

(a) Lateral forces

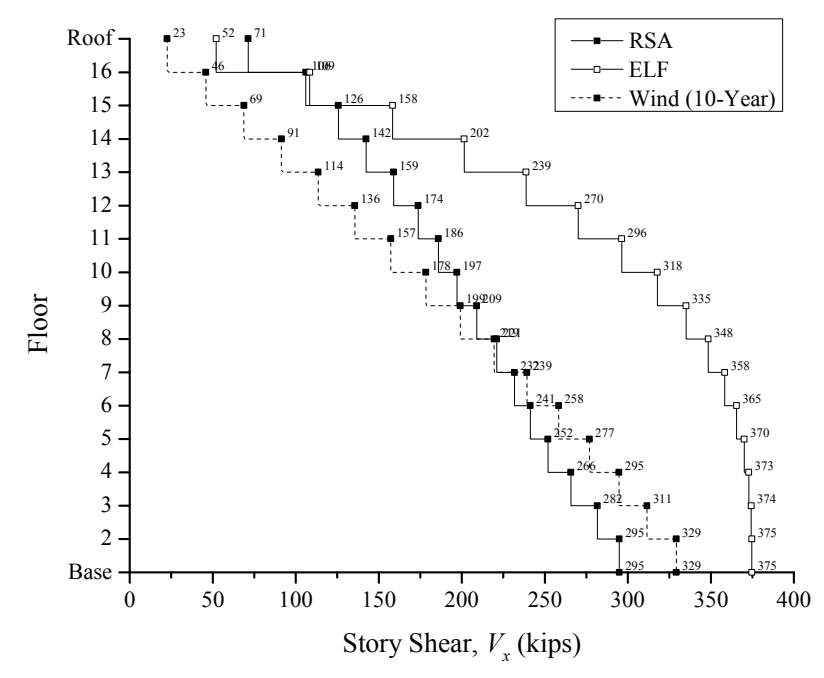

(b) Story Shears

Figure B-8. Drift Design Lateral Forces and Story Shears 


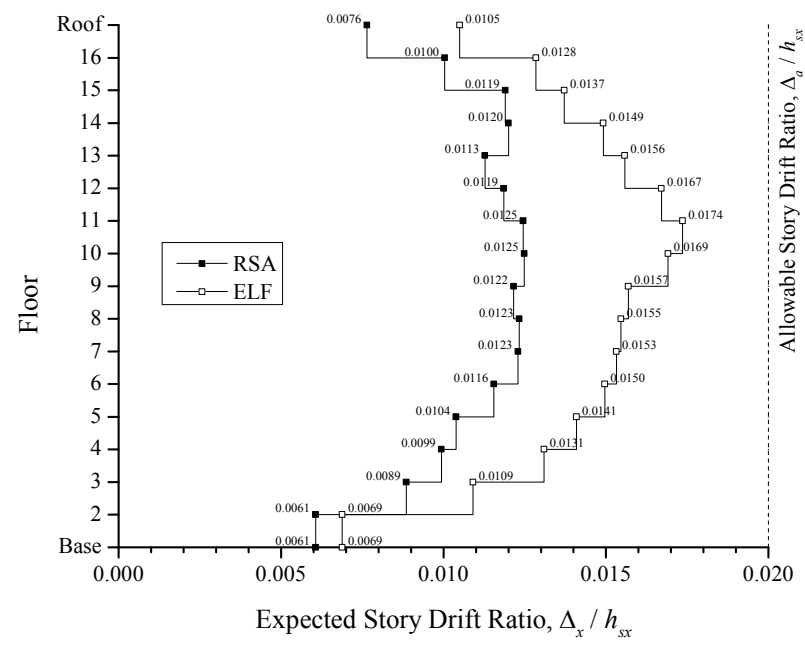

(a) Story Drift ratios at Center of Mass

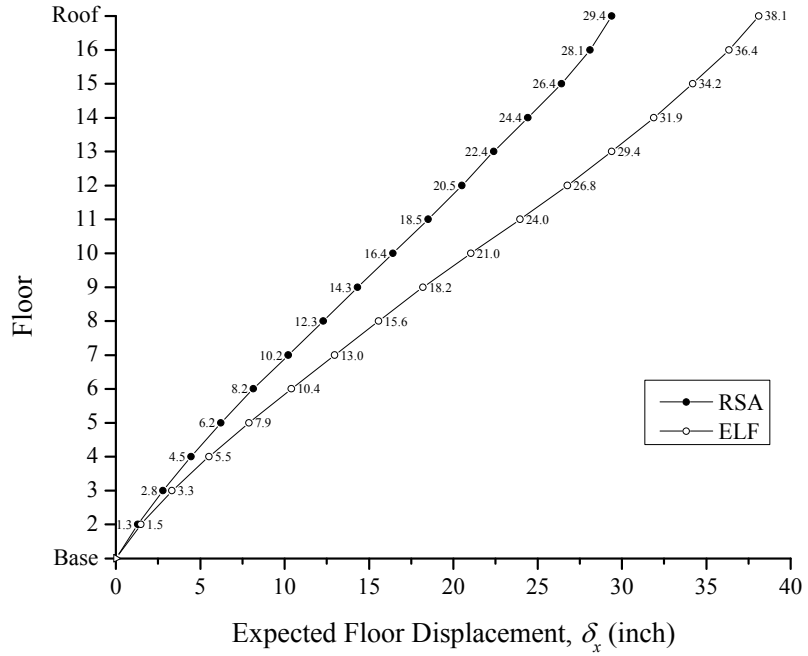

(b) Floor Displacements at Center of Mass

Figure B-9. Story Drift Ratios and Deflected Shape

Table B-17 and Table B-18 provide the tabulated data for verifying the allowable story drifts and the seismic stability coefficient per ASCE 7 \$12.8.6 and ASCE 7 \$12.8.7 for each building design.

Table B-17. ASCE 7 Allowable Drift and Stability Verification, E-W ME16 RSA

\begin{tabular}{cccccccccc}
\hline Level, $x$ & $\begin{array}{c}V_{i} \times C_{d} \\
\text { (kips) }\end{array}$ & $\begin{array}{c}P_{x} \\
\text { (kips) }\end{array}$ & $\begin{array}{c}\Delta_{i} \\
\text { (inch) }\end{array}$ & $\begin{array}{c}h_{s x} \\
\text { (inch) }\end{array}$ & $\Delta_{i} / h_{s x}$ & $\theta_{2 i}$ & $\theta_{1 i}$ & $\beta_{i}$ & $\theta_{\max , i}$ \\
\hline ROOF & 392 & 1080 & 1.28 & 168 & 0.008 & 0.021 & 0.021 & 0.52 & 0.173 \\
16 & 583 & 2444 & 1.69 & 168 & 0.010 & 0.042 & 0.040 & 0.68 & 0.133 \\
15 & 692 & 3812 & 2.00 & 168 & 0.012 & 0.066 & 0.062 & 0.78 & 0.116 \\
14 & 783 & 5184 & 2.02 & 168 & 0.012 & 0.079 & 0.074 & 0.81 & 0.112 \\
13 & 874 & 6570 & 1.89 & 168 & 0.011 & 0.085 & 0.078 & 0.65 & 0.139 \\
12 & 956 & 7960 & 1.99 & 168 & 0.012 & 0.099 & 0.090 & 0.67 & 0.137 \\
11 & 1022 & 9355 & 2.09 & 168 & 0.012 & 0.114 & 0.102 & 0.68 & 0.134 \\
10 & 1084 & 10754 & 2.10 & 168 & 0.012 & 0.124 & 0.110 & 0.68 & 0.134 \\
9 & 1149 & 12160 & 2.04 & 168 & 0.012 & 0.129 & 0.114 & 0.67 & 0.135 \\
8 & 1216 & 13570 & 2.07 & 168 & 0.012 & 0.138 & 0.121 & 0.67 & 0.135 \\
7 & 1275 & 14986 & 2.07 & 168 & 0.012 & 0.145 & 0.126 & 0.68 & 0.133 \\
6 & 1327 & 16406 & 1.94 & 168 & 0.012 & 0.143 & 0.125 & 0.68 & 0.134 \\
5 & 1386 & 17837 & 1.75 & 168 & 0.010 & 0.134 & 0.118 & 0.63 & 0.143 \\
4 & 1462 & 19276 & 1.67 & 168 & 0.010 & 0.131 & 0.116 & 0.61 & 0.149 \\
3 & 1549 & 20726 & 1.49 & 168 & 0.009 & 0.118 & 0.106 & 0.60 & 0.150 \\
2 & 1622 & 22207 & 1.31 & 216 & 0.006 & 0.083 & 0.077 & 0.54 & 0.169 \\
\hline
\end{tabular}


Table B-18. ASCE 7 Allowable Drift and Stability Verification, E-W ME16 RSA

\begin{tabular}{cccccccccc}
\hline Level, $x$ & $\begin{array}{c}V_{i} \times C_{d} \\
\text { (kips) }\end{array}$ & $\begin{array}{c}P_{x} \\
\text { (kips) }\end{array}$ & $\begin{array}{c}\Delta_{i} \\
\text { (inch) }\end{array}$ & $\begin{array}{c}h_{s x} \\
\text { (inch) }\end{array}$ & $\Delta_{i} / h_{s x}$ & $\theta_{2 i}$ & $\theta_{1 i}$ & $\beta_{i}$ & $\theta_{\max , i}$ \\
\hline ROOF & 287 & 1081 & 1.76 & 168 & 0.010 & 0.040 & 0.038 & 0.47 & 0.194 \\
16 & 597 & 2444 & 2.16 & 168 & 0.013 & 0.053 & 0.050 & 0.61 & 0.150 \\
15 & 870 & 3822 & 2.30 & 168 & 0.014 & 0.060 & 0.057 & 0.58 & 0.157 \\
14 & 1108 & 5208 & 2.51 & 168 & 0.015 & 0.070 & 0.065 & 0.64 & 0.142 \\
13 & 1313 & 6601 & 2.62 & 168 & 0.016 & 0.078 & 0.073 & 0.69 & 0.131 \\
12 & 1486 & 7997 & 2.81 & 168 & 0.017 & 0.090 & 0.082 & 0.74 & 0.123 \\
11 & 1630 & 9399 & 2.92 & 168 & 0.017 & 0.100 & 0.091 & 0.78 & 0.117 \\
10 & 1748 & 10806 & 2.84 & 168 & 0.017 & 0.104 & 0.095 & 0.78 & 0.116 \\
9 & 1843 & 12221 & 2.64 & 168 & 0.016 & 0.104 & 0.094 & 0.71 & 0.128 \\
8 & 1916 & 13641 & 2.60 & 168 & 0.015 & 0.110 & 0.099 & 0.69 & 0.131 \\
7 & 1971 & 15066 & 2.57 & 168 & 0.015 & 0.117 & 0.105 & 0.71 & 0.127 \\
6 & 2010 & 16496 & 2.51 & 168 & 0.015 & 0.123 & 0.109 & 0.72 & 0.126 \\
5 & 2036 & 17935 & 2.37 & 168 & 0.014 & 0.124 & 0.110 & 0.75 & 0.121 \\
4 & 2051 & 19381 & 2.20 & 168 & 0.013 & 0.124 & 0.110 & 0.73 & 0.125 \\
3 & 2058 & 20843 & 1.83 & 168 & 0.011 & 0.110 & 0.099 & 0.71 & 0.128 \\
2 & 2061 & 22341 & 1.49 & 216 & 0.007 & 0.075 & 0.069 & 0.59 & 0.154 \\
\hline
\end{tabular}

\section{B.1.2.3 Horizontal Seismic Forces, N-S Direction}

\section{B.1.2.3.1 ME4 (Eccentrically Braced Frame)}

Table B-19 and Table B-20 provide the horizontal seismic forces and story shears for each archetype building design for the seismic strength analysis and the drift analysis. The data are also graphically illustrated in Figure B-10 through Figure B-12. In these tables, $F_{y}$ represents the seismic forces acting in the N-S direction only. The equivalent story forces for the RSA design are backed out from the story shears computed via a modal combination procedure. Although not theoretically correct, the forces provide a comparison basis for evaluating variations in the vertical distribution.

Table B-19. Seismic Strength Design Forces, N-S ME4

\begin{tabular}{ccc|ccc|cc}
\hline & \multicolumn{2}{c|}{ RSA } & \multicolumn{3}{c|}{ ELF } & \multicolumn{2}{c}{ Wind (700-Year) } \\
Level, $x$ & $F_{y}$ & $V_{i}$ & $F_{y}$ & $V_{i}$ & $V_{E L F} /$ & $F_{y}$ & $V_{i}$ \\
& (kips) & (kips) & (kips) & (kips) & $V_{R S A}$ & (kips) & (kips) \\
\hline Roof & 138 & 138 & 153 & 153 & 0.90 & 52 & 52 \\
4 & 100 & 238 & 137 & 291 & 0.82 & 50 & 103 \\
3 & 70 & 308 & 89 & 380 & 0.81 & 48 & 150 \\
2 & 51 & 359 & 45 & 425 & 0.85 & 50 & 200 \\
\hline
\end{tabular}




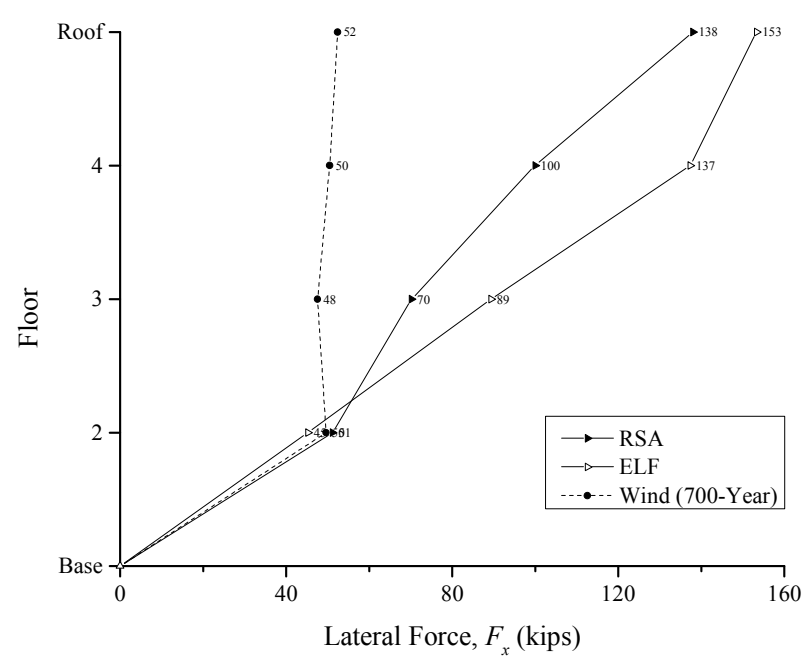

(a) Lateral forces

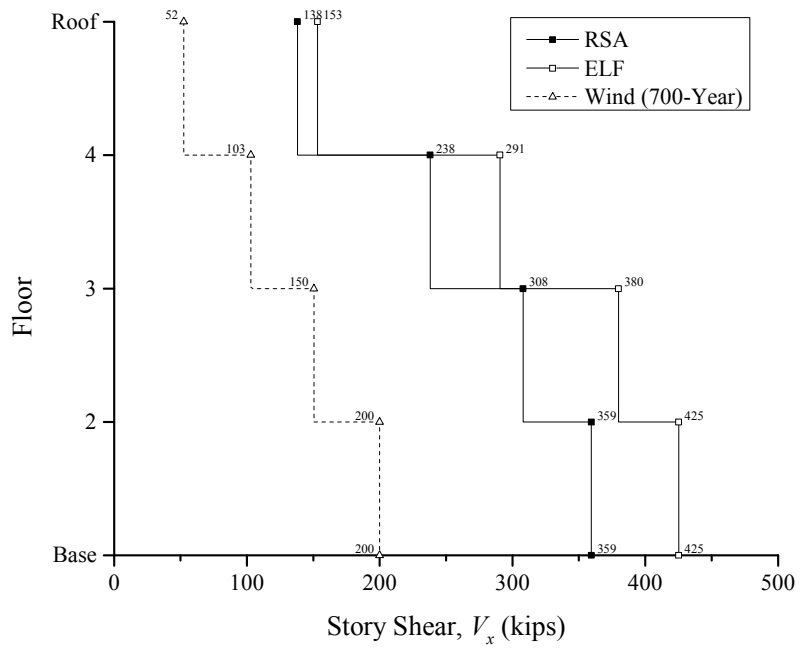

(b) Story Shears

Figure B-10. Strength Design Lateral Forces and Story Shears

Table B-20. Seismic Drift Forces, N-S ME4

\begin{tabular}{|c|c|c|c|c|c|c|c|c|c|c|c|c|}
\hline \multirow{3}{*}{ Level, $x$} & \multicolumn{5}{|c|}{ RSA } & \multicolumn{5}{|c|}{ ELF } & \multicolumn{2}{|c|}{ Wind (10-Year) } \\
\hline & $F_{y}$ & $V_{i}$ & $\delta_{x}$ & $\Delta_{i}$ & $\Delta_{i} / h_{s x}$ & $F_{y}$ & $V_{i}$ & $\delta_{x}$ & $\Delta_{i}$ & $\Delta_{i} / h_{s x}$ & $F_{y}$ & $V_{i}$ \\
\hline & (kips) & (kips) & (inch) & (inch) & & (kips) & (kips) & (inch) & (inch) & & (kips) & (kips) \\
\hline Roof & 120 & 120 & 4.35 & 1.02 & 0.006 & 137 & 137 & 5.14 & 1.22 & 0.007 & 0.02 & 22 \\
\hline 4 & 87 & 207 & 3.43 & 1.15 & 0.007 & 120 & 257 & 3.92 & 1.40 & 0.008 & 0.02 & 22 \\
\hline 3 & 61 & 268 & 2.34 & 1.11 & 0.007 & 76 & 333 & 2.52 & 1.24 & 0.007 & 0.02 & 20 \\
\hline 2 & 44 & 313 & 1.25 & 1.26 & 0.006 & 37 & 370 & 1.28 & 1.28 & 0.006 & 0.02 & 21 \\
\hline
\end{tabular}

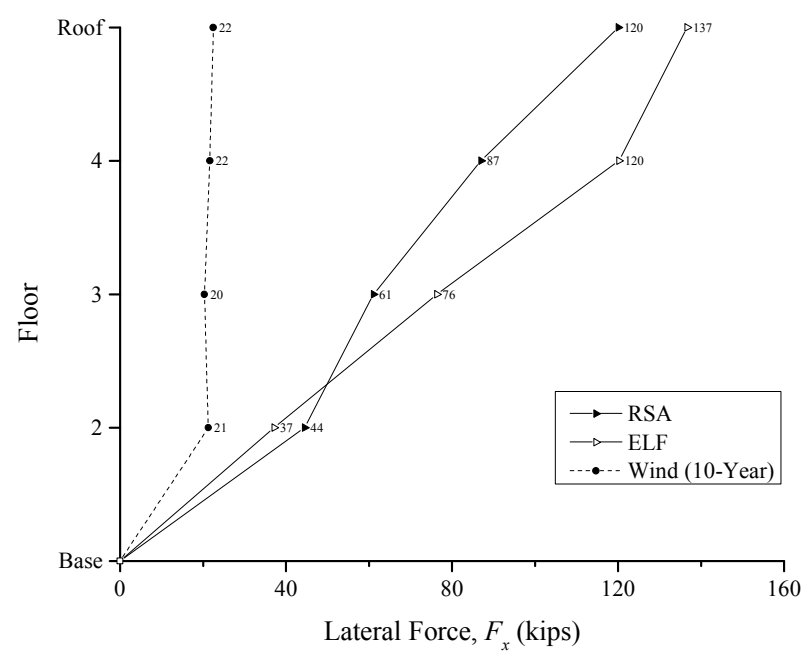

(a) Lateral forces

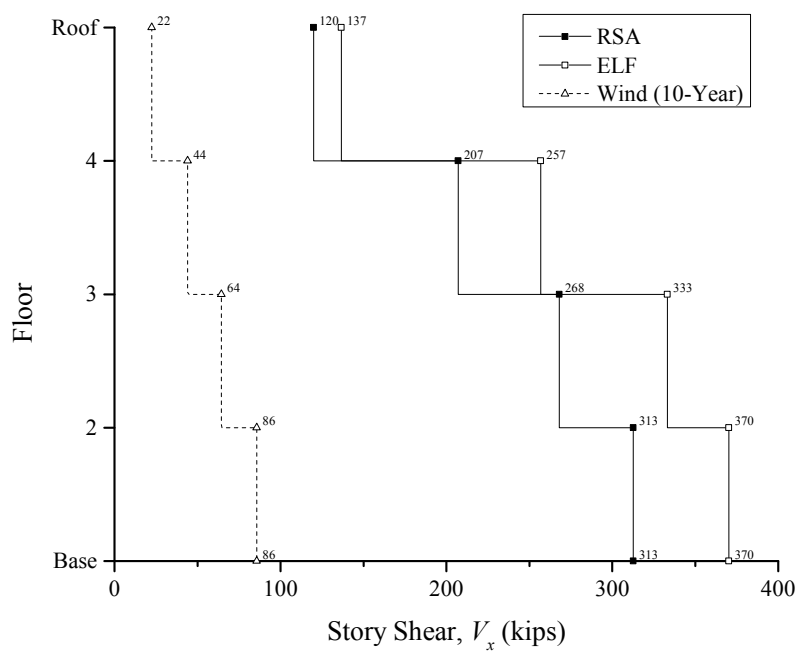

(b) Story Shears

Figure B-11. Drift Design Lateral Forces and Story Shears 


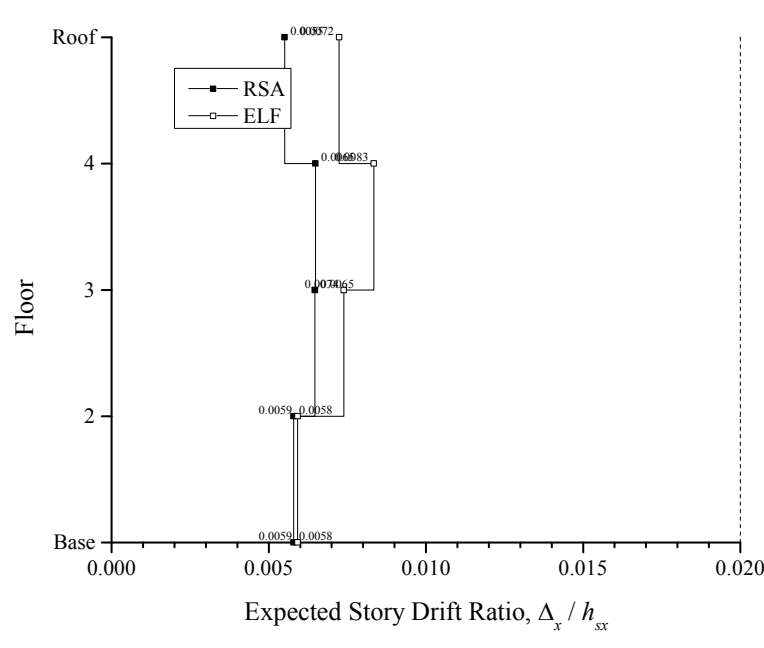

(a) Story Drift Ratios at Center of Mass

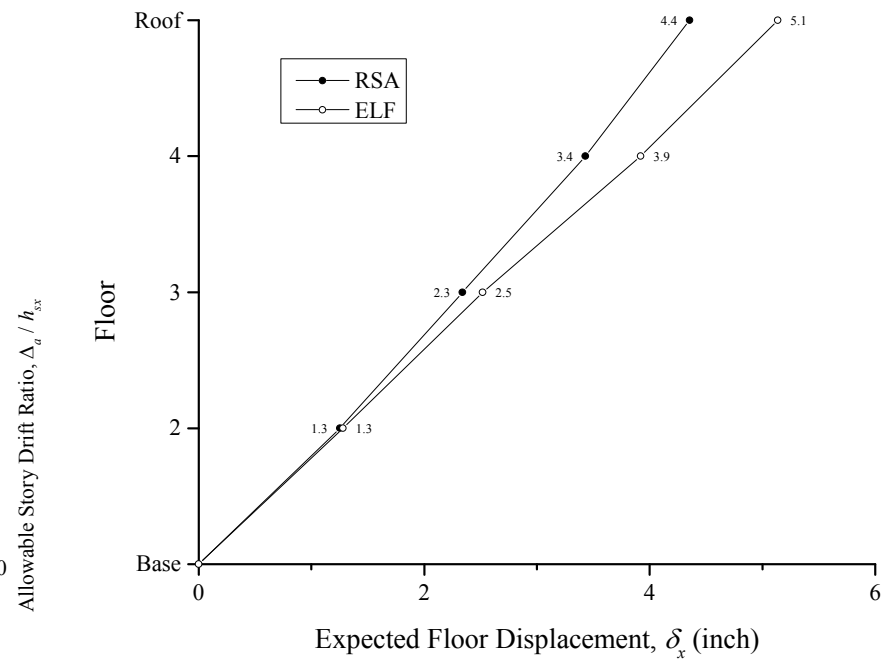

(b) Floor Displacements at Center of Mass

Figure B-12. Story Drift Ratios and Deflected Shape

Table B-21 and Table B-22 provide the tabulated data for verifying the allowable story drifts and the seismic stability coefficient per ASCE $7 \S 12.8 .6$ and ASCE $§ 12.8 .7$ for each archetype building design.

Table B-21. ASCE 7 Allowable Drift and Stability Verification, N-S ME4 RSA

\begin{tabular}{cccccccccc}
\hline Level, $x$ & $\begin{array}{c}V_{i} \times C_{d} \\
\text { (kips) }\end{array}$ & $\begin{array}{c}P_{x} \\
\text { (kips) }\end{array}$ & $\begin{array}{c}\Delta_{i} \\
\text { (inch) }\end{array}$ & $\begin{array}{c}h_{s x} \\
\text { (inch) }\end{array}$ & $\begin{array}{c}\Delta_{i} / h_{s x} \\
\theta_{2 i}\end{array}$ & $\theta_{1 i}$ & $\beta_{i}$ & $\theta_{\max , i}$ \\
\hline ROOF & 480 & 1085 & 1.02 & 168 & 0.006 & 0.012 & 0.012 & 1.00 & 0.125 \\
4 & 828 & 2457 & 1.15 & 168 & 0.007 & 0.019 & 0.019 & 1.00 & 0.125 \\
3 & 1073 & 3834 & 1.11 & 168 & 0.007 & 0.023 & 0.023 & 1.00 & 0.125 \\
2 & 1250 & 5223 & 1.26 & 216 & 0.006 & 0.024 & 0.024 & 1.00 & 0.125 \\
\hline
\end{tabular}

Table B-22. ASCE 7 Allowable Drift and Stability Verification, N-S ME4 ELF

\begin{tabular}{cccccccccc}
\hline Level, $x$ & $\begin{array}{c}V_{i} \times C_{d} \\
\text { (kips) }\end{array}$ & $\begin{array}{c}P_{x} \\
\text { (kips) }\end{array}$ & $\begin{array}{c}\Delta_{i} \\
\text { (inch) }\end{array}$ & $\begin{array}{c}h_{s x} \\
\text { (inch) }\end{array}$ & $\Delta_{i} / h_{s x}$ & $\theta_{2 i}$ & $\theta_{1 i}$ & $\beta_{i}$ & $\theta_{\text {max }, i}$ \\
\hline ROOF & 547 & 1086 & 1.22 & 168 & 0.007 & 0.014 & 0.014 & 1.00 & 0.125 \\
4 & 1027 & 2465 & 1.40 & 168 & 0.008 & 0.020 & 0.020 & 1.00 & 0.125 \\
3 & 1333 & 3851 & 1.24 & 168 & 0.007 & 0.021 & 0.021 & 1.00 & 0.125 \\
2 & 1481 & 5253 & 1.28 & 216 & 0.006 & 0.021 & 0.021 & 1.00 & 0.125 \\
\hline
\end{tabular}

\section{B.1.2.3.2 ME8 (Eccentrically Braced Frame)}

Table B-23 and Table B-24 provide the horizontal seismic forces and story shears for each archetype building design for the seismic strength analysis and the drift analysis. The data are also graphically illustrated in Figure B-13 through Figure B-15. In these tables, $F_{y}$ represents the seismic forces acting in 
the N-S direction only. The equivalent story forces for the RSA design are backed out from the story shears computed via a modal combination procedure. Although not theoretically correct, the forces provide a comparison basis for evaluating variations in the vertical distribution.

Table B-23. Seismic Strength Design Forces, N-S ME8

\begin{tabular}{ccc|ccc|cc}
\hline & \multicolumn{2}{c|}{ RSA } & \multicolumn{3}{c|}{ ELF } & \multicolumn{2}{c}{ Wind (700-Year) } \\
Level, $x$ & $F_{y}$ & $V_{i}$ & $F_{y}$ & $V_{i}$ & $V_{E L F} /$ & $F_{y}$ & $V_{i}$ \\
& (kips) & (kips) & (kips) & (kips) & $V_{R S A}$ & (kips) & (kips) \\
\hline Roof & 139 & 139 & 118 & 118 & 1.18 & 66 & 66 \\
8 & 76 & 215 & 119 & 237 & 0.91 & 68 & 133 \\
7 & 27 & 242 & 97 & 334 & 0.72 & 66 & 199 \\
6 & 21 & 264 & 75 & 409 & 0.64 & 64 & 263 \\
5 & 35 & 299 & 55 & 464 & 0.64 & 62 & 325 \\
4 & 48 & 347 & 37 & 502 & 0.69 & 59 & 385 \\
3 & 57 & 404 & 22 & 524 & 0.77 & 56 & 441 \\
2 & 45 & 449 & 9 & 533 & 0.84 & 59 & 500 \\
\hline
\end{tabular}

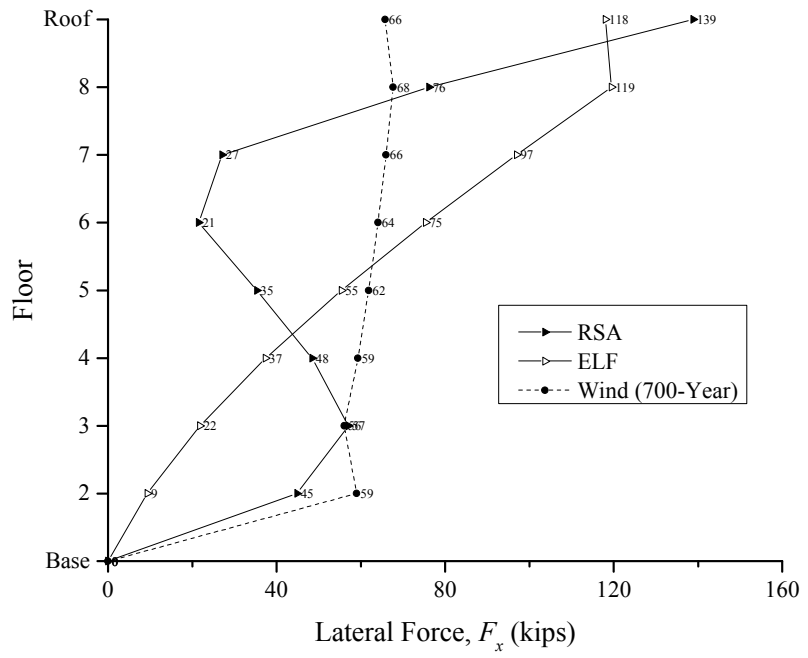

(a) Lateral forces

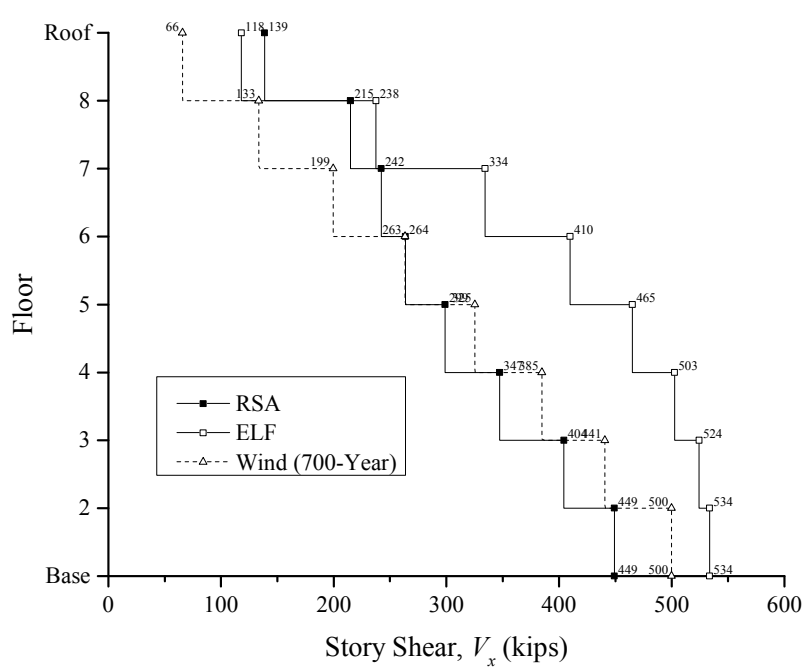

(b) Story Shears

Figure B-13. Strength Design Lateral Forces and Story Shears

Table B-24. Seismic Drift Forces, N-S ME8

\begin{tabular}{|c|c|c|c|c|c|c|c|c|c|c|c|c|}
\hline \multirow[b]{2}{*}{ Level, $x$} & \multicolumn{5}{|c|}{ RSA } & \multicolumn{5}{|c|}{ ELF } & \multicolumn{2}{|c|}{ Wind (10-Year) } \\
\hline & $\begin{array}{c}F_{y} \\
\text { (kips) }\end{array}$ & $\begin{array}{c}V_{i} \\
\text { (kips) }\end{array}$ & $\begin{array}{c}\delta_{x} \\
\text { (inch) }\end{array}$ & $\begin{array}{c}\Delta_{i} \\
\text { (inch) }\end{array}$ & $\Delta_{i} / h_{s x}$ & $\begin{array}{c}F_{y} \\
\text { (kips) }\end{array}$ & $\begin{array}{c}V_{i} \\
\text { (kips) }\end{array}$ & $\begin{array}{c}\delta_{x} \\
\text { (inch) }\end{array}$ & $\begin{array}{c}\Delta_{i} \\
\text { (inch) }\end{array}$ & $\Delta_{i} / h_{s x}$ & $\begin{array}{c}F_{y} \\
\text { (kips) }\end{array}$ & $\begin{array}{c}V_{i} \\
\text { (kips) }\end{array}$ \\
\hline Roof & 97 & 97 & 10.72 & 1.77 & 0.011 & 91 & 91 & 13.71 & 1.94 & 0.012 & 0.02 & 27 \\
\hline 8 & 53 & 151 & 9.15 & 1.97 & 0.012 & 89 & 180 & 11.77 & 2.18 & 0.013 & 0.02 & 27 \\
\hline 7 & 19 & 170 & 7.46 & 1.83 & 0.011 & 69 & 249 & 9.59 & 2.17 & 0.013 & 0.02 & 26 \\
\hline 6 & 15 & 185 & 5.88 & 1.62 & 0.010 & 50 & 299 & 7.42 & 2.00 & 0.012 & 0.02 & 26 \\
\hline 5 & 25 & 210 & 4.43 & 1.48 & 0.009 & 35 & 334 & 5.42 & 1.82 & 0.011 & 0.02 & 25 \\
\hline 4 & 34 & 244 & 3.08 & 1.25 & 0.007 & 21 & 355 & 3.60 & 1.48 & 0.009 & 0.02 & 24 \\
\hline 3 & 40 & 284 & 1.90 & 1.04 & 0.006 & 11 & 366 & 2.12 & 1.14 & 0.007 & 0.02 & 22 \\
\hline 2 & 31 & 315 & 0.89 & 0.90 & 0.004 & 4 & 370 & 0.98 & 0.98 & 0.005 & 0.02 & 24 \\
\hline
\end{tabular}




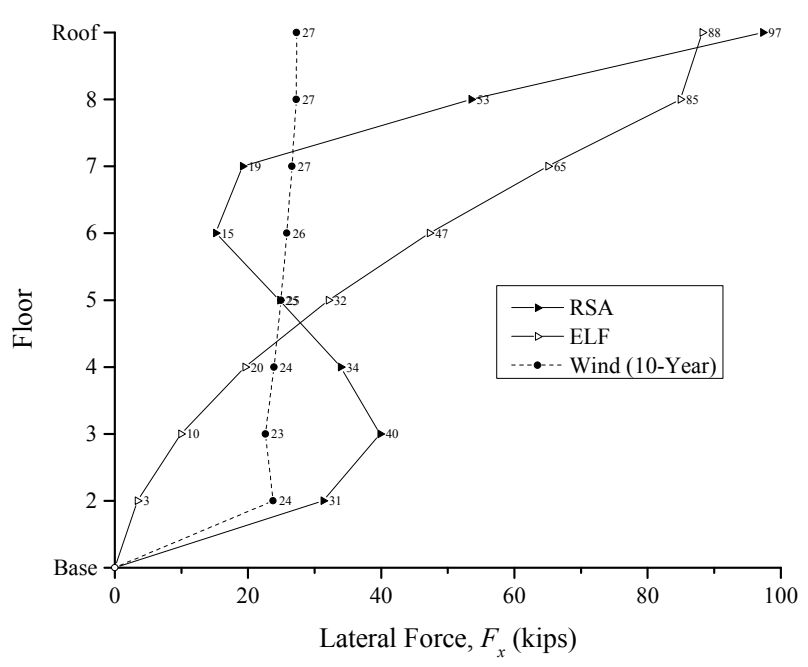

(a) Lateral forces

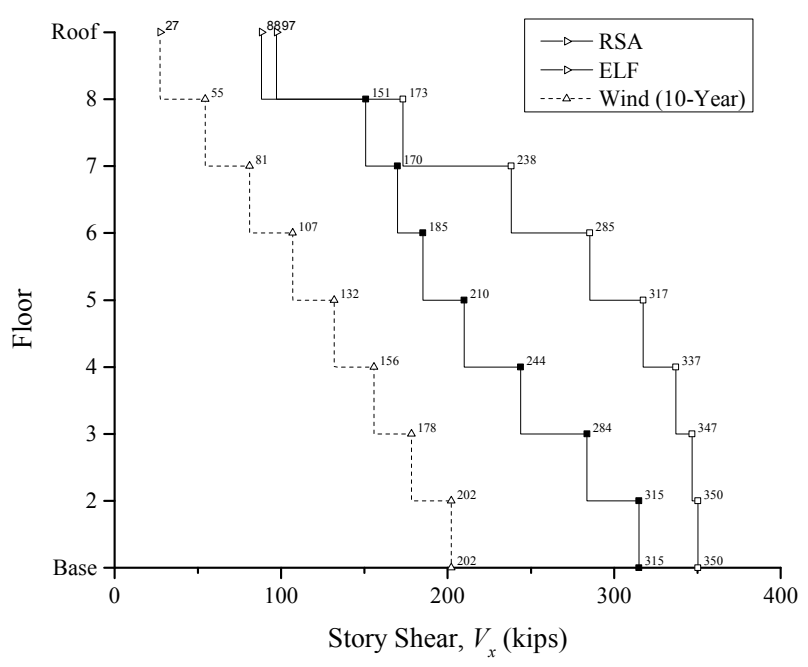

(b) Story Shears

Figure B-14. Drift Design Lateral Forces and Story Shears

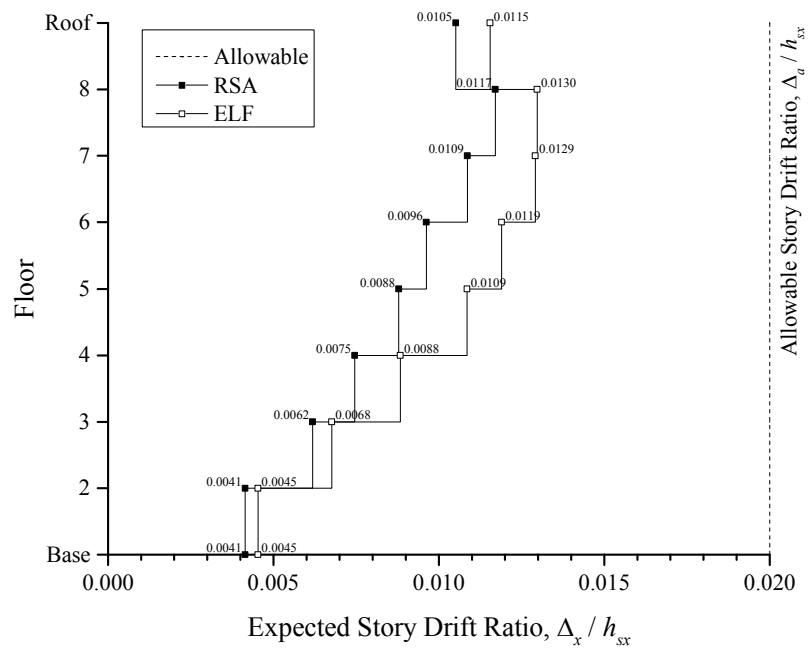

(a) Story Drift ratios at Center of Mass

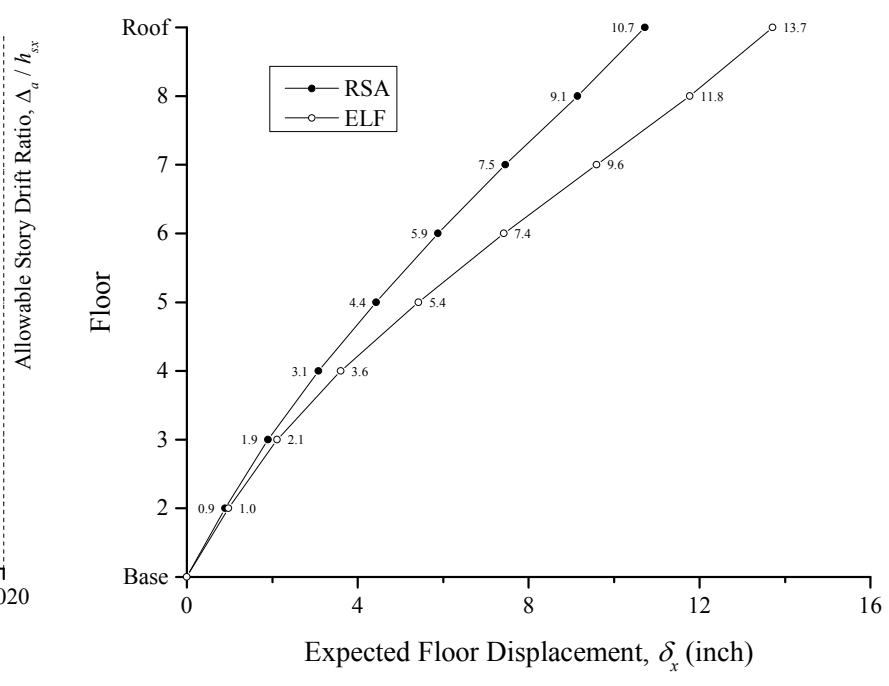

(b) Floor Displacements at Center of Mass

Figure B-15. Story Drift Ratios and Deflected Shape

Table B-25 and Table B-26 provide the tabulated data for verifying the allowable story drifts and the seismic stability coefficient per ASCE $7 \S 12.8 .6$ and ASCE $\$ 12.8 .7$ for each archetype building design. 
Table B-25. ASCE 7 Allowable Drift and Stability Verification, N-S ME8 RSA

\begin{tabular}{cccccccccc}
\hline Level, $x$ & $\begin{array}{c}V_{i} \times C_{d} \\
\text { (kips) }\end{array}$ & $\begin{array}{c}P_{x} \\
\text { (kips) }\end{array}$ & $\begin{array}{c}\Delta_{i} \\
\text { (inch) }\end{array}$ & $\begin{array}{c}h_{s x} \\
\text { (inch) }\end{array}$ & $\Delta_{i} / h_{s x}$ & $\theta_{2 i}$ & $\theta_{1 i}$ & $\beta_{i}$ & $\theta_{\max , i}$ \\
\hline ROOF & 389 & 1078 & 1.77 & 168 & 0.011 & 0.026 & 0.025 & 1.00 & 0.125 \\
8 & 603 & 2439 & 1.97 & 168 & 0.012 & 0.041 & 0.039 & 1.00 & 0.125 \\
7 & 680 & 3805 & 1.83 & 168 & 0.011 & 0.053 & 0.050 & 1.00 & 0.125 \\
6 & 740 & 5175 & 1.62 & 168 & 0.010 & 0.060 & 0.057 & 1.00 & 0.125 \\
5 & 839 & 6555 & 1.48 & 168 & 0.009 & 0.063 & 0.059 & 1.00 & 0.125 \\
4 & 975 & 7941 & 1.25 & 168 & 0.007 & 0.057 & 0.054 & 1.00 & 0.125 \\
3 & 1134 & 9333 & 1.04 & 168 & 0.006 & 0.049 & 0.047 & 1.00 & 0.125 \\
2 & 1260 & 10740 & 0.90 & 216 & 0.004 & 0.035 & 0.034 & 1.00 & 0.125 \\
\hline
\end{tabular}

Table B-26. ASCE 7 Allowable Drift and Stability Verification, N-S ME8 ELF

\begin{tabular}{cccccccccc}
\hline Level, $x$ & $\begin{array}{c}V_{i} \times C_{d} \\
\text { (kips) }\end{array}$ & $\begin{array}{c}P_{x} \\
\text { (kips) }\end{array}$ & $\begin{array}{c}\Delta_{i} \\
\text { (inch) }\end{array}$ & $\begin{array}{c}h_{s x} \\
\text { (inch) }\end{array}$ & $\Delta_{i} / h_{s x}$ & $\theta_{2 i}$ & $\theta_{1 i}$ & $\beta_{i}$ & $\theta_{\max , i}$ \\
\hline ROOF & 353 & 1081 & 1.94 & 168 & 0.012 & 0.042 & 0.040 & 1.00 & 0.125 \\
8 & 693 & 2446 & 2.18 & 168 & 0.013 & 0.052 & 0.050 & 1.00 & 0.125 \\
7 & 953 & 3825 & 2.17 & 168 & 0.013 & 0.058 & 0.055 & 1.00 & 0.125 \\
6 & 1142 & 5211 & 2.00 & 168 & 0.012 & 0.058 & 0.055 & 1.00 & 0.125 \\
5 & 1270 & 6606 & 1.82 & 168 & 0.011 & 0.060 & 0.056 & 1.00 & 0.125 \\
4 & 1348 & 8006 & 1.48 & 168 & 0.009 & 0.055 & 0.052 & 1.00 & 0.125 \\
3 & 1388 & 9411 & 1.14 & 168 & 0.007 & 0.047 & 0.045 & 1.00 & 0.125 \\
2 & 1401 & 10829 & 0.98 & 216 & 0.005 & 0.033 & 0.032 & 1.00 & 0.125 \\
\hline
\end{tabular}

\section{B.1.2.3.3 ME16 (Eccentrically Braced Frame)}

Table B-27 and Table B-28 provide the horizontal seismic forces and story shears for each archetype building design for the seismic strength analysis and the drift analysis. The data are also graphically illustrated in Figure B-16 through Figure B-18. In these tables, $F_{y}$ represents the seismic forces acting in the N-S direction only. The equivalent story forces for the RSA design are backed out from the story shears computed via a modal combination procedure. Although not theoretically correct, the forces provide a comparison basis for evaluating variations in the vertical distribution.

Table B-27. Seismic Strength Design Forces, N-S ME16

\begin{tabular}{|c|c|c|c|c|c|c|c|}
\hline \multirow[b]{2}{*}{ Level, $x$} & \multicolumn{2}{|c|}{ RSA } & \multicolumn{3}{|c|}{ ELF } & \multicolumn{2}{|c|}{ Wind (700-Year) } \\
\hline & $\begin{array}{c}F_{y} \\
\text { (kips) }\end{array}$ & $\begin{array}{c}V_{i} \\
\text { (kips) }\end{array}$ & $\begin{array}{c}F_{y} \\
\text { (kips) }\end{array}$ & $\begin{array}{c}V_{i} \\
\text { (kips) }\end{array}$ & $V_{E L F} / V_{R S A}$ & $\begin{array}{c}F_{y} \\
\text { (kips) }\end{array}$ & $\begin{array}{c}V_{i} \\
\text { (kips) }\end{array}$ \\
\hline Roof & 170 & 170 & 133 & 133 & 1.28 & 83 & 83 \\
\hline 16 & 120 & 290 & 144 & 277 & 1.04 & 89 & 172 \\
\hline 15 & 56 & 346 & 127 & 405 & 0.85 & 88 & 260 \\
\hline 14 & 20 & 365 & 111 & 516 & 0.71 & 87 & 347 \\
\hline 13 & 9 & 375 & 96 & 611 & 0.61 & 86 & 432 \\
\hline 12 & 14 & 389 & 81 & 692 & 0.56 & 85 & 517 \\
\hline 11 & 23 & 412 & 68 & 760 & 0.54 & 83 & 600 \\
\hline 10 & 30 & 442 & 55 & 815 & 0.54 & 82 & 682 \\
\hline 9 & 32 & 474 & 45 & 860 & 0.55 & 80 & 762 \\
\hline 8 & 34 & 508 & 35 & 895 & 0.57 & 79 & 841 \\
\hline 7 & 41 & 548 & 26 & 921 & 0.60 & 77 & 918 \\
\hline 6 & 50 & 598 & 19 & 939 & 0.64 & 75 & 993 \\
\hline 5 & 58 & 657 & 12 & 951 & 0.69 & 72 & 1065 \\
\hline 4 & 62 & 719 & 7 & 959 & 0.75 & 70 & 1135 \\
\hline 3 & 55 & 774 & 4 & 962 & 0.80 & 66 & 1201 \\
\hline 2 & 36 & 810 & 1 & 964 & 0.84 & 70 & 1271 \\
\hline
\end{tabular}




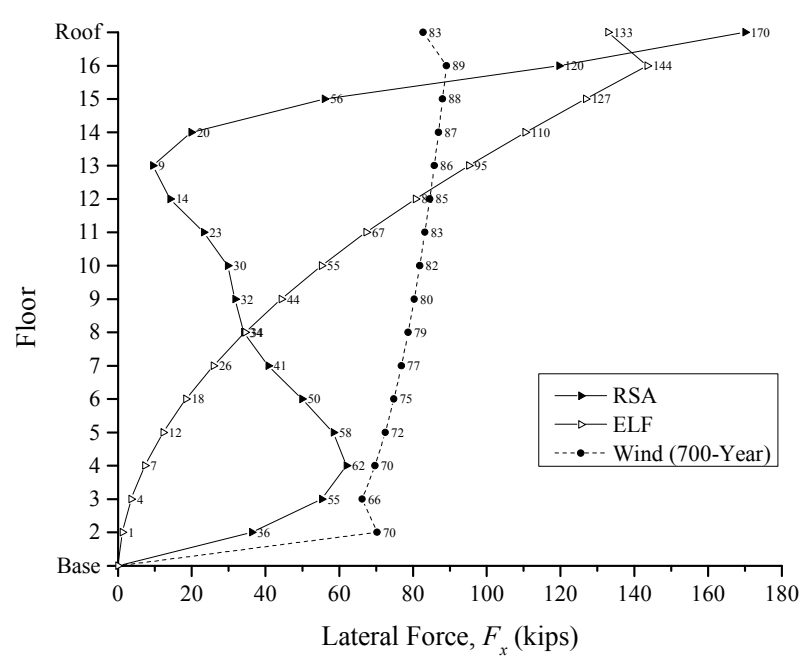

(a) Lateral forces

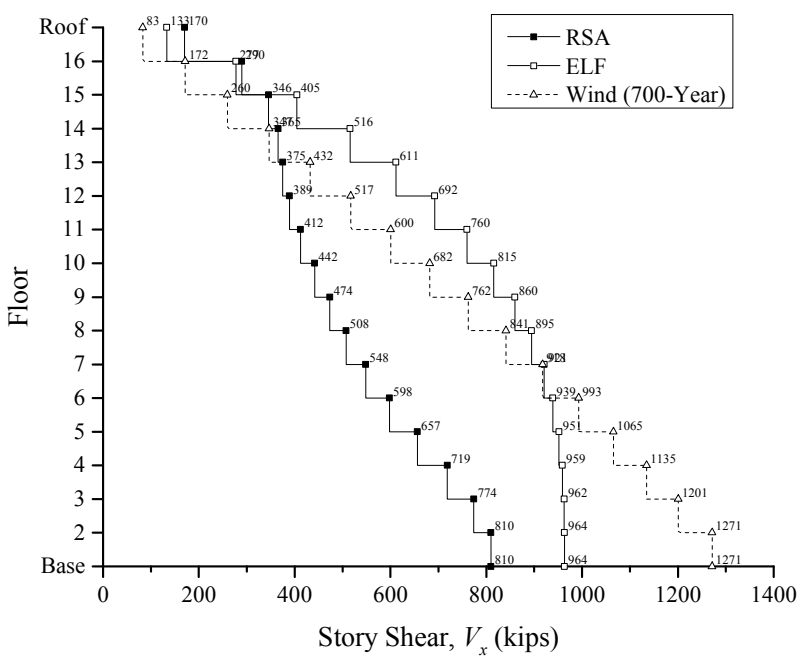

(b) Story Shears

Figure B-16. Strength Design Lateral Forces and Story Shears

Table B-28. Summary of Seismic Drift Forces, N-S ME16

\begin{tabular}{|c|c|c|c|c|c|c|c|c|c|c|c|c|}
\hline \multirow{3}{*}{ Level, $x$} & \multicolumn{5}{|c|}{ RSA } & \multicolumn{5}{|c|}{ ELF } & \multicolumn{2}{|c|}{ Wind (10-Year) } \\
\hline & $F_{y}$ & $V_{i}$ & $\delta_{x}$ & $\Delta_{i}$ & $\Delta_{i} / h_{s x}$ & $F_{y}$ & $V_{i}$ & $\delta_{x}$ & $\Delta_{i}$ & $\Delta_{i} / h_{s x}$ & $F_{y}$ & $V_{i}$ \\
\hline & (kips) & (kips) & (inch) & (inch) & & (kips) & (kips) & (inch) & (inch) & & (kips) & (kips) \\
\hline Roof & 99 & 99 & 17.56 & 1.59 & 0.009 & 70 & 70 & 22.68 & 1.69 & 0.010 & 0.02 & 34 \\
\hline 16 & 70 & 169 & 16.09 & 1.72 & 0.010 & 76 & 146 & 20.99 & 1.83 & 0.011 & 0.02 & 34 \\
\hline 15 & 33 & 202 & 14.57 & 1.77 & 0.011 & 67 & 213 & 19.15 & 1.89 & 0.011 & 0.02 & 34 \\
\hline 14 & 12 & 214 & 13.02 & 1.74 & 0.010 & 58 & 271 & 17.27 & 1.92 & 0.011 & 0.02 & 34 \\
\hline 13 & 6 & 220 & 11.50 & 1.65 & 0.010 & 50 & 322 & 15.34 & 1.88 & 0.011 & 0.02 & 33 \\
\hline 12 & 8 & 228 & 10.05 & 1.53 & 0.009 & 42 & 364 & 13.47 & 1.82 & 0.011 & 0.02 & 33 \\
\hline 11 & 14 & 242 & 8.70 & 1.39 & 0.008 & 35 & 400 & 11.65 & 1.73 & 0.010 & 0.02 & 32 \\
\hline 10 & 17 & 259 & 7.47 & 1.28 & 0.008 & 29 & 428 & 9.93 & 1.62 & 0.010 & 0.02 & 32 \\
\hline 9 & 19 & 278 & 6.32 & 1.19 & 0.007 & 23 & 452 & 8.30 & 1.52 & 0.009 & 0.02 & 31 \\
\hline 8 & 20 & 297 & 5.26 & 1.08 & 0.006 & 18 & 470 & 6.78 & 1.37 & 0.008 & 0.02 & 30 \\
\hline 7 & 24 & 321 & 4.28 & 0.98 & 0.006 & 13 & 483 & 5.41 & 1.25 & 0.007 & 0.02 & 30 \\
\hline 6 & 29 & 350 & 3.38 & 0.88 & 0.005 & 10 & 493 & 4.16 & 1.12 & 0.007 & 0.02 & 29 \\
\hline 5 & 34 & 384 & 2.56 & 0.78 & 0.005 & 6 & 499 & 3.04 & 0.99 & 0.006 & 0.02 & 28 \\
\hline 4 & 36 & 420 & 1.82 & 0.70 & 0.004 & 4 & 503 & 2.06 & 0.85 & 0.005 & 0.02 & 27 \\
\hline 3 & 32 & 453 & 1.14 & 0.61 & 0.004 & 2 & 505 & 1.21 & 0.67 & 0.004 & 0.02 & 26 \\
\hline 2 & 21 & 474 & 0.54 & 0.54 & 0.003 & 1 & 505 & 0.54 & 0.54 & 0.002 & 0.02 & 27 \\
\hline
\end{tabular}




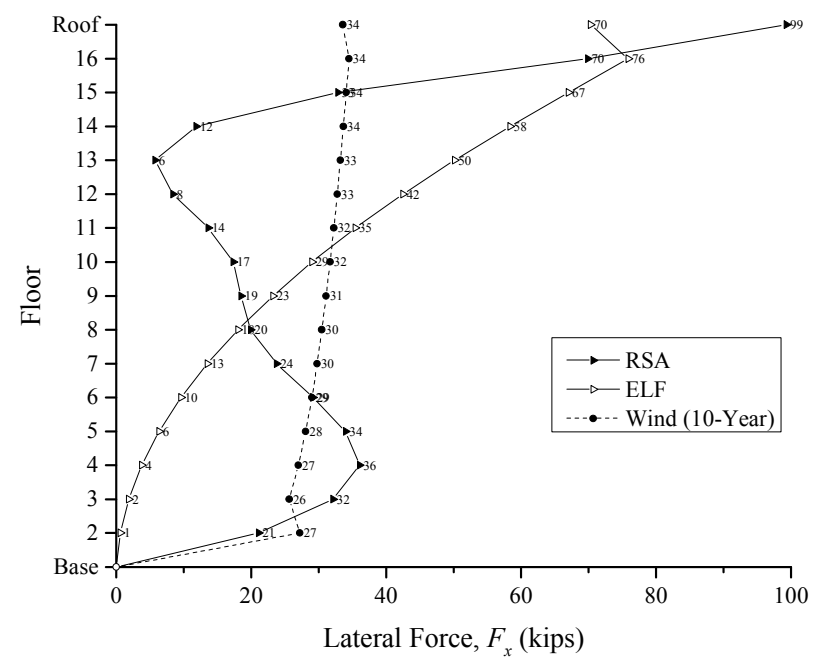

(a) Lateral forces

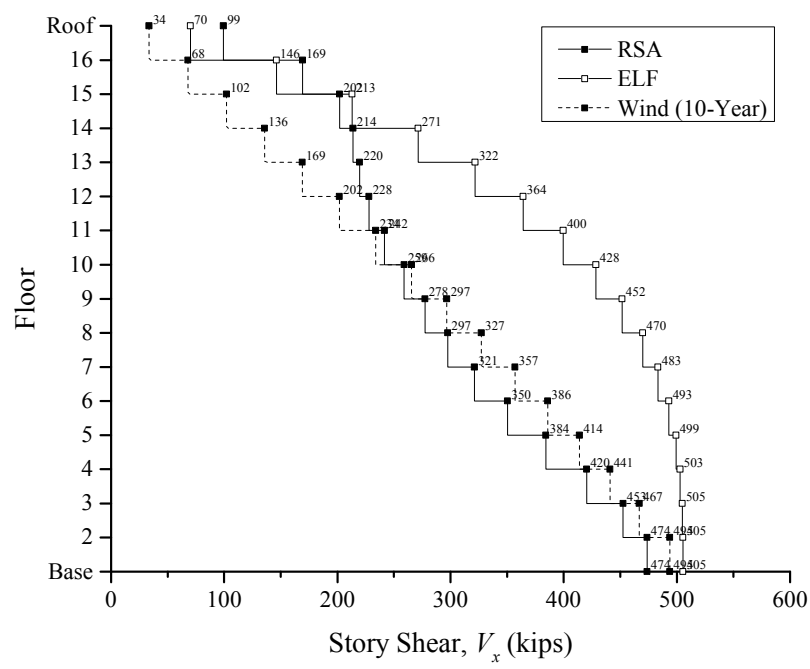

(b) Story Shears

Figure B-17. Drift Design Lateral Forces and Story Shears

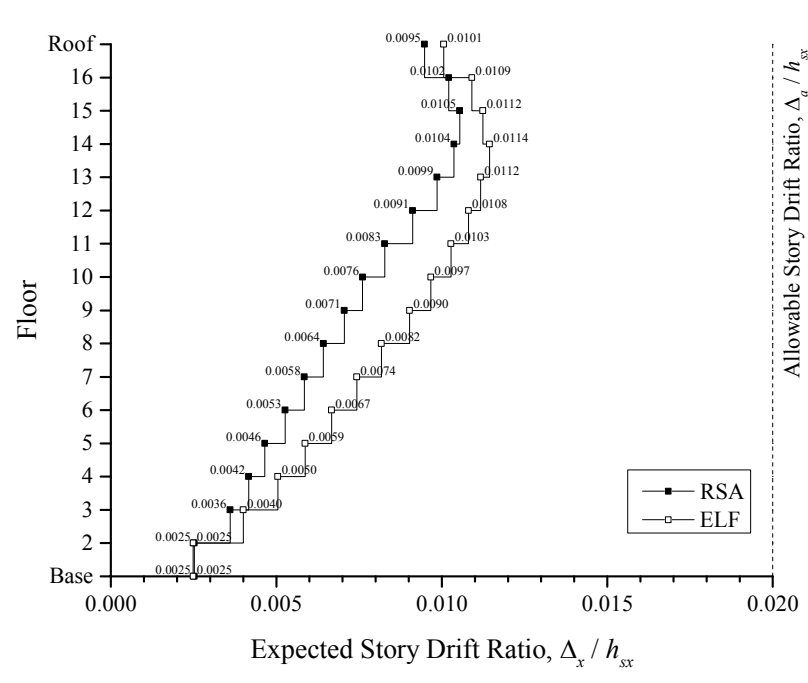

(a) Story Drift ratios at Center of Mass

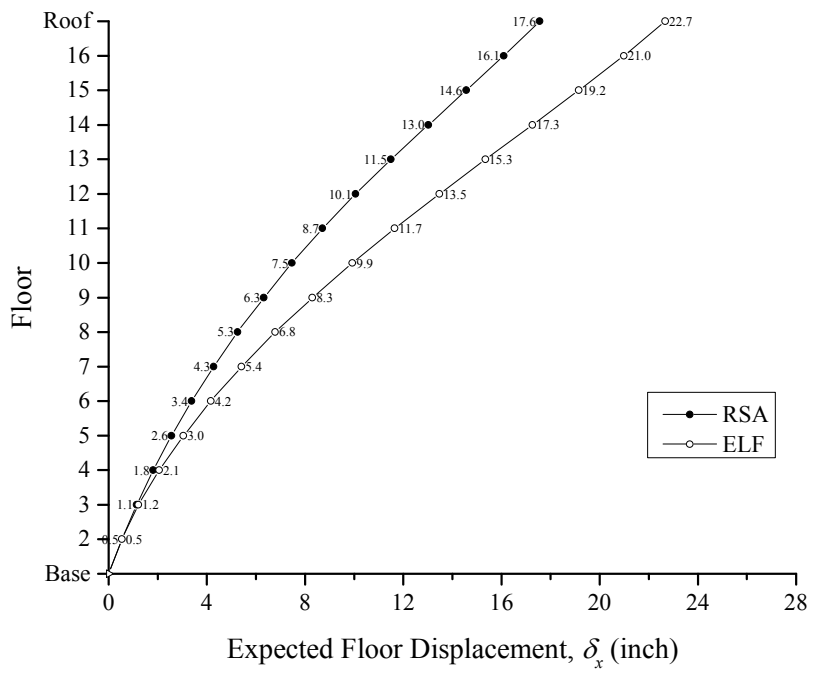

(b) Floor Displacements at Center of Mass

Figure B-18. Story Drift Ratios and Deflected Shape

Table B-29 and Table B-30 provide the tabulated data for verifying the allowable story drifts and the seismic stability coefficient per ASCE $7 \S 12.8 .6$ and $\S 12.8 .7$ for each archetype building design. 
Table B-29. ASCE 7 Allowable Drift and Stability Verification, N-S ME16 RSA

\begin{tabular}{cccccccccc}
\hline Level, $x$ & $\begin{array}{c}V_{i} \times C_{d} \\
\text { (kips) }\end{array}$ & $\begin{array}{c}P_{x} \\
(\mathrm{kips})\end{array}$ & $\begin{array}{c}\Delta_{i} \\
\text { (inch) }\end{array}$ & $\begin{array}{c}h_{s x} \\
\text { (inch) }\end{array}$ & $\Delta_{i} / h_{s x}$ & $\theta_{2 i}$ & $\theta_{1 i}$ & $\beta_{i}$ & $\theta_{\max , i}$ \\
\hline ROOF & 397 & 1084 & 1.59 & 168 & 0.009 & 0.024 & 0.023 & 1.00 & 0.125 \\
16 & 677 & 2452 & 1.72 & 168 & 0.010 & 0.033 & 0.032 & 1.00 & 0.125 \\
15 & 808 & 3823 & 1.77 & 168 & 0.011 & 0.044 & 0.042 & 1.00 & 0.125 \\
14 & 855 & 5197 & 1.74 & 168 & 0.010 & 0.055 & 0.052 & 1.00 & 0.125 \\
13 & 878 & 6585 & 1.65 & 168 & 0.010 & 0.065 & 0.061 & 1.00 & 0.125 \\
12 & 912 & 7980 & 1.53 & 168 & 0.009 & 0.070 & 0.066 & 1.00 & 0.125 \\
11 & 967 & 9379 & 1.39 & 168 & 0.008 & 0.071 & 0.067 & 1.00 & 0.125 \\
10 & 1036 & 10781 & 1.28 & 168 & 0.008 & 0.071 & 0.066 & 1.00 & 0.125 \\
9 & 1110 & 12190 & 1.19 & 168 & 0.007 & 0.070 & 0.065 & 1.00 & 0.125 \\
8 & 1190 & 13605 & 1.08 & 168 & 0.006 & 0.066 & 0.062 & 1.00 & 0.125 \\
7 & 1285 & 15024 & 0.98 & 168 & 0.006 & 0.063 & 0.059 & 1.00 & 0.125 \\
6 & 1401 & 16447 & 0.88 & 168 & 0.005 & 0.058 & 0.054 & 1.00 & 0.125 \\
5 & 1537 & 17883 & 0.78 & 168 & 0.005 & 0.051 & 0.049 & 1.00 & 0.125 \\
4 & 1682 & 19327 & 0.70 & 168 & 0.004 & 0.046 & 0.044 & 1.00 & 0.125 \\
3 & 1810 & 20779 & 0.61 & 168 & 0.004 & 0.041 & 0.039 & 1.00 & 0.125 \\
2 & 1894 & 22258 & 0.54 & 216 & 0.003 & 0.030 & 0.029 & 1.00 & 0.125 \\
\hline
\end{tabular}

Table B-30. ASCE 7 Allowable Drift and Stability Verification, N-S ME16 ELF

\begin{tabular}{cccccccccc}
\hline Level, $x$ & $\begin{array}{c}V_{i} \times C_{d} \\
\text { (kips) }\end{array}$ & $\begin{array}{c}P_{x} \\
\text { (kips) }\end{array}$ & $\begin{array}{c}\Delta_{i} \\
\text { (inch) }\end{array}$ & $\begin{array}{c}h_{s x} \\
\text { (inch) }\end{array}$ & $\Delta_{i} / h_{s x}$ & $\theta_{2 i}$ & $\theta_{1 i}$ & $\beta_{i}$ & $\theta_{\text {max }, i}$ \\
\hline ROOF & 249 & 1084 & 1.69 & 168 & 0.010 & 0.059 & 0.056 & 1.00 & 0.125 \\
16 & 517 & 2452 & 1.83 & 168 & 0.011 & 0.068 & 0.064 & 1.00 & 0.125 \\
15 & 753 & 3833 & 1.89 & 168 & 0.011 & 0.074 & 0.068 & 1.00 & 0.125 \\
14 & 959 & 5221 & 1.92 & 168 & 0.011 & 0.078 & 0.072 & 1.00 & 0.125 \\
13 & 1136 & 6617 & 1.88 & 168 & 0.011 & 0.081 & 0.075 & 1.00 & 0.125 \\
12 & 1286 & 8018 & 1.82 & 168 & 0.011 & 0.083 & 0.077 & 1.00 & 0.125 \\
11 & 1411 & 9424 & 1.73 & 168 & 0.010 & 0.084 & 0.077 & 1.00 & 0.125 \\
10 & 1513 & 10834 & 1.62 & 168 & 0.010 & 0.083 & 0.077 & 1.00 & 0.125 \\
9 & 1595 & 12252 & 1.52 & 168 & 0.009 & 0.082 & 0.076 & 1.00 & 0.125 \\
8 & 1659 & 13677 & 1.37 & 168 & 0.008 & 0.079 & 0.073 & 1.00 & 0.125 \\
7 & 1706 & 15106 & 1.25 & 168 & 0.007 & 0.076 & 0.070 & 1.00 & 0.125 \\
6 & 1740 & 16542 & 1.12 & 168 & 0.007 & 0.071 & 0.067 & 1.00 & 0.125 \\
5 & 1762 & 17985 & 0.99 & 168 & 0.006 & 0.066 & 0.062 & 1.00 & 0.125 \\
4 & 1775 & 19436 & 0.85 & 168 & 0.005 & 0.058 & 0.055 & 1.00 & 0.125 \\
3 & 1782 & 20899 & 0.67 & 168 & 0.004 & 0.047 & 0.045 & 1.00 & 0.125 \\
2 & 1784 & 22393 & 0.54 & 216 & 0.002 & 0.028 & 0.028 & 1.00 & 0.125 \\
\hline
\end{tabular}

\section{B.2 Horizontal and Vertical Irregularities}

\section{B.2.1 Special Moment Frame}

Table B-31 through Table B-34 provide the results for the horizontal (type 1) and vertical (type 1 and 5) irregularity verifications. 
Table B-31. Horizontal Irregularity Type 1 (a and b) Verification

\begin{tabular}{ccccccc}
\hline & \multicolumn{4}{c}{$\Delta_{\max } / \Delta_{\text {avg }}{ }^{1}$} & & \multicolumn{2}{c}{ ME16 } \\
Floor $(x)$ & ELF & RSA & ELF & RSA & ELF & RSA \\
\hline Roof ME16 & - & - & - & - & 1.015 & 1.012 \\
16 & - & - & - & - & 1.011 & 1.009 \\
15 & - & - & - & - & 1.012 & 1.008 \\
14 & - & - & - & - & 1.011 & 1.007 \\
13 & - & - & - & - & 1.010 & 1.008 \\
12 & - & - & - & - & 1.010 & 1.007 \\
11 & - & - & - & - & 1.009 & 1.007 \\
10 & - & - & - & - & 1.009 & 1.007 \\
9 (Roof ME8) & - & - & 1.016 & 1.011 & 1.008 & 1.007 \\
8 & - & - & 1.013 & 1.009 & 1.009 & 1.007 \\
7 & - & - & 1.012 & 1.008 & 1.008 & 1.006 \\
6 & - & - & 1.011 & 1.007 & 1.008 & 1.007 \\
5 (Roof ME4) & 1.007 & 1.006 & 1.009 & 1.006 & 1.007 & 1.006 \\
4 & 1.006 & 1.004 & 1.008 & 1.005 & 1.007 & 1.007 \\
3 & 1.005 & 1.004 & 1.008 & 1.006 & 1.011 & 1.009 \\
2 & 1.004 & 1.003 & 1.006 & 1.004 & 1.004 & 1.005 \\
\hline
\end{tabular}

Table B-32. Vertical Irregularity Type 1 (a and b) Verification (Exception 1)

\begin{tabular}{ccccccc}
\hline & \multicolumn{4}{c}{$\left(\Delta / h_{s x}\right)_{x} /\left(\Delta / h_{s x}\right)_{x+1}^{1}$} & \multicolumn{2}{c}{ ME16 } \\
Floor $(x)$ & ELF & RSA & ELF & RSA & ELF & RSA \\
\hline Roof ME16 & - & - & - & - & - & - \\
16 & - & - & - & - & 1.22 & 1.27 \\
15 & - & - & - & - & 1.07 & 1.13 \\
14 & - & - & - & - & 1.09 & 0.97 \\
13 & - & - & - & - & 1.04 & 0.89 \\
12 & - & - & - & - & 1.07 & 1.02 \\
11 & - & - & - & - & 1.04 & 1.02 \\
10 & - & - & - & - & 0.97 & 0.98 \\
9 (Roof ME8) & - & - & - & - & 0.93 & 0.95 \\
8 & - & - & 1.26 & 1.20 & 0.99 & 1.00 \\
7 & - & - & 0.99 & 1.07 & 0.99 & 0.98 \\
6 & - & - & 1.07 & 1.02 & 0.98 & 0.93 \\
5 (Roof ME4) & - & - & 1.02 & 0.95 & 0.94 & 0.88 \\
4 & 1.32 & 1.35 & 1.02 & 1.00 & 0.93 & 0.94 \\
3 & 1.11 & 1.19 & 0.95 & 0.94 & 0.83 & 0.90 \\
2 & 0.82 & 0.67 & 0.77 & 0.60 & 0.63 & 0.67 \\
\hline
\end{tabular}

$$
\begin{gathered}
V_{y, i}=\frac{2 \sum_{j=1}^{n} M_{p r, j}}{h} \\
h=\frac{h_{s x}+h_{s x+1}}{2}
\end{gathered}
$$


Table B-33. Vertical Irregularity Type 5 (a and b) Verification, ELF (kip, feet)

\begin{tabular}{|c|c|c|c|c|c|c|c|c|c|c|}
\hline \multirow[b]{2}{*}{ Floor $(x)$} & \multirow[b]{2}{*}{$h^{c}$} & \multicolumn{3}{|c|}{ MC4 } & \multicolumn{3}{|c|}{ MC8 } & \multicolumn{3}{|c|}{ MC16 } \\
\hline & & $M_{p r}{ }^{\mathrm{a}}$ & $V_{y, x} \mathrm{~b}$ & $V_{y, x+1} / V_{y, x}$ & $M_{p r}{ }^{\text {a }}$ & $V_{y, x}{ }^{\mathrm{b}}$ & $V_{y, x+1} / V_{y, x}$ & $M_{p r}{ }^{\text {a }}$ & $V_{y, x}{ }^{\mathrm{b}}$ & $V_{y, x+1} / V_{y, x}$ \\
\hline Roof MC16 & 7 & - & - & - & - & - & - & 491.1 & 421.0 & - \\
\hline 16 & 14 & - & - & - & - & - & - & 491.1 & 210.5 & 0.50 \\
\hline 15 & 14 & - & - & - & - & - & - & 951.8 & 407.9 & 1.94 \\
\hline 14 & 14 & - & - & - & - & - & - & 951.8 & 407.9 & 1.00 \\
\hline 13 & 14 & - & - & - & - & - & - & 1242.1 & 532.3 & 1.31 \\
\hline 12 & 14 & - & - & - & - & - & - & 1242.1 & 532.3 & 1.00 \\
\hline 11 & 14 & - & - & - & - & - & - & 1242.1 & 532.3 & 1.00 \\
\hline 10 & 14 & - & - & - & - & - & - & 1242.1 & 532.3 & 1.00 \\
\hline 9 (Roof MC8) & $14 / 7$ & - & - & - & 491.1 & 421.0 & - & 1662.3 & 712.4 & 1.34 \\
\hline 8 & 14 & - & - & - & 491.1 & 210.5 & 0.50 & 1662.3 & 712.4 & 1.00 \\
\hline 7 & 14 & - & - & - & 951.8 & 407.9 & 1.94 & 1662.3 & 712.4 & 1.00 \\
\hline 6 & 14 & - & - & - & 951.8 & 407.9 & 1.00 & 1662.3 & 712.4 & 1.00 \\
\hline 5 (Roof MC4) & $14 / 7$ & 491.1 & 421.0 & - & 1161.6 & 497.8 & 1.22 & 1662.3 & 712.4 & 1.00 \\
\hline 4 & 14 & 679.7 & 291.3 & 0.69 & 1161.6 & 497.8 & 1.00 & 1662.3 & 712.4 & 1.00 \\
\hline 3 & 14 & 754.6 & 323.4 & 1.11 & 1161.6 & 497.8 & 1.00 & 1662.3 & 712.4 & 1.00 \\
\hline 2 & 16 & 754.6 & 283.0 & 0.88 & 1161.6 & 435.6 & 0.88 & 1662.3 & 623.3 & 0.88 \\
\hline $\begin{array}{l}M_{p r} \text { is } \\
V_{y x} \text { is } \\
h \text { is ta }\end{array}$ & $\begin{array}{l}\text { uted } \mathrm{r} \\
\text { s the }\end{array}$ & $\begin{array}{l}\text { m plastic } 1 \\
\text { cting the } p \\
\text { nce from } n\end{array}$ & $\begin{array}{l}\text { nge at the } \\
\text { istic mom } \\
\text { dheight of }\end{array}$ & $\begin{array}{l}\text { er of the RBS. } \\
\text { trength of the } \\
\text { ory to mid-heis }\end{array}$ & the sto & ove $\left(\neq h_{\mathrm{xx}}\right)$ & & & & \\
\hline
\end{tabular}

Table B-34. Vertical Irregularity Type 5 (a and b) Verification, RSA

\begin{tabular}{|c|c|c|c|c|c|c|c|c|c|c|}
\hline \multirow[b]{2}{*}{ Floor $(x)$} & \multirow[b]{2}{*}{$h^{c}$} & \multicolumn{3}{|c|}{ MC4 } & \multicolumn{3}{|c|}{ MC8 } & \multicolumn{3}{|c|}{ MC16 } \\
\hline & & $M_{p r}{ }^{a}$ & $V_{y, x}{ }^{\mathrm{b}}$ & $V_{y, x+1} / V_{y, x}$ & $M_{p r}{ }^{\mathrm{a}}$ & $V_{y, x}{ }^{\mathrm{b}}$ & $V_{y, x+1} / V_{y, x}$ & $M_{p r}{ }^{\mathrm{a}}$ & $V_{y, x} \mathrm{~b}$ & $V_{y, x+1} / V_{y, x}$ \\
\hline Roof MC16 & 7 & - & - & - & - & - & - & 491.1 & 421.0 & - \\
\hline 16 & 14 & - & - & - & - & - & - & 491.1 & 210.5 & 0.50 \\
\hline 15 & 14 & - & - & - & - & - & - & 491.1 & 210.5 & 1.00 \\
\hline 14 & 14 & - & - & - & - & - & - & 491.1 & 210.5 & 1.00 \\
\hline 13 & 14 & - & - & - & - & - & - & 951.8 & 407.9 & 1.94 \\
\hline 12 & 14 & - & - & - & - & - & - & 951.8 & 407.9 & 1.00 \\
\hline 11 & 14 & - & - & - & - & - & - & 951.8 & 407.9 & 1.00 \\
\hline 10 & 14 & - & - & - & - & - & - & 951.8 & 407.9 & 1.00 \\
\hline 9 (Roof MC8) & $14 / 7$ & - & - & - & 358.7 & 307.5 & - & 1242.1 & 532.3 & 1.31 \\
\hline 8 & 14 & - & - & - & 358.7 & 153.7 & 0.50 & 1242.1 & 532.3 & 1.00 \\
\hline 7 & 14 & - & - & - & 491.1 & 210.5 & 1.37 & 1242.1 & 532.3 & 1.00 \\
\hline 6 & 14 & - & - & - & 491.1 & 210.5 & 1.00 & 1242.1 & 532.3 & 1.00 \\
\hline 5 (Roof MC4) & $14 / 7$ & 491.1 & 421.0 & - & 679.7 & 291.3 & 1.38 & 1662.3 & 712.4 & 1.34 \\
\hline 4 & 14 & 491.1 & 210.5 & 0.50 & 679.7 & 291.3 & 1.00 & 1662.3 & 712.4 & 1.00 \\
\hline 3 & 14 & 491.1 & 210.5 & 1.00 & 754.6 & 323.4 & 1.11 & 1662.3 & 712.4 & 1.00 \\
\hline 2 & 16 & 491.1 & 184.2 & 0.88 & 754.6 & 283.0 & 0.88 & 1662.3 & 623.3 & 0.88 \\
\hline $\begin{array}{l}M_{p r} \\
V_{y x} \text {. } \\
h \text { is }\end{array}$ & $\begin{array}{l}\text { single } \\
\text { suted } n\end{array}$ & $\begin{array}{l}\text { h plas } \\
\text { ting t }\end{array}$ & tic mon & $\begin{array}{l}\text { er of the RB } \\
\text { trength of th }\end{array}$ & & 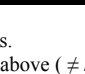 & & & & \\
\hline
\end{tabular}

\section{B.2.2 Eccentrically Braced Frame}

Table B-35 through Table B-34 provide the results for the horizontal (type 1) and vertical (type 1 and 5) irregularity verifications. 
Table B-35. Horizontal Irregularity Type 1 (a and b) Verification

\begin{tabular}{ccccccc}
\hline & \multicolumn{4}{c}{$\Delta_{\max } / \Delta_{\text {avg }}{ }^{\mathrm{a}}$} & \multicolumn{2}{c}{ ME16 } \\
Floor $(x)$ & ELF & RSA & ELF & RSA & ELF & RSA \\
\hline Roof ME16 & - & - & - & - & 1.073 & 1.069 \\
16 & - & - & - & - & 1.066 & 1.066 \\
15 & - & - & - & - & 1.065 & 1.067 \\
14 & - & - & - & - & 1.070 & 1.066 \\
13 & - & - & - & - & 1.070 & 1.067 \\
12 & - & - & - & - & 1.069 & 1.068 \\
11 & - & - & - & - & 1.072 & 1.070 \\
10 & - & - & - & - & 1.071 & 1.068 \\
$9($ Roof & & & & & & \\
ME8) & - & - & 1.070 & 1.073 & 1.075 & 1.071 \\
8 & - & - & 1.067 & 1.073 & 1.070 & 1.069 \\
7 & - & - & 1.067 & 1.073 & 1.074 & 1.071 \\
6 & - & - & 1.067 & 1.074 & 1.074 & 1.073 \\
$5($ Roof & & & & & & \\
ME4) & 1.054 & 1.064 & 1.072 & 1.075 & 1.076 & 1.072 \\
4 & 1.077 & 1.075 & 1.070 & 1.073 & 1.075 & 1.068 \\
3 & 1.072 & 1.079 & 1.084 & 1.087 & 1.129 & 1.100 \\
2 & 1.080 & 1.081 & 1.087 & 1.090 & 1.161 & 1.097 \\
\hline a. Values include accidental torsion with $A_{x}=1.0$ & & &
\end{tabular}

Table B-36. Vertical Irregularity Type 1 (a and b) Verification (Exception 1)

\begin{tabular}{ccccccc}
\hline & \multicolumn{4}{c}{$\left(\Delta / h_{s x}\right)_{x} /\left(\Delta / h_{s x}\right)_{x+1}{ }^{\mathrm{a}}$} & \multicolumn{2}{c}{ ME16 } \\
Floor $(x)$ & ELF & RSA & ELF & RSA & ELF & RSA \\
\hline Roof ME16 & - & - & - & - & - & - \\
16 & - & - & - & - & 1.08 & 1.08 \\
15 & - & - & - & - & 1.03 & 1.03 \\
14 & - & - & - & - & 1.02 & 0.98 \\
13 & - & - & - & - & 0.98 & 0.95 \\
12 & - & - & - & - & 0.97 & 0.93 \\
11 & - & - & - & - & 0.95 & 0.91 \\
10 & - & - & - & - & 0.94 & 0.92 \\
9 (Roof ME8) & - & - & - & - & 0.93 & 0.93 \\
8 & - & - & 1.12 & 1.11 & 0.91 & 0.91 \\
7 & - & - & 1.00 & 0.93 & 0.91 & 0.91 \\
6 & - & - & 0.92 & 0.89 & 0.90 & 0.90 \\
5 (Roof ME4) & - & - & 0.91 & 0.91 & 0.88 & 0.88 \\
4 & 1.15 & 1.13 & 0.81 & 0.85 & 0.86 & 0.90 \\
3 & 0.89 & 0.96 & 0.77 & 0.83 & 0.79 & 0.87 \\
2 & 0.80 & 0.68 & 0.67 & 0.52 & 0.62 & 0.70 \\
\hline a. $\Delta$ is taken at the center of mass per ASCE 7 \$12.8.6. & & & & &
\end{tabular}

$$
V_{y, i}=V_{n, \operatorname{link}} \frac{b}{h_{s x}}
$$

$[b=20 \mathrm{ft}$. for ME4 and ME8, $b=30 \mathrm{ft}$. for ME16] 
Table B-37. Vertical Irregularity Type 5 (a and b) Verification, ELF (kip, feet)

\begin{tabular}{|c|c|c|c|c|c|c|c|c|c|c|}
\hline \multirow[b]{2}{*}{ Floor $(x)$} & \multirow[b]{2}{*}{$h_{s x}$} & \multicolumn{3}{|c|}{ ME4 } & \multicolumn{3}{|c|}{ ME8 } & \multicolumn{3}{|c|}{ ME16 } \\
\hline & & $V_{n}$ & $V_{y, x}{ }^{\mathrm{a}}$ & $V_{y, x+1} / V_{y, x}$ & $V_{n}$ & $V_{y, x}{ }^{\mathrm{a}}$ & $V_{y, x+1} / V_{y, x}$ & $V_{n}$ & $V_{y, x}{ }^{\mathrm{a}}$ & $V_{y, x+1} / V_{y, x}$ \\
\hline Roof ME16 & 14 & - & - & - & - & - & & 128.6 & 275.6 & - \\
\hline 16 & 14 & - & - & - & - & - & & 128.6 & 275.6 & 1.00 \\
\hline 15 & 14 & - & - & - & - & - & & 159.2 & 341.2 & 1.24 \\
\hline 14 & 14 & - & - & - & - & - & & 159.2 & 341.2 & 1.00 \\
\hline 13 & 14 & - & - & - & - & - & & 197.0 & 422.2 & 1.24 \\
\hline 12 & 14 & - & - & - & - & - & & 209.3 & 448.5 & 1.06 \\
\hline 11 & 14 & - & - & - & - & - & & 240.6 & 515.5 & 1.15 \\
\hline 10 & 14 & - & - & - & - & - & & 254.5 & 545.4 & 1.06 \\
\hline 9 (Roof ME8) & 14 & - & - & - & 99.9 & 142.7 & - & 269.2 & 576.8 & 1.06 \\
\hline 8 & 14 & - & - & - & 121.6 & 173.6 & 1.22 & 304.8 & 653.2 & 1.13 \\
\hline 7 & 14 & - & - & - & 154.9 & 221.3 & 1.27 & 304.8 & 653.2 & 1.00 \\
\hline 6 & 14 & - & - & - & 197.0 & 281.5 & 1.27 & 304.8 & 653.2 & 1.00 \\
\hline 5 (Roof ME4) & 14 & 99.9 & 142.7 & - & 209.3 & 299.0 & 1.06 & 304.8 & 653.2 & 1.00 \\
\hline 4 & 14 & 121.6 & 173.6 & 1.22 & 240.6 & 343.7 & 1.15 & 304.8 & 653.2 & 1.00 \\
\hline 3 & 14 & 171.5 & 244.9 & 1.41 & 240.6 & 343.7 & 1.00 & 304.8 & 653.2 & 1.00 \\
\hline 2 & 18 & 237.0 & 263.4 & 1.08 & 304.8 & 338.7 & 0.99 & 371.9 & 619.9 & 0.95 \\
\hline
\end{tabular}

Table B-38. Vertical Irregularity Type 5 (a and b) Verification, RSA (kip, feet)

\begin{tabular}{|c|c|c|c|c|c|c|c|c|c|c|}
\hline \multirow[b]{2}{*}{ Floor $(x)$} & \multirow[b]{2}{*}{$h_{s x}$} & \multicolumn{3}{|c|}{ ME4 } & \multicolumn{3}{|c|}{ ME8 } & \multicolumn{3}{|c|}{ ME16 } \\
\hline & & $V_{n}$ & $V_{y, x}{ }^{\mathrm{a}}$ & $V_{y, x+1} / V_{y, x}$ & $V_{n}$ & $V_{y, x}{ }^{\mathrm{a}}$ & $V_{y, x+1} / V_{y, x}$ & $V_{n}$ & $V_{y, x}{ }^{\mathrm{a}}$ & $V_{y, x+1} / V_{y, x}$ \\
\hline Roof ME16 & 14 & - & - & - & - & - & & 121.6 & 260.5 & - \\
\hline 16 & 14 & - & - & - & - & - & & 121.6 & 260.5 & 1.00 \\
\hline 15 & 14 & - & - & - & - & - & & 121.6 & 260.5 & 1.00 \\
\hline 14 & 14 & - & - & - & - & - & & 121.6 & 260.5 & 1.00 \\
\hline 13 & 14 & - & - & - & - & - & & 121.6 & 260.5 & 1.00 \\
\hline 12 & 14 & - & - & - & - & - & & 154.9 & 332.0 & 1.27 \\
\hline 11 & 14 & - & - & - & - & - & & 179.6 & 384.8 & 1.16 \\
\hline 10 & 14 & - & - & - & - & - & & 197.0 & 422.2 & 1.10 \\
\hline 9 (Roof ME8) & 14 & - & - & - & 99.9 & 142.7 & - & 224.9 & 482.0 & 1.14 \\
\hline 8 & 14 & - & - & - & 99.9 & 142.7 & 1.00 & 237.2 & 508.4 & 1.05 \\
\hline 7 & 14 & - & - & - & 123.9 & 177.0 & 1.24 & 254.5 & 545.4 & 1.07 \\
\hline 6 & 14 & - & - & - & 137.2 & 195.9 & 1.11 & 254.5 & 545.4 & 1.00 \\
\hline 5 (Roof ME4) & 14 & 99.9 & 142.7 & - & 159.2 & 227.5 & 1.16 & 269.2 & 576.8 & 1.06 \\
\hline 4 & 14 & 121.6 & 173.6 & 1.22 & 182.4 & 260.6 & 1.15 & 269.2 & 576.8 & 1.00 \\
\hline 3 & 14 & 154.9 & 221.3 & 1.27 & 197.0 & 281.5 & 1.08 & 269.2 & 576.8 & 1.00 \\
\hline 2 & 18 & 204.5 & 227.2 & 1.03 & 304.8 & 338.7 & 1.20 & 371.9 & 619.9 & 1.07 \\
\hline
\end{tabular}

\section{B.3 SMF AISC Frame Stability (Effective Length Method)}

Table B-39 through Table B-44 provide the results from AISC 360, appendix 7 Effective Length Method verifications. 
Table B-39. AISC 360 Frame Stability (Effective Length Method), 4-Story SMF ELF (kip, inch)

\begin{tabular}{ccccccc}
\hline$P_{\text {story }}{ }^{\mathrm{a}}$ & $\Delta_{H}{ }^{\mathrm{b}}$ & $H$ & $L$ & $R_{m}$ & $P_{\text {estory }}$ & $B_{2}$ \\
\hline 1314 & 2.24 & 499.9 & 168 & 0.954 & 35716 & 1.04 \\
2936 & 2.92 & 897.8 & 168 & 0.954 & 49223 & 1.06 \\
4574 & 3.21 & 1120.0 & 168 & 0.954 & 55854 & 1.09 \\
6235 & 3.38 & 1208.0 & 216 & 0.954 & 73615 & 1.09 \\
\hline $1 . P_{\text {story }}$ is computed from $1.2 \times$ Dead $+0.25 \times$ Floor Live. & & &
\end{tabular}

2. $\Delta_{H}$ and $H$ are determined from a first-order static analysis using ASCE $7 \S 12.8 .6$.

Table B-40. AISC 360 Frame Stability (Effective Length Method), 4-Story SMF RSA (kip, inch)

\begin{tabular}{ccccccc}
\hline$P_{\text {story }} \mathrm{a}^{\mathrm{a}}$ & $\Delta_{H}{ }^{\mathrm{b}}$ & $H$ & $L$ & $R_{m}$ & $P_{\text {estory }}$ & $B_{2}$ \\
\hline 1309 & 2.51 & 435.5 & 168 & 0.954 & 27764 & 1.05 \\
2925 & 3.50 & 765.8 & 168 & 0.954 & 35055 & 1.09 \\
4547 & 3.97 & 938.0 & 168 & 0.954 & 37806 & 1.14 \\
6192 & 4.13 & 999.6 & 216 & 0.954 & 49870 & 1.14 \\
\hline
\end{tabular}

2. $\Delta_{H}$ and $H$ are determined from a first-order static analysis using ASCE $7 \$ 12.8 .6$

Table B-41. AISC 360 Frame Stability (Effective Length Method), 8-Story SMF ELF (kip, inch)

\begin{tabular}{ccccccc}
\hline$P_{\text {story }}{ }^{\mathrm{a}}$ & $\Delta_{H}{ }^{\mathrm{b}}$ & $H$ & $L$ & $R_{m}$ & $P_{\text {estory }}$ & $B_{2}$ \\
\hline 1300 & 2.15 & 392.5 & 168 & 0.954 & 29312 & 1.05 \\
2901 & 2.68 & 764.5 & 168 & 0.954 & 45678 & 1.07 \\
4525 & 2.64 & 1045.2 & 168 & 0.954 & 63361 & 1.08 \\
6158 & 2.79 & 1244.9 & 168 & 0.954 & 71414 & 1.09 \\
7803 & 2.81 & 1377.1 & 168 & 0.954 & 78453 & 1.11 \\
9452 & 2.83 & 1455.0 & 168 & 0.954 & 82282 & 1.13 \\
11115 & 2.68 & 1493.0 & 168 & 0.954 & 89151 & 1.14 \\
12806 & 2.66 & 1505.3 & 216 & 0.954 & 116595 & 1.12 \\
\hline $1 . P_{\text {story }}$ is computed from 1.2 $\times$ Dead $+0.25 \times$ Floor Live. \\
2. $\Delta_{H}$ and $H$ are determined from a first-order static analysis using ASCE 7 $\S 12.8 .6$. &
\end{tabular}

Table B-42. AISC 360 Frame Stability (Effective Length Method), 8-Story SMF RSA (kip, inch)

\begin{tabular}{ccccccc}
\hline$P_{\text {story }}{ }^{\mathrm{a}}$ & $\Delta_{H}{ }^{\mathrm{b}}$ & $H$ & $L$ & $R_{m}$ & $P_{\text {estory }}$ & $B_{2}$ \\
\hline 1297 & 5.76 & 665.9 & 168 & 0.954 & 18506 & 1.08 \\
2893 & 7.62 & 1297.1 & 168 & 0.954 & 27284 & 1.12 \\
4501 & 8.56 & 1770.7 & 168 & 0.954 & 33134 & 1.16 \\
6114 & 8.74 & 2107.1 & 168 & 0.954 & 38612 & 1.19 \\
7741 & 8.11 & 2330.3 & 168 & 0.954 & 46054 & 1.20 \\
9373 & 7.72 & 2462.1 & 168 & 0.954 & 51065 & 1.22 \\
11021 & 6.83 & 2526.7 & 168 & 0.954 & 59299 & 1.23 \\
12697 & 6.44 & 2547.5 & 216 & 0.954 & 81474 & 1.18 \\
\hline $1 . P_{\text {story }}$ is computed from 1.2×Dead $+0.25 \times$ Floor Live.
\end{tabular}

2. $\Delta_{H}$ and $H$ are determined from a first-order static analysis using ASCE $7 \$ 12.8 .6$. 
Table B-43. AISC 360 Frame Stability (Effective Length Method), 16-Story SMF ELF (kip, inch)

\begin{tabular}{ccccccc}
\hline$P_{\text {story }}{ }^{\mathrm{a}}$ & $\Delta_{H}{ }^{\mathrm{b}}$ & $H$ & $L$ & $R_{m}$ & $P_{\text {estory }}$ & $B_{2}$ \\
\hline 1297 & 1.62 & 278.1 & 168 & 0.954 & 27566 & 1.05 \\
2895 & 1.97 & 578.3 & 168 & 0.954 & 47016 & 1.07 \\
4509 & 2.09 & 843.4 & 168 & 0.954 & 64592 & 1.08 \\
6133 & 2.26 & 1073.9 & 168 & 0.954 & 76131 & 1.09 \\
7766 & 2.34 & 1272.1 & 168 & 0.954 & 86953 & 1.10 \\
9403 & 2.49 & 1439.8 & 168 & 0.954 & 92517 & 1.11 \\
11046 & 2.57 & 1579.7 & 168 & 0.954 & 98355 & 1.13 \\
12695 & 2.50 & 1694.0 & 168 & 0.954 & 108744 & 1.13 \\
14355 & 2.31 & 1785.7 & 168 & 0.954 & 123781 & 1.13 \\
16020 & 2.27 & 1856.8 & 168 & 0.954 & 131214 & 1.14 \\
17691 & 2.24 & 1909.9 & 168 & 0.954 & 136851 & 1.15 \\
19369 & 2.18 & 1947.6 & 168 & 0.954 & 143441 & 1.16 \\
21056 & 2.05 & 1972.5 & 168 & 0.954 & 154360 & 1.16 \\
22754 & 1.91 & 1987.2 & 168 & 0.954 & 167096 & 1.16 \\
24469 & 1.60 & 1994.5 & 168 & 0.954 & 199929 & 1.14 \\
26227 & 1.32 & 1996.8 & 216 & 0.954 & 311432 & 1.09 \\
\hline $1 . P 3$
\end{tabular}

2. $\Delta_{H}$ and $H$ are determined from a first-order static analysis using ASCE $7 \S 12.8 .6$.

Table B-44. AISC 360 Frame Stability (Effective Length Method), 16-Story SMF RSA (kip, inch)

\begin{tabular}{ccccccc}
\hline$P_{\text {story }}{ }^{\mathrm{a}}$ & $\Delta_{H}{ }^{\mathrm{b}}$ & $H$ & $L$ & $R_{m}$ & $P_{\text {estory }}$ & $B_{2}$ \\
\hline 1296 & 2.83 & 390.9 & 168 & 0.954 & 22100 & 1.06 \\
2893 & 3.88 & 812.9 & 168 & 0.954 & 33576 & 1.09 \\
4496 & 4.75 & 1182.8 & 168 & 0.954 & 39868 & 1.13 \\
6105 & 4.89 & 1503.8 & 168 & 0.954 & 49305 & 1.14 \\
7728 & 4.56 & 1781.0 & 168 & 0.954 & 62558 & 1.14 \\
9358 & 4.71 & 2015.7 & 168 & 0.954 & 68592 & 1.16 \\
10993 & 4.83 & 2211.3 & 168 & 0.954 & 73302 & 1.18 \\
12634 & 4.73 & 2371.3 & 168 & 0.954 & 80383 & 1.19 \\
14282 & 4.45 & 2499.2 & 168 & 0.954 & 90046 & 1.19 \\
15936 & 4.34 & 2598.5 & 168 & 0.954 & 95899 & 1.20 \\
17595 & 4.17 & 2672.7 & 168 & 0.954 & 102632 & 1.21 \\
19260 & 3.78 & 2725.3 & 168 & 0.954 & 115496 & 1.20 \\
20939 & 3.25 & 2760.2 & 168 & 0.954 & 136170 & 1.18 \\
22627 & 2.94 & 2780.8 & 168 & 0.954 & 151337 & 1.18 \\
24328 & 2.50 & 2790.8 & 168 & 0.954 & 178505 & 1.16 \\
26067 & 2.16 & 2794.1 & 216 & 0.954 & 267012 & 1.11 \\
\hline $1 . P$ &
\end{tabular}

2. $\Delta H$ and $H$ are determined from a first-order static analysis using ASCE $7 \$ 12.8 .6$

Table B-45. Adjusted Effective Length Factors - 4-Story SMF

\begin{tabular}{ccc||cc}
\hline \multirow{2}{*}{ Story } & \multicolumn{2}{c||}{ ELF } & \multicolumn{2}{c}{ RSA } \\
& $K_{2 x \text { (note a) }}$ & $K_{2 x \text { (note a) }}$ & $K_{2 x \text { (note a) }}$ & $K_{2 x \text { (note a) }}$ \\
& Exterior & Interior & Exterior & Interior \\
\hline 4 & 2.23 & 2.98 & 2.46 & 3.08 \\
3 & 2.44 & 3.26 & 2.99 & 3.74 \\
2 & 2.54 & 3.37 & 3.08 & 3.86 \\
1 & 2.42 & 3.21 & 2.58 & 3.23 \\
\hline a. & $x$ designates in-plane buckling about $x$-axis as depicted in AISC 360 Part 1.
\end{tabular}


Table B-46. Adjusted Effective Length Factors - 8-Story SMF

\begin{tabular}{ccc||cc}
\hline \multirow{2}{*}{ Story } & \multicolumn{2}{c||}{ ELF } & \multicolumn{2}{c}{ RSA } \\
& $K_{2 x \text { (note a) }}$ & $\begin{array}{c}K_{2 x \text { (note a) }} \\
\text { Exterior }\end{array}$ & $\begin{array}{c}K_{2 x \text { (note a) }} \\
\text { Interior }\end{array}$ & $\begin{array}{c}K_{2 x \text { (note a) }} \\
\text { Exterior }\end{array}$ \\
\hline 8 & 2.18 & 2.75 & 2.59 & 2.74 \\
7 & 2.12 & 2.67 & 2.61 & 2.76 \\
6 & 2.09 & 2.80 & 2.26 & 3.21 \\
5 & 2.04 & 2.74 & 2.16 & 3.08 \\
4 & 2.04 & 2.88 & 2.20 & 3.38 \\
3 & 1.99 & 2.81 & 2.27 & 3.48 \\
2 & 2.09 & 3.21 & 2.44 & 3.82 \\
1 & 2.03 & 3.11 & 2.24 & 3.51 \\
\hline a. $x$ designates in-plane buckling about $x$-axis as depicted in AISC 360 Part 1.
\end{tabular}

Table B-47. Adjusted Effective Length Factors - 16-Story SMF

\begin{tabular}{ccc||cc}
\hline \multicolumn{1}{c||}{} & \multicolumn{2}{c||}{ ELF } & \multicolumn{2}{c}{ RSA } \\
Story & $K_{2 x \text { (note a) }}$ & $K_{2 x \text { (note a) }}$ & $K_{2 x \text { (note a) }}$ & $K_{2 x \text { (note a) }}$ \\
& Exterior & Interior & Exterior & Interior \\
\hline 16 & 3.09 & 2.46 & 2.51 & 2.64 \\
15 & 3.33 & 2.65 & 2.41 & 2.53 \\
14 & 2.66 & 2.80 & 2.11 & 2.73 \\
13 & 2.46 & 2.59 & 2.02 & 2.61 \\
12 & 2.29 & 2.52 & 2.17 & 2.66 \\
11 & 2.20 & 2.43 & 2.29 & 2.81 \\
10 & 2.05 & 2.49 & 2.11 & 2.90 \\
9 & 2.01 & 2.43 & 2.05 & 2.81 \\
8 & 1.97 & 2.70 & 2.21 & 2.89 \\
7 & 2.07 & 2.84 & 2.32 & 3.04 \\
6 & 2.03 & 3.21 & 2.22 & 3.43 \\
5 & 1.97 & 3.12 & 2.09 & 3.22 \\
4 & 2.08 & 3.21 & 2.88 & 3.11 \\
3 & 2.07 & 3.20 & 3.79 & 4.09 \\
2 & 2.69 & 3.25 & 3.48 & 4.20 \\
1 & 2.62 & 3.17 & 2.62 & 3.17 \\
\hline a. $x$ designates in-plane buckling about $x$-axis as depicted in AISC 360 Part 1.
\end{tabular}

\section{B.4 Eccentrically Braced Frame Plastic Link Rotation}

Figure B-19 through Figure B-21 illustrate the design link rotation angles for the 4-, 8-, and 16-story frames, respectively. The links in the 8- and 16-story frames have high link rotation demands in the upper stories using the approach prescribed by the code. Research (Richards and Thompson 2009) has shown how current procedures based on elastic analysis can overestimate the expected link rotation angle, as compared to results from a nonlinear analysis. Further, included in the figures for comparison is the acceptance criterion for the Life Safety SPL for link rotation angle from ASCE 41; note that the seismic hazard for BSE-1 given the BSO criteria and design per ASCE 7 are identical at maximum demand (i.e., ${ }^{2} / 3 \times \mathrm{MCE}_{\mathrm{R}}$ ). 
Table B-48. 4-Story EBF ELF, Shear Link Parameters

\begin{tabular}{|c|c|c|c|c|c|c|c|c|}
\hline \multirow{2}{*}{ Floor } & \multirow{2}{*}{$\begin{array}{l}\text { Link } \\
\text { Member }\end{array}$} & \multirow{2}{*}{$\begin{array}{c}e \\
\text { (inch) }\end{array}$} & \multirow{2}{*}{$\begin{array}{l}1.6 M_{p} / V_{p} \\
\text { (inch) }\end{array}$} & \multirow{2}{*}{$\mathrm{e} V_{p} / M_{p}$} & \multicolumn{2}{|c|}{$\begin{array}{c}\text { Demand-to-Capacity Ratio } \\
\text { (DCR) }\end{array}$} & \multirow{2}{*}{$\begin{array}{l}\text { Plastic Story } \\
\text { Drift, } \delta_{x p, s} \\
\quad \text { (inch) }\end{array}$} & \multirow{2}{*}{$\begin{array}{c}\text { Link } \\
\text { Rotation } \\
\text { Angle } \\
\text { (rad) } \\
\end{array}$} \\
\hline & & & & & EQ & Wind & & \\
\hline Roof & W14X26 & 30 & 32.2 & 1.49 & 0.64 & 0.19 & 0.91 & 0.043 \\
\hline 4 & W14X38 & 30 & 40.5 & 1.19 & 1.00 & 0.29 & 1.05 & 0.050 \\
\hline 3 & W16X50 & 30 & 42.9 & 1.12 & 0.93 & 0.29 & 0.93 & 0.044 \\
\hline 2 & W16X89 & 30 & 59.1 & 0.81 & 0.97 & 0.35 & 0.96 & 0.036 \\
\hline
\end{tabular}

Table B-49. 4-Story EBF RSA, Shear Link Parameters

\begin{tabular}{|c|c|c|c|c|c|c|c|c|}
\hline \multirow{2}{*}{ Floor } & \multirow{2}{*}{$\begin{array}{l}\text { Link } \\
\text { Member }\end{array}$} & \multirow{2}{*}{$\begin{array}{c}e \\
\text { (inch) }\end{array}$} & \multirow{2}{*}{$\begin{array}{l}1.6 M_{p} / V_{p} \\
\text { (inch) }\end{array}$} & \multirow{2}{*}{$\mathrm{e} V_{p} / M_{p}$} & \multicolumn{2}{|c|}{$\begin{array}{c}\text { Demand-to-Capacity Ratio } \\
\text { (DCR) }\end{array}$} & \multirow{2}{*}{$\begin{array}{c}\text { Plastic Story } \\
\text { Drift, } \delta_{x p, s} \\
\text { (inch) }\end{array}$} & \multirow{2}{*}{$\begin{array}{c}\text { Link } \\
\text { Rotation } \\
\text { Angle } \\
\text { (rad) }\end{array}$} \\
\hline & & & & & EQ & Wind & & \\
\hline Roof & W14X26 & 30 & 32.2 & 1.49 & 0.58 & 0.19 & 0.77 & 0.036 \\
\hline 4 & W14X38 & 30 & 40.5 & 1.19 & 0.83 & 0.29 & 0.86 & 0.041 \\
\hline 3 & W16X45 & 30 & 42.5 & 1.13 & 0.84 & 0.33 & 0.83 & 0.040 \\
\hline
\end{tabular}

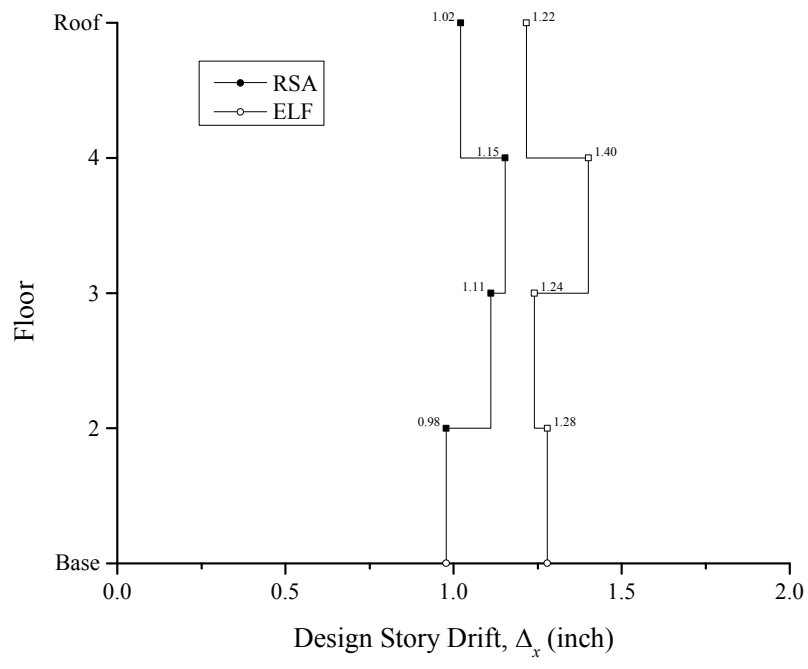

(a) design story drift at center of mass

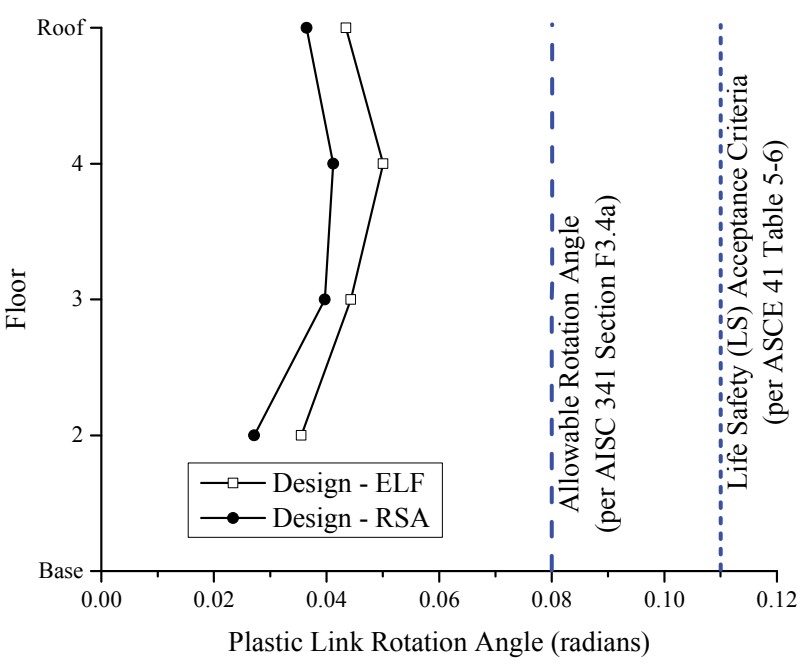

(b) plastic rotation of link beams

Figure B-19. Link Rotation Angle, 4-Story EBF ELF and RSA 
Table B-50. 8-Story EBF ELF, Shear Link Parameters

\begin{tabular}{ccccccccc}
\hline Floor & $\begin{array}{c}\text { Link } \\
\text { Member }\end{array}$ & $\begin{array}{c}e \\
\text { (inch) }\end{array}$ & $\begin{array}{c}1.6 M_{p} / V_{p} \\
\text { (inch) }\end{array}$ & $\mathrm{e} V_{p} / M_{p}$ & $\begin{array}{c}\text { Demand-to-Capacity Ratio } \\
\text { (DCR) }\end{array}$ & $\begin{array}{c}\text { Plastic } \\
\text { Story } \\
\text { Drift, } \delta_{\text {pp,s }} \\
\text { (inch) }\end{array}$ & $\begin{array}{c}\text { Link } \\
\text { Rotation } \\
\text { Angle } \\
\text { (rad) }\end{array}$ \\
\hline Roof & W14X26 & 30 & 32.2 & 1.49 & 0.50 & 0.21 & 1.45 & 0.069 \\
8 & W14X38 & 30 & 40.5 & 1.19 & 0.82 & 0.35 & 1.63 & 0.078 \\
7 & W16X45 & 30 & 42.5 & 1.13 & 0.91 & 0.41 & 1.63 & 0.078 \\
6 & W18X55 & 30 & 45.5 & 1.06 & 0.87 & 0.43 & 1.50 & 0.071 \\
5 & W18X60 & 30 & 47.0 & 1.02 & 0.93 & 0.49 & 1.37 & 0.065 \\
4 & W21X57 & 30 & 42.9 & 1.12 & 0.88 & 0.52 & 1.11 & 0.053 \\
3 & W21X57 & 30 & 42.9 & 1.12 & 0.92 & 0.59 & 0.85 & 0.041 \\
2 & W21X83 & 30 & 51.4 & 0.93 & 0.95 & 0.67 & 0.73 & 0.027 \\
\hline
\end{tabular}

Table B-51. 8-Story EBF RSA, Shear Link Parameters

\begin{tabular}{|c|c|c|c|c|c|c|c|c|}
\hline \multirow{2}{*}{ Floor } & \multirow{2}{*}{$\begin{array}{c}\text { Link } \\
\text { Member }\end{array}$} & \multirow{2}{*}{$\begin{array}{c}e \\
\text { (inch) }\end{array}$} & \multirow{2}{*}{$\begin{array}{l}1.6 M_{p} / V_{p} \\
\text { (inch) }\end{array}$} & \multirow{2}{*}{$\mathrm{e} V_{p} / M_{p}$} & \multicolumn{2}{|c|}{$\begin{array}{c}\text { Demand-to-Capacity Ratio } \\
\text { (DCR) }\end{array}$} & \multirow{2}{*}{$\begin{array}{c}\text { Plastic } \\
\text { Story } \\
\text { Drift, } \delta_{x p, s} \\
\text { (inch) }\end{array}$} & \multirow{2}{*}{$\begin{array}{c}\text { Link } \\
\text { Rotation } \\
\text { Angle } \\
\text { (rad) }\end{array}$} \\
\hline & & & & & EQ & Wind & & \\
\hline Roof & W14X26 & 30 & 32.2 & 1.49 & 0.59 & 0.22 & 1.33 & 0.063 \\
\hline 8 & W14X26 & 30 & 32.2 & 1.49 & 0.92 & 0.44 & 1.47 & 0.070 \\
\hline 7 & W16X31 & 30 & 34.9 & 1.38 & 0.84 & 0.53 & 1.37 & 0.065 \\
\hline 4 & W18X46 & 30 & 39.8 & 1.21 & 0.81 & 0.69 & 0.94 & 0.045 \\
\hline 3 & W18X55 & 30 & 45.5 & 1.06 & 0.87 & 0.73 & 0.78 & 0.037 \\
\hline 2 & W21X83 & 30 & 51.4 & 0.93 & 0.80 & 0.68 & 0.52 & 0.019 \\
\hline
\end{tabular}

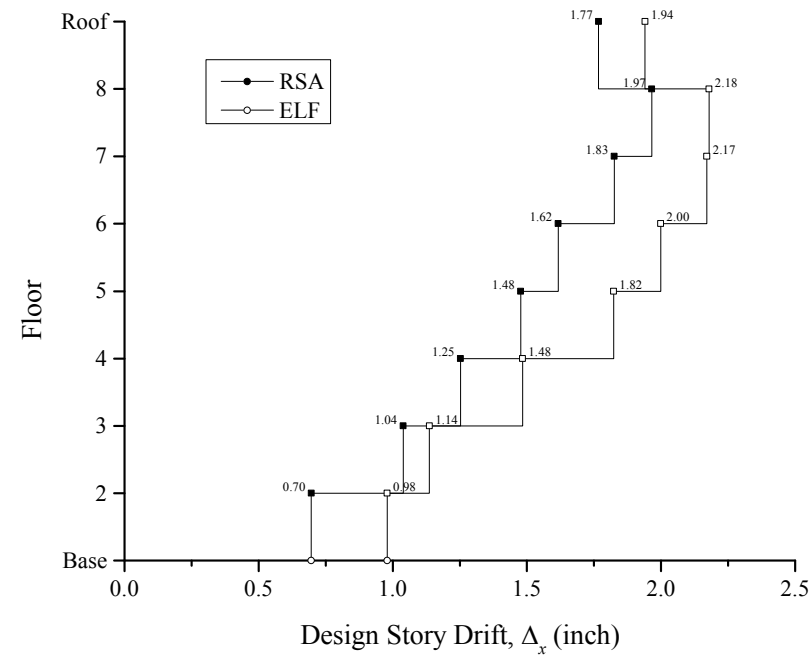

(a) design story drift at center of mass

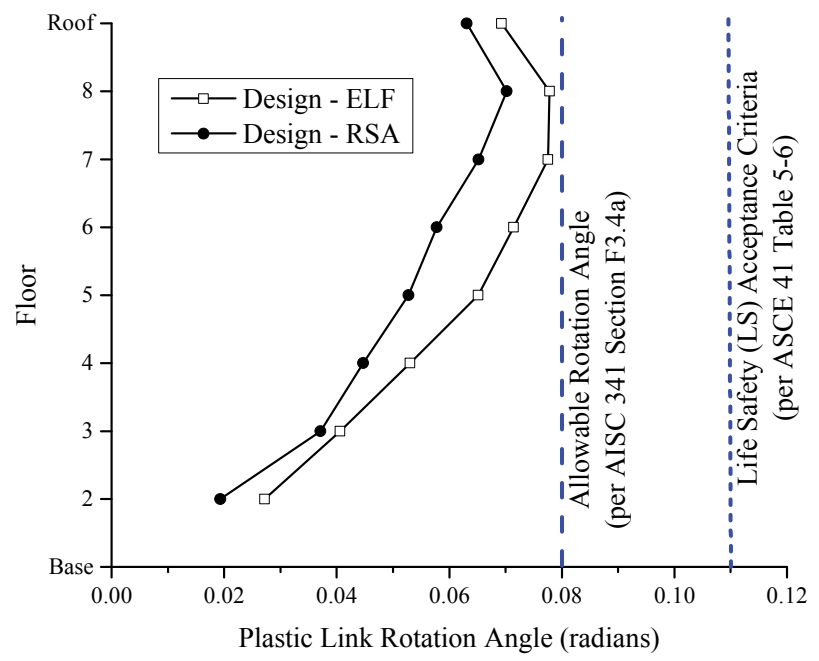

(b) plastic rotation of link beams

Figure B-20. Link Rotation Angle, 8-Story EBF ELF and RSA 
Table B-52. 16-Story EBF ELF, Shear Link Parameters

\begin{tabular}{|c|c|c|c|c|c|c|c|c|}
\hline \multirow{2}{*}{ Floor } & \multirow{2}{*}{$\begin{array}{c}\text { Link } \\
\text { Member }\end{array}$} & \multirow{2}{*}{$\begin{array}{c}e \\
\text { (inch) }\end{array}$} & \multirow{2}{*}{$\begin{array}{l}1.6 M_{p} / V_{p} \\
\text { (inch) }\end{array}$} & \multirow{2}{*}{$\mathrm{e} V_{p} / M_{p}$} & \multicolumn{2}{|c|}{$\begin{array}{c}\text { Demand-to-Capacity Ratio } \\
\text { (DCR) }\end{array}$} & \multirow{2}{*}{$\begin{array}{c}\text { Plastic } \\
\text { Story } \\
\text { Drift, } \delta_{x p, s} \\
\text { (inch) }\end{array}$} & \multirow{2}{*}{$\begin{array}{c}\text { Link } \\
\text { Rotation } \\
\text { Angle } \\
\text { (rad) }\end{array}$} \\
\hline & & & & & EQ & Wind & & \\
\hline Roof & W14X48 & 39 & 48.8 & 1.28 & 0.27 & 0.13 & 1.27 & 0.070 \\
\hline 16 & W14X48 & 39 & 48.8 & 1.28 & 0.56 & 0.28 & 1.37 & 0.076 \\
\hline 15 & W18X40 & 39 & 39.4 & 1.58 & 0.66 & 0.35 & 1.42 & 0.078 \\
\hline 12 & W18X60 & 39 & 47.0 & 1.33 & 0.85 & 0.51 & 1.36 & 0.075 \\
\hline 11 & W21X57 & 39 & 42.9 & 1.45 & 0.82 & 0.53 & 1.29 & 0.071 \\
\hline 10 & W21X68 & 39 & 50.3 & 1.24 & 0.83 & 0.56 & 1.22 & 0.067 \\
\hline 9 & W21X73 & 39 & 51.1 & 1.22 & 0.82 & 0.59 & 1.14 & 0.062 \\
\hline 8 & W21X83 & 39 & 51.4 & 1.21 & 0.76 & 0.57 & 1.03 & 0.057 \\
\hline 3 & W21X83 & 39 & 51.4 & 1.21 & 0.81 & 0.81 & 0.50 & 0.028 \\
\hline 2 & W24X103 & 39 & 60.2 & 1.04 & 0.86 & 0.91 & 0.40 & 0.017 \\
\hline
\end{tabular}

Table B-53. 16-Story EBF RSA, Shear Link Parameters

\begin{tabular}{|c|c|c|c|c|c|c|c|c|}
\hline \multirow{2}{*}{ Floor } & \multirow{2}{*}{$\begin{array}{l}\text { Link } \\
\text { Member }\end{array}$} & \multirow{2}{*}{$\begin{array}{c}e \\
\text { (inch) }\end{array}$} & \multirow{2}{*}{$\begin{array}{l}1.6 M_{p} / V_{p} \\
\text { (inch) }\end{array}$} & \multirow{2}{*}{$\mathrm{e} V_{p} / M_{p}$} & \multicolumn{2}{|c|}{$\begin{array}{c}\text { Demand-to-Capacity Ratio } \\
\text { (DCR) }\end{array}$} & \multirow{2}{*}{$\begin{array}{c}\text { Plastic } \\
\text { Story } \\
\text { Drift, } \delta_{x p, s} \\
\text { (inch) }\end{array}$} & \multirow{2}{*}{$\begin{array}{c}\text { Link } \\
\text { Rotation } \\
\text { Angle } \\
\text { (rad) }\end{array}$} \\
\hline & & & & & EQ & Wind & & \\
\hline Roof & W14X38 & 39 & 40.5 & 1.54 & 0.40 & 0.17 & 1.19 & 0.066 \\
\hline 16 & W14X38 & 39 & 40.5 & 1.54 & 0.69 & 0.34 & 1.29 & 0.071 \\
\hline 15 & W14X38 & 39 & 40.5 & 1.54 & 0.87 & 0.52 & 1.33 & 0.073 \\
\hline 12 & W16X45 & 39 & 42.5 & 1.47 & 0.84 & 0.81 & 1.15 & 0.063 \\
\hline 11 & W18X50 & 39 & 45.0 & 1.39 & 0.80 & 0.82 & 1.04 & 0.057 \\
\hline 10 & W18X55 & 39 & 45.5 & 1.37 & 0.78 & 0.84 & 0.96 & 0.053 \\
\hline 9 & W21X50 & 39 & 39.1 & 1.59 & 0.73 & 0.83 & 0.89 & 0.049 \\
\hline 8 & W21X62 & 39 & 48.6 & 1.29 & 0.76 & 0.86 & 0.81 & 0.044 \\
\hline 3 & W21X73 & 39 & 51.1 & 1.22 & 0.99 & 1.01 & 0.45 & 0.025 \\
\hline 2 & W24X103 & 39 & 60.2 & 1.04 & 0.90 & 0.93 & 0.41 & 0.017 \\
\hline
\end{tabular}




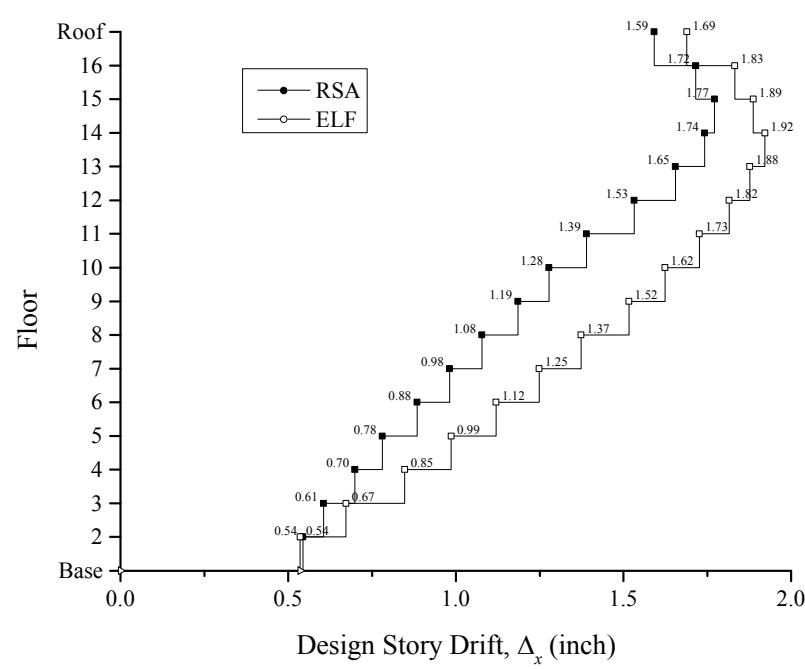

(a) design story drift at center of mass

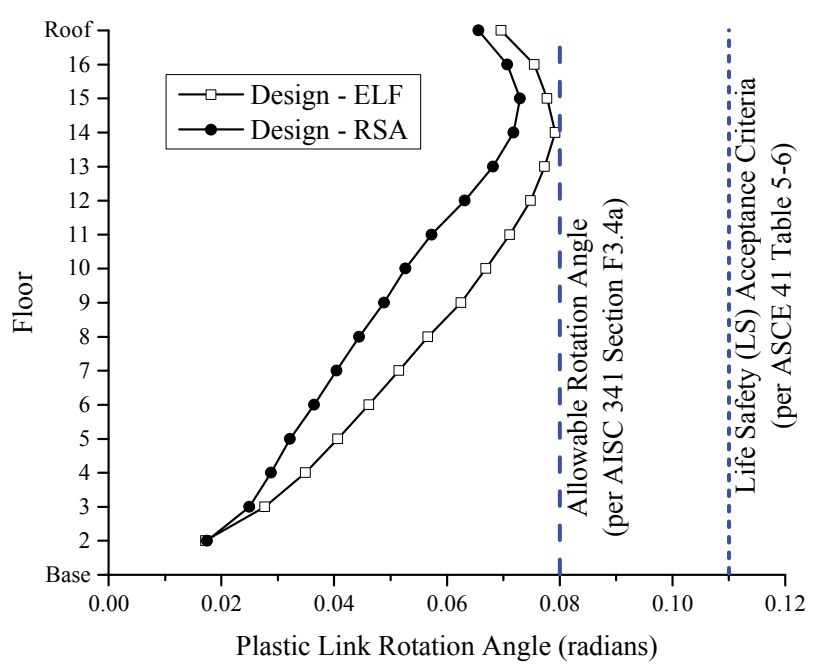

(b) plastic rotation of link beams

Figure B-21. Link Rotation Angle, 16-Story EBF ELF and RSA 


\section{B.5 Example Design Calculations}

The examples presented detail the strength design calculations for the following members and components of each SFRS for the RSA-designed 8-story buildings (ME8):

- EBF shear link, ME8 RSA

- EBF brace, ME8 RSA

- $\quad$ EBF column, ME8 RSA

Example calculations for components of the SMF can be found in Volume 1 (Harris and Speicher 2015a). Example calculations of columns and braces (ductile) can also be found in Volume 2 (Harris and Speicher 2015b).

\section{B.5.1 Eccentrically Braced Frame Example}

\section{B.5.1.1 Member Selection}

After several design and analysis iterations, the final member sizes are shown in Figure B-22.

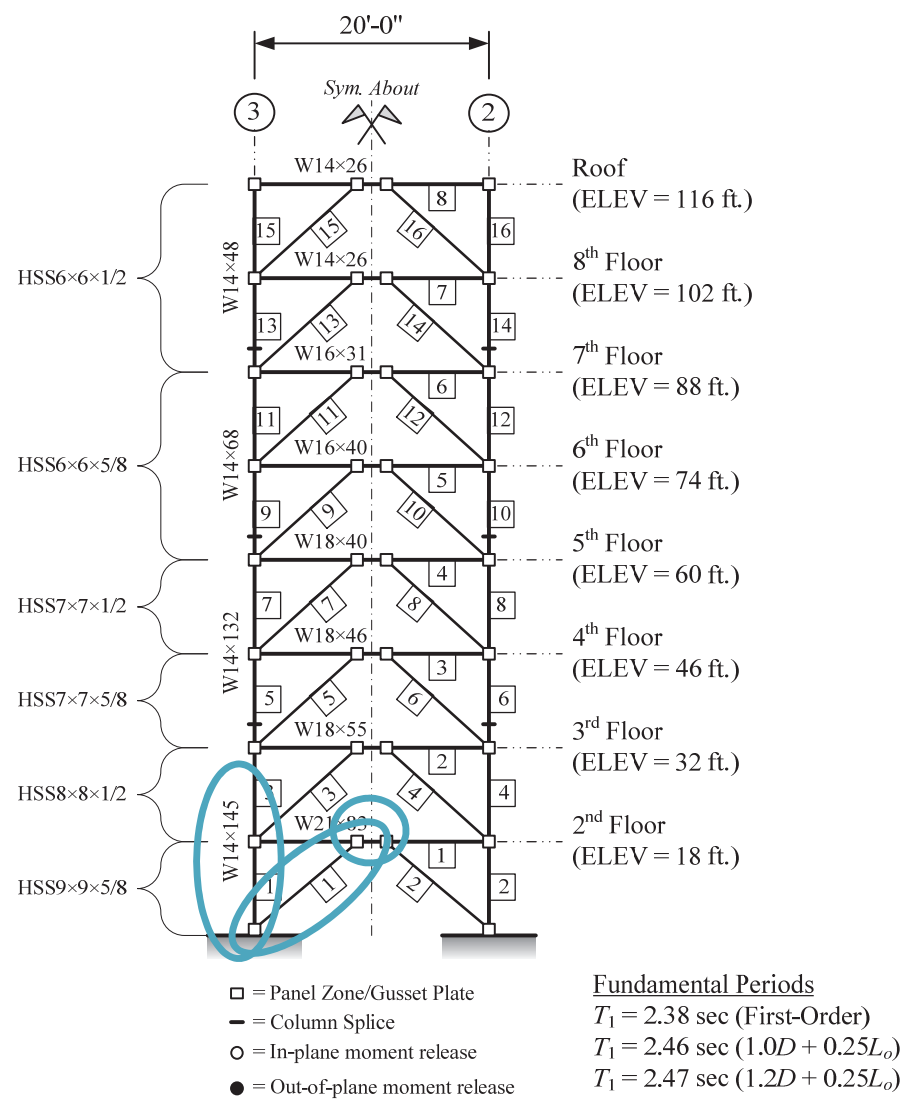

Figure B-22. EBF member sizes, 8-story RSA 


\section{B.5.1.2 EBF Link Beam}

The second floor link beam along grid line 1 between grid lines B and $\mathrm{C}$ is selected for this example (see circled elements in Figure B-22):

- $\mathrm{W} 21 \times 83$

- $e=30$ in

- $\quad$ A992 Steel: $F_{y}=50 \mathrm{ksi}, R_{y}=1.1, E=29000 \mathrm{ksi}, G=11200 \mathrm{ksi}$

\section{B.5.1.2.1 Shear Demand}

Based on the controlling load combination from ASCE 7, the demand on the link is as follows:

$$
V_{u}=247 \text { kips (governed by seismic) }
$$

\section{B.5.1.2.2 Shear Strength}

Check to see if the link is short.

$$
\begin{aligned}
& M_{p}=F_{y} Z_{x}=50(196)=9800 \text { kip-in. } \\
& A_{l w}=\left(d-2 t_{f}\right) t_{w}=(21.4-2(0.835))(0.515)=10.16 \text { in. }^{2} \\
& V_{p}=0.6 F_{y} A_{l w}=0.6(50)(10.25)=305 \mathrm{kips} \\
& e=30<1.6 \frac{M_{p}}{V_{p}}=1.6\left(\frac{9800}{305}\right)=51.4 \therefore \text { the link is short. }
\end{aligned}
$$

The nominal design shear strength of the link is determined by AISC $341 \S \mathrm{F} 3.5 \mathrm{~b}$ :

$$
\phi_{v} V_{n}=\phi_{v} V_{p}=0.9(305)=275 \text { kips }
$$

\section{B.5.1.2.3 Strength Check}

$$
\frac{V_{u}}{\phi V_{n}}=\frac{247}{275}=0.89 \leq 1.0 \therefore \mathrm{OK}
$$

\section{B.5.1.2.4 Link Rotation Angle Verification}

The allowable plastic rotation angle for a short link beam in accordance with AISC 341 is 0.08 radians. 


$$
\begin{aligned}
& \Delta_{x p, s}=\frac{3}{4} \Delta_{x}=0.75(0.7)=0.52 \text { in. (see Chapter 2) } \\
& \gamma_{p}=\frac{L_{b}}{e} \frac{\Delta_{x p, s}}{h_{s x}}=\frac{20(12)}{30} \frac{0.52}{216}=0.019 \mathrm{rad} \text { ( (see Chapter 2) }
\end{aligned}
$$

\section{B.5.1.3 EBF Brace}

The first story brace along grid line 1 between grid lines $\mathrm{B}$ and $\mathrm{C}$ was selected for this example (see circled elements in Figure B-22):

- HSS $9 \times 9 \times 5 / 8$

- $L=213$ in

- $\quad$ A500 Gr. B, $F_{y}=46 \mathrm{ksi}, R_{y}=1.4, E=29000 \mathrm{ksi}, G=11200 \mathrm{ksi}$

\section{B.5.1.3.1 Axial and Flexure Demand}

The demands in the brace from the controlling load combinations from ASCE 7 are presented in Table B-54-maximum/minimum values for an individual action displayed with bolded text.

Table B-54. Controlling Load Combinations (kips, feet)

\begin{tabular}{llllll}
\hline LC Index & $P_{u, A}$ & $P_{u, B}$ & $M_{u, A, x}$ & $M_{u, B, x}$ & Max/Min Action \\
\hline 179 & 295.39 & $\mathbf{2 9 5 . 3 9}$ & 27.58 & 33.66 & $P_{u, \min }$ \\
133 & $\mathbf{- 3 3 3 . 6 1}$ & -332.20 & -31.38 & -31.39 & $P_{u, \max }$ \\
179 & 294.68 & 295.39 & $\mathbf{2 7 . 5 8}$ & 33.66 & $M_{u, A, \min }$ \\
133 & -333.61 & -332.20 & $\mathbf{- 3 1 . 3 8}$ & -31.39 & $M_{u, A, \max }$ \\
123 & 278.92 & 280.34 & 26.15 & $\mathbf{3 4 . 7 0}$ & $M_{u, B, \min }$ \\
189 & -317.85 & -317.14 & -29.95 & $\mathbf{- 3 2 . 4 2}$ & $M_{u, B, \max }$ \\
\hline
\end{tabular}

$P_{r}=334$ kips and $M_{r, x}=32 \mathrm{kip}$-ft.- - from the load combination selected for this example (\#133 in Table).

The flexural demand contribution to the $P-M$ interaction verification is small $(\leq 10 \%)$ compared to the axial demand contribution. Therefore, $P-M$ interaction is neglected in this example for brevity.

EBF brace design is also governed by the capacity design requirements specified in AISC $341 \S \mathrm{F} 3.3$. AISC $341 \S \mathrm{F} 3.3$, exception (2) permits flexural forces resulting from seismic drift of the frame to be neglected.

$$
P_{r, C D}=564 \text { kips }
$$

\section{B.5.1.3.2 Axial Strength}

The axial compression strength of the brace is calculated per AISC 360 § 3 -weak-axis (y) governs.

$$
P_{n, y}=A_{g} F_{c r, y}
$$




$$
\begin{aligned}
& \frac{K_{y} L_{y}}{r_{y}} \sqrt{\frac{F_{y}}{E}}=\frac{1.0(213)}{3.40} \sqrt{\frac{46}{29000}}=2.50 \leq 4.71 \\
& F_{e, y}=\frac{\pi^{2} E}{\left(\frac{K_{y} L_{y}}{r_{y}}\right)^{2}}=\frac{\pi^{2}(29000)}{\left(\frac{1.0(213)}{3.40}\right)^{2}}=72.9 \mathrm{ksi} \\
& \therefore F_{c r, y}=\left[0.658^{\left(F_{y} / F_{e, y}\right)}\right] F_{y}=35.4 \mathrm{ksi} \\
& \phi_{c} P_{n, y}=0.9(18.7) 35.4=0.9(660)=595 \mathrm{kips}
\end{aligned}
$$

\section{B.5.1.3.3 Strength Check}

$$
\frac{P_{r, C D}}{\phi_{c} P_{n, y}}=\frac{564}{595}=0.95 \leq 1.0 \therefore \mathrm{OK}
$$

\section{B.5.1.4 EBF Column}

The first story column along grid line 3 was selected for this example (see circled elements in Figure B-22):

- $\mathrm{W} 14 \times 145$

- $h_{s x}=216$ in, $L_{\text {clear }}=197.4$ in

- $\quad$ A992 Steel: $F_{y}=50 \mathrm{ksi}, R_{y}=1.1, E=29000 \mathrm{ksi}, G=11200 \mathrm{ksi}$

\section{B.5.1.4.1 Axial and Flexural Demand}

The demands in the column from the controlling load combinations from ASCE 7 are presented in Table B-55-maximum/minimum values for an individual action displayed with bolded text.

Table B-55. Controlling Load Combinations (kips, feet)

\begin{tabular}{llllll}
\hline LC Index & $P_{u, A}$ & $P_{u, B}$ & $M_{u, A, x}$ & $M_{u, B, x}$ & Max/Min Action \\
\hline 179 & 528.58 & $\mathbf{5 2 8 . 5 8}$ & 101.33 & 43.23 & $P_{u, \min }$ \\
133 & $\mathbf{- 9 8 7 . 6 8}$ & -984.39 & -112.00 & -22.81 & $P_{u, \max }$ \\
179 & 526.93 & 528.58 & $\mathbf{1 0 1 . 3 3}$ & 43.23 & $M_{u, A, \min }$ \\
133 & -987.68 & -984.39 & $\mathbf{- 1 1 2 . 0 0}$ & -22.81 & $M_{u, A, \max }$ \\
123 & 358.95 & 362.24 & 97.43 & $\mathbf{5 0 . 6 8}$ & $M_{u, B, \min }$ \\
189 & -819.70 & -818.06 & -108.11 & $\mathbf{- 3 0 . 2 7}$ & $M_{u, B, \max }$ \\
\hline
\end{tabular}

$P_{r}=988$ kips and $M_{r, x}=112 \mathrm{kip}-\mathrm{ft}$.—-from the load combination selected for this example (\#133 in Table B-55). 
The flexural demand contribution to the $P-M$ interaction verification is small $(\leq 10 \%)$ compared to the axial demand contribution. Therefore, $P-M$ interaction is neglected in this example for brevity.

EBF column design is also governed by the capacity design requirements specified in AISC $341 \S$ F3.3. AISC $341 \S \mathrm{F} 3.3$, exception (2) permits flexural forces resulting from seismic drift of the frame to be neglected. AISC $341 \S$ F3.3 exception (1) permits demands to be multiplied by 0.88 for columns in frames over three stories.

$$
P_{r, C D}=0.88(1668)=1468 \text { kips }
$$

\section{B.5.1.4.2 Axial Strength}

The axial compression strength of the column is calculated per AISC 360 § 3 -weak-axis (y) governs.

$$
\begin{aligned}
& P_{n, y}=A_{g} F_{c r, y} \\
& \frac{K_{y} L_{y}}{r_{y}} \sqrt{\frac{F_{y}}{E}}=\frac{1.0(216)}{3.98} \sqrt{\frac{50}{29000}}=2.25 \leq 4.71 \\
& F_{e, y}=\frac{\pi^{2} E}{\left(\frac{K_{y} L_{y}}{r_{y}}\right)^{2}}=\frac{\pi^{2}(29000)}{\left(\frac{1.0(216)}{3.98}\right)^{2}}=97.18 \mathrm{ksi} \\
& \therefore F_{c r, y}=\left[0.658^{\left(F_{y} / F_{e, y}\right)}\right] F_{y}=\left[0.658^{(50 / 97.18)}\right] 50=40.3 \mathrm{ksi} \\
& \phi_{c} P_{n, y}=0.9(42.7)(40.3)=0.9(1721)=1549 \mathrm{kips}
\end{aligned}
$$

\section{B.5.1.4.3 Strength Check}

$$
\frac{P_{r, C D}}{\phi_{c} P_{n, y}}=\frac{1468}{1549}=0.95 \leq 1.0 \therefore \mathrm{OK}
$$





\section{Appendix C Example Assessment Calculations}

The examples presented in this appendix detail the assessment calculations for the following primary members and connections of each SFRS for the RSA-designed 8-story buildings (ME8):

- $\quad$ EBF shear link, ME8 RSA

Linear assessment example calculations are provided in C.1, and examples for the nonlinear assessment example calculations are provided in C.2.

\section{C.1 Linear Assessment Examples}

The following example provides guidance to how the linear assessment calculations were conducted in this study. Linear assessment of the selected components is performed for the following criteria:

- Linear Dynamic Procedure (LDP)

- Collapse Prevention (CP) Building Performance Level (BPL) for the BSE-2 Earthquake Hazard Level (EHL)

\section{C.1.1 EBF Link}

The second floor link along grid line 1 between grid lines B and $\mathrm{C}$ is selected for this example (see circled elements in Figure B-22):

- $\mathrm{W} 21 \times 83$

- $e=30$ in

- A992 Steel: $F_{y, L B}=F_{y}=50 \mathrm{ksi}, F_{y e}=55 \mathrm{ksi}, E=29000 \mathrm{ksi}, G=11154 \mathrm{ksi}$

\section{C.1.1.1 Shear Demand}

Based on the controlling load combination, the demand on the link is as follows:

$$
V_{U D}=V_{G} \pm V_{E}=1705 \text { kips }
$$

\section{C.1.1.2 Shear Strength}

The flexural and shear strength of the link beam is determined per ASCE 41 §5.5.3.3.2.

$$
M_{C E}=M_{p e}=F_{y e} Z_{x}=55(196)=10776 \text { kip-in. }
$$




$$
\begin{gathered}
V_{C E}=0.6 F_{y e} A_{w}=0.6(55)(10.16)=335 \mathrm{kips} \\
A_{w}=\left(d-2 t_{f}\right) t_{w}=10.16 \mathrm{in}^{2} \\
e=30<1.6 \frac{M_{C E}}{V_{C E}}=1.6 \frac{10776}{335}=51.5 \mathrm{in} .
\end{gathered}
$$

\section{C.1.1.3 Acceptance Criteria}

The $m$-factor is taken from ASCE 41 Table 5-5 (Primary Component).

$$
e \leq 1.6 \frac{M_{C E}}{V_{C E}} \rightarrow \therefore m=13
$$

\section{C.1.1.4 Acceptance Criteria Check}

The link acceptance criteria check is:

$$
D C R_{N}=\frac{D C R}{\kappa m}=\frac{V_{U D}}{\kappa m V_{C E}}=\frac{1705}{13(335)}=0.39<1.0
$$

Therefore, the link beam satisfies the CP BPL acceptance criteria at the BSE-2 EHL.

\section{C.2 Nonlinear Assessment Examples}

The following example provides guidance to how the nonlinear assessment calculations were conducted in this study. Nonlinear assessment of the selected components is performed for the following criteria:

- Nonlinear Dynamic Procedure (NDP) or Nonlinear Static Procedure (NSP)

- Median value of the record set is used for the NDP

- Collapse Prevention Building Performance Level for the BSE-2 EHL

\section{C.2.1 EBF Link}

The second floor link along grid line 1 between grid lines $\mathrm{B}$ and $\mathrm{C}$ is selected for this example (see circled elements in Figure B-22):

- $\mathrm{W} 21 \times 83$

- $e=30$ in.

- A992 Steel: $F_{y, L B}=F_{y}=50 \mathrm{ksi}, F_{y e}=55 \mathrm{ksi}, E=29000 \mathrm{ksi}, G=11154 \mathrm{ksi}$

The demands for the EBF beam are taken from the median value of the record set from the NDP. 


\section{C.2.1.1 Deformation Demand}

The demand is given in terms of plastic shear strain of the link beam is:

$$
\theta_{U D} \equiv \gamma_{p}=0.125 \mathrm{rad}
$$

\section{C.2.1.2 Acceptance Criteria}

The acceptance criteria in terms of plastic shear strain are given in ASCE 41 Table 5-6.

$$
\begin{aligned}
& e=30<1.6 \frac{M_{C E}}{V_{C E}}=1.6 \frac{10776}{335}=51.5 \\
& \therefore \theta_{p, A C} \equiv \gamma_{p, A C}=0.14 \mathrm{rad} .
\end{aligned}
$$

\section{C.2.1.3 Acceptance Criteria Check}

The link acceptance criteria check is:

$$
D C R_{N}=\frac{\gamma_{p}}{\kappa \gamma_{p, A C}}=\frac{0.125}{1.0(0.14)}=0.89<1.0
$$

Therefore, the link satisfies the CP BPL acceptance criteria at the BSE-2 EHL. 
C-4 


\section{Appendix D References}

AISC (1989). Specification for Structural Steel Buildings: Allowable Stress Design and Plastic Design. American Institute of Steel Construction, Chicago, IL.

AISC (1999). Wide-Flange Column Stiffening at Moment Connections - Design Guide 13. American Institute of Steel Construction, Chicago, IL.

AISC (2002). Seismic Provisions for Structural Steel Buildings - ANSI/AISC 341-02. American Institute of Steel Construction, Chicago, IL.

AISC (2005). Specification for Structural Steel Buildings - ANSI/AISC 360-05. American Institute of Steel Construction, Chicago, IL.

AISC (2010a). Specification for Structural Steel Buildings - ANSI/AISC 360-10. American Institute of Steel Construction, Chicago, IL.

AISC (2010b). Seismic Provisions for Structural Steel Buildings - ANSI/AISC 341-10. American Institute of Steel Construction, Chicago, IL.

AISC (2010c). Prequalified Connections for Special and Intermediate Steel Moment Frames for Seismic Applications - ANSI/AISC 358-10. American Institute of Steel Construction, Construction, Chicago, IL.

AISC (2013). Stability Design of Steel Buildings - Design Guide 28. American Institute of Steel Construction, Chicago, IL.

ASCE (2005). Minimum Design Loads for Buildings and Other Structures - ASCE/SEI 7-05. American Society of Civil Engineers, Reston, VA.

ASCE (2006). Seismic Rehabilitation of Existing Buildings - ASCE/SEI 41-06. American Society of Civil Engineers, Reston, VA.

ASCE (2010). Minimum Design Loads for Buildings and Other Structures - ASCE/SEI 7-10. American Society of Civil Engineers, Reston, VA.

ASCE (2014). Seismic Evaluation and Retrofit of Existing Buildings - ASCE/SEI 41-13. American Society of Civil Engineers, Reston, VA.

ASCE (2016). Minimum Design Loads for Buildings and Other Structures - ASCE/SEI 7-16. American Society of Civil Engineers, Reston, VA. In Progress. 
CBSC (2010) California Code of Regulations, Part 2 - California Building Standards Code - Title 24. California Building Standards Commission, Sacramento, California.

CSI (2011a). ETABS (Version 9.7.4) [Computer Software]. Computers and Structures, Inc., Berkeley, CA.

CSI (2011b). PERFORM-3D (Version 5.0) [Computer Software]. Computers and Structures, Inc., Berkeley, CA.

CSI (2011c). Components and Elements for PERFORM-3D and PERFORM-COLLAPSE (Version 5.0).

Computers and Structures, Inc., Berkeley, CA.

CSI (2011d). User Guide PERFORM-3D (Version 5.0). Computers and Structures, Inc., Berkeley, CA.

Engelhardt, M.D., Winneberger, T., Zekany, A.J., and Potyraj, T.J. (1998). "Experimental Investigation of Dogbone Moment Connections". Engineering Journal, AISC, $4^{\text {th }}$ Quarter, pp. 128-139.

FEMA (1997). NEHRP Guidelines for the Seismic Rehabilitation of Buildings - FEMA 273. Federal Emergency Management Agency, Washington, D.C.

FEMA (2000a). Recommended Seismic Design Criteria for New Steel Moment Frame Buildings - FEMA 350. Federal Emergency Management Agency, Washington, D.C.

FEMA (2000b). Recommended Seismic Evaluation and Upgrade Criteria for Existing Welded Steel Moment Frame Buildings - FEMA 351. Federal Emergency Management Agency, Washington, D.C.

FEMA (2000c). State of the Art Report on Connection Performance - FEMA 355D. Federal Emergency Management Agency, Washington, D.C.

FEMA (2000d). State of the Art Report on Performance Prediction and Evaluation - FEMA 355F. Federal Emergency Management Agency, Washington, D.C.

FEMA (2000e). Prestandard and Commentary for the Seismic Rehabilitation of Buildings - FEMA 356. Federal Emergency Management Agency, Washington, D.C.

FEMA (2000f). Global Topics Report on the Prestandard and Commentary for the Seismic Rehabilitation of Buildings - FEMA 357. Federal Emergency Management Agency, Washington, D.C.

FEMA (2005). Improvements of Nonlinear Static Seismic Analysis Procedures - FEMA 440. Federal Emergency Management Agency, Washington, D.C.

FEMA (2009a). Quantification of Building Seismic Performance Factors - FEMA P695. Federal Emergency Management Agency, Washington, D.C.

FEMA (2009b). NEHRP Recommended Seismic Provisions for New Buildings and Other Structures FEMA P-750. Federal Emergency Management Agency, Washington, D.C. 
FEMA (2009c). Effects of Strength and Stiffness Degradation on Seismic Response - FEMA P-440A. Federal Emergency Management Agency, Washington, D.C.

FEMA (2012). Seismic Performance Assessment of Buildings - FEMA P-58. Federal Emergency Management Agency, Washington, D.C.

FEMA (2015). NEHRP Recommended Seismic Provisions for New Buildings and Other Structures. Federal Emergency Management Agency, Washington, D.C. In Progress.

GSA (2012). Facilities Standards for the Public Buildings Service - PBS-P100. U.S. General Services Administration, Washington, D.C.

Harris, J.L. and Speicher, M.S. (2015a). Assessment of First Generation Performance-Based Design Methods for New Steel Buildings, Volume 1: Special Moment Frames - NIST TN 1863-1. National Institute of Standards and Technology, Gaithersburg, MD.

Harris, J.L. and Speicher, M.S. (2015b). Assessment of First Generation Performance-Based Design Methods for New Steel Buildings, Volume 2: Special Concentrically Braced Frames - NIST TN 1863-2. National Institute of Standards and Technology, Gaithersburg, MD.

Haselton, C.B., Kircher, C.A., and Liel, A.B. (2009). "Concept Paper on Utilizing the FEMA P695 (ATC63) Ground Motion Spectral Shape Guidelines to Adjust the Target Displacement in the ASCE/SEI 41 Nonlinear Static Procedure" Proc.: ATC \& SEI 2009 Conference on Improving the Seismic Performance of Existing Buildings and Other Structures, San Francisco, CA Dec. 9-11, 2009.

ICC (2012a). International Existing Building Code (IEBC). International Code Council, Washington, DC.

ICC (2012b). International Building Code (IBC). International Code Council, Washington, DC.

Koboeviv, S., Rozon, J., and Tremblay, R. (2012). "Seismic Performance of Low-to-Moderate Height Eccentrically Braced Steel Frames Designed for North American Seismic Conditions." Journal of Structural Engineering, ASCE, 12(138), pp. 1465-1476.

Krawinkler, H., Bertero, V.V., and Popov, E.P. (1971). Inelastic Behavior of Steel Beam-to-Column Subassemblages - Report No. UCB/EERC-71/07. Earthquake Engineering Research Center, University of California, Berkeley, CA, October 1971.

Krawinkler, H. (1978). "Shear in Beam Column Joints in Seismic Design of Steel Frames". Engineering Journal, AISC, $3^{\text {rd }}$ Quarter, pp. 82-91.

Lee, D., Cotton, S.C., Dexter, R.J., Hajjar, J.F., Ye, Y. and Ojard, S.D. (2002). Column Stiffener Detailing and Panel Zone Behavior of Steel Moment Frame Connections - Report No. ST-01-3.2. Dept. of Civil Engineering, University of Minnesota, Minneapolis, MN, 2002. 
NIBS (2013). National Performance-Based Design Guide for Buildings. National Institute of Building Sciences, Washington, D.C.

NIST (2009a). Research Required to Support Full Implementation of Performance-Based Seismic Design - NIST GCR 09-917-2. Produced by the Building Seismic Safety Council for the National Institute of Standards and Technology, Gaithersburg, MD.

NIST (2009b). Seismic Design of Steel Special Moment Frames: A Guide for Practicing Engineers, NEHRP Seismic Design Technical Brief No. 2 - NIST GCR 09-917-3. Produced by the NEHRP Consultants Joint Venture, a partnership of the Applied Technology Council and the Consortium of Universities for Research in Earthquake Engineering, for the National Institute of Standards and Technology, Gaithersburg, MD.

NIST (2010a). Nonlinear Structural Analysis for Seismic Design: A Guide for Practicing Engineers, NEHRP Seismic Design Technical Brief No. 4 - NIST GCR 10-917-5. Produced by the NEHRP Consultants Joint Venture, a partnership of the Applied Technology Council and the Consortium of Universities for Research in Earthquake Engineering, for the National Institute of Standards and Technology, Gaithersburg, MD.

NIST (2010b). Evaluation of the FEMA P-695 Methodology for Quantification of Building Seismic Performance Factors - NIST GCR 10-917-8. Produced by the NEHRP Consultants Joint Venture, a partnership of the Applied Technology Council and the Consortium of Universities for Research in Earthquake Engineering, for the National Institute of Standards and Technology, Gaithersburg, MD.

NIST (2010c). Applicability of Nonlinear Multi-Degree-of-Freedom Modeling for Design - NIST GCR 10917-9. Produced by the NEHRP Consultants Joint Venture, a partnership of the Applied Technology Council and the Consortium of Universities for Research in Earthquake Engineering, for the National Institute of Standards and Technology, Gaithersburg, MD.

NIST (2011a). Standards of Seismic Safety for Existing Federally Owned and Leased Buildings: ICSSC Recommended Practice 8 (RP 8) - NIST GCR 11-917-12. Produced by the Building Seismic Safety Council of the National Institute of Buildings Sciences for the National Institute of Standards and Technology, Gaithersburg, MD.

NIST (2011b). Seismic Design of Composite Steel Deck and Concrete-filled Diaphragms: A Guide for Practicing Engineers, NEHRP Seismic Design Technical Brief No. 5 - NIST GCR 11-917-10. Produced by the NEHRP Consultants Joint Venture, a partnership of the Applied Technology Council and the Consortium of Universities for Research in Earthquake Engineering, for the National Institute of Standards and Technology, Gaithersburg, MD.

NIST (2011c). Research Plan for the Study of Seismic Behavior and Design of Deep, Slender Wide-Flange Structural Steel Beam-Column Members - NIST GCR 11-917-13. Produced by the NEHRP Consultants Joint Venture, a partnership of the Applied Technology Council and the Consortium of Universities for Research in Earthquake Engineering, for the National Institute of Standards and Technology, Gaithersburg, MD. 
NIST (2011d). Selecting and Scaling Earthquake Ground Motions for Performing Response-History Analyses - NIST GCR 11-917-15. Produced by the NEHRP Consultants Joint Venture, a partnership of the Applied Technology Council and the Consortium of Universities for Research in Earthquake Engineering, for the National Institute of Standards and Technology, Gaithersburg, MD.

NIST (2012). Tentative Framework for Development of Advanced Seismic Design Criteria for New Buildings - NIST GCR 12-917-20. Produced by the NEHRP Consultants Joint Venture, a partnership of the Applied Technology Council and the Consortium of Universities for Research in Earthquake Engineering, for the National Institute of Standards and Technology, Gaithersburg, MD.

NIST (2013). Seismic Design of Steel Special Concentrically Braced Frame Systems: A Guide for Practicing Engineers, NEHRP Seismic Design Technical Brief No. 8 - NIST GCR 13-917-24. Produced by the NEHRP Consultants Joint Venture, a partnership of the Applied Technology Council and the Consortium of Universities for Research in Earthquake Engineering, for the National Institute of Standards and Technology, Gaithersburg, MD.

Paret, T.F., Searer, G.R., and Freeman, S.A. (2011). “ASCE 31 and 41: Apocalypse Now”. Proc.: Structures Congress 2011, Las Vegas, NV, April 14-16, 2011.

Pekelnicky, R. and Poland, C. (2012). "ASCE 41-13: Seismic Evaluation and Retrofit Rehabilitation of Existing Buildings”. Proc.: 2012 SEAOC-SEANM Convention, Santa Fe, NM, Sept. 12-15, 2012.

Richards, P., Taichiro, O., Engelhardt, M., and Uang, C.-M. (2007). "Impact of Recent Research Findings on Eccentrically Braced Frame Design”. Engineering Journal, AISC, $1^{\text {st }}$ Quarter, pp. 41-53.

Richards, P. and Thompson, B (2009). "Estimating Inelastic Drifts and Link Rotation Demands in EBFs". Engineering Journal, AISC, $3^{\text {rd }}$ Quarter, pp. 123-135.

SEAONC (2010) "Perspectives on ASCE 41 for Seismic Rehabilitation of Building - Survey by the Structural Engineers Association of Northern California”. Structures Magazine, Oct. 2010.

Toranzo-Dianderas, L.A. (2009). "Evaluation of the ASCE 41 Linear Elastic Procedure for Seismic Retrofit of Existing Structures: Pros and Cons of the Method.” Proc.: ATC \& SEI 2009 Conference on Improving the Seismic Performance of Existing Buildings and Other Structures, San Francisco, CA Dec. 9-11, 2009.

Troup, E. (1999). "Effective Contract and Shop Drawings for Structural Steel”. Proc.: North American Steel Construction Conference, Toronto, Ontario, Canada, May 19-21, 1999.

White, D.W. and Hajjar, J.F. (1997). "Design of Steel Frames without Consideration of Effective Length". Engineering Structures, Vol. 19(10), pp. 797-810. 